

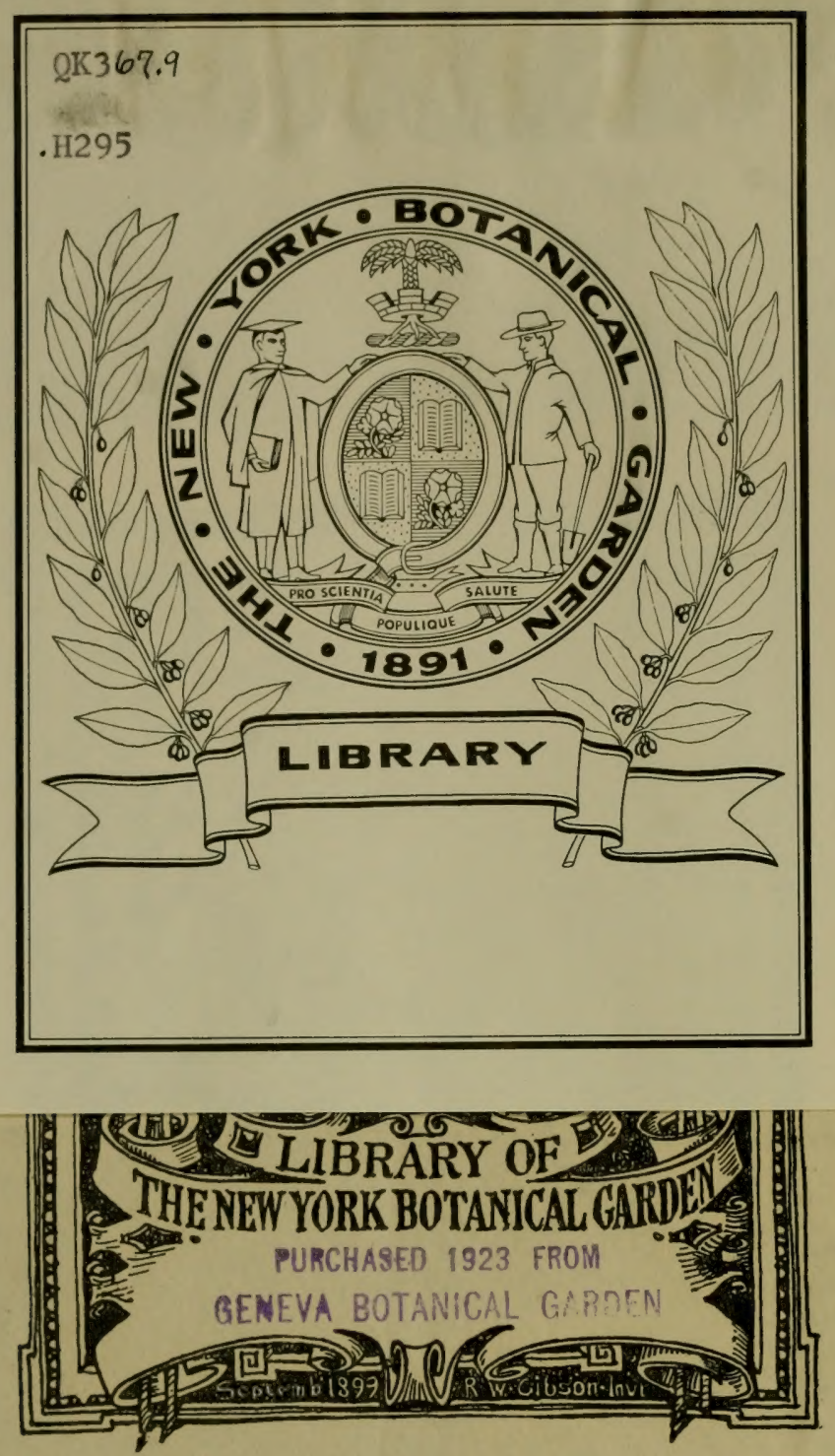


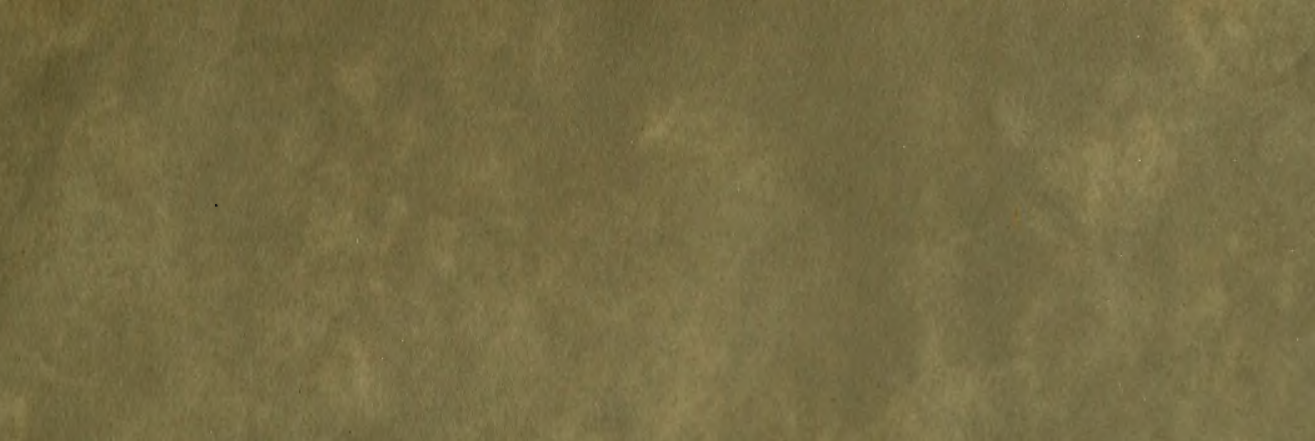

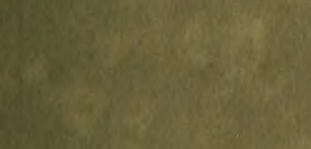

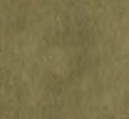

are

ats.

19.0.

tons

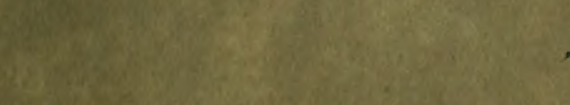

conger

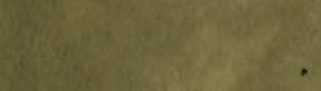

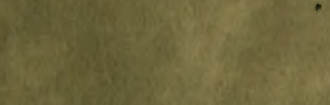

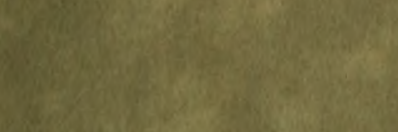

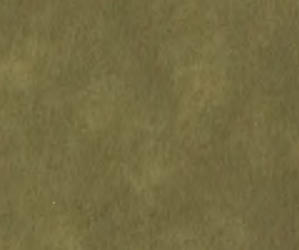

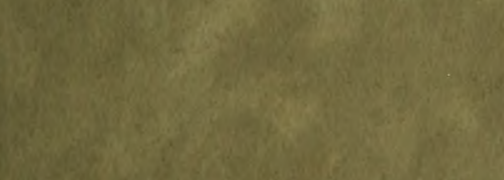

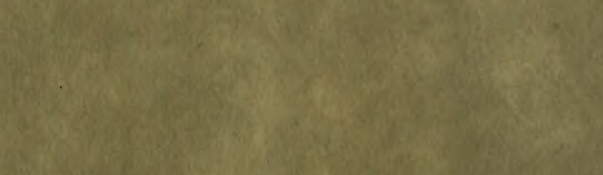

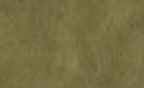

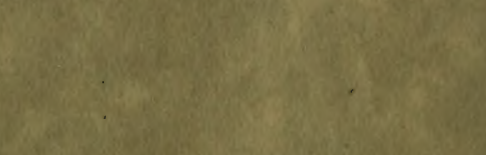

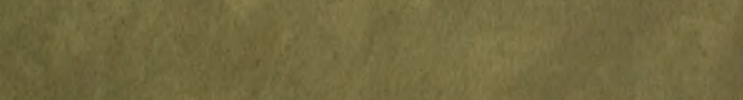

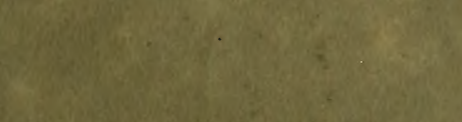

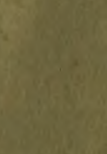

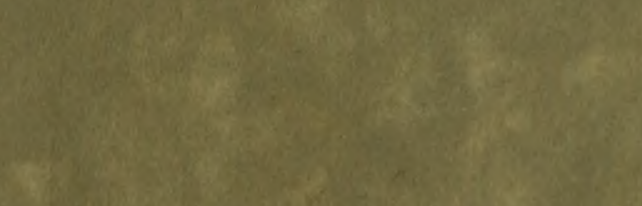

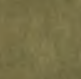

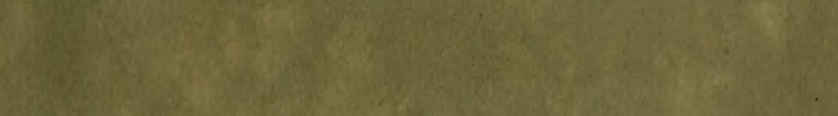

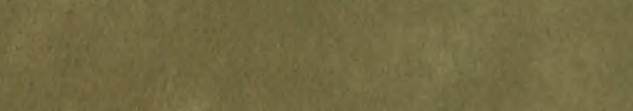

xis

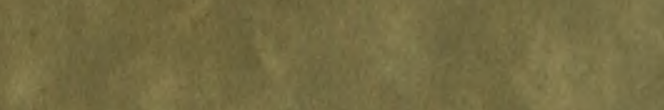

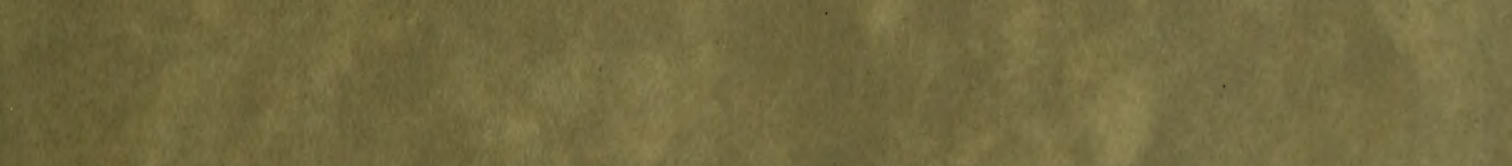

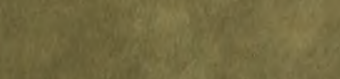

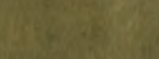
ate

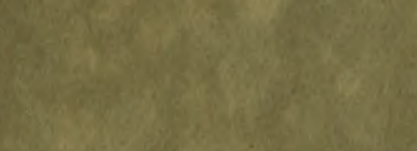

S.

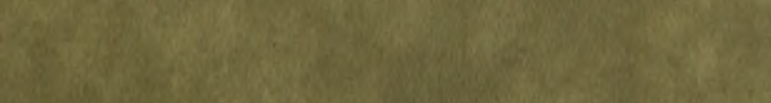

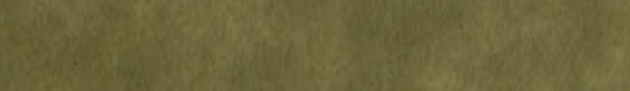

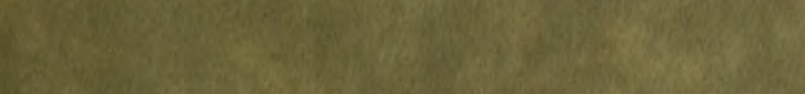

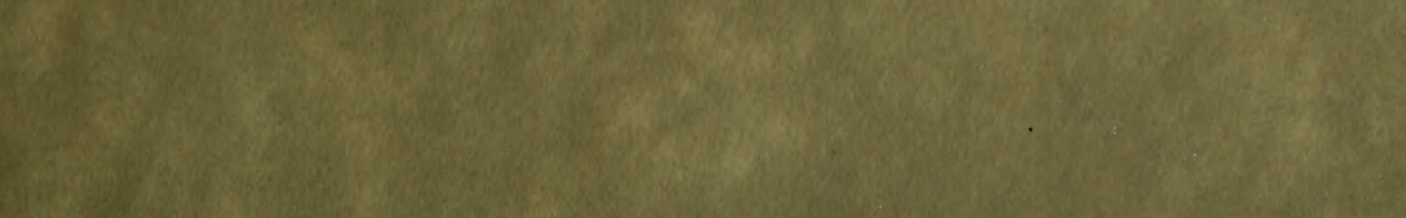
? 
40

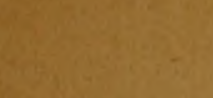




\title{
FLORA MONTANA FORMOSAE
}

\author{
An \\ Enumeration of the Plants found on Mt. Morrison, \\ the Central Chain, and Other Mountainous \\ Regions of Formosa at Altitudes \\ of $3,000-13,000 \mathrm{ft}$.
}

By

B. Hayata, Rigaluhakushi.

REPRTNTED FROM THE JOURNAL OF THE COLLEGE OF SOIBNOB

IMPERIAL UNIVERSITY, TŪKYŌ, JAPAN.

VOL. XXV, ARTIOLE 19.

1908 


\section{$Q K 367.9$ . $\mathrm{H} 295$}

\section{Publishing Committee.}

Prof. J. Sakurai, LL. D., Rigakuhakushi, Director of the College, (ex officio)

Prof. I. ljima, Ph. D., Rigakuhakushi.

Prof. F. Ōmori, Rigakuhakushi.

Prof. S. Watasé, Ph. D., Rigakuhakushi.

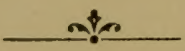

\section{N OTICE.}

In this Journal, each article has a paging of its own; and the position of an article in a volume is indicated by the number placed at its head.

It is hoped that this arrangement, which enables us to print papers independently of one another, will ensure a rapid publication of the material.

All communications relating to this Journal should be addressed to the Director of the College of Science. 


\section{Corrections.}

P. 8, line 12 from bottom, for Then brachycarps, rend Thea brevistyla.

P. 10, line 2 from top, after Vidal, omit the comma.

P. 11, line 10 from bottom, for Fatsia multicarpa, rend Fatsia polycarpa.

P. 13, line 12 from bottom, for Artemisia paucicarpa, read Artemisia oligocarpa.

P. 29, line 9 from bottom, for Fatsia multicarpa, read Fatsia polycarpa.

P. 31, line 8 from bottom, after genera and Conandron, omit the commas.

P. 32, line 8 from bottom, after ages 3 ), omit the ".

P. 32, line 7 from bottom, nfter Conifers $\mathrm{s}^{3}$, add ".

P. 56, line 11 from top, instead of the semicolons, put in commas.

P. 157, line 11 from bottom, for Sysimachia, read Lysimachia. 


\section{$Q K 367.9$ \\ . $H 295$}

\section{Publishing Committee.}

Prof. J. Sakurai, LL. D., Rigakuhakushi, Director of the College, (ex officio)

Prof. I. ljima, Ph. D., Rigakuhakushi.

Prof. F. Ōmori. Riaaleuthalaseli:

All communications relating to this Journal should be addressed to the Director of the College of Science. 


\section{JOURNAL OF THE COLLEGE OF SOIENOE, IMPERIAL UNIVERSITY, TŌKY $\overline{0}$, JAPAN.}

VOL. XXV., ARTICLE 19.

\section{LIERART NEW YORK \\ BOTANICAL. \\ (IARDEN}

\section{Flora Montana Formosæ}

An

Enumeration of the Plants found on Mt. Morrison, the Central Chain, and Other Mountainous

Regions of Formosa at Altitudes of $3,000-13,000 \mathrm{ft}$.

By

B. Hayata, Rigakuhakushi.

Assistant in the Botanical Institute, College of Science,

Imperial University, Tökȳo.

With 41 plates and 16 woodouts.

\section{Introduction.}

In the year 1905, Professor Matsumura and I jointly published an enumeration ${ }^{1)}$ of plants found in Formosa, comprising about two thousand species of flowering plants, ferns and their allies. At that time, our collections, with the exception of a few sets of plants found on Mt. Morrison, did not extend to elevations of any great altitude. It is, therefore, quite proper to regard it as an enumeration of the flora of the low districts.

On the botany of the montane zone, there exists no special

1) Matsumura, J., and Harata, B.-Enumeratio Plantarum Formosanarum, in Journ. Sei. Coll., Imp. Univ. Tōkyō, XXய., 702 pages, with 18 plates, 1906. 
publication, except a few papers ${ }^{1)}$ that have appeared in the Tokyō Botanical Magazine.

In the present work, it is my desire to give some complete information relating to the montane zone of the island. The majority of the collections upon which this work is based, were made by the officers of the Government of Formosa. Some materials were collected by Prof. S. Honda in 1896, and by Mr. R. TorII in 1900, both from Mt. Morrison.

In November 1905, a botanical excursion was carried out for the first time on the above mountain by Messrs. T. Kawakami, S. Nagasawa and G. Nakahara, and numerous specimens were sent to me for determination by Mr. S. Nagasawa. In October 1906 , Messrs. T. Kawakami and U. Mori made another excursion to the same mountain, and collected quite a number of plants. Another ascent of the same peak was made by Mr. G. NaKaHara who had equal success. Other mountainous districts of Taito and the central ranges were botanically explored by Mr. U. Mori in the same year. Some parts of the hill regions of Taihoku, Taichū, Tainan and Kōshūn have been little by little botanized for some years; but the greater part of the island remains as yet unexplored.

All the determinations of the species have been worked out by me; but a few families with which I am not yet thoroughly acquainted are almost entirely omitted in the present paper. The

1) Matsumura, J.--On Coniferæe of Loo-choo and Formosa, in Tōkyō Bot. Mag. XV. pp. 137-141.

Hayata, B.-On the Distribution of the Formosan Conifers, in Tōkyō Bot. Mag. XIX. pp. 43-61.

Haxata, B.-Contributions to the Alpine Flora of Formosa, in Tōkyō Bot. Mag. XX. pp. 14-22, Pl. I.

Hayata, B.-Contributions to the Flora of Mt. Morrison, in Tōkyō Bot. Mag. XX. pp. 52-58, and pp. 73-75. 
work on these families will be specially treated in the near future.

Although the materials here treated are rather limited and further exploration will reveal many new features, the present work will, I hope, throw some light upon the study of the montane flora of the island.

In conclusion, I wish to tender my sincere thanks to Prof. J. Matsumura under whose oversight this work has been carried out. Thanks are also due to Messis. T. Kawakinr, S. NAGasawa and G. Nakahara, who all have generously put their important collections at my disposal. Lastly, I desire to expresis my cordial gratitude to Mr. N. Konishi whose valuable collections have enabled me to make most interesting discoveries on Coniferæ and Quercineæ. A new species of Coniferæ is named Cunninghamia Konishii in recognition of his kindness.

Narch 14th., 1908.

B. Hayata,

Botanical Institule, College of Science, Tokigy. 


\section{I.) Elements of the Flora of the Montane Zone.}

The montane zone treated in the present paper embraces a most extensive area from $3,000 \mathrm{ft}$. up to $13,000 \mathrm{ft}$. above sea level,

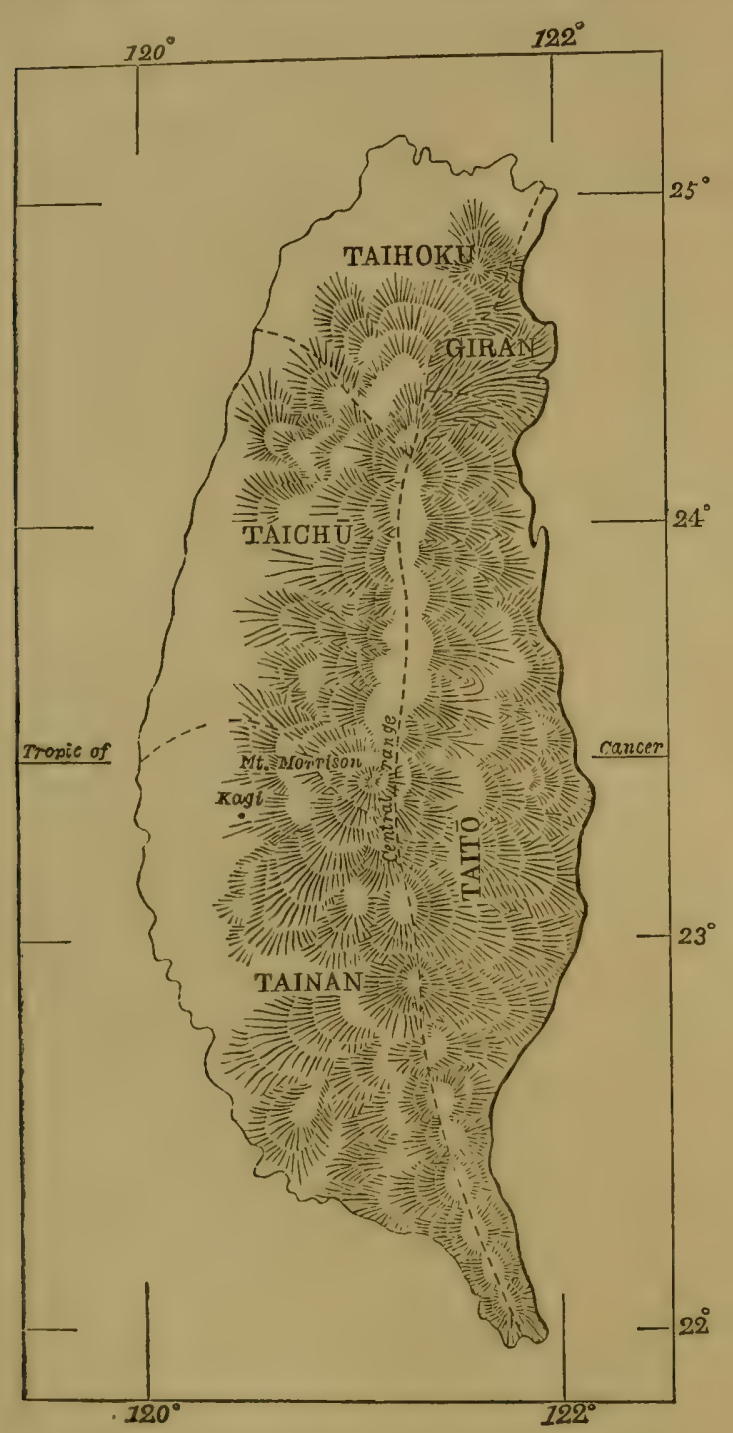

Fig. 1. Slietcl-map of Formosn.

Prefectural boundaries. including in its centre Mt. Morrison, the highest peak of the Japanese Empire. This mountain, lying a little within the tropic of Cancer, attains a height of $13,120 \mathrm{ft}$. It presents various climatal features from tropical through temperate up to those of cold regions, and in winter snow is frequently seen on the summit.

The term flora used in this work includes flowering plants, ferns and their allies. The lower cryptogams are entirely omitted. I have enumerated 392 species belonging to 79 families and 266 genera. Most of the species are northern elements. 
It is a very interesting matter to compare this flora with the floras of the neighbouring countries, and to consider their relations. So far as I am aware, no attempt of this kind has yet been made.

In the year 1905, I published a paper" "On the Distribution of the Formosan Conifers" in which I pointed out that the flora of the Conifers of Formosa has a far closer relation to that of Japam than to that of China, regardless of geographical proximity. In this paper, I have compared all families of flowering plants in the montane zone, and found that the conclusion I had made from the study of Conifers holds good for the general featuies of the flora. I shall refer to this matter later on.

Before we go further in the discussion of this interesting subject, it is necessary to consider the elements of the montane flora. A list of plants, therefore, should properly be given here, with indications as to their distribution.

Regarding the remarks as to distribution given in this list, I have referred to the following literature and herbarium.

1) The Malay peninsula and archipelago:

Blume :-Flora Javæ, 4 vols.

MrQUeL :-Flora van Nederlandsch Indië, 3 vols.

VIdal:--Ravision de Plantas Vasculares Filipinas.

:- Phanerogame Cumingianæ Philippinarum.

Mernitu:-New or Noteworthy Philippine Plants, de, ... in

Publications from the Depurtment of the Interior;

Bureau of Government Laboratories.

'The Philippine Jonrual of Science, I. Suppl. Botany.

Hook. f.:--Flon of British India, 7 vols.

1) Hayata, B., On the Distribntion of the Formosßu Conifers, in Tókyó liot. Mag XIX. pp. 13-61. 
Trimen :-A Hand Book of the Flora of Ceylon, 5 parts.

2) The Himalayas:-

Hoor. f. :--Flora of British India, 7 vols.

3) Central and southern China (including Tibet) :-

Diels :-Die Flora von Central-Cbina, in Engl. Bot. Jahrb. XXIX., pp. 169-657.

" :-Beiträge zur Flora des Tsin ling shan und andere

Zusätze zur Flora 'von Central-China, in Beiblatt zu den Bot. Jahrb. XXXVI. pp. 1-138.

Frá́chet :- Plantæe Davidianæe ex Sinarum Imperio.

Forbes and HFarsey :- Index Floræ Sinensis, 3 vols.

4) Northern China (including Korea, Manchuria, Amurland, and Saghalien :-

Forbes and Hemsley :-Index Floræ Sinensis, 3 vols.

Maxinowicz :-Primitire Flore Amurensis.

Schmidt :-Reisen im. Amur-lande und anf der Insel Sachalin.

Komarov :-Flora Manshurie, 2 rols.

PaLibin :-Conspectns Floræ Koreæ.

NAKAI:--Polygonaceæ Koreanæ, in Journ. Sci. Coll. XXIII. Art. 11.

5) Japan :-

Franche't et Savatier :-Enumeratio Plantarum Japonicarum, 2 rols.

Herbarium of the Rotanical Institute, College of Science, Imperial University, Tōkyō.

Itro et matsumura :- Tentamen Florae Lutchuensis, in Jomrn.

Sci. Coll. XII. pp. 263-541.

Matsumura :-Index Plantarum Japonicarum, I. et II.-1.

" :-Enumeration of selected scientific Names of both native and foreign Plants.

Makino:-Observations on the Flora of Japan, 6 Fascicles, (1901-’06). 
LIST OF THE ELEMENTS OF THE MIONTANE FLORA.

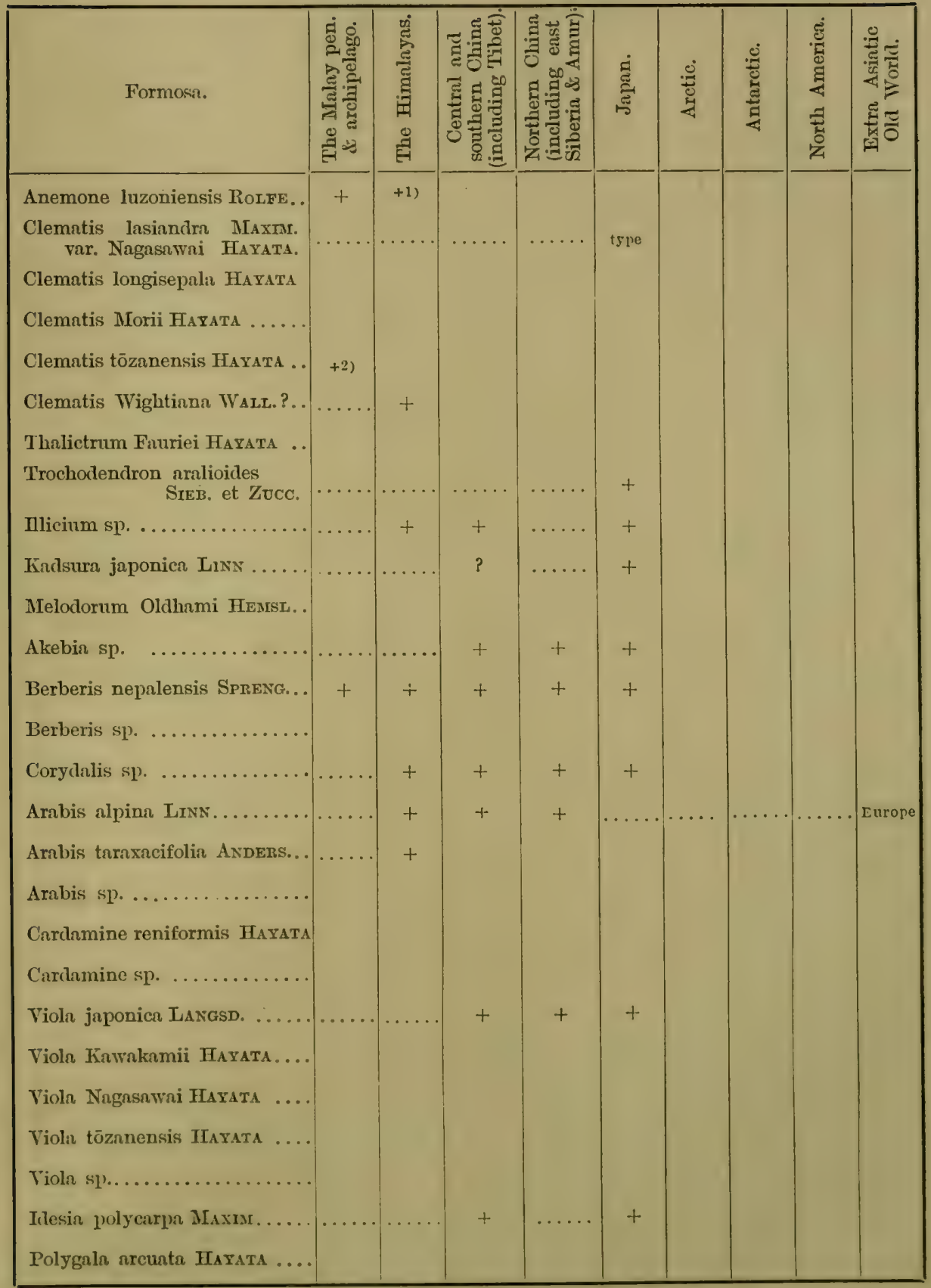

1) Anemone vitifolin Hasr. 2) Clemntis smilacifolia WaLx. 


\begin{tabular}{|c|c|c|c|c|c|c|c|c|c|}
\hline Formos & 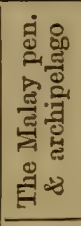 & 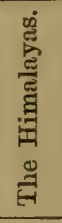 & 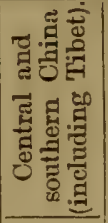 & 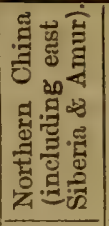 & 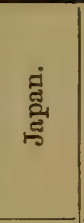 & 胥 & 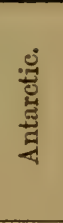 & 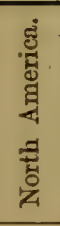 & 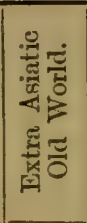 \\
\hline Polygala japonica Hourт. .. & & + & + & + & + & & & & Austr. \\
\hline Dinnthus superbus LINN. . & & .... & + & + & + & & & & Europe \\
\hline Dianthus sp. .......... & & & & & & & & & \\
\hline Silene Fortunei Vrs. .... & & & + & + & & & & & \\
\hline Cucubalus baceifer Lins. .... & & + & + & + & + & & & + & Europe \\
\hline $\begin{array}{l}\text { Cernstium morrisonense } \\
\text { H.YATA. }\end{array}$ & & & & & & & & & \\
\hline Cerastium pilosum LEDEB. ... & & & $\ldots \ldots$ & + & & & & & \\
\hline Stellaria stellato-pilosa HAYATA $_{\text {A }}$ & & & & & & & & & \\
\hline $\begin{array}{l}\text { Hypericum attenuatum } \\
\qquad \text { CHorsY. }\end{array}$ & & & + & + & & & & & \\
\hline Ternstromia japonica ThunB. & + & $\cdots$ & + & & + & & & & \\
\hline Eurya japonica THuns. ..... & + & $?$ & + & & + & & & & \\
\hline Eurya strigillosa НАХATA .... & & & & & & & & & \\
\hline Actinidia callosa Lunde. ..... & & + & + & .... & + & & & & \\
\hline $\begin{array}{l}\text { Strchyurus præcox Sres. } \\
\text { et ZucC. }\end{array}$ & & & + & & + & & & & \\
\hline Schim Noronhæ REINw..... & + & & & & $\begin{array}{l}\text { Loo- } \\
\text { clioo. }\end{array}$ & & & & \\
\hline Thea brachycarpa HAYATA .. & & & & & & & & & \\
\hline Ther caudat $\left(W_{\Delta L L}\right.$ ) ....... & & + & + & & & & & & \\
\hline Triumfetta pilosa Rотн. ..... & + & & + & & & & & & Africa \\
\hline Elæocarpus decipiens HeMrsL. & & & + & & $\begin{array}{l}\text { Loo- } \\
\text { clioo. }\end{array}$ & & & & \\
\hline $\begin{array}{r}\text { Geranium Robertianum } \\
\text { LTNN. }\end{array}$ & & & + & + & + & & & & + \\
\hline Geranium uniflorum FAYATA. & & & & & & & & & \\
\hline $\begin{array}{l}\text { Oxnlis Griffithii EDGEW. } \\
\text { et HK. f. } \\
\text { Impatiens uniflora HAXATA.. }\end{array}$ & & + & + & & & & & & \\
\hline $\begin{array}{l}\text { Boenninghausenia albiflora } \\
\text { REICHB. }\end{array}$ & + & + & + & & + & & & & \\
\hline Evodia meliæfolia BENTH.... & & + & + & $\ldots \ldots$ & Soutl & & & & \\
\hline Skimmia japonica THuxs.... & + & + & + & $\cdots \cdots$ & + & & & & \\
\hline Murrnya exoticr Lins. .... & + & & + & & & & & & \\
\hline
\end{tabular}




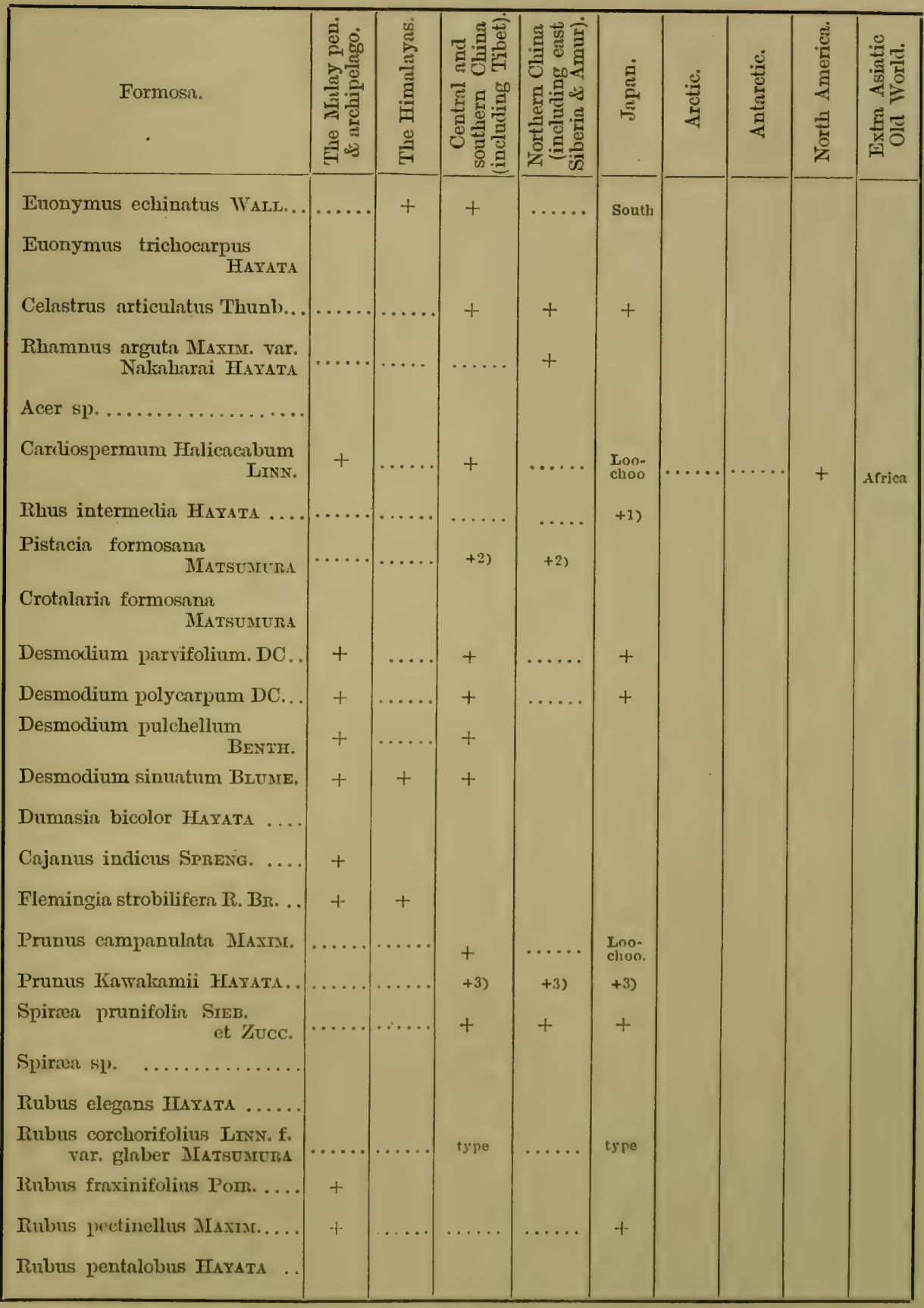

1) Thus Toxicolendron LINs, var. raliens ENot.

3) Prunus japonien 'I'rux».

2) Pistncin chinensis 13UxaE. 


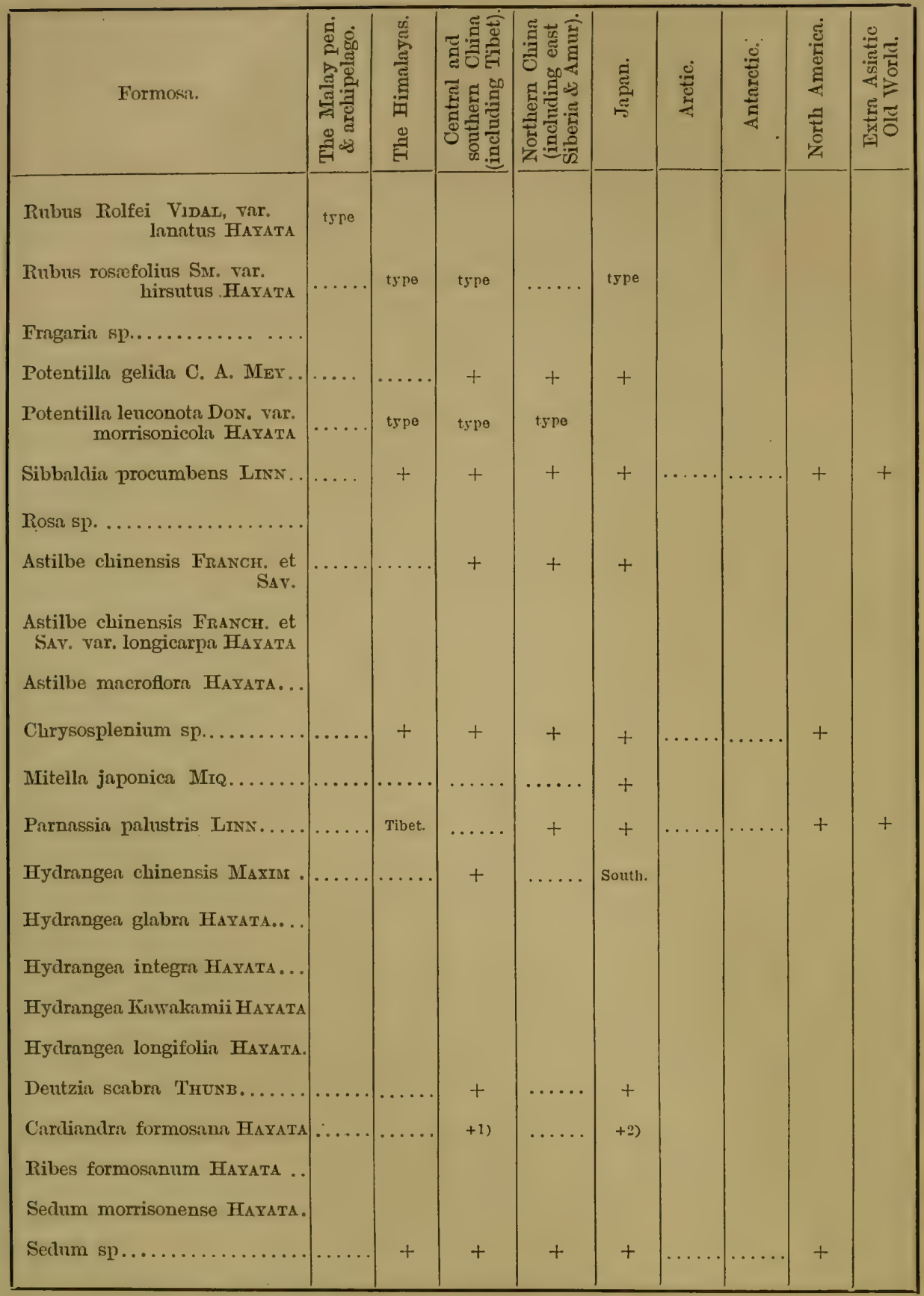

1) Cardiandra sinensis Hemsiex

2) Cardinndra alternifolin Steb, et Zucc. 


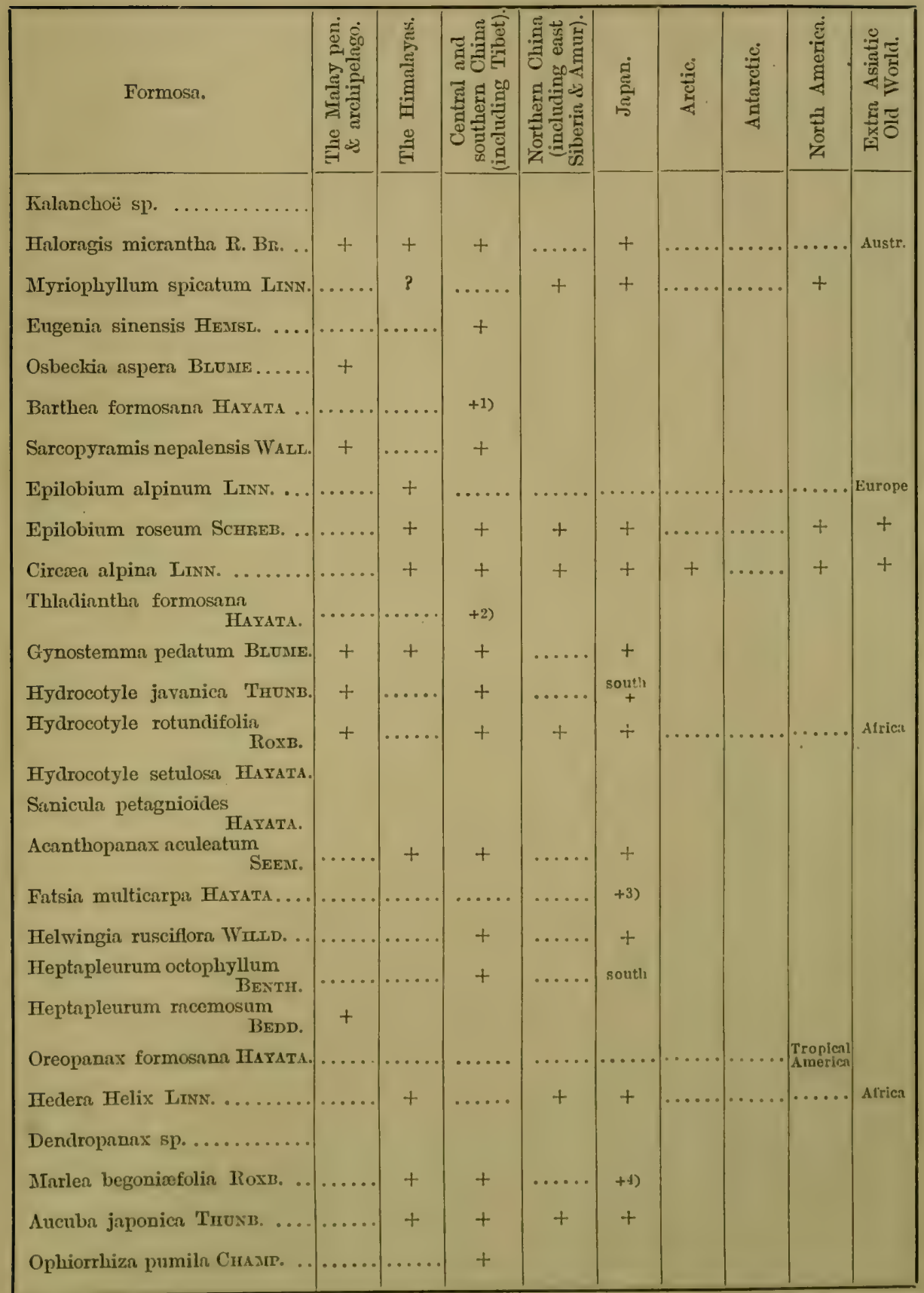

1) Barthen chinensis Hoor.

3) Fatsir japonica Decri. et Prascr.
2) 'Thlntinnthe nucliflorn IIEмsi.

4) Mrrten platonifolin S. et Z. 


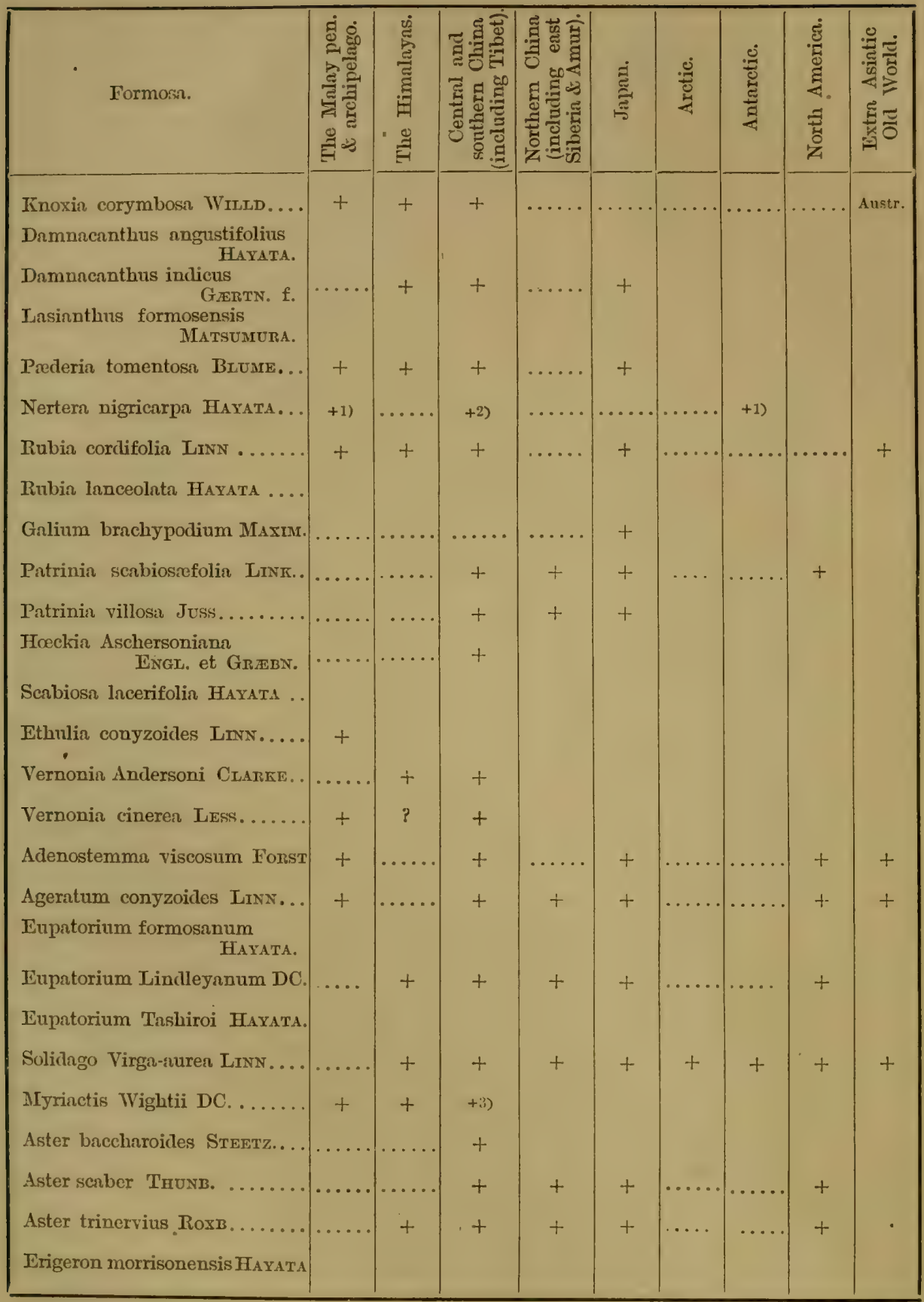

1) Nertera depressa Banks et Sou.

2) Nertern sinensis Hexsu.

3) Myrirctis nepalensis LEss. 


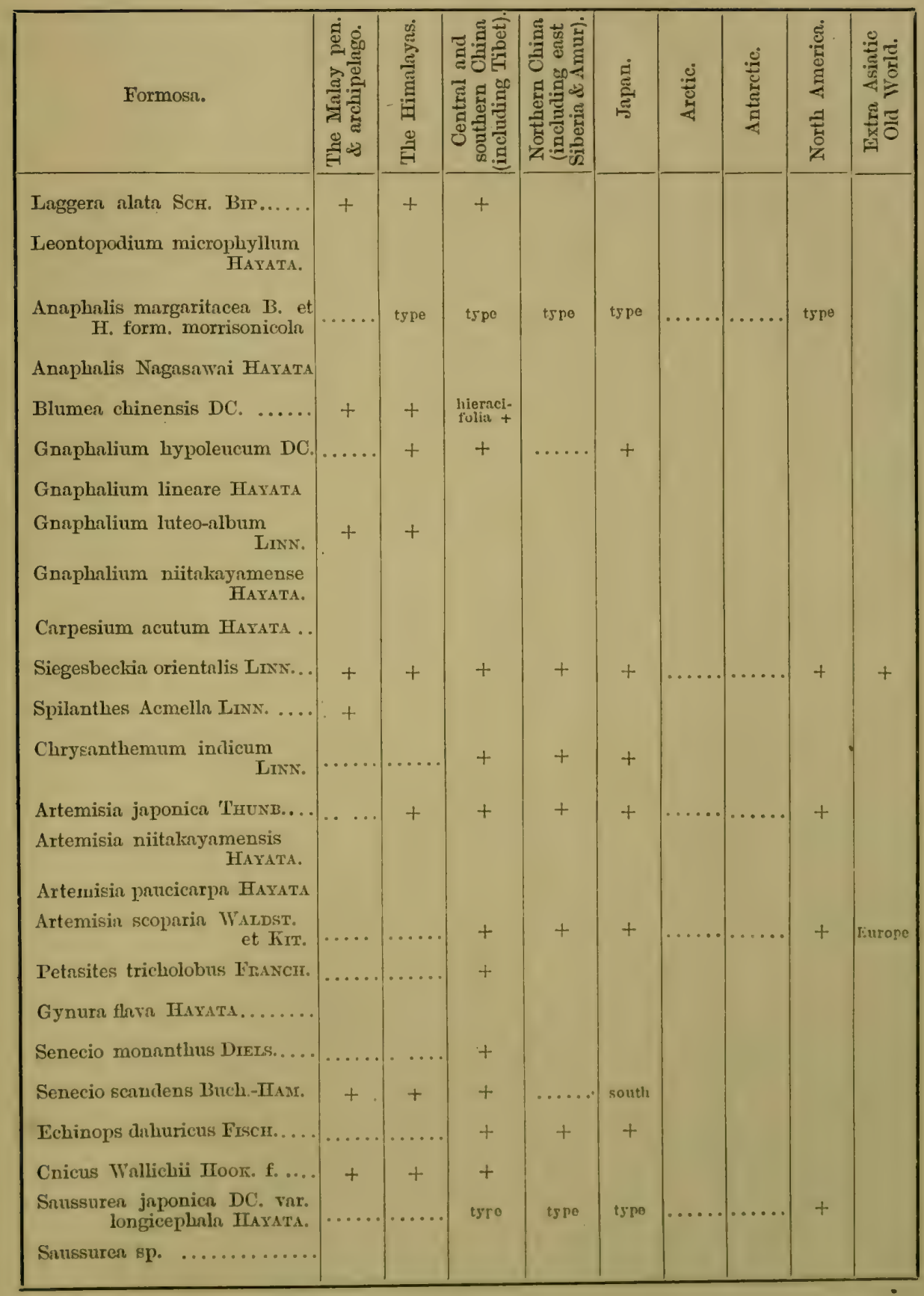




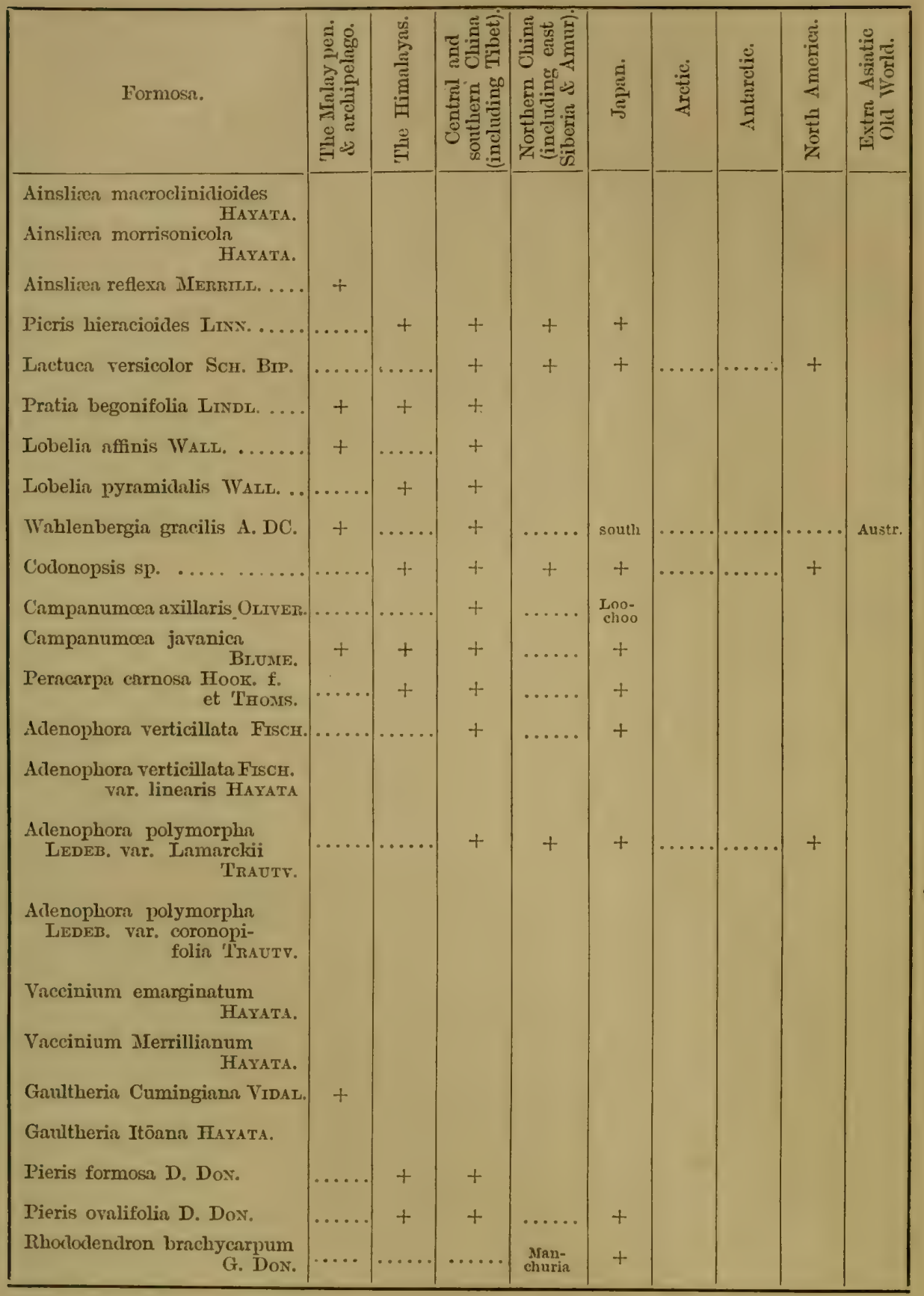




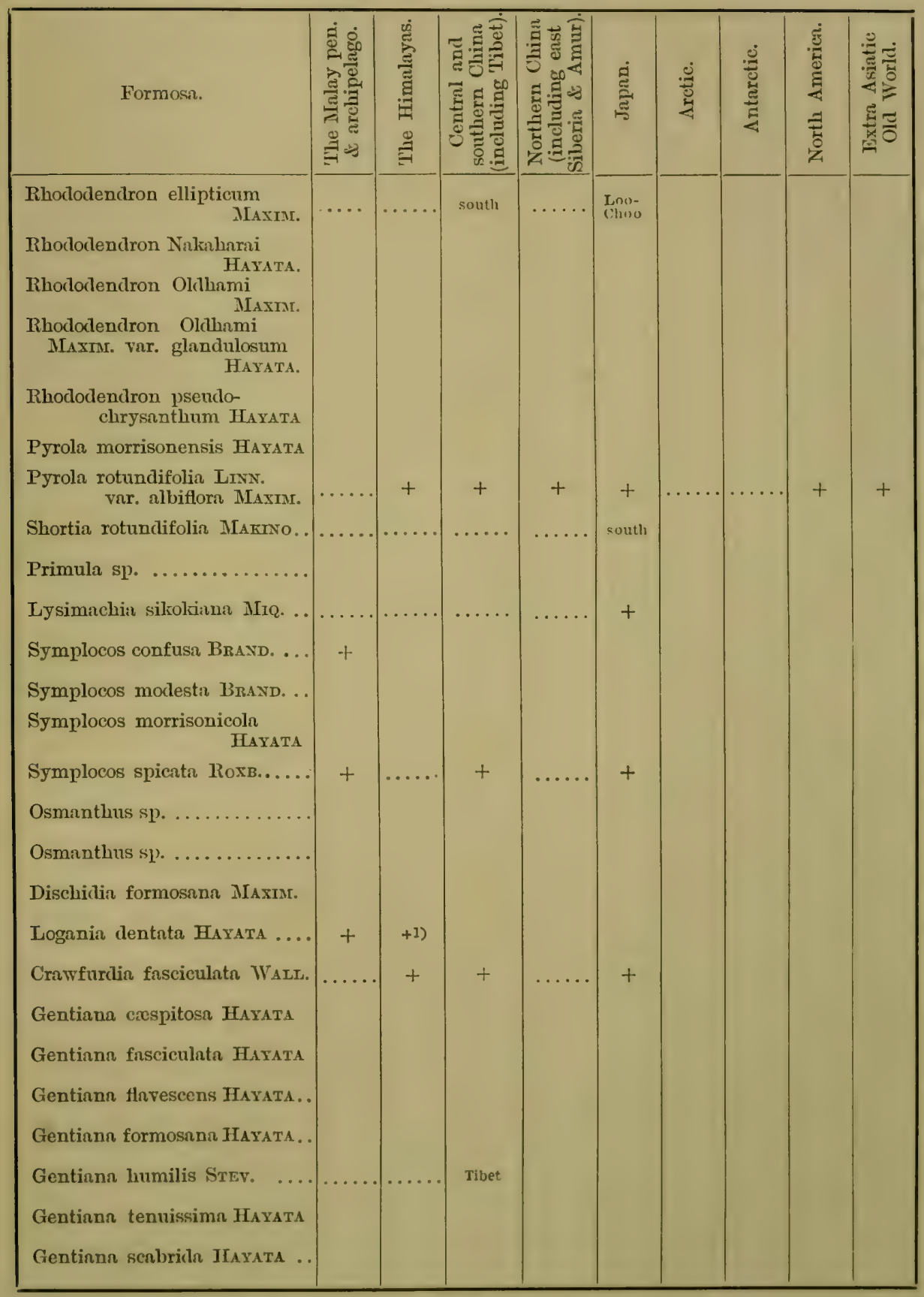

1) Hemiphrngmn heterophyllum WaLLicr. 


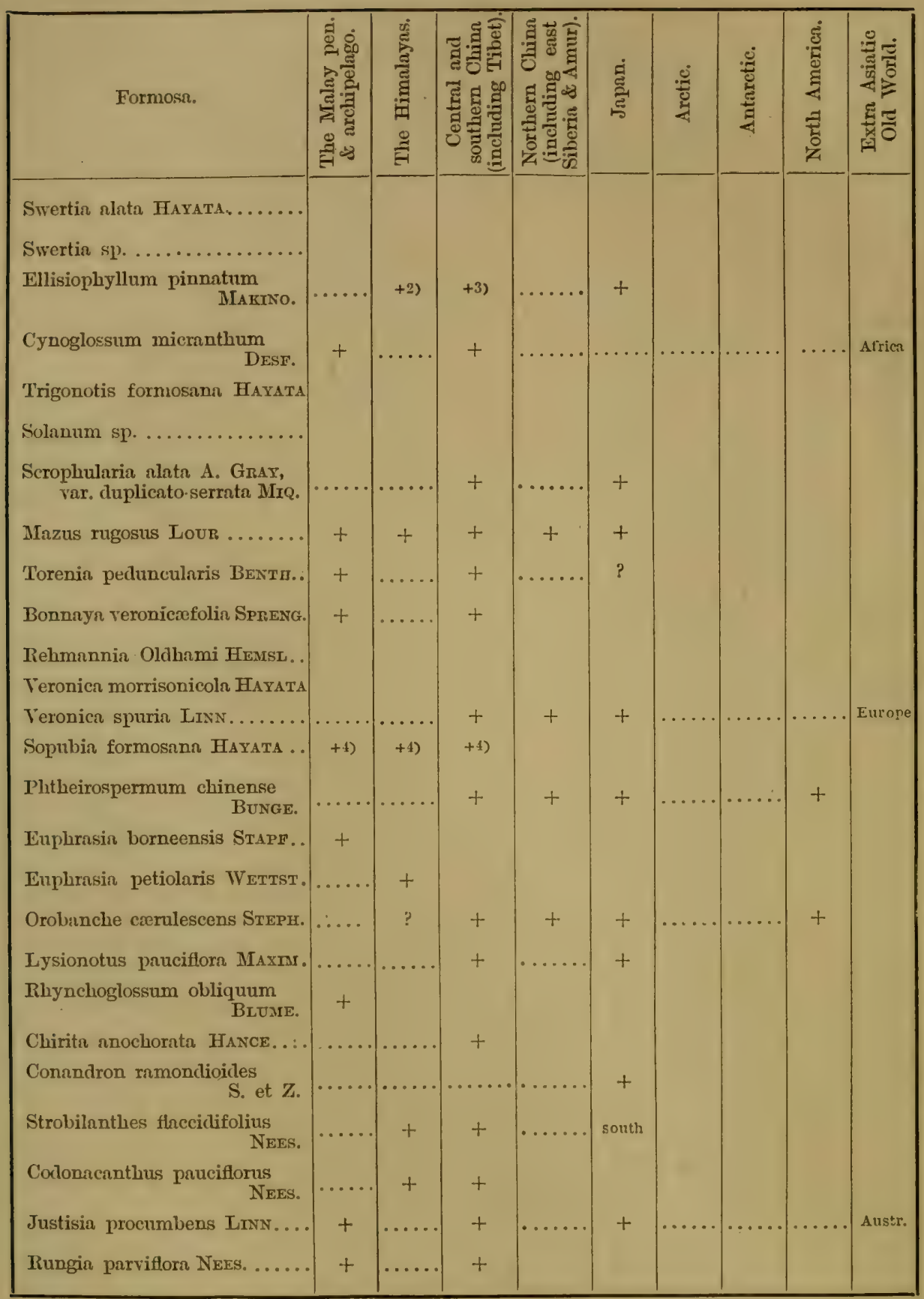

1) Ellisiophyllum pinmatum MaKINo.=Ellisiophyllum reptans Mastar.

=2) Moseleya pinnata HeMsL. =3) Hornemannia pinnata Benth. = Sibthorpia pinnata Besth. 4) Sopubia trifida Buch.-Hax. 


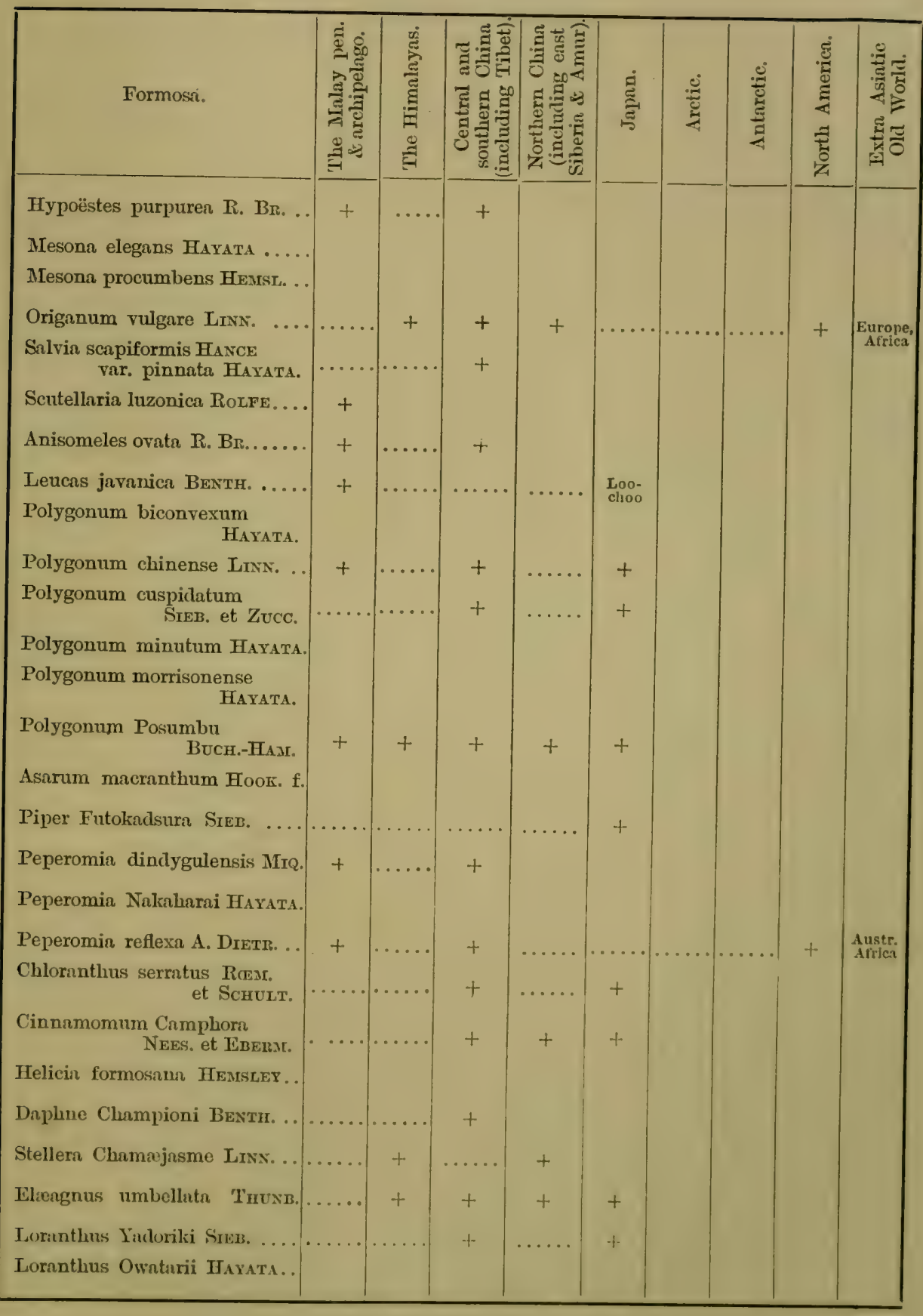




\begin{tabular}{|c|c|c|c|c|c|c|c|c|c|}
\hline Formosn. & 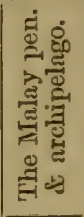 & 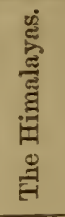 & 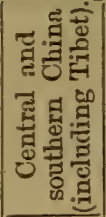 & 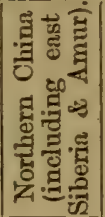 & $\stackrel{\Xi}{\grave{\Xi}}$ & 㞭 & 递 & 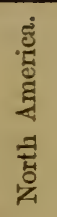 & 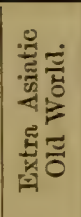 \\
\hline $\begin{array}{l}\text { Viscum articulatum BURa.... } \\
\text { Viscum orientale WILLD. var. } \\
\text { multinerve HAYATA. }\end{array}$ & $\begin{array}{l}+ \\
+\end{array}$ & + & + & & south & & & & \\
\hline $\begin{array}{l}\text { Balanophora parvior НАхАтA. } \\
\text { Balanophora spicata НАгАтA. }\end{array}$ & & & & & & & & & \\
\hline $\begin{array}{l}\text { Buxus sempervirens Liss.... } \\
\text { Glochidion formosanum }\end{array}$ & . & + & + & & + & & & & Europe \\
\hline $\begin{array}{r}\text { HAYATa. } \\
\text { Glochidion zeylanicum A. JUss. }\end{array}$ & + & & & & & & & & \\
\hline Aleurites cordata Steud .... & & & + & & + & & & & \\
\hline $\begin{array}{r}\text { Mercurinlis lasiocarpa SiEb. } \\
\text { et ZucC. }\end{array}$ & & & + & + & + & & & & \\
\hline IInllotus cochinchinensis Lour. & + & & + & & & & & & \\
\hline Fatoua pilosa GAUd. ......... & + & . & + & & + & & & & \\
\hline IIorus alba Lins........... & ‥ & ... & + & + & + & & & & \\
\hline $\begin{array}{r}\text { Urtica Thunbergiana } \begin{array}{r}\text { SIEs et } \\
\text { ZUCC. }\end{array}\end{array}$ & & & + & & + & & & & \\
\hline Girardinia heterophylla DeCNe & + & + & & & & & & & \\
\hline Pilea stipulosa MrQ......... & + & + & + & & $\begin{array}{l}\text { I.oo- } \\
\text { chooo }\end{array}$ & & & & \\
\hline Pilen Wattersii Haxce....... & & & & & & & & & \\
\hline Lecanthus Wightii WEDD. .... & + & + & & & & & & & \\
\hline Elatostem 2 minutum НАгАтА. & & & & & & & & & \\
\hline $\begin{array}{l}\text { Elatostema sessile FonST. } \\
\text { var. cuspidatum WEDD. }\end{array}$ & + & & + & & + & & & & \\
\hline Procris læevigatr BLUME. ..... & + & & + & & & & & & \\
\hline Juglans sp.................... & & + & + & + & + & & & + & \\
\hline $\begin{array}{l}\text { Engelhardtia spicata BLuMre. } \\
\text { var. formosana HAYATA. }\end{array}$ & + & + & & & & & & & \\
\hline $\begin{array}{l}\text { Alnus maritima NutT. var. } \\
\text { formosana BurkILL. }\end{array}$ & & & & + & + & & & + & \\
\hline
\end{tabular}




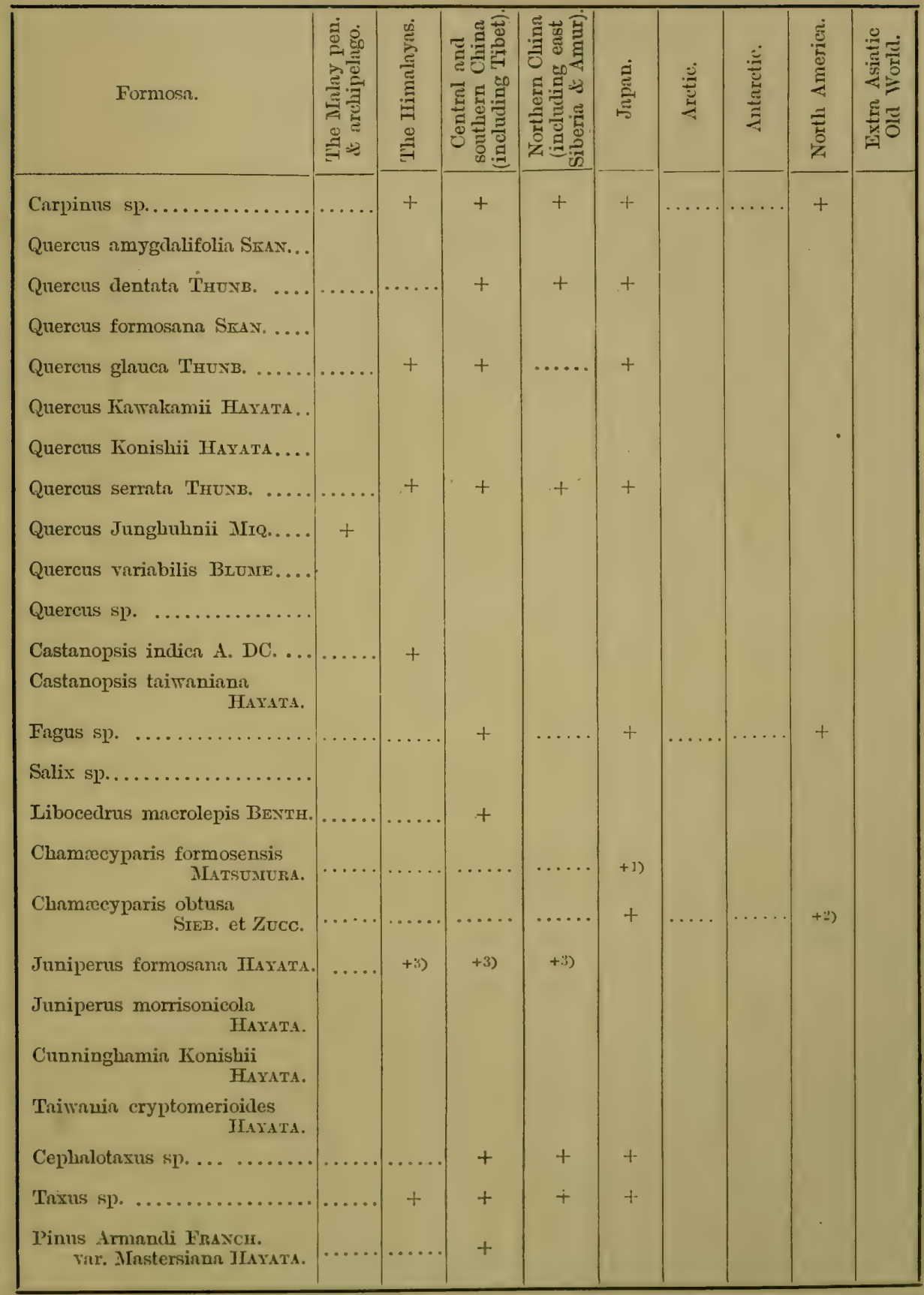

1) Chamrecyparis pisifera $S$, et $Z$.

3) Juniperus communis Lns. 


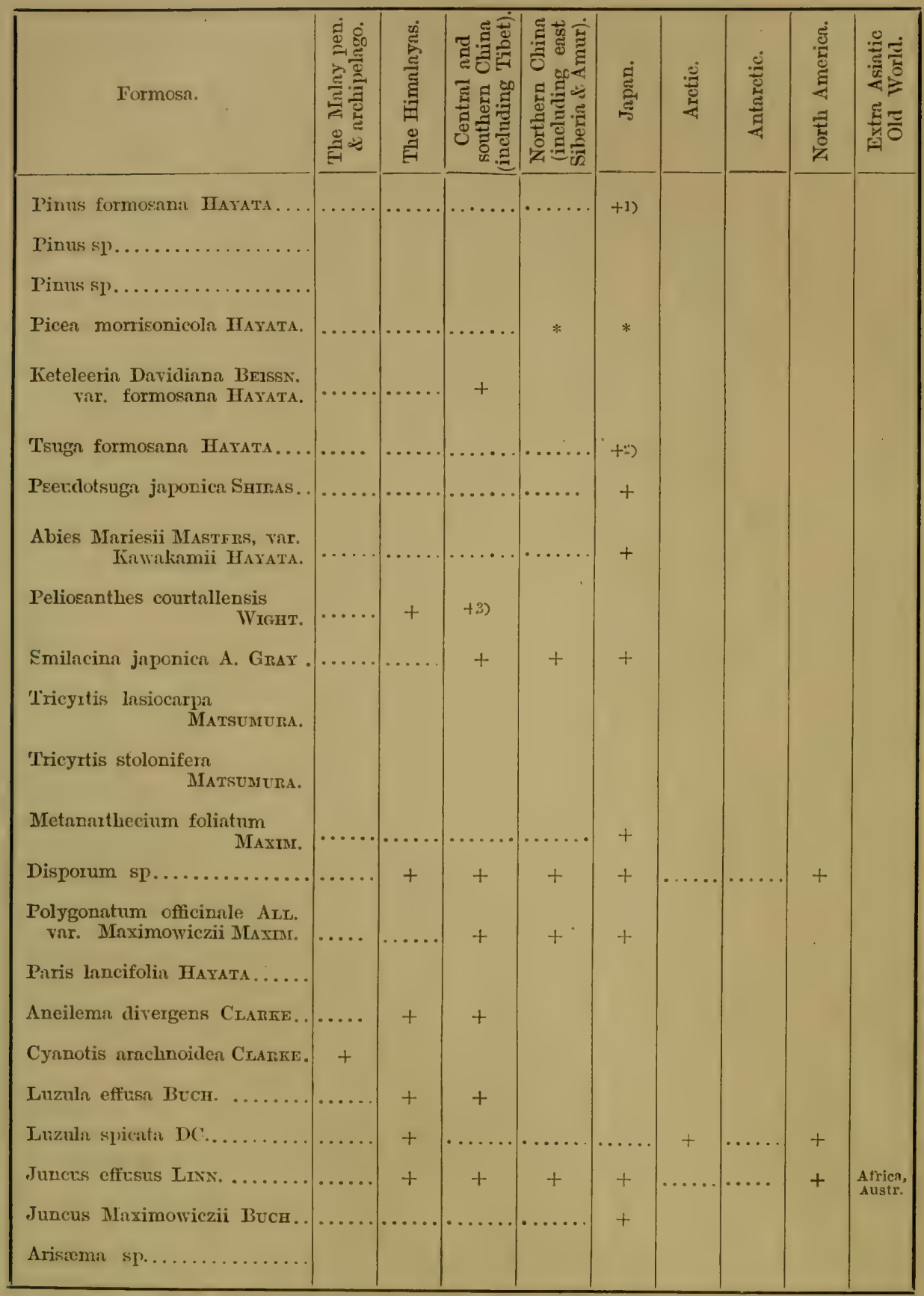

1) Pinus parvittorn $S$. et $Z$.

3) Peliosanthes Delarayi Fraxch.

2) Tsuga diversifolia Maxis.

* Picea Glehni Fr. Scmur. 


\begin{tabular}{|c|c|c|c|c|c|c|c|c|c|}
\hline Formosa. & 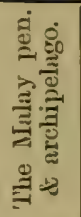 & 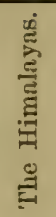 & 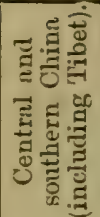 & 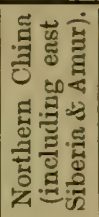 & 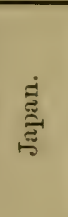 & $\stackrel{3}{3}$ & 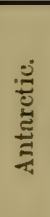 & 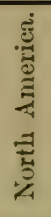 & 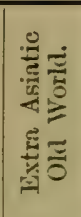 \\
\hline Alocasia sp. ..... & & & & & & & & & \\
\hline $\begin{array}{l}\text { Bulbostylis capillaris Kunth, } \\
\text { var. trifila CuarKe. }\end{array}$ & & + & + & & + & & & & \\
\hline Scirpus morrisonensis HaYata & & & & & & & & & \\
\hline Carex sp. ....... & & & & & & & & & \\
\hline Isachne Clarkei Пfоок. f. .... & & + & & & & & & & \\
\hline Panicum montanum RoxB. & + & & + & & & & & & \\
\hline $\begin{array}{l}\text { Oplismenus undulatifolius } \\
\text { BEAUv. var. imbecillis } \mathrm{H}_{A C K} \text {. }\end{array}$ & + & + & + & $+?$ & + & & & & \\
\hline Arundinella setosa Triv. .... & + & & + & & & & & & \\
\hline $\begin{array}{l}\text { Misennthus sinensis } A \text { NDERss. } \\
\text { var. formosanus HACK. }\end{array}$ & type & & type & type & tspe & & & & \\
\hline $\begin{array}{c}\text { Saccharum Narenga Buch.- } \\
\text { Hair. }\end{array}$ & & + & + & & & & & & \\
\hline $\begin{array}{l}\text { Sporliopogon Kawakamii } \\
\text { НАуATA }\end{array}$ & & & & & & & & & \\
\hline $\begin{array}{l}\text { Spodiopogon tainanensis } \\
\text { HAYATA }\end{array}$ & & & & & & & & & \\
\hline $\begin{array}{l}\text { Pollinir ciliatn 'Tress. var. } \\
\text { Wallichinn HAcs. }\end{array}$ & + & & + & & & & & & \\
\hline $\begin{array}{l}\text { Cymbopogon Nardus RENDLE } \\
\text { subsp. marginatus var. } \\
\text { Gœringii REXDLE. }\end{array}$ & + & & + & & + & & & & \\
\hline Agrostis Clarkei Ноок. f. .... & 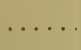 & + & & & & & & & \\
\hline $\begin{array}{l}\text { Calnmagrostis arundinacen } \\
\text { Rотн. }\end{array}$ & & + & & & + & & & & Europe \\
\hline $\begin{array}{l}\text { Calnmagrostis arundinacen } \\
\text { RotH. var. nipponica H.ACK. }\end{array}$ & + & & & & + & & & & \\
\hline Deschampsia crespitosa BEauv. & & + & + & + & + & + & + & & \\
\hline Desehampsia flexuosia Tris... & & & & & + & & & & Europe \\
\hline 'I'risetum subspicatum BEAUv. & & + & + & + & + & + & + & & \\
\hline Arundlo formosana JАСK. . & & & & & & & & & \\
\hline $\begin{array}{l}\text { Brachypodium Kawakamii } \\
\text { HAYATA }\end{array}$ & & & & & & & & & \\
\hline
\end{tabular}




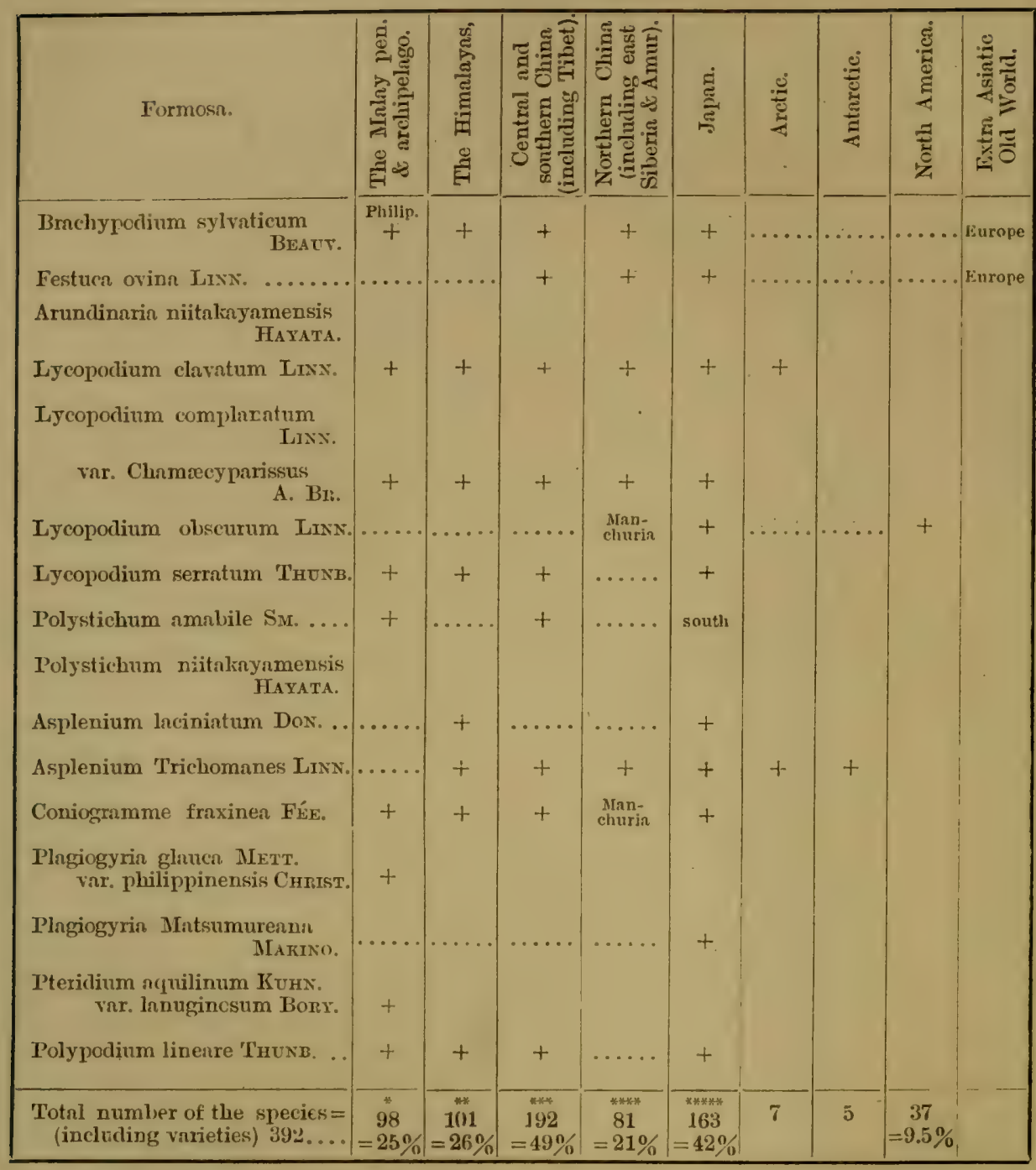

* Of these species, three are here represented by allied species, Sopubia trifida, Nertera depressa and Clematis smilacifolia.

** One of these species, is represented by an allied species, Sopribia trifula.

*** Of these species, ten are here represented by allied species, Thladiantha nudiflor $\alpha, M y$ riactis nepalensis, Blumea hieracifolia, Peliosanthes Delarayi, Cephalotaxus Fortunei, Burthea clinensis, Nertera sinensis, Cardiandra sinensis, Prunus juponica and Pistacia chinensis.

***: Of these species, two are here represented by allied species, Pistaciu chinensis and Prunus japomica.

****** Of these species, nine are here represented by allied species, Rhus toxicodendron var. radicans, Cardiandra alternifolia, Fatsia japonica, Marlea platanifolir, Chamacyparis pisifera, Cephalotaaus pedunculata, Taxus cuspidatu, Pinus paviflora and Tsuga dicersifolia. 
The following points of importance are taken from the above list.
a) Arctic Elements.

Arctic elements are represented by the following species:Asplenium Trichomanes Lins.

Circcea alpina LINN.

Solidago Virga-aurea LINs.

Leontopodium R. Br.

Luzula spicata DC.

Deschampsia coespitosa BEAUv.

Trisetum subspicatum BEAUv.

b) Antarctic Elements.

Antarctic elements are represented by the following species :-

Asplenium Trichomanes LINN.

Nertera Banks.

Deschampsia caespitosa Beaur.
Trisetum sulspicatum BeaUT. Solidayo Virgct-aurea Lnx.

\section{c) Alpine Elements.}

Alpine elements, by which I mean those plants that are found at elevations above $10,000 \mathrm{ft}$. in Asia or Europe, are represented by the following species :-

Aralis alpina LINN.

Aralis taraxacifolia ANDEns.

Potentilla gelida C. A. MEY.

Potentilla Teuconota DoN.

Sibbaldia procumbens LNx.

Epilobium alpinum LINs.

Circcea alpina Lisx.

\section{Leontopodium R. B13.}

Peracarpa carnosa Ноок. f. et 'Tномs. Rhododendron brachycarpem G. Dox.

Gentiana humilis STEr.

Origanum vulgare LINx.
Stellera Chamojasme Lisx.

Juniperus LrNx.

Luzula effusa Bucr.

Luzule spicata DC.

Agrostis Clarkci Hook. f.

Deschampsia caspitosa Benur.

Deschampsia flexuosa 'Inn.

Trisetum subspicatum BEAUr.

Brachypodium sylvaticum BEAvr.

Fesinca ovina LINN.

Lycopodium olscurum Lisx.

Asplenium Trichomanes LINs. 


\section{d) Tropical American Elements.}

These elements are very few in number, but among them we have Orcopanax, a genus of Araliacex. The occurrence of this genus, which is all but peculiar to the flora of Tropical America, is exceptionally remarkable. It is perhaps the most anomalous case we meet with in the Formosan flora. This genus is here represented by a large tree, Oreopanax formosana Hayata. As far as I am aware, we have had no representative of this American genus in any other region on the globe. The tree is found in the mountainous districts on the north-western side of the central ranges, and also on Mt. Morrison, both localities having elevations varying from $6,000-8,000 \mathrm{ft}$. As the plots are quite inaccessible, it does not seem probable that the tree was planted there by human agency.

\section{e) Malay Elements.}

These elements are rather less numerous. There are in all 98 , or $25 \%$ of the whole number mentioned in the list. Among them, we have 45 tropical elements or $12 \%$ of the whole number. None of these species ascends to an altitude higher than 3,000 ft. An exceptional case is that of the tropical genus, Hcptapleurum, which is found at an elevation of about $7,000 \mathrm{ft}$.

\section{f) North American Elements.}

These elements are comparatively well represented in this flora. As has been seen in the foregoing list, we have as many as 37 species, or $9.5 \%$ of the whole number. Of these American representatives, almost no species, except a very few cosmopolitans, extends any farther south than Formosa, not even to 
Luzon. It is, therefore, certain that the flora under consideration has a far closer affinity to that of North America than the flora of the Philippines has to that of the new world.

It is very interesting to notice that the montane flora has some genera which are found in North America, North China, and Japan, but nowhere else. They are :-

Mlitella (Formosa, Japan, North China, Siberia, and North America). Chamcecyparis (Formosa, Japan, and North America).

Pseudotsuga (Formosa, Japan, and North America).

\section{g) Himalayan Elements.}

These elements are here represented by as many. as 101 species, or $26 \%$ of the whole number. Nost of them are found in Japan and also in China.

Those plants which are confined to Formosa, the Himalayas, and China are as follows :-

Arabis alpina LINN.

Thea caudata (W ALL.)

Oxalis Griffithii EDGEW.

Desmodium sinuatum BuUne.

Vernonia Andersoni Clanke.

Pieris formosa D. Dox.

Sopubia

Laggera alata $\mathrm{ScH}$. Brr.
Codonacanthus pauciflorus NEES. Origanum vulgare (also in Europe, Ameriea, and Africa) Stellerce Chamajasme LINv. Juniperus communis LINs. (represented by $J$. formosana HaYita. Peliosanthes courtallensis Wight. Aneilema divergens Cramke. Luzule effusa Bucr.

Those plants, which are found in the Himalayas and Formosa, but nowhere else, are :-

Arabis taraxacifolia ANDEnS.

Epilobium alpinum Lisv. (also in Europe).

Leconthus Wightii WEDD (also in the Malay Archip.).
Castanopsis indice A. DC.

Luătle spicate DC. Alyrostis Clarkei Hook, f. Isache Clarkei Hook. f. 
h) Elements of central and southern China (including T'ibet).

This is the class best represented in the flora, comprising as many as 192 species, or $49 \%$ of the whole number. Most of them are also found in Japan.

The plants which are confined to this region and Formosa, are as follows :-

Hoeckia Aschersoniana Exat. et Græbx.

Senecio monanthus DIELS.

Petasites tricholobus Fraxch.

Gentiance humilis STEr.
Salvia scapiformis HAXEE.

Daphene Championi BeNTH.

Libocedrus macrolepis Вехтн.

Pinus Armandi Fraxch.

Keteleeria Daridiana BeLssx.

They are in all 9 species, among which we have 2 genera, Hockia and Keteleeria, which are found in this region and Formosa, but nowhere else.

Here we see that the strong affinity between the two regions is as clearly shown by the plants of peculiar character as by the number of the elements.

\section{i) Fapanese Elements.}

The Japanese elements are, next to the Chinese, best represented in the flora. They comprise in all 163 species or $42 \%$ of the whole number.

The plants, which are known to exist only in Formosa and Japan, are as follows :-

Clematis lasiandra Maxns.

Mitella japonica Miq.

Trochodendron aratioides $\mathrm{S}$. et $\mathrm{Z}$.

Fatsia

Galium brachypodium MAxts.
Lysimachice sikokiance MIQ.

Conandron ramondioides $\mathrm{S}$. et $\mathrm{Z}$.

Tsuga diversifotia MAxnr.

Psoudotsuga japonica Shmasawa.

Abies IMaviesii Masters. 
Chamocyparis pisiferce $\mathrm{S}$. et $\mathrm{Z}$. represented by $C$. formosensis Mitsum.

Chamcecyparis obtusa S. et Z.

Pinus parvifora S. etZ. (represented by $P$. formosana HiYatı.)
Iletanarlhesium foliatum Mixur. Juncus Maximoviczii Fr. et SAr. Plargiogyria Malsumureana MakiNo.

They are in all 16 species, among which we have some genera which are all but peculiar to Japan and Formosa. They are as many as 4 genera :-

$$
\begin{array}{l|l}
\text { Trochodentron } & \text { Conandrom } \\
\text { Fatsia } & \text { Iletanarthesirm. }
\end{array}
$$

The Japanese elements are, on the whole, a little less numerously represented in the Formosan flora than the elements of central and southern China, so far as the figures of the elements are concerned. We see, however, that the number of the plants peculiar to both islands far exceeds the number of those which are confined to the continent and Formosa.

Origanum vulgare Liss., which ranges over high mountains of the northern hemisphere, is wanting in Japan.

Luzula spicata DC., which spreads over the Itimalayas, North America and the arctic regions, has not yet heen found. in Japan.

\section{j) Elcments of nortinem China (induding Mranchuria,} Saghtatien, Amurtand, and east Siberia).

These are the least numerously represented in the flora. They number 81 species or $21 \%$ of the whole.

Those plants, which are only found in North China and the island, are as follows :- 


\section{Cerastium pilosum LEDEB.}

Rilcmmus arguta Maxis. (represented by a variety.)

Here we see that the relation is far less close.

\section{k) Endemic Elements (excepting varieties.)}

Endemic plants are comparatively numerous as is to be expected in an island. There are as many as 99 species, or $25 \%$ of the total number of the plants found in the high elevations. This richness in endemic plants seems to indicate that the island has been entirely separate from neighbouring countries since geological epochs.

However opulent Formosa is in peculiar plants, the figure representing the number of the endemic species is not so large in the case of this island as it is in that of the Philippines.") This fact shows that the flora of Formosa is of continental character, while that of the archipelago is insular.

The numbers of the plants of endemic character under each genera are shown in the following list.

\begin{tabular}{|ll|ll|ll|}
\hline Clematis & 2 & Eurya & 1 & Astilbe & 1 \\
Thatictrum & 1 & Thea & 1 & Hydrangea & 4 \\
Melodorum & 1 & Geranium & 1 & Ribes & 1 \\
Cardamine & 1 & Impations & 1 & Sedum & 1 \\
Viola & 3 & Euonymus & 1 & Hydrocotyle & 1 \\
Polygala & 1 & Crotalaria & 1 & Sanicula & 1 \\
Cerastium & 1 & Dumasia & 1 & Fatsia & 1 \\
Stellaria & 1 & Rubus & 2 & Oreopanax & 1 \\
\hline
\end{tabular}

1) Mr. E. D. Merrill states that $41 \%$ of the total number of the plants found in the Lamao Forest Reserve is endemic to the Philippines....(see Philipp. Journ. Sci. Vol. I. Suppl. p. 9.) 


\begin{tabular}{|c|c|c|c|c|c|}
\hline Damnacanthers & 1 & Symplocos & 2 & Elatostema & 1 \\
\hline Lasianthus & 1 & Dischidia & 1 & Quercus & 4 \\
\hline Rubice & 1 & Gentiance & 6 & Castanopsis & 1 \\
\hline Scabiosa & 1 & Sucertia & 1 & Chamcecyparis & 1 \\
\hline Eupatorium & 2 & Trigonotis & 1 & Juniperus & 1 \\
\hline Erigeron & 1 & Relemannia & 1 & Cunninghamia & 1 \\
\hline Anaphatis & 1 & Teronica & 1 & Taimania & 1 \\
\hline Gnapliatiun & 1 & Mesonce & 2 & Pinus & 1 \\
\hline Carpesium & 1 & Potygonum & 3 & Tsuge & 1 \\
\hline Artemisia & 2 & Asarum & 1 & Faris & 1 \\
\hline Gymure & 1 & Peperomia & 1 & Scivpus & 1 \\
\hline Ains'ioece & 2 & Helicia & 1 & Spoctiopogon & 2 \\
\hline Vaccinium & 2 & Lovantleus & 1 & Avrundo & 1 \\
\hline Gaultheria & 1 & Balanoplionce & 2 & Brachypodium & 1 \\
\hline Rhododendron & 3 & Gloctidion & 1 & Arundinarice & 1 \\
\hline Pyrola & 1 & Piler & 1 & Polystichum. & 1 \\
\hline
\end{tabular}

Among the above species, the most striking plants, with the endemic genus, Tainania, are as follows :-

Fatsia multicarpe HaYata.

Oreopanax formosana HAYATA.

Damnacanthers angustifolia HAXsTs.

Leontopodium microplygllum HAYsTs.

Pyrola morrisonicole HaYaTA.

Heticia formosana HexstuE.
Chamacyparis formosensis Marsurum. Cumninghamia Körishii HAYıTı. 'Iaimania cryptomerioides Fixıt. Pinus formosance Hirata. Brachyppodium Kancalsamii Hayata.

l) General Character of the Eloments.

The general features of the elements are shown in the following table. 
Temperate elements

Tropical elements

Arctic, antarctic \& alpine

elements

Total

$$
\begin{aligned}
320 & =81 \% \text { of the whole number } \\
45 & =12 \% \quad " \quad, \quad \\
27 & =7 \% \quad, \\
392 & =100 \%
\end{aligned}
$$

Thus, the flora is, in general, temperate, having as many as 320 species of temperate character, or $81 \%$ of the whole number of the elements.

The total number of the species in the flora is 392 , belong$\mathrm{ng}$ to 79 families and 266 genera.

\section{2) Floristic Relationship between Formosa and Neighbouring Countries.}

The numbers of the elements in the regions under comparison are shown in the following table.

\begin{tabular}{|l|c|c|}
\hline \multicolumn{1}{|c|}{ Regions } & Number of elements & Ratio \\
\hline The Nalaypen. \& archip. & 98 & $25 \%$ \\
\hline The Himalayas & 101 & $26 \%$ \\
\hline Central \& southern China & 192 & $49 \%$ \\
\hline Northern China & 81 & $21 \%$ \\
\hline Japan & 163 & $42 \%$ \\
\hline North America & 37 & $9.5 \%$ \\
\hline Endemic & 99 & $25 \%$ \\
\hline
\end{tabular}

As shown in the above table, the island has the strongest affinity to central and southern China and Japan; next, to the 
Himalayas; then, to the Malay peninsula and archipelago, and North China; and lastly, to North Ameriea.

As to central and southern China and Japan, the comparative strength of their floristic relationship to Formosa is not to be measured by the number of elements only; the character of the elements must also be taken into account.

So far as the number of the elements is concerned, it appears that the most striking affinity obtains between the island and central and southern China. It is not so, however, when we compare those elements which give the flora its peculiar features. The comparison of this class of elements, which plays so important a part in the study of phytogeography, is worthy of special attention.

As we have already seen, the species the distribution of which is limited to Formosa and Japan are far more numerous than those confined to Formosa and China. We have also observed that the number of the genera, which are found in the islands and nowhere else, is double that of such kinds in Formosa and Chini.

When we consider these species of peculiar character, we are forced to think that the flora of Formosa has a striking affinity to that of Japan. And it is even more so, when the ggenera, Trochodendron, Futsia, Conandron, and Metanarthesium are? taken into account.

Thus, I am much inclined to eonclucle that the montane flonir of Formosa is nearest to that of Jipan, regartless of geographical proximity to China.

A ferv lines should be here devoted to the cause of this similarity between the islands." It is a very remarkable fact that so many plants of peeuliar character are found in both regions.

1) Jnpan and Formosa. 
This fact has led me to think that these plants once ranged over all the continent but became extinct there, while they have still survived in the islands, owing to their insular conditions ${ }^{1)}$.

This opinion will, however, not satisfactorily explain why the plants, which are found still living in the islands, do not also suluvive in so sheltered a place as Tein-ling-shan², where the flora is quite as rich as it is in Japan and Formosa. It is very reasonable to think that in the so called coast provinces of China, the disturbances were so severe as to destroy these inhabitants of peculiar character. But, why in the protected centre of China?

It seems to me that insular conditions are not the only cause of the floristic affinity of the two regions, (Japan and Formosa) and I have wondered if this affinity were not due to a land-mass or mountain chains, which are by some geologists conjectured to have existed between the islands in former ages ${ }^{3)}$."

In my paper "On the Distribution of the Formosan Conifers", I referred to the probable derivation of the coniferous flora of Formosa, and came to the conclusion that the floris of Japan and Formosa have been dereloped in the border regions of the former continent, the extention of which reached from Japan southwards to the Loo-choo islands as far as Formosa; while the flora of central China has been formed in the centre of the continent.

1) I am much impressed by the opinion of Mr. WAILACE who made the following conclusion in his "Island Life" ed-3, p. 404:- "It is clear, therefore, that before Formosa was separated from the mainland the above named animals or their ancestral types must have ranged over the intervening country as far as the Himalayas on the west, Japan on the north, and Borneo or the Philippines on the south; and that after that event occurred the conditions were so materially changed as to lead to the extinction of these species in what are now the coast provinces of China, while they or their modified descendants continued to exist in the dense forests of the Himalnyas and the Malay Islands, and in such detached islands as Formoosa and Japan."

2) DicLs, L.-Flora von Central-China, in Engl. Bot. Jahrb. XXIX. pp. 169-659.

3) Hayata, B.-On the Distribution of the Formosan Conifers, in Tokyö Bot. Mag. XIX. p1. 43-61. 
Thus, I divided the Chino-Japanese flora into two florulæ, one is the central florula, the other, the border florula.

Regarding the present subject of the montane flora of the island, I see that my former conchusion will hold equally good of the formation of this flora.

Taking all these cases into account, I have come to the conclusion that the similarity of the floras of Formosa and Japan may have been caused, on the one hand, by the existence formerly of a land-mass between the islands, and, on the other, by the same insular conditions caused by the depression forming the inner seas in more recent geological ages.

\section{3) General Aspect of the Vegetation.}

The vegetation of the montane zone of Formosa raries considerably according to the height. As Mit. MIorrison presents various climatal features from subtropical through temperate up to cold regions, the vegetation of this mountain will give us a fair idea of the general aspect of the growth in the hill regions of the island.

As I have already mentioned, a botanical excursion was carried out on the mountain by Messi's. T. KAWaKanr, S. NAGASAWA and G. NAKAhara, and a report' ${ }^{12}$ of the journey was published by Mr. T. Kawakani in the 'Tokyó Botanieal Magazine. As his report is the only publication relating to that momtain, I take the liberty of drawing some descriptions from it.

It was on the 28th of October, 1905 , that the party left Kagi, a small town on the north-western foot of the mountain.

1) Kawaka w, 'T. :- Botnnieal Excursion to Mt. Morrison, in Tökyō Bot. Mng. XX. 11., 30-36, (Japrnese). 
Passing over many hilly passes, they found themselves on an elevation of abont 2,400 ft., where they saw a beautiful forest of Dendrocalamus") and Bambusa").

From a height of $3,000 \mathrm{ft}$. upwards, beautiful arbors of camphor $^{31}$ and Quercus s. $^{4}$ made a dense forest with a liana formation, and many epiphytes of ferns, orchids and mosses. In this forest, the undergrowth is also beautiful; large tree ferns $s^{5)}$, graceful stellate ferns $^{(6)}$, wild Musa $a^{i)}$, Calamus ${ }^{5)}$, Alocasia $a^{9)}$, all combining to form a glorious example of tropical vegetation.

At the height of $3,700 \mathrm{ft}$., a pretty her $\mathrm{b}^{10)}$ of the Urtica family occurred plentifully, and on it two species ${ }^{11)}$ of Balanophoracere were found attached to the host.

Further, at the height of $4,200 \mathrm{ft}$, the trees of various Querci|' ${ }^{12)}$ densely covered the plot, while many climbing plants hanging from the top of trees made the forest still more beautiful.

Ascending a little higher, they came to the boundary of the savage districts. In a grassy plot near by, they found an Adenophor ${ }^{13)}$, wild pinkss ${ }^{14)}$, and violets ${ }^{15)}$, in full bloom. Aleurites ${ }^{16)}$ and $I$ desia ${ }^{17)}$ were found in bloom in the village.

Crossing this savage belt, they entered the virgin forest of the western slope. Here at an elevation of $4,500 \mathrm{ft}$., the camphor

1) Dendrocalamus latiflorus Muxno.

2) Bambusa Oldhami Mrsro.

3) Cinnamomum Camphora N. et L.

1) Quercus \& Castanopsis.

5) Alsophila, Cibotium, \& Dicksonia.

6) Asplenirm Nidus Lrss.

7) Irusa paradisiana Lrss. subsp. seminifera BAKER, var. formosana WARB.

8) Calamus formosanus BECC. \& Calamus Margarita HANCE.

9) Alocasia macromhiza Sснотт.
10) Pilea Wattersii Hasce?

11) Balanophora spicata Hayata \& B. parvior Haxata.

12) Quercus Junglurthnii MIQ., Q. Farcakamii HAYATA, and other species of the genus:

13) Adenophora verticillata Fiscr.

14) Dianthus supertus LINN.

15) Viola japonica LAvGSD.

16) Aleurites coriata STEUd.

17) Idesia polycarpa MIaxnr. 
trees $^{1)}$ and various kinds ${ }^{2}$ of Quercus were so enormously great that the trunks attained a circumference of eren $8 \mathrm{ft}$.

At the height of $6,800 \mathrm{ft}$, a dark forest of Chamacyparis ${ }^{3}$ was first met with. The Conifer was here wonderfully large attaining a diameter of even $10 \mathrm{ft}$. Intermixed with the tall trunks of the five leaved pine ${ }^{4)}$, with the gregarious undergrowth of a pretty variety of bamboo, this Chamceyparis ${ }^{5)}$ occupies the greatest part of the Conifer regions and constitutes the most peculiar feature of the flora of Formosa. The regetation of this kind is seen only in the mountainous districts of Japan, though the growth is there far less luxurious. This spot is, it is said, frequently haunted by deer ${ }^{(6)}$ of the peculiar species of the Formosan fauna.

The climatal features here were temperate. The thermometer indicated $59^{\circ} \mathrm{F}$. The familiar Polygonum (near P. Thunbergii), Smilacina $a^{8}$, and Rhus were all weleomed as old friends. The red tinted leaves of the Rhus twining about the trunk of the pine reminding them of the autumnal scenery of Japan. The season of flowers was past; still there remained a few flower's and some fruits.

From the elevation of about $8,000 \mathrm{ft}$., a full sight of the peak was clearly obtained. On the north, the immense forest of Mt. Arizan was seen far below the foot. T'suga $a^{(1)}$ was here first met with, intermixed with shrubberies of Pieris ${ }^{11)}$ and various kinds ${ }^{12)}$

1) Cinnamomum Campleore $\mathrm{N}$. et $\mathbf{E}$.

2) Quercus \& Castamopsis.

3) Chamacyparis oblusa $S$. et $\%$. form. formosenue

4) Pinus Amandi Franch. var. Mastersiane HAYATA.

5) Chemacyperis obluse S. et Z. form. formosana.

6) Centus tavanus = Formusatn spottell Deer. Allied to $C$. Silia of Jnjam.
7) I'olygonum biconexum MaYAтA.

8) Smilacine japonied $\Lambda$ Grax.

9) lihus intermedie Harats.

10) Tstega formosane ITAтAтA.

11) l'ieris formose D. Dox.

12) Rhedodendron Ohlliami Masms, var. glanulue lestem Hasata. 
of Rhododendron. Here, Trochodendron ${ }^{\text {) }}$ of a enormous size was found most abundantly.

Further on, there was a grassy hillside with pines scattered here and there. Various plants including Thymelæaceæ"), Ruta-

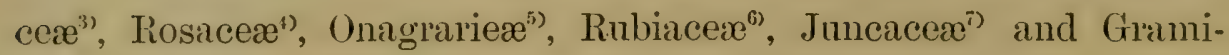
nex $e^{8)}$ were also found. A large tree of Juglandace $x^{9)}$ was met with, and the first example of a deciduous arbor ${ }^{10)}$ on this elevation of $8,250 \mathrm{ft}$.

Then, the slope became more and more gentle. At the height of 9,000 ft., a red berried Vaccinium ${ }^{11)}$ was found, and a kind of Sphagnum, Campanulace ${ }^{12)}$ and Orobanchace ${ }^{13)}$; atso a forest of Picea ${ }^{14}$, with an undergrowth of Compositæ ${ }^{15)}$, Rubiacex ${ }^{16)}$, Saxifragace ${ }^{17)}$, and Geraniacex ${ }^{18}$. A curious species of Impatiens ${ }^{197}$, Mitell $\left(^{20)}\right.$ and a long-leaved Rubia $a^{21)}$ were just coming into flower. The white Parnassia ${ }^{22)}$ was in full bloom. Nothing could be

1) Trochodendron aralioides SrEB. et Zucc.

2) Daphne Championi Bextr. \& Stellera Chamajasme LrNx.

3) Boenningharsenia albiflora Rвтсн, \& Skimia japonice 'Thusв.

4) Rubus pectinellus Maxim.

5) Epilobium sp.

6) Ophiorrhiza pumila Chaмp., Damnacentluts angustifolia Hayata, \& D. indicus G正rTx. f.

7) Juncus effisus Livs.

8) Panicum montanum Roxв., Cymbopogon Narlus Renden., Arumlinella setosa 'TRIN., Calamagrostis arundinucea RoTH. \& Mi: canthus sinensis ANDErss. var. formosanus HACK.

9) Juglans sp.

10) Carpinus sp.

11) Vaccinium Mervilliana Hayata \& $V$. emarginatum HAXATA.

12) Peracarpa curnosa H. E. et'T. \& Adenophora polymorph LEDED.

13) Orobanche carulescens STEPHx.

14) Picea morrisonicola Hayata.
15) Eupatorirm formosanum HАYАтA, Solidago Virga-aurea LnNo, Myriactis Wightii DC. Aster baccharoides STEETz, Aster scaber 'Thuns., \& A. trinervius Roxn. Laggerc clate Schultz-Bip., Gnaphalium lineare Hayata, G. luteo-album LiNs., Carpesirm acutum HAyata, Artemisiu scoparia WALdst. et KIT. Petasites tricholobus Fraxcr., Senecio monathrs DrELs., Senecio scandens HAMr, Cnicus IVallichii DC., Ainsliaa macroclinidioides Hayata, \& Picris hieracioides Lins.

16) Damnacanthus.

17) Astilbe chinensis Fr. et $\mathrm{S}_{\mathbf{A v}}$, Astilbe chinensis var. longicarpo HAYATA, Chrysosplenirm, Mitella japonica, Mre, IIydrangea, Deutzia Scabra Thuxe. \& Ribes formosana Haxata.

18) Impatiens, Geraninum, \& Oxalis.

19) Impatiens rmifloris Hayata.

20) Milella japonica MrQ.

21) Rubia lanceolata Hayata.

22) Parnassia palustris Lixx. 
more interesting than to see these flowers of cold regions on this mountain lying within the tropies. A kind of Fragaria with a yellow fruit and a beautiful flower $^{2}$ of Caryophyllese were seen among the rocks.

At the height of $10,500 \mathrm{ft}$., the climate was rather' cold. 'The temperature fell at night to $43^{\circ} \mathrm{F}$. The frost was very heavy at that season of the year. There a dark forest of Abies ${ }^{3)}$, T'suga ${ }^{4)}$, and Chamcecyparis ${ }^{5)}$ was very beautiful.

Passing through this forest, they came to a brook on the banks of wheh they found two species ${ }^{6)}$ of Umbellifere and a kind of Primula $a^{i}$. It was here that the beantiful flowers of Thalictrum $^{8)}$ were found. Epilobium9) of a very small size was also growing along the brook.

Further on, the coniferous forest gradually gave way to a shrubby formation of Juniperus ${ }^{10)}$, intermixed with dwarf trees of the Berberis ${ }^{11)}$ family. The ground was all covered with debris of clay slate fallen from the peak above.

From the height of $12,000 \mathrm{ft}$ and upwards, the mountain sirles were grassy, where Potentilla ${ }^{12)}$, Astilbe ${ }^{132}$, Lyycopodium.1 and Adenophora $^{15)}$ were growing abundantly. A small swampy plot was found, there were beantiful groups of various flowers of the blue Scabiosa ${ }^{16)}$, yellow Hypericum ${ }^{17)}$ and Sedum ${ }^{18)}$, Oxalis ${ }^{19}$ of

1) Fragaria sp.

2) Cucubalus baccifer LnN.

3) Abies Mariesii MAst. var. Iäuculamii Haxata.

4) Tsuga formosana IIAyata.

5) Chamacyparis formosensis Matsumura.

6) Sanicula petagnioides Haysta \& Chidium sp.

7) Primula sp.

8) Thalietrum Fauriei Ifarata.

9) Epilobium alpinum Lixx. \& E. \%oseun ScunEB.

10) Juniperus formosenu HAYata \&. Jomisomicola Haxati.
11) Berberis sp.

12) Potentilla geliul C. A. MEx., P. lenconota Dox. var. morrisonicole HAYA of Siblutelie mocrmbers Lrxx.

13) Astilbe chinensis In. et Sav.

11) Incoportium olseverum Isxx., $L$. clecatum Lxxs. of $I_{\text {s }}$ servelum 'I'Huxis.

15) Adenophore polymoriplere LF:DriB.

16) Senbiose lacerifolie Haxata.

17) IIypericum attenutum Cuosx.

18) Sedum morrisonense Haxata.

19) Orelis Gritflhii Enckw, et 11ouk. f. 
violet color, red Epilobium" and snow white Spircea". The flowering season was over, but still a few flowers remained.

On the top $(13,120 \mathrm{ft}$. $)$ of the peak, the ground is covered with blocks of rocks. The flora is here extremely scanty, and the flowers were all gone. The scene was very desolate. No sound save the humming of an insect broke the profound silence.

Thus ended the trying excursion carried out by Messi's. T. Kawakami, S. Nagasawa and G. Natiahara. It was a joumey of more than a week. From this trip, they all brought back very good collections. The report of the botanical tour written by Mr. T. Kawakani must, I infer, have thrown some light upon the study of the regetation of the island.

Summarizing the description above referred to, the montane zone of the island may be divided into four regions.

1) Broad leaved tree region (Trochodendron, Cinnamomum, and Quercus) from $2,000 \mathrm{ft} .-6,000 \mathrm{ft}$.

2) Coniferous region (Abies, Picea, Pinus, T'aiwania, Cunninghamia, and Chamcecyparis) from $6,000 \mathrm{ft}$. up to $10,000 \mathrm{ft}$.

3) Shrubbery region (Juniperus and Berberis) from 10,000 ft. up to $12,000 \mathrm{ft}$.

4) Grass region (Leontopodium, Potentilla, Origanum, Sibbaldia Lu:ula, Trisetum, Festuca, Brachypodium, and Lycopodium) from $12,000 \mathrm{ft}$. up to $13,100 \mathrm{ft}$. 


\section{4) Enumeration of the Plants.}

\section{Dicotyledones.}

\section{Polypetalæ.}

\section{Ranunculaceæ.}

\section{Anemone Lixs.}

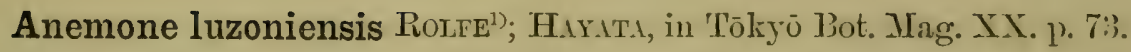
HAB. Shintiku: Goshōrin, leg. 'T. KAwarianr, Mai. 1906. Distrub. The Philippine islands.

Anemone sp). Herbacea peremis, hasi apiceque longe sericenpubescens, mndique pilis hrevioribus alpressis tenuiter obtecta. Folia radicalia magna cum petiolis circ. $30 \mathrm{~cm}$. longa trifoliolata palmatinervia, superne læete viridia subtus glauca; foliola petiolulata, circumscriptione cordato-rotundata, tri- vel multiloba vel profunde incisa, margine inapualiter inciso-scrrata vel duplicato-scrulata, serraturis mucronatis, apice acuta $7-10 \mathrm{~cm}$. longa totidemque lata; foliola terminalia. majuscula valde obliqua, profunde cordata. Caulis $50-60 \mathrm{~cm}$. longus erectus teres: Folia caulina opposita breviter petiolata, petiolis villosis basi raginatis, patentia trifoliolata, foliolis petiolulatis basi cuncatis

1) After completing this manuscript, I nm informed by $\mathrm{Ir}$. L. D. Mrenrm $\mathrm{L}$ of the Burenu of Science, Manila, that when he was in the Tiew Merbarium he and Mr. Rowre compared the Philippine materinl with the Mimalnyan specimen, nnd crme to the conclusion that the Luzon plant is not distinctive from Anemone vitifolia His. The deseription of Anemone luzoniensis RoLFe has never been published. I think that the Formosan plunt above mentioned is exnetly the same as the Luzon plant, and Mr. MEnrm. las the samo opinion too. After considering ull the abovo eases, I am much inclined to think thint my plnnt should be referrer to Anemone: vilifolia Havr. 
oblongo-lanceolatis, sursum attenuatis sub-3-lobatis, inæqualiter duplicato-scrratis. Pedunculi plerumque 2 valde clongati tenues erecti.

Hab, in monte Morrison, leg. T. KaWaKanr et U. Mori, ad 8000 ped. alt., Nov. 1906, (No. 1869).

Very near the preceding species, but possibly different from it. The specimens, howerer, being in too imperfect a state, are not determinable.

\section{Clematis Lins.}

Clematis lasiandra MaxnI. var. Nagasawai Harata, n. r. Caulis lignoso-scandens glaber sulcatus. Folia pinnata 3-5-foliolata, cum petiolis $10-15 \mathrm{~cm}$. longa $5-9 \mathrm{~cm}$. lata, foliolis longe petiolulatis simplicibus vel interdum trilobatis oratis r. orato-lanceolatis acuminatis inæqualiter argute serratis. Paniculæ axillares panciflorata, vel quasiterminales, folio breviores vel longiores, basin pedicelli bracteatr, bracteis trilobatis rel elobatis linearibus minoribus, pedicellis gracilibus flore 2-3-plo longioribus. Flores nutantes, $2 \mathrm{~cm}$. in diametro æquantes et totidem longi. Sepala 4, connirentia apice reroluta, oblonga r. orato-oblonga obtusa r. emarginata, $23 \mathrm{~mm}$. longa $8 \mathrm{~mm}$. lata, utraque pagine subglabra leviter purpurea, margine relutinoso-tomentosa. Stamina 4-seriata, extima longissima, filamentis planis linearibus $17 \mathrm{~mm}$. longis dorso longe denseque sericeo-pilosis, antheris $2 \mathrm{~mm}$. longis apice non appendiculatis, stamina intima breviora, filamentis $9 \mathrm{~mm}$. longis, antheris $3 \mathrm{~mm}$. longis. Pistilla $8 \mathrm{~mm}$. longa sericeo-plumoso-caudata. Receptaculum fructiferum erectum globosum 4 11mm. in diametro requans pilosum; carpellis sub maturitate numerosis ovato-lanceolatis acuminatis compressis $3 \mathrm{~mm}$. longis marginatis rubescentilus pilosis, longe plumoso-caudatis, caudis $3 \frac{1}{2} \mathrm{~cm}$. longis. 
НАв. in valle Shūkoran, ad 11117 ped. alt., in monte Morrison, leg. S. Nagasawa, Nov. 1905, (No. 611).

Distris. Type : Kiūshiū, southern part of Japan.

The present variety differs from the type in having manyflowered perluncles; this never has uni-flowered peduncles as the type.

Clematis longisepala Harata, sp. nor. Caulis lignoso-scandens glaber striatus. Folia pimnatim 5-foliolata longe petiolata glaberrima, cum petiolis $15-20 \mathrm{~cm}$. longa, petiolis rolubilibus striatis, foliolis petiolulatis, petiolulis 1.5-2 $\mathrm{cm}$. longis, oppositis ovatooblongis apice acutis basi rotundatis integris 3-5-11erviis submembranaceis subtus pallidioribus $5 \mathrm{~cm}$. longis $2 \frac{1}{2} \mathrm{~cm}$. latis, stipulis connatis ad nodos caulis peltam formantibus. Panicula axillares, folio subæquilongæ $\tilde{y}-8$-floratr. Flores majuseuli patentes, 6- $7 \mathrm{~cm}$. in diametro æquantes, ad basin pedicelli 1-bracteati, bracteis pinnatim 3 -sectis $3-4 \mathrm{~cm}$. longis longe stipitatis, segmentis oblongo-lanceolatis, pedicellis $7-10 \mathrm{~cm}$. longis. Sepala 4, linearilanceolata $3 \mathrm{~cm}$. longa vel longiora apice ohtusal carinato-mucrenata, intus subglabra atro-purpurea, extus ebumea velutinosia parallelimulti-nervia. Petala 0. Stamina multi-seriata (circ. 5-seriata), extima longiora $2 \mathrm{~cm}$. longa, filamentis linearihus sursum leviter crassiusculis ad basin antherarum constrictis deorsum tenuibus dilatis glabris, antheris linearibus - mm longis apiculatis, stanima intima sensim breviora $7 \mathrm{~mm}$. longa. Carpella longe sericeoplumoso-caudata, circ. $1 \mathrm{~cm}$. longa.

HaB, in monte Morrison, ad 6000 ped. alt., leg. 'T. KaWakanI et U. Moni, Oct. 1906, (No. 2018).

Comes very closely to Clematis crassifolia Bexar. Fl. Hongk. p. 7 ; but differs from that in having leaves of the round base. 
Clematis Morii IItrats, sp. nov. Caulis lignoso-scindens glaber striatus. Folia opposita vel quaternata trifoliolata, cum petiolis circ. $15 \mathrm{~cm}$. longa, petiolis volubilibus, subcoriacea subtus glaucescentia exstipulata, foliolis terminalibus petiolulatis longe caudatoacuminatis leorsum obscure lobatis basi rotundatis margine subintegris vel remote mucronato-scriulatis subtus venis prominentibus supra venis impressis venulis prominentibus 5 -nerviis $11 \mathrm{~cm}$. longis $3 \frac{1}{2} \mathrm{~cm}$. latis, petiolulis $1 \mathrm{~cm}$. longis, foliolis lateralibus multo brevioribus sæpe elobatis caudato-ovatis remote mucronato-serrulatis petiolulatis, petiolulis $3 \mathrm{~mm}$. longis, rel subsessilibus. Flores majusculi cernui semi-clausi, $1 \stackrel{1}{2} \mathrm{~cm}$. in diametro æquantes totidem longi, axillares solitarii pedicellati, ad basin pedicelli 2-bracteati, loracteis minutissimis pubescentibus, peclicellis $3 \mathrm{~cm}$. longis pubescentibus. Sepala 4, latiora $1.8 \mathrm{~cm}$. longa $9 \mathrm{~mm}$. lata, ovata mucronato-acuta, intus atro-purpurea, extus velutinoso-pilosa basi leviter cordata venulis parallelis, semi-clausa numquam patentia. Stamina $3-4$-seriata, extima longissima, filamentis linearibus planis longe barbatis $13 \mathrm{~mm}$. longis, antheris circ. $2 \mathrm{~mm}$. longis apice emarginatis, stamina intima brevissima, filamentis $7 \mathrm{~mm}$. longis, antheris $3 \mathrm{~mm}$. longis. Carpella circ. $1 \mathrm{~cm}$. longa longe plumosocaudata.

HAB. im montibus centralibus, ad 10000 ped. alt., leg. U. Nor', Nov. 1906, (No. 1854).

Comes near to Clematis barbellata EDGEw., and still more to Clematis lasiande Maxm.; but differs from them in having silky pubescent sepals and subentire leaflets.

Clematis tozanensis Marata, sp. nor. Caulis lignoso-scandens glaber striatus. Folia majuscula pimnatim 5-3-foliolata longe petiolata glaberrima, cum petiolis circ. $20 \mathrm{~cm}$. longa totidem 
lata, petiolis striatis rolubilibus, foliolis oblongo-oratis rel cordato-ovatis, basi cordatis vel truncatis $8 \mathrm{~cm}$. longis $6 \mathrm{~cm}$. latis palmatim $9-\bar{\tau}$-nerriis apice obtusis integerrimis leviter repandis submembranaceis vel crassiusculis, petiolulis $2-3 \mathrm{~cm}$. longis ralde rolubilibus, stipulis latis comnatis ad nodos caulis peltam formantibus. Panieula axillares panci-florate folio subrquilonga. Flores najusculi patentes $6 \mathrm{~cm}$. in diametro aquantes, ad basin pedicelli 1-bracteati, bracteis majusculis foliaceis simplicibus stipitatis ellipticis apice acutis. Sepala 4 , angusta $3 \frac{1}{2} \mathrm{~cm}$. longa $8 \mathrm{~mm}$. lata acuta vel obtusa intus glabra atro-purpurea, extus eburnea velutinoso-pubescentia multi-striata patentes. Stamina multi-seriata, extima longissima filamentis ㄴ. $\mathrm{cm}$. longis dilatis linearibus tenuibus antheris linearibus $2 \mathrm{~mm}$. longis apiculatis, stamina intima lrevissima filamentis ¿ $\mathrm{mm}$. longis antheris $3 \mathrm{~mm}$. longis longiuscule apiculatis. Carpella longe serice()plumoso-caudata, circ: $9 \mathrm{~mm}$. longa.

Нав, in Tōzan, in montibus Morrison, leg. G. Namaнira, Oct. 1906 .

Comes closely to Clematis smilacifolia WALL., and still more to the preceding species. The present species differs from them in the shape of the bracts and stipules. In this new Clematix, the filaments of the stamens of the outer-most series are the longest, while the anthers of the same series are the shortest.

Clematis Wightiana Watr. ? ; J Ind. I. p. 5 ; Wigrt', Ic. Pl. Ind. or. t. 935.

Haв. Taitō: Iryoknkakusha, leg. 'T'. Kawakame et U. Monr, 1)ec. 1906.

This Clematis is very like C. Wighliama Was.. ; lut, owing to the imperfectness of the slecimen, the determination is rather conjectural. 


\section{Clematis sp.}

We have one more species belonging to this genus from Rakurakusha; but the specimen is very imperfect.

\section{Thalictrum Lisn.}

Thalictrum Fauriei HaYata ${ }^{1)}$, in Matsumura et Hayata, Enumeratio Plautarum Formosanarum, in Journ. Sci. Coll. Imp. Univ. Tōkyō, XXII. p. 7.

Hab. in monte Morrison, leg. T. Kawakami, 1906.

As the specimen is imperfect, the determination is rather conjectural.

\section{Magnoliaceæ.}

\section{Trochodendron SIEB, et ZuCC.}

Trochodendron aralioides Sieb. et Zucc. Fl. Jap. I. p. 83, tt. 39 et 40 ; Mir. Prol. Fl. Jap. p. 146 ; Franch. et Savat. Enum. Pl. Jap. I. p. 19 ; Hexny, List Pl. Formos. p. 16 ; MLatsux. in Tōkyō Bot. Mag. XII. p. 54; Matsum. et HaYata, Enum. Pl. Formos. in Journ. Sei. Coll. XXII. p. 11.

HAB. Suizan, ad 7702 ped. alt., in montibus Morrison, leg. S. Nagasawa, Oct. 1905, (No. 648); Arizan, in montibus Morrison, leg. G. Nakahara, Nov. 1906 ; in monte Morrison, ad 6000 ped. alt., leg. T. Kawakami et U. Mori, Oct. 1906, (No. 1710).

Distrib. Japan and the Loo-choo islands.

The plant spreads over from the main-island of Japan through Kiūshiu to the Loo-choo islands as south as Formosa. It grows the most luxuriously in this region of the island, forming

1) In the year 1905, the present Thalictrim of. Formosa was described by myself as a new species. 'The publication, however, in which my new plant is nppenred had not been issued until Augnst, 1906. In the same yenr, another new species from Japan was described by M. LÉverLLÉ under the same name. As it is not desirable to maintrin one and the same name for two different plants, Prof. J. Matsumura advisably proposes to change the name of the Formosan species to Thalictrum Urbaini Matsumura. 
a pure forest on the boundary between the Conifer and broad leaved tree regions. The trunk is here so large as to attain a diameter of even $15 \mathrm{ft}$.

\section{Illicium Lins.}

In this genus, I. anisatum LrNs." has been the only species recorded from Formosa. We have another plant from the high elevations of the island. The specimens, howerer, are all wanting of flowers, so that accurate identification is impossible.

Illicium sp. Folia versus apicem ramorum plerumque approximata opposita vel sub-verticillata petiolata, petiolis $1 \frac{1}{2} \mathrm{~cm}$. longis, superne sulcatis subtus convexis crassis, laminis oblongis oblongoellipticis vel obovatis utrinque attenuatis rel apice cuspidatoacuminatis integris coriaceis, superne nitidis et lrete viridibus subtus pallidioribus, $10-12 \mathrm{~cm}$. longis $3-4 \mathrm{~cm}$. latis, nervo medio crasso leviter prominulo, venis lateralibus evanidis. Pedunculi circ. $4 \mathrm{~cm}$. longi. Carpella plerumque 13, libera, stylo breviore extrorsum recurvato. Semina oblonga compressia $9 \mathrm{~mm}$. longa $6 \mathrm{~mm}$. lata.

Пнав. in montibus centralibus, ad 8000 pecl., alt., leg. U. Monr, Nov. 1906, (No. 1918); in monte Morrison, ad 6.500 ped. alt., legr. T. KAwaknur et U. Mori, (No. 2028); Tōzan et Arisan, in montibus Morrison, leg. G. Nakahara, Oct. 1906.

The carpels are, in most cases, thirteen, in which respect, it resémbles very much $I$. T'ashiroi Maxn..")

\section{Kadsure Juss.}

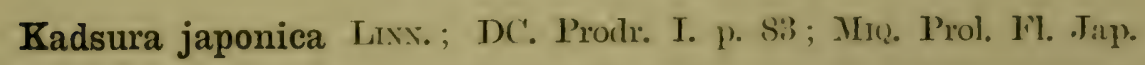

1) Miatsum, et Hirata, Enum. I'l. Formos. 1. 9.

2) S. Matsuba, in 'Iôkyö Jot. Mng. XXI. p. 21:3. 
p. 255 ; Fraver, et Sat. Eunm. Pl. Jap. I. p. 18; Hexnx, List Pl. Formos. p. 16 ; Iтō et Matsunr. Tent. Fl. Lutch. in Journ. Sci. Coll. XU. p. 285 ; Matsumr. in Tōkyō Bot. Mag. XV. p. 85; Matsum. et Hayata, Enum. Pl. Formos. p. 12.

Kadsura clinensis Haxce, in Benth. Fl. Hongk. p. 8 ; Fonses et Heyst. Ind. Fl. Sin. I. p. 25.

Uvaria japoinca LrNo. Sp. Pl. ed-2, p. 756 ; Thurs. Fl. Jap. p. 237. Hab. Kagi : Kishirēi, leg. 'T. Kawakami et U. Mori, Oct. 1906. Distrib. Japan and China.

The plant is found commonly in the low districts. But sometimes it ascends to the hilly regions in the prefecture of Kagi.

\section{Anonaceæ.}

\section{Melodorum Dus.}

Melodorum Oldhami Hexss. in Fonises et Henss. Iud. Fl. Sin. I. p. 27 ; Hexry, List Pl. Formos. p. 16 ; Matsum. in Tökyō Bot. Mang. XV. p. 86 ; Matsur. et Hayata, Enum. Pl. Formos. p. 13.

Hab. Nanto: Kashinokiyama, leg. G. Nakahara, Feb. 1907.

Distrib. An endemic plant.

\section{Berberideæ.}

\section{Akebia DECNE.}

In this genus, Akebia longeracemosa Matsun. has been the only species recorded from Formosa. On the high elevations, we have another, probably new, species. The specimen is, however, in too imperfect a state for exact determination.

Akebia sp. Folia trifoliolata subcoriacea, petiolis circ. $2 \mathrm{~cm}$. longis, foliolis petiolulatis, terminalibus longioribus lanceolatis obtusis basi truncatis $4 \mathrm{~cm}$. longis $1.3 \mathrm{~cm}$. latis, petiolulis $\frac{1}{2} \mathrm{~cm}$. 
longis, lateralibus pauce minoribus breviter petiolulatis, renis et venulis utraque pagine valde prominentibus.

Haв. in monte Morrison, ad 10000 ped. alt., leg. T. KAWAKAMI et U. Morr, Oct. 1906, (No. 2288).

Distrib. A genus confined to Japan and China.

Near A. longeracemosa Matsum., but differ's from it in having trifoliolate leaves.

\section{Berberis Linn.}

Berberis nepalensis 'Spreng. has been the only species recorded from the island. Two more species are found in the hilly districts.

Berberis nepalensis SPRexa.; Hayce, "in Journ. IBot. (1882) p. 2"; Hook. f. Fl. Brit. Ind. I. p. 109 ; Fonbes et Hexrst. Ind. Fl. Sin. I. p. 31 ; Matsun. in Tōkyō Bot. Mag. XII. p. 54 ; Matsux. et Hayata, Enum. Pl. Formos. p. 18.

Mahonia nepantensis DC. Prodr. I. p. 109 ; Diers, Fl. Centr. China, in ENGL. Bot. Jahrb. XXIX. p. 338.

Berberis Bealei Fontune, Bot. Mag. t. 4852.

Ilex japonica Thuns. Fl. Jap. p. 79, et Ic. Pl. Jap. t. 12.

HAв. Ganzan, in montibus Morrison, ad 8012 ped. alt., leg. S. Nagasawa, Oct. 1905, (No. 567); Arizan, in isdem montibus, leg. G. Narahara, Nor. 1906 ; in monte Morrison, ad 7000 ped. alt., leg. 'T. Kawakani et U. Mori, Oct. 1906, (Nos. 177 et 1870).

Distrib. Khasia, central China, Japan and the Philippine islands. Mr. E. D. Merrilu remarks that this Formosan form is just the same as the Luzon one.

Berberis sp. Frutex erectus ramosissimus, ramulis spinis termatis. Folia fasciculata corracea ovata spathulatare apiece rotunclata aristato-mucronata vel obtusia margine remote spinulosodentata basi cuncata sessilia vel breve petiolata, $1.5 \mathrm{~cm}$. longa 7 
mm. lata. Bacce 3-fasciculatx globoso-ellipsoidales, utrinque obtusæ rubræ, $9 \mathrm{~mm}$. longæ 3 -spermæ, stigmatibus parvis sessilibus, seminibus lunaribus $4 \mathrm{~mm}$. longis, pedunculis $1 \frac{1}{2} \mathrm{~cm}$. longis.

$\mathrm{H}_{\mathrm{AB}}$. ad verticem montis Morrison, ad 13094 ped. alt., leg. S. Nagasawa, Nov. 1905, (No. 595); in monte Morrison; ad 12500 ped. alt., leg. T. Kawakami et U. Mori, Oct. 1906, (Nos. 2289 et 2297 ).

The leaves and fruits of this plant are beautifully tinted by the autumnal coloration.

Berberis sp. Frutex erectus ramosissimus, ramulis angulatis sulcatis, spinis ternatis $2-3 \mathrm{~cm}$. longis. Folia fasciculata coriacea obovata oblanceolata vel lanceolata acuta basi cuneata subsessilia remote spinuloso-dentata 5-3 cm. longa 2-1 c.m. lata, renis supra impressis subtus prominulis, venulis utraque pagine prominentibus, subtus pallidiora. Baccæ 5-10-fasciculatæ nigricantes oblongoovulares $7 \mathrm{~mm}$. longæ, utrinque obtusæ, 2-3-spermæ seminibus łunaribus curvis $5 \mathrm{~mm}$. longis pedunculis $1 \mathrm{~cm}$. longis.

HAB. Seizan, in montibus Morrison, ad 11579 ped. alt., leg. S. Nagasawa, Nov. 1906, (No. 712); in monte Morrison ad 12000 ped. alt., (No. 2133); ad 9000 ped. alt., in eodem monte, (No. 1941), leg. T. Kawakam, Oct. 1906 ; Arizan, in montibus Morrison, leg. G. NAKahara, Oct. 1906.

The plant is very near $B$. barandana VidaL.

\section{Papaveraceæ.}

\section{Corydatis DC.}

In this genus, two species have hitherto been known from the island. We have another species from the hilly regions, 
which is quite different from the others. The specimens are, however, in too imperfect a state either for determination or description.

Corydalis sp.

Нав. Taitō: Daironkōsha, leg. T. Kawakaßri et U. Morr, Nor. 1906, (Nos. 2170 et 2183).

\section{Cruciferæ.}

\section{Arabis LinN.}

In the lowland flora we have had nothing of Arabis. On the high elevations, there are found three species belonging to the genus.

Arabis alpina LINs. Sp. Pl. ed-2. p. 928; DC. Prodr. I. p. 142 ; Hook. f. et Thous. in Jouru. Tinn. Soc. V. p. 141; Hook. f. et ANDEns. in Ноок. f. Fl. Brit. Ind. I. p. 135 ; Drets, Fl. Centr. Chin. in Exal. Bot. Jahro. XXIX. p. 359 ; Ledeb. Fl. Ross. I. p. 117 ; Hook. et Ans. Bot. Beech. Voy. p 112; Wagnen, Deut. Fl. ed-3, p. 290 ; Thoxre, Fl. Deut. Ost. u. Schw. II. p. 175.

Arabis albida Stev.; DC. Prodr. I. p. 142.

Arabis pterosperma EDGEw. in 'Tran. Linn. Soc. XX. p. 39.

Hab, in monte Morrison, leg. T. Kawakami et U. Mori, Oet. 1906, (No. 223).

Distrib. Asia, from Altai westward to Europe; east Himalaya, central China northward to east Siberia.

My specimen does not quite agree with the description of A. alpina Lrxs. It seems to me that the plant differs at little from the type in its individual character only. The leaves of the present form are subentire, while those of the type are more or less dentate.

Arabis taraxacifolia Axners, Hook, f. et Axmbls. in IJook. f. Fl. Brit. Ind. I. p. 136. 
HAB. ad verticem montis Morrison, ad 13094 ped. alt., leg. S. Nagasawa, Nov. 1905, (No. 680); eodem loco, leg. T. Kawakani et G. Nakahara, Nov. 1905; in monte Morrison, ad 11000 ped. alt., leg. T. Kawakani et U. Mori, (No. 1864).

Distrib. Punjab, the Himalayas.

My specimen is quite agreeable with the description of the species above referred, written in Ноoк. f. Fl. Brit. Ind. I. p. 136, and also very like the European A. arenosa Scop. Although I have not yet seen an Indian specimen, I think that the plant should be referred to this species. The specimen bearing No. 680 is of a rather elongated form and of prostrate habit. This form has much more runners and more remote leaves than the type.

Arabis sp. Caulis stellato-tomentosus, 14-15 cm. longus, basi 2-3 foliolatus ramosus. Folia radicalia longe petiolata, petiolis $2 \mathrm{~cm}$. longis, laminis obovatis apice rotundatis integris basi cuneatis et remote serratis, utrinque stellato-tomentosis, $9 \mathrm{~mm}$. longis $4 \mathrm{~mm}$. latis. Siliquæ lineares $2 \mathrm{~cm}$. longæ.

Hab. in monte Morrison, ad 12000 ped. alt., leg. T. Kawaikanir et U. Mori, Oct. 1906, (No. 2234).

As the specimens are wanting of flowers, they are not specifically determinable.

\section{Cardamine Lins.}

Of this genus, two species have been recorded from the island. We have two more species from the high elevations of Mt. Morrison.

Cardamine reniformis Hayata, sp. nov. Caulis humilis tenuis 8-9 cm. longus crectus glaber. Folia radicalia longe petiolata, petiolis circ. $5 \mathrm{~cm}$. longis basi leviter dilatis, laminis rotundato- 
reniformibus apice obtusis basi reniformibus repandis palmatim6-7-nerviis, $4 \mathrm{~cm}$. longis totidem latis utraque pagine subglabris pauce ciliolatis. Folia canlina brere petiolata radicali similia. Scapi panciflorati. Flores parvi, $3 \mathrm{~mm}$. longi pediecllati. Sepali t, oblongoelliptica, utrinque obtusa, 롤 $\frac{1}{2} \mathrm{~mm}$. longa. l'etala spathulata $2 \mathrm{~mm}$. longa. Stamina $6,2 \frac{1}{2} \mathrm{~mm}$. longa. Ovarium cylindraceum $2 \mathrm{~mm}$. longum, stylo brevi, stigmate globoso. Silique lineares 2 cm. longx $\frac{1}{2} \mathrm{~mm}$. latæ, seminibus oblongis compressis $\frac{1}{2} \mathrm{~mm}$. longis.

Hab. in monte Morrison, ad 7500 ped. alt., leg. T. KawatadI et U. Mori, Oct. 1906, (No. 1982).

The present species differs from the other species of this genus in having reniform leaves. The leaves are somewhat like C. asarifolia Lins., in which species they are never cordate or reniform.

Cardamine sp. Caulis glaber 10-15 cm. longus. Folia ratlicalia $15 \mathrm{~cm}$. longa $2 \mathrm{~cm}$. lata bi-pimata longe petiolata, pimis laterailibus breve petiolulatis, pimulis partis superioris sessilibus majoribus lobatis, lobis rotundatis. Folia caulina multo minora.

HAB. in monte Morrison, ad 11000 ped. alt., leg. 'T. KaWAKamI et U. Mori, Oct. 1906, (No. 2252).

As the specimens are sterile, the accurate identification is impossible.

\section{Violaceæ.}

\section{Viola Lins.}

Six species belonging to this genus are known from the low districts. In the mountainous regrions, we have five species, among which two are new. 
Viola japonica Iañasd.; DC. Prodr. I. p. 295 ; MiQ. Prol. Fl. Jap. p. 86 ; Maxnr. in Mél. Biol. Ix. (1877) p. 724 ; Franch. et Sarat. Euum. Pl. Jap. I. p. 42, et II. p. 287; Fonbes et Hexss. Ind. Fl. Sin. I. p. 53 ; Hentry, List Pl. Formos. p. 18; Itō et Matsum. Tent. Fl. Luteh. p. 207 ; Paumin, Conspect. Fl. Korer, I. p. 32 ; Matsum. et Hayata, Enum. Pl. Formos. p. 29.

Viola japonica rar. pekinensis Maxrm. "Bull. Soc. Nat. Mose. (1879) p. 4."

Viola kamtschatica var. pekinensis REGEL, Pl. Rad. I. p. 230.

Haв. Tappansha, ad 3138 ped. alt., leg. S. Nagasawa, Oct. 1905, (No. 774) ; eodem loco, leg. T. Kawakami et U. Mori, Oct. 1906, (No. 1759); in monte Morrison, ad 7000 ped. alt., leg. 'T. KawaKAMII et U. Moni, Oct. 1906, (No. 2011).

Distrib. Northern and central China, and Japan.

The same as the Japanese form.

Viola Kawakamii HaYata, sp. nov. Herba acamlis. Folia longe petiolata stipulata, petiolis gracilibus $10-15 \mathrm{~cm}$. longis, laminis hastato-cordatis acuminatis vel obtusis crenulatis ad sinus crenæ pilosis ceterum glabris, subtus glauco-violascentibus circ. $3 \mathrm{~cm}$. longis $2 \mathrm{~cm}$. latis, stipulis laciniatis ad petiolum parce adnatis. Flores patentes $1.5 \mathrm{~cm}$. in diametro xquantes, longe pedunculati, pedunculis petiolo æquilongis, bracteis 2 subulatis remote sitis, $5 \mathrm{~mm}$. longis. Sepala subrqualia obtuso-acuminata $4 \mathrm{~mm}$. longa $1 \mathrm{~mm}$. lata, basi ultra insertionem $\frac{1}{2} \mathrm{~mm}$. producta glabra. Petala superiora et lateralia subæqualia ovato-cuneata apice emarginata, $12 \mathrm{~mm}$. longa $5 \mathrm{~mm}$. lata, inferius majus $15 \mathrm{~mm}$. longum $9 \mathrm{~mm}$. latum apice valde emarginatum vel leviter 2-lobatum basi longe calcaratum, calcare $6 \mathrm{~mm}$. longo leviter curvo. Antheræ subsessiles, connectivo complanato apice in membranam $1 \mathrm{~mm}$. longam producto; stamina 2 inferiora dorso basi calcarata, calcare $3 \mathrm{~mm}$. 
longo. Stylus fere rectus, stigmate terminali. Capsula ignota. Hab. Suizan, in montibus Morrison, ad 7702 ped. alt., leg. S. Nagasaiwa, (No. 649); in monte Morrison, ad 7000 ped. alt., leg. T. Kawakami et U. Mori, (No. 2010).

Very near $V$. formosana Hayata, but differ's from it in having more elongate leaves.

Viola Nagasawai Makino et Hay.ta, in Matsux. et Haytu, Eumm. Pl. Formos. p. 30.

Hab, in monte Morrison, leg. G. Nakahara, Oct. 1906.

Viola tozanensis Harata, sp. nov. Herba accaulis. Folia longe petiolata stipulata, petiolis $4 \mathrm{~cm}$. longis, laminis late cordatis rotundatis crenulatis setoso-pubescentibus subtus glaucis violascentibus, circ. $2 \mathrm{~cm}$. longis totidem latis, stipulis laciniatis ad petiolum parce adnatis. Flores patentes reflexi $1 \frac{1}{2} \mathrm{~cm}$. in diametro arquantes longe pedunculati, pedunculis petiolo xquilongis, bracteis 2 subulatis oppositis $5 \mathrm{~mm}$ longis. Sepala sub-æequilonga obtuso-acuminata $4 \mathrm{~mm}$. longa $1 \mathrm{~mm}$. lata basi ultra insertionem $1 \mathrm{~mm}$. producta glabra. Petala superiora et lateralia subaqualia ovato-cuneata apice truncata $10 \mathrm{~mm}$. longa $5 \mathrm{~mm}$. lata, inferius majus $18 \mathrm{~mm}$. longum $8 \mathrm{~mm}$. latum apice valde emarginatum hasi longe calcaratum, calcare $4 \mathrm{~mm}$. longo leviter curvo. Anthera subsessiles, connectivo complanato apice in membranam $1 \mathrm{~mm}$. longam producto; stamina 2 inferiora dorso basi calcarata, calcare $3 \mathrm{~mm}$. longo. Stylus fore reetus, stigmate sub-terminali. Capsula ignota.

Hab. Tōzan, in montibus Morrison, leg. G. Nakimara, Nov. 1906.

This Viola is found attaching on the large trunk of a tree. The flower is patent and of a whitish purple colour.

Viola sp. 
Hab. Tōzan et Arizan, in montibus Morrison, leg. G. Nakahara. No flower, indeterminable.

\section{Bixineæ}

\section{Idesia Maxnr.}

Idesia polycarpa Maxur. in MIél. Biol. VI. p. 9 ; Franch. et Sarat. Enum. Pl. Jap. I. p. 45; Bot. Mag. t. 6794; Henrix, List Pl. Formos. p. 18; Matsum. in Tökyó Bot. Mag. XII. p. 67 ; Diels, Fl. Centr. Chin. in Engr. Bot. Jahrb. XXIX. p. 478 ; Matsur. et Hayata, Enum. Pl. Formos. p. 32.

Hab. Taitō: Iryokukakusha, leg. T. Kawakani et U. Mori, Dec. 1906, (No. 2165); Kagi: Burakukansha, leg. T. KawaKani et U. Mori, Oet. 1906, (No. 1756).

Distrib. Central China and Japan.

\section{Polygalex.}

\section{Polygala I.tNx.}

Polygala arcuata Haysta, sp. nov. (PI. I.). Tota, præeter ramulos novellos tenuiter pubescontes, glaberrima, caule lignescente plerumque simplici. Folia petiolata, laminis membranaceis crassiusculis elliptico-lanceolatis acuminatis integris $10 \mathrm{~cm}$. longis $3 \mathrm{~cm}$. latis basi in petiolum $1 \mathrm{~cm}$. longum cuneatum attenuatis supra opacis subtus glancescentibus, venis transrersis primariis utrinque circ. 4-5 arcuatis prope marginem anastomosantibus. Racemi caulis apicem rersus axillares rel terminales $5-6 \mathrm{~cm}$. longi densiuscule multiflorati folium haud superantes. Flores lutei pediccllis brevibus $2 \mathrm{~mm}$. longis. Sepala 5, exteriora 3, interiora 2, decidua, supremum exteriorum sepalorum globosum majusculum 
rotundatum saccatum $3 \mathrm{~mm}$. longum, 2 inferiora exteriorum sepalorum late rotundata basi obliqua $1 \frac{1}{2} \mathrm{~mm}$. longa ; 2 interiora petaloidea oblique oblonga $5 \frac{1}{2} \mathrm{~mm}$. longa. Petala alte coalita, lateralia arte imbricata $7 \mathrm{~mm}$. longa apice rotundata, carina breviter cucullata dorso longe cristata incrassata bisaccata. Ovarium glabrum breviter stipitatum, disco late annulari. Stylus apice dilatus, infra stigma appendiculatus. Capsula $5 \mathrm{~mm}$. lata $4 \mathrm{~mm}$. longa, membranacea compressa obreniformis v. late crbiculata emarginata ad margines loculicide dehiscens. Semina ovoidea $2 \mathrm{~mm}$. longa, pendula pilosa strophiolata, strophiolis in utroque latere $1.2 \mathrm{~mm}$. longis.

Hab. Taichũ: Kashigatani, leg. G. Nakahara, Feb. 1907.

Somewhat resembles P. Wattersii Haxce, in Journ. Bot. (1881) p. 209; but differs from it in having obreniform fruits, much smaller flowers, divided crests, and in many other points.

Polygala japonica Houtr.; DC. Prodr. I. p. 324 ; BaKer et S. Moore, in Journ. Linn. Soc. XVII. p. 379 ; Franchet, PI. David. p. 45; Fraxch. et Sayat. Enum. Pl. Jap. I. p. 45 ; Hennx, List Pl. Formos. p. 18 ; Itō et Matsum. Tent. Fl. Lutch. p. 311 ; PaLibin, Conspect. Fl. Koreæ, I. p. 37 ; Matsum. et Hayata, Enum. Pl. Formos. p: 34.

Polygala sibirica Linn. Sp. PI. ed-2, p. 987; DC. Prodr. I. p. 324 ; A. W. Benn. in Journ. Bot. (1878) p. 277 ; HANCE, in "Journ. Bot. (1882) p. 257 "; Forbes et HeMsi. Ind. Fl. Sin. I. p. 61.

Hab: in monte Morrison, leg. G. Nakahara, Oct. 1906.

Distrib. From Siberia to Japan and India, and occurs also in Australia.

\section{Caryophylleæ.}

\section{Dinnthes LINN.}

Dianthus superbus I.r.x. Sp. 11. ed-2, p. $589 ;$, )(. I'rodr. I. p. 365 ; HAxce, in Journ. Bot. (1883) 1. 296 ; Frixcher, Pl. 1)aviul. 1. 46; Leder. 
F1. Ross. I. p. 533 ; MLxis. Prim. Fl. Amur. p. 52 ; lieger, Pl. Radd. I. p. 288 ; Mir. Prol. Fl. Jap. p. 9; Schirdt, Reis. in Amur. p. 116 ; Franch. et Savat. Enum. Pl. Jap. I. p. 46 ; Evgl. in Engu. Bot. Jahrb. VI. p. 57 ; Fonbes et Henst. Ind. Fl. Sin. I. p. 64 ; Diels, Fl. Centr. Chin. in ExGit. Bot. Jahrb. XXIX. p. 316 ; Palibn, Conspect. Fl. Koreæ, I. p. 39 ; MIatsux. et Hayata, Enum. Pl. Formos. p. 35.

Hab. Ganzan, in montibus Morrison, ad 9141 ped. alt., (No. 700) ; Suizan, in isdem montibus, ad 7702 ped. alt., (No. 664); in monte Morrison, ad 9000 ped. alt., (Nos. 2040 et 1895); in montibus centralibus, ad 7000 ped. alt., (No. 1861) ; Taitō : Taironkōsha, (Nos. 1883 et 1950); Toroku : Gunkei ; leg. T. KawaKanI et U. Mori, Nov. 1906 ; Nanto: Hinokiyama, leg. G. Nakahara, Feb. 1907.

Distrib. Europe to Mongolia; China throughout, Saghalien and Japan.

\section{Dianthus sp.}

HAB. in monte Morrison, ad 12000 ped. alt., leg. T. KawaKanI et U. Mori, Oct. 1906, (No. 2259).

This differs from $D$. superbus Lins. in having elongate bracts, and especially two inferior ones.

\section{silene LINN.}

Silene Fortunei VIs. in Linnæa, XXIV. p. 181, et XXXVI. p. 688; Francher, Pl. David. p. 47 ; Forbes et Hearst. Ind. Fl. Sin. I. p. 65 ; Henry, List Pl. Formos. p. 19 ; Diels, Fl. Centr. Chin. in ENGL. Bot. Jahrb. XXIX. p. 318 ; Bot. Mag. t. 7649 ; Matsuar. et Hayata, Enum. Pl. Formos. p. 35.

Haв. in monte Morrison, ad 7000 ped. alt., leg. T. KawaKanI et U. Morr, (No. 1917).

Distrib. Central and southern China.

The present plant found in the high regions is very like the 
specimen (No. 20, A. Tashiro) collected on the sea shore. Excepting that the flowers of the former is almost as half as those of the latter, I can find no distinction whatever between the two. My specimen should, therefore, be regarded as a form of the shore plant.

\section{Cucubalus Linn.}

Cucubalus baccifer LnN. Sp. P1. ed-2, p. 591 ; DC. Prodr. I. p. 367 ; Ledeb. Fl. Ross. I. p. 332 ; Maxtr. Prim. Fl. Amur. p. 56 ; Reget, Fl. Radd. p. 333 ; Franch. et Savat. Enum. Pl. Jap. I. p. 48 ; Koxrarov, Fl. Manshurie, II. p. 205 ; Wagner, Deut. Fl. ed-3, p. 244 ; Drets, Fl. Centr. Chin. in Exgi. Bot. Jahrb. XXIX. p. 319 ; Thомє́, Fl. Dent. Öst. n. Schw. IT. p. 98.

$\mathrm{H}_{\mathrm{AB}}$. in monte Morrison, ad 13000 ped. alt., et ad 7500 ped. ait., leg. T. Kawakani et U. Mori, Oct. 1906, (Nos. 2253 et 1984); Rakurakusha, leg. G. Nakahara, Aug. 1905, (No. 454).

Distrib. The Himalayas, China throughout, eastward to Japan as far as North America; westward to Europe.

\section{Cerastiume LinN.}

We have hitherto had no species belonging to this genus from Formosa. The following Cerastium is the first recorder from the island.

Cerastium morrisonense Hayte, sp. nov. Herba diffusæ $v$. crspitosæ, glanduloso-pubescentes demum glabræ. Folia plerumulue remota interdum approximata lineari-lanceolata vel spathulatoacuminata basi interdum attenuata dilata semi-amplexicaulia apice calloso-acuta vel aristato-acuta 1-20 cm. longa 느-21 $\mathrm{mm}$. lata. Cyma terminales, nunc uni-florx, nunc tri-flora, bracteatr, bracteis scariosis, pedicellis $2-1 \frac{1}{2} \mathrm{~cm}$. longris glanduloso-pubescentibus. Sepala 5, lanceolata $7 \frac{1}{2} \mathrm{~mm}$. longa margine scariosa extus glan- 
duloso-pubescentia intus glabra. Petala 5, oblanceolata $13 \mathrm{~mm}$. longa apice 2-lobata, lobis oblongis obtusis $6 \mathrm{~mm}$. longis, basi angusta. Stamina 10, filamentis petalo 2-plo brevioribus ad basin filamentorum sepalis oppositorum 2-glanduliferis. Ovarium ovoideum $2 \mathrm{~mm}$. longum 1-loculare $\infty$ ovulatum. Styli 5 , sepalis oppositi orario longiores $3 \mathrm{~mm}$. longi. Capsula cylindracea $7 \mathrm{~mm}$. longa $2 \frac{1}{2} \mathrm{~mm}$. lata erecta apice in dentes 10 dehiscens, dentibus obtusis truncatis. Semina subreniformi-globosa, $1 \mathrm{~mm}$. longa a latere plus minus compressa dorso granulata.

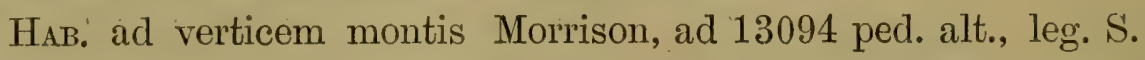
Nagasatwa, Nov. 1905, (Nos. 681 et 619); in montis Morrison, ad 12000 ped. alt., leg. T. Kawakami et U. Mori, Oct. 1906, (Nos. 2127 et 2261).

The plant comes nearest to $C$. grandiflor but differs from it in having lanceolate leaves and smaller flowers.

Cerastium pilosum Ledeb. in "Mém. Acad. Pétersb. V. p. 539," “Fl. Alt. II. p. 173 "; Fl. Ross. I. p. 398, et "Ic. Pl. Fl. Ross. t. 351 "; FonbeS et Hewsi. Ind. Fl. Sin. I. p. 67.

$\mathrm{H}_{\mathrm{AB}}$. in monte Morrison, ad 13000 ped. alt., leg. S. NAGASAWA, Nov. 1905 , (No. 626).

Distrib. Ural eastward to North China, Manchuria and Korea.

In the imperfectness of the specimen, the determination is rather conjectural.

Stellaria LinN.

Two species belonging to this genus have been known from Formosa. We have here a more plant from the hilly regions.

Stellaria stellato-pilosa HAxATA, sp. nov. (PI. II). Herbæ diffusæ ramosæ laxe adscendentes in totum præter caulem densissime 
stellato-pilosæ. Folia sessilia orato-lanceolata basi cordata apice aristato-acuminata $12 \mathrm{~mm}$. longa $3 \mathrm{~mm}$. lata rarius ovato-cordata, supra pilis stellatis subtus pilis longioribus dense tectis, costis prominentibus. Flores $3-4$, in cymas terminales v. rarius axillares dispositi pedicellati, perlicellis circ. $1 \mathrm{~cm}$. longis, basi pedicelli bracteati, bracteis 2 oppositis ovato-lanceolatis. Sepala $\tilde{5}$, lanceolata extus dense stellato-pilosa demum glabra $3 \frac{1}{2} \mathrm{~mm}$. longa $1 \mathrm{~mm}$. lata. Petala profunde 2 -fida, lobis lanceolatis obtusis sepalo longioribus. Stamina 10 ; discus inter stamina in glandulas prominentes plus minus expansus. Ovarium 1-loculare, pauci-ovulatum. Styli 3. Capsula oblongo-ovoidea, $4 \mathrm{~mm}$. longa in 3 valvas dehiscens, valvis 2-fidis. Semina globoso-reniformia a latere compressa, $1 \mathrm{~mm}$. in diametro æequantia granulata v. muricata.

HAB. ad verticem montis Morrison, ad 13094 ped. alt., leg. S. NAgasatwa, Nov. 1905, (No. 622) ; in monte Morrison, ad 12000 ped. alt., leg. T. Kawakanir et U. Moni, Oct. 1906, (No. 2258).

Near Stellaria saxatilis Buch.-HAм.; but differ's from it in having lanceolate leaves and entirely separate sepals.

\section{Hypericineæ.}

\section{Hypericum LINx.}

Among this genus, eight species were previously reeorted from the island. A more species is found in the high regions.

Hypericum attenuatum Crrorsy, in DC. Prodr. I. p. 548 ; H.s.'e, in Journ. Bot. (1874) p. 259, (1878) p. 10.t, et (1885) p. 321 ; Maxin. in Mé́. Biol. XI. p. 166 ; Fraxchet, PI. David. p. 56 ; Fonbes et Hexst. Ind. Fl. Sin. I. p. 72.

$\mathrm{H}_{\mathrm{AB}}$. ad rerticem montis Morrison, ad 13094 ped. alt., leg. S. NagasaWa, Nov. 1905, (No. 754); in monte Morrison, ad 12001 
perl. alt., (No. 2260) ; et ad 8000 ped. alt., leg. T. KawasanI et U. Morr, Oct. 1906, (No. 1800).

Distrib. Baical eastward to central and northern China.

\section{Ternstromiaceæ.}

\section{Ternstromia LinN.}

Ternstrœmia japonica Thuxs. in Trans. Linn. Soc. II. p. 335 ; Sies. et Zucc. Fl. Jap. p. 148, t. 80 ; Bехтн. Fl. Hongk. p. 27 ; Dyer, in Hook. f. Fl. Brit. Ind. I. p. 280 ; Mre. Prol. Fl. Jap. p. 202 ; ENGL. in ENGL. Bot. Jahrb. VI. p. 60 ; Fonees et Hearst. Ind. Fl. Sin. I. p. 75 ; Hexrry, List PI. Formos. p. 19 ; Itũ et Matsuir. Tent. Fl. Lutch. p. 324 ; Mátsux. et Hayatı, Enum. Pl. Formos. p. 45.

Cleyera japonica Thuns. Fl. Jap. p. 224.

Cleyera fragrans et Cleyerce dulia CHasrr. in Trans. Linn. Soc. XXI. p. 115.

Taonabo japonica Szysz. in Evgu. et Prantu. Nat. Pf.-fam. III. 6. p. 188.

Hab. Nantō: Mushazan, ad 7500 ped. alt., leg. T. Kawakani et U. Morr, Aug. 1906, (No. 1141).

Distrib. South China and Japan. Western peninsula of India to Ceylon, and Khasia mountains to Sumatra and the Philippine islands.

\section{Eurya 'THUNB.}

Eurya japonica Truuse. FI. Jap. p. 191, t. 25 ; DC. Prodr. I. p. 525 ; Bexth. Fl. Hongk. p. 28 ; Dyen, in Hook. f. Fl. Brit. Ind. I. p. 284; Hoor. et Anv. Bot. Beech. Voy. p. 260 ; Buume, MIus. Bot. Lugd.-Bat. II. p. 105; Mre. Prol. Fl. Jap. p. 202 ; Fraxch, et S.ritr. Enum. Pl. Jap. I. p. 57 ; Fonbes et Hexss. Ind. Fl. Sin. I. p. 77 ; Hevry, List Pl. Formos. p. 19 ; Diels, Fl. Centr. Chin. p. 474; Putibiv, Conspect. Fl. Koreæ, I. p. 46 ; Matsum. et Hayata, Enum. Pl. Formos. p. 46. 
$\mathrm{H}_{\mathrm{AB}}$. in monte centrali, ad 8000 ped. alt., leg. T. KAWAKAMII et U. Mori, (No. 1866) ; inter Arizan et Tōzan, in montibus Morrison, leg. G. Nakahara, Oct. 1906 ; in monte Morrison, arl 8000 perl. alt., (No. 1788), et ad 10000 ped. alt., (No. 2121), leg. T. KАWAKAмI et U. Mori, Oct. 1906 ; eodem loco, ad 11000 ped. alt., (No. 623), Ganzan, in montibus Morrison, ad 9041 ped. alt., (No. 623), leg. S. Nagasawa, Nov. 1905 ; Rinkiho, ad 4500 ped. alt., leg. N. Koxishi, Feb. 1904 ; Taitō : Inikufuku, et Iryokukakusha, (No. 1840), leg. T. Kawakani et U. Mori, Dec. 1906.

Distrib. The Malay archipelago, southern and central China, and Japan.

The same as the Luzon form.

Eurya strigillosa Hayata, sp. nov. Ramuli graciles strigillosi primum sericeo-villosi. Folia disticha approximata sessilia coriacea oblongo-acuminata vel lanceolata $10 \mathrm{~cm}$. longa $2 \frac{1}{2} \mathrm{~cm}$. lista basi rotundata sæpiusque leviter inæqualia apice acuminata margine præter basin serrulata, serrulis acutis, supra glabra nitida subtus strigillosa, costis prominentibus, venis utraque inconspicuis. Flores $\hat{\jmath}$ breve pedicellati, pedicellis $1 \mathrm{~mm}$. longis vel longioribus, ar axillas foliorum 2-3 congesti, patentes $8 \mathrm{~mm}$. in diametro arquantes. Sepalia $5-6$, valde inrqualia late rotundata $1 . \frac{1}{2} \mathrm{~mm}$. longa totidem lata extus pilosula. Petala plerumque 5, obovato-oblonga $4 \mathrm{~mm}$. longa $21 \mathrm{~mm}$. lata glabra basi leviter connata. Stamina circe 15, glabia petalo breviora, filamentis planis $2 \frac{1}{2} \mathrm{~mm}$. longis, antheris unclulatis filamento duplo vel triplo brevioribus. Ovarii rudimentum hrevissimum conicum apice barbatum. Flores o ignoti.

HAB. in monte Morrison, ad 8000 ped. alt., leg. T. KAWAKAMI et U. Morr, Oet. 1906, (No. 2023).

The plant is very like $E$. distichoploylla IIExiss.; but differs 
from it in having serrulate leaves and larger flowers which attain a diameter of even $8 \mathrm{~mm}$.

\section{Actinidia LINDL.}

Actinidia callosa Linde. Walp. Ann. I. p. 15; Dyer, in Hook. f. Fl. Brit. Ind. I. p. 286 ; Fonibes et Henst. Ind. Fl. Sin. I. p. 78 ; Henrx, List Pl. Formos. p. 20 ; Diels, Fl. Centr. Chin. p. 470 ; Matsuñ. et Hayata, Enum. Pl. Formos. p. 47.

Нав. Kagi : in monte Kishirei, leg. T. Kawakaur et U. Morr, Oct. 1906 .

Distrib. Himalaya, central China to Japan.

Stachyurus SiEв. et ZUCC.

Stachyurus præcox SiEB. et Zucc. Fl. Jap. I. p. 43, t. 18 ; MIL. Prol. Fl. Jap. p. 204 ; Franch。 et SAvat. Enum. Pl. Jap. I. p. 59 ; Forbes et Hemsi. Ind. Fl. Sin. I. p. 79 ; Mratsusr. et Hayata, Enum. Pl. Formos. p. 48 .

Stachynurus himalaicus Hоок. f. et THохs. in Hоок. f. Fl. Brit. Ind. I. p. 288; Diels, Fl. Centr. Chin. p. 475.

HAB. in monte Morrison, ad 7500 ped. alt., leg. T. Kawakani et U. Mori, Nov. 1906, (Nos. 1873 et 1810); Kōshūn : Naibun, leg. G. Natihara, Feb. 1907.

Distrib. The Himalayas through central China to Japan.

This is somewhat different from what we have in Japan. The plant should, I think, be regarded as a form of the Japanese species.

\section{Schime ReINw.}

Schima Noronhæ Rieıvw.; Mið. Fl. Ind. Bat. I.-2, p. 492 ; Bevтrr. Fl. Hongk. p. 29 ; Mraxnr. Mél. Biol. XII. p. 426 ; Foribes et Hemst. Ind. 
Fl. Sin. I. p. 80 ; Henry, List PI. Formos. p. 20 ; Matsus. in Tōkyō Bot. Mag. XII. p. 63 ; Itō et Matsuxr. Tent. FI. Lutch. p. 328 ; MLatsurro et Hayata, Enum. Pl. Formos. p. 49 :

Gordonia javanica Hoor. f. Bot. Mag. t. 4539.

$\mathrm{H}_{\mathrm{AB}}$, in monte Morrison, ad 6500 ped. alt., leg. T. KawaKanI et U. MonI, Oct. 1906, (No. 1711).

Distrib. The eastern frontier of India; from Cochinchina through the Nalay archipelago, and South China to the Loo-choo islands.

\section{Thea LINN.}

Thea brevistyla Hayata, sp. nov. (PI. III.). Ramuli graciles primum pubescentes demum glabri. Folia breve petiolata, petiolis $5 \mathrm{~mm}$. longis semi-teretibus supra sulcatis pubescentibus, laminis elliptico-oblongis $4-5 \mathrm{~cm}$. longis $2 \mathrm{~cm}$. latis basi apiceque acutis vel obtusis margine crenulatis leviter repandis basin versus sæpissime integris utraque pagine glabris, costis prominulis venis impressis coriaceis. Flores ad axillas foliorum semper solitarii sessiles patentes $3 \mathrm{~cm}$. in diametro xquantes. Sepala decidua 45 valde inæqualia 2 -seriata late ovata obtusa vel mucronata 6-8 mm. longa totidem lata extus medio pilosiuscula. Petali 5 alba obovato-cuneata apice sinuato-emarginata vel 2-lobata circ. $1 \frac{1}{2} \mathrm{~cm}$. longa $1 \mathrm{~cm}$. lata. Stamina cire. 30, 2-seriata, exteriora longiora, filamentis plerumque petalis duplo brevioribus hasi comnatis. Ovarium globosum sericeo-pilosum 1 1 12 mm. longum. Styli 4 , hrevissimi basi connati apice recurvi $1 \mathrm{~mm}$. longi. Fructus ignotus.

Haв. Arizan et Tōzan, in montibus Morrison, leg. G. NAkAHARA, Nov. 1906.

Thea caudata (WALI.).

Camellia cendete WALL. "PI. As. Rar. III. p. 36 "; Dyen, in Hook. f. Fl. Brit. Ind. I. p. 293. 
Hab. Taitõ : Iryokukakusha, leg. T. Kawakani et U. Mori, Dec. 1906, (No. 2157).

Distrib. Himalaya, Khasia mountains, and South China.

Although I have seen no specimen of the Indian plant, my plant is, I think, quite referable to this species.

\section{Tiliaceæ.}

\section{Triumfette LINN.}

Triumfetta pilosa Roth; DC. Prodr. I. p. 506 ; Bexth. Fl. Hongk. p. 41 ; Fonbes et Hemst. Ind. Fl. Sin. I. p. 93 ; Henry, List Pl. Formos. p. 23 ; Matsumr. et Hayata, Enum. Pl. Formos. p. 63.

Hab. Sanchōki, leg. S. Nagasawa, Nov. 1905, (No. 726).

Distrib. Tropical Asia and Africa; South China.

\section{Elceocarpus LinN.}

Elæocarpus decipiens HexrsL. Ind. Fl. Siц. I. p. 94; Hexny, List Pl. Formos. p. 24; ITō et Matsum. Tent Fl. Lutch. p. 349 ; Matsum. et Hayata, Enum. Pl. Formos. p. 65.

Hab. Biōritsu : Sensuikō, leg. T. Kawarami et U. Mori, Juli. 1906, (No. 1101).

Drstrib. Sonth China and the Loo-choo islands.

Geraniaceæ.

Geranium LINN.

Geranium Robertianum Linx.; DC. Prodr. I. p. 644; Miaxnr. Mlél. Biol. X. p. 613 ; Franch. et Sarat. Enum. Pl. Jap. II. p. 307. (var. glabrum); 
Hоoк. f. Fl. Brit. Ind. I. p. 432 ; Diels, Fl. Centr. Chin. p. 419 ; Ledeb. “Fl. Alt. III. p. 233 ”; ThомÉ, Fl. Deut. Öst. u. Schw. III. p. 201 ; WagNer, Deut. Fl. ed-3, p. 431.

Hab. Taitō: Bunshisekisha, leg. T. Kawakasir et U. Mori, Dec. 1906, (No. 2152).

Distrib. Southern part of Japan, China throughout, and westward to Europe.

This exactly agrees with the Japanese form.

Geranium uniflorum Hayata, sp. nov. Caulis 1-2 ped. altus erecto-patens glaber superne pilosus, ramis articulato-nodosis. Folia longe petiolata piloso-pubescentia, petiolis $2-4 \mathrm{~cm}$. longis, laminis ambitu late orbicularibus vel pentagonis $5-7 \mathrm{~cm}$. in diametro æquantibus profunde 5-partitis, segmentis acuminatis, pinnatifidis inciso-serratis, stipulis oblongis abrupte acuminatis $1 \mathrm{~cm}$. longis extus pilosiusculis. Flores axillares vel subterminales longe pedunculati uniflori 2 -bracteati, pedunculis $5-6 \mathrm{~cm}$. longis pubescentibus, bracteis subulatis oppositis circ. $1 \mathrm{~cm}$. longis. Sepala 5, elliptica $12 \mathrm{~mm}$. longa $5 \mathrm{~mm}$. lata extus distincte 5 nervia ad nervos pilosula apice aristato-acuta intus glabra. Petala 5, obovata cuneata integra $2 \mathrm{~cm}$. longa vel longiora apice rotundata emarginata basi supra unguem ciliata. Glandule 5. Stamina 10, 2-seriata, filamentis basi dilatis berissime ciliatis antheris oblongis deciduis. Ovarium pilosum. Capsule lobi oblongi pilosi $5 \mathrm{~mm}$. longi $2 \frac{1}{2} \mathrm{~mm}$. lati, caudis $14 \mathrm{~mm}$. longis.

$\mathrm{H}_{\mathrm{AB}}$ ad verticem montis Morrison, ad 13094 ped. alt., leg. S. Nagasawa, Nov. 1905, (No. 684); in eodem monte, ad 13000 perl. alt., leg. T. Kawamane et U. Morr, Oet. 1906, (No. 2254).

This new plant is somewhat like $G$. pratense Lins. in its foliage and flowers; but greatly differs from it in having uni- 
flowered peduncles. The flowers of the present species are always solitary as is the case with $G$. sanguineum Livs. and G. sibiricum Lins.

\section{Oxalis LinN.}

In this genus, Oxalis corniculata Lins. has been the only species recorded from the island. We have here another species found in the montane zone.

Oxalis Griffithii EDGEw. et Hook. f. in Hook. f. Fl. Brit. Ind. I. p. 436 ; S. Moone, in Journ. Bot. (1875) p. 230 ; FonBes et Heust. Ind. Fl. Sin. I. p. 99 ; Drels, Fl. Centr. Chin. p. 420.

$\mathrm{H}_{\mathrm{AB}}$. Suizan, in montibus Morrison, ad 7700 ped. alt., leg. S. Nagasawa, Oct. 1905, (No. 654) ; in monte Morrison, ad 7500 ped. alt., leg. T. Kawakani et U. Morr, Oct. 1906, (No. 1792) ; Arizan, in isdem montibus, leg. G. NaKahara, Nov. 1906.

Distriв. Eastern Himalaya and the Khasia mountains; also in central China.

\section{Impatiens LinN.}

Impatiens uniflora $\mathrm{H}_{A Y A T A}$, sp. nov. Herbæ erectæ circ. $30 \mathrm{~cm}$. altæ pance ramosæ sursum flexuosæ, caulibus (exsiccatis) stramineis præter apicem glabris. Folia versus apicem caulis approximata breviter petiolata, laminis oblongis ellipticis vel lanceolato-ellipticis circ. $8 \mathrm{~cm}$. longis $2 \frac{1}{2} \mathrm{~cm}$. latis apice candato-acuminatis basi cuneatis in petiolum attenuatis margine serratis, serraturis setosis incurvis. Flores majusculi rosei terminales vel ex axillis superioribus, pedunculis gracilibus circ. $4 \mathrm{~cm}$. longis 1-floris (rarius 2-) nudis medio bracteola minuta incurva instructis. Sepala $3: 2$ lateralia oblique ovata acuminata integra $6 \mathrm{~mm}$. longa $2 \frac{1}{2} \mathrm{~mm}$. lata, posticum longe saccatum apice ad orem acutum, ore $1 \frac{1}{2} \mathrm{~cm}$. in 
diametro, basi subito calcar breve incurvum apice incrassatum ac bilobum abeuns, a basi usque ad apicem calcaris $3 \frac{1}{2} \mathrm{~cm}$. longum. Petala : vexillum alis duplo brevius late subreniforme medio dorso cristatum in cornu recurvum maculosum attenuatum; alæ ambitu elliptice $2 \frac{1}{3} \mathrm{~cm}$. longæ latere superiore 2-lobatx, lobo basilari exteriore lato, lobo apicali longiore oblongo. Stamina 5, filamentis inæqualibus brevioribus circ. $4 \mathrm{~mm}$. longis complanatis medio appendiculatis, antheris ovatis apiculatis circa pistillum coherentibus, loculis introrsum dehiscentibus. Ovarium oblongum $4 \mathrm{~mm}$. longum, stigma sessile 5-dentatum. Capsula elongata $2 \mathrm{~cm}$. longa 5 -ralvata, valvis elastice dissilientibus, columna persistente. Semina longe elliptica vix longiora quam $2 \mathrm{~mm}$., testa glabra sub microscopium minute papillosa.

НАв. Tozan et Arizan, in montibus Morrison, leg. G. NAKaнana, Oct. 1906 ; in monte Morrison, ad 7500 ped. alt., leg. T. KAWAraMII et U. Mori, (No. 1724).

\section{Rutaceæ.}

\section{Bonninghausenia REICHB.}

Bonninghausenia albiflora Reichr. "Conspect. Reg. Veg. p. 197"; Hoor. f. Fl. Brit. Ind. I. p. 486 ; Hance, in Journ. Bot. (1874) p. 259; Frinchet, Pl. David. p. 66 ; Franch. et Savat. Euum. Pl. Jap. I. p. 71 ; Mre. Prol. Fl. Jap. p. 209 ; ExGL. in Exat. et Pr.iNT. Nat. Pf.-fam. III.-4, p. 130 ; Fonses et Hexss. Ind. FI. Sin. I. p. 102 ; Driss, II. Centr. ('hin. p. 42!3 ; HAYATA, in Tōkyō Bot. Mağ. XX. p. 52.

Haв. Tappansha, leg. T. Kawarame et U. Monr, Oct. 1906 , (Nos. 1738 et 1772 ).

Distrib. Himalaya to Japan and China; recently found in Luzon. 


\section{Evodia Forst.}

Evodia meliæfolia Lenth. Fl. Hongk. p. 58; Hoor. f. Fl. Brit. Ind. I. p. 490 ; Fonbes et Hearst. Ind. Fl. Sin. I p. 104 ; Henny, List Pl. Formos. p. 24 ; Diels, Fl. Centr. Chin. p. 423 ; Matsum. et Hayata, Enum. Pl. Formos. p. 70 .

Megazotrya meliaefolia HANCE, in WALP. Ann. II. p. 259.

Evodia glauca MiQ. in Ann. Mus. Bot. Iugd.-Bat. IIT. p. 23.

HAв. Taitō: Dakunsha, leg. T. KawaKanI et U. Mori, Dec. 1906, (No. 2177).

Distrib. South China and southern part of Japan.

\section{SFimmia Thunb.}

Skimmia japonica Thunb. Fl. Jap. pp. 4 et 62 ; Frarch. et Savat. Enum. Pl. Jap. II. p. 311 ; DC. Prodr. II. p. 18 ; ITō et Matsurr. Tent. Fl. Lutch. p. 357 ; Drels, Fl. Centr. Chiu. p. 424 ; Hayata, in Tōkyō Bot. Mag. XX. p. 56 ; Menrin, in Philipp. Journ. Sci. I. Supp. Bot. p. 201.

Hab. in monte Morrison, ad 7500 ped. alt., leg. T. KawaKamr et U. Moni, Oct. 1906, (Nos. 2060 et 2059); Tōzan, in montibus Morrison, leg. G. Nakahara, Oct. 1906.

Drstrib. Himalaya, central China and Japan throughout; recently found in the Philippine islands.

\section{MIurraya Linn.}

Murraya exotica LinN.; DC. Prodr. I. p. 537 ; ВеNтh. Fl. Hougk. p. 56 ; Fonbes et Hexss. Ind. Fl. Sin. I. p. 159 ; Hook. et Arix. Bot. Beech. Voy. p. 260 ; WIght, Ic. Pl. Ind. or. t. 96 ; Bentr. Fl. Austral. I. p. 369 ; Ourv. in Journ. Linn. Soc. V. Supp.-2, p. 28 ; Hoor. f. Fl. Brit. Ind. I. p. 502 ; Maxmr. in Mél. Biol. XII. p. 429; Matsunr. et Hayata, Enum. Pl. Formos. p. 74.

Hab. Taitō: Kōshūsha, leg. T. Kawakami et U. Mori, Dec. 1906, (No. 1837). 
Distrib. Widely spread in tropical Asia and Polynesia.

\section{Ilicineæ.}

There are three species belonging to this family ; the specimens are all very imperfect and therefore they are not determinable.

\section{Celastrineæ.}

\section{Euonymus LiNN.}

In this genus, we had previously four species from the lowland. Twormore species are found in the montane zone.

Euonymus echinatus WALL.; LAwson, in Hoor. f. Fl. Brit. Ind. I. p. 610 ; Fonbes et Hemst. Ind. Fl. Sin. I. p. 119 ; Itō et Matsur. Tent. FI. Lutch. p. 371.

$\mathrm{H}_{\mathrm{AB}}$. in monte Morrison, ad 7000 ped. alt., (No. 1721); in eodem monte, ad 9000 ped. alt., (No. 2004), log. T. Kawar.un et U. Morr, Nov. 1906 ; Tōzan, in montibus Morrison, leğ. G. Nakahara, Oct. 1906 ; Nantō: Mushazan, leg. T. Kawakaır et U. МГor, Augr. 1906, (No. 1122).

Distrib. The Himalayas, central China and the Loo-choo islands.

Euonymus trichocarpus IIAYATA, sp. nov. Ramuli trichotome divaricati sub-tetragoni glabri. Folia opposita petiolata, petiolis circ. $1 \mathrm{~cm}$. longis semi-teretibus, laminis oblongo-ellipticis $6-7 \mathrm{~cm}$. longis $4 \mathrm{~cm}$. latis apice obtusis vel acutis basi rotundatis vel obtusis rarius leviter angustis margine serrulatis, serrulis obtusis, venis utraque prominulis. Cyma (ar ramulos terminales) laterales oppositx pauciflore. Flores ignoti. Capsula depresso-globosa 6-7 mm. in diametro aequans hreve echinulata, echinulis $1 \mathrm{~mm}$. longis. 
Hab. in monte Morrison, 7000 ped. alt., leg. T. Kawakami et U. Mori, Oct. 1906, (No. 1791).

This new plant resembles $E$. echinatus WALL. ; but differs from it in having very short and densely, covering spines on the fruit.

\section{Celastrus LinN.}

Celastrus articulatus Thuns. Fl. Jap. p. 97 ; DC. Prodr. II. p. 7 ; Maxnr. in Mél. Biol. XI. p. 200 ; Franchet, Pl. David. p. 70 ; Mir. Prol. Fl. Jap. p. 17 ; Fraxch. et Savat. Enum. Pl. Jap. I. p. 80 ; Sieb. et Zucc. Fl. Jap. Fam. Nat. I. p. 149 ; A. GraY, Bot. Jap. p. 384 ; Forbes et Heust. Ind. Fl. Sin. I. p. 122 ; Hennx, List Pl. Eormos. p. 27 ; Drels, Fl. Centr. Chin. p. 446 ; Palimin, Conspect. Fl. Korex, I. p. 54 ; Matsumr. et Hayata, Enum. Pl. Formos. p. 84 .

Häв. Taitō: Iryokukakusha, leg. T. KawaKanI et U. Mori, Dec. 1906, (No. 2178).

Distrib. Central and northern China, and Japan northward to Saghalien.

\section{Rhamneæ.}

\section{Rhamuns LinN.}

Rhamnus arguta MAxis. val. Nakaharai ILAYATA, n. v. Rami glabenimi, subalterne divaricati. Folia rotundato-orata basi rotunclata rel acuta apice acuminata vel cuspidata $7 \mathrm{~cm}$. longa $3 \frac{1}{2} \mathrm{~cm}$. lata margine proter basin apicemque crenulato-serrata, serraturis setaceo-acuminatis recurvatis, venis utrinque 5-6 omnibus angulo acuto emergentibus leviter arcuatis, membranacea, petiolis circ. 1 $\mathrm{cm}$. longis. Flores $f$ ignoti. Flores $q$ in axillis foliorum inferiorum circ. 5-6 fasciculati, fasciculis paulo supra-axillaribus cum ramulo connatis interdum 5-6 mm. supra axillam quasi insertis, pedicellis tenuibus petiolum paulo superantibus $1 \mathrm{~cm}$. longis apice incrassa- 
tis in tubum calycis turbinatum gradatim abeuntibus. Calycis lobi tubum triplo superantes circ. $3 \mathrm{~mm}$. longi lanceolati apice callosi trinervii. Rudimenta petalorum staminaque filiformia minutissima $\frac{1}{2} \mathrm{~mm}$. longa. Orarium globosum $1 \mathrm{~mm}$. longum e tubo exsertum in stylum cylindraceum $2 \mathrm{~mm}$. longum attenuatum, stylo apice $3-4$ fido, ramis $1 \frac{1}{2} \mathrm{~mm}$. longis stigmatosis recurvatopatentibus. Fructus ignotus.

Hab. Taichū: Binōshō, leg. G. Nakahara, Feb. 1907.

Distrib. Type: North China.

The present variety differs from the type in having slender flowers and longer styles, and especially in the supra-axillary inflorescence.

\section{Sapindaceæ.}

Acer Linn.

Three species belonging to this genus have been known hitherto from the low districts. We have here more four species, all confined to the high regions. The specimens are all in too imperfect a state for exact determination.

Acer sp. (aff. A. micrantho S. et Z.). Ramuli glabri. Folia ambitu orbicularia $7 \mathrm{~cm}$. in diametro xquantia palmatim 7 -loba, lobis lanceolatis acuminatis, duplicato-dentatis, lobo terminali $\check{5} \mathrm{~cm}$. longo $1 \frac{1}{2} \mathrm{~cm}$. lato, lobis infimis brevioribus $2 \frac{1}{2} \mathrm{~cm}$. longis, petiolis $2 \mathrm{~cm}$. longis.

$\mathrm{H}_{\mathrm{AB}}$. Taitō: Bataiankei, in monte Lagalan, ad 5000 ped. alt., leg. N. Konishi, Juni. 1902.

Acer sp. (aff. A. cratcegifolio S. et $/$.). Ramuli glabri, atropurpurei Folia ovato-cordata 5 -nervia, $8 \mathrm{~cm}$. longa $5 \mathrm{~cm}$. lata leviter 3 -loba, 
lobis inconspicuis obtusissimis, margine præter apicem duplicatoserrata, apice acuminata vel cuspidata, cuspidibus serrulatis, petiolis circ. $3 \mathrm{~cm}$. longis.

HАв, in monte Morrison.

Acer sp. (aff. A. rufinervi S. et Z.). Ramuli palliduli exsiccato nigricantes. Folia ambitu cordata octagona leviter 5loba, lobis brevissimis cuspidatis, cuspidibus terminalibus angustis linearibus, lateralibus latioribus serrulatis, infimis brevissimis, basi cordata, margine præter cuspidem duplicato-serrata, 9-10 cm. longa $7 \mathrm{~cm}$. lata coriacea longe petiolata, petiolis $6-7 \mathrm{~cm}$. longis.

Nom. iNDrG. : Lankas-lain=Acer foliis magnis.

HAB. Taitō: Bataiankei, ad 7600 ped. alt., leg. N. KonishI, Juni. 1902, (No. A. 11) ; in monte Morrison, ad 7500 ped. alt., (No. 1874); ad 6000 ped. alt., (No 1798), leg. T. Kawakami et U. Mori, Nov. 1906 ; Tōzan, in montibus Morrison, leg. G. Nakahara, Oct. 1906.

Acer sp. (aff. A. picto Thuns.). Ramuli palliduli glabri. Folia ambitu late orbicularia $7 \mathrm{~cm}$. longa $10 \mathrm{~cm}$. lata palmatim 5 -loba basi cordata, lobis triangularibus cuspidatis $3-3 \frac{1}{2} \mathrm{~cm}$. longis $2 \mathrm{~cm}$. latis vel latioribus, margine serrulatis, serraturis acutis, venis subtus pilosiusculis demum glabris. Cymæ terminales. Flores ignoti. Carpella elliptico-oblonga lenticularia $4 \mathrm{~mm}$. longa, alis dimidiatoobovatis divaricantibus cum carpello $2-2 \frac{1}{2} \mathrm{~cm}$. longis.

Hab. Chōsōkei, leg. G. Nakahara, Juli. 1905, (No. 161); Shintiku, in rivulos Taitō, leg. T. Kawakami et U. Mori, Juli. 1906, (No. 1426) ; Taitō : Taironkōsha, leg. T. Kawakaiı et U. Mori, Nov. 1906 , (No. 1842). 


\section{Cardiospermum LrNs.}

Cardiospermum Halicacabum Linn. Sp. PI. ed-2, p. 525 ; DC. Prodr. I. p. 601 ; BeNth. Fl. Hongk. p. 46, et Fl. Austral. I. p. 453 ; HANCE, in Journ. Bot. (1878) p. 226 ; Bot. Mag. t. 1049 ; Hiens, in Hook. f. Fl. Brit. Ind. I. p. 670 ; Forbes et Hexsu. Ind. Fl. Sin. I. p. 138 ; Henry, List. Pl. Formos. p. 28 ; Diels, FI. Cent. Chin. p. 450 ; Matsur. et Hayata, Enum. Pl. Formos. p. 94.

Cardiospermum microcarpum H. B. K.; HANcE, in Journ. Linn. Soc. XIII. p. 101, et in Journ. Bot. (1878) p. 226 ; DC. Prodr. I. p. 601.

Hab. 'Toroku, leg. T. KawaKami et U. Mori', Nov. 1906, (No. 176).

Distrib. Common in the warm regions of Asia, Africa, and America, and also in Australia.

\section{Anacardiaceæ.}

\section{Rhus Lins.}

Rhus intermedia Hayata, sp. nov. radicans vel volubilis. Folia trifoliolata cum petiolis circ. $30 \mathrm{~cm}$. longa longe petiolata, petiolis $9-10 \mathrm{~cm}$. longis puberulis foliolo terminali æquilongis, foliolis lateralibus oblongis acutis basi rotundatis obliquis $13 \mathrm{~cm}$. longis breviter petiolulatis, petiolulis $3 \mathrm{~mm}$. longis, foliolis terminalibus longe petiolulatis, petiolulis $3 \mathrm{~cm}$. longis, laininis oblongo-ovatis apice acutis vel breviter acuminatis $15 \mathrm{~cm}$. longis $7 \frac{1}{2} \mathrm{~cm}$. latis, integris, subtus imprimis costis et nervis pilosis demum glabris Drupæ late globosæ compressæ breve apiculatæ $5 \mathrm{~cm}$. latæ, totidem longæ viridi-flavescentes pilis setaceis brevioribus dense obtectre.

$\mathrm{H}_{\mathrm{AB}}$. in monte Morrison, ad 7500 ped. alt., leg. 'T. Kawakami et U. Morr, Oct. 1906, (No. 2024). 
The present plant is in every respect very like Rluus Toxicodendron Lins., but differs from it in having densely bristled fruits. At first sight, this new species appears to be referable to Trichocarpce, on account of its bristled exocarpium. Studying the plant carefully, I have found that it should be referred to Venenatce by reason of the mesocarpium and general characters of the fruit. The plant may better be placed between the two sections.

\section{Pistacia LinN.}

Pistacia formosana Matsuivura, in Tōkyō Bot. Mag. XV. p. 40; Matsum. et Hayata, Enum. Pl. Formos. p. 99, t. 9.

Häв. in montibus Akō, leg. N. Konishi, Oct. 1903, (No. A. 5.).

Distrib. An allied species $P$. chinensis Bunge is found in central and northern China.

\section{Leguminosæ.}

\section{Crotalaria LINN.}

Crotalaria formosana Matsumura, in Itö et Matsurs. in Tent. Fl. Lutch. p. 395 ; Matsum. et Hayata, Enum. Pl. Formos. p. 103.

HaB. Tappansha, leg. T. KaWakayi et U. Mori, Oct. 1906, (No. 1760).

Closely resembles $C$. linifolia Lins. ; probably a form of it.

\section{Desmodium Desv.}

Desmodium parvifolium 1)C. ; Iтü et Matsum. 1. c. p. 418 ; Matsusr. et Hayata, Enum. Pl. Formos. p. 107.

Haв. Suizan, in montibus Morrison, ad 7702 ped. alt., leg. S. Nagasawa, Oct. 1905, (No. 669); in monte Morrison, leg. T. Kawakami et U. Mori, Nor. 1906, (No. 1916). 
Dis'rRib. Widely distributed in India, Malay, and through central and southern China eastward to Japan.

Desmodium polycarpum DC. ; ITō et Matsun. 1. c. p. 416 ; MTASUM. et HaYata, 1. c. p. 107.

Hab. Sanchōki, ad 3000 ped. alt., Oct. 1905, (No. 728), et Tōhosha, Nor. 1905, (No. 709), leg. S. Nagasawa.

Distrib. Tropical Asia, Polynesia, through southern China to Japan.

Desmodium pulchellum Benth. ; Itō et MLatsus. l. c. p. 412 ; Matsesw. et Hayata, l. c. p. 107.

Hab. Kagi: Shukukōshō, leg. T. KaWtaKani et U. Mori, Oct. 1906, (No. 1976).

Distrib. Tropical Asia and the Philippine islands.

Desmodium sinuatum Bucire; Itō et Mitsuxr. I. c. p. 416 ; Matscir. et Hayata, 1. c. p. 108.

Haв. Kagi : Shukukōshō, leg. T. Kawakami et U. Mori, Oct. 1906, (No. 1746).

Distrib. Tropical Asia and southern China.

\section{Dumasia DC.}

Dumasia bicolor Harata, sp. nov. Herba rolubiles in totum pubescentes. Folia bicoloria pubescentia pimatim 3-foliolata 12 $\mathrm{cm}$. lata $18 \mathrm{~cm}$. longa longe petiolata, petiolis basi crassiusculis $9 \mathrm{~cm}$. longis foliolo terminali subrequilongis, foliolis lateralibus brevissime petiolulatis, petiolulis $3 \mathrm{~mm}$. longis, laminis rotundatooratis basi truncatis vel acutis apice rotundatis minutissime aristatemucronatis 3-nervis, foliolis terminalibus is lateralibus conformilus longe petiolulatis, petiolulis 20 (m. longis, laminis ovatis 
basi acutis majoribus $6 \frac{1}{2} \mathrm{~cm}$. longis $4 \frac{1}{2} \mathrm{~cm}$. latis, stipulis setaceis, stipellis filiformibus minutis, interdum folia superiora simplicia. Flores in racemos circ. $10 \mathrm{~cm}$. longos axillares dispositi, bracteis parvis angustis, bracteolis minutis. Calycis tubus cylindraceus, $9 \mathrm{~mm}$. longus basi postice gibberosus, ore valde oblique truncato antice acuto. Vexillum obovatum apice emarginatum $14 \mathrm{~mm}$. longum $7 \mathrm{~mm}$. latum late unguiculatum, lamina unguem subrquanti supra medium postice inflexa basin angusta ad unguem abeunti et auriculata. Alæ longe unguiculatæ $14 \mathrm{~mm}$. longæ, laminis oblongis, unguibus linearibus laminam duplo superantibus, carina adhærentes. Carina alis brevior obtusa. Stamen vexillare liberum, cætera connata. Antheræ uniformes. Ovarium villosum substipitatum, stipite $1 \mathrm{~mm}$. longo ; stylus supra ovarium filiformis erectus supra medium dilatus, superne inflexus subulatus imberbis, stigmate terminali. Legumen subsessile villosum semper monospermum.

HAB. Suizan, in montibus Morrison, ad 7702 ped. alt., leg. S. Nagasawa, Oct. 1905, (No. 667); Tõzan, in montibus Morrison, leg. G. Nakahara, Oct. 1906.

The present plant is, in all respects, like D. villosa DC. But, in this new species, the seed is always one in each pod and the standard has distinct spurs on both sides of the lamina. In these respects, I think the plant is specifically separable from $D$. villosa DC. The leaves of the specimen, upon which the above description is based, are of a thinly hairy form. Here is another form with villose leaves which are much smaller than the leaves of the other form. 'The villose form seems to be a young stage of the other. 
Cajanus DC.

Cajanus indicus Sprexa.; Itō et Matsum. 1. c. p. 431 ; Matsum. et HAYATA, 1. c. p. 113.

Hab. Tōhosha, ad 2930 ped. alt., leg. S. Nagasawa, Nor. 190z, (No. 708).

Distrib. All over the tropics.

\section{Flemingia RoxB.}

Flemingia strobilifera R. Br.; Itū et Matsusr. 1.c. p. 432 ; Miststar. et Hayata, 1. c. p. 113.

HAB. Suizan, in montibus Morrison, ad 7702 ped. alt., leg. S. Nagasatwa, Oct. 1905, (No. 782).

Distrib. The Himalayas, Ceylon, cast Bengal, Burma, Malacca, Malay, and the Philippine islands.

There is a little doubt about identifying my plant with the above species. In the present plant, the flowers in each bract are tor) few. In $F$. strobilifera $\mathrm{R}$. Br., the flowers are arranged in a raceme or a short spike within a large bract, while in my plant the flowers are not so numerous as to form either raceme or spike.

\section{Rosaceæ.}

\section{Prumus Lins.}

Prunus campanulata Mixar. in Mél. Biol. XI. p. 6998; Foribes et Hemsi. Ind. Fl. Sin. I. p. 218; Itō et Matsurr. Tent. Tl. Lutch. p. 446 ; Matsum. et Hayata, Enum. Pl. Formos. p. 117.

HАв. Taichū : Kashigatani, leg. G. Natshara, Feb. 1907.

Distrib. South China: Fokien; the Luo-choo islands.

Prunus Kawakamii Ilaratı, sp. nov. Frutex, ramis novellis virgatis cortice cincreo-fusco glabro restitis. Folial hysteranthat 
ammua alterna (novella) brevissime petiolata, petiolis $4 \mathrm{~mm}$. longis semi-teretibus glabris, laminis basi acutis ovato-oblongis apice acutis margine glanduloso-serrulatis utraque pagine glabris, stipulis lanceolatis glanduloso-ciliolatis. Flores $5-6$ fasciculati pedunculati, pedunculis $7 \mathrm{~mm}$. longis teretibus glabris. Calyx hypogynus persistens; tubus breviter campanulatus glaber, intus disco adnato suffultus; lobis 5 ovatis $3 \mathrm{~mm}$. longis obtusis glanduloso-ciliatis horizontaliter patentibus. Petala calycis fauci affixa, ex ungue hreve cuneato-obovata, apice rotundata integerrima radiatim venosa tenera glabra horizontaliter patentia $6 \frac{1}{2} \mathrm{~mm}$. longa $4 \mathrm{~mm}$. lata. Stamina calycis fauci affixa longe exserta petalo vix longiora. Ovarium superum, oroideum cum stylo $6 \mathrm{~mm}$. longum, stigmate capitato-peltato.

Hab. Toroku : Kanōsha, leg. T. Kawatianr et U. Mori, Nov. 1906, (No. 1904).

Distrib. An allied species $P$. japonica Thuns. occurs in Japan and China.

Closely resembles $P$. japonica Thunb.; but differs from it in having peltately capitate stigmas, longer stamens and smaller petals.

\section{Spivaca LinN.}

Spiræa prunifolia Suez. et Zucc. fl. simplici ; Matsusr. et Hayat, Enum. Pl. Formos. p. 119.

HaB. in monte Morrison, leg. T. Kawakani et U. Mori, Oct. 1906, (No. 1795); Nantō: Hinokiyama, leg. G. NaKahara, Feb. 1907 ; Toroku : Küreikiyaku, leg. T. KawaKàr et U. Mori, Nov. 1906, (No. 1902).

Distrib. China throughout, Japan and Korea.

Spiræa sp. Suffintices nani glabri. Folia alterna subsessilia 
ovata apice obtusa basi acuta vel cuneata $1 \frac{1}{2} \mathrm{~cm}$. longa medio sursum denticulata basin versus integra, venis supra impressis subtus prominentibus. Fructus in cymas racemosas terminales dispositi. Carpella $2 \mathrm{~mm}$. longa breve rostrata.

HAB. in monte Morrison, ad 12000 ped. alt., leg. 'T. KaWAKAגI et U. Mori, Oct. 1906, (Nos. 2233 et 2296).

\section{Rubus Lins.}

Rubus corchorifolius LrNx. f. var. glaber MLıTsuxr. in Tōkyú l'ot. Mag. XV. p. 157 ; Matsun. et Hayata, Enum. PI. Formos. p. 120.

Haв. Taichū : Kashigatani, leg. G. Nakahara, Feb. 1907.

Distrib. Type: Japan and China.

Rubus elegans Hayata, (Pl. IV.), in Tōkyō Bot. Mag. XX. p. 7. Caulis humilis herbaceus basi suffruticosus simpliusculus suberectus 1-rarius 2-florus. Folia in totum subradicalia ambitu oblonga vel oblongo-lanceolata cum petiolis $6-7 \mathrm{~cm}$. longa $2 \mathrm{~cm}$. lata, petiolis $15 \mathrm{~cm}$. longis, pinnata $13-15$-foliolata, foliolis oboratis 1 $1.5 \mathrm{~cm}$. longis $\frac{1}{2} \mathrm{~cm}$. latis, terminalibus interdum tri-lobatis, serratis, serris acutis, ad petiolum et costas pinnarum aculeolata, stipulis adnatis subulatis linearibus circ. $1 \mathrm{~cm}$. longis. Flores majusculi, pedunculati, peduneulis $5-6 \mathrm{~cm}$. longis, 1-bracteati, bracteis minutis acutis $2 \mathrm{~mm}$. longis. Flores patentes $22 \mathrm{~mm}$. in diametro aquantes. Calycis lobi ovato-triangulares aculeato-acuminati $8 \mathrm{~mm}$. longi extus pubescentes. Petala late ovata $9 \mathrm{~mm}$. longia apice obtusissima, basi brevissime unguiculata. Stamina numerosa, filament is planis glabris. Capitulum fructiferum ovato-globosmm $1 \mathrm{~cm}$. longum vel longins. Receptaculum ovato-globosum.

HAB. Ganzan, ad 9141 ped. alt., in montibus Morrison, leg. S. Nagasawa, Oet. 1905, (No. 691); in monte Morrison, ad 10000 ped. alt., leg. T. KaWakani et U. Mori, Oet. 1906, (No. 1956). 
As the original description is drawn from an imperfect specimen, I have taken the liberty of repeating the description of this plant, basing the above account upon the most perfect materials.

Rubus fraxinifolius Porr.; MrQ. Fl. Ind. Bat. I. p. 376 ; Hook. f. Fl. Brit. Ind. II. p. 342 ; Maxis. in Mél. Biol. VIII. p. 391 ; Matsuxr. in Tōkyō Bot. Mag. XVI. p. 4 ; Matsunr. et Hayata, Enum. Pl. Formos. p. 121.

Hab. Tōhosha, ad 2930 ped. alt., leg. S. Nagasawa, Nov. 1905, (No. 605).

Distrib. Java and the Philippine islands. The occurrence of this Rubus in India is a little doubtful.

Rubus pectinellus Maxir. in Mél. Biol. VIII. p. 374; Franch, et Savat. Enum. Pl. Jap. I. p. 122 ; Hayata, in Tōkyō Bot. Mag. XX. p. 55.

Hab. Tōzan, in montibus Morrison, leg. G. Nakahara, Oct. 1906 ; in monte Morrison, ad 7500 ped. alt., leg. T. KawaKanr et U. Mori, Oct. 1906, (No. 1799).

Distrib. Southern part of Japan, and also recently found in Litzon.

Rubus pentalobus Harata, sp. nov. Suffirtex scandens in totum rilloso-tomentosus. Folia longe petiolata tomentoso-villosa, petiolis $5-10 \mathrm{~cm}$. longis, laminis ambitu cordato-rotundatis $5-7 \mathrm{~cm}$. in diametro æquantibus læviter 5-lobis apice rotundatis basi cordatis, lobis rotundatis irregulariter denticulatis palmatim 5-7 nerviis supra pilosiusculis subtus villoso-tomentosis pallidioribus, venis subtus prominentibus, stipulis laciniatis $13 \mathrm{~mm}$. longis. Flores axillares solitarii rel gemini pedunculati, peclunculis $1 \mathrm{~cm}$. longis, 2-3 bracteolatis, bracteolis minutis laciniatis. Calycis lobi orati apice laciniati tomentosi $1 \mathrm{~cm}$. longi. Achænia drupacea.

$\mathrm{H}_{\mathrm{AB}}$. in monte Morrison, ad 10000 ped. alt., leg: T. KawaKamI 
et U. Morr, Oct. 1906, (Nos. 2123 et 2265); Biōritsu : Hakkeirin, leg. T. Kawakami et U. Mori, Juli. 1906, (No. 1096).

The present Rubus is near $R$. pectinellus Maxn.; but differs from it in having unármed sepals and five lobed leaves. The leaves are much more tomentose, and somewhat tuberculate on the upper surface.

Rubus Rolfei VIDAL var. lanatus Hayata, n. v. Suffrutices erecti dense lanati demum glabri. Folia 5- rarius 3-loba, ambitu cordato-orbicularia $3-5 \mathrm{~cm}$. in diametro æquantia, lobis rotundatis rel obtusis irregulariter denticulatis, utraque pagine dense lanata supra demum glabra intra venulas prominente tuberculata subtus dense albo- vel ferrugineo-lanata, petiolis $2-3 \mathrm{~cm}$. longis, stipulis oratis laceratis $12 \mathrm{~mm}$. longis. Flores ad apicem ramulorum 2-3 fasciculati vel subaxillares, ad basin calycis $2-3$ bracteati, bracteis majusculis truncatis laceratis $9 \mathrm{~mm}$. longis totidem latis submembranaceis. Calyx turbinatus $1 \frac{1}{2} \mathrm{~cm}$. longus, lobis ovatis $9 \mathrm{~mm}$. longis acuminatis extus villosis intus pubescentibus crassis. Fructus ignotus.

Hab. Seizan, in montibus Morrison, leg. S. Nagasawa, Nor. 1905, (No. 574); in monte Morrison, ad 10000 ped. alt., leg. T. Kawakami et U. Mori, Oct. 1906, (No. 2263).

The present variety differs from the type in having more densely woolly leaves and much larger flower's.

Distrib. type: the Philippine islands.

Rubus rosæfolius S.r. var. hirsutus HaYaT, 11, v. Ramuli aculeati pilosi, pilis validiusculis, aculeis parvis falcatis acuminatis. Folia ambitu ovato-acuminata hirsuta cum petiolis 5-(i $\mathrm{cm}$. longa 5-foliolata, foliolis lateralibus subsessilibus vel hreve petiolulatis oblongoellipticis $1 \mathrm{~cm}$. longis vel longioribus dentatis, dentibus acutis, 
foliolis terminalibus petiolulatis, petiolulis $5 \mathrm{~mm}$. longis, laminis ovato-lanceolatis duplicato-dentatis, clentibus acuminatis, petiolis et costis aculeatis, stipulis subulatis ciliolatis. Flores terminales vel laterales sæpe solitarii, pedunculati. Calycis lobi ovato-triangulares longe caudati, caudis linearibus $6 \mathrm{~mm}$. longis, extus pubescentes. Petala ovata $11 \mathrm{~mm}$. longa $8 \mathrm{~mm}$. lata apice rotundata basi acuta. Stamina numerosa, filamentis planis. Fructus ignotus.

Hab. in monte Morrison, ad 12000 ped. alt., leg. T. KaWaKanI et U. Mori, Oct. 1906, (No. 2264).

The present variety differs from the type in having hirsute leaves and in all respects is much smaller than the type.

Distrirs. Type : common in the warmer parts of India, China and Japan.

\section{Fragaria LiNN.}

Fragaria sp. Caulis brevis validiusculus longe stoloniferus. Folia in totum radicalia longe petiolata, petiolis $3-4 \mathrm{~cm}$. longis tomentosis, trifoliolata, foliolis lateralibus rotundatis $13 \mathrm{~mm}$. longis $11 \mathrm{~mm}$. latis apice truncatis basi valde obliquis inferne rotundatis superne acutis et integris, foliolis terminalibus late obovatis $15 \mathrm{~mm}$. longis $14 \mathrm{~mm}$. latis apice rotundatis vel truncatis basi acutis præter basin dentatis, subsessilibus subtus sericeo-pilosis supra pilosiusculis, dentibus rotundato-acutis, venis supra plicato-impressis subtus prominentibus, stipulis membranaceis ad basin petiolorum connatis $11 \mathrm{~mm}$. longis $4 \mathrm{~mm}$. latis aristato-acutis extus pilosis. Pedunculi uniflori tenues piloso-tomentosi $1 \frac{1}{2} \mathrm{~cm}-2 \mathrm{~cm}$. longi.

Hab. in monte Momison, leg. T. Kawakami et U. Moni, Oct. 1906, (No. 2236); Tōzan, in montibus Morrison, leg. G. Nakahari, Nov. 1906. 
Mr. T. Kawakanir tells me that the fruit of this Fragaria is ver'y delicious.

\section{Potentilla LinN.}

Potentilla gelida C. A. MIex.; Ledeb. Fl. Ross. II. p. 59 ; Hook. f. Fl. Brit. Ind. II. p. 357; Diels, Fl. Tin ling shan, in ExGr. Bot. Jahrb. XXXVI. Beibl. p. 56 ; HАYaTA, in Tōkyō Bot. Nag. XX. p. 73.

Potentilla grandiflore Linx.; Wagner, Deut. FI. ed-3, p. 359; Thoxé, FI. Deut. Öst. 1. Schw. III. p. 70.

HAB. in monte Morrison, ad 13094 ped. alt., leg. S. NAGASAwA, Nov. 1905, (No. 683).

Distrib. Extends to Europe, North India, central ('hina, Japan, East Siberia, and the Kurile and Aleutian islands.

The species seems to vary over a very wide range, and especially so in the size of flowers.

Potentilla leuconota Don, "Prodr. p. $230 "$; Hoor. f. Fl. Brit. Ind. II. p. 352 ; DIELS, Fl. Centr. Chin. p. 403.

rar. morrisonicola HAYATA, n. จ. (Pl. V).

Potentilla leuconota Hayata, in Tōkyō Bot. Mag. XX. p. 74.

Caulis sericeo-pilosus erectus circ. $15 \mathrm{~cm}$. longus. Folia subradicalia pinnata in ambitu oblanceolata obtusa $10 \mathrm{~cm}$. longa cire. 19-foliolata, foliolis sessilibus obovatis obtusis $1 \mathrm{~cm}$. longis argute dentatis supra pilosiusculis subtus sericeo-pilosis, petiolis appresse pilosis, stipulis majusculis seariosis cire. $3 \mathrm{~cm}$. longis an basin petiolorum adnatis integris. Folia caulina radicali conformia ser multo minora, panca sxpe ad medium canlis 1-2. F'lores ad apicem canlis 9-8 fasciculati subumbellati 1 -2-bracteati, pedicellis $1 \mathrm{~cm}$. longis. Flores patentes $8 \mathrm{~mm}$. in diametro xquantes, bracteolis angustis integris. Calycis lohi ovati acuti sericei. Potala late ob- 
orata, basi leviter angusta, apice rotundata. Stamina $10(-20$ ?). Achænia circ. 15 glabra.

Haß. in monte Morrison, ad 13094 ped. alt., leg. S. NaGaSawa, Nor. 1905 , (No. 732); in eodem loco, T. Kawakami et G. Nakahara.

Distrib. The type is rather of the alpine character, being found in high mountains of Asia such as the Himalayas, and those of Borneo and west central China.

In my former paper above cited, I referred this Formosan species to Potentilla leuconota Don. After studying more carefully, I found that there is a little difference between this and that. It is not, however, without hesitation, that I have described it as a new variety. The present plant differs from the type mainly in the absence of whorled leaves at the base of an umbel.

\section{Sibbaldia LinN.}

Sibbaldia procumbens Lins. Sp. Pl. ed-2, p. 406 ; Diels, Fl. Centr. Chin. p. 404, et Fl. Tsin ling shan, in ExGL. Bot. Jahrb. XXXVI. Beibl. p. 56 ; Thomé, Fl. Deut. Öst. u. Schw. III. p. 60; Aschenson et Græbn. Syn. Mitt. Fl. VI.-1, p. 661 ; Wagker, Deut. Fl. ed-3, p. 361 ; Hayata, in Tōkyō Bot. Mag. XX. p. 58; Makño, in Tōkyō Bot. Mag. XV. p. 98.

Potentilla Sibbaldi HaLler f. in "Ser. Mus. Helvet. I. p. 51 "; Ноок. f. F1. Brit. Ind. II. p. 345.

Sibbaldia cuneata KunzE, in Linnæa, XX. p. 59 ; EDGEW. in Journ. Linn. Soc. XX. p. 44 .

HAB. ad verticem montis Morrison, 13094 ped. alt., leg. S. Nagasawa, Nor. 1905, (No. 757) ; in eodem loco, ad 13000 ped. alt., leg. T. Kawakami et U. Mori, Oct. 1906, (No. 2256).

Distrim. This plant having had a wide range in the glacial period is now found here and there in the polar and alpine regions of Europe and Asia. 


\section{Rosu LinN.}

Rosa sp. Suffrutices spinosissimi, aculeis albidis rectis subulatis, ramis patentibus gracilibus. Folia pinnata 7 -11-foliolata. glabra ambitu elliptica cum petiolis $5 \mathrm{~cm}$. longa $2 \frac{1}{2} \mathrm{~cm}$. lata, petiolis gracilibus minutissime aculeolatis, foliolis subsessilibus firmis late obovatis vel subrotundatis $13 \mathrm{~cm}$. longis $7 \mathrm{~mm}$. latis medio denticulatis apicem versus dentatis, dentibus acutis, stipulis petiolo adnatis apice liberis acutis glabris glanduloso-scrulatis, serrulis argutis. Flores secus ramulos quasi racemosi, e gemmis solitarii breviter pedunculati, pedunculis $1 \frac{1}{2} \mathrm{~cm}$. longis apice in calycis tubo abeuntibus. Calycis tubi post anthesin pyriformes $8 \mathrm{~mm}$. longi apice constricti basi attenuati, lobis integris lanceolatis longe acuminatis intus lanato-pubescentibus extus parce pubescentibus marginibus parcissime glandulosis. Petala ignota. Carpella 4-5 trigona $5 \mathrm{~mm}$. longa apice hirsuta, stylis persistentibus.

Hab. in monte Morrison, leg. S. Nagasawa, Nov. 1905, (Nos. 572 et 618$)$; eodem loco, ad 11000 ped. alt., leg. T. Kawak.un et U. Mori, Nov. 1906, (No. 2293).

The present $R$ osa is very like $R$. xanthina. Lindu and $R$. Willmottice Henss. in Bot. Mag. t. 8186 ; but the serrature of the leaves of this plant is far too acute for those species. My specimen has the spines arranged in opposite position as $R$. Willmottice.

\section{Rosa sp.}

Hab. Arizan, in montibus Morrison, leg. G. Natimara, Oct. 1906.

\section{Saxifragaceæ.}

Astilbe Hus.

No species belonging to this genus has been recorded hitherto 
from the island. The following two species and rariety are the first recorded from Formosa, and come from the hilly regions. Astilbe chinensis Franch. et SAvat. Eumm. Pl. Jap. I. p. 144 (var. japonica); Franchet, Pl. David. p. 121 (var. Davidi); Fonbes et Hessst. Ind. Fl. Sin I. p. 265 ; Diets, Fl. Centr. Chin. p. 363.

Hoteia chinensis Mraxtr. Prim. Fl. Amur. p. 120 ; Wate. Ann. VII. p. 889. Astilbe odontophylla MIQ. in Ann. Mus. Bot. Lugd.-Bat. III. p. 96.

HAB. Ganzan, in montibus Morrison, ad 9141 ped. alt., leg. S. Nagasawa, Oct. 1905, (No. 636).

Distris. Amurland, Japan, and central and northern China. There is a little doubt about this being A. chinensis Fr. et SAv.

Astilbe chinensis F'tanch. et Sat. var. longicarpa HaYata, n. $\mathrm{r}$. Herba circ. $1 \mathrm{~m}$. alta vel altior erecta, caulibus glabris. Folia radicalia ignota. Folia caulina longe petiolata ternato-bipinnata, foliolis lateralibus ovatis terminalibus acuminato-ovatis duplicato serratis, serris acuminatis, petiolulatis. Raceni $30 \mathrm{~cm}$. longi basi $12 \mathrm{~cm}$. lati pyramidales, racemulis deorsum pedunculatis sursum subsessilibus. Flores brevissime pedicellati basi calycis bracteolati. Calyces $1 \frac{1}{2} \mathrm{~mm}$. longi, lobis ovatis truncatis. Petala spathulatoobovata vix $1 \mathrm{~mm}$. longa apice minute apiculata margine integra diffusa. Stamina 10 multo exserta, petalum duplo superantia. Carpella 2, distincta. Fructus cylindraceo-oroidei breve rostrati cum rostris $4 \mathrm{~mm}$. longi.

Hab. Tappansha, leg. T. KawaKami et U. Mori, Oct. 1906, (No. 1743); in monte Morrison, ad 8500 ped. alt., leg. T. Kawakani et U. Morr, Oct. 1906, (No. 1990).

Very like the type; but the fruits of this variety are much longer than those of the type.

Astilbe macroflora Hayata, sp. nor. Mferba circ. $30 \mathrm{~cm}$. alta, 
erecta basi squamis membranaceis fuscis late ovatis acuminatis numerosis obvallata, rhizomate crasso fibris numerosissimis ohtecto, caulibus petiolisque pilis fuscis parce restitis. Folia radicalia et caulina ternato-bipinnata, petiolis lamina subæqualibus ad ramificationes parce longe pilosis, foliolis ovatis vel oblongis basi cordatis rarius leviter 3 -lobatis $3 \mathrm{~cm}$. longis $2 \frac{1}{2} \mathrm{~cm}$. latis supra parcissime pilis scabris vestitis subtus ad costas pilosiusculis biserratis, serraturis primariis majusculis patulis breviter acuminatis secundariis minutis seta brevi terminatis, foliolis terminalibus longe, eis lateralibus brevissime petiolulatis, stipulis membranaceis ovatis acuminatis fuscis erectis. Racemi folio florali minuto instructi $10 \mathrm{~cm}$. longi basi $5 \mathrm{~cm}$. lati pyramidales, rhachibus fusco-hirtis, bracteis stipula conformibus erectis. Flores versus apicem racemulorum subpedunculatorum dense congesti, breviter pedicellati. Calyx $3 \frac{1}{2} \mathrm{~mm}$. longus campanulatus lutescens, lobis ovatis obtusis $3 \mathrm{~mm}$. longis trinerviis crassiusculis tubo multo longioribus. Petala spathulata $4 \mathrm{~mm}$. longa $1 \mathrm{~mm}$. lata ralde exserta margine minute ciliato-serrata, utraque latere $2-3$ serris, diffusa. Stamina 10 disco calycis inserta. Carpella 2, distincta. Fructus breviter rostrati cum rostro $5 \mathrm{~mm}$. longi, rostris carpello æquilongis. Semina scobiformia oblique fusiformia $1 \mathrm{~mm}$. longa.

НАB. in monte Morrison, leg. G. NaKahara (?), Oct. 1906.

Remarkable for the large form of flowers and the capitate racemés.

\section{Chrysospleninm LINN.}

Chrysosplenium sp. Herbe humiles piloso-pubescentes. Folia membranacea longe petiolata, petiolis $1 \frac{1}{2} \mathrm{~cm}$. longis lamina vix longioribus planis subalatis pilosis basi dilatis, laminis late ovatis rel orbicularibus $1 \frac{1}{2} \mathrm{~cm}$. longis apice rotundatis basi abrupte acutis vel 
truncatis brevissime attenuatis in petiolum abeuntibus margine crenatis, crenis emarginatis apice callis crassatis minutis suffultis, supra pilosiusculis, pilis subsetaceis, subtus subglabris. Flores ignoti.

$\mathrm{H}_{\mathrm{AB}}$. in monte Morrison, ad 8000 ped. alt, leg. T. KawaKami et U. MonI, Oct. 1906, (No. 1993).

This is the only Chrysosplenium found in the island and must be a very interesting one. In the imperfectness of the specimen, the specifical determination is impossible.

\section{Mitella LinN.}

Mitella japonica Miq. in Ann. Mus. Bot. Lngd.-Bat. III. pp, 96 et 201, et Prol. Fl. Jap. pp. 260 et 365 ; Franch. et SAv. Enum. Pl. Jap. I. p. 147, excl. syn. (non Maxnr.); Makino, in Icon. Fl. Jap. Imp. Uni. Tōkyō, I.-2, p. 7, tt. IV. et V.; HaYaT. in Tōkyō Bot. Mag. XX. p. 54.

HAB. in monte Morrison, ad 8000 ped. alt., (No. 2035), et eodem loco, ad 12000 ped. alt., (No. 2131), leg. T. KawaKani et U. Mori, Oct. 1906 ; Tōzan, in monte Morrison, leg. G. Nakahara, Oct. 1906.

DisTRIB. In Japan ; very common in the valleys of the lowland hills. The distribution of the genus Mitella is rather limited, being found only in North America, Manchuria, East Siberia, Japan and Formosa.

The Morrison specimens are of a form having rather more divaricate and fimbriate petals and less tuberculate seeds. A more diverged form of this species was found in Japan by Mr. T. Makino who described it in Tōkyō Bot. Mag. XIX. p. 17 as a new variety integripetala. Another species $M$. acerina Makiso was found also in Japan.

\section{Parnassia LinN.}

Parnassia palustris Linn. Sp. Pl. ed-2, p. 391 ; DC. Prodr. I. p. 320 ; Maxrm. Ind. Fl. Pek. in Prim. Fl. Amur. p. 469 ; Hoэк. f. Fl. Brit. 
Ind. II. p. 401 ; Drude, in Limnea, XXXIX. p. 307 ; Fonbes et Hexst. Ind. Fl. Sin. I. p. 272 ; Hayata, in Tōkyō Bot. Mag. XX. p. 19.

$\mathrm{H}_{\mathrm{AB}}$. Ganzan, in montibus Morrison, ad 9141 ped. alt., leg. S. Nagasawa, Oct. 1905 ; in monte Morrison, ad 9000 ped. alt, leg. T. Kawakami et U.' Mori, Oct. 1906, (No. 2209).

Distrib. West Asia to Europe, and eastward to Japan; but not yet found in central China.

\section{Hydrangea Lins.}

Hydrangea chinensis Maxur. Revis. Hydrang. As. or. p. 7 ; Haxce, in Journ. Bot. (1878) p. 11; FonBes et HemsL. Ind. Fl. Sin. I. p. 273 ; Henry, List Pl. Formos. p. 41; Itō et Mímsum. Tent. Fl. Lutch. p. 461 ; Matsum. et Hayata, Enum. PI. Formos. p. 131.

HAB. in monte Morrison, ad 8000 ped. alt., leg. 'T. KAWAKAMI et U. Mori, Oct. 1906, (No. 2073) ; Taitō : Bushiseki, leg. T. Kaw.кnari et U. Mori, (No. 2185); Toroku : Nanshikiyakumansha, leg. T. KaWakani et U. Mori, Nov. 1906, (No. 1906).

Distrib. The Loo-choo islands and south central China.

Hydrangea glabra IIAYATA, sp. nov. (PI. VI.). Frutices al truncos scandentes, ramis glaberrimis cortice cinereo-fusco tectis. Folia oblonga petiolata, petiolis lamina 3-plo lnevioribus, laminis oblongis $11 \mathrm{~cm}$. longis $7 \mathrm{~cm}$. latis apice acutis basi obtusis rel acutis margine serratis, serraturis acutis, utraque pagine glabedrimis. Cymæ corymbosæe ternato-ramosæ, rhachibus tomentosis demum glabris. Flores exteriores steriles, sepalis 4 petaloideis late obovatis nerrosis basi breve euneatis apice rotundatis rel truncatis interdum emarginatis $15 \mathrm{~mm}$. longis $17 \mathrm{~mm}$. latis. Flores interiores fertiles. Capsula depresso-globosa apice truncata latere leviter compressa. Semina oblonga plana ala angusta cincta. 
HAB. in monte Morrison, ad 7500 ped. alt., leg. T. KaWAKAMI et U. MonI; Nov. 1906, (No. 1787).

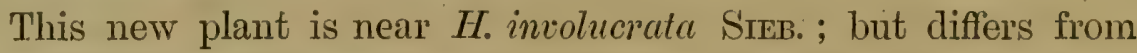
it in having quite glabrous leaves and more expanded cymes; from H. Kacakamii, this differs in having entire sepals of radial flowers and broad wings of seeds.

Hydrangea integra HaYata, sp. nov. (PI. VII). Frutices ad trumcos scandentes, ramis subglabris cortice fusco-rubro tectis. Folia petiolata oblongo-elliptica $22 \mathrm{~cm}$. longa $8 \mathrm{~cm}$. lata acuta rel cuspidato-acuta basi obtusa vel angusta integra utraque pagine glabra, laminis petiolum 3-plo superantibus, petiolis ferrugineorubris. Cymæ corymbosæ umbellato-fasciculatæ terminales $14 \mathrm{~cm}$. longæ $15 \mathrm{~cm}$. in diametro æquantes, rhachibus tomento subfloceoso dense tectis. Flores exteriores steriles, sepalis 2 petaloideis late rotundatis valde nervosis, altero minore $1 \frac{1}{2} \mathrm{~cm}$. lato altero majore $2 \mathrm{~cm}$. lato. Capsula hemispherica latere compressa $3-4 \mathrm{~mm}$. lata, calycis limbis obscuris, stylis persistentibus apice recurvis. Semina fusiformia longitudinaliter striata.

Hab, in monte Morrison, leg. T. Kawakanir et U. Mori, Oct. 1906, (No. 1723).

Somewhat resembles II. integrifolia, but easily distinguished in having narrowed base of the leaves.

Hydrangea Kawakamii Harata, sp. nov. (Pl. VIII). Frutices ad truncos scandentes, ramis tomentoso-pubescentibus demum subglabris, cortice cincreo-fuscente tectis. Folia tomentoso-hirsuta petiolata, petiolis lamina 3-plo brevioribus, laminis oblongo-ovatis circ. $14 \mathrm{~cm}$. longis $7 \mathrm{~cm}$. latis apice acuminatis basi acutis vel rotundatis margine irregulariter subbiserratis, serris aristato-acutis, 
supra pilis scaberrimis sparce subtus dense tectis. Cyma corymbosæ umbellato-fasciculatx terminales circ. $12 \mathrm{~cm}$. longa $14 \mathrm{~cm}$. in diametro æquantes, rhachibus tomentosis. Flores exteriores steriles, sepalis 4 petaloideis nerrosis fere orbicularibus $2 \mathrm{~cm}$. longis totidem latis utraque latere præter basin apicemque serratis, scrris acutis; interiores fertiles. Capsula hemispherica apice leviter constricta 10-costata, calycis lobis persistentibus acuminatis, stylis persistentibus valde divaricatis apice leviter recurvis. Semina fusiformia utrinque producta longitudinaliter striata intra strias transrersum reticulata.

HAB. in monte Morrison, ad 7500 ped. alt., leg. 'T. KaWaKAMII et U. Mori, Nor. 1906, (No. 1875).

Somewhat near H. involucrata SIEв. ; but differs from that in its scandent habit, and in having serrate sepals of the rarlial flower's.

Hydrangea longifolia HayATA, sp. nor. Frutices erecti? ramis tomentosis, cortice cinereo-fusco tectis. Folia hreve petiolata, petiolis lamina 10-plo brevioribus, laminis oblongo-lanceolatis acuminatis basi obtusis cire. $20 \mathrm{~cm}$. longis $4 \frac{1}{2} \mathrm{~cm}$. latis margine remote serrulatis, serraturis acuminatis, supra scabro-pilosis subtus ad costas tomentosis. Cymæ corymbosa umbellato-fasciculati terminales circ. $9 \mathrm{~cm}$. longa $14 \mathrm{~cm}$. lata, rhachihus tomentulosis. Flores exteriores steriles, sepalis petaloideis utraque pagine tomentulosis late orbicularibus nervosis apice rotundatis basi lrevissime contractis $17 \mathrm{~mm}$. longis totidem latis. Cilpsula hemispherical tomentulosa latere leviter compressa, stylis persistentilus apiee recurvis. Semina fusiformia utrinque producta.

Hab. Taitō: Torokusha, leg. T. Kawakamr et G. Nakahara, Januar. 1906, (No. 690).

Somewhat resembles II. Karealemii IIAYats; but differ's from it 
in having long lanceolate leaves, hairy capsules and entire hairy sepals of marginal flowers.

\section{Deut:ia Thuns.}

Deutzia scabra Thuns. Fl. Jap. p. 185 ; DC. Prodr. IV. p. 17 ; Maxtr. Revis. Hydrang. As. or. p. 24 ; Mre. Prol. Fl. Jap. p. 263; Franch. et Savat. Enum. Pl. Jap. I. p. 155; Hance, in Journ. Bot. (1878) p. 11 ; S. Moone, in Journ. Bot. (1878) p. 138 ; Bot. Mag. t. 38 ; Fonbes et HeMst. Ind. Fl. Sin. I. p. 276 ; Henrry, List Pl. Formos. p. 41 ; Irō et Matsunr. Tent. Fl. Lutch. p. 463 ; Diets, Fl. Centr. Chin. p. 372 ; Matsum. et Hayata, Enum. Pl. Formos. p. 132.

Deutzia crenata Sieb. et Zucc. Fl. Jap. p. 19, t. 6 ; Maxim. Revis. Hydrang. As. ox. pp. 22. et 45.

Deutzic crenata ò taiwanensis MAXIMr. 1. c.

$H_{\mathrm{AB}}$. in monte Morrison, ad 11000 ped. alt., (No. 2287); ad 9000 ped. alt., (No. 1701), et ad 7500 ped., alt., (No. 1703), in montibus centralibus, (No. 2198), leg. T. Kawakami et U. Mori, Nov. 1906.

Distrib. Common in Japan and central China.

It is a little doubtful whether my plant is referable to this species. In the imperfectness of the specimens, the determination is rather conjectural.

\section{Cardiandra SIEB. et ZUCC.}

Cardiandra sinensis Hensst. in Gard. Chronic. Feb. 7, 1902, p. 81.

Cardiandra formosana Hayata, in Tōkyō Bot. Mag. XX. p. 54 ; Matsux. et Hayata, Enum. Pl. Formos. p. 132.

Hab. Taitō : Tōkeizan, leg. T. Kawakamr et U. Mori, Dec. 1906, (No, 2201) ; Kagi : Kōden, leg. T. KawaKami et U. Moni, Oct. 1906, (No. 1735).

Distrib. Central China: Kiangsi.

In the year 1902, Mr. W. B. Hemscey described a new species 
of Cardiandra from Central China in the foot note of the Gardener's Chronicle above cited. Unfortunately, owing to an error on my part, the description had been carelessly overlooked by myself when I studied the Cardiandra from Formosa. I found afterward that my plant is entirely referable to the species described by the above authority. C. formosana Harata should, thercfore, he rendered to a synonym of $C$. sinensis Heuss.

\section{Ribes LinN.}

Ribes formosanum HaYati, (Pl. IX.), in Tókyō Bot. Mag. XX. p. 56. Frutices, ramulis angularibus spinis infra-axillaribus ternatis validis patentibus. Folia simplicia vernatione plicata late rotundata basi truncata vel cordata $2 \frac{1}{2} \mathrm{~cm}$. longa 5-6-lobata, lobis obtusis grosse pance serratis, petiolata, petiolis pance glandulososetosis laminam æquantibus. Flores subsolitarii, pedicellis $1 \mathrm{~cm}$. longis basi bracteatis medio articulatis $2-4$ bracteolatis. Calycis tubus ovoidens ovario adnatus, lobis 5 subpatentibus $1 \mathrm{~cm}$. longis oblongo-ovatis apice rotundatis petalum 3-plo superantibus. Petala 5 calycis fauce inserta oblonga parra squamiformia inclusa $4 \mathrm{~mm}$. longa. Stamina $5,4 \mathrm{~mm}$. longa, antheris oblongis. Ovarium inferum 1-loculare, ovulis numerosis, placentis 2, parictalibus; styli 2 distincti basi leviter connati, stigmatibus simplicibus. Baccæ globosæ roser pulposa $18 \mathrm{~mm}$. in diametro rquantes, sepalis persistentibus. Scmina subangulata, testa cxtus gelatinosa intus crustacea $3 \mathrm{~mm}$. longa leviter compressa minute reticulata.

HAB. Tōhokei, ad 5907 ped. alt., in montibus Morrison, leg.. . Nagasawa, Nor. 1905, (No. 592); in monte Morrison, ad 10000 ped. alt., leg. T. Kawakanir et U. Mori, Nov. 1906, (Nos. 2141 et 2132).

As the original description is drawn from an imperfect specimen, I have taken the liberty of repeating the description 
of the plant, basing the above account upon the most perfect material.

\section{Crassulaceæ.}

\section{Sedum LinN.}

Sedum morrisonense Harata, sp. nov. Perennis? erecta humilis circ. $8 \mathrm{~cm}$. alta a basi ramosa glabra, caulibus solitariis interdum subsurculigeris. Folia approximata dense disposita adpresse imbricata crassa carnosa oblongo-lanceolata apice obtusa basi obtusissima ulta insertionem $\frac{1}{2} \mathrm{~mm}$. producta $6 \mathrm{~mm}$. longa $1 \frac{1}{2} \mathrm{~mm}$. lata vel latiora. Cymæ trifidæe plurifloræ bracteatæ, bracteis folio conformibus flore brevioribus; floribus ad ramos cymarum sessilibus, parvis $7 \mathrm{~mm}$. longis campanulatis. Sepala oblongo-linearia obtusa petalum $\frac{7}{2}$ æquantia crassiuscula. Petala oblongo-acuta apice obtuse breve carinata $6 \frac{1}{2} \mathrm{~mm}$. longa; stamina episepala petalum requantia, epipetala paulo breviora, antheris oblongis; squamis hypogynis minutis late quadratis. Folliculi membranacei basi breviter conmati crecto-patuli oblongi in stylos "2-plo breviores attenuati cum stylis $6 \mathrm{~mm}$. longi, stigmatibus punctiformibus. Semina oblonga obseure striata, striis minutissime muriculatis.

HAB. ad summam montis Morrison, ad 13094 ped. alt., leg. S. Nigasatwa, Nor. 1905, (No. 566); codem loco, ad 12000 ped. alt., leg. 'T. Kawakami et U. Mori, Oct. 1906, (No. 2281) ; in montibus centralibus, leg. T. Kawarkani et U. Mori, Nov. 1906, (No. 1892).

The present plant comes near S. Roborouskii Maxır.; but differs from it in having smaller calcarate leaves and larger sessile flowers. 


\section{Sedum sp.}

HAB. in monte Morrison, ad 6000 ped. alt., leg. 'T. KaWakanI et U. Moni, Oct. 1906, (No. 2019).

No fruit, indeterminable.

\section{Kalanchö̈ ADANs.}

\section{Kalanchoë sp.}

HAB. in monte Morrison ad 6000 ped. alt., leg. 'I. KawaKanI et U. Mori, Nov. 1906, (No. 1881) ; Kagi : Kishirei, leg. T. Kawıтамг et U. Mori, Oct. 1906, (No. 1779).

No flower, indeterminable.

\section{Halorageæ.}

\section{Haloragis Forst.}

Haloragis micrantha R. Br.; Bextr. Fl. Anstril. II. p. 482 ; Cr.sikil, in Hoor. f. Fl. Brit. Ind. II. p. 430; Haxce, in Joun. Bot. (1870) p. 276 ; Sied. et Zucc. Fl. Jap. Fam. Nat. I. p. 133 ; MIQ. Prol. Fl. Jap. p. 261; Franch, et Savat. Enum. Pl. Jap. I. p. 164; Fonbes et Hearst. Ind. Fl. Sin. I. p. 292 ; Iтō et Matsuy. Ten. Fl. Lutch. p. 471; Diels, F1. Centr. Chin. p. 486 ; Matsuxr. et Hayata, Enum. PI. Formos. p. 138.

Gonocarpus micranthets THuxis. Fl. Jap. p. 69 t. 15 ; DC. Prodr. III. p. 66 .

Hab. Ganzan, in montibus Morrison, ad 9141 ped. alt., leg. S. NAGaSAwa, Oct. 1905, (No. 638).

Distrus. India, Malay, Australia, New realand; China throughout, and Japan.

\section{I!y}

Myriophyllum spicatum IJNX. Sp. PJ. ed.-2, p. 1409; DC. Prodl: III. p. 68 ; Ledrer. Fl. Ross. IT. p. 119 ; Maxms. Ind. F1. Pelin. in Prim. lil. 
Amur. p. 471 ; Clarke, in Hook. f. Fl. Brit. Ind. II. p. 433 ; Franch. et Savat. Enum. Pl. Jap. I. p. 165 ; Forbes et HeMst. Ind. Fl. Sin. I. p. 293 ; Itū et Matsum. Tent. Fl. Lutch. p. 471 ; Matsum. et Hayata, Enum. Pl. Formos. p. 138.

Haв. Kagi : Rokuryōshō, leg. T. KawaKamI et U. Mori, Oct. 1906, (No. 1974).

Distrib. Generally spread over the cool and temperate regions of the northern hemisphere.

\section{Myrtaceæ.}

\section{Eugenia LinN.}

Eugenia sinensis Hemst.? in Forbes et Hexss. Ind. Fl. Sin. I. p. 298; Henny, List Pl. Formos. p. 43 ; Matsum. in Tōkyō Bot. Mag. XII. p. 64; Irō et Matsur. Tent. Fl. Lutch. p. 481; Diers, Fil. Centr. Chin. p. 484 ; Matsunr. et Hayata, Enum. Pl. Formos. p. 143.

Eugenia Grijsii HANCE, in Journ. Bot. (1871) p. 5, et (1879) p. 10.

Engenia pyxopleylla HaNce, in Journ. Bot. (1871) p. 6.

Syzygium Zuxifolium Ноок, et ArN. Bot. Beech. Voy. p. 187; WaLP. Ann. IT. p. 180 ; Benth. Fl. Hongk. p. 118.

Hab. Nantō: Shojidaizan, leg. T. Kawakami et U. Moni, Aug. 1906, (No. 1158).

Distrib. South central China.

Sp. Hab. Kōshūn: Marihasha leg. T. KawaKamr, Juli. 1906, (No. 1631).

Under this family, I have a very interesting plant which it is difficult for me to determine even its genus. The specimen in my hands is only a male and therefore it remains as yet undetermined. 


\section{Melastomaceæ.}

\section{Osbeckia Lins.}

Osbeckia aspera Blune; Wrght, Ic. Pl. Ind. or. t. 377 ; Clanke, in Hоoк. f. Fl. Brit. Ind. II. p. 519 ; Cogs. in DC. Monogr. Phanerog. VII. p. 315 ; Matsum. et Hayata, Enum. Pl. Formos. p. 146.

Melastoma asperum LINN.; DC. Prodr. II. p. 145.

HAB, in monte Morrison, ad 8000 ped. alt., leg. T. KaWAKaMr et U. Mori, Nov. 1906, (No. 1923).

Distrib. Deccan peninsula and Ceylon.

Barthea Hook. f.

Barthea formosana Harıtı, sp. nov. (Pl. X.). Frutex scandlens? ramosus, ramis gracillimis glaberrimis v. partibus novellis sub microscopio glanduloso-lepidotis. Folia breve petiolata, petiolis lamina 10-plo brevioribus laminis subcoriaceis (novellis memlranaceis) oblongis circ. $10 \mathrm{~cm}$. longis $3 \mathrm{~cm}$. latis apice oblique caudato-acuminatis, caudis linearibus, basi acutis xqualibus margine subintegris vel remote obscure serrulatis $3-5$-nerviis supra sub lente minute sparce setulosis subtus palliclioribus venis prominentibus sub microscopio minute densinscule Iepidotis. Flores ad apicem ramorum terminales 1-3-congesti majusculi patentes $3 \mathrm{~cm}$. in diametro xquantes breve pedicellati, pedicellis $3-4 \mathrm{~mm}$. longis minutissime lepidotis. Calycis tubus glaberrimus obpyramidalicampanulatus $9 \mathrm{~mm}$. longus 4 -quetrus, limbus dilatus 4 lobus, lobis brevibus triangularibus apice sctoso-carinato-acuminatis. Petala 4, ampla oblique obovata $18 \mathrm{~mm}$. longal $16 \mathrm{~mm}$. lata apice rotundato-apiculata glaberrima margine sub lente ciliato-scrrulata. Stamina 8, valde inarualia ; anthera majores lineari-elongitæ obtusæ 1-porosæ incurva, connectivis basi antice longe 
2-setosis (setis bi-fidis) postice in calcaria crassa ascendentia productis; antheræ minores breves oblongæ recurvæ, connectivis basi antice 2 -setosis (setis simplicibus) postice breve calcaratis. Ovarium calyci adherens (inter ipsum et calycem longitudinaliter 4-canaliculatum) 4-loculare vertice leviter productum; stylus filiformis declinatus ovarium 2-plo superans, stigmate punctiformi. Capsula oblongo-globosa sub-tetragona basi in stipitem abrupte attenuata $7 \mathrm{~mm}$. longa $5 \mathrm{~mm}$. lata 4 -valvata. Semina cuneiformia latere compressa, cum alis vix $2 \mathrm{~mm}$. longa, alis subcultriformibus.

Hab. Suihenkiaku? leg. T. Kawakani et G. NaTiahara, Jan. 1906, (No. 41); Nantō: Mushazan, ad 6000 ped. alt., leg. T. KawakauII et U. Mori, Oct. 1906, (No. 1148).

Closely resembles Barthea chinensis Hook. f. ; but differs from it in having no glandular crown over the ovary and in having subentire leaves.

Distrib. The only congener $B$. chinensis Hoor. f. occurs in Hongkong.

\section{Sarcopyramis. WALL.}

Sarcopyramis nepalensis Warc. "Tent. Fl. Nep. t. 23 "; Clarke, in Hook. f. Fl. Brit. Ind. II. p. 541 ; Forbes et HemsL. Ind. Fl. Sin. I. p. 302 ; CoGN. in DC. Monogr. Phanerog. VII. p. 517.

Sarcopyramis lanceolata WALL. ; KuRz, in Journ. Bot. (1873) p. 193 ; Hance, in Journ. Bot. (1878) p. 107.

Haв. Suizan, in montibus Morrison, ad 7702 ped. alt., (No. 652); et Ganzan, in isdem montibus, ad 9141 ped. alt., Oct. 1905. (No. 637) ; Taitō: Tōkeizan, leg. T. Kawakani et U. Mori, Dec. 1906 , (No. 1913).

Distrib. India and Malay. 


\section{Onagrarieæ.}

\section{Epitobium Lins.}

Epilobium alpinum Lrss.; DC. Prołr. III. p. 41 ; Ноoк. f. FI. Prit. Ind. II. p. 586 ; WAGNeR, Deut. Fl. ed.-3, p. 480.

HAB. in monte Morrison, ad 11000 ped. alt., leg. S. NAGASAwA, Nov. 1905, (No. 610); eodem loco, leg. T. Kawakani et U. Moni, Nov. 1906, (Nos. 2118, 2276 et 1893).

Distrib. The Himalayas and alpine regions of Europe.

Epilobium roseum Schred.; DC. Prodr. III. p. 41 ; MLıxir. Ind. Iit. Pek. in Prim. Fl. Amur. p. 471; Clarke, in Hook. f. Fl. Brit. Ind. II. p. 584 ; Forbes et Hears.. Ind. Fl. Sin. I. p. 308 ,

HAB. Ganzan, in montibus Morrison, ad 9141 ped. alt., leg. S. Nagasatwa, (No. 699); in monte Morrison, ad 9000 ped. alt., leg. T. Kawakami et U. Moni, Oct. 1906, (No. 2067); Nantō: Mushazan, 8000 ped. alt., leg. T. Kawakami et U. Mori, (No. 1130); Taitō: Daironkōsha, leg. T. Kawarkanr et U. Moni, Nov. 1906, (No. 22175).

Distrib. Broadly speaking, this species extends from Europe eastward to eastern Asia and North-West America. But not yet found in Japan.

\section{Circaed Linn.}

Circæa alpina Lisv. Sp. Pl. ed.-2, p. 12 ; 1)C. 1'rodr. III. p. 6:3 ; Cr.Iriks, in Hook. f. Fl. Brit. Ind. II. p. 589 ; Franchet, PI. David. p. 134; Fonbes et Hexrst. Ind. Fl. Sin. I. p. 310 ; Diers, Fl. Centr. Chin. p. 485.

Haв. in monte Morrison, leg. T. Kawakamr et U. Morr, Oct. 1906, (No. 2030).

Distrib. Generally spread in the nortli temperate regions. 


\section{Cucurbitaceæé.}

\section{Thladiantha BuNGE.}

Thladiantha formosana Hayata, sp. nov. (Pl. XI.). Herbæ scandentes vel volubiles, ramulis floriferis graciliusculis sulcatis pilosis, pilis longis, cirrhis 2-partitis. Folia longe petiolata, petiolis lamina xquilongis vel brevioribus pilosis, laminis crassiusculis membranaceis angularibus rotundato-cordatis rel orato-cordatis $10 \mathrm{~cm}$. longis $7 \mathrm{~cm}$. latis acute acuminatis margine minute remoteque denticulatis supra scabridis subtus villoso-pubescentibus. Flores $\hat{\delta}$ puberuli racemosi, racemis longis petiolo 2-3-plo longioribus, bracteis parvis cito deciduis. Calycis tubus brevis late campanulatus, lobis orato-lanceolatis $5 \mathrm{~mm}$. longis. Petala patentia oratolanceolata $11 \mathrm{~mm}$. longa. Stamina 5 xqualia, antheris 1 -locularibus rectis, filamentis liberis. Ovarii rudimentum globosum. Appendicula brevis petaloidea obtusa discum centrale horizontaliter tegens.

Hab. Tōzan, in montibus Morrison, leg. G. Nakahara, Oct. 1906.

Distrib. An allied species, 'T. mudiflora Henst., occurs in central China.

Very much resembles $T$. nudifiora Henss. ; but differs from it in having peduncled racemes, and smaller flowers which are as half as those of the allied species.

\section{Gynostemma BLUME.}

Gynostemma pedatum Buune; Watr. Rep. T. 1).98; MIr. Fl. Iud. Bat. T.-1, p. 683 ; Clanke, in Ноок, f. Fl. Brit. Ind. II. p. 633 ; CoGN. in DC. Monogr. Phanerog. III. p. 913 ; Franchet, Pl. Darid. I. p. 136 ; Fonbes et Hemst. Ind. Fl. Sin. I. p. 320 ; Itō et Matsux. Tent. Fl. Lutch. p. 519 ; Drets, Fl. Centr. Chin. p. 604 ; Hayata, in Tōkșō Bot. Mag. XX. p. 53. 
Gynostemma cissoides Benth. et Hook. f. Gen. PI. I. p. 839 ; Francr. et Savat. Enum. Pl. Jap. I. p. 176.

Enkylia trigyna Grifrtт.; ; Miq. Prol. F1. Jap. pp. 15 et 142.

Pestalozria pedata Zolt. et Mor.; W WLP. Ann. I. p. 316.

Zanonia cissoides WALL. ; WaLP. Rep. II. p. 194.

Zanonia pedata MIQ. FI. Ind. Bat. I.-1, p. 6S3.

Hab. Arizan, in montibus Morrison, leg. G. Nakahara, Nov. 1906 ; in eodem monte, ad 6000 ped. alt., (No. 2001), et ad 6500 ped. alt., (No. 2049), leg. T. Kawakami et U. Mori, Oct. 1906.

Distrib. India, Malay archipelago, central and eastern China, Japan and the Loo-choo islands.

\section{Begoniaceæ.}

The three species of Begoniacer. The specimens are all imperfect and can not be satisfactorily determined.

\section{Umbelliferæ.}

\section{Hydrocotyle LinN.}

Hydrocotyle javanica Thuxs. "Dissertatio p. 415, t. :3"; 1)(. Proils. IV. p. 67 ; MTe. Fl. Ind. Bat. I.-1, p. 734; HANce, in Joum. Bot. (1883) p. 321 ; Clarke, in Hoor. f. Fl. Brit. Ind. II. p. 667 ; Muxim. in Mél. Biol. XII. p. 246 ; Fonbes et Hexst. Ind. Fl. Sin. I. p. 325 ; Hexst. et Colt. in Journ. Linn. Soe. XXVIII. p. 61; Henny, List Pl. Formos. p. 47; I Tō et Matsusr. Tent. Fl. Lutch. p. 260 ; Dies, Fl. Centr. Chin. p. 490 ; Yabe, Revis. Umbell. Jap. p. 10 ; Matsun. et Hayata, Enum. Pl. Formos. p. 170. Hydrocotyle nepalensis Hook.; DC. Prodr. IV. p. 65; Mro. Fl. Ind. Bat. I.-1, p. 735 (var.) ; Mre. Prol. Fl. Jap. p. 243; Francir. et Savat. Enum. Pl. Jap. I. p. 178. 
Hydrocotyle polycephala Wrght et ArN.; Wight, Ic. PI. Ind. or. t. 1003. Hydrocotyle zeylanica DC. Prodr. IV. p. 67; MrQ. Fl. Ind. Bat. I.-1, p. 734.

Hydrocotyle lirta R. Br. var. acutiloba F. MueLt. in BeNth. Fl. Austr. III. p. 340.

Haz. Tappansha, ad 3139 ped. alt., leg. S. Nagasawa, Oct. 1905, (No. 719) ; Tōzan, in montibus Morrison, leg. G. Nakahara, Dec. 1906.

Distrib. South China and Japan. Tropical Asia to eastern Africa and Australia.

Hydrocotyle rotundifolia Roxr. Fl. Ind. II. p. 88; DC. Prodr. IV. p. 64; Wight, Ic. Pl. Ind. or. t. 564 ; Benth. Fl. Hongk. p. 134; Clanke, in Hоoк. f. Fl. Brit. Ind. II. p. 668; Maxim. in Mél. Biol. XII. p. 461; Forbes et Hemsl. Ind. Fl. Sin. I. p. 825 ; Henrx, List. Pl. Formos. p. 47 ; Iтō et Matsur. Tent. Fl. Lutch. p. 259 ; Diels, Fl. Centr. Chin. p. 491 ; Yabe, Revis. Umbell. Jap. p. 12 ; Matsum. et Hayata, Enum. Pl. Formos. p. 171.

Hydrocotyle nitidula RicH.; DC. Prodr. IV. p. 66 ; MiQ. Prol. Fl. Jap. p. 243.

Hydrocotyle Sibthorpioides Lam.; DC. Prodr. IV. p. 66 ; Franch. et Savat. Enum. Pl. Jap. I. p. 178.

Hydrocotyle puncticulata MiQ. Fl. Ind. Bat. I.-1, p. 733.

Hydrocotyle Zollingeri MoLKENB.; MrQ. F1. Ind. Bat. I.-1, p. 733.

Hydrocotyle tenella Dov, in DC. Prodr. IV. p. 64.

HaB. Ganzan, in montibus Morrison, ad 9141 ped. alt., Oct. 1905.

Distrib. Asia and Africa.

Hydrocotyle setulosa Hayata, sp. nor. Herba perennis, prostrata ad nodos radicans. Caules prostrati, ramis innovationibus erectis pilosis. Folia longe petiolata, petiolis circ. $2 \mathrm{~cm}$. longis 
piloso-puberulis, pilis descendentibus, laminis reniformi-cordatis $10 \mathrm{~mm}$. longis $13 \mathrm{~mm}$. latis 7 -lobis, lobis obtusis late rotundatis rarius contiguis leviter marginatis crenatis, crenis acutis, supra setulosis subtus piloso-setosis, setis longiusculis; stipulis late rotundatis integris rel bilobis membranaceis. Pedunculi $2-3 \mathrm{~cm}$. longi piloso-pubescentes, pilis descendentibus. Umbellæ simplices multiflores, floribus breve pedicellatis, pedicellis $1 \mathrm{~mm}$. longis, dense capitatis. Fructus late cordato-orbiculati compressi utrinque costati $1 \mathrm{~mm}$. longi $1 \frac{1}{2} \mathrm{~mm}$. lati.

Hab. Arizan, in montibus Morrison, leg. G. NaKahara, Nov. 1906.

Somewhat resembles $H$. Wilfordi Maxnr., but differs from it in the fruits and setulose leaves.

\section{Sanicula LINN.}

Sanicula petagnioides Hayata, sp. nor. (Pl. XII.). Herba perennes humiles glaberrimx. Caules erecti circ. 12 cm. alti pauciramosi. Folia radicalia longe petiolata, petiolis gracilibus $6 \mathrm{~cm}$. (rarius $12 \mathrm{~cm}$.) longis glabris basi dilatis, laminis ambitu late pentagonis $2-2 \frac{1}{2} \mathrm{~cm}$. in diametro æquantibus 5 -partitis, segmentis 2-3-lobatis rhomboideis basi cuneatis aristato-serratis albo-lamelligeris breve petiolulatis. Folia caulina sessilia folis radicalibus conformia 3-5-partita, segmentis cuncato-lanceolatis. Umbella compositæ ad apicem caulis 2-3, vel axillares solitariæ, longe pedunculatæ, pedunculis $3 \mathrm{~cm}$. longis, $5-7$-radiate $6 \mathrm{~mm}$. longæ, 10 $\mathrm{mm}$. in diametro æquantes, bracteis paucis setaceo-dentiformibus vel subfoliaceis minutis. Umbellulæ minores $2 \frac{1}{2} \mathrm{~mm}$. longa $3 \mathrm{~mm}$. in diametro rquantes basi bracteolate, lracteolis 5-6 minutissimis setaceo-dentiformibus, ,, 6 -flore, flonibus exterioribus masculinis (=sterilibus) longe perlicellatis, centralilus mifloris perfectis ferti- 
libus. Flores $\hat{\delta}$ : minutissimi circ. $\frac{2}{3} \mathrm{~mm}$. in diametro æquantes longe pedicellati, pedicellis $1 \frac{1}{2} \mathrm{~mm}$. longis ; calycis lobis prominulis setaceo-dentiformibus; petalis albis ovatis apice obtuse acutis inflexis; staminibus 5, filamentis petalo duplo longioribus; ovarii rudimentis convexis. Flores 오 : sessiles minuti, florem $\hat{\delta}$ in magnitudine 3-plo superantes $2 \mathrm{~mm}$. longi; calycis tubis ellipsoidalibus echinatis, lobis suberectis prominente setaceis ; petalis staminibusque iis fl. $\hat{\delta}$ conformibus; stylis suberectis leviter recurvis. Fructus obovato-orbiculares $1 \frac{2}{3} \mathrm{~mm}$. lati valde compressi multicostati secus costam echinati, calycis lobis persistentibus erectis, stylis persistentibus valde reflexis.

$\mathrm{H}_{A B}$. in monte Morrison, ad 7500 ped. alt., (No. 2026), et ad 8000 ped. alt., (No. 1988), leg. T. KaWaKanr et U. Mori, Oct. 1906 ; Tōzan, in montibus Morrison, leg. G. NaKahara, Nov. 1906.

The present plant comes very near S. satsumana Maxnr.; but differs from it in having 5-parted leaves, less spined fruits, smaller flowers, and in the presence of cauline leaves; and still more in the structure of the fruits. As seen in the section of the fruit (Pl. XII.), this plant differ's greatly from the congener in the position of vittæ. The vittr in my species are very few, while in the other they are as many as eight. An allied genus, Petagnia Guss., has no vitta in its fruits, in which respect the present plant is the nearest to it.

The five species of the distinct Umbelliferæ. In the absence of the mature fruits, the genera can not be determined with certainty.

\section{Araliaceæ.}

Acanthopanax Done et PL.

Acanthopanax aculeatum SeExr. in Journ. Bot. (1867) p. 238 ; HaxcE, 
in Journ. Linn. Soc. XIIT. p. 105 ; Fraxch. Pl. Darid. p. 146 ; Clanke, in Hoor. f. Fl. Brit. Ind. II. p. 726 ; Fonbes et Hexsis. Ind. Fl. Sin. I. p. 339 ; Henry, List Pl. Formos. p. 47 ; Diels, Fl. Centr. Chin. p. 489 ; Matsum. et Hayata, Eumm. Pl. Formos. p. 176.

Panax aculeatum Aтt. Hort. Kew. ed-2, V. p. 482 ; DC. Prodr. IV. p. 252.

Panax Loureirianum DC. Prodr. IV. p. 252.

Zanthoxylum trifoliatum Lins. Sp. Pl. ed-2, p. 1455.

Plectronic chinensis Lour. FI. Cochinch. ed-WnLD. p. 201.

Hab. Tappansha, leg. S. Nagasawa, Oct. 1905, (No. 717); in monte Morrison, ad 6000 ped.alt., leg. 'T. Kawakami et U. Mori, Oct. 1906, (No. 2022).

Distris. Eastern India, central China, and Japan.

Fatsia DCxe et PL.

Fatsia polycarpa Hayatı, sp. nor. (Pl. XIII.). Arbor?' Riamuli primum valde fulvo-lanati, pilis longiuseulis, demum subglabri. Folia (norella fulvo-lanate demum glabra) ampla longe petiolata, petiolis in longitudine laminam æquantibus rel brevioribus basi valde dilatis ciliatis, stipulis intra petiolum parum prominulis, laminis ambitu late orbicularibus $15-30 \mathrm{~cm}$. in diametro equantibus profunde ad $\frac{2}{3}$ partem laminæ palmatim 7 -fidis inter lohes valde sinuatis, lobis oblongo-oboratis apice caudato-acuminatis lasi attenuatis margine præter acumen partes basilares et sinuatas dentato-serratis, serraturis mueronat(r-acuminatis ascendentibus, palmatim 7 -nerriis, sultus nervis prominentibus, pallidioribus. Umbelle paniculata. Panicule terminales $30-40 \mathrm{~cm}$. longe dense fulvo-lanater, ramis infimis $14 \mathrm{~cm}$. longis umbellis pedunculatis remote instructis; pedunculi umbellarum $1-\frac{1}{2} \mathrm{~cm}$. longi umbellam in longitudine

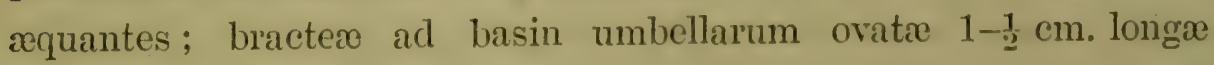


membranaceæ caducissimæ dense lanatæ; bracteolæ minutissimæ lineares. Umbellæ circ. 20 -floræ in alabastro globosæ $1 \mathrm{~cm}$. in diametro æquantes indumento lanato obtectæ; umbellæ demum patentes $1 \frac{1}{2} \mathrm{~cm}$. longæ $2 \frac{1}{2} \mathrm{~cm}$. in diametro æquantes. Flores patentes longe pedicellati, pedicellis $1 \mathrm{~cm}$. longis gracilibus flore continuis sed basi pedunculo articulatis, majusculi $7 \mathrm{~mm}$. in diametro æquantes. Calycis tubus brevis leviter sulcatus, margine vix prominulo. Petala 5 membranacea valvata revoluta longe triangularia apice mucronato acuta $3 \frac{1}{2} \mathrm{~mm}$. longa basi vix latiora quam $2 \mathrm{~mm}$. Stamina 5, filamentis filiformibus petalo longioribus horizontaliter patentibus reflexis, antheris oblongis. Discus convexus margine integer. Ovarium 10-loculare, loculis 1-ovulatis. Styli 10 a basi distincti brevissimi $\frac{1}{2} \mathrm{~mm}$. longi, stigmatibus terminalibus parvis.

HaB. in monte Morrison, ad 8500 ped. alt., leg. T. KaWaKalII et U. Morr, Nov. 1906, (No. 1868).

Distrib. 'The only congeners we have at present are $F$. japonica Dcne. et PL. and $F$. papyriferc Bentн. et Hoor. f. The former occurs in Japan, and the latter, in Formosa.

The present species is a very interesting addition to the Formosan flora. The plant is by far the most remarkable species in having 10 -celled ovaries in which respect it appears to be referable to Trevesia VIS. All other characters of the plant, however, quite agree with Fatsia Dcne. et PL. I think, therefore, it will be better to refer the new plant to this genus, and at the same time to extend the limit of the generic character.

\section{Helvingia WILLD.}

Helwingia rusciflora WrLtd. Sp. Pl. IV. p. 716 ; Sirb. et ZucC. Fl. Jap. I. p. 164, t. 86 ; Fonbes et HeMrst. Ind. Fl. Sin. I. p. 341 ; Diels, Fl. Centr. Chin. p. 505. 
Helvingia japonica Dietr.; DC. Prodr. XVI.-2, p. 680 ; Fraxch. et Savat. Enum. PI. Jap. I. p. 195.

Osyris japonica Thuмв. Fl. Jap. p. 31, et Tc. Pl. dec. III. t. 1. Distrib. Japan and central China.

\section{Heptaplenvim G-RTTX.}

Heptapleurum octophyllum Вехти. in Bexтr. et Hоок. f. Gen. Pl. I. p. 942 ; Hance, in Journ. Linn. Soc. XIII. p. 105 ; Fonbes et Hexss. Ind. Fl. Sin. I. p. 342 ; Henry, List Pl. Formos. p. 48 ; Matsunr. et Hayata, Enum. P1. Eormos. p. 178.

Aralia octophylla Lour. Fl. Cochinch. ed.-Wmi.d. p. 233 ; DC. Prodr. IV. p. 258.

Paratropia cantoniensis Hook. et AnN. Bot. Beech. Voy. p. 189; Watr. Rep. IV. p. 433 ; BeNth. Fl. Hongk. p. 136.

Agalma octophyllum SeEnr. in Journ. Bot. (1864) p. 298.

Haв. in monte Morrison, ad 9000 ped. alt., leg. T. Kawakauг et U. Mori, Nov. 1906, (Nos. 1899 et 1879).

Distrib. South China and the Loo-choo islands.

The plant grows usually in the low regions and sometimes ascends to such an elevation almost ten thousand feet high.

Heptapleurum racemosum BEDD. FI. Sylv. t. 214; CI.MTKK, in Hook. f. FI. Brit. Ind. II. p. 729; HaYats, in Tōkyō Bot. Mag. XX. p. 53.

Hedera racemosa Wight, Ic. Pl. Ind. or. t. 1015.

Agalma racemosum SeEnr. "Rer. Heder. p. 24."

HAB. Tōzan, in montibus Morrison, leg. ('. Nakanarı, Oct. 1906; Arizan, in isdem montibus, leg. G. Numamara, Nov. 1906 ; in monte Morrison, 7000 ped. alt., leg. 'T. KAwakar et U. Mon, Oct. 1906, (No. 1709).

Distris. The present plant grows in the mountainous districts 
of sonthern India such as Nilghiries at elevations of $3000-5000 \mathrm{ft}$., and also in Ceylon at altitudes of 3000-5000 ft., (after BeDdone); but not found in Himalaya. It is very remarkable case that such a southern element is found in the high regions of Formosa. No species belonging to this genus has yet been known from central China:

\section{Oreopanax DCNE. et PL.}

Oreopanax formosana Hayata, sp. nov. (Pl. XIV). Arbor? Rami cinereo-stellato-tomentosi et adpresse pilosi. Folia ampla longe petiolata, petiolis primum adpresse stellato-pilosis demum subglabris lamina 1-2-plo longioribus basi dilatis, stipulis parim prominulis acutis ad basin petiolorum connatis, laminis ambitu late orbicularibus $20 \mathrm{~cm}$. longis $23 \mathrm{~cm}$. latis vel minoribus basi, late truncatis vel cordato-truncatis margine obsolete $3-5$-lobatis vel grosse irregulariter dentatis, dentibus acutis inter dentes sinuatis, 5-7-nerviis, supra subglabris renis impressis, subtus pilis stellatis et simplicibus dense obtectis venis et venulis prominentibus. Florum capitula corymboso-paniculata. Paniculæ terminales $15 \mathrm{~cm}$. longæ petiolum a fuantes $13 \mathrm{~cm}$. latæ, pilis stellatis et simplicibus dense obtectx, ramis alternis angulo obtuso divaricantibus, capitulis florum pedunculatis remote instructis, et ad apicem ramorum capitulis ternatis; bractex basi pedunculorum late ovatæ $4 \mathrm{~mm}$. longæ; bracteolæ florum squamelliformes crassiusculæ sub singulo flore 3 -næ, 1 subtendente majore late ovata $3 \mathrm{~mm}$. longa, 2 lateralibus minoribus oppositis, pilis longis dense tectis et barbis validis nigris sparce insertis. Capitula florum subglobosa. 6-7 mm. in diametro æquantia, circ. 15-flora, pedunculis capitulum 2-plo superantibus. Flores omnio villosi intra bracteas arcte sessiles in alabastro subturbinati $2 \frac{1}{2} \mathrm{~mm}$. longi. Calycis margo() 
obsolete dentatus. Petala 5 valvata intus glabra ovato-triangularia $1 \frac{1}{2} \mathrm{~mm}$. longa vel longiora caclucissima. Stamina 5, filamentis brevissimis, antheris oblongis; discus explanatus. Ovarium 2. loculare, loculis 1-orulatis, stylis 2 brevibus distinctis erectis, stigmatibus terminalibus. Fructus late globosi $4 \frac{1}{2} \mathrm{~mm}$. longi $5 \mathrm{~mm}$. lati læres abortu 1 -spermi compressiusculi tomentulosi drupacei, stylis persistentibus valde recurvis. Semina ovoidea subtriquetra $4 \mathrm{~mm}$. longa, albuminibus ruminatis. Embryo hilo proximus minutus.

HAB. Taitō: Bataiankei, ad 6000 ped. alt., leg. N. Konishi, Juni. 1902, (No. 34, A); Arizan, in montibus Morrison, leg. G. NAKaHARA, Oct. 1906 ; in monte Morrison, ad 8000 ped. alt., leg. T. Kawakami et U. Morr, Nov. 1906, (Nos. 1871 et 1709); Taitō : in monte Iryokukaku, leg. T. Kawakani et U. Mori, Dec. 1906, (No. 1914.

In " ВеNтн. et Hоoк. f. Gen. Pl. I. p. 939 ", it is stated that the ovary of the genus Orcopanax is 5-celled. But, in "EvgL. und Praxts, Nat. Pfl.-fam. III.-8, p. 39 ", the generic character of this genus is a little broadened in such an extent that the ovary is sometimes 2celled and the flower is usually hermaphrodite. Accepting the latter' statement, the present plant which has two celled-oraries should be referred to Oreopanax, on account of capitate and sessile flowers, ruminate albumens, simple leaves, and the existence of three bracts under each flower. The style of this plant is exceptionally short. The occurrence of this genus in the island is very remarkable. So far as I am aware, we have had no representative of this American genus in any, other region on the globe. 


\section{Hedera Lins.}

Hedera Helix Lrvx. Sp. I'l. ed-2. p. 292; DC. Prodr. IV. p. 261; CuAnKe, in Hoor. f. Fl. Brit. Ind. IT. p. 739 ; "HANCE, in Journ. Bot. (1882) p. 6 "; Franch. et Savat. Enum. Pl. Jap. I. p. 194; Fonbes et Heass. Ind. Fl. -Sin. T. p. 343 ; Dreus, Fl. Centr. Chin. p. 487.

Hedera colchica Koch; Seem. in Journ. Bot. (1864) p. 307.

Hederca rhombea Steb, et Zucc. Fl. Jap. Fam. Nat. I. p. 94.

HAB. Kagi: Tappansha, (No. 1907), in monte Morrison, ad 6000 ped. alt., (No. 2017), et ad 8000 ped. alt., (No. 1889), leg. T. KawAKaNI et U. MorI, Oct. 1906.

Distrib. Western Europe and North Africa eastward to Japan.

\section{Dendropanax DCNE.}

Dendropanax sp. Rami glabri cortice cineraceo-flaro tectis. Folia longe petiolata, petiolis lamina 1-2-plo longioribus, basi dilatis stipulis fere obsoletis, laminis profunde 3 -lobatis in circumscriptione obtriangularibus $9 \mathrm{~cm}$. longis totidem latis basi abrupte cuneatis apice trilobis, lobis lateralibus terminali conformibus ascendentibus lanceolato-acuminatis, 3-nerviis utraque pagine inter reticula punctatis, interdum laminis simplicibus oblongo-ellipticis cuspidato-acuminatis basi cuncatis. Umbellæ ad apicem ramulorum solitarix pauciflorae breve pedunculatæ, pedunculis $7 \mathrm{~mm}$. longis, pedicellatx, pedicellis $8 \mathrm{~mm}$. longis. Flores ignoti. Fructus globosi $4 \mathrm{~mm}$. in cliametro æquantes multisulcati.

$H_{A B}$. in monte Morrison, ad 6000 ped. alt., (No. 2056), et in eodem monte, ad 7000 ped. alt., (No. 2041), leg. 'T. KawaKani et U. Mori, Oct. 1906. 
The specimens are wanting of flowers and it is rather questionable whether this belongs to Dendropanax.

\section{Cornaceæ.}

\section{Marlea Roxs.}

Marlea begoniæfolia Roxr. Fl. Ind. ed.-Catiex, II. p. 261; DC. Prodr. IV. p. 267 ; ВеNтr. Fl. Hongk. p. 183 ; Ноок. et ARN. Bot. Beech. Voy. p. 187; Clanke, in Hoor. f. Fl. Brit. Ind. II. p. 743; Forbes et Henst. Ind. Fl. Sin. I. p. 344.

Stylidium chinense Lour. Fl. Cochinch. ed.-WHLt. p. 273.

Hab. Kagi : Murōensha, leg. T. Kawakài et U. Mori, Nov. 1906, (No. 1750).

Distrib. India, Malay, south central China and Japan.

\section{Aucuba Lins.}

Aucuba japonica Thuxs. Fl. Jap. plp. 4 et 64 , tt. 12 et 13; Writd. Sp. Pl. IV. p. 328 ; Bot. Mag. tt. 1197 et 5512; DC. Prodr. IV. p. 274; Min. Prol. F1. Jap. p. 92 ; Franch. et Savat. Enum. P1. Jap. p. 197 ; Henry, List. PI. Formos. p. 48; Fonbes et Hexsst. Ind. FI. Sin. I. p. 346 ; ITō et Matsur. Tent. Fl. Lutch. p. 541 ; Diels, Fl. Centr. Chin. p. 507 ; Palibn, Conspect. Fl. Korex, I. p. 102 ; Hayata, in Tōkyō Bot. Mag. XX. p. 55.

Ancuba himalaica Hook. f. Fl. Brit. Ind. II. p. 747.

Aucuba chinensis Bentr. Fl. Hongk. p. 138.

$\mathrm{H}_{\mathrm{AB}}$, in monte Morrison.

Distrib. From middle Himalaya through central China to Japan and the Korean archipelago.

In my specimen, the flower's are much larger, and the leaves are narrower, oblanceolate, and with more diverging veinlets. I think the plant may be regarded as a form of the Japanese species. 


\section{Dicotyledones}

\section{Gamopetalæ}

\section{Caprifoliaceæ.}

\section{Lonicer LINN.}

\section{Lonicera sp.}

$\mathrm{H}_{\mathrm{AB}}$, in monte Morrison, (No. 1816).

The four species of Viburnum; but not yet determined.

\section{Rubiaceæ.}

\section{Ophiorvhiac LINN.}

Ophiorrhiza pumila Ch.urr.; Bextr. Fl. Hongk. p. 147 ; Watr. Amm. V. p. 117; Fonbes et Hemsl. Ind. Fl. Sin. I. p. 378; Henny, List Pl. Formos. p. 50 ; Matsur. in Tōkyō Bot. Mag. XIV. p. 147; MLatsum. et HAYATA, Enum. Pl. Formos. p. 187.

Haв. Tappansha, ad 3138 ped. alt, leg. S. NAgasawa, Oct. 1905, (No. 580); in monte Morrison, ad 7500 ped. alt., leg. T. KAWaKani et U. Moni, Oct. 1906, (No. 1713); Tōzan et Arizan, in montibus Morrison, leg. G. Narahara, Oct. 1906; Taitō: Busshisckisha, leg. T. Kawakami et U. Mori, Dec. 1906, (No. 2182).

Distrib. South China.

\section{Knoxia Lins.}

Knoxia corymbosa WrLLD. Sp. Pl. I. p. 582 ; Bextr. Fl. Hongk. p. 164; Ноок. f. Fl. Brit. Ind. III. p. 128; WтGнт, Ic. Pl. Ind. or. t. 128; 
WmLd. Sp. Pl. I. p. 582 ; Miq. FI. Ind. Bat. II. p. 330 ; Fories et Hemst. Ind. Fl. Sin. I. p. 384 ; Matsuær. in Tōkyō Bot. Mag. XVI. p. 13 ; Matsum. et Fayata, Enum. Pl. Formos. p. 189.

Spermacoce teres RoxB. Fl. Ind. ed.-CAnEY, I. p. 367.

НАв, in monte Morrison, ad 6000 ped. alt., leg. T. Kawakam et U. Mori, Oct. 1906, (No. 1945).

Distrib. Malay, North Australia, and South China.

\section{Damnacanthus GARTN.}

Damnacanthus angustifolius HAYATA, sp. nor. (Pl. XV.). Frtltices glaberrimi dichotome ramosissimi, ramulis cinereo-fulvis nitentibus 4-gonis. Folia opposita brevissime petiolata lanceolata rel lanceolato-linearia $13 \mathrm{~cm}$. longa $1 \frac{1}{2} \mathrm{~cm}$. lata basi acuta apice acuminata margine integra vel remote minuteque denticulata subtus pallidiora, venis utrinque prominulis, venulis fere transversum divergentibus, stipulis interpetiolaribus multi-dividis minute digitaliformibus. Flores parvi in axillis $5-6$ fasciculatim vel umbellatim dispositi, pedicellis gracilibus $2-3 \mathrm{~mm}$. longis. Calycis tubus obovoideus $1 \mathrm{~mm}$. longus limbum in longitudine xquans, limbo campanulato 4-lobo, lobis triangularibus persistentibus. Corolla cylindrico-campanulata $5 \mathrm{~mm}$. longa, tuto fauceque piloso, limbo 4 lobo, lobis valvatis ovato-triangularibus tubo 3-plo brevioribus minute apiculatis. Stamina 4, lobis corollæ alterna fere libera, filamentis longiusculis, antheris oblongis obtusis dor'so connectivo lato affixis. Discus pulvinatus. Orarium 4-loculare loculis 1ovulatis, ovulis in loculis solitariis al, apice loculi pendulis amphitropis, stylo filiformi, stigmate clavellato 4 -fido, ramis latiusculis erecto-patentibus. Drupe rubre late globosx $6 \mathrm{~mm}$. in diametro æquantes 4-pyrenæ, pyrenis dorso rotundatis subtrigonis cartilagineis 1-spermis. Semina sulbtrigona, testa tenuissima, albumine 
corneo; embryo parvus, cotylidonibus subplanis crassis, radicula brevi infra.

$\mathrm{HAB}_{\mathrm{AB}}$ in Suizan, in montibus Morrison, ad 7703 ped. alt., leg. S. NAGASAwa, Oct. 1905, (No. 784); Tōzan et Arizan, in isdem montibus, leg. G. NaKahara, Dec. 1906 ; in monte Molrison, ad 6500 ped. alt., (No. 1794), ad 7500 ped. alt., (No. 2055), et ad 9000 pert. alt., (No. 1926), leg. T. Kawamami et U. Mori, Oct. 1906.

This new species is very remarkable for very small flowers and very narrow leaves.

Damnacanthus indicus G ERTx. f. "Fruct. III. p. 18, t. 182 "; DC. Prodr. IV. p. 473 ; S. Moone, in Journ. Bot. (1875) p. 231 ; Ноок. f. Fl. Brit. Ind. III. p. 158; Maxim. in Mél. Biol. XI. p. 795; Fonbes et Hexss. Ind. Fl. Sin. I. p. 386.

Hab. Suizan, in montibus Morrison, leg. S. Nagasawa, Oct. 1905, (No. 658); in monte Morrison, leg. T. Kawakanir et U. MonI, Oct. 1906, (No. 1704); Nantō: Hinokiyama, leg. G. Naizahara, Feb. 1907.

Distrib. Eastern India, central China, the Loo-choo islands, and Japan.

It is stated by Mr. W. B. Hexrscer that D. macrophyllus Sreb. is a form of $D$. indicus G Grisv. f., as he has very many transitional stages between the two species. Mr. T. Makrio refers also to this interesting species in the Tökyō Botanical Magazine, XVIII. pp. 1214, where he mentions that the above two species and $D$. major Sieb, et Zucc. do not show any specifical distinction and they should be regarded as one and the same species accordingly. He also proposes to make many varieties, $\alpha, \beta, \gamma$, and $\delta$, including various forms. To my opinion, however, the plants do not seem to vary from one to another. It present, I am much inclined to regard the 
former two as specifically distinct species. The precerling new plant bears much resemblance to $D$. macropluyllus Sies. from which it differs in having much smaller flowers and more narrowed leaves.

\section{Lusianthus JACK.}

Lasianthus formosensis ML.ıTsurr. in Töleyó Ioot. MIag. XV. p. 17; Matsux. et Fayata, Enum. PI. Formos. p. 195, t. XV. A.

Huв. Taitō: Dakunsha, leg. T. Kawakisi et U. Mori, Dec. 1906, (No. 2159).

\section{Prederia Lins.}

Pæderia tomentosa Buuxe; DS. Proitr. IV. 1. 471 ; ITook. f. Fl I3rit. Ind. III. p. 197 ; Maxmr. in Mél. Biol. XI. p. 798 ; MIǫ. Fl. Tnd. Bat. II. p. 258 ; Fonbes et Heirst. Ind. Fl. Sin. I. p. 389 ; Mratsurr. in Tōkyō Bot. Mag. XV. p. 38 ; Diels, Fl. Centr. Chin. p. 582 ; PıLiprs, Conspect. Fl. Tiore:e, I. p. 106 ; Matsun. et Hayate, Enum. Pl. Formos. p. 197.

Pacderia foetida Thuns. Fl. Jap. p. 106 ; Hook. et Anx. Bot. Beech. Voy. p. 194; Benth. Fl. Hongk. p. 162; Fraxch. et Sivat. Enum. Pl. Jap. I. p. $210 ;$ HANCE, in Journ. Bot. (1874) p. 261.

Pcederic chinensis Haxce, in Journ. Bot. (1878) p. 228, et (1879) p. 12 ; Franchet, PI. David. p. 155.

Haв. Taitō: Daironkōsha, leg. T. Kawakaıı et U. Mori, Nov. 1906, (No. 2163).

Distrmb. Eastern India, Malay peninsula and arehipelago, China, and Japan.

\section{Nerterce Banks et Sor.}

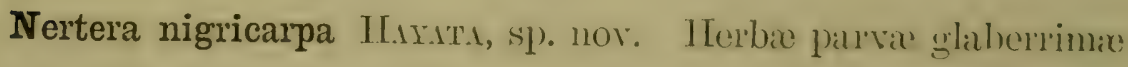
exsiccato nigricantes, caulibus repentilus ad norlos radicantibus, internodiis 1-3 cm. Iongis, ramulis depressis. Folia petiolatal, petiolis laminam in longitudine superantibus, laminis late ovatis vel ovato-reniformibus obtusis minute mucronatis basi abrupte 
attenuatis ad petiolum abeuntibus margine integerrimis $5 \mathrm{~mm}$. latis $4 \mathrm{~mm}$. longis, stipulis interfoliaceis validiusculis triangularibus vel late cuspidatis. Flores quasiterminales sessiles solitarii $2 \frac{1}{2} \mathrm{~mm}$. longi. Calycis tubus oroideus, limbo integro annulato. Corolla tubulata 4-loba, lobis integerrimis margine sub microscopio minute cristatis. Stamina 4, filamentis disco insertis, antheris late oratis brevissime apiculatis. Ovarium 2-loculare, stylis 2 elongatis suberectis intus cristato-stigmatosis. Drupæ nigricantes globosæ $4 \frac{1}{2} \mathrm{~mm}$. in diametro æquantes carnosæ dicoccæ (rariusmono coccæ), calycis limbo annulari coronatæ, coccis coriaceis ovatis intus plano-concavis extus convexis 1 -spermis. Semina orata plano-convexa circ. $2 \mathrm{~mm}$. longa intus leviter sulcata.

Hab. Tōzan, in montibus Morrison, Oct. 1906 ; in monte Morrison, leg. S. Nagasatwa, Nov, 1905.

Distrib. According to Mr. E. D. Merricu, an allied Australian species $N$. depressa Baxks et Sou. occurs in the Philippine islands.

Very much like $N$. depressa Banks et Sou. ; but differs from it in having entire lobes of corolla, black colored berries, and cristate stigmas. The occurrence of this Australian genus is very remarkable.

\section{Rubia LINN.}

Rubia cordifolia LrNv.; DC. Prodr. IV. p. 588; Francher, PI. David. p. 155 ; Maxnr. Mél. Biol. IX. p. 266 ; Hook. f. Fl. Brit. Ind. III. p. 202 ; Miq. Fl. Ind. Bat. II. p. 337; FonBes et Hearst. Ind. Fl. Sin. I. p. 393 ; Drezs, Fl. Ceutr. Chin. p. 583 ; Matsuxr. in Tōkyō Bot. Mag. XV. p. 39 ; Patibn, Couspect. Fl. Koreæ, I. p. 106 ; Matsusr. et Hayata, Enum. PI. Formos. p. 199.

Haв. Toroku : Tōhozan, Nov. 1905, (No. 1827), et in monte Morrison, ad 7000 ped. alt., leg. T. Kawaikani et U. Mori, Oct. 1906 , (No. 2058). 
Distrib. Japan to central China and Dahuria; mountains of India southward to Ceylon and Nalacca; tropical Africa.

Rubia lanceolata Harata, sp. nov. Herhe minute acnleolatx, caulibus elongatis 4-gonis. Folia t-natim verticillata longe petiolata, petiolis lamina in longitudine 1-2-plo longioribus minute: uncinato-aculeolatis, laminis lanceolatis ovato-lanceolatis vel lincarilanceolatis apice acuminatis basi rotundatis vel leviter cordatis $9 \mathrm{~cm}$. longis $1 \mathrm{~cm}$. latis supra ad nervos hispirlulis sulutus ad nervos et margines uncinato-asperis trinerviis. Flores minuti in cymas paniculatas circ. $20 \mathrm{~cm}$. longas axillares et terminales dispositi, bracteis angusto-lanceolatis 4-natim verticillatis. Flores ignoti. Fructus didymi $5 \mathrm{~mm}$. lati $3 \mathrm{~mm}$. longi.

HAB. Ganzan, in montibus Morrison, ad 9141 ped. alt., leg. S. Nagasatwa, Oct. 1905, (No. 687); in monte Morrison, ad 7000 ped. alt., leg. T. Kawakami et U. Mori, Oet. 1906, (Nos. 1715 et 1989?).

Closely resembles $R$. cordifolia Lrsv.; but differs from it in having lanceolate and trinerved leaves; from $R$. Schumanniuna li. PritzeL, this differs in having minutely aculeolate stems and mcinately asperous petioles.

Galiume Linn.

Galium brachypodium MAxtr. in Mél. Biol. IX. p. 260.

НАв. in monte Morrison, ad 9000 ped. alt., leg. 'T. KAwakim et U. MorI, Oct. 1906, (No. 1805).

Distris. Northern part of Japan.

A more species of Galium; not yet determined. 


\section{Valerianeæ.}

\section{Patrinia Juss.}

Patrinia scabiosæfolia Fisch.; DC. Prodr. IT. p. 624; Ledez. Fl. Ross. II. p. 427 ; HaNCE, in Journ. Bot. (1S70) p. 225, et (1883) p. 322 ; Franch, et Sayat. Enum. Pl. Jap. I. p. 216 ; Franchet, Pl. David. p. 158; Forbes et Hemst. Ind. FI. Sin. I. p. 397.

Patrinic parviflora Sieb. et Zucc. Fl. Jap. Fam. Nat. n: 678; MrQ. in Ann. Murs. Bot. Lugd.-Bat. III. p. 115.

Hab. Nantō: Mushazan, ad 6000 ped. alt., leg. T. Kawakami et U. Moni, Aug. 1906, (No. 1162).

Distrib. Japan through China westward to Dahuria.

Patrinia villosa Juss.; DC. Prodr. IV. p. 624 ; Franch. et Savat. Enum. Pl. Jap. I p. 216; Fonbes et Hexsl. Ind. Fl. Siv. I. p. 398; Henry, List Pl. Formos. p. 51 ; Diels, Fl. Centr. Chin. p. 597 ; Partbin, Conspect. Fl. Korese, I. p. 108.

Patrinia ovata Bunge; Franchet, Pl. David. p. 157.

Valeriana villosa Thuxb. Fl. Jap. p. 32, t. 6 .

Hab. Suizan, in montibus Morrison, ad 7702 ped. alt, Oct. 1905, (No.672) ; Toroksu : Tōhokei, leg. T. Kawamani et U. Moni, Nov. 1906, (No. 1905).

Distrim. Japan and China.

Hoectia ENGL. et Grans.

Hœckia Aschersoniana Engt. et Grizin. in Diets, Fl. Centr. Chin. p. 598 ; Hayata, in Tờyó Bot. Mag. XX. p. 57.

Haв. Ganzan, in montibus Morrison, ad 9141 ped. alt., leg. S. Nagasatwa, Oct. 1905, (No. 635); in monte Morrison, ad 11000 ped. alt., leg. T. Kawainami et U. Monr, Oct. 1906, (No. 2272). 
Distrib. Central China. This monotypic genus is found in central China and Formosa, but nowhere else.

\section{Dipsaceæ.}

\section{Scabiosn Linn.}

Scabiosa (Sect. Sclerostemma) lacerifolia HıYaTı, (PI. XYT.), in Tōkyō Bot. Mag. XX. p. 16. Herbæ perennes pubcscentes rel glabriusculæ, caulibus validiusculis $10-20 \mathrm{~cm}$. altis pauci-ramosis. Folia sessilia semi-amplexicaulia linearia oblanceolata obsolete pinnatisecta rel lacerata, segmentis irregulariter serratis, radicalia circ. $12 \mathrm{~cm}$. longa 5-6 mm. lata, caulina opposita breviora minora 4-5 cm. longa apico acuta basi longe attenuata sensim dilata. Capitula terminalia depressa $3.5-4 \mathrm{~cm}$. in diametro æquantia longe pedunculata, pedunculis 5-6 cm. longis vel longioribus. Involucri bractex 3 -seriatre herbacex liberæ sublanceolatæ plerumque 1 -florem gerentes extimæ $15 \mathrm{~mm}$. longæ interiores minores ; receptaculi palex spathulate 5 mm. longr. Involucellum 4-costatum 8-foveolatum apice 4-lobatum, lobis obtusis. Calycis limbus in 5 setas radiato-patentes diversus. Flores radiantes involucrum fere excedentes; corolla $18 \mathrm{~mm}$. longa, limbis 5-fidis 2-labiatis. Stamina 4, omnia perfecta. Stylus filiformis. Achænium involucello basi adnatum calycis limbo persistente coronatum obovoideum $3 \mathrm{~mm}$. longum; setx breves $1 \mathrm{~mm}$. longæ.

Hab. in monte Morrison, ad 13094 ped. alt., leg. S. Nagasawa; in montibus centralibus, ad 110.0 ped. alt., leg. T. Kawarani et U. Mori, Nov. 1906, (No. 1852).

As the original description is based upon an imperfect specimen, I have taken the liberty of repeating the description of the plant, basing the above diagnosis upon a most perfect specimen. 
Distrib. The present genus is mainly distributed in Europe, West Asia, and Africa. In East Asia, four species are found in the alpine regions of the Himalayas, one species in North China, and also one in Japan. No species has ever been known from either central or southern China.

\section{Compositæ.}

\section{Ethulia LINN.}

Ethulia conyzoides Linv. DC. Prodr. V. p. 12; Clarke, Comp. Ind. p. 1; Ноок. f. Fl. Brit. Ind. III. p. 226.

Ethulia ramosa Roxb. Fl. Ind. ed.-CaRey, p. 413.

Ethullia gracilis Deltue; DC. Prodr. V. p. 12.

Ethulia angustifolia BoJER; DC. Prodr. V. p. 12.

Haв. Tikusan, ad 150 ped. alt., leg. S. Nagasawa, Oct. 1905, (No. 763).

Distrib. Tropical Africa and Asia. The Philippine islands and Java.

The present plant does certainly not belong to the mountain flora of the island. As the species is new to the Formosan flora, I have taken this occasion to mention it.

\section{Vernonia SCHREB.}

Vernonia Andersoni Clarise, Comp. Ind. p. 26 ; Hoor. f. Fl. Brit. Ind. III. p. 241 ; Fonbes. et Hemsl. Ind. Fl. Sin. I. p. 400 ; Hexry, List Pl. Formos. p. 51 ; Hayata, Compos. Formos. p. 4 ; Matsun. et Hayata, Enum. Pl. Formos. p. 201.

Haв. Taichū: Kashigatani, leg. G. Nakahara, Feb. 1907.

Distrib. South China; Assam, Burma, and Tenasserim.

Vernonia cinerea Less. in Linnrea, IV. p. 291 et VI. p. 673; DC. Prodr. V. p. 24 ; Mir. Fl. Ind. Bat. II. p. 11; BeNtr. Fl. Hongk. p. 169 ; 
Grisebach, Fl. Brit. W. Ind. Isl. p. 353 ; Bexth. Fl. Austral. III. p. 459 ; Clarke, Comp. Ind. p. 20 ; Ohrver, Fl. Tropic. Afric. p. 275 ; Ноок. f. Fl. Brit. Ind. III. p. 233 ; Fonbes et Hexsis. Ind. Fl. Sin. I. p. 401 ; Drels, Fl. Centr. Chin. p. 608; Hayata, Comp. Formos. p. 5 ; Mratsusr. et Hayata, Enum. Pl. Formos. p. 201.

Hab. Tōzan, in montibus Morrison, leg. G. NaKahara, Oct. 1906.

Distrib. Tropical Asia, Africa and Australia.

Adenostemma Forst.

Adenostemma viscosum Forst; Less. Synop. Comp. p. 156 ; DC. Prodr. V. p. 111 ; Sieb. et ZucC. Fl. Jap. Fam. Nat. p. 181 ; Bextr. Fl. Hongk. p. 171, et Fl. Austral. III. p. 462 ; Franch. et Savat. Enum. Pl. Jap. I. p. 219 ; Clarke, Comp. Ind. p. 23 ; Oliver, Fl. Tropic. Afric. p. 299 ; Franchet, Pl. David. p. 159 ; Mrq. Fl. Ind. Bat. II. p. 23 ; Ноок. f. Fl. Brit. Ind. III. p. 242 ; Forbes et Heass. Ind. Fl. Sin. I. p. 403 ; Diels, Fl. Centr. Chin. p. 608 ; Hayata, Comp. Formos. p. 7 ; Matsur. el Hayata, Enum. Pl. Formos. p. 202.

Spilanthes tinctorius Lour. Fl. Cochinch. ed-WmLd. p. 590.

Hab. Toroku: Washa, leg. 'T. Kawakani et U. Moni, Nov. 1906 , (Nos. 1825).

Distrib. South China; Tropical Asia, Africa, America, and Australia.

\section{Ageratum Lins.}

Ageratum conyzoides Lisx. Sp. Pl. ed-2, p. 1175; Lasss. Symup. Comp. p. 155 ; DC. Prodr. V. p. 108; MIR. Fl. Ind. Bat. II. p. 23 ; Bextir. Fl. Hongk. p. 171 ; Sonder, Fl. Capens. III. p. 57 ; Bехтн. Fl. Austr. III. p. 462 ; Grisebach, Fl. Brit. W. Ind. Isl. p. 356 ; Seemanx, Tl. Vit. p. 140 ; Clarike, Comp. Ind. p. 30 ; Oliver, Fl. Tropic. Afric. IIT. p. 300 ; Heisst. Voy. Chall. Bot. I. p. 40, et in Biol. Centr.-Americ. II. p. 81 ; Hoor f. Fl. Brit. Ind. III. p. 243 ; Fonbes et Herst. Ind. Tl. Sin. I. p. 403 ; Hexry, 
List Pl. Formos. p. 51. Hayata, Comp. Formos. p. 7 ; Matsus. et Hayata, Enum. Pl. Formos. p. 202.

Haв. Taitō : Bokusekikaku, leg. T. Kawakani et U. Mori, Dec. 1906, (No. 1831).

Distrib. Generally spread over all the warm regions, though often existing only as a colonist. Perhaps, an American origin.

\section{Eupatorium LiNN.}

Eupatorium formosanum HaIATA, sp. nor. Herbæ basi suffiruticosæ præter inflorescentiam subsimplices villoso-pubescentes. Folia opposita tri-partita, segmentis lateralibus subsessilibus lanceolatis basi obliquis rel latere inferiore ad petiolum decurrentibus segmentis terminalibus lanceolatis breve petiolulatis iis lateralibus longioribus, apice acuminata basi acuta serrulata supra scabra subtus pallidiora pubescentia ad costas venulasque villosa, petiolis villoso-pubescentibus segmento terminali 2-plo brevioribus. Capitulum parvulum $7 \mathrm{~mm}$. Iongum corymbosum 4-5-florum. Involucrum oblongo-campanulatum, bracteis 3 -seriatis imbricatis scariosis, intimis oblongo-angustis $5 \mathrm{~mm}$. longis, exterioribus gradatim minoribus, extimis parvissimis ovatis $1 \frac{1}{2} \mathrm{~mm}$. longis. Receptaculum parvum convexum foveolatum. Corollæ tubulatæ $4 \mathrm{~mm}$. longæ, tubis tenuibus limbis parum ampliatis tubo 2-plo brevioribus lreviter 5-dentatis, dentibus triangularibus marginatis. Anthere appendiculate basi obtusæ integræe. Styli basi disco dentato annulari coronati, ramis elongatis obtusis. Achænia 5 -angulata apice truncata basi attenuata $2 \mathrm{~mm}$. longa ; pappi setæ 1 -seriatæ rigidulx scabræ $3 \frac{1}{2} \mathrm{~mm}$. longæ.

Hab. in monte Morrison, ad 7000 ped. alt., leg. T. KatwaKanm et U. Moni, Oct. 1906, (No. 1961) ; Arizan in montibus Morrison, leg. 
G. Naknhara, Nor. 1906 ; in montibus centralibus, ad 9000 ped. alt., leg. T. Kawakant et U. Mori, Nor. 1906, (No. 1898).

The present plant comes near E. chinensis Lins. ; and resembles especially its rariety, tripartitum of Mrever; but differs from them in having distinctly triparted leares and hairy stems and leares.

Eupatorium Lindleyanum DC. Prodr. T. p. 180; 13натr. Fl. Hongk. p. 172, et Fl. Austr. III. p. 462 ; Fonbes et Henss. Iud. Fl. Sin. I. p. 404; Hexry, List Pl. Formos. p. 52 ; Diels, Fl. Centr. Chin. p. 608 ; Hayata, Comp. Formos. p. 9 ; Matsung. et Hayata, Euum. Pl. Formos. p. 203.

Hab. Kagi : Shakkōshō, leg. T. Kawakanir et U. Mori, Nor. 1906, (No. 1978); Goryō, ad 350 ped. alt., leg. S. NAGASAWA, Oct. 1905, (No. 760).

Diștrtb. China, Manchuria and Japan.

Eupatorium Tashiroi Hayat, Compos. Formos. p. 9; M.atsix. et Hayata, Enum. Pl. Formos. p. 203.

Haв. Kōshuin: Naibun, leg. G. Nakahara, Feb. 1907.

\section{Solidago Lins.}

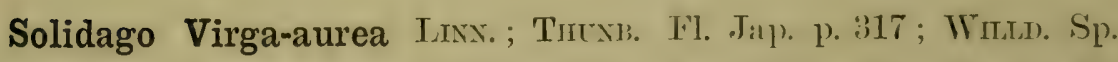
P1. p. 2065 ; Less. Synop. Comp. p. 163; DC. Prodr. V. p. 338 ; Bentr. TI. Hongk. p. 179 ; Fraxch. et Satat. Enum. Pl. Jap. I. p. 228 ; Clanke, Comp. Ind. p. 35 ; Hoor. f. Fl. Brit. Ind. III. p. 245 ; FonBes et Hexst. Ind. Tl. Sin. I p. 406 ; Hexry, List Pl. Formos. p. 52 ; Hayata, Comp. Formos. p. 10; Matsurr. et Hayata, Enum. P1. Formos. p. 203.

Solidago cantoniensis et $S$. decurvens Lour. Fl. Cochinch. ed-WmLD. p. 612 ; DC. Prodr. V. pp. 341-342.

HAB. Suizan, in montibus Morrison, ad Tio2 perl. alt., leg. S. NA-

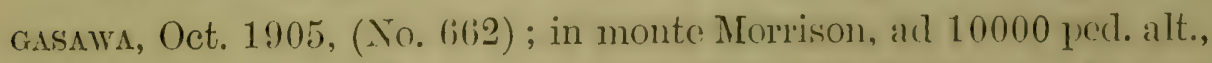
leg. T. Kawamasir et T'. Mori, Oct. 1906(i, (No. 2146); Nanto : Musha- 
zan, ad 7500 ped. alt., leg. T. Kawakani et U. Mori, Aug. 1906 , (No. 1132); in montibus centralibus, ad 10000 ped. alt., leg. T. Kawakami et U. Morr, Nov. 1906, (Nos. 2208 et 2207).

Distrib. Cosmopolitan; North America to Japan, westward to temperate Asia and Europe.

\section{Myriactis Less.}

Myriactis Wightii DC. Prodr. V. p. 308 ; WIGHT, Ic. PI. Ind. or. t. 1091 ; Cuanke, Comp. Ind. p. 38 ; Hook. f. Fl. Brit. Ind. III. p. 247 ; Tnnnen, Fl. Ceyl. III. p. 15.

Myriactis javanica DC. Prodr. V. p. 308; Clarke, Comp. Ind. p. 38.

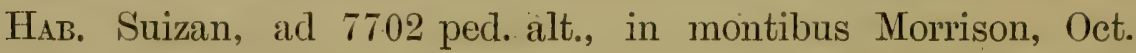
1905, (No. 657), eodem loco, ad 11579 per., alt., (No. (920), et Tōzan, in isdem montibus, leg. G. Nagasawa, Oct. 1906 ; in isdem montibus, ad 7000 ped. alt., leg. T. Kawakani et U. Moni, Oct. 1906, (No. 1790), Taitō: Daironkōsha, leg. T. Kawakani et U. Morr, Nov. 1906, (No. 1844).

Distrib. The Nilghiry Mountains : at an altitude of $8000 \mathrm{ft}$; Ceylon : the central province, at elevations of 5-8000 ft.

According to Sir. J. D. Hooker, this species is very variable. He states that three species belonging to this genus mentioned in "The Flora of British India " are all similar. Accepting his statement, the Formosan plant may be referred to this species.

\section{Aster Linn.}

Aster baccharoides SteETz; Bexth. Fl. Hongk. p. 75; Fonbes et Hemsi. Ind. Fl. Sin. p. 409 ; Henny, List Pl. Formos. p. 52 ; Diels, Fl. Centr. Chin. p. 610 ; Hayata, Comp. Formos. p. 13 ; Matsuxr. et Hayata, Enum. Pl. Formos. p. 204.

HAB. in monte Morrison, ad 8000 ped. alt., Oct. 1906, (No. 1959), et in montibus centralibus, ad 10000 ped. alt., leg. 'T. Kaws- 
Kami et U. Mori, Nor. 1906, (No. 2206); Nantō: Nankōkei, leg. 'T. Kawakairi et U. Mori, Aug. 1906, (No. 1174).

Distrib. South central China.

Aster scaber Thunb. Fl. Jap. p. 316 ; Foribes et Heass. Ind. FI. Sin. I. p. 415 ; DieLs, Fl. Centr. Chin. p. 611.

Biotia discolor Maxtr. in Prim. Fl. Amur. p. 146.

Doellingeria scalna NeEs; DC. Prodr. V. p. 263; Mro. in Ann. Mus. Bot. Lugd.-Bat. II. p. 169.

HAB. in montibus centralibus, ad 10000 ped. alt., leg. T. KAWAKaMi et U. Moni, Nor. 1906, (No. 1850).

Distrib. China, Manchuria eastward to Japan as far as North America.

Aster trinervius Roxb. Fl. Ind. ed.-Carey, p. 433 ; Bextr. Fl. Hongk. p. 174; Franch. et Savat. Enum. Pl. Jap. I. p. 222 ; Ноoк. f. Fl. Brit. Ind. III. p. 252 ; Franchet, Pl. David. p. 161 ; Maxim. in Exgl. Bot. Jahrb. VI. p. 68 ; Forbes et Hemst. Ind. Fl. Sin. I. p. 416 ; Henrr, List Pl. Formos. p. 52 ; Hayata, Comp. Formos. p. 13 ; Matsum. et Hayata, Enum. Pl. Formos. p. 204.

Diplopappus asperrimus DC. Prodr. V. p. 277 (fide Hexst.).

Aster ageratoides Tuncz; Maxtr. Prim. Fl. Amur. p. 144.

HAB. Ganzan, in monte. Morrison, ad 9141 ped. alt., leg. S. NAGASAWA, Nov. 1905, (No. 695); in monte Morrison, ad 7000 ped. alt., (Nos. 1870 et 1960), et ad 8000 pecl. alt., (No. 1958), leg. T. KawAKanI et U. Mori; Suizan in montibus Morrison, ad 7702 pecl. alt., (No. 559), Oct. 1905 ; Tappansha, ad 3138 pecl. alt., leg. s. Nıcissawa, Oct. 1905, (No. 715); Tōzan, in montibus Morrison, leğ. (i. Nakahara, Oct. 1906 ; Tōhosha, ad 2930 ped. alt., (Nos. 607 et lioti) Nov. 1905, leg. S. Nagasawa; Nantō: Minokiyama leg. (i. Nakanaid, Feb. 1907. 
Distrib. Japan to Manchuria, westward to the mountains of northern India.

This species is, so far as I am aware, exceptionally variable, and many apparently different specimens in my hands are mentioned under a single name.

\section{Erigeron Lins.}

Erigeron morrisonensis HaxaTA, sp. nov. Herbæ perennes parve, caulibus hirsutis subsimplicibus circ. $10 \mathrm{~cm}$. altis erectis. Folia radicalia longe spathulata cum petiolis $6 \mathrm{~cm}$. longa $5 \mathrm{~mm}$. lata apice rotundata vel brevissime apiculata basi longe attenuata in petiolum $3 \mathrm{~cm}$. longum abeuntia, caulina alterna sessilia angusta breviola. Capitula mediocria $13 \mathrm{~mm}$. in cliametro squantia, solitaria heterogama, floribus radialibus $q$, disci $\not{q}$, omnibus fertilibus. Involucrum late campanulatum, bracteis sub-3-seriatis angustis linearibus circ. $6 \mathrm{~mm}$. longis apice coloratis margine scariosis numerosis parum inæqualibus cxtus pilosissimis. Receptaculum conrexum nudum. Corollæ o ligulatæ angustissime tubis limbo vix brevioribus; ㅎ tubulatæ $4 \mathrm{~mm}$. longæ, tubis pilosiusculis, limbis parum ampliatis 4-dentatis. Antheræ basi obscure sagittatæ. Styli fl. ఫ̧ rami complanati, appendicibus triangularibus. Achænia compressa angusta $2 \mathrm{~mm}$. longa pilosa, margine nervio, facie cnervia; pappi setæ tenues 1 -seriatæ scahrinscule rufo-albæ $3 \mathrm{~mm}$. longæ:

HAB. in summam montis Morrison, ad 13094 ped. alt., leg. S. Nagasawa, Nov. 1905; in codem loco, leg. T. Kamamani et U. Moni, Oct. 1906, (No. 2247).

Near E. Thunbergi Girsy ; but distinguished hy the spathulate linear leaves and small ray florets. 
Blumen DC.

Blumea chinensis DC. Protr. V. p. 444 ; Bexтir. Fl. Hongk. p. 177 ; Hook. f. Fl. Brit. Ind. III. p. 268; Forbes et HensL. Ind. Fl. Sin. I. p. 420 ; Henry, List Pl. Formos. p. 53 ; Hayata, Comp. Formos. p. 30 ; MLatsum. et HaYata, Enum. P1. Formos. p. 209.

Conyza chinensis Livv. Sp. P1. ed.-2, p. 1208 ; MIr. Fl. Ind. Bat. II. p. 52. Hab. Washakei, leg. T. Kawakami et U. Mori, Nov. 1906, (No. 1823) ; Kagi: Shitōsha, leg. T. KaтаталI et U. Mori, Oct. 1906, (No. 1979).

Distrib. South China, eastern India, and Java.

Laggera SсH.-Bip.

Laggera alata Sch.-Br.. ; Clanke, Comp. Ind. p. 91 ; Hoor. f. Fl. Brit. Ind. III. p. 271; Fonbes et Heusc. Ind. Fl. Sin. I. p. 422 ; Matsux. et Hayata, Enum. Pl. Formos. p. 210.

Blumea alata DC. Prodr. V. p. 448 ; Bevth. FI. Hongk. p. 177 ; Wighr, Ic. Pl. Ind. or. t. 1101.

Hab. in monte Morrison, ad 10000 ped. alt., leg. T. Kawakami et U. Mori, Nov. 1906, (No. 2114).

Distrib. Generally found in tropical Asia, Africa, and Madagascar; South China.

It is rather an anomalous case that we have such a tropical species in the high regions of Formosa.

\section{Leontopodium Br.}

Leontopodium microphyllum IIArati, sp. nov: (PI. XY'II). IIerbo suffruticosa peremes cespitosæ lanata, caulibus ascenclentibus erectisve simplicibus $\tilde{5}-(; \mathrm{cm}$. altis. Folia radicalia caulinis conformia spathulato-angusta integerrima $1 \mathrm{~cm}$. longa $2 \mathrm{~mm}$. lata, supra laxe subtus dense lanata. Capitula parrula $3 \mathrm{~mm}$. longal 
ad apicem caulis in cymas densas foliis floralibus quasi involucratas 4-5-conferta ; foliis floralibus 5-6 in cyma quisque, horizontaliter patentibus dense lanatis linearibus acuminatis $1 \mathrm{~cm}$. longis; fl. 우 fertilibus in ambitu pancis; fl. $\not{q}$ sterilibus in disco paucis. Inrolncrum campanulatum, bracteis circ. 2 -seriatis imbricatis scariosis, intimis angusto-acutis apice lanatis circ. $3 \mathrm{~mm}$. longis, extimis latioribus dorso lanatis. Receptaculum convexum fere epaleaceum foreolatum. Corollæ ㅇf filiformes truncatæ; $\not{\gamma}$ regulares $2 \mathrm{~mm}$. longæ tubulosæ, limbis ampliatis subcampanulatis apice 5 -fidis pance pilosis. Antheræ basi sagittatæ, auriculis tenuissime caudatoappendiculatis. Stylus breviter 2-fidus, ramis obtusis. Achænia parra oblonga subteretia ecostata glabra; pappi setæ 1 -seriatie tenues breviter valdeque barbellatæ basi subconnatæ.

HaB. in monte Morrison, ad 12000 ped. alt., leg. T. Kawakami et U. Morr, Oct. 1906, (No. 246).

Very distinct species having very small leaves.

\section{Anaphatis DC.}

Anaphalis margaritacea Bехтн. et Hook. f. Gen. Pl. II. p. 303; Clarke, Comp. Ind. p. 103 ; Maxmr. in Mél. Biol. XI. p. 235 ; Fonbes et HeMss. Ind. Fl. Sin. I. p. 425.

Antemaria margaritacea R. BR.; DC. Prodr. VI. p. 270; Ledeb. Fl. Ross. II. p. 613.

Gnaphatium margaritaceum LINx. Sp. Pl. ed-2, p. 1198; Franch. et Savat. Enum. Fl. Jap. I. p. 242.

var. angustifolia (Franch. et SAvat.).

Gnaphalium margaritaceum LINN. var. angustifolium Fraxch. et SAVAT* Enum. Pl. Jap. I. p. 242.

Antemaria japonica Mro. Prol. Fl. Jap. p. 110.

forma nana. Humiles circ. $5 \mathrm{~cm}$. altæ, caulibus simplicibus. 
Folia parvissima oblonga vel oblongo-linearia if mm. longa $1 \mathrm{~mm}$.$1 \frac{1}{2} \mathrm{~mm}$. lata apice crassiusculo-apiculata lanata.

HAB, in monte Morrison, ad 10000 ped. alt.

forma morrisonicola. Altiores $10-15 \mathrm{~cm}$. altæ, caulibus simplicibus. Folia parviora oblongo-lanceolata rel oblongo-linearia circ. $2 \frac{1}{2} \mathrm{~cm}$. longa $3 \mathrm{~mm}$. lata aristato-apiculata rel obtusa supra glabra subtus lanata.

HAB. In monte Morrison, ad 5000 ped. alt., leg. R. TonIr, 1899 ; Giyokusan, ad 10634 ped. alt., (No. 711), et Ganzan, ad 9141 ped. alt., (No. 705), in montibus Morrison, leg. S. Nagasawa, Nov. 1905 ; Tōzan, in isdem montibus, leg. G. NAKshata, Oct. 1906 ; in monte Morrison, ad 8000 ped. alt., (No. 1797), et ad 10000 ped. alt., (No. 2277), leg. T. Kawakani, et U. Mori, Oct. 1906 ; in montibus centralibus, ad 10000 ped. alt., leg. T. Kawanane et U. Mozi, Nov. 1906, (No. 1848); Nantō : Hinokiyama, leg. G. Nakahara, Feb. 1907; Mushasan, ad 8000 ped. alt., leg. T. Kawakasi et U. Mori, Aug. 1906, (No. 1128).

There is a little doubt about identifying the all above plants with $A$. margaritacea var. angustifolia. The plant represented by var. angustifolia is exceptionally variable. I have examined a considerable number of various forms of this variety collected in Tapan and Formosa, and found that they show no specifical distinction though they seem apparently different.

Distrib. Type: Japan and China northward to Amurland and Kamtchatka; also in North America and nationalized in Europe.

Anaphalis Nagasawai HaxıTA, (PI. XTIII.), in Tōkyō T3ot. Mage. XX. p. 15. Herbæ peremes crectæ lanatie stoloniferæe $4 \mathrm{~cm},-5 \mathrm{~cm}$. altæ tenuiusculx, radicibus lignescentilus. Folia alterna integerrima decurrentia obovato-spathulata apice rotundata vel obtusa 
$1 \mathrm{~cm} .-1 \frac{1}{2} \mathrm{~cm}$. longa $3 \mathrm{~mm} .-5 \mathrm{~mm}$. lata lanata. Capitula majuscula ad apicem caulis subsimplicis solitaria $1.5 \mathrm{~cm} .-2 \mathrm{~cm}$. in diametro æquantia heterogama; floribus 우 fertilibus in ambitu $\infty$-seriatis,

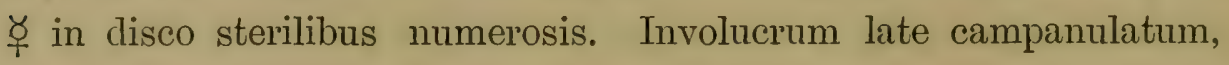
bracteis radiantibus niveis $\infty$-seriatis imbricatis scariosis, intimis oblongo-lanceolatis $7 \mathrm{~mm}$. longis $1 \mathrm{~mm}$. latis, interioribus oblongis petaloideis patentibus $10 \mathrm{~mm}$. longis $3 \mathrm{~mm}$. latis, exterioribus brevioribus, extimis ovatis dorso lanatis $8 \mathrm{~mm}$. longis $3 \mathrm{~mm}$. latis. Receptaculum convexum epaleaceum. Corollæ fl. 우 filiformes, 4-dentatæ apice glanduloso-pilosæ; fl. $\not{q}$ tubulosæ, limbo ampliato subcampanulato apice 5-fido minuto papilloso-marginato. Antheræ apice appendiculatæ, appendicibus obtusis, basi sagittatæ caudatæ. Styli fl. ㅇ filiformes minute 2-lobi; fl. ㅎ apice breve 2-lobi, ramis truncatis. Achænia oblonga minutissime hirtello-papillosa subteretia $1 \mathrm{~mm}$. longa $\frac{1}{2} \mathrm{~mm}$. in sectione æquantia; pappi setæ tenues 1 -seriatæ scabræ liberæ caducæ $6 \mathrm{~mm}$. longæ.

HAB. in monte Morrison, ad 13000 ped. alt., leg. T. KawaKam et U. Morr, (Nos. 2126 et 2239).

In general appearance, this species resembles very much G. niitakayamense HAYATA. The difference by which the genera, Gnaphalium and Anaphalis, are separated from each other is indeed a very slight one. The separation lies on the point whether the disc flowers are stirile or not. So far as my knowledge extends, this does not seem to be a fixed character. I am, therefore, much inclined to think that the separation of Anaphalis from Gnaphatium is rather artificial.

Gnaphatium LiNs.

Gnaphalium hypoleucum DC. Prodr. VI. p. 222 ; Hoor. f. FI. Brit. 
Ind. III. p. 2SS ; Wight, Ic. Pl. Ind. or. t. 1114; Fonbes et Hevrst. Ind. F1. Sin. I. p. 426 .

Gnaphatium amoyenss HAxcE, in Journ. Bot. (1868) p. 174, et (1878) p. $10 \mathrm{~S}$; BeNTH. Fl. Hongk. p. 187.

HAB. Ganzan, ad 9141 ped. alt., in montibus Morrison, Oct. 1905, (No. 630), in monte Morrison, ad 11000 ped. alt., (No. 2077), et ad 12000 ped. alt., (No. 2234), leg. T. Kawakami et U. Mori, Oct. 1906 .

Distrib. South China and Japan; and also in the mountains of India and Abyssinia.

Gnaphalium lineare Hayata, sp nov. (Pl. XIX.). Herba graciles sericeo-lanatæ. Caules erecti tenuissimi simplices $20 \mathrm{~cm}$. alti foliis caulinis remote instructi. Folia radicalia sessilia linearia $7 \mathrm{~cm}$. longa $1 \frac{1}{2} \mathrm{~mm}$. lata apice obtusa basi decurrentia, caulina radicali conformia alterna breviora basi semi-amplexicaulia exsiccato revoluta supra subglabra subtus sericeo-lanata. Capitula ad apicem caulis in cymas densas foliis floralibus quasi involucratas circ. 10 conferta. Cyma capitulorum depresso-globosa 2! $\mathrm{cm}$. in diametro æquans, foliis floralibus 5-10, 2-seriatis linearibus circ. $1 \mathrm{~cm}$. longis $1 \frac{1}{2} \mathrm{~mm}$. latis apice obtusis basin versus dilatis intus subglabris extus plus minus lanatis. Involucrum ovoideum $\neq \mathrm{mm}$. longum $2 \mathrm{~mm}$. latum, bracteis $2-3$-seriatis scaliosis apice fuscis, interioribus spathulatis integris vel pauce laceratis $t \mathrm{~mm}$. longis $\frac{2}{3} \mathrm{~mm}$. latis, exterioribus brevioribus latioribus obovatis dorso lanatis. Receptaculum leviter concarmm minute muricatum. Fl. 우 in ambitu co-seriati; corolla liliformes $3 \mathrm{~mm}$. longa apice breviter 2-3-fidx; styli rami elongati recurvi. Fl. $\not$ i in disco paucissimi 1-4, tubulosi temes, limbo parum ampliato 5-rlentato, dentibus triangularibus marginatis; anthera basi sagittata, auriculis sape paucifidis temuiter caudato-appendiculatis; styli 
rami subteretes apice subcapitati. Achænia oblonga $\frac{1}{2} \mathrm{~mm}$. longa teretiuscula ecostata minute pauceque glanduloso-tuberculata, pappi setis 1 -seriatis tenuibus caducis scariosis $3 \mathrm{~mm}$. longis.

Haв. Tōzan, in montibus Morrison, leg. G. NaKahara, Oct. 1906 ; in monte Morrison, leg. T. KawaKamr et U. Mori, Oct. 1906, (No. 1995).

Near G. japonicum Thunb.; but easily distinguished from it by the linear leaves; from $G$. collinum LABrLL, by the compound heads; from $G$. Thomsoni Hoor. f., by the not papillose achenes and smaller clusters of the heads; and from G. uliginosum Liss., by the extremely narrow leaves.

Gnaphalium luteo-album I.rx. Sp. Pl. ed-2, p. 1196 ; I.Ess. Synop. Comp. p. 331 ; DC. Prodr. VI. p. 230 ; MIQ. Fl. Ind. Bat. II. p. 91 ; ВеNTH. Fl. Austr. III. p. 653 ; Sonder, Fl. Capons. III. p. 262 ; Cuarke, Comp. Ind. 1. 114 ; Hexst. Biol. Centr.-Americ. II. p. 139 ; Ноок. f. Fl. Brit. Ind. III. p. 288 ; Henriy, List Pl. Formos. p. 53 ; Hayata. Comp. Formos. p. 32 ; Matsuar. et Hayata, Enum. Pl. Formos. p. 210.

Hab, in monte Morrison, ad 7500 ped. alt., leg. 'T. KawaKami et U. Morr, Oct. 1906, (Nos. 1980 et 2044); Nantō: Mushasan, ad 8000 ped. alt, leg. T. Kawamani et U. Momi, Aug. 1906, (No. 1133).

Distrib. Himalaya, mountains of Java and Australia.

Gnaphalium niitakayamense Haxatı, in Tökyó Bot. Nag. XX. 1). 14. Herbæ perennes erectæ dense lanatæ $6-\tau \mathrm{cm}$. altæ validiusculæ. I'olia alterna integerrima sessilia oblanceolata vel spathulato-oborata 1-1 $\frac{1}{2} \mathrm{~cm}$. longa $5 \mathrm{~mm}$. lata. Capitula parva oboroidea $1 \mathrm{~cm} .-1 \frac{1}{2} \mathrm{~cm}$. in diametro æquantia ad apicem caulis 2-3 conferta vel solitaria, floribus in ambitu 우 $\infty$-serratis, $\not$ p paucioribus, ommibus fertilibus. Involucrum oboroideo-campanulatum, bracteis 4-5 seriatis scariosis albis, extimis dorso lanatis, intimis linearibus, interioribus 
oblongis $8 \mathrm{~mm}$. longis. Receptaculum planum foreolatum. Corollæ 우 filiformes, minute 4 -dentatx; $\not{\gamma}$ regulares tubulosæ tenues, limbo parum ampliato 5-fido. Intheræ apice appendiculate basi sagittatæ, auriculis caudato-appen(liculatis. Styli fl. $\not{q}$ rami sul)teretes apice subcapitati. Achænia oblongo-oboroidea teretiuscula ecostata glabra minute papillosa $\frac{7}{8} \mathrm{~mm}$. in sectione requantia ; pappi setæ 1 -seriatæ tenues caducissimæ $4 \mathrm{~mm}$. longæ.

HAB. in monte Morrison, ad 13000 ped. alt., (Nos. 2241, 2244 et 2128), et in montibus centralibus, ad 11000 ped. alt., (No. 1853), leg. T. Kawakaum et U. Moni, Nov. 1906.

The present plant is remarkable in having general appearance of Anaphalis. As the disc flower's are all fertile, however, this should be referred to Gnaphalium.

Carpesium Lins.

Carpesium acutum Harata, sp. nov. IIerbe rigide basi suflirtticosæ erectæ ramosæ puberulæ vel tomentosæ 2-3-ped. altæ. Folia alterna ovata vel lanceolata cum petiolis $7 \mathrm{~cm}$. longa apice acuminata basi acuta vel rotundata abrupte attenuata ad petiolum $2 \mathrm{~cm}$. longum abeuntia, rel superiora lanceolata sessilia, margine obscure dentata, lentibus mucronatis, supra pubescentia subtus tomentoso-hirsuta palliclinscula. Capitula mediocria $8 \mathrm{~mm}$. longa $10 \mathrm{~mm}$. in diametro xquantia cernua ad axillas foliorum longe pedunculata, floribus in ambitu o $\infty$-seriatis discique $\not$ fertilibus. Involucrum campanulato-semiorbiculatum, bracteis sub-t-seriatis, interioribus angustis $4 \mathrm{~mm}$. longis obtusis rel acutis searriosis, extimis 5-6 foliaceis lanceolatis dentatis 20 cm. longis. Receptaculum planum muricatum. Corollæ fl. o l lreve tululose $1 \frac{1}{2} \mathrm{~mm}$. longæ 5-dentata ; fl. ̧ longiores $8 \mathrm{~mm}$. longa achanium axuantes, limbo paulo latiore $\tilde{5}$-dentato. Antheræ apice appendiculatæ 
rotundato-truncatæ basi sagittatæ, auriculis caudis setaceo-sub-

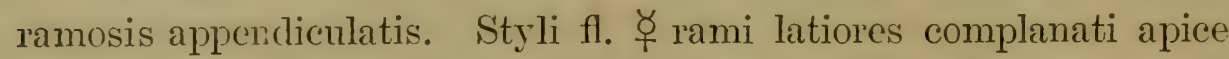
rotundati. Achænia elongata teretia circ. $4 \mathrm{~mm}$. longa 10 -striata apice in rostrum glanduloso-punctatum longum vel breve contracta, annulo obscuro coronata; pappus 0 .

HAB. Ganzan, in montibus Morrison, ad 9141 ped. alt., leg. S. Nagasawa, Oct. 1905, (No. 694) ; Arizan, in isdem montibus, leg. G. Nakahara, Nov. 1906 ; in eodem monte, ad 8000 ped. alt., leg. T. KатакамI et U. Mori, Oct. 1906, (No. 1991) ; Toroku: Washa, leg. T. KawaKanit et U. Mori, Nov. 1906, (No. 1937).

Remarkable for the acute foliose bracts of involucres.

\section{Siegesbeckia LINN.}

Siegesbeckia orientalis Lrnv. Sp. P1. ed.-2, p. 1269 ; Roxb. Fl. Ind. ed.-CArex, III. p. 439 ; Lour. Fl. Cochinch. ed.-WILLD. p. 616 ; DC. Prods. V. p. 495 ; Siez. et Zucc. Fl. Jap. Fam. Nat. p. 185; Miq. Fl. Ind. Bat. II. p. 67 ; Benth. Fl. Hongk. p. 182, et Fl. Austr. III. p. 535 ; Sonder, Fl. Capens. III. p. 132 ; Seemann, Fl. Vit. p. 142 ; Franch. et Satat. Enum. Pl. Jap. I. p. 231 ; Clanke, Comp. Ind. p. 133 ; Oliver, Fl. Tropic. Afric. III. p. 372 ; Hoor. f. Fl. Brit. Ind. III. p. 304 ; Franchet, Il. Darid. p. 164; Fonbes et Henrst. Ind. Fl. Sin. I. p. 433 ; Henry, List Fl. Formos. p. 54 ; Drets, Fl. Centr. Chin. p. 615; Hayata, Comp. Formos. p. 17 ; Matsumr. et Hayata, Enum. Pl. Formos. p. 205.

Hab. Toroku : Kureikiaku, leg. T. Kawakami et U. Mori, Nov. 1906, (No. 1818).

Distrib. Ccsmopolitan in the warm and temperate regions.

\section{Spilanthes LiNN.}

Spilanthes Acmella Linn.; Thuns. Fl. Ja1. p. 321 ; DC. Prodr. V. p. 623 ; Mir. Fl, Ind. Bat. II. p. 79 ; Clarke, Comp. Ind. p. 138; Oliver, Fl. Tropic. Afric. III. p. 384; Hoor. f. Fl. Brit. Ind. III. p. 307; Henry, 
List Pl. Formos. p. 54 ; Hayata, Comp. Formos. p. 20 ; Matsurr. et Hayata, Enum. Pl. Formos. p. 205.

HАв. Kōdenshō : ad 2623 ped.alt., leg. S. Nagasawa, Oct. 1905; Tappansha, leg. T. KawaKani et U. Mori, Oct. 1906, (No. 1761).

Distrib. India throughout, and extends to all warm countries.

\section{Chrysanthemum LINN.}

- Chrysanthemum indicum Linx. Sp. Pl. ed.-2, p. 1253 ; Thuxв. Fl. Jap. p. 320 ; Forbes et Hexsis. Ind. Fl. Sin. I. p. 437; Diels, Fl. Cents. Chin. p. 617.

Pyrethrum idicum Cass.; Maxtr. in Mél. Biol. VIII. p. 516.

Haв. Suizan, in montibus Morrison, ad 7702 ped. alt., leg. S. Nagasawa, Oct. 1905, (No. 734); in monte Morrison, ad 7500 ped. alt., Oct. 1906, (No. 1814), et in montibus centralibus, Nor. 1906, (No. 1885), leg. T. Kawakani et U. Mori ; Toroku : Tōhozan, leg. T. KawaKani et U. Mori, Nov. 1906, (No. 1828).

Distris. China and Japan.

The Formosan plant appears at first sight to be difierent from the Japanese species. But, after comparing various forms of the plants from both regions, I have found that they do not show any specifical distinction, and thought it better to regard them as the same species.

\section{Artemisin Linv.}

Artemisia japonica Thexs. Til. Jap. 1. :310; .DC. Prohl. VI. p. 100 ; Benth. Fl. Hongk. p. 186 ; Franchet, Pl. David. p. 168 ; Maxms. in Mél. Biol. VIII. p. 526, (varietates, japonica et desertorum) ; Fonmes et Hrasst. Ind. FI. Sin. I. p. 443.

Artemisia parviflora Buch. ex Roxв. Fl. Ind. ed.-Carex, III. p. 420 ; Hоoк. f. Fl. Brit. Ind. III. p. 322.

Artemisia cuneifolia DC. Prodr. VI. p. 126. 
HAB. Tappansha, ad 3138 ped. alt., leg. S. NAGasatwa, Oct. 1905, (No. 786) ; Kagi : Kōdenshō, leg. T. Kawakami et U. Mori, Oct. 1906, (No. 1740) ; Tōzan, in montibus Morrison, leg. G. NaKaHARA, Oct. 1906.

Distrib. Japan and China; westward to North India, from Kashmir to Khasia, Pulney mountains in the Madras Peninsula (after HemsL.); also in North America.

Artemisia niitakayamensis HAYATA, (Pl. XX.), in Tōkyō Bot. Mag. XX. p. 16 . Herbæ perennes basi suffiticosæ hirsutæ odoratæ multicaules, caulibus simplicibus $10 \mathrm{~cm} .-16 \mathrm{~cm}$. altis. Folia alterna ambitu obovata $2 \mathrm{~cm}$. longa $1 \mathrm{~cm}$. lata busi longe angusta decurrentia semi-amplexicaulia bipinnatisecta, segmentis utrinque 2-3 obovatis 2-3 lobatis, lobis ovato-acutis. Capitula majuscula $1 \mathrm{~cm}$. in diametro æquantia erecta rarius cernua racemosa longe pedicellata, pedicellis $2 \mathrm{~cm}$. longis. Involucrum late campanulatum, bracteis 3 -seriatis margine scariosis, intimis $5 \mathrm{~mm}$. longis spathulatis, interioribus oblongis margine denticulatis, exterioribus gradatim minoribus ovato-acutis. Receptaculum semiorbiculatum sparce pilosum. FI. $ᄋ$ in ambitu 1-seriati fertiles; corollæ tenues tubulosæ apice breviter 4-fidæ. Fl. 支 fertiles; corollæ teretes limbis parum ampliatis basi limbi constrictis apice 5 -fidis. Antheræ apice appendiculatæ basi obtusæ. Styli rami apice truncati dilati penicillati. Achænia obovoidea compressa $2 \mathrm{~mm}$. longa $1 \mathrm{~mm}$. in sectione xquantia leviter incurva apice truncata facie dorsali sæpe ecostata ventrali prominente 4 -costata.

HAB. in monte Morrison, ad 13000 ped. alt., (Nos. 2282 et 2130.), et in montibus centralibus, leg. T. Kawakair et U. Mori, Nov. 1906 , (No. 2195).

As the original description is drawn from an imperfect 
specimen, I have repeated the description basing it upon a most perfect specimen.

Artemisia oligocarpa HaYata, sp. nor. (PI. XXI.). Herbæ perennes basi suffruticosæ. Caules erectæ glabrescentes $15 \mathrm{~cm}$.$30 \mathrm{~cm}$. alti. Folia radicalia caulino conformia ambitu obovata basi longe attenuata $3 \mathrm{~cm}$. longa $1 \mathrm{~cm},-1 \frac{1}{2} \mathrm{~cm}$. lata bipinnatisecta, segmentis linearibus $3-5$-lobis vel -sectis, lobis linearibus apice obtusis vel acutis, primum pubescentia demum slabra. Capitula parviora $4 \mathrm{~mm}$. in diametro æquantia erecta racemosa vel paniculata pedicellata, pedicellis $\frac{1}{2} \mathrm{~cm} .-1 \mathrm{~cm}$. longis. Involucrum late campanulatum, bracteis 2-seriatis margine scariosis oboratis apice rotundatis basi constrictis $3 \mathrm{~mm}$. longis $2 \mathrm{~mm}$. latis. Receptaculum semiorbiculatum $1 \frac{1}{3} \mathrm{~mm}$. in diametro aequans nudum. Flores $q$ in ambitu 1 -seriati 15-20, fertiles; corollæ breve tubulosæ $1 \mathrm{~mm}$. longæ apice 2-3-fidæ; styli rami elongati re-

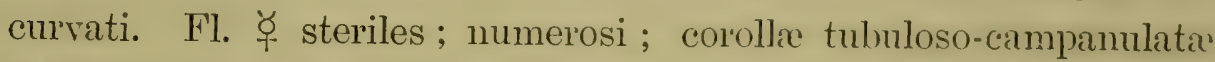
$2 \frac{1}{2} \mathrm{~mm}$. longx, limbis longe ampliatis tubum in longitudine rquantibus apice 5 -fidis, lobis triangularibus acutis marginatis; anthere apice appendiculatx apiculatæ cuspidato-acuminatx, hasi leviter brevissime candatæ; stylus indivisus apice peltato-dilatus minute et longiuscule papillosus. Achænia oblique obovoidea obscure reticulato-striata $1 \frac{1}{2} \mathrm{~mm}$. longa.

НАв. in monte Morrison, ad 12000 ped. alt, leg. T. KAwАкмп et U. Moni, Nov. 1906, (Nos. 2280 et 2140).

The present plant is somewhat near A. salsoloides WILLD.; but differs from it in having narrowly pimatisected leaves. Is stated by Penthin, in the Genera Plintarum Vol. Il. p. 551, the anthers of the genus Aitemisia are wanting of any tail. Nevertheless, I have observed in this species that there is in almost all 
cases a very small tail at the base of anthers. Moreover, the group to which this plant belongs is far different from other groups in having flowers, which are perfect and sterile, and even more different in having simple peltate stigmata. Upon considering the above cases, I am much inclined to raise this group to a genus separated from Artemisia.

Artemisia scoparia WaLdst. et Krt.; DC. Prodr. VI. p. 99 ; MLıxn. in Mé́l. Biol. VIII. p. 523 ; Franchet, Pl. David. p. 167; Hook. f. Fl. Brit. Ind. III. p. 323 ; Forees et Hexss. Ind. Fl. Sin. I. p. 445.

HAB. Ganzan, in montibus Morrison, ad 9141 ped. alt., leg. S. Nagasatia, Oct. 190כ. (No. 631) ; Tōzan, in montibus Morrison, leg. G. Nakahara (Nor. 1906); in monte Morrison, ad 11000 pecl. alt., leg. T. Katwakami et U. Mori, Oct. 1906, (No. 2043).

Distrib. Japan and China, northward to Kamtchatka and North America; westward to North India and extends to central Europe.

\section{Petrisites GrentN.}

Petasites tricholobus Fraxchet, Pl. Darid. p. 173; Diels, Fl. Centr. Chin. p. 618 ; Fonbes et Hexss. Ind. Fl. Sin. I. p. 447.

HAB. in monte Morrison, ad 8000 ped. alt., (Nos. 1880 ct 2078), et in montibus centralibus, leg. T. Kawakani et U. Mori, Nor. 1906.

Distrib. Central China.

\section{Gymura CAss.}

Gynura flava Hayata, sp. nov. Herbæ sparce hirtellæ' vel subglabiæ. Caules validiusculi sulcati pauci-ramosi 2-3 ped. alti. Folia alterna in ambitu oblonga vel oblongo-ovata $18 \mathrm{~cm}$. longa (i cm. lata grosse irregulariter dentata vel infra medium profunde dissecta, lobis argute dentatis, apice acuta basi angusta in petiolum 
$2 \mathrm{~cm}$. longum sensim attenuata, aurieulis basin petioli distinctis oblongis dentatis, utraque pagine sparce hirtella ad costas et renulas hirsuta subtus pallidiora. Capitula mediocria $1 \frac{1}{2} \mathrm{~cm}$. longa ad apicem ramorum corymbosa, pedicellis $1 \mathrm{~cm} .-2 \mathrm{~cm}$. longis hirsutis, bracteis ad basin pedicelli linearibus $1 \mathrm{~cm}$. longis, homogama, floribus omnibus $\not{q}$ fertilibus. Involucrum campanulatum, bracteis 1-seriatis angustis xqualibus $11 \mathrm{~mm}$. longis $2 \mathrm{~mm}$. latis margine scariosis plus minus cohærentibus, additis nounullis exterioribus parvis linearibus $5 \mathrm{~mm}$. longis ciliolatis. Receptaculum planum foveolatum. Corollæ flaræ tenuiter tubulosæ $13 \mathrm{~mm}$. longre, limbis parum ampliatis tubo in longitudine 2-plo hrevioribus apice breviter 5 -fidis, lobis oblongo-triangularibus. Antheræ subexsertæ, elongatæ $2 \frac{1}{2} \mathrm{~mm}$. longæ basi integræ. Styli exserti cum ramis 17 $\mathrm{mm}$. longi, ramis elongatis erecto-patentibus $5 \mathrm{~mm}$. longis in appendices longas tereti-subulatas hirtellas desinentibus. Ichænia glabra angusta 10-striata; pappi setis copiosis tenuibus $10 \mathrm{~mm}$. longis albis.

HAB. Suizan, in montibus Morrison, ad 7702 ped. alt., leg. S. Nagasawa, Oct. 1905, (No. 562); in monte Morrison, ad 6500 ped. alt., leg. T. Kawananir et U. Morr, Oct. 1906, (No. 2012); 'Toroku : Gunkei, leg. T. Kawakami et U. Mori, Nov. 1906, (No. 1951).

\section{Senecio LinN.}

Senecio monanthus Diets, Fl. Ceutr. Chin. p. 621.

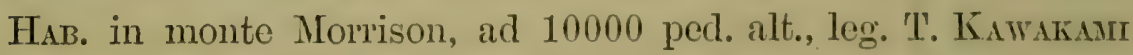
et U. Mori, Nor. 1906, (No. 2133); eodem loco, et G. Nafihara, Nov. 1905.

Distrib. Central China.

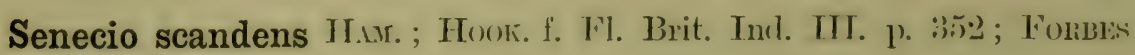
et Hexsu. Ind. FI. Sin. I. p. 457 ; Dn:s, Fl. Centr. Chin. p. 620. 
Senecio campylodes DC. Prodr. VI. p. 370 ;

Senecio stipulatus WaLL。; DC. Prodr. VI. p. 370.

Senecio Wightianus DC.; Wighт, Ic. Pl. Ind. or. t. 1136.

Senccio chinensis DC. Prodr. VI. p. 363 ; Вехтн. Fl. Hongk. p. 190 ; Maxnr. in Mél. Biol. VIII. p. 16.

Senecio intermedius WIGHT, Ic. Pl. Ind. or. t. 1135.

Cineraria repanda Loun. Fl. Cochinch. ed-WmL. p. 613.

Haв. Tōzan, in montibus Morrison, leg. G. NaKahara, Oct. 1906 ; in monte Morrison, ad 8000 ped. alt., (No. 1877), et ad 7000 perl. alt., (No. 2081), leg. T. Kawakami et U. Mori, Nov. 1906 ; Toroku : Hōōsan, leg. T. Kawakani et U. Mori, Nov. 1906, (No. 1927); 'Tōhosha, leg. S. Nagasawa, Nov. 1905, (No. 608).

Distrib. South China and southern part of Japan; north and sonth India, and Ceylon.

\section{Echinops LinN.}

Echinops dahuricus Fisch.; DC. Prodr. VI. p. 523 ; Fr.txcheT, I'l. David. p. 176 ; Henry, List Pl. Formos. p. 55 ; Fonizes et Henst. Ind. Fi. Sin. I. p. 459 ; Hayata, Comp. Formos. p. 33 ; Matsuar. et Hayata, Enum. Pl· Formos. p. 221.

Echinops Gmelini Ledeb. Fl. Ross. II. p. 653 ; Mixtr. Prim. Fl. Amur. p. 167.

Echinops sphcerocephalus MIQ. in Aun. Mus. Bot. Lugd.-Bat. II. p. 182; Franch. et Savat. Enum. Pl. Jap. I. p. 253.

Hab. Taitō: Bokusekikaku, leg. T. Kawakani et U. MonI, Dec. 1906, (No. 1832); Arōkonsha, leg. T. Kawakani et U. Mori, Oct. 1906, (No. 1741).

Distrib. Siberia to Japan and China.

\section{Cnicus Lins.}

Cnicus Wallichii DC. Prodl. VI. p. 643; Hoor. f. Fl. Brit. Ind. IIT. p. 363. 
Cirsium Wallichii DC. ; DIELs, F1. Centr. Chin. p. 627.

НАв. Ganzan, in montibus Morrison, ad 9141 ped. alt., Oct. 1905, (No. 778), in monte Morrison, ad 12000 ped. alt., (No. 2245), et in eodem monte, ad 10000 ped. alt., (No. 2279), leg. T. KAWAKaMII et U. Mori, Oct. 1906.

Distrib. Sonth China to the Philippine islands, and Himalaya.

\section{Saussurea DC.}

Saussurea japonica DC. in Prodr. VT. p. 536 ; Вехтн. Fl. Hongk. p. 167 ; Maxnr. in Mél. Biol. IX. p. 337; Haxce, in Journ. Linn. Soc. XIII. p. 108; Franchet, Pl. David. p. 181; Fonbes et Heust. Ind. FI. Sin. I. p. 464 ; Drets, Fl. Centr. Chin. p. 627.

var. longicephala Harata, 11. r. Capitula longiuscula oroidea, squamis involucri apice appendicula petaloidca rotundata instructis.

HАB. loco non indicato.

Drstrib. Type: China, Japan, and Manchuria to North America.

The type of Saussurea japonica. DC. has a globose head, while the present variety has a oblong head. Besides, the bracts of the involucres of the variety which are crowned with a petaloidal appendage, are slightly different from those of the type.:

\section{Saussurea sp.}

Hab. Taitō: Bushisekisha, leg. T. Kawatsani et U. Mroni, Dec. 1906, (No. 2158).

\section{Ainstian DC.}

Ainsliæa macroclinidioides ILATat, sp. nor. (PI. XXII.). IIcrba

* After completing this manuscript, I hnve had the opportunity of exлmining n Chinese specimen nnmed S. japonica DC. collected by Dr. Hexrx. The specimen is exnetly the snme as the Formosan one, and is certainly not the type of $S$. juponice DC. 
perennes basi suffruticosæ pilosiusculæ elatiores $60 \mathrm{~cm}$. altæ. Folia alterna ad medium caulis conferta pilosiuscula longe petiolata, petiolis lamina 3-plo brevioribus $4 \mathrm{~cm}$. longis, laminis ovatis vel ovato-lanceolatis circ. $9 \mathrm{~cm}$. longis $3 \frac{1}{2} \mathrm{~cm}$. latis acuminatis basi rotundatis truncatis vel cordatis margine remote aristato-serrulatis albo-lamelligeris 3 -nerviis subglabratis ad costas breve pilosiusculis, subtus pallidioribus. Capitula angusta mediocria $2 \mathrm{~cm}$. longa $2-3$-flora secus caulem subspicata. Involucrum angustum circ. $1 \mathrm{~cm}$. longum, bracteis $3-4$-seriatis valde inæqualibus unicostatis rigidis scariosis intimis oblanceolatis acutis $1 \mathrm{~cm}$. longis $2 \mathrm{~mm}$. latis, exterioribus gradatim brevioribus, extimis brevissimis late ovatis $1 \frac{1}{2} \mathrm{~mm}$. longis. Receptaculum parvum nudum. Flores normales ignoti. Flores cleistogami: corollæ tubulosæ pappi setis 2-plo breviores, apice 5-fidx; antheræ basi longe sagittatæ, auriculis in caudas longas 2-fidas productis; styli rami obtusi basi incrassati. Achænia oblongo-obovoidea subcompressa $7 \mathrm{~mm}$. longa apice contracta 10-costata pilosa, stipitibus parvis persistentibus; pappi setæ 1 -seriatæ plumosæ $8 \mathrm{~mm}$. longæ rufo-albæ.

Hab. Suizan, ad 7702 ped. alt., leg. S. Nagasawa, Oct. 1905, (No. 665); Kagi : Tappansha, leg. T. KawakauI et U. Mori, Oct. 1906, (No. 1971).

Remarkable for the fascicled leaves at the middle of the stem just like those of Macroclinidium robustum Maxn.

Ainsliæa morrisonicola Hayata, n. n. (Pl. XXIII).

Ainslicea elegans Hayatı, (non Hexrsu.) in Tōkyō Bot. Mag. XX. p. 14. Herbæ laxe pilosæ, caulibus erectis $5-6 \mathrm{~cm}$. altis simplicibus aphyllis. Folia omnia radicalia oblanceolata $15 \mathrm{~mm} .-20 \mathrm{~mm}$. longa $8 \mathrm{~mm}$. lata apice apiculato-acuta basi subito angusta ad petiolum attenuata vel basi rotundata margine remote ciliato- 
aristata, petiolis $10 \mathrm{~mm}$. longis basi dilatis. Capitula 3-4-flora secus caulem spicata sessilia demum pendula. Involucrum angustum 3-4 mm. longum, bracteis 3 -seriatis rigidis subpaleaceis aristato-acutis, interioribus elongatis, exterioribus gradatim brevioribus, intimis longissimis $\frac{1}{2} \mathrm{~cm}$. longis. Receptaculum nudum. Flores normales ignoti. Flores cleistogami : corollæ tubuiosie pappi setis breviores circ. $4 \mathrm{~mm}$. longæ, apice leviter $\overline{5}$-fidæ clausæ; antheræ basi sagittatæ, auriculis in caudas longas productis; styli basi dilati, ramis angustis, complanatis apice rotundatis. Achænia obovoideo-oblonga subteretia $5 \mathrm{~mm}$. longa $1 \mathrm{~mm}$. in sectione æquantia 10-costata apice truncata glabra; pappi setæ 1 seriatæ plumosæ $5 \frac{1}{2} \mathrm{~mm}$. longæ rufo-albæ.

Hab. Seizan, in montibus Morrison, ad 11707 ped. alt., leg. S. Nagasawa, Nov. 1905, (No. 628); Tōzan, in montibus Morrison, leg. G. Nakahara, Nov. 1906.

The plant is originally named Ainslicea elegans, which name is, however, found afterward in the Chinese plants. As it is not desirable to maitain one and the same name for two different plants, I have taken this occasion to change the name of the Formosan plant to a new name, A. morrisonicola Haysa.

Ainsliæa reflexa Mernul. in Philipp. Jouru. Sci. I. Suppl. Bot. p. 242.

HAB. Nantō: Mushazan, ad 7500 ped. alt., leg. T. KawakanI et U. Mori, Aug. 1906, (No. 1138) ; Hinokiyama, leg. (*. NAnimili.l, Feb. 1907.

Distrib. The Philippine islands.

\section{Picris Linv.}

Picris hieracioides Lixx. Sp. Pl. ed.-2. p. 111; ; 1)C. Protl. VII. 1). 
128 ; Hoor. f. Fl. Brit. Tud. IIT. p. 393 ; Franchet, Pl. David. p. 185 ; Fondes et Hexss. Ind. Fl. Sin. I. p. 474.

Picris japonica Thuns. Fl. Jap. p. 299; DC. Prodr. VII. p. 130.

Hab, in monte Morrison, ad 10000 ped. alt., Nov. 1906, (No. 2.94), et in montibus centralibus, ad 10000 ped. alt., Dec. 1906 , (No. 2204), leg. T. Kawakami et U. Mori.

Distrib. Japan and central China ; eastward to America and western Europe ; northward to Kamtchatka ; also in Australia and New zealand.

\section{Lactuca Lins.}

Lactuca versicolor Sch.-Bir.; Maxim. in Mél. Biol. IX. p. 362 ; BAKen et Moone, in Jouim. Linn. Soc. XVII. p. 383 ; Franchet, Pl. David. p. 188; Fonbes et Hemst. Ind. Fl. Sin. I. p. 485 ; Diets, Fl. Centr. Chin. p. 631 ; Hayata, Comp. Formos. p. 39 ; Matsun. et Hayata, Enum. Pl. Formos. p. 212.

Ixeris versicolor DC. Prodr. VII. p. 151; BENTH. Fl. Hongk. p. 193; Mir: in Ann. Mus. Bot. Lugd.-Bat. II. p. 191 ; Leded. Fl. Ross. II. p. 817. Prenanthes chinensis Thuns. Fl. Jap. p. 301.

Youngia chinensis DC. Prodr. VII. p. 194.

Hab. Tappansha, ad 3138 ped. alt., leg. S. Nagasatwa, Oct. 1905, (No. 775) ; Taitō : Daironkōsha, leg. T. Kawarami et U. Moni, Nov. 1906, (No. 2220).

Distrib. Japan and China northward to Dahuria ; also in North America.

\section{Campanulaceæ}

Pratia GAUD.

Pratia begonifolia LiNdL.; Cl.ınie, in Hook. f. Fl. Brit. Ind. III. p. 
422 ; Forbes et Heist. Ind. Fl. Sin. II. p. 2; Hennr, List Pl. Formos. p. 56 ; Difls, Fl. Centr. Chin. p. 607 ; Matsuxr. et Hayatı, Enum. Pl. Formos. p. 213.

Piddingtonia Nummularia DC. Prodr. VII. p. 341 ; BeNtH. Fl. Hongk. p. 196.

Lobelia Horsfictdiana MrQ. Fl. Ind. Bat. ПI. p. 577.

НАв. Kōdenshō, ad 2623 ped. alt., leg. S. NAGısıiwa, Oct. 1905, (No. 742).

Distrib. South China, Malay, and eastern India.

\section{Lobelia LINN.}

Lobelia affinis WaLl. in DC. Prodl. VII. p. 360; Bexth. Fl. Hongk. p. 197, (in nota sub L. trigona); Clarke, in Hoor. f. Fl. Brit. Ind. III. p. 424 ; Hance in Journ. Linn. Soc. XIII. p. 109 ; Fonbes et HemsL. Ind. Fl. Sin. II. p. 2.

Haв. Tōzan, in montibus Morrison, leg. G. Nakahırı, Oct. 1906 ; in monte Morrison, ad 7500 ped. alt., leg. T. K.ıwasir et U. MLni, Oct. 1906, (No. 1983).

Distrib. Widely diffused in India, Ceylon, and Malay; South China.

Lobelia pyramidalis WALL.; DC. Prodr. VII. p. :381; CLARKF, in Hоок. f. Fl. Brit. Ind. III. p. 426 ; Bot. Mag. t. 2387; Fonbes et Hexsi. Ind. Fl. Sin. II. p. 3.

Lobelia Davidi Franchet, Pl. David. p. 191.

Нав. Taitō : Bunshisekisha, leg. T. Kawanum et U. Monr, Dece. 1906, (No. 1911).

Distrib. Mountains of North and East India, and Burma; South China.

Wahlenbergia ScHrad.

Wahlenbergia gracilis .1. .)C. "Monogr. Camp. 1. 112." et Prodr. 
VII. p. 433 ; BеNтн. Fl. Austral. IV. p. 137 ; Cranke, in Hook. f. Fl. Brit. Ind. III. p. 429 ; Franchet, Pl. David. p. 192 ; Henry, List Pl. Formos. p. 56 ; Fonbes et Hemss. Ind. Fl. Sin. II. p. 4 ; Matsum. in Tōkyō Bot Mag. XIV. p. 58 ; Diels, Fl. Centr. Chin. p. 606 ; Matsum. et Hayata, Enum. Pl. Formos. p. 215.

Wahlenbergica agrestis A. DC. Prodr. VII. p. 434; BentH. Fl. Hongk. p. 197.

Wahlenbergia marginata A. DC. Prodr. VII. p. 433 ; Franch. et Savat. Enum. Pl. Jap. I. p. 277.

Wahlenbergia dehiscens A. DC., W. Sieberi A. DC., W. lavanduldofolica A. DC. et $W$. quadrifida A. DC., ex Clarke, in Hоoк. f. Fl. Brit. Ind. II. p. 429.

HAB. Suizan, in montibus Morrison, ad 7702 ped. alt., leg. S. Nagasawa, Oct. 1905, (No. 729); in monte Morrison, ad 7500 ped. alt., leg. T. Kawakamr et U. Momi, Oct. 1906, (No. 2063).

DisTRIB. South China and southern part of Japan; India throughout; Australia, and also found in New Zealand and South Africa.

\section{Codonopsis WALL.}

Codonopsis sp. nov.?

Hab. in monte Morrison, leg. T. Katvakami et G. Nakahara, Nov. 1905.

Remarkable for small leaves and seeds.

\section{Campanumøa BL.}

Campanumœa axillaris OrIv. in Hook. Ie. Plant. XVIII. t. 1775; Fonbes et HeMsL. Ind. Fl. Sin. II. p. 7.

Codonopsis truncata WALL. DC. Prodr. VII. p. 423.

Cyclocodon truncatus Hоок. f. et Thомs. in Journ. Limn. Soc. II. p. 18. Hab. Kagi : Tappansha, leg. T. Kawakani et U. Mori, Oct. 1906 , 
(No. 1780); Taitō: Bunshisekisha, leg. T. K.ıwakanı et U. Mori, Dec. 1906, (No. 2162).

Distrib. The Loo-choo islands, westivard to central and southern China, and Burma.

Campanumœa javanica BludE ; DC. Prodr. VII. p. 423; Cl.1TKe, in Hook. f. Fl. Brit. Ind. III. p. 435 ; Fonbes et Hexsu. Ind. Fl. Sin. II. p. 8. Codonopsis cordata Hook. f. Bot. Mag. t. 5372.

Campanumoea japonica Maxir. in Mél. Biol. VI. p. 268.

Hab. Taitō: Rokurō, leg. G. Nakahara, Jan. 1906, (No. 752).

Distrib. Frequently found in Japan and central China; also in mountains of East Burma and Java.

\section{Peracarpa Ноок. f. et Тномs.}

Peracarpa carnosa Ноoк. f. et Thous. in Journ. Limn. Soc. II. p. 26 ; Clarke, in Hook. f. Fl. Brit. Ind. III. p. 437.

Peracarpa circceoides H. FEer, in ENGI. Bot. Jahnb. XII. p. 621.

Campanula carnosa WaLL. "in RoxB. Fl. Ind. II. p. 102 "; DC. Prodr. VII. p. 474.

Campanula circcooides F. Schurdt. Reis. in Amur. pp. 154 et 222, t. III. fig. 14-19 ; MIQ. in Ann. Mrus. Bot. Lugd.-Bat. III. pp. 195 et 201; Fr.uvcir • et Savat. Enum. Pl. Jap. I. p. 278; Forbes et Hexrst. Ind. Fl. Sin: II. p. 9.

Hab. in monte Morrison, leg. 'T. Kawakasi, 1905 ; Tōzan, in montibus Morrison, leg. G. Nakahara, Oct. 1906.

Distrib. Japan, Manchuria, Saghalien; westward to central China as far as Himalaya.

Upon considering the various forms of this species, I am much inclined to think that the Indian Pcracarpa is quite identical with the Japanese one, and in this Mr. T. Makivo concurs. 


\section{Adenophora Fisch.}

*Adenophora verticillata FIsch.; DC. Prodr. VII. p. 492 ; Herder, Pl. Radd. IV.-1, p. 28 ; Franch. et Savat. Eumm. Pl. Jap. II. p. 422 ; Hance, in Journ. Bot. (1885), p. 325 ; Forbes et Herrs. Ind. Fl. Sin. II. p. 14.

Har. Suizan, in montibus Morrison, ad 7702 ped. alt., Oct. 1905, (No. 650).

Distrib. Type: Japan and China; Manchuria to Dahuria.

var. linearis HAYATA, v. n. Folia opposita vel 4-natim verticillata linearia $6 \mathrm{~cm}$. longa $2 \mathrm{~mm}$. lata subglabra integra.

Hab. Nantō: Mushasan, ad 7500 ped. alt., leg. T. Kawakami et U. Mori, Aug. 1906, (No. 1131).

Adenophora polymorpha LEDEE. var. Lamarckii TrauTv.; Herder, P1. Radd. IV.-1, p. 27.

Hab. in monte Morrison, ad 13000 ped. alt., leg. T. Kawakanr et U. Moni, Oct. 1906, (No. 2266).

Adenophora polymorpha LEDEB. var. coronopifolia Trautr.; HERDER, P1. Radd. IV.-1, p. 27.

HAB. Ganzan, in montibus Morrison, ad 9141 ped. alt., leg. S. Nagasatwa, Oct. 1905, (No. 733); in montibus Morrison, ad 800010000 ped. alt., leg. T. Kawakami et U. Mori, (Nos. 2290, 1942, 1943, et 2290.)

Distrib. Type: China, Japan, and North America.

* It seems to me that the plant is extremely variable, althongh I am not sure about the Lalbit of this plant in Formosa, as I have never stayed so long in the island as to study the living state of the plant. As $I$ have observed in Japan, the range of the variation of this plant is very wide. In ordinary condition, the lentes are disposed in whorls, but on the branches shooting out from a stumip after the stem is cut they are arranged alternately. Mr. T. Marivo also refers to this point in 'Tökyō Bot. Mng. Xx. p. 39, where he expresses his opinion that A. verticillata Fisch. $\gamma$. alternifolic Fraxch. et Savat. is nothing but a sport of the type. 


\section{Vacciniaceæ.}

\section{Vrecinium LrNo.}

Vaccinium emarginatum IIATAт, sp. nor. Frutices ramosi cortice cinereo vestiti, ramulis glabris in exsiccato rufescentibus. Folia oblonga vel oblongo-obovata circ. $5 \mathrm{~cm}$. longa $2 \mathrm{~cm}$. lata margine integerrima revoluta apice emarginata basi sensim attenuata crasse coriacea nitida pallida subtus pallidiora breve petiolata, petiolis $4 \mathrm{~mm}$. longis. Flores in racemos breves axillares folio 2-plo breviores fasciculatim dispositi, bracteis 2 subulatis. Calycis tubus globosus, limbo 5-lobo, lobis longe triangularibus. Corollæe ignotæ. Baccæe oblongo-globosæ $8 \mathrm{~mm}$. longæ 10-loculares, loculis polyspermis. Semina minuta ovoidea $1 \frac{1}{2} \mathrm{~mm}$. longa $1 \mathrm{~mm}$. lata compressa angulata testa coriacea reticulata.

Haв. Tōzan, in monte Morrison, leg. G. Nakahara, Oct. 1906 ; eodem loco, leg. T. KawaKami et U. Mori, Oct. 1906, (No. 1947); Nantō: Mushasan, ad 7500 ped. alt., leg. T. Kawakisir et U. Mori, Aug. 1906, (Nos. 71461, 1178 et 1143) ; Taitō: Iryokukakusha, legr. T. Kawakami et U. Mori, Dec. 1906, (No. 2166).

Vaccinium Merrillianum Harata, sp. nov. (Pl. XXIT.). Frutices ramosissimi, ramulis ad innovationem squamatis rufescentibus hispido-tomentosis. Folia parvula approximata oborata circ. $8 \mathrm{~mm}$. longa $5 \mathrm{~mm}$. lata apice rotundata emarginata basi cuncata breve petiolata, petiolis $1 \mathrm{~mm},-2 \mathrm{~mm}$. longis, integerrima margine albolamelligera supra nitida in exsiccato profunde rugosa subtus lievia pallidiora crasse coriacea. Flores in racemos terminales dispositi. Calycis lobi 5, lobis late triangularibus. Bacex globosa, circ. $1 \mathrm{~cm}$. in diametro xquantes 10-loculares, loculis oligospermis. Semina 
minuta oboroidea compressa testa coriacea minute reticulata $1 \frac{1}{2} \mathrm{~mm}$. longa $1 \mathrm{~mm}$. lata.

Haв. Seizan, in montibus Morrison, ad 11707 ped. alt., leg. S. Nagasawa, Nor. 1905, (No. 573); Tōzan, in isdem montibus, leg. (i. Namahara, Oct. 1906 ; in eodem monte, ad 10000 ped. alt., (No. 2286), et ad 6000 ped. alt., (No. 1730), leg. T. KawaKami et U. Moni, Oct. 1906.

\section{Ericaceæ.}

\section{Gaultheria LINN.}

Gaultheria Cumingiana Vrdal, Plianerog. Cuming. p. 184, et Rer. P1. Vascul. Filip. p. 170 ; HaYata, in Tōkyō Bot. Mag. XX. p. 72.

$H_{A B}$. Suizan, in montibus Morrison, ad 7702 ped. alt., leg. S. NAGASAWA, Oct. 1905 , (No. 738); in eodem monte, ad 7500 ped. alt., (No. 1714), et ad 9000 ped. alt., (No. 1554), leg. T. KawaKalII et U. Mori, Nov. 1906.

Distrib. The Philippine islands.

Gaultheria Itōana Hayata, (Pl. XXV.), in Tōkyō Bot. Mag. XX. p. 74. Gauttheric repens Hayata, in Tökyō Rot. Mag. XX. p. 18. Suffrititices decumbentes v. erecti $10-15 \mathrm{~cm}$. alti sparce pubescentes. Folia coriacea brevissime petiolata oblonga $1 \mathrm{~cm}$. longa $3 \mathrm{~mm}$. lata utrinque acuta serrulata, supra glabra venis impressis subtus minute setulosa venis prominentibus. Flores parvi in racemos terminales dispositi cernui rosei? Recemi $2 \mathrm{~cm}$. longi, pedicellis $6 \mathrm{~mm} .-7 \mathrm{~mm}$. longis apicem rersus glanduloso-ciliatis bracteatis bracteolatisque, bracteolis oratis $2-3 \mathrm{~mm}$. longis acutis marginibus scariosis alternis persistentibus. Calyx 5-partitus $2 \mathrm{~mm}$. longus, lobis acutis. Corolla 
lata globosa urceolata $6 \mathrm{~mm}$. in diametro rquans $4 \mathrm{~mm}$. longa apice 5 -loba, lobis brevissimis $0.5 \mathrm{~mm}$. longis erectis recurvis. Stamina 10 basi tubi corollæ adherentia inclusa, filamentis glabris supra basin dilatis quam anthera longioribus, antheris 2-locularibus, loculis in tubos recurvos apice furcatos productis. Discus non conspicuus. Ovarum 5-loculare 5-lobum leviter pubescens, stylo columnari $4 \mathrm{~mm}$. longo apice truncato. Calyx fructifer accrescens persistens carnosus depresso-globosus $6 \mathrm{~mm} .-7 \mathrm{~mm}$. in diamctro æquans. Capsulæ sparce pubescentes intra calycem inclusæ, loculicide 5 -valvatæ polyspermæ. Semina minuta $0.5 \mathrm{~mm}$. longa obtuse angulata, testis crustaceis minute reticulatis.

Hab. Seizan, in montibus Morrison, ad 11707 ped. alt., leg. S. Nagasatva, Nov. 1905, (No. 625); Tōzan, in isdem montibus, leg. G. Nakahara, Nov. 1906 ; in monte Morrison, ad 12000 ped. alt., Oct. 1906, (No. 2134), ad 9000 ped. alt., (No. 1727), et in montibus centralibus, Nor. 1906, (No. 1887), leg. T. KAWAKAlI et U. MORI.

As the original description is based on an imperfect specimen, it would not be superfluous if I have repeated the description, basing it upon the most perfect material.

\section{Pieris Don.}

Pieris formosa D. Dox. ; DC. Prodr. VII. p. 599 ; Charke, in Hook. f. FI. Brit Ind. III. p. 461 ; Wight, Ic. Pl. Ind. or. t. 1200 ; Fonbes et Hrast. Ind. Fl. Sin. II. p. 16; Matsum. in Tōkgō Bot. Mag. XIV.p. 59 ; Matsuar. et Hayata, Enum. Pl. Formos. p. 219.

HAb. Suizan, in monte Morrison, ad 7702 ped. alt., leg. S. NAGASAWA, Oct. 1905, (No. 735); in eodem monte, ad 10000 ped alt., (Nos. 1890, 2136 et 2068), leg. T. Kawakasi et U. MonI, Nov. 1906; Taitō: Daironsan, leg. T. Kawaranı et U. Monr, Nor. 1906, (No. 
2184) ; Nantō: Mushazan, ad 7000 ped. alt., leg. T. KawaKanir et U. Morr, Aug. 1906, (No. 1145).

Distrib. South China; North and East India.

Pieris ovalifolia D. Don. ; DC. Prodr. VII. p. 599 ; Clanke, in Hook. f. Fl. Brit. Ind. III. p. 460 ; Fonbes et Hexss. Ind. Fl. Sin. II. p. 17; Diels, Fl. Centr. Chin. p. 515 ; Matsunr. et Hayata, Enum. Pl. Formos. p. 219.

Andromeda ovalifolia WaLt.; Maxim. in Mél. Biol. VIII. p. 620 ; Wight, Ic. PI. Ind. or. t. 1199.

Andromeda elliptica SIEB. et Zucc. Fl. Jap. Fam. Nat. n. 433.

Hab. Nantō: Shojōdaizan, ad 6000 ped alt., leg. T. Kawakaxi et U. Mori, Aug. 1906, (No. 1124).

Distrib. Japan and China ; common in North India, extending from Kashimir to the Khasia mountains and Burma.

\section{Rhododendron LINN.}

Rhododendron brachycarpum G. Don.; DC. Prodr. VII. p. 723 ; A. Gray, Bot. Jap. p. 400 ; Maxtr. Rhod. As. or. p. 22.

HaB. in monte Morrison? leg. T. KaWaKami? 1907.

Distrib. Manchuria and Japan.

Rhododendron ellipticum MAxrm. in Mél. Biol. XII. p. 742 ; Forbes et Hemst. Ind. Fl. Sin. II. p. 22 ; TAshino, in Tōkyō Bot. Mag. III. p. 201 ; MAKזvo, in Tōkyō Bot. Mag. XVIII. p. 47, et Icones Floræ Japonic. I.-3, p. 15, t. 9 .

HAB. Akō, ad 8000 ped. alt, leg. T. KawaKani ? 1907 ; Biōritsu : Bahozan, leg. T. Kawakamr et U. Mori, Juli. 1906, (No. 1112).

Distrib. South China and the Loo-choo islands.

The specimens here mentioned have not so long a bract as the Loo-choo plant which is excellently figured by Mr. T. Manivo in the Icones above cited. 


\section{Rhododendron Nakaharai HAYATA, sp. nov.}

Rhododendron serpyllifolium Hayats, in Tōkyō Bot. Mag. XX. p. 72.

Frutices humiles ramosi, ramis divaricantibus cinereo-fuscis novellis badio-strigosis. Folia in apices ramulorum conferta obovata vel oblonga $1 \mathrm{~cm}$. longa $5 \mathrm{~mm}$. lata apiculata basi attenuata breve petiolata supra adpresse strigosa subtus ad costas et margines dense longeque strigosa. Flores ad apices ramulorum lateralium brevissimorum 2-3-nati, pedunculis $7 \mathrm{~mm}$. longis strigosis basi squamis ovatis apiculatis circumvallatis. Sepala obovata spathulata $4 \mathrm{~mm}$. longa extus et margine longe strigoso-ciliata. Corolla campanulata $2 \frac{1}{2} \mathrm{~cm}$. longa 5 -fida, lobis obovatis apice rotundatis tubo brevioribus. Stamina 10, corollam superantia, filamentis $2 \frac{1}{2} \mathrm{~cm}$. longis infra medium pilosiusculis, antheris obovoideis apice truncatis 2-porosis. Ovarium longe et dense setosum. Capsula ignota.

Hab. Monte Shichiri, leg. G. Nakahara, Juli. 1905.

In my paper above cited, I make an error of identifying the present plant with $R$. scrpyllifolium. MrQ. In sterile specimens. the two come so closely that it is difficult to distinguish one from the other. On comparing flower's, I have found that both plants entirely differ from each other. The new species differs from the other in having much larger flowers, long hairy sepals, and in the number of stamens.

Rhododendron Oldhami Maxm. Rhor. As. or. p. 34; Fonises et Hearst. Ind. FI. Sin. II. p. 28 ; Henry, List Pl. Formos. p. 57 ; Matsum. et Hayata, Enum. Pl. Formos. p. 218.

Haв. Toroku : Kasōgri, leg. 'T. IŚmakani et U. Morr, Nov. 1906, (No. 1821).

Distrib. An endemic plant:

Rhododendron Oldhami M.ırns. val. glandulosum ILArata n. v. 
Rami in totum pilis longis mollissimi atque pubibus teneribus brevioribus glandulosis badio-villosi. Folia crasse membranacea breve petiolata oblonga vel ovato-oblonga utrinque acuta apice mucrone longiusculo glanduloso apiculata obscure 7-8-nervia. Flores ad apices ramorum 3-4-nati basi squamis circumvallati 4-bracteati, bracteis lanceolatis vel ovatis $5 \mathrm{~mm}$. longis pilosis rol glabris, pedunculis corollam $\frac{1}{2}$ æquantibus glanduloso-villosis. Calyx 5-partitus, lobis lanceolatis longissime acuminatis $5 \mathrm{~mm}$.$15 \mathrm{~mm}$. longis. Corolla campanulato-infundibuliformis $4 \mathrm{~cm}$. longa, tubo lobum in longitudine æquante. Stamina 10, corollam subæquantia, filamentis basin versus pubescentibus, antheris oblongis apice truncatis 2-porosis. Ovarium glanduloso-setosum.

HaB. Suizan, in montibus Morrison, ad 7702 ped. alt., leg. S. Nagasawa? Oct. 1905, (No. 668); in monte Morrison, ad 8000 ped. alt., (No. 2219), ad 9000 ped. alt., Oct. 1906, (No. 1807), et in montibus centralibus, ad 10000 ped. alt., Nov. 1906, (No. 1860), leg. T. Kawakami et U. Mori.

The present plant is very like $R$. Oldhami Maxnr. The veins of leaves and the proportion of sepals to peduncles are the principal points in which the new variety is separated from the type.

Rhododendron pseudo-chrysanthum Hayata, sp. nov. (Pl.XXVI.). Frutices humiles dichotome ramosi, cortice cinereo obtecti. Folia in tertium annum persistentia oblonga crasse apiculata basi attenuata $6 \mathrm{~cm}$. longa $2 \mathrm{~cm}$. lata glabra rigide coriacea, supra costis et venulis reticulatis profunde impressis, subtus ad costas prominentes floccoso-tomentosa cæeterum glabra, margine integerrima leviter revoluta, petiolis $5-7 \mathrm{~mm}$. longis. Gemma florifera terminalis strobiliformis, squamis multiseriatis imbricatis, exterioribus sensim brevioribus late ovatis apiculatis, interioribus sensim 
angustis, intimis linearibus. Flores ad apices ramorum corymbosi, pedunculis glanduloso-tomentosis circ. $2 \mathrm{~cm}$. longis florem in longitudine æquantibus. Calycis lobi acuti. Corolla rotato-campanulata, lobis rotundatis leviter emarginatis. Stamina 10, antheris oblongis apice truncatis, filamentis inæqualibus basi leviter dilatis pubescentibus. Ovarium ovoideum 5-loculare 5-sulcatum pubescens, styo corollam vix superanti basi glanduloso-piloso declinato, stigmate capitato 5-lobo. Capsula oblonga $1 \mathrm{~cm}$. longa rel longiora lignosa 5 -locularis apice septicede dehiscens. Semina scobiformia $1 \frac{1}{2} \mathrm{~mm}$. longa, nucleis parvis oblongis, testis reticulatis utrinque in appendiculam laceram productis.

Hab. in monte Morrison, ad 13000 ped. alt., (Nos. 2144 et 2240), legg. T. Kawarani et U. Morr, Nov. 1906 ; Nantõ : Mushazan, ad 6000 ped. alt., leg. T. Kawakami et U. Mori, Aug. 1906, (No. 1144).

Near R. chrysanthum PALL; but differs from it in having conspicuously 5-lobed calyces and apiculate leaves.

\section{Pyrola Linn.}

\section{Pyrola morrisonensis Hayata, n. 1 .}

Pyrola elliptica NutT. var. morrisonensis HaYaTA, in Tōkyō Bot. Mag. XX. p. 18.

Folia late ovata $2 \mathrm{~cm}$. longa totidem lata apice obtusa vel acuta basi truncata rel rotundata coriacea glanduloso-scrrulati longe peteolata, petiolis $3-5 \mathrm{~cm}$. longis supra impresso-reticulata sul)tus prominente venosa glaberrima. Racemi $1 \tilde{\mathrm{cm}} \mathrm{cm}$. longi. Flores ignoti. Capsula depresso-globosa cernua $5-6 \mathrm{~mm}$. in diametro rquans. Semina minutissima $1.1 \mathrm{~mm}$. longa angusta reticulati.

НАв. in monte Morrison, ad 10000 ped. alt., leg. T. KAwakanr et U. Morr, Oct. 1906, (No. 2273); in montibus centralibus arl 
10000 ped. alt., leg. T. Kawakani et U. Mori, Nov. 1906, (No. 1896).

The present plant is originally described by myself as a variety of $P$. clliptica Nutr. On examining carefully the leaves with impressed veins and most minute seeds, I have thought it better to regard the plant as specifically distinct from that species.

Pyrola rotundifolia LivN. Sp. Pl. ed-2, p. 567 ; DC. Prodr. VII. p. 772, Maxtr. Mél. Biol. VIII. p. 622 ; Clanke; in Hook. f. Fl. Brit. Ind. III. p. 475 ; Haxce, in Joumn. Bot. (1878) p. 109 ; Fravchet, Pl. David. p. 197 ; Forbes et Hexss. Ind. Fl. Sin. II. p. 33.

НАB. in monte Morrison, leg. T. KawaKaMI, Oct. 1906.

Distrib. In the temperate and cold regions all around the North Hemisphere. Japan and China.

\section{Diapensiaceæ.}

\section{Shortic Tonr. et Gr.}

Shortia rotundifolia (Maxmr.) Makino, in Tōkyō Bot. Mag. XV. p. 149. Schizocodon rotundifolius Maxins. in Mél. Biol. XIT. p. 743 ; Fonbes et Hexrsu. Ind. Fl. Sin. II. p. 34 .

HAB. in monte Morrison, ad 12000 ped. alt., leg. T. KawaKAMI et U. Morr, Oct. 1906, (No. 2283); Nantō: Mushasan, ad 7000 ped. alt., leg. T. Kawaknмi et U. Mori, Aug. 1906, (No. 1150); Shintilin: Karesan, leg. T. Kawakani et U. Moni, Juli. 1906, (No. 1422).

Distrib. The Loo-choo islands.

It is no matter of dispute that the plant should be referred to Shortia, as stated by Mr. T. Makino. 


\section{Primulaceæ.}

\section{Primula Lins.}

Primula sp. Herbæ annux. Folia radicalia spathulata circ. $13 \mathrm{~cm}$. longa $2 \frac{1}{2} \mathrm{~cm}$. lata apice rotundata ad medium deorsum sensim attenuata basi leviter dilata sursum argute denticulata deorsum subintegra. Scapi clongati $30 \mathrm{~cm}$. longi. Flores in rerticillos 2-3 superpositos dispositi, bracteis linearibus, pedicellis $3 \mathrm{~cm}$. longis. Capsula obconica $7 \mathrm{~mm}$. longa.

$\mathrm{H}_{\mathrm{AB}}$, in monte Morrison.

This very interesting, if not new, rare Primula is the only species found in the island. The plant resembles very much $P$. Cockburniana Henss. (in Gard. Chronic. May 27, 1905, p. 331 Fig. 137) in having two or three whorls of flowers towards the top of the scape.

\section{Lysimachia Lins.}

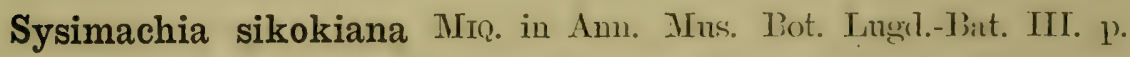
121, et Prol. Fl. Jap. p. 285; Fravch. et Savat. Eumm. PI. Jap. I. p. 302 ; Matsum. in Tōkyō Bot. Mag. XIV. p. 84; Exal. Bot. Jahru. VI. p. 64; Forbes et Henss. Ind. Fl. Sin. II. p. 57 ; Mitsum, et Hayata, Enum. Pl. Formos. p. 223.

Hab. Taitō: Tökeisha, leg. T. Kawaraur et U. Mori, Dec. 1905, (No. 1929).

Distrin. Japan.

Myrsineæ.

There is three distinct species belonging to Ardisia and two species to Mcesa; but they are not yet determined. 


\section{Styraceæ.}

\section{Symplocos Linn.}

Symplocos confusa Brand. in ExgL. Pfl-reich, IV.-242, Symploc. p. 88.

$\mathrm{H}_{\mathrm{AB}}$. in monte Morrison, ad 7000 ped. alt., Oct. 1906, (No. 2006), et ad 8000 ped. alt., leg. T. KawaKami et U. Mori, Nov. 1906, (No. 1785).

Distrib. The Philippine islands.

The above cited description of Mr. Brand is not very clear and even wanting of any account about fruits. It is, therefore, most desirable to give some additional account about this plant.

Frutices? ramulis pubescentibus badio-fuscis. Folia ad apices ramorum approximata alterna breve petiolata ovata vel oblonga utrinque obtusa vel apice obtuse acuminata $7 \mathrm{~cm}$. longa $3 \mathrm{~cm}$. lata margine integra rarius obscure crenata supra costis impressis venulis obscuris, subtus prominentibus petiolis circ. $5 \mathrm{~mm}$. longis intus basi hirtellis. Flores ad axillas foliorum ramulorum superiorum solitarii vel 2-3 racemoso-fasciculati breve pedicellati, bracteis minutis. Calyx per anthesin campanulatus basi pedicellum abeuns pubescens $3 \mathrm{~mm}$. longus obscure 5-lobus, lobis latis truncatis. Corolla cylindraceo-campanulata $1 \mathrm{~cm}$. longa 5-loba, lobis patentibus oblongis apice rotundatis crassiusculis. Stamina numerosa $\infty$-seriata corollæ tubo adnata, exterioribus longioribus filamentis complanatis apice abrupte angustis fere usque ad medium in tubum coalitis, antheris brevibus facie dorsoque minute papillosis, loculis parallelis longitudinaliter dehiscentibus. Ovarium apice pilosum semi-inferius 2loculare, loculis 2-ovulatis, ovulis pendulis ex angulo superiore, 
stylo filiformi pilosissimo, stigmate capitato. l'ructus (immaturi ?) carnosi cylindracei apice truncati.

Symplocos modesta Br.nd, in Exal. Pfl.-reich, IV. 242 Symploc. p. 66. Symplocos myrtacea Henst. in Fonbes et Hexsu. Ind. Fl. Sin. II. p. 73 ; Matsun. et Hayata, Enum. Pl. Formos. p. 230.

HAB. Taitō : Iryokukakusha, Dec. 1906, (No. 2151), in monte Morrison, ad 6500 ped. et ad 7000 ped. alt., (Nos. 2020 et 1712), leg. T. Kawakayi et U. Mori, Oct. 1906; Arizan, in montibus Morrison, leg. G. NaKahara, Nov. 1906.

Distrib. An endemic plant.

As the original description of $M r$. Brand seems to have been drawn from an imperfect specimen, a complete account relating to flowers is much desirable. The following description is, therefore, may properly be added here.

Frutices arborescentes glaberrimi, ramulis gracillimis. Folia chartacea oblonga longe cuspidato-acuminata rel abrupte ad acumen $2 \frac{1}{2} \mathrm{~cm}$. longum attenuata $7 \mathrm{~cm}$. longa $2 \mathrm{~cm}$. lata basi cuneata, supra costis impressis venulis leviter prominentibus, subtus costis et venulis prominentibus. Racemi ad axillas foliorum inferiorum ramulorum novellorum solitarii laxiflori vel densiflori folio breviores gracillimi. Flores longe pedicellati, pedicellis $1-2 \mathrm{~cm}$. longis basi pedicelli 2 -bracteatis, bracteis parvis ciliatis triangularibus costatis. Calyx longe campanulatus glaber viridis $3 \mathrm{~mm}$. longus, lobis 5 rotundatis margine ciliatis. Corolla patens $5 \frac{1}{2} \mathrm{~mm}$. longa, tubo brevissimo, 5-loba, lobis ovatis apice rotundatis margine obscure ciliolatis imbricatis. Stamina numerosa oo-seriata, exterioribus longioribus corollam excedentibus, ad basin corolla adnata basi connatil, filamentis subcomplanatis. Ovarium inferius 3-loculare, loculis 2-ovulatis, stylo filiformi, stagmate truncato. Fructus ignoti. 
Symplocos morrisonicola Hayata, sp. nov. (Pl. XXVIl). Frutices ramosi, ramis pubescentibus cinereo-fuscis, ramulis novellis badio-villosis. Folia parviora alterna coriacea breve petiolata ovata rel oblonga $3 \frac{1}{2} \mathrm{~cm}$. longa $1 \frac{1}{2} \mathrm{~cm}$. lata apice aristato-acuta vel abrupte acuminata basi acuta vel rotundata margine obscure serrulata rel subintegra supra nitida venis et venulis prominentibus subtus obscure renosa, petiolis brevissimis $2 \mathrm{~mm}$. longis. Racemi panciflori axillares folio 2-plo brevioribes pubescentes, bracteis ovatis parvis $\frac{1}{2} \mathrm{~mm}$. longis. Calyx campanulatus $2 \mathrm{~mm}$. longus, tubo ovario adnato, limbo 5-lobo, lobis imbricatis latis subtruncatis extus et margine pubescentibus. Corolla campanulata $5 \mathrm{~mm}$. longa 5 -loba, lobis oratis apice rotundatis imbricatis. Stamina circ. 20, sub-2-seriata ad basin corollæ adnata exterioribus longioribus, filamentis complanatis basi connatis corollam excedentibus, antheris brevibus, loculis parallelis longitudinaliter dehiscentibus. Ovarium inferius 3 -loculare, loculis 2-ovulatis, ovulis pendulis ex angulo superiore, stylo filiformi, stigmate capitato obscure 3-lobato. Fructus ignoti.

$\mathrm{H}_{\mathrm{AB}}$. Suizan, in montibus Morrison, ad 7702 ped. alt., leg. S. Nagasawa, Oct. 1905, (No. 737) ; Tōzan, in isdem montibus, leg. G. Nakahara, Oct. 1906 ; in monte Morrison, ad 7500 ped. alt., leg. T. KawaKami et U. Mori, Oct. 1906, (No. 1702),

Near S. modesta Brand; but differs from it in having shorter racemes, subentire leaves and pubescent branchlets.

Symplocos spicata Roxr. Fl. Ind. ed.-C.1rey, II. p. 541 ; A. DC. Prodr. VIII. p. 254; Bемth. Fl. Hongk. p. 212 ; Clarke, in Hook. f. Fl. Brit. Ind. III. p. 573 ; Fonbes. et Hemsi. Ind. Fl. Sin. II. p. 75 ; Henrx, List Pl. Formos. p. 58; Matsun. in Tōkyō Bot. Mag. XV. p. 77; Diels, Fl. Centr. Chin. p. 528 ; Matsur. et Hayata, Enum. Pl. Formos. p. 231.

Lodhra spicata.Muens, in Journ. LINT. Soc. XVII. p. 298. 
Haв. Taichū : Kashigatani, leg. G. Nanahara, Feb. 1907.

Distrib. Japan, south central China, and North and East India.

\section{Oleaceæ.}

\section{Osmanthus Lour.}

Osmanthus sp. nov.? Folia alterna rigide coriacea lanceolota $10 \mathrm{~cm}$. longa $2 \frac{1}{2} \mathrm{~cm}$. lata longe acuminata basi acuta petiolata, petiolis $1 \mathrm{~cm}$. longis. Flores ad axillas foliorum circ. 20-fasciculati, fasciculis squamis $2-3$ ovatis acutis coriaceis $5 \mathrm{~mm}$. longis instructis, pedicellis $1 \mathrm{~cm}$. longis. Calyx brevis late 4-lobus. Corolla subcampannlata $4 \mathrm{~mm}$. longa 4 loba, lobis $2 \frac{1}{2} \mathrm{~mm}$. longis late ovatis subclausis. Stamina 2 ad medium tubi corollæ affixa, antheris suborbicularibus, connectivis latis apiculatis. Orarium conicum, stylo ovario longiore, stigmate peltato crasso 2-lobo. Fructus ignoti.

Hab. Tōzan, in monte Morrison, leg. G. Natihara, Oct. 1906.

Osmanthus sp. nov.? Folia alterna oblonga 5 $\mathrm{cm}$. longa $2 \mathrm{~cm}$. lata apice aristato-acuta basi obtusa rigide coriacea supra (exsiccato) reticulato-rugosa subtus obscure renosa, petiolata, petiolis $1 \mathrm{~cm}$. longis. Flores ad axillas foliorum circ. 10 fasciculati, fasciculis squamis 2-3 late ovatis minutis $1 \mathrm{~mm}$. longis instructis, pedicellis $1 \mathrm{~cm}$. longis. Calyx brevis $1 \mathrm{~mm}$. longus + lobus, lobis late ovatis. Corolla late campanulata $4 \mathrm{~mm}$. longa, tubo brevissimo, limbo 4 lobo lobis late ovatis obtusis. Stamina ¿ supra basin corolla affixa, filamentis brevibus, antheris oblongis apiculatis. Ovarium conicum, stylo brevi, stigmate conico 2-lobo. Fructus ignoti.

HАв. in monte Morrison, ad 8000 ped. alt., leg. 'I'. Kawakami et U. Mori, Oct. 1906, (No. 2003). 


\section{Asclepiadeæ.}

Dischidia R. BR.

Dischidia formosana Maxtr. in Mél. Biol. IX. p. 822; Fondes et Heusu. Ind. Fl. Sin. II. p. 116 ; Matsum. et Hayata, Enum. Pl. Formos. p. 240.

Hab. Tappansha, ad 3138 ped. alt., leg. S. NAGASawn, Oct. 1905, (No. 581).

Distrib. An endemic plant.

\section{Loganiaceæ.}

Logania R. BR.

Logania dentata HAYATA, n. n. (Pl. XXVIII.).

Nertera dentata ELmer, in Leafl. Philipp. Bot. I.-1, p. 15.

Herbæ humiles repentes in totum hispidae, ramis ad nodos ascendentibus $7-8 \mathrm{~cm}$. longis superiore proliferis. Corpora ad axillas foliorum solitaria squamis teretibus glabris crassiusculis $\infty$-seriatis instructa $3-4 \mathrm{~mm}$. longa stipitata, stipitibus $\frac{1}{2} \mathrm{~cm} .-1 \mathrm{~cm}$. longis. Folia opposita petiolata late ovata vel orbicularia circ. $10 \mathrm{~mm}$. longa 13 mm. lata apice obtusa basi rotundata truncata margine serrata, serraturis crassiuscule mucronatis, supra setuloso-hispida subtus sparce hispida, petiolis lamina 2-plo brevioribus $6 \mathrm{~mm}$. longis, internodiis circ. $1 \mathrm{~cm}$. longis, stipulis 0 . Flores parvissimi $3 \mathrm{~mm}$. longi axillares solitarii breve pedicellati, pedicellis $1 \mathrm{~mm}$. longis glabratis. Calyx $3 \mathrm{~mm}$. longus 4-partitus, segmentis lanceolatis extus glabratis, intus et margine sericeo-setulosis crassiusculis. Corollæ tubus urceolatus, limbo 4-lobo, lobis patentibus rotundatis. 
Stamina 4 infra faucem tubi affixa, filamentis brevibus, antheris oblongis. Ovarium globosum 2-lobum stylum subæquans 2-loculare, loculis $\infty$-ovulatis. Capsula globosa $6 \mathrm{~mm}$. in diametro æquans rubra 4 valvis dehiscens. Semina numerosa oroidea $\frac{1}{2} \mathrm{~mm}$. longa lævia minute reticulata.

HAB. Ganzan, in montibus Morrison, ad 9141 ped. alt., leg. S. Nagasawa, Oct. 1905, (No. 639); in isdem montibus, leg. G. NaKaHARA, Oct. 1906 ; in monte Morrison, ad 8000 ped. alt., Oct. 1906 , (No. 1803), et in montibus centralibus, ad 10000 ped. alt., Nov. 1906, (No. 1847), leg. T. KawaKanI et U. MorI.

It is not without hesitation that I have identified the present plant with Elner's Nerterc dentata, transferring it to the genus Logania. There is a little doubt about this being similar to Mr. ELMer's. In his diagnosis, the fruits are described as follows: " berries shining vermilion red, 2 lin. in diameter"; globose, frequently with a short persistent style..." But, in my specimens, they are decidedly capsules, $5 \mathrm{~mm}$. in diameter, opening with two valves, each valve being afterward divided into two. After considering his description and my specimens, I have wondered if he had not made an error of regarding the fruits as "berries." Mr. E. D. Merrill affirmed me that my plant is exactly the same as the Philippine plant. In superficial observation, it appear's very much to be a rubiaceous plant as Mr. Etarer writes in his paper. Nevertheless, the plant has superior ovaries, by which character it should not be regarded as belonging to Rubiacere, but must be referred to Loganiacer. This species is greatly different from any other species of Logania known to us, and it is perhaps the smallest of all the plants belonging to this genus. The plant agrees with the generic character's of Logania in its floral structure, infloreseence and habit, and it is somewhat like the 
Australian L. pusilla R. Br. It also bears some resemblance to Polypremum Liss., but differs from the latter in the absence of glands and in having entirely supericr ovaries. This new Loganic is very remarkable in its prostrate habit and in having strikingly deformed flowers. The occurrence of this Australian genus in Formosa and the Philippines is exceptionally interesting. So far as I am aware, no representative of this genus has ever been known from any other regions on the North Hemisphere.":

\section{Gentianaceæ.}

\section{Craufurdia WALL.}

Crawfurdia fasciculata WALL.; DC. Prodr. IX. p. 120 ; CLAnTE, in Hoor. f. Fl. Brit. Ind. IV. p. 107; S. Moore, in Journ. Bot. (1875) p. 231 ; Bot. Mag. t. 4838; Forbes et Heyst. Ind. Fl. Sin. II. p. 122 ; Henry, List PI. Formos. p. 61 ; Diels, Fl. Centr. Chin. p. 538 ; Matsum. et Hayata, Enum. Pl. Formos. p. 243.

Cravfurdia japonica SreB. et Zucc. Fl. Jap. Fam. Nat. II. p. 36, n. 516 ; Franch. et Savat. Enum. Pl. Jap. I. p.324; Maxim. in Mél. Biol. IX. p. 399 ; S. Moone, in Journ. Rot. (1880), p. 4.

Golowninia japonica Maxtr. "in Bull. Acad. Imp. Sc. Pétersb. IV. p. 251."

Hab. Suizan, in montibus Morrison, ad 7702 ped. alt., leg. S. Nagasatwa, Oct. 1905, (No. 660).

Distrib. Japan, China, and the mountains of North East India.

* After completing this manuseript, I am informed by Mr. E. D. MERkILL who have just returned to Manila from his trip, that he thinks the Philippine plant Nerterc dentata ELmen to be referable to Ilemiphragma heterophylla WALL. of the Himalayns. 'I'he Formosan species in my hands does, however, not agree with the description of that genus. In Ifemiphragma, the corolla is 5-lobed, and stamens are affixed to the base of the tube, while in my plant, corolla is 4-lobed and stamens are affixed to the middle of the tube. Accordingly, the Formosan plant is, I think, not identical with the Himulayan species, though the latter may be the same as the Philippine one. It remains still questionable whether the Formosan plant should be referred to Logania or Tlemipliragma. 


\section{Gentiana LINN.}

Gentiana (§ Chondrophylla) cæspitosa Haratı, sp. nor. IIerbie humillimæ perennes rigidiusculæ glabræ cæspitosæ, caulibus ascendentibus $4 \mathrm{~cm}$. altis simplicibus multifoliatis. Folia approximata opposita plicata leviter recurva prominente costata ovata $5 \mathrm{~mm}$. longa $2 \frac{1}{2} \mathrm{~mm}$. lata aristato-acuta basi truncata, vaginis interpetiolaribus $1 \mathrm{~mm}$. longis hyalinis, crasse cartilaginex margine albo-lamellatá apicem versus obscure basin argute denticulata. Flores terminales solitarii $17 \mathrm{~mm}$. longi. Calyx campanulato-tubuliformis $7 \mathrm{~mm}$. longus 5 -lobus, lobis $3 \mathrm{~mm}$. longis angustis acutis crassinsculis, sinibus inter lobos acutis. Corolla tubalosa campanulata $17 \mathrm{~mm}$. longa 5 -loba, lobis $3 \mathrm{~mm}$. longis late oratis, sinibus inter lobos plicatis, appendiculatis triangularibus. Stamina כ̃ inclusa, filamentis ad medium adnatis. Capsulæ compresso-obovatæ $6 \mathrm{~mm}$. longæ ad suturas 2-valvis dehiscentes, valvis margine denticulatis, stipitibus longis capsulam in longitudine ?-plo superantibus, stylis persistentibus 2-fidis $2 \mathrm{~mm}$. longis spiraliter recurvis. Semina oblonga angulata $1 \mathrm{~mm}$. longa longitudinaliter reticulata.

HAB, in monte Morrison, ad 12500 ped. alt., leg. 'T'. KaWarann et U. Mori, Oct. 1906, (No. 2242).

Very near G. micans Cuarise (in Hook. f. Fl. Brit. Ind. IV. p. 112); but differs from it in having undivided folds of corollas.

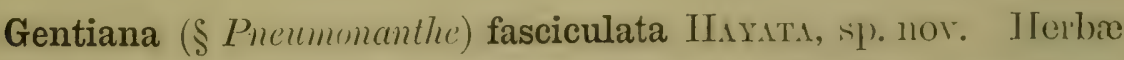
humiles glabre, caulibus repentibus apiee asecndentibus gracilibus $8-10 \mathrm{~cm}$. longis, foliis basin versus remote apicem versus approximate dispositis. Folia canlina remota opiposita sessilia uninorvia lanceolato-linearia obtusa $1.5 \mathrm{~cm} .-2 \mathrm{~cm}$. longa $3 \mathrm{~mm}$. lata valginis interpetiolaribus $3 \mathrm{~mm}$. longis; folia floralia ad basin fisciculi florum 
conferta folio caulino conformia sed 3-plo majora. Flores terminales sessiles erecti 5-fasciculati ebracteati. Calyx tubuliformis $12 \mathrm{~mm}$. longus 5-lobatus, lobis linearibus $3 \mathrm{~mm}$. longis, sinibus latis truncatis. Corolla campanulato-tubuliformis $2.5 \mathrm{~cm}$. longa, fauce tubi nuda 5-loba, lobis patentibus contortis rotundato-acutis, sinibus plicatis, appendiculis 0 . Stamina 5 tubo corollæ affixa inclusa; antheris linearibus, connectivis apiculatis. Ovarium stipitatum 1 loculare apice in stylum brevem pubescens 2-divisum attenuatum, stigmatibus lamellatis revolutis. Capsulæ oblongæ cum stylo stipiteque $22 \mathrm{~mm}$. longæ, stipite capsula breviore $6 \mathrm{~mm}$. longo, ad suturas 2-valvis revolutis dehiscentes. Semina ovoidea compressiuscula $\frac{2}{3} \mathrm{~mm}$. longa elegante reticulata.

HAB. Seizan, in montibus Morrison, ad 11707 ped. alt., leg. S. Nagasawa, Nov. 1905, (No. 624); ibidem, leg. T. Kawakami et G. NaKahara.

Gentiana ( Chondrophylla) flavescens Harata, sp. nor. Herbæ annuæ minute scabriusculæ vel glabratæ, caulibus gracilibus. Folia opposita angusto-obovata $8-\mathrm{mm}$. longa $2 \frac{1}{2} \mathrm{~mm}$. lata apice aristatoacuta basi confluentia, vaginis interpetiolaribus $1 \mathrm{~mm}$. longis, margine obscure albo-lamellata integra basin versus minute ciliato-denticulata crassiuscula. Flores ad apices ramorum solitarii. Calyx campanulatus $4 \frac{1}{2} \mathrm{~mm}$. longus 5 -lobatus, lobis $1 \frac{1}{2} \mathrm{~mm}$. longis cuspidato-acuminatis. Corolla tubuloso-campanulata $12 \mathrm{~mm}$. longa, lobis primariis $5,21 \mathrm{~mm}$. longis triangularibus, lobis secundariis $5 \mathrm{ad}$ sinus plicatos lobo primario xquilongis cuspidato-acutis tenuissimis. Stamina 5 tubo corollæ affixa, filamentis ad medium adnatis, antheris oblongis $1 \mathrm{~mm}$. longis basi sagittatis apice emarginatis. Capsulæ oboratæ $6 \mathrm{~mm}$. longæ longe stipitatæ corollam excedentes, stipitibus $12 \mathrm{~mm}$. longis, stylis linearibus 2-divisis complanatis 
pilosiusculis spiraliter revolutis. Semina oblonga angulata $\frac{2}{3} \mathrm{~mm}$. longa minute longitudinaliter reticulato-striata.

Haв. Suizan, in montibus Morrison, ad 7702 ped. alt., (No. 646), et Ganzan, in isdem montibus, ad 9141 ped. alt., leg. S. Nagasawa, Oct. 1905 .

Gentiana formosana Hayata, in Matsus. et Hayata, Enum. Pl. Formos. p. 242.

HAB. in monte Shichiseitonzan, leg. B. Hayata, Aug. 1900 ; ibidem, leg. G. NaKahara, 1905. (No. 5.)

Gentiana humilis Stev. ; Cranke, in Hoor. f. Fl. Brit. Ind. 1V. p. 111. Gentiana aquatica PaLL. Fl. Ross. II. t. 97, fig. 2 (non LrNv.).

Hab. Tōzan, montibus Morrison, leg. S. Nagasatwa, Nov. 1905.

Distrib. Western Tibet.

Gentiana (§Chondrophylla) tenuissima HAYATA, sp. nor. Herbæ humillimæ $6 \mathrm{~cm}$. altæ glabræ tenues. Folia radicalia conferta lanceolato-ovata $1 \mathrm{~cm}$. longa $5 \mathrm{~mm}$. lata apice acuta basi abrupte angusta sessilia margine minutissime denticulata; folia caulina paucissima; folia floralia radicali conformia minora. Flores paniculato-cymosi. Calyx tubuliformis $3 \mathrm{~mm}$. longus 5-lobatus, lobis circ. $1 \mathrm{~mm}$. longis late ovatis aristato-acutis, sinibus inter lobos latis truncatis. Corolla tubuloso-campanulata $5 \mathrm{~mm}$. longa 5 -lobata, lobis $1 \mathrm{~mm}$. longis rotundato-acutis, sinibus plicatis obscure lobatis. Stamina 5 tubo corollæ affixa inclusa, filamentis supra medium liberis. Ovarium stipitatum oblongo-obovoideum cum stipitibus $3 \mathrm{~mm}$. longum, stylo brevi $\frac{1}{2} \mathrm{~mm}$. longo stigmate 2-lamellato recurvo. Capsulæ obovatæ $3 \mathrm{~mm}$. longre longe stipitatæ, stipitibus capsulam 2-plo in longitudine supcrantibus, ad suturas 2-valvis dehiscentes, valvis patento-rorolutis marginibus 
minute serratis. Semina oblonga $1 \mathrm{~mm}$. longa longitudinaliter reticulata utrinque attenuata.

Haв. Taitō : Gozenjō, leg. T. Katwakami.

Neer G. aquatica Linn. and G. delicata Hance, but differs from them in having much smaller flowers and reticulated seeds.

Gentiana scabrida Hayata, sp. nov. Herbæ annuæ pancifoliatæ scabriusculæ, caulibus $20 \mathrm{~cm}$. longis atropurpureis pauciramosis. Folia radicalia caulino conformina oblongo-lanceolata $11 \mathrm{~mm}$. longa $4 \mathrm{~mm}$. lata apice aristato-acuta, vaginis interpetiolaribus $2 \mathrm{~mm}$. longis, margine et ad costam subtus scaberrima albo-lamellata, supra minute scaberrima subtus præter costas glabra. Flores ad apices ramorum solitarii pedunculati, pedunculis $\frac{1}{2} \mathrm{~cm}$. longis. Calyx campanulatus obscure 5-costatus, costis scaberrimis, $1 \mathrm{~cm}$. longus 5-lobatus, lobis spathulatis supra basin leviter constrictis basi paullo dilatis $5 \mathrm{~mm}$. longis $2 \mathrm{~mm}$. latis 1 -costatis, costis serrulatis, margine albo-lamellatis intus minute scaberrimis. Corolla campanulata $2 \mathrm{~cm}$. longa 5 -lobata, lobis late ovatis $4 \mathrm{~mm}$. longis aristatoacutis, sinibus plicatis, appendiculis triangularibus $3 \mathrm{~mm}$. longis. Stamina 5, supra medium tubi corollie affixa, filamentis ad insertionem dorso dilatis, antheris angustis basi sagittatis apice obtusis. Capsulæ oratæ $7 \mathrm{~mm}$. longæ longe stipitatæ, stipitibus $20 \mathrm{~mm}$. longis capsulam 3-plo superantibus, stigmatibus lamellatis revolutis. Semina oblonga $1 \mathrm{~mm}$. Iongr longitudinaliter minute reticulato-striata.

HAB. Ganzan, in montibus Morrison, ad 9141 ped. alt., leg. S. Nagasatwa, Oct. 1905, (Nos. 701 et 702); in monte Morrison, leg. T. Kawakani et U. Mori, Oct. 1906, (No. 2275).

\section{Swertia LinN.}

Swertia alata Hayata, sp. nov. Herbæ annuæ 2-3 ped. altæ 
glabræ, caulibus tetragonis tetrapteris, alis $2 \mathrm{~mm}$. latis. Folia opposita sessilia membranacea oblongo-lanceolata $7 \mathrm{~cm}$. longa $2 \mathrm{~cm}$. lata apice breve obtuse acuminata basi angusta ad insertionem dilata $6 \mathrm{~mm}$. Iata trinervia supra nervis impressis subtus prominentibus. Flores paniculato-racemosi, ramis oppositis, pedicellis oppositis per anthesin $\frac{1}{2} \mathrm{~cm}$. longis bracteis lanceolatis. Calyx 4-partitus, lobis lanceolatis $5 \mathrm{~mm}$. longis trinerviis. Corollæ tubus brevis, limbo 4-partito, lobis laciniatis oblongis $7 \mathrm{~mm}$. Iongis apice obscure serratis prope basin foveolatis, foveolis glandulosis margine ciliato-fimbriatis supra foreolam squamulis fimbriatis. Stamina 4 basi corollæ affixa, filamentis complanato-filiformibus basi dilatis, antheris subsagittatis apiculatis. Ovarium ovoideum apice attenuatum, stylo subnullo, stigmate 2-lamellato, lobis brevissimis latisque leviter recurvis. Capsulæ oblongo-ovatæ circ. $1 \mathrm{~cm}$. longæ ad suturas 2 -valvis dehiscentes. Semina parvissima $\frac{1}{2} \mathrm{~mm}$. longa compressa ovata reticulato-muricatar.

Hab. Arizan, in montibus Morrison, leg. G. NaKahara, Oct. 1906.

Near S. tetraptera Maxni., but differs from it in having mostly 3-nerved and sessile leaves and paniculate racemes; from S. pulchellu HANCE, in having prominent wings and much smaller petals.

Swertia sp. Herbæ basi suffruticosæ circ. 1 ped. altæ. Folia oblonga $3 \mathrm{~cm}$. longa apice obtusa basi angusta al petiolum attenuata. Flores paniculato-racemosi. Capsulæ $6 \mathrm{~mm}$. longæ.

HАв. Ganzan, in montibus Morrison, ad 9140 ped. alt., leg. S. Nagasatwa, Oct. 1905, (No. 642) ; Tōzan, in isdem montibus, leg. G. Nakahara, Nov, 1906. 


\section{Hydrophyllaceæ.}

\section{Ellisiophyllum MaXIM.}

Ellisiophyllum pinnatum Makino, in Tōkyō Bot. Mag. XX. p. 92. Hornemannia pinnata BENTH. in DC. Prodr. X. p. 428.

Sibthorpia pinnata Benth. in BeNth. et Hook. f. Gen. P1. II. p. 959 ; Hоoк. f. Fl. Brit. Ind. IV. p. 288.

Moseleya pinnata HeMsL. in Hoor. Ic. PI. XXVI. t. 2592.

Ellisiophyllum reptans Maxm. in Mél. Biol. VIII. p. 18; FravoH. et Safat. Enum. Pl. Jap. I. p. 329.

Нав. in monte Morrison, ad 8000 ped. alt., leg. T. KawakanIr et U. Mori, Oct. 1906, (No. 1781).

Distrib. Japan, China and the Philippines, westward to the Himalayas.

As stated by Mr. T. MAKino, the plant should properly be called E. pimatum Makino, assuming that Moseleya pimnata Henrsi. is the same as Ellisiophyllum reptans Maxñ.; for Ellisiophyllum is older name than Moseleya, and the specific name pinnata is much so than reptans.

\section{Boragineæ.}

\section{Cynoglossum LINN.}

Cynoglossum micranthum Desf.; DC. Prodr. X. p. 149 ; Maxm. in Miél. Biol. VIII. p. 555 ; MrQ. Fl. Ind. Bat. II. p. 931; FrancheT, Pl. David. p. 215 ; Clarke, in Hook. f. Fl. Brit. Ind. IV. p. 156 ; Forbes et HersL. Ind. Fl. Sin. II. p. 150 ; Hexnx, List Pl. Formos. p. 63 ; Matsuxr. in Tōkyō Bot. Mag. XII. p. 108; Diels, Fl. Centr. Chin. p. 546 ; Matsuxr. et Hayata, Enum. P1. Formos. p. 257.

Cynoglossum racemosum Roxв. Fl. Ind. I. ed.-CAREY, p. 456.

Cynoglossum furcatum WALL. ; WIGHT, Ic. Pl. Ind. or. t. 1395. 
Haв. Kagi : Shakkzōshō, leg. T. KawaKani et U. Mori, Oct. 1906, (No. 1746).

DistrIB. South China; widely spread in India Malay and also found in East Africa.

\section{Trigonotis STEV.}

Trigonotis formosana Hayata, sp. nov. Herbæ perennes pilosæ scaberrimæ, caulibus ascendentibus $10 \mathrm{~cm}$. longis stoloniferis. Folia radicalia et inferiora longe petiolata, petiolis $5 \mathrm{~cm}$. longis laminam æquantibus setuloso-pilosis, laminis oblongis vel spathulatis $7 \mathrm{~cm}$. longis $2 \frac{1}{2}-3 \mathrm{~cm}$. latis apice truncato- vel retuso-mucronatis basi acuminatis membranaceis crassiusculis margine integris repandis supra pilis setulosis brevissimis scaberrimis subtus hispidulis pallidioribus, venis transversis primariis utrinque obscure 5-6 ad margines arcuatis 2-3-seriatim anastomosantibus. Folia superiora inferioribus conformia sed minora. Flores in cymas scorpioidales ramosas vel simplices racemosas elongatas dispositi, breve pedicellati pedicellis $1 \mathrm{~mm}$. longis. Calyx 5 -fidus, lobis obovatis $1 \frac{1}{2} \mathrm{~mm}$. longis obtusis intus et extus pilosiusculis, fructifer vix anctus. Corolla rotata $2 \frac{1}{2} \mathrm{~mm}$. longa, tubo brevi $1 \mathrm{~mm}$. longo fance squamis brevibus crassis subfornicatis subclausis instructo, lobis 5 imbricatis patentibus late orbicularibus basi breve angustis $1 \frac{1}{2} \mathrm{~mm}$. longis. Stamina 5 supra medium tubi affixa inclusa, filamentis brevibus, antheris oblongis obtusis. Ovarium depresso-globosum ${ }_{4}^{1} \mathrm{~mm}$. longum $\frac{1}{2} \mathrm{~mm}$. latum 4-lobatum, lobis distinctis, stylo brevi $\frac{1}{2} \mathrm{~mm}$. longo, stigmate capitato. Nuculæ 4, obpyramidales ad angulas costatæ $1 \mathrm{~mm}$. longæ vel vix longiores erectæ læves facie superiore planx truncatæ pance setulosæ, cæterum glabri.

HAB. Sanchōki, ad 4000 ped. alt., leg. S. NAGAsıw.., Oct. 1905, (No. 724); Kagi : Kishirei, leg. 'T. Kawatani et U. Moni, Oet. 1906, 
(No. 1969) ; Taitō: Dakumsha, leğ. T. Kawatraji ct U. Mori, Dec. 1906, (No. 2230).

\section{Solanaceæ}

\section{Solanem LINN.}

Solanum sp. Herbre tenues scandentes pubescentes ad nodos radicantes. Folia ovata $5 \mathrm{~cm}$. longa $3 \mathrm{~cm}$. lata petiolata, petiolis lamina 2-plo brevioribus, apice obtusa acuta basi rotundata subito attenuata ad petiolum abeuntia membranacea utrinque ad costas pubescentia cxterum glabra. Klores axillares longe pedunculati, solitarii, pedunculis petiolum vix superantibus. Bacca globosa $1 \mathrm{~cm}$. in diametro aequans.

Haв. Nantō: Rakurakusha, leg. G. Nakahara, Aug. 1905, (No. 466) ; Kagi : Tappansha, leg. T. Kawaramir et U. Mori, Oct. 1906, (No. 1737).

Very interesting plant, on account of its intermediate form between Solanum and Capsicum. It may constitute a new genus when the floral structures are fully known.

\section{Scrophularineæ.}

\section{Scrophularia LiNN.}

Scrophularia alata A. GraY, Bot. Jap. p. 401 ; MiQ. Prol. Tl. Jap. p. 47 ; Diets, Fl. Centr. Chin. p. 565 ; Francer. et Savat. Enum, Pl. Jap. I. p. 342.

var: duplicato-serrata MiQ. Prol. Fl. Jap. p. 47; Franch. et SAvat. Enum. Pl. Jap. T. 343.

Hab. in monte Morrison, leg. G. Nakahara, Nov. 1905.

Distriв. Type: Central China and Japan. 


\section{Mraves Lour.}

Mazus rugosus Lovr. Fl. Cochinch. ed.-IVILLD. p. 468; Bextir. in DC. Prodr. X. p. 375, et Fl. Hongk. p. 247; Maxnr. Prim. F'l. Amur. pp. 205 et 475, et in Mél. Biol. TX. p. 402 ; Fonbes et Hexrst. Ind. Fl. Sin. II. p. 183 ;' Franchet, Pl. David. p. 222 ; Henry, List Pl. Formos. p. 67; Diels, Fl. Centr. Chin. p. 566; Patibin, Conspect. Fl. Koree, II. p. 20 ; Matsusr. et Hayata, Enum. Pl. Formos. p. 275.

Mazus vandellioides HaNce, in WaLP. Ann. III. p. 193.

Lindernia japonica Thuns. Fl. Jap. p. 253.

MIazus japonica O. Kuntze, "Revis. Geu. Pl. II. (1891) p. 462"; Makino, in Tōkyō Rot. Mag. XIV. p. 170 ;

Hab. Tōzan, in montibus Morrison, leg. G. Nakahara, Nov. 1906.

Distrib. South China and Japan; Afghanistan to Manchuria; and also in the Malay archipelago.

Here we do not follow O. Kuntze, for M. migosa Lour. is too commonly used to be suppressed.

\section{Torenia LinN.}

Torenia peduncularis Benth.; Hоoк. f. Fl. Brit. Ind. IV. p. 276 ; Forbes et Henss. Ind. Fl. Sin. II. p. 188; Hexry, List Pl. Formos. p. 67. Diels, Fl. Centr. Chin. p. 567 ; Matsum, et Hayata, Enum. Pl. Formos. p. 278.

Torenia edentula Bentri. in DC. Prodx. X. p. 410 ; Bot. Mag. t. 4229.

$\mathrm{H}_{\mathrm{AB}}$. Suizan, in montibus Morrison, ad 7702 ped. alt., leg. S. Nagasawa, Oct. 1905, (No. 670).

Distrib. South central China, India and Malay.

\section{Bomnaya LINK. et OтTо.}

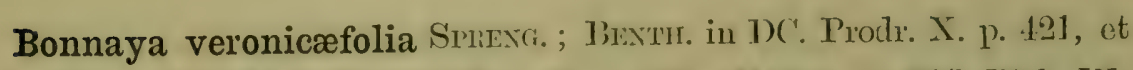

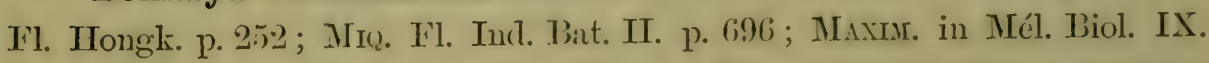
p. 421 ; Hooк. f. Fl. Brit. Ind. IV. p. 285; Wignt, Ic. PI. Ind. or. t. 1411; 
Forbes et Hexrst. Ind. Fl. Sin. II. p. 192 ; Henrix, List Pl. Formos. p. 68 ; Matsum. et Hayata, Enum. Pl. Formos. p. 281.

HAB. Tappansha, ad 3139 ped. alt., leg. S. NaGasawa, Oct, 1905, (No. 718).

Distrib. South China, India throughout, Ceylon and Malay.

\section{Rehmannia LiBosch.}

Rehmannia 0ldhami HexsL. in Ann. Bot. IX. p. 154, et in FonBEs et Hexss. Ind. Fl. Sin. II. p. 194 ; Henry, List Pl. Formos. p. 68 ; Matsus. et Hayata, Fnum. PI. Formos. p. 282.

Hab. Sanchōki, ad 4000 ped. alt., leg. S. NaGasawa, Oct. 1905, (No. 723) ; Kagi : Tappansha, leg. T. Kawakam et U. Mori, Oct. 1906, (No. 1745).

Distrib. An endemic plant.

\section{Veronica LINN.}

Veronica morrisonicola HaYata, sp. nov. (Pl. XXIX). Herbæ validæ basi suffruticosæ repentes ad nodos radicantes, ramis ascendentibus $10-20 \mathrm{~cm}$. longis præter inflorescentiam simplicibus hispidulis demum glabratis. Folia opposita spathulato-Ianceolata sessilia $2 \mathrm{~cm}$. longa $6 \mathrm{~mm}$. lata apice acuta basi sensim attenuata margine sursum serrata deorsum integra subtus costis prominentibus venulis inconspicuis. Racemi pubescentes ad axillas foliorum superiorum ramorum siti, longe pedunculati ramos excedentes, pedunculis $5 \mathrm{~cm}$. longis partem florigeram rquantibus, bracteis angustis $4 \mathrm{~mm}$. longis pedicello vix longioribus. Calyx 5-partitus, lobis angustis acutis $4 \mathrm{~mm}$. longis. Corolla rotata circ. $5 \mathrm{~mm}$. longa, tubo brevissimo limbo 4-lobato, lobo postico majore late ovato obtuso, lobis lateralibus mediocribus, lobo antico minore. Stamina 2 tubo ad latere lobi postici affixa, filamentis exsertis, antheris loculis divergentibus 
obtusis apice confluentibus. Ovarium pilosum. Capsulæ compressæ apice emarginatæ bisulcatæ $6 \mathrm{~mm}$. longæ totidem latæ loculicide dehiscentes, valvis columna placentifera adherentibus. Semina numerosa ovata $1 \mathrm{~mm}$. longa facie interna plano-cymliformi affixa, dorso rugulosa.

$\mathrm{H}_{\mathrm{AB}}$. Ganzan, in montibus Morrison, ad 9141 ped. alt., (Nos. 704 et 633), Seizan, in isdem montibus, ad 11579 ped. alt., (No. 634), et Suizan, in isdem montibus, ad 7702 ped. alt., Oct. 1905, (No. 736), leg. S. Nagasawa ; in monte Morrison, ad 9000 ped. alt., Oct. 1906, (No. 2278), et in montibus centralibus, ad 10000 ped. alt., leg. T. Kawakami et U. Mori, Nov. 1906, (No. 2213).

Very like $V$. serpyllifolia Lins.; but differs from that species in having long pedunculate racemes and very small bracts. The habit of this plant is like that of $V$. Onoei Fravch. et Savat., but differs from it in having obovate lanceolate leaves.

Veronica spuria Lrnn. Sp. Pl. ed.-2, p. 13 ; LEDEb. Fl. Ross. III. p. 236 (var.) ; Fonbes et Hexss. Ind. Fl. Sin. II. p. 200 ; PaLibin, Conspect. Fl. Koreæ, II. p. 21 ; Diets, Fl. Centr. Chin. p. 267 ; HayatA, in Tökyō Bot. Mag. XX. p. 72.

Veronica angnstifolia Frsch.; HANCE, in Jonn. Linn. Soc. XIII. p. 84.

Veronica paniculata Lrnn.; Bentr. in DC. Prodr. X. p. 465; Franch. et Savat. Enum. PI. Jap. I. p. 348.

$\mathrm{H}_{\mathrm{AB}}$. in monte Morrison, ad 8500 ped. alt., (Nos. 1809, 2080 et 1811), et eodem monte, ad 12000 ped. alt, leg. T. KAWAKaMI et U. Morr, Oct. 1906, (No. 2295) : Tikuzan, ad 150 ped. alt., leg. S. NaGaSaTw, Oct. 1905, (No. 764).

Distrib. Japan to central Asia, and westward to central Europe.

Sopubia Harr.

Sopubia formosana Intrats, sp. nov. Herbæ ammuæ erectæ circ. 
$30 \mathrm{~cm}$. altæ pubescentes siccitate nigricantes, caulibus ramosis rel simplicibus tetragonis sulcatis. Folia opposita sessilia oblonga $2 \mathrm{~cm}$. longa $8 \mathrm{~mm}$. lata dentata deorsum integra apice acuta rel obtusa basi acuta rel cuneata supra exsiccato nigricantia subtus pallicliora distincte nervosa. Flores ad apices ramorum dense subspicati, ad axillas bractearum solitarii, bracteis lanceolatis dentatis calycem 2-plo superantibus, pedicellis $1 \mathrm{~mm}$. longis 2-bracteolatis, bracteolis linearibus calycem æquantibus. Calyx campanulato-semiorbiculatus $7 \mathrm{~mm}$. longus 10 -nervatus breviter 5-lobus, lobis valvatis triangularibus acuminatis. Corolla ignota. Stamina 4 didynamia subinclusa: 2 longiora, antheris majoribus coherentibus, filamentis longe barbatis ; 2 breviora, antheris parvioribus liberis, filamentis glabris ; loculis antherarum omnibus ovatis basi muticis. Stylus apice stigmatoso-incrassatus sublinguiformis obtusus. Capsulæ depressoglobosæ $5 \mathrm{~mm}$. in diametro rquantes apice retusæ, loculicide deliscentes, valvis demum 2-fidis columnam placentiferam liberantibus. Semina numerosa minutissima angusta $1 \mathrm{~mm}$. longa truncata testa laxiuscula.

HAB. Suizan, in montibus Morrison, ad 7702 ped. alt., leg. S. Nagasatwa, Oct. 1905, (Nos. 661 et 673); Kagi: Kōdenshō, leg: 'T. Kawakami et U. Mori, Oct. 1906, (No. 1739).

Distrim. An allied species S. trifida Han. occurs in India and Ceylon; and is also found in Kwangtung in the opposit continent.

Near $S$ trifida Hux.; but differs from it in having ovatolanceolate leaves.

\section{Phtheirospermum Bunge.}

Phtheirospermum chinense Busge; Bexтн. in DC. Prodr. X. p. 391 ; Maxur. Prim. Fl. Amur. p. 208 ; HANCE, "in Journ. Bot. (1882), p. 292 "; Franchet, Pl. David. p. 225; Fonbes et Hemst. Ind. Fl. Sin. II. p. 204 ; Drels, Fl. Centr. Chin. p. 570. 
Pltheirospermum japonicum Kavinz, "Anthoph. Jap. p. 12 "; MAזrNo, in Tōkyō Bot. MLag. XV.p. 72.

Gerardia japonica Thunb. Fl. Jap. p. 251, et Ien. P1. Jap. Decas-5, t. X ; Wmi.d. Sp. Pl. III. p. 224 : Benth. in DC. Prodr. X. p. 519.

HAB. Toroku : Tōhozan, (No. 2082); Taitō: Dakunsha, leg. T. KaWaKaMI et U: Mori, Dec. 1906, (No. 2169).

Distris. Japan and China northward to Nanchuria; and also in North America.

Here we do not follow Mr. T. Makino, for P. chinense Bunge is too generally used to be supressed.

\section{Euphrasia LiNN.}

Euphrasia borneensis STAPF in "Traus. Linn. Soc. II. 4, (1794) 210 , t. 16, f. 1-16"; Merrit, in Philipp. Journ. Scie. I. Suppl. Bot. p. 236.

Hab. Nanto: Mushazan, ad 8000 ped. alt., leg. T'. KawakanII et U. MonI, Aug. 1906, (No. 1129).

Distrib. The Philippine islands and also in Borneo.

Euphrasia petiolaris WETTST. Monogr. Gatt. Euphris. p. 199, t. IV. Figs. 321-325, et t. XI. Fig. 8 .

Hab. Tōzan, in montibus Morrison, leg. G. Nakahara, Nor. 1906 ; in monte Morrison, ad 8000 ped. (No. 1728), et collem monte, ad 10000 ped. alt., leg. T. Kawakisi et U. Moni, Oct. 1906, (Nos. 1894 et 2274).

Distrib, in Himalaya.

\section{Orobanchaceæ.}

\section{Orobanche LinN.}

Orobanche cœrulescens StTEiri.; WIILD. Sp. Pl. 1IT. p. 349; DC: Prodr. XI. p. 34 ; Leder. Fl. Ross. III. p. 322 ; PaLibI. Conspect. Fl. 
Koreæ, II. p. 22 ; Biblioth. Bot. IV. p. 138 ; 认̂́asum. et Hayat.1, Enum. PI. Formos. p. 285.

HAB. in montibus Morrison, ad 9000 ped. alt., (No. 2238), et ad 8000 ped. alt., (No. 2071), leg. T. K.wakanI et U. Mori, Oct. 1906.

Distrib. Asia, Europe, and North America.

\section{Gesneraceæ.}

\section{Lysiomotus Dox.}

Lysionotus pauciflorus Maxis. in Ị!él. Biol. IX. p. 366 ; Fravor. et Savat. Enum. Pl. Jap. I. p. 327 ; S. Moore, in Journ. Bot. (1875) p. 231 ; Clarke, in DC. Monogr. Phanerog. V. p. 59 ; Fonbes et Hemss. Ind. Fl. Sin. II. p. 225 ; Henry, List Pl. Formos. p. 68 ; Matsun. et Hayata, Enum. Pl. Formos. p. 287.

HAB. Suizan, in montibus Morrison, ad 7702 ped. alt., leg. S. Nagasawa, Oct. 1905, (No. 556) ; Arizan, in isdem montibus, leg. G. Nakahara, Oct. 1906; in monte Morrison, ad 6000 ped. alt., leg. T. Kawakaui et U. Moni, Oct. 1906, (No. 1719).

Distrib. Japan and central China.

Rhynchoglossum BLUMIE.

Rhynchoglossum obliquum Bluste; DC. Prołr. IX. p. 274; Cl.1rise, in Hoor. f. Fl. Brit. Ind. IV. p. 367.

Rhynchoglossum Blumei DC. Prodr. IX. p. 274.

Hab. Kōdenshō : ad 2623 ped. alt., legg. S. N'Agasawa, Oct. 1906, (No. 745) ; Kagi: Kishirei, leg. T. KaWarani et U. Morr, Oct. 1906, (No. 1777).

Distrib. The Philippine islands throughout, and the other islands of the Malay archipelago. 
Chivita Hà̃.

Chirita anachorata HAXCE; DC. in DC. Monogr. Phanerog. V.-1, p. 115 ; Maxtr. in Mél. Biol. IX. p. 370 ; Foribes et Hexrst. Ind. Fl. Sin. II. p. 231 ; Henry, List Pl. Formos. p. 68; Matsux. et Hayata, Enum. PI. Formos. p. 288.

НАв. in monte Morrison, ad 7000 ped. alt., leg. T. KawaKam et U. Monr, Oct. 1906, (No. 2048).

DistriB. South China.

Conandron SIEB. et Zucc.

Conandron ramondioides SIEB. et Zucc. Fl. Jap. Fam. Nat. II. p. 730, t. 3, f. 1 ; Mre. Prol. Fl. Jap. p. 55 ; Maxmu. Mél. Biol. IX. p. 370 ; Franch. et Savat. Enum. Pl. Jap. I. p. 328 ; Bot. Mag. t. 6484.

Hab. Biyōritsu : Banahōzan, leg. T. Kawakasir et U. Mori, Juli. 1906, (No. 1093).

Distrib. Japan.

Acanthaceæ.

Strobilanthes BLUME.

Strobilanthes flaccidifolius NEES, in DC. Prodr. XI. p. 194; T. ANDERs. in Journ. Linn. Soc. IX. p. 481; Hance, in Journ. Linn. Sos. XII. p. 116 ; CumKe, in Hoor. f. Fl. Brit. Ind. IV. p. 468; Bot. Mag. t. 6947; Fonbes et Hemsi. Ind. Fl. Sin. II. p. 239 ; Matsuxr. et Hay.atı, Enum. Pl. Formos. p. 291.

Strobilanthes Championi T. Axdens.; Bextir. Fl. Hongk. p. 261.

Goldfussic Cusia NeEs, in DC. Prodr. XI. p. 175.

Hab. Kōdenshō, ad 2623 ped. alt., leg. S. Nagasaivı, Oct. 1905, (No. 727); in monte Morrison, ad 5000 ped. alt., leg. 'T. KawakanI et U. Morr, Oct. 1906, (No. 2064).

Distrib. South China, Burma and eastern India. 


\section{Codonacanthus NEES.}

Codonacanthus pauciflorus NeEs, in DC. Prodr. XI. p. 103; Crarke, in Hoor. f. Fl. Brit. Ind. IV. p. 500 ; Benth. Fl. Hongk. p. 267 ; Fonbes et Henrst. Ind. Fl. Sin. II. p. 244 ; Henry, List Pl. Formos. p. 69 ; Matsurr. et HaYata, Enum. Pl. Formos. p. 292.

Hab. Tappansha, ad 3138 ped. alt., leg. S. Nagasawa, Oet. 1905, (No. 787) ; Taitō : Inikufukusha, leg. T. Kawarim et U. Mori, Dec. 1906, (No. 2156).

Distrib. South China and eastern India.

\section{Justicia Lins.}

Justicia procumbens LINN.; HANCE, in Jomrn. Limn. Soc. XIII. p. 116 ; Clanie, in Ноor. f. Fl. Brit. Ind. IV. p. 539 ; Fories et Hemst. Ind. Fl. Sin. II. p. 246 ; Henry, List Pl. Formos. p. 69 ; Diels, Fl. Centr. Chin. p. 579 ; Matsujr. et Hayata, Enum. Pl. Formos. p. 293.

Rostellularia procumbens Nees, in DC. Prodr. XI. p. 371; Benth. Fl. Hougk. p. 265; Wight, Ic. Pl. Ind. or. t. 1539 ; FrancheT, Pl. David. p. 230.

Hab. in monte Morrison, leg. T. Kawakamr et U. Mori, Nov. 1906, (No. 1886).

Distrib. China and Japan; Malay, India, Ceylon, and also in Australia.

\section{Rungia NeEs.}

Rungia parviflora NeEs, in DC. Prodr. XI. p. 469 ; Cuarke, in Hоок. f. Fl. Brit. Ind. IV. p. 550 ; Fonbes et Henst. In J. FI. Sin. II. p. 247.

rar. pectinata Clurke, in Hook. f. Fl. Brit. Ind. IV. p. 550 ; Matsur. et Hayata, Enum. Pl. Formos. p. 294.

Rungia pectinata NeEs, in DC. Prodr. XI. p: 470 ; WIGHT. Ic. Pl. Ind. or. t. 1547 ; T. ANDERs. in Journ. Linn. Soc. IX. p. 517.

Rangia polygonoides NeEs, in DC. Prodr. XI. p. 471. 
Hab. Kagi : Tappansha, leg. T. Kawakami et U. Moni, Oct. 1906, (No. 1967).

Distrib. South China; East and South India and Ceylon.

Hypostes $\mathrm{R}$. BR.

Hypœstes purpurea R. Br.; NeEs, in DJ. Prodr. XI. p. 509; Mre. II. Ind. Bat. II. p. 851 ; Benth. Fl. Hongk. p. 265 ; Forbes et Hearst. Ind. FI. Sin. II. p. 249; Henry, List Pl. Formos. p. 70 ; Matsux. et Hayata, Enum. PI. Formos. p. 295.

Hab. Tappansha, ad 3138 ped. alt., leg. S. NAGASAwa, Oct. 1905, (No. 577) ; Kagi : Kishirei, leg. T. Katwanari et U. Morr, Oct. 1906, (No. 1768).

Distrib. South China and in the Philippine islands.

\section{Labiatæ}

\section{Mesona BLume.}

Mesona elegans Hayati, in Matsuar. et Hiyatı, Enmm. Pl. Formos. p. 306, t. 16.

Нав. in monte Morrison, ad 6000 ped. alt., leg. 'T. Kawakasr et U. Mori, Oct. 1906.

Distrib. An endemic plant.

Mesona procumbens Heirst. in Anu. Bot. IX. p. 155; Jevny, List Pl. Formos. p. 72 ; Matsurr. et Hayata, Enum. Pl. Formos. p. 306.

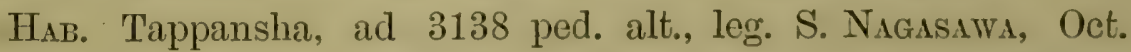
1905, (No. 777) ; Taitō: 'Tabari, leg. G. Naminara, Jan. 1906, (No. 736); in monte Morrison, ad 6000 ped. alt., (No. 2211), et arl 7000 ped. alt., leg. T. KawaKam et U. Mori, Oct. 1906, (No. 2212).

Distrib. An endemic plant. 


\section{Origanum LiNs.}

Origanum vulgare Lnx. Sp. Pl. ed.-2, p. 824 ; Bextrr. in DC. Prodr. XII. p. 193 (varietates); Hance, in Journ. Bot. (1880) p. 300 ; Fraxchet, P1. David. p. 235 ; Hoor. f. Fl. Brit. Ind. IV. p. 648; Fonbes et HexsL. Ind. Fl. Sin. II. p. 282 ; DieLs, Fl. Centr. Chin. p. 559.

Origanum heracleoticum et 0 . creticum Lour. Fl. Cochmeh, ed-.WmLd. p. 453.

HAB. Ganzan, in montibus Morrison, ad 9141 ped. alt., leg. S. Nagasaw, Oct. 1905, (No. 632); in monte Morrison, ad 8000 ped. alt., (No. 1957), et eodem monte, ad 11000 ped. alt., (No. 2292), leg. 'T. Kawakani et U. Mori.

Distrib. Central China to Manchuria; from North Africa to West Europe.

This species has dimorphic flowers; the larger flowers are bisexual, and the smaller ones are female which are one third long: as the perfect flowers.

\section{Salvia Lins.}

Salvia scapiformis HAYce, in Jouru. Bot. (1885) p. 368; Pot. Mag. t. 6980 ; Fonizes et Hexrst. Ind. Fl. Sin. II. p. 287 ; Hexriy, List Pl. Formos. p. 73 ; Dress, Fl. Centr. Chin. p. 559.

Hab. Taitō: Dakunsha, leg. T. Kawakanit et U. Mori, Dec. 1906, (No. 1934).

var. pinnata Hayata, in Matsurr. et Hayata, Enum. Pl. Tormos. p. 312, t. 17 .

HAB. Suizan, in monte Morrison, ad 7702 ped. alt., leg. S. Nagasawa, Oct. 1905, (No. 663); in montibus centralibus, ad 9000 ped. alt., (No. 2194), et Toroku : Gunkei, (No. 1820), leg. T. KawAKami et U. Moni, Nov. 1906.

forma hirsuta. caules basi subglabri, inflorescentix hirsutx. Folia hirsuta. 
Hab. Tōzan, in montibus Morrison, leg. G. Nakahara, Nov. 1906 ; 'Taitō: Shūkoranzan, leg. T. Kawarani et U. Mori, Nor. 1906, (No. 1884).

forma gracilis. Folia minora, foliolis rhomboideis acutis dentatis, dentibus acutis vel obtusis.

HAB. Tōzan, et Arizan, in montibus Morrison, leg. G. NAKAHARA, Oct. 1906 ; in monte Morrison, ad 8000 ped. alt., leg. T. KAWAKAMI et U. MorI, Oct. 1906, (No. 2050).

Distrib. Type: Central China.

\section{Scutellaria LinN.}

Scutellaria luzonica Roufe, in Journ. Linn. Soc. XXI. p. 315 ; Fonbes et Hensr. Ind. Fl. Sin. II. p. 296 ; Henry, List Pl. Formos. p. 73 ; Mratsus. et Hayata, Enum. Pl. Formos. p. 314.

HAB. in montibus centralibus, ad 7000 ped. alt., leg. T. $K_{A}-$ WAKaMi et U. Mori, Nov. 1906, (No. 1862).

Distrib. The Philippine islands.

Anisomeles R. Br.

Anisomeles ovata R. Br. AIr. Hort. Kew. ed.-2. II. p. 364 ; Mir. Fl. Ind. Bat. II. p. 975 ; ВеNтH. in DC. Prodr. XII. p. 455, et Fl. Hongk. p. 278; Hoor. f. Fl. Brit. Ind. IV. p. 672 ; Fonbes et Hexss. Ind. Fl. Sin. II. p. 299 ; Hexry, List Pl. Formos. p. 73 ; Matsurr. et Hayat., Enmm. Pl. Formos. p. 315.

Hab. Tappansha, leg. T. Kawakami et U. Mori, Oct. 1906, (No. 1770).

Distrib. South China, tropical and subtropical India.

\section{Leucas R. BR.}

Leucas javanica I3EXTIr. in DC. Prodr. XII. p. 528 ; Fonmes et Hexist. Ind. Fl. Sin. II. p. 304 ; Matsum. et HaYata, Enum. Pl. Formos. p. 317.

Hав. Taitō: Hinansha, leg. T. Kawaknзi et U. Mori, Dec. 1906 , (No. 1835).

Distrib. Java and the Philippines. 


\section{Dicotyledones}

\section{Monochlamydeæ}

\section{Polygonaceæ}

\section{Polygonum LinN.}

Polygonum biconvexum Hayata, sp. nov. Herbæ graciles decumbentes $15 \mathrm{~cm} .-20 \mathrm{~cm}$. altæ inæqualiter angulatæ retrorsum aculeatæ. Folia alterna hastato-triloba $3 \mathrm{~cm}$. longa basi $2.5 \mathrm{~cm}$. lata apice caudato-acuminata pilis setulosis atque pilis stellatis brevissimis sparce tecta margine integra dense setulosis, petiolis lamina 2-plo brevioribus aculeolatis, ochreis truncatis hirsutis ciliatis. Flores ignoti. Achænia biconvexa, ovata $3 \frac{1}{2} \mathrm{~mm}$. longa $2 \frac{1}{2} \mathrm{~mm}$. lata lævia.

HaB. in monte Morrison, leg. G. NaKahara, 1905.

This is very near $P$. Thunbergii S. et $Z$. ; differs from it in having biconvex seeds.

Polygonum chinense LnN. Sp. Pl. ed.-2, p. 520 ; MeIss. in DC. Prodr. XIV. p. 130 ; MIQ. Fl. Ind. Bat. I:-1, p. 1008 ; LINN. Fl. Cochinch. ed.-WILld. p. 297 ; Hoor. f. Fl. Brit. Ind. V. p. 44 ; BENтн. Fl. Hongk. p. 289 ; Roxb. Fl. Ind. ed.-Carey, p. 289 ; Bot. Mag. t. 5238 ; Wight. Ic. Pl. Ind. or. t. 1806 ; Ноoк. et ArN. Bot. Beech. Voy. p. 208 ; Franch. et Savat. Enum. Pl. Formos. I. p. 398, et II. p. 480 ; Fonbes et HersL. Ind. Fl. Sin. II. p. 335 ; Diels, Fl. Centr. Chin. p. 314 ; Matsuxr. et Hayata, Enum. Pl. Formos. p. 334.

Polygonum sinense Hook. et ArN. Bot. Beech. Voy. p. 269.

HAB. Suizan, in montibus Morrison, ad 7702 ped. alt., leg. S. 
Nagasawa, Oct. 1905, (No. 653); in monte Morrison, ad 7500 ped. alt., leg. T. Kawamami et U. Mori, Oct. 1906, (No. 1789).

Distris. Japan, south central China, the Philippines, India throughout, and Malay to Ceylon.

Polygonum cuspidatum SIEB. et Zucc. Fl. Jap. Fam. Nat. n. 731 ; Metsr. in DC. Prodr. XIV. p. 136 ; Franch. et Savat. Enum. Pl. Jap. I. p. 402, et II. p. 481; S. Moore, in Journ. Bot. (1875) p. 231 ; Franchet, Pl. David. p. 256 ; Bot. Mag. t. 6503 ; Forbes. et HeMsi. Ind. Fl. Sin. II. p. 336.

HAB. Suizan, in monte Morrison, ad 11707 ped. alt., leg. S. Nagasawa, Nov. 1905, (No. 575); in monte Morrison, ad 8000 pecl. alt., leg. T. Kawakani et U. Moni, Oct. 1906, (No. 2071).

Distrib. Japan and North China.

Polygonum minutum Hayata, sp. nov. (PI. XXX.). Herbæ perennes? minutæ scandentes ramosæ, caulibus glabratis. Folia parva ovato-triangularia $4 \frac{1}{2} \mathrm{~mm}$. longa $4 \mathrm{~mm}$. lata apice acuta vel obtusa utraque pagine breve setulosa integra, petiolis $1 \mathrm{~mm}$.$2 \mathrm{~mm}$. longis, ochreis $1 \frac{1}{2} \mathrm{~mm}$. longis setulosis laceratis. Flores 3-4 conferti, pedicellis $1 \mathrm{~mm}$. longis, bracteolis hyalinis connatis 2-3-fidis. Perianthium late campanulatum $1 \frac{1}{3} \mathrm{~mm}$. latum tubo brevissimo, limbo 5 lobato, lobis oratis obtusis glandulis sparce punctatis, discis basi perianthii connatis. Stamina 8 subrqualia. Achænia ovato-trigona ad angulas prominente costata $2 \frac{1}{2} \mathrm{~mm}$. longa $1 \frac{1}{2} \mathrm{~mm}$. lata, styli ramis brevissimis.

$\mathrm{H}_{\mathrm{AB}}$, in monte Morrison, ad 12000 ped. alt., leg. 'T. KawamaxiI et U. Mori, Oct. 1906, (No. 2267).

Polygonum morrisonense ILarat, sp. nov. (PI. XXXI.). Herha annuæ ?' caulibus erectis vel adscendentibus panciramosis ad nodos 
retrorsum pilosis cxterum glabris, ochreis pilosis truncatis $7 \mathrm{~mm}$. longis. Folia obcuneata $4 \mathrm{~cm}$. longa $2 \mathrm{~cm}$. lata apice cuneatoacuminata basi abrupte angusta ad petiolum $1 \mathrm{~cm}$. longum attenuata rarius infra medium profunde sinuata, folia superiora subsessilia obcuneato-cordata, margine albo-lamellata sursum integra deorsum ciliata utraque pagine glandulis subpellucidis punctata. Flores dense spicato-capitati, capitulis suboroideis $8 \mathrm{~mm}$. longis $6 \mathrm{~mm}$. latis ad apices ramorum vel axillas foliorum superiorum solitariis pedunculatis, pedunculis $1 \mathrm{~cm}$. longis apice glanduloso-hispidulis, bracteis scariosis ovato-acutis $3 \frac{1}{2} \mathrm{~mm}$. longis $2 \mathrm{~mm}$. latis. Flores 2 ad axillas bractearum siti, bracteolis subconnatis hyalinis, pedicellis brevissimis. Perianthium urceolatocampanulatum $4 \mathrm{~mm}$. longum corollinum, 5-lobum segmentis subæqualibus oblongis $2 \frac{1}{2} \mathrm{~mm}$. longis. Stamina 8 , inæqualia; 3 longiora prope basin perianthii affixa, filamentis basi utrinque glandulosis; 5 breviora tubo perianthii affixa, filamentis basi non-g]andulosis. Styli rami 3. Achænia ovato-globosa subtrigona vix $2 \mathrm{~mm}$. longa.

HaB. in monte Morrison, ad 10000 ped. alt., leg. T. Kawakami et U. Mori, Oct. 1906, (No. 2074).

Somewhat near $P$. alatum Ham. ; but differs from it in laving no involucral leaf; from $P$. miorocephalum Dos, in having no auricled leaf.

Polygonum Posumbu Hax.; MIQ. Fl. Ind. Bat. I. p. 1000 ; Hoor. f. Fl. Brit. Ind. V. p. 38 ; Franchet. Pl. David. p. 258; Franch. et Savat. Euum. Pl. Jap. I. p. 394, et II. p. 473; Fonbes et Henss. Ind. Fl. Sin. II. p. 345 ; Henny, List Pl. Formos. p. 76. Diets, Fl. Centr. Chin. p. 312 ; Mitsuni. et Hayata, Enum. Pl. Formos. p. 340.

$\mathrm{H}_{\mathrm{AB}}$, in monte Morrison, ad 8500 ped. alt., leg. T. KatwaKamI et U. Morr, Nov. 1906, (No. 1872). 
Distrib. Japan and China; Java to East. Himalaya and Khasia.

\section{Aristolochiaceæ.}

\section{Asarum Lins.}

Asarum macranthum Hoor. f. in Bot. Mag. t. 7022; Heirst. in Gard. Chron. 3rd. Ser. VII. p. 421; Fonbes et Hexss. Ind. Fl. Sin. II. p. 359 ; Matsum. et Hayata, Enum. Pl. Formos. p. 343.

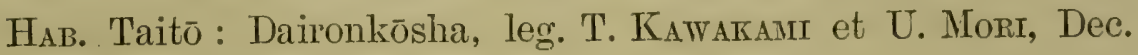
1906, (No. 2186).

Distrib. An endemic plant.

\section{Piperaceæ.}

\section{Piper Linn.}

Piper Futokadsura StEb. et Zucc. Fl. Jap. Fim. Nat. II. p. 2:31, џ. 811; MiQ. in Ann. Mus. Bot. Lugd.-Bat. III. p. 139 ; C.DC. in DC. Prodr. XVI-1, p. 346 ; Fraxch. et Savat. Enum. Pl. Jap. I. p. 443 ; Maxno. in Mél. Biol. XII. p. 532 ; Fonbes et Hesist. Ind. Fl. Sin. I. p. 365 ; Hexry, List Pl. Formos. p. 77 ; Matsun. et Hayata, Enum. Pl. Formos. p. 346.

Haв. Taitō: Tōkeisha, leg. T. Kawakani et U. Morr, Dec. 1906, (No. 1910).

Distrib. Japan.

\section{Peperomia R. et PAY.}

Peperomia dindygulensis MIO.; C.1)C. in 1)C. Proll. XTI.-1, p. 442; Ноок. f. Fl. Brit Ind. V. p. 98; WIGrrT, Ic. Pl. Ind. or. t. 1921; Fonbes et Heust. Ind. Fl. Sin. II. p. 366 ; Hexry, Iist Pl. Formos. p. 77 ; Mratsuar. et Hayata, Enum. Pl. Formos. p. 346. 
Hab. Suizan, in montibus Morrison, leg. S. Nagasawa, Oct. 1905, (No. 558); in monte Morrison, ad 6000 ped. alt., leg. T. Katwakani et U. Mori, Oct. 1906, (No. 2045).

Disiris. South China, and the western peninsula of India.

Peperomia Nakaharai HayatA, sp. nor. (XXXII.). Herbæ perennes ramosissimæ glabræ procumbentes, ramis ascendentibus 8-9 cm. longis glaberrimis. Folia opposita rarius $3-4$ verticillata cuneato-obovata apice profunde emarginata basi obtusa, circ. $1 \mathrm{~cm}$. longa $6 \mathrm{~mm}$. lata integerrima crassiuscula obscure marginata subtus pallidiora. Flores o spicati, spicis clavatim incrassatis $1 \frac{1}{2}$ $\mathrm{cm}$. longis. Ovarium sessile ovoideum, bracteis peltatis glandulosopunctatis.

Hab. in monte Arizan, in montibus Morrison, leg. G. NaKaHara, Nov. 1906 ; in monte Morrison, ad 8000 ped. alt., T. KawAKAMII et U. Mori, Oct. 1906, (No. 1997).

Peperomia reflexa A. Dietr. ; Miq. Fl. Ind. Bat. I.-2, p. 436 ; Wrair, Ic. Pl. Ind. or. t. 1923 ; Hook. f. Fl. Brit. Ind. V. p. 99 ; DC. Prodl. XVI.-1, p. 451; Fonbes et Henst. Ind. Fl. Sin. II. p. 366 ; Matsunr. et Hayats, Enum. Pl. Formos. p. 347.

Haв. in monte Morrison, ad 6000 ped. alt, leg. T. KawakanI et U. Mori, Oct. 1906, (No. 2033).

Distrib. Subtropical Asia, Africa, America, and Australia, Sonth China.

\section{Chloranthaceæ.}

Chloranthus Sw.

Chlorantus serratus Roexr. et Schult.; Soldrs, in DC. Prodr. XVI.-1. p. 475 ; MiQ. Fl. Ind. Bat. I.-1. p. 802 ; Fravien. et SAVAT. Enum. 
Pl. Jap. I. p. 444'; Fonbes et Hexrst. Ind. Fl. Sin. II. p. 369 ; Diets, Fl. Centr. Chin. p. 273 ; Matsum. et Hayata, Enum. Pl. Formos. p. 348.

Нав. Kagi : Kōdenshō, leg. T. Kawakanir et U. Mori, Oct. 1906 , (No. 1774).

Distrib. Japan and central China.

\section{Laurineæ.}

Cinnamomum Burnan.

Cinnamomum Camphora Nees et Eberar.; Meisx. in DC. Prodr. XV.-1. p. 24 ; Franch. et SAVAT. Enum. PI. Jap. I. p. 411; MIQ. Fl. Ind. Bat. I.-1, p. 892 ; Forees et Hemsi. Ind. Fl. Sin. II. p. 371 ; Diets, Fl. Centr. Chin. p. 347 ; Matsuar. et Hayata, Enum. Pl. Formos. p. 349.

Laurus Campliora Lins. Sp. Pl. ed.-2, p. 528; Thuns. Fl. Jap. p. 172. $\mathrm{H}_{\mathrm{AB}}$. in montibus Morrison.

Distrib. Japan and China.

\section{Proteaceæ.}

\section{Helicia Lour.}

Helicia formosana Hersir. in Am. Bot. IX. p. 1nth, et in Fonises et Henss. Ind. Fl. Sin. II. p. 394 ; Henry, List Pl. Formos. p. 80 ; Matsuar, et Hayata, Enum. Pl. Formos. p. 354.

forma subintegra, foliis subintegris.

Haв. Kōshun ; Botansha, leg. G. NakiHara, 1905.

forma acuminata, foliis apice abrupte acuminatis.

HAB. Nantō: Shichikwaikutsuzan, leg. N. KonısHI, (No. 39); in monte Momison, ad 6500 ped. alt., leg. 'T. Kiwakani et U. Mom, Oct. 1906, (No. 2013).

Distrib. An endemic plant. 


\section{Thymelæaceæ.}

\section{Daphene Lins.}

Daphne Championi Bexth. Fl. Hongk. p. 296 ; Forbes et Heyst. Ind. Fl. Sin. II. p. 395 ; Matsuxr. et. Hayata, Enum. Pl. Formos. p. 355. Hab. Taitō: Shinrōzan, leg. T. Kawakani et U. Mori, Deç. 1906, (No. 1836).

Distrib. Hongkong.

Stellera Lins.

Stellera Chamæjasme LrNv.; Meisn. in DC. Prodr. XTV. p. 549; MLAxחr. Ind. Fl. Pels. in Prim. Fl. Ammr. p. 476 ; Hoor. f. Fl. Brit. Ind. V. p. 196 ; "Ledeb. Te. Pl. Ross. t. 374"; Fonbes et Hexsst. Ind. Fl. Sin. II. p. 401.

HAB. in monte Morrison, ad 8000 ped. alt., Oct. 1906, (No. 1708), et eodem monte, ad 7500 ped. alt., (No. 1999), leg. T. KawAKaMI et U. Moni.

Distrib. Caucasus through central Asia eastward to North India, Malay, and central China.

\section{Elæagnaceæ.}

\section{Elaragnes Lins.}

Elæagnus umbellata Thund. Fl. Jap. p. 66, t. 14; Schlecht. in DC. Prodr. XIV. p. 614 ; Maxtr. in MIél. Biol. VII. p. 559; Hook. f. Fl. Brit Ind. V. p. 201 ; S. Moonie, in Journ. Bot. (1878), p. 138; Fories et Hexss. Ind. Fl. Sin. II. p. 404.

Has. Ganzan, in montibus Morrison, ad 9141 ped. alt., leg. S. Nagasatwa, Oct. 1905, (No. 675) ; Arizan et Tōzan, in isdem montibus, leg. G. Nanahara, Oct. 1906; in monte Morrison, ad 9000 
ped. alt., leg. T. Kawakami et U. Mori, Oct. 1906, (Nos. 1952 et 1891); in montibus centralibus, ad 10000 ped. alt., leg. T. KawdKAMI et U. Mori, Nov. 1906, (No. 1856).

Distrib. China to Japan, North India westward to Afghanistan.

\section{Loranthacee.}

\section{Loranthus LiNs.}

Loranthus Yadoriki Sreb. in SIEb. et Zucc.'Fl. Jap. Fam. Nat. n. 398 ; Franch. et Savat. Enum. Pl. Jap. II. p. 481; Forbes et Hemst. Ind. Fl. Sin. II. p. 407 ; Maxır. in Mél. Biol. IX. p. 609 ; Henrr, List Pl. Formos. p. 80 ; Diels, Fl. Centr. Chin. p. 305 ; Matsumr. et Hayata, Euum. Pl. Formos. p. 357.

HAB. Tōzan, in montibus Morrison, leg. G. Nakahara, Oct. 1906 ; in monte Morrison, ad 7500 ped. alt., (Nos. 2038, 1786 et 2007), leg. T. Kawakairi et U. Mori, Oct. 1906.

DistrIb. Japan and south central China.

Loranthus: Owatarii Hayata, in Matsuir. et Hayatı, Enum. Pl. Formos. p. 357.

HaB. in monte Morrison, ad 8000 ped. alt., leg. 'T. Kawakani et U. Mori, Oct. 1906, (No. 1948); in montibus centralibus, ad 8000 ped. alt., leg. T. Kawakaji et U. Mori, Nov. 1906, (No. 1865).

\section{Viscum LINN.}

Viscum articulatum Btria.; Mrę. Iil. Int. Jat. I.-1, p. 806; 1)C. Prodr. IV. p. 284; Hoor. f. Fl. Brit. Ind. V. p. 226 ; Fonses et Hexss. Ind. Fl. Sin. II. p. 407 ; Hexriy, List Pl. Formos. p. 81; Diers, Fl. Centr. Chin. p. 305 ; Matsux. et Hayata, Enum Pl. Formos. p. 358.

HAB. Suizan, in montibus Morrison, ad 7700 ped. alt., leg. S. 
Nagasatwa, Oct. 1905, (No. 751); in monte Morrison, ad 7000 ped. alt., leg. T. KawaKani et U. Mori, Oct. 1906, (No. 2014).

Distrib. Widely spread in India and Malay.

Viscum orientale WnLd. var. multinerve HAY.ıT, in Tōkyō Rot. Mag. XX. p. 72.

Hab. Jitsugetsutan, leg. G. Nakahara, Aug. 1905.

Distrib. Type: Java.

\section{Balanophoreæ.}

\section{Balanophora Forst.}

Balanophora spicata Hayıtı, sp. nor. (Pl. xxxIII). Planta 우 gracilis $7 \mathrm{~cm}$. alta. Pedunculus per partem inferiorem bracteatus, bracteis sæpius suboppositis. Inflorescentia ovata $1 \frac{1}{2} \mathrm{~cm}$. longa. Flores of brevissime stipitati circa stipites graciles spadicellorum capitatorum apiculatorum dispositi, stylis gracillimis quam ovario circ. 2-plo longioribus. Planta $\hat{\delta}$ graciliuscula circ. $14 \mathrm{~cm}$. alta. Pedunculus infra medium bracteatus, bracteis sub-oppositis. Inflorescentia incrassata circ. $5 \mathrm{~cm}$. longa. Flores $\hat{\jmath}$ sessiles laxiuscule spicati. Perianthium crassiusculum, segmentis 6 inæqualibus, postice et antice latissimis apice truncatis, lateralibus ovatis obtusis minoribus. Antherarum loculi circ. 15, 2-seriatim dispositi.

Hab. Kishirei, ad 4000 ped. alt., leg. S. Nagasawa, Nov. 1905.

The present plant is very like $B$. laxifora Henss. in its halit; but differs from it in having multi-locular anthers and in the segments of perianths.

Balanophora parvior HAYaT, sp. nov. (Pl. XxxIT). Planta 우 parvior $7 \mathrm{~cm}$. alta. Pedunculus per totam fere longitudinem lracteatus, bracteis oratis. Inflorescentia oblongo-cylindracea $3 \frac{1}{2}$ 
cm. longa. Flores o o brevissime stipitati, circa stipites graciles spadicellorum clavato-capitatorum dispositi, stylis gracillimis quam ovario 3-plo longioribus. Planta $\hat{\delta}$ gracilis cire. $14 \mathrm{~cm}$ alta. Pedunculus fere per totam partem bracteatus, bracteis suboppositis. Inflorescentia incrassata circ. $4 \mathrm{~cm}$. longa. Flores $\hat{\delta}$ sessiles, laxiuscule spicati. Perianthium crassiusculum, segmentis 6 inæqualibus, postice et antice majoribus ovatis truncatis, lateralibus parvioribus, oblongo-ovatis acutis. Antherarum loculi circ. 20, irregulariter dispositi.

Haв. in monte Morrison, ad 6000 ped. alt., leg. T. KawaKam et U. Mori, Oct. 1906, (No. 2227).

This differs from the preceding species in the shape of the segments and the cells of the authers.

\section{Euphorbiaceæ.}

\section{Buxus LinN.}

Buxus sempervirens LinN.; MUdeld. Arit. in DC. Mrodr. XTI.-I, p. 18; Hook. f. Fl. Bxit. Ind. V. p. 267 ; Bextr. Fl. Hongk. p. 315, (excl. syn. B. sinensis Lrnr.); Forbes et Herrsu. Ind. Fl. Sin. II. p. 418.

Buxus japonica MuedL. Arg. in DC. Prodr. XVI.-1. p. 20 ; Francr. et Sayat. Enum. Pl. Jap. I. p. 428.

Buxus sempervirens LnNo var. japonica Maкino, in Tōkyō Bot. Mag. IX. p. 281, et XV. p. 169 ; HaYatA, Revis. Euphorb. et Bux. Jap. in Journ. Scie. Coll. XX.-3. p. S2.

Haв. loco non indicata.

Distris. This plant extends from Japan and China through North Africa and Europe to the Canary islands.

The plant presents a great range of variation. Although my plant does not exactly agree with this species, still I think it may be regarded as a form of it. 
Glochidion Fonst.

Glochidion formosanum HAYatı, in Journ. Scie. Coll. XX.-3. p. 201, t. 2, G. ; Matsum. et Hayata, Enum. Pl. Formos. p. 360.

НАв. loco non indicato.

Glochidion zeylanicum A. Juss.; MUtzr. AnG. in Linnæea, XXXII. p. 60, et in DC. Prodr. XV.-2. p. 281; Hoor. f. Fl. Brit. Ind. V. p. 311 Matsum. et Hayata, Enum. Pl. Formos. p. 360.

Distrib. Malay archipelago and Deccan Peninsula.

\section{Alemites Fonst.}

Aleurites cordata Steud. M Metr. Arig. in DC. Protr. XV.-2, p. 724 ; Fonbes et Henss. Ind. Fl. Sin. II. p. 433 ; Diets, Fl. Centr. Chin. p. 430 ; Hayata, in Journ. Scie. Coll. XX.-3, p. 55 ; Matsum. et Hayata, Enum. Pl. Formos. p. 366.

Hab. Kagi : Tappansha, leg. T. Kawakani et U. Mori, Oct. 1906, (No. 1757).

Distrib. Japan and south central China.

\section{Mercurialis Lrsy.}

Mercurialis leiocarpa Steb. et Zucc. Fl. Jap. Fam. Nat. I. p. 145, u. 78; Muelt. Arg, in DC. Prodr. XV.-2, p. 795 ; MrQ. Prol. Fl. Jap. p. 291 ; Franch. et Savat. Enum. Pl. Jap. I. p. 425 ; Fonbes et Hexisl. Ind. Fl. Sin. II. p. 436 ; DirL, Fl, Centr. Chin. p. 428 ; P.utbin, Conspect. Fl. Koreæ, II. p. 43 ; Huyata, in Journ. Scie. Coll. XX.-3, p. 38, t. 3, D ; Matsum. et Hayata, Enum. Pl. Formos. p. 363.

Hab. Tōzan, in montibus Morrison, leg. G. NaKahara, Oct. 1906 ; in monte Morrison, ad 7000 ped. alt., leg. T. Kawaram et U. Moni, Oct. 1906, (No. 1716).

Distrib. Japan and south central China. 
Mallotess Tour.

Mallotus cochinchinensis Lour. Fl. Cochinch. el.-Wחis. p. 635 ; Mueti. Arg. in Linnea, XXXIV. p. 189; Hoor. f. Fl. Brit. Ind. V. p. 430 ; Forbes et Henst. Ind. Tl. Sin. II. p. 439 ; Henry, List Pl. Formos. p. 84; Hiyte, in Journ. Scie. Coll. XX.-3, p. 45, t. 3, J ; Matsur. et Huyata, Enum. Pl. Formos. p. 364.

HAв. Tappansha, ad 3138 ped. alt., leg. S. Nıgısawı, Oct. 1906 , (No. 713).

Distrib. South China, Malay peninsula and archipelago.

\section{Urticaceæ.}

\section{Fatoua G.1UD.}

Fatoua pilosa Gavd. ; Bureat, in DC. Prolx. XVII. p. 256 ; Fravor. et Savit. Enum. Pl. Jap. I. p. 434 ; Fondes et Henst. Ind. Fl. Sin. II. p. 454.

Fatous japonica Budne, in Muss. Bot. Lngd.-Bat. II.-1, p. 38 ; Hance, in Journ. Bot. (1878) p. 232.

HAB. in monte Morrison, ad 9000 ped. alt., leg. 'T. KawaKanI et U. Morr, Oct. 1906, (No. 2065).

Distrib. Japan, South China, and Malay archipelago.

\section{Momes Lins.}

Morus alba Linv. Sp. Pl. ed.-2. p. 1398; BunEut, in DC. Prodl.

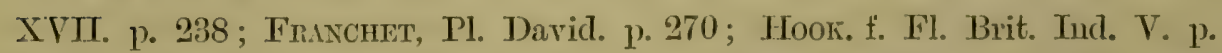
492 ; Fonbes. et Henss. Ind. Fl. Sin. II. p. 455 ; Drets, Fl. Centr. Chin. p. 297 ; Pajibin, Conspect. Fl. Koree, II. p. 45 ; Mitsuar. et Hiyıti, Enum. Pl. Formos. p. 373.

Haв. Tappansha, leg. T. Kaw.shain et U. Mori, Oet. 1906, (No. 1778) ; Taichū : Kashigatani, leg. G. NaкuHuR., Feb. 1907.

Distrib. China and Japan; temperate and subtropical Asia. 


\section{:Ficus LinN.}

The plants belonging to this genus are very few in the flora of the hilly regions. The specimens brought to me are all wanting of receptacles and therefore they are not determinable.

\section{Urtica Lins.}

Urtica Thunbergiana Sпвв. in Siez. et Zicc. Fl. Jap. Fam. Nat. II. p. 21.4, n. 756 ; DC. Prodr. XVI.-1. p. 55 ; Fonbes et Henss. Ind. Fl. Sin. II. p. 472; Diets, Fl. Centr. Chin. p. 301 ; Matsur. et Hayata, Enum. Pl. Formos. p. 390.

Urtica dioica Thuxb. Fl. Jap. p. 69.

HaB. Tappansha, leg. T. KawakanI et U. Moni, Oct. 1906, (No. 1747).

Distrib. Japan and central China.

\section{Girardinia GAUD.}

Girardinia heterophylla Dcxi. ; DC. Prodr. XVT.-1, p. 100; Buture, Mrrs. Bot. Lugd.-Bat. II. p. 158; Hoor. f. Fl. Brit. Ind. V. p. 550.

* The following Ficus was found on the sea shore in the island of Kōto. As the plant is very remarkable for the smallest size of receptacles and the full description of the species was not previously made, I take this occasion to give the following description of the plant.

Ficus vaccinioides Herss, et KnNG. (Pl. XXXV.), in Ann. Bot. Gard. Calc. I,-2, p. 126, t. 159, A; Folibes et Henrse. Ind. Fl. Sin. II. p. 468; Matsun. et Hainata, Enum. Pl. Formos. p. 379. Suffrutices. Rami reptuntes vel scandentes graciles ferrugineo-tomentoso-pubescentes remote foliati teretes. Folia subrhombea vel obovata obtusa vel acuta basi rotunda vel subcordata $1 \frac{1}{5}-2 \mathrm{~cm}$. longa $1 \mathrm{~cm} .-8 \mathrm{~mm}$. lata 1 -nervia, venis primariis lateralibus utrinqne $3-4 \mathrm{sub}$ angulo $60^{\circ}$ egressis, venulis reticulatis inconspicpis, margine minute serrulata, petiolis $2-3 \mathrm{~mm}$. longis, stipulis subulatis 1-nerviis caducis scariosis ferrugineis $3 \mathrm{~mm}$. longis. Receptncula axillaria solitnria breve stipitata globosa apice leviter contracta basi rotundata rarius leviter attenuata hirsuta, ore rotundo concavo extus bracteis latioribus clausis et intus bracteis triangularibus vel lanceolatis instructo, basi bracteis 3-4 late oratis acutis. Fl. $\delta$ : sessiles, perianthia 4-5 partita, segmentis linenribus, purpureo-fuscentibus; stamina $3-\mathbf{4}$, flamentis brevioribus. Fl. galliferi srepe longe peciicellati ; ovarium stipitatum. Fl. $\&$ : breve pedicellati ; perianthia 4-6-partita, segmentis purpureo-fuscentibus, subulato-linearibus; ovarium obovoideum basi contractum, stylo laternli filiformi.

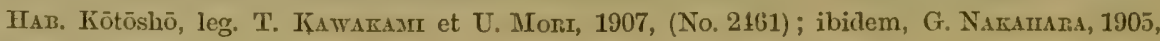
(No. 974)

Distrib. An endemic, and very local plant.

'This curious and beautiful Ficus was found creeping on the walls or rocks along the sea shore. It is very remarkable for its smallest form of the receptacles. Gall and male flowers are found mixed in one receptacle, while female flowers are in a different receptrcle. 
Urtica heterophygla V.hL; WIGHT. Ic. Pl. Ind. or. t. 687.

Girardinice palmata GaUd. ; Fonbes et Hexss. Ind. Fl. Sin. II. p. 475.

Haв. Tappansha, leg. T. Kawaкuп et U. Мoni, Oct. 1906, (No. 1769).

Distrib. Java, Ceylon, and Himalaya.

\section{Pilea Linde.}

Pilea stipulosa MIQ.; Hoor. f. Fl. Brit. Ind. V. p. 555 ; Miq. F1. Ind. Bat. I.-2, p. 236 ; Fonbes et Hearst. Ind. Fl. Sin. II. p. 478; Deels, Fl. Centr. Chin. p. 303 ; Matsusr. et Hayata, Enum. Pl. Formos. p. 383.

Pilea angulata Buune, Mris. Bot Lugd.-Bat. $\Pi$. p. 55; WEDD. in DC. Prołr. XVI.-1, p. 131 ; Maxmr. in Mél. Biol. TX. p. 631.

НАв. in monte Morrison, ad 8000 ped. alt., leg. T. Kawıinain et U. Moni, Oct. 1906, (No. 1985).

Distrib. Central China, North India, Ceylon, and Java.

Pilea Wattersii IIINce, in Jouru. Bot. (1885) 1). :327 ; Fonbes et HIxrst. Ind. Fl. Sin. II. p. 479 ; IIAtsun. et HAYati, Enum. Pl. Formos. p. 383.

Нів. Tappansha, ad 3108 ped. alt., (No. 590), et Sanchōki, ad 4000 ped. alt., leg. S. Nagasawa, Oct. 1905.

Dis'rrib. An endemic plant.

\section{Leccuthus WEDD.}

Lecanthus Wightii Wedn.; Mro. Fl. Ind. 13at. I.-2, p. 2:38; J Iook. f. Fl. B3rit. Ind. V. p. 559 ; Foribes et HexsL. Ind. Fl. Sin. II. p. 480.

Lecanthas peduncularis Wedv. in DC. Prodr. XVI.-1, p. 164.

Lecanthus Wallichii WrDD. in DC. Protr. XVI.-1, p. 164; Fondes et Hexrst. Ind. Fl. Sin. II. p. 480.

Lecentlues major WEDD. in DC. Prodr. XVI.-1, p. 164.

Elutostemc ovctum. WiGir, Ic. PI. Ind. or. t. 1985.

Hab. Arizan, leg. G. Nakamara, Nov. 1906. 
Distrib. Central China: Szechuen and Yumnan. The Himalayas, Java, and Tropical Africa.

Very small form of the specis.

\section{Elatostema Fonst.}

Elatostema minutum Hмiт, sp. nov. (Pl. XXXVI.). Неrba parvissima, canlilus decumbentibus basi ad nodos radicantibus tomentosis: Folia alterna obovata $14 \mathrm{~mm}$. longa $7 \mathrm{~mm}$. lata ralde obliqua apice obtusa basi inferiore latere rotundata superiore latere angusta, petiolis 1-2 mm. longis, utraque pagina pilis minutissimis dense atque pilis setulosis sparce obtecta margine serrata, serraturis acutis setulosis, viridissima, stipulis lanceolatis. Flores in receptaculo campanulato fasciculati. Receptacula in axillis foliorum solitaria sessilia, bracteis parvis. Achænia ovata minuta punctata.

HAB, in monte Morrison, ad 7500 ped. alt., leg. T. KAwakamI et U. Моri, Oct. 1906, (No. 1986).

Elatostema sessile Fonst.; WEDD, in DC. Prodr. XVI.-1, p. 172; Hoor. f. Fl. Brit. Ind. V. p. 563 ; FonBes et Hemst. Ind. F1. Sin. II. p. 483. Procris sessilis Hook. et Anx. Bot. Beech. Voy. p. 70.

var. cuspidatum WEDD. in DC. Prodr. XVI.-1, p. 173; Hook. f. FI. Brit. Ind. V. p. 564 ; Maxrn. in Mél. Biol. IX. p. 634; Fraxch. et Satat. Enum. Pl. Jap. II. p. 495.

HAB. in monte Morrison, ad 7000 ped. alt., leg. T. KaWAKAMII et U. Mori, Oct. 1906, (No. 1987).

Distrib. Type : central China through Japan to the Pacific islands and also in Malar.

\section{Procris Juss.}

Procris lævigata L1,ME; MI?. FI. Ind. 13it. I.-2, p. 248 ; WEDD. in DC: Prodr. XVI.-1, p. 192 ; Hook. f. Fl. Brit. Ind. V. p. 575 ; Fonbes et Hexst. 
Ind. Fl. Sin. II. p. 484 ; Ноок. Ic. P1. Ind. or. t. 1295 ; Matsum. et HaYatu, Enum. Pl. Formos. p. 385.

Hab. Tappansha, ad 3138 ped. alt., leg. S. Nagasawa, Oct. 1905. (No. 591).

Distrib. South China, India throughout, and extending from Java to the Comoro islands.

Juglandaceæ.

Tuglaus LINN.

Juglans sp.

НАв. Tōhosha, ad 2930 ped. alt., leg. S. Nagasawı, Nov. 1905 , (No. 604).

\section{Engelhavatia LESCH.}

Engelhardtia spicata Brure, Fl. Jav. Juglumd. p. 8, t. 1, et t. 5, A ; DC. Prodr. XVI-2. p. 140 ; Mre. Fl. Ind. Bat. I.-1. p. 842 ; Hoor. f. Til. Brit. Ind. V. p. 595 ; Mennпl, in Philipp. Journ. Scie. I. Supp. Bot. p. 41.

var. formosana Harats, n. v. Bracter fructiferx parviores 2.1 cm. longx 3 -lobatx, lobis lateralibus $1 \frac{1}{2} \mathrm{~cm}$. longis, terminale $2 \frac{1}{2} \mathrm{~cm}$. longo lineari-oblongo, scariosis reticulatis.

Hab. Kusshaku, (Nov. 6), et Bunsanho, (No. 25), leg. N. Konishi, Sept. 1902 ; Giran : Chūrei, leg. T. Kawakam et U. Monx, Juni. 1906, (No. 1359), Nantō; Nankōkei, leğ. 'T. KAwAK.ın et U. Morr, Aug. 1906, (No. 1173).

Distrib. Type: Java, Cochinchina, subtropical Himalaya.

\section{Cupuliferæ.}

Almus GizRTX.

Alnus maritima NinT. var. formosana BunK, in Fon:sis et HExst. Tnd. Fl. Sin. II. p. 500 ; Hexny, List Pl. Formos. p. 90 ; M Mrsum. Revis. 
Aln. Jap. in Journ. Scie. Coll. XVI.-5, p. 8 ; Matsurr. et Hayata, Enum. Pl. Formos. p. 391.

Hab. in monte Morrison, ad 8000 ped. alt., leg. T. KawakanI et U. Morr, Oct. 1906, (Nos. 1731, 1926 et 1047).

Distrib. Type: Japan, North China, Manchuria, and extending to North America.

Carpinus LinN.

Carpinus sp.

Hab, in monte Morrison, ad 7500 ped. alt., leg. T. Kawakani et U. Moni, Oct. 1906, (No. 1998).

\section{Queveus LinN.}

Quercus amygdalifolia Skav, in Fonbes et Hexsst. Ind. Fl. Sin. II. p. 506 ; Matsum. et Hayata, Enum. Pl. Formos. p. 393.

Hab. Nantō: Mushazan, ad 6000 ped. alt., leg. T. Kawakani et U. Mori, Aug. 1906, (Nos. 1139 et 1194).

Quercus dentata Thuns. Fl. Jap. p. 177, et Ic. Pl. Jap. Dec.-V. t. 6 ; DC. Prodr. ẊVI-2, p. 13 ; Fraveh. et Savat. Enum. Pl. Jap. I. p. 445; Fonbes et Hemst. Ind. Fl. Sin. II. p. 511; Deris, Fl. Centr. Chin p. 288 ; Palibin, Conspect. Fl. Korex, II. p. 51 ; Matsum. et Hayata, Enum. Pl. Formos. p. 393.

Quercus obovata Bunge; DC. Prodr. XVI.-2, p. 13.

HAB. Taichū: Dainankō, leg. T. Kawamani et U. Mori, Aug. 1906, (No. 1220).

Distrib. China and Japan.

Quercus formosana Srin, in Fonbes et HexsL. Ind. FI. Sin. II. p. 513 ; Matsuar. et Hayata, Enum. Pl. Formos. p. 393.

HАв. Kōshūn; Kōbutsuzan, leg. N. Konishr, April. 1898.

Quercus glauca Thunb. Fl. Jap. p. 175 ; Hook. f. Fl. Brit. Ind. V. 
p. 604 ; Fonbes et Hevist. Ind. Fl. Sin. II. p. 515 ; Diets, Fl. Centr. Chin. p. 293 ; Matsuir. el Hayata, Enum. Pl. Formos. p. 392.

Hıв. Nantō: Nankōkei, (No. 1172), et Kwantōzan, (No. 1120), leg. T. Kawakam et U. Mori, Aug. 1906.

Distrib. Japan, China, and Himalaya.

Quercus (Pasania) Kawakamii ILsist, sp. nor. Ramuli ralidiusculi sulcati cinereo-flavescentes. Folia oblongo-obovata 13-15 $\mathrm{cm}$. longa $6-7 \mathrm{~cm}$. lata apice abrupte obtuse acuminata basi atte nuata integra apicem versus obscure undulato-serrata coriacea supra nitida, costis planis leviter sulcatis, subtus pallidiora, costis valde prominentibus venis primariis lateralibus utrinque 13 prominulis sub angulo $50^{\circ}$ egressis prope margines curratis anastomosantibus tenuibus obscuris, petiolis semiteretibus supra leriter sulcatis basin versus incrassatis circ. $3 \mathrm{~cm}$. longis. Flores ignoti. Fructus juniore ternatim aggregati sed proventu solitarii ad pedunculum incrassatum remote dispositi. Cupula subpatelliformis $2 \frac{1}{2} \mathrm{~cm}$. in diametro $7 \mathrm{~mm}$. longa intus margine depressa medio convexa extus squamis adpressis latis apice cuspidatis pubescentibus 7-8 seriatis suffulta. Glans depresso-glohosa brere apiculata $2 \mathrm{~cm}$. longa $2 \frac{1}{4} \mathrm{~cm}$. in diametro, cicatrici depressa.

Haв. in monte Morrison, leg. T. Kawamami et U. Mori, Oct. 1906.

The present Quercus bears some resemblance to $Q$. spicata SiIrth, Q. formosana SkAx, and Q. Trevicaudatu Sk.1x; but is easily distinguished by the shape of the leares. M[r. S. A. Sk.tr informs me that this new plant is the nearest to Quercus spicata Surru, but sufficiently distinct in the long stallied leaves loroad and rounded at the apex.

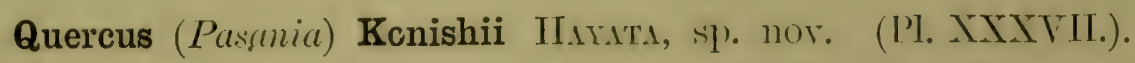
Ramuli juniores dense pubescentes angulati. Folia petiolata re- 
motiuscula oblongo-elliptica $7-9 \mathrm{~cm}$. longa 2-3 cm. lata apice cuspidato-acuminata basi angusta integra apicem rersus serrata, scrris obtusis, supra nitila, nervis et renis supra impressis subtus valde prominentibus, venis primariis lateralibus utrinque $\tau$ sub, angulo $45^{c}$ egressis ad apices serrarum attingentibus, venulis tenuibus rix prominulis, petiolis sub-gracilibus $1-2 \mathrm{~cm}$. longis dense pubescentibus. Amenta ad apicem ramuli ?-3-fasciculata erecta antrogyna 5-8 cm. longa validiuscula erecta, floribus inferioribus fomineis remotis solitariis, superioribus masculinis dense aggregatis. Fl. $\hat{\delta}$ : perianthium 6-lobatum, lobis obtusis incrassatis rotundatis, intus hirsutum extus tomentosum. Stamina 8-12, filamentis perianthio 3-plo-longioribus; anthere globosx glabrx; bracteis oratis hasi incrassatis concavis. Ovarii rudimentum dense albotomentosnm. Fl. 우 : inrolucrum globosum tomentosum squamis incrassatis triangularibus obtusis multiseriatim suffultum; orarium globoso-conicum, stylis trifidis incrassatis; bracteis subulatis incrassatis. Fructus secus pedunculum incrassatum foliis paullo breviorem dense dispositi. C'upula subpatelliformis medio depressa \& mm. longa $3 \mathrm{~cm}$. in diametro rquans intus pallida rubra leviter glauca pubescens extus griseo-pubescens, squamis latis basi 2-sulcatis minute cuspidatis arete adpressis circ. 10-seriatis suffulta. Clans $2 \mathrm{~cm}$. longa $3 \mathrm{~cm}$. in diametro semiglobosa nitida brevissime apiculata, cicatrici margine depressa medio convexa.

Hab. Taitō: 'Tōkwasan, Shinshōshō, leg. N. Koxishr, 1906, 'T’ōōgyōho, ad ¿̋00 ped. alt., Juli. 1906 ; Kōshun : Botansha, leg. G. Nakahara, Dec. 1906, (No. 747.); Nantō: Hinokiyama, leg. G. NAKahara, Feb. 1907.

Mr. S. A. Srix informs me that this new plant appears to be a distinct new species and there is nothing at Kew near it among the Asiatic material. 
Quercus serrata Thuxb. Fl. Jap. p. 176 ; DC. Prodr. XVI.-2, p. 50; Fnaxch. et Sivat. Enum. P. Jap. I. p. 447 ; Hook. f. Fl. Brit. Ind. T. p. 601 ; Fonbes et Hewsi. Ind. Fl. Sin. II. p. 520.

Haв. Taichū: Toshikaku, leg. Y. Tashiro, April. 1896.

Distrib. Japan, China, and Himalaya.

Quercus Junghuhnii MrQ. (Fig. 2.); Fl. Ind. Bat. I.-1, p. 853 ; Amn. Bot. Gard. Cale. II. p. 78 , t. 73 .

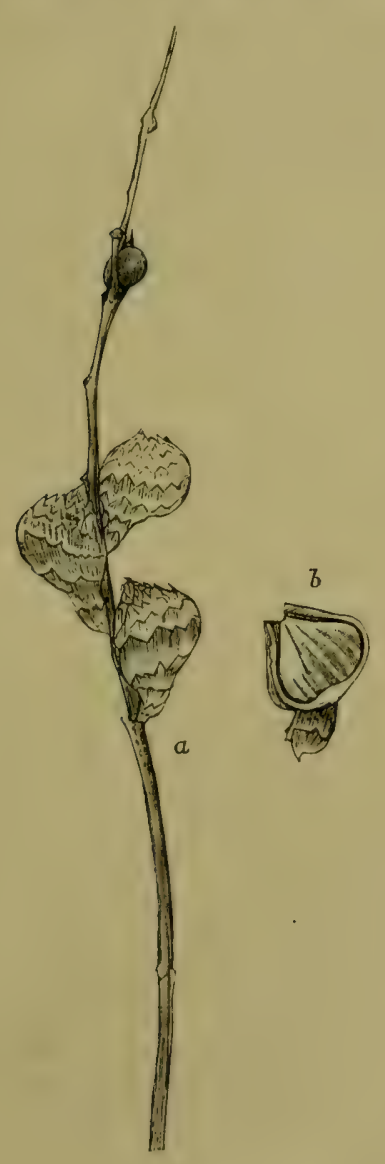

Fig. 2.

Quercus Jungluthii Mre. a- a branch of an inflorescence bearing fruits; $b-\mathbf{a}$ fruit, in rertical section, glans clearly seen.

Ramuli graciles griseo-fuscentes patentes. Folia petiolata patentia coriacea elliptico-ovata vel ovato-lanceolata $7 \mathrm{~cm}$. longa $2 \mathrm{~cm}$. lata margine integra extra medium grosse serrata apice longe cuspidata vel caudata leviter recurva basi rotundata rquilateralia rarius obliqua supra viridia costis impressis subtus ferrugineo-albicantia sub lente integumento tenuissimo lepidoto obtecta, costis prominentibus, venis primariis lateralibus utrinque circ. 10 apicem versus inconspicuis sub angulo 60 egressis, petiolis supra sulcatis semiteretibus $8 \mathrm{~mm}$. longis. Fructus secus pedunculum gracilem erectum remote dispositi solitarii. Cupula turbinata valde obliqua $1 \mathrm{~cm}$. in diametro sursum acuta deorsum abrupte attenuata in stipitem $\tau$ mm. longum abeuns, glandem includens, extus squamis dentoideis in annulum dispositis $7-\infty$-seriatis sursum erectis deorsum adpressis, extus tenuiter 
cinero-tomentosa, intus sericea, crustaceo-fragilis. Glans subglobosa sursum attenuata acutiuscula glabra brunnea $8 \mathrm{~mm}$. longa $9 \mathrm{~mm}$. lata.

HaB, in monte Morrison, leg. T. KawaKanI et U. Morr, Oct. 1906.

Distrib. Java.

I am informed by Mr. S. A. SkaN that this plant is certainly very near Quercus cuspidata Thuns., and still nearer Q. Carlesii HemsL. in Ноок. Ic. Pl. t. 2591, from Foo-chow. But, in $\mathrm{Mr}$. Hemsley's species, the leaves are not so much rounded at the base, the fruit is not so distinctly stipitate, and it is broadest in the middle, not at the base. To my opinion, however, this Formosan plant is quit referable to the Javan species, although I have not yet seen the specimen of it.

Quercus variabilis Puune, Mrus. Bot. Lugd.-Bat. I. p. 297 ; DC. Prodr. XVI.-2, p. 50 ; Franch. et Savat. Enum. Pl. Jap. I. p. 447 ; Matsunt. et HaYata, Enum. Pl. Formos. p. 394.

HАв. Taichū : Suiteiryō, leg. Y. Tashino, 1896, (No. 77); Biyōritsu : Banahōzan, leg. T. Kawakani et U. Mori, Juli. 1906, (No. 1106).

Distrib. Japan.

Quercus sp.

Hab. Bunsanho (No. 3), et Heirinbi (No. 21), leg. N. Konishi, Oct. 1899.

Very near $Q$. glauca Thuns.; but the leaves are much narrower and the veins are more elevated. The leaves of dried specimens are of a color of neutral tint.

Castanopsis SPACH.

Castanopsis indica A. DC. in SEEn. Journ. Bot. (1864) p. 182; DC. 
Prodr. XVI.-2, p. 109 ; Mrn. in Anu. Mrus. Bot. Lugd.-Bat. I. p. 119 ; Ноок. f. Fl. Brit. Ind. V. p. 620.

Castanea indica Roxb. Fl. Ind. ed.-Caner, III. p. 643 ; Buune, Mrus. Bot. Lugd.-Bat. I. p. 284; Wight, Ic. Pl. Ind. or. t. 417.

Castanopsis tribuloides rar. formosma Hayata, in Matsux. et Hayata, Enum. Pl. Formos. p. 394.

Hab. Banchoryō: Rokurisha, leg. G. Nakahara, Oct. 1905, (No. 593).

Distris. Tropical Himalaya.

Castanopsis taiwaniana Harata, sp. nov. (Fig. 3). Ramuli teretes dense ferrugineo-pubescentes. Folia breviter petiolata patentia oblongo-lanceolata $8-14 \mathrm{~cm}$. longa $31-5 \mathrm{~cm}$. lata integerrima apice obtuse acuminata basi rotundata vel leviter attenuata coriacea rigida bicoloria supra glabra nitida pallidoviridia costis impressis venis inconspicuis, subtus ochraceolepidota costis prominentibus venis primariis lateralibus utrinque $10-13 \mathrm{sub}$ angulo $60^{\circ}$ egressis intra mar-

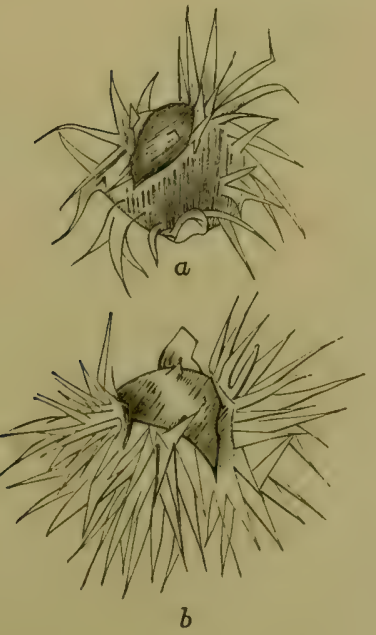

Fig. 3.

Castunopsis taicrenime Marata. $a$ and $b$ - fruits. gines curvatis anastomosantibus, petiolis $1 \mathrm{~cm}$. longis basi leviter incrassatis sulcatis semiteretibus; folia novella conduplicativa subtus dense ferrugineotomentosa, stipulis caducis lateralibus sub-obliquis obtusissimis integerrimis sub-coriaceis venis parallelis sericeis ab initio erectis dein revolutis. Amenta $10 \mathrm{~cm}$. longa ex axillis foliorum superiorum solitaria simplicia erecta remotiflora. Fructus sessiles solitarii globosi magnitudine cum spinis $3 \mathrm{~cm}$. æquantes. Cupula clausa intus spadiceo-sericea 
extus echinata, spinis confertis erectis subulatis rigidis rufescentibus sericeis. Cilans solitaria globoso-trigona basi truncata leviter convexa ochraceo-rufescens pubescens vel subglabra.

Hab. Nantō, leg. T. Kawakani et U. Mori, Oct. 1906, (Nos. $118 \tilde{5}$ et 1165$)$; Shintiku : Goshizan, leg. 'T. KawaKuI, Dec. 1905, (No. 1294); Hokuho, leg. N. Koxishi, 1899, (No. 8).

This Castanopsis is very like C.javanica A. DC.; but differs from it in having subglabrous nuts and more strong spines on the involucres.

I am informed by Mr. S. A. Srax that this new plant is very near to C. Hystrix A. DC., in which the leaves, though usually toothed, are sometimes quite intire, and that there is no specimen at kew of $C$. Hystrix with the spines of the involucre so stout and that is apparently the only difference.

\section{Fagues Linu.}

Fagus sylvatica Lrxx. Sp. Pl. ed.-2, p. 1416; DC. Prodr. XVI.-2. p. 118.

var. ?

Hab. Kushaku : Sōtenzan, ad 5600 ped. alt., leg. N. Konishi, Feb. 1906, (spec. steril.).

\section{Salicaceæ.}

Salix Lixx.

\section{Salix sp.}

Haв. in monte Norrison, ad 13094 ped. alt., leg. S. Nagasatwa, Nov. $1905,($ No. 596).

It somewhat resembles the Japanese S. Sicboldiana Buuse. The specimens being all sterile are not yet determined. 


\section{Gymnospermeæ.}

\section{Coniferæ.}

\section{Libocedrus ENDL.}

Libocedrus macrolepis BexrH. (Fig. 4). BЕхтн. in Bexth. et Hоoк. Gen. Pl. TI. p. 426 ; Fonbes et Hersu. Ind. Fl. Sin. II. p. 540 ; Mastens, in Journ. Linn. Soc. XXXVII p. 411; M.yr, Fremdland. Wald-und Park-

Bäume, p. 316.

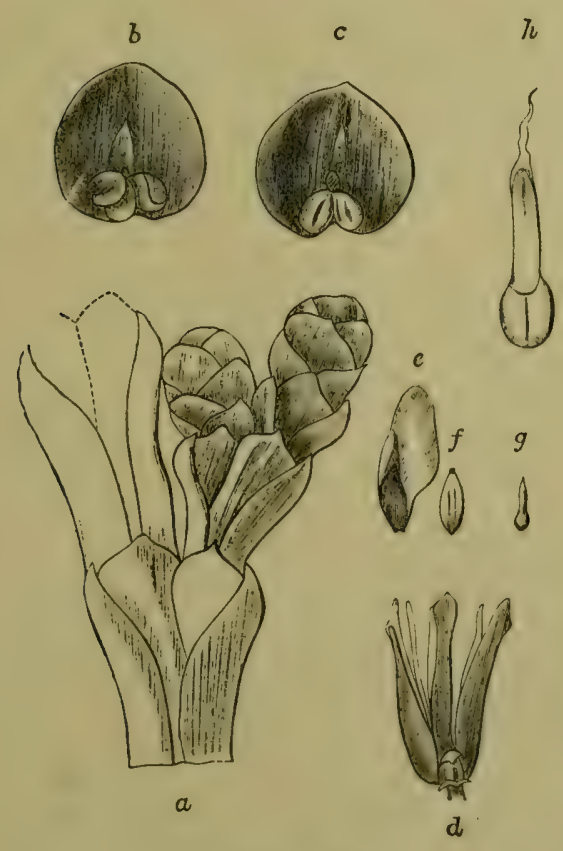

Fig. 4.

Lilocedrus macrolepis Bшхти. a- male flowers; $b$ and $c-$ stamens; $d-$ a cone; $e-$ $a$ seed; $f$ - an albumen; $t-$ an embryo ; $h$ - the same, highly magnified.
Calocedrus macrolepis Kunz, in Journ. Bot. (1873) p. 196.

HАв. Rinkōshō: leg. 'T. Kawakami et U. Moni, Sept. 1906 , (No. 1675).

As the descriptions of the male flowers and seeds were not sufficiently made previously, the following accounts may be properly added here.

Flores masculini ad ramulos brevissimos terminales oblongi, staminibus circ. 8 , filamentis ad basin squamarum subpeltatis, squamis suborbicularibus vel subcordatis, loculis antheræ $2-3$. Strobilus cylindraceo-oblongus. Semina samaroidea, alis cultriformibus. 


\section{Chameeyparis SPACH.}

Chamæcyparis formosensis Matsuxr. in Tükyō Bot. Mrag. XV. p. 137; Matsum. et Hayata, Enum. PI. Formos. p. 402.

Hab. in montibus Morrison, leg. R. TonIr, 1900 ; Giyokusan, in montibus Morrison, ad 10634 ped. alt., (No. 554), ct Seizan, in isclem montibus, ad 11579 ped. alt., (No. 584), leg. S. Nagasatwa, Nov. 1905; in monte Morrison, ad 7000 ped. alt., leg. T. Kawaisair et U. Mort, Oct. 1906, (No. 2103) ; Taitō: Bunshiseki, leg. T. KawaKaMi et U. Mori, Dec. 1906, (No. 2104).

Distrib. An allied species, C. pisifera S. et Z., ocenrs in Japan.

Chamæcyparis obtusa Sieb. et Zucc. in "Eddu. Conif. p. 63"; Part. in DC. Prodr. XVI.-2, p. 466 ; Franch. et Savat. Enum. PI. Jap. I. p. 471 ; Warburg, Monsunia, I. p. 190 ; Beissn. Handb. Nadelh. p. 92.

Retinispora obtusa Steb. et Znce. Fl. Jap. II. p. 38 ; MIQ. Prol. Fl. Jap. p. 332.

Thuya obtusa MasT. Journ. Linn. Soc. XVII. p. 491, Fig. 4.

forma formosana, (Fig. 5) ; Hayata, in Gard. Chron. (1908) p. 194. Strobili ut typicx multo minores $8 \mathrm{~mm}$. in diametro xquantes, seminibus minoribus cum alis $2 \mathrm{~mm}$. in diametro xquantibus.

Shinkō: Shirakku, leg. T. Kawakami et U. Mori, Juni. 1906, (No. 1329) ; Arizan, in montibus Morrisson, leg. G. NaKahara, Nov. 1906.

Distrib. Japan.

This Formosan Chamcecyparis is in all respects the same as the Japanese species. But, the cone of the present plant is always much smaller than the Japanese one, and the sced is also smaller. Native botanists in the island suggest to me to separate it from the Japanese species as a new variety. For the present, 


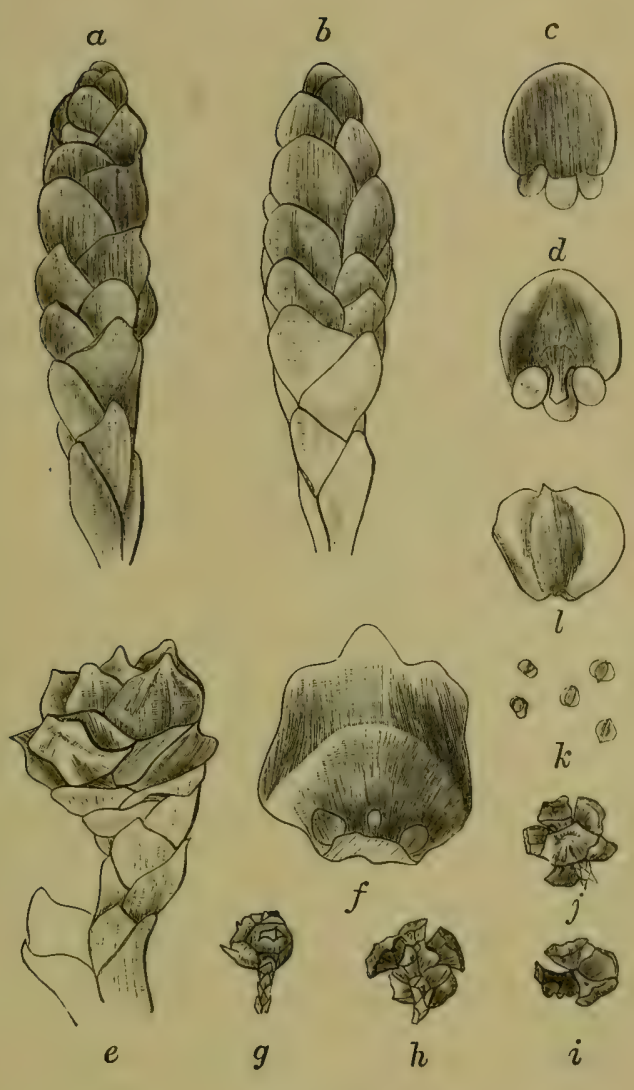

Fig. 5.

Chamaryparis obtusa $\mathrm{S}$. et $Z$. form. formosana $a-$ a male flower; $l$ - the same, seen from a different side; $c-a$ stamen, Eeen from the onter side; $d-$ the same, seen from the inner side; $e-$ a female flower in an advanced stage ; $f$ - a scale; $g$ - a cone; $h-$ another cone, after opening; $i$-another form; $j$ another one ; $k$ - seeds; $l-$ a seed, magnificd. however, it would be better to regard it as a form of the Japanese species.

\section{Tuniperus LINN.}

\section{Juniperus formosana HAYA-} TA. (PI. XXXVIII.) in Gard. Chron. (1908) p. 194.

Juniperus taxifolic HaYATA, in Tōkyō Bot. Mag. XX. p. 46; Matsum. et Hayata, Enum. PI. Formos. p. 403.

Ramuli novelli triquetri in angulis prominentibus marginati glabri. Folia in totum lineari-angusta $14 \mathrm{~mm}$. longa $2 \mathrm{~mm}$. lata verticillatoternata pátentia integerrima acuminato-pungentia glabra supra concava glauca stomatifera subtus valde carinata prominentia convexa. Flores ignoti. Galbuli ad axillas foliorum inferiorum ramulorum novellorum solitarii, basi squamıs ovato-acutis rerticillatis ¿-scriatim dispositis, globosi $7 \mathrm{~mm}$. longi carnosi rufo-fuscentes supra medium vestigiis acutis bractearum intimarum notati. Semina 3, erecta trigono-elliptica apice mucronata, testa ossca, facie dcrsali glandulis $3-4$ resiniferis oblongis insculpta, facie ventrali pauce notata. 
Haв. in monte Morrison, leg. R. Torir, 1900 ; Seizan, in montibus Morrison, ad 11579 ped, alt., leg. S. Nagasatwa, Nov. 190う, (No. 752); in isdem montibus, ad 13000 ped. alt., (No. 2262), et ad 8000 ped. alt., (No. 2039), in montibus centralibus, (No. 2099), leg. T. Kawakami et U. Mori, Nov. 1906.

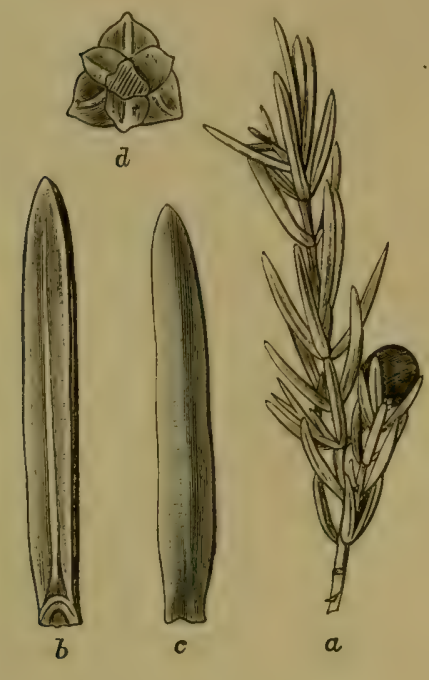

Fig. 6.

Juniperus texifoliz Hoor. et Ars. from the Bonin islands.

$a-a$ branch; $b-$ a leaf, seen from within; $c$ - the same, seen from without; $a$ - bracts at the base of a fruit, seen from belorr.

Very like J. communis Lins. ; but differs from it in having pointed vestiges on the fruits ; also near J. taxifolia Ноок. et ARx., but differs from it in having acute leaves; from $J$. rigida Sieb. et Zucc.; in having less narrowed leaves and acnte vestiges on the fruits.

The present plant has erroneously been regarded by myself and also by some other botanists as the same species as the Bonin J.taxifolia. On re-examining carefully the all specimens of the genus, Juniperus, from China, Formosa, Japan, the Bonin islands and Loo-choo archipelago, I have found that J.taxifolia is confined to the latter two archipelagos, but does not extend to either Formosa or China. The Formosan plant differs from the Bonin spccies in having pungent leaves. As to the Juniperus of China, I was so fortunate as to examine the same specimens which were studied by the late Dr. M. T. Masters, F. R. S., F. L. S. The Chinese plant collected by Mr. E. II. Wrusox in West China, Changyang (IIupeh, No. 4.28), which is referred to J. taxifolia by that ominent authority ${ }^{21}$

1). M. T. Masters: Chinese Conifers collected by E. H. WilsoN, in the Journal of Botany, Vol. XLI. 1. 267, \& On Chinese Conifers, in the Journal of the Linnean Society. XXXVII. p. 113. 
is not to my opinion the same as the Bonin plant in which the leaves are altogether blunt, and the male flowers are much longer (see Fig. 6). Nor is the specimen" which was collected by M. A. Hexry (Hupeh, No. 2067, A !), referable to the juneper of the archipelago. The Chinese plant has pungent leaves and much shorter male flowers. Whether the Juniperi of Formosa and China are similar or not, demands further investigation. It present, I can only state that J. taxifolia does not extend to either Formosa or China, and that it is found in the Bonin, and Loo-choo islands, but nowhere else.

Juniperus morrisonicola HistT1, (Fig. 7), in Gart. Chron. (1908) p. 194. Rami teretes, ramulis novellis viridibus trigonis. Folia omnia lanceolata apice acerosa patentiuscula $3-4 \mathrm{~mm}$. longa $1 \mathrm{~mm}$. lata verticillato-ternata supra cancara glancescentia subtus leviter carinata. Flores masculini terminales ad ramulos brerissimos oroidei $4 \mathrm{~mm}$. longi $2 \mathrm{~mm}$. lati, staminibus 8 , filamentis in squamam peltatis, squamis suborbicularibus $1 \frac{1}{2} \mathrm{~mm}$. in diametro xquantibus, loculis antherr 3. Flores forminei ad ramulos brevissimos terminales basi foliis squamiformibus bracteisque o-9 ternato-rerticillatis suffulti, squamis intimis 3 ovatis acutis verticillatis patentibus. Ovulum terminale solitarium squamis intimis circumdatum oblongum apice attenuatum. Galbuli solitarii globosi vel paullo longiores $6 \mathrm{~mm}$. longi, sul maturitate nigricantes glaluri medio restigiis squamarum notati. Semina solitaria globoso-ovoidea $5 \mathrm{~mm}$. long. $4 \mathrm{~mm}$. lata, testa ossea sulcata. Embryo normalis; cotyledones 2.

HАB. ad summam montis Morrison, 13200 ped. alt., leg. Shrmorma, 1899 ; ibidem, leg. R. Toni, 1900 ; ibidem, leg. S. Nagasawa. 1905, (No. 585), et leg. T. Kawakam, 1906, (No. 2142, fr.). 

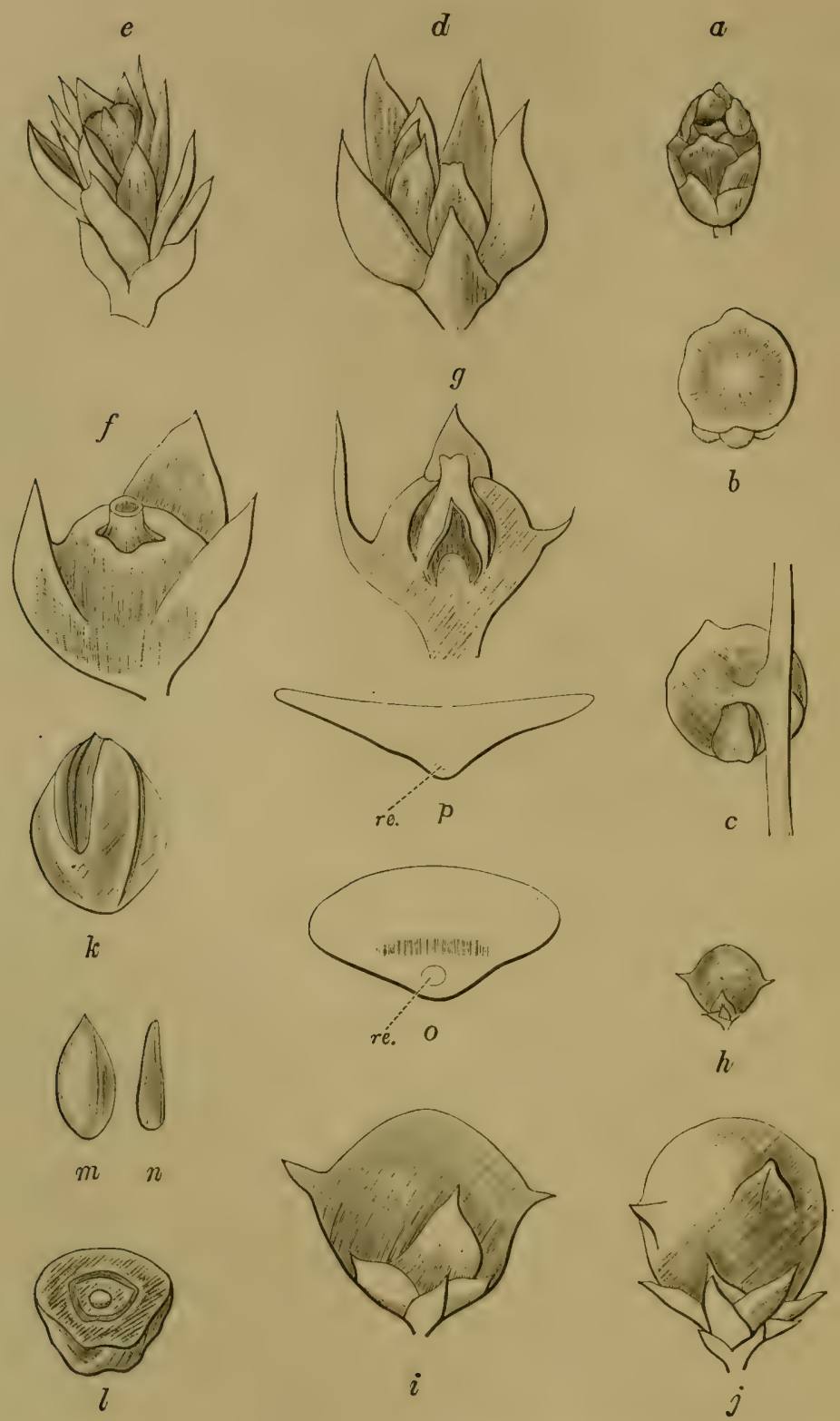

Fig. 7. Juniperus morrisonicolu Harata.

$u$ - a male flower; $b$ - a stamen; $c$ - the same, seen frcm the inner side; $a-\Omega$ female flower; $e$ - the same, in a little advanced stage; $f$ - the same, in a more advanced stage; $g$ - the same, in vertical section, one side taken cff, showing the hollow receptacle, ovule and nucellus (as the figure is drawn from a dry specimen, the nucellus is probably much more contracted than it really is); $h$ - a fruit (natural size); $i$ - the same, magnified; $j$ - the same, seen from a different side; $k-a$ seed; $l$ - the same, in section, the upper half taken awny, showing the hard seed coat, albumen and embryo; $m$ - an albumen; $n$ - an embryo; 0 and $p$ - sections of different parts of a leaf, $r e=$ resin-chnal. 
The Juniperus described above ivas first found by Mr. Shmorama on the top of Mit. Morrison at an altitude of abont $4000 \mathrm{~m}$., in the year 1899. Although the same mountain was since botanized by several collectors, the specimens brought back to me were but fragments of a sterile branch. I could not get any more idea about this plant than that it is something like Juniperus chinensis Lins., until Mrr. T. Kawar.um gave me a most perfect specimen of it. Examing the material, I have found that the plant is far different from J.chinensis Lixx., in having a solitary ovule on a short branchlet, and in the shape of its cone. The leaves have a large single resin-canal near' the phloëm. So far, the plant does not seem to have dimorphic leaves, all the specimens we have at present possessing but one kind of leaf.

Cunninghamia R. BR.

Cuminghamia, being a monotypic genus, implies only $C$. sinensis R. Br. It is, therefore, the most remarkable matter that we have here an addition of one more species belonging to this interesting genus. The new Cunninghamia was kindly sent to me by Mr. T. Kawakari, Goremment Expert of Formosa. It was obtained by Mr. N. Koxisur on Mit. Randaisan at an altitude of about $2000 \mathrm{~m}$. It is rery rarely found in the coniferous forests, and attains a considerable height. It affords a good timber which bears some resemblance to Chamcectparis.

Cunninghamia Konishii IIArat, in (iart. ('hron. (1!)18) p). 194. Arbor, ramis omnibus teretibus glabris foliorum spiraliter confertorum cicatricibus notatis. Gemmæ florifere nudx dejesso-glolosa, hracteis represso-ovatis hrevissime aristato-acutis. 
Folia ramorum retorum spiraliter conferta adnato-decurrentia anguste lineari-falcata incurvo-erecta acuta dorso leviter carinata, ramulorum jurenium longiora ascendento-patentia anguste linearilanceolata $15 \mathrm{~mm}$. longa $2 \frac{1}{2} \mathrm{~mm}$. lata ad basin oblique torta apice obtusiuscula margine sub lente serrulata rigida coriacea utraque pagine glaucescentia stomatibus multiseriatis instructis octarum in annum virentia demum exarida sensim soluta. Strobili sub maturitate ovato-globosi $20 \mathrm{~mm}$. longi $15 \mathrm{~mm}$. lati. Squamæ rotundatre mucronatx basi distincte unguiculate, unguibus brevibus, laminis dilatis, cordatis late depresso-oratis margine integris lignescentibus sursum coriaceis et marginem versus subundulatis dorso apice leviter carinatis glabris. Bractex obsoletx. Squamulx 3 ad medio laminæ squamæ distinctæ fimbriatæ crenulatæ. Semina 3 ad medium squamularum affixa reversa libera ovato-clliptica, testis corioceis duriusculis, alis angustis. Embryo ignotus.

HAB. Nantō: in monte Randaisan, ad 7000 ped. alt., leg. N. Konishi, Mai. 1907.

Mr. T. Kawakam informs me that the habit of this new plant is an intermediate between those of Cunninghamia and I'airania. On examining the specimen carefully, I find that the cone of the plant has a secondary squama. Therefore, this should undoubtedly be referred to Cunninghamia. The leaf of this plant has stomata on both surfaces, while that of $C$. sinensis has no stoma on the upper surface, or a very few if at all. In the case of 'Taiuania, the stomata are found on both surfaces. The new Cumninghamia differs mainly from the other species in the arrangement and the shape of the leaves, and in having smaller cones and broader squame. The timber is like other Conifers, the bark is reddish brown and in all respects is very like that of Chamcecyparis, but it has an odour peculiar to itself. The leaf of the present plant is more persistent 
than that of the other'; the former lasts for cight years, while the latter only lasting five years.

Taiwania HATATs.

Taiwania cryptomerioides HAYATA, in Jovrn. Limn. Soc. XXXVII. p. 331, t. 16, et in Tōkyō Bot. Mag. XXI. p. 2, t. 1.

Cryptomeria japonica HaYATA, (non Don.) in Tökyō Bot. Mag. XX. p. 46.

HAB. Arizan, in montibus Morrison, ad 7500 ped. alt., leg. T. Kawakami et U. Mori, Oct. 1906, et ibidem, leg. G. NaKahara; Taitō: Taironkōsha, ad 8000 ped. alt., leg. U. Mori, Nov. 1906.

Distrib. An endemic monotypic genus.

\section{Cephalotaxus ZuCC.}

\section{Cephalotaxus sp.}

HAB. in monte Morrison, leg. R. TorIr, 1900; Ganzan, in montibus Morrison, ad 8012 ped. alt., leg. S. Nagasawa, Nor. (1905), (No. 568); in monte Morrison, ad 8500 ped. alt., leg. T. KawaKaмI et U. MonI, Nov. 1906, (No. 2106); Taitō : Dakunsha, leg. T. KawAKaMI et U. Mori, Dec. 1906, (No. 2105).

Distrib. The genus extends from Japan to China.

In the absence of cones, the species is indeterminable.

\section{Taxus sp.}

\section{Taxus LinN.}

Hab. Arizan, in montibus Morrison, leg. G. NaKahara, Oct. 1906 ; 'Taitō : Bunshisekisha, leg. 'T. Kawanani et U. Mori, Dec. 1906, (No. 2107).

In the absence of cones, it is impossible to determine it specifically.

\section{Pimes Linn.}

Pinus Armandi Fraxchet, Pl. David. I. p. 285, t. 12; MLasteris, in Forbes et Heuss. Ind. Fl. Sin. 1I. p. 549. 

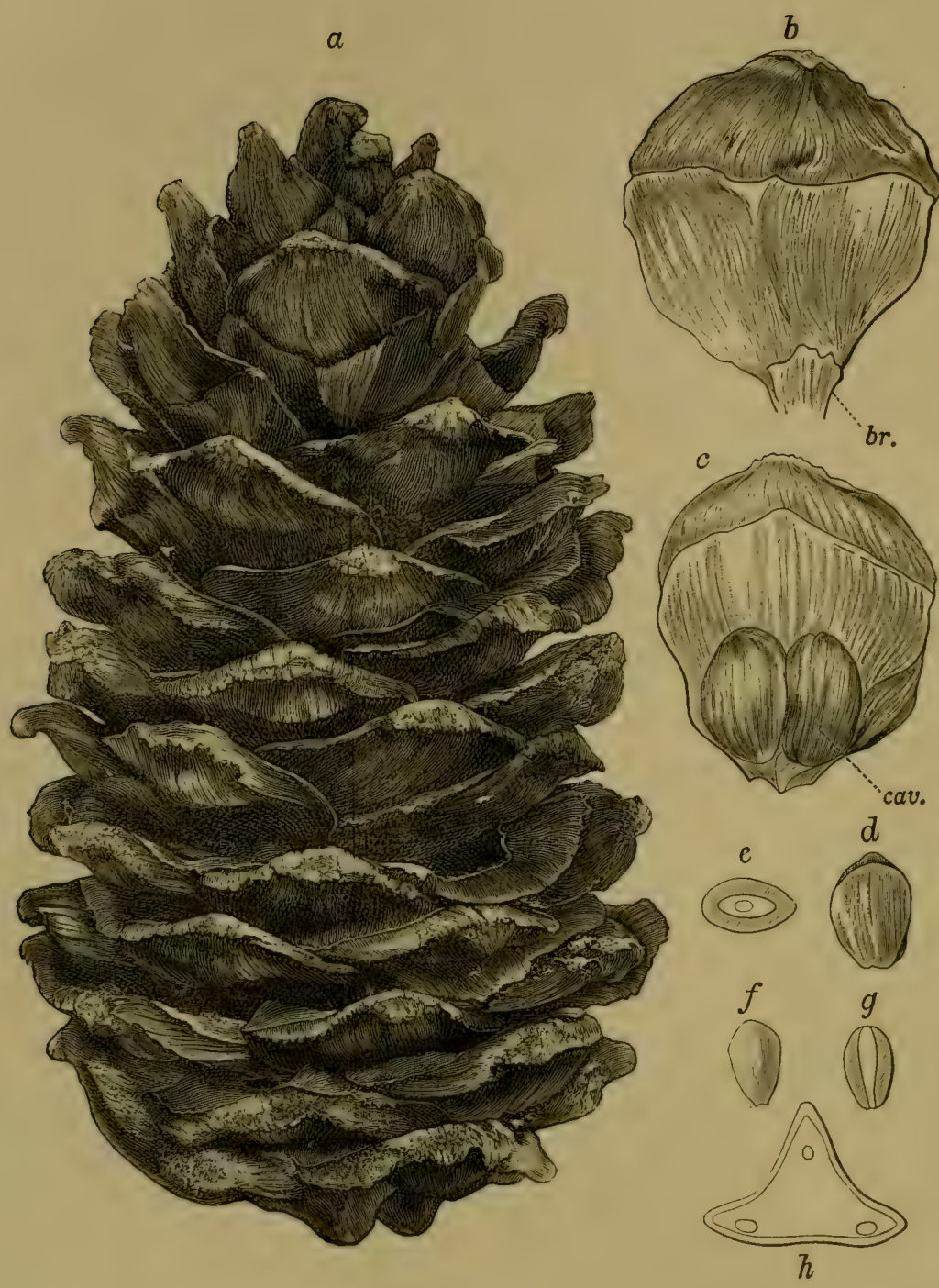

Fig. 8.

I'imus dimandi Fraxch. var. Mastersina Harata.

( - in cone, (slightly reduced); $b-n$ scale detached from the middle part of the same cone, seen from the outer sicle; $c$ - the same, seen from the inner side, seeds taken off, cav.=impression of the seels; $d-\Omega$ seed; $e$ - cross section of the same; $f$ - an albumen; $g$ - the same, in vertical section; $h$ - cross seciion of a leaf. 
var. Mastersiana HaYstA, (Fig. 8), in Gard. Chron. (1908) p. 194. Rami teretes fusci novelli glabri cicatricibus perularum et foliorum notati. Folia in fasciculo quinque acerosa filiformia tenuia acuta dorso plana triangularia in sectione margine et in carina remote serrulata $10 \mathrm{~cm}$. longa. Strobili erecti ovato-cylindracei obtusi $14 \mathrm{~cm}$. longi $7 \mathrm{~cm}$. lati. Bractex minutissimæ. Squamæ numerosæ orbiculares v. subrhombeæ acutæ sursum reflexæ basi breviter cuneatæ lignescentes longitudinaliter rugosæ fuscæ dispermæ. Semina obovata $12 \mathrm{~mm}$. longa $9 \mathrm{~mm}$. lata apice leviter apiculata aptera compressiuscula, testa ossea crassa fusca glabra. Albumen crassum oleosum. Embryo cotyledonibus 5 verticillatis.

Haв. in monte Morrison, leg. R. Toris, 1900 ; Hatsukwanzan, ad pedem montis Morrison, leg. Yarishiтs; Gyokusan, in montibus Morrison, ad 10634 ped. alt., leg. S. NAGAsawA, Nov. 1905, (No. 582); in monte Morrison, ad 8000 ped. alt., (No. 2088), et in eodem monte, ad 9000 ped, alt., (No. 2095), leg. T. KAwaKaxi et U. Mori, Oct. 1906 ; in eodem monte, leg. G. NaKahara, Oct. 1905.

The present variety differs from the tipe in its reflexed squamie and longer cones.

Distrib. Type: west central China.

Pinus formosana HaytT, (Fig. 9).

Pinus morrisonicola HaYst从, in Gard. Chron. (1908) p. 194.

liamuli teretes perularum rudimentis notati, novelli pubescentes. Gemmæ ovatæ perulatx, perulis acutis membranaceis margine fractis. Folia in fasciculo quinque, fasciculis approximatis

* The Pinus is for the first time printed under the numo, $P$. morvisonirole, which is, Lowever, an unfortunate name which is erroneonsly copied from my mnutscript. 'The oceurrence of the plant in the Mount Morrison is minther doubtful, for the Tinus is very local plant, being found only in some parts of the 'Thichñ district. 
acerosa rigidula $6-8 \mathrm{~cm}$. longa arcuata sed non torta apice acuta dorso plana facie acute carinata triangularia in sectione margine (et in carina remote serrulata. Strobili erecti ovato-elliptici obtusi, e squamis circ. 40 compositi $7-9 \mathrm{~cm}$. longi $4-6 \mathrm{~cm}$. lati, squamis ellipticis basi cuneatis sursum rotundatis leviter reflexis $3 \mathrm{~cm}$. longis $1 \frac{1}{2}$ cm. latis coriaceo-crassis sublignescentibus concavis badio-fuscis dispermis semper abortu monospermis. Bractex brevissimæ. Semina orata apice obtusa $10 \mathrm{~mm}$. longa $6 \mathrm{~mm}$. lata, testa coriacea pallide ferruginea glabra, ala membranacea tenu cultriformi $2 \mathrm{~cm}$. longa $8 \mathrm{~mm}$. lata.

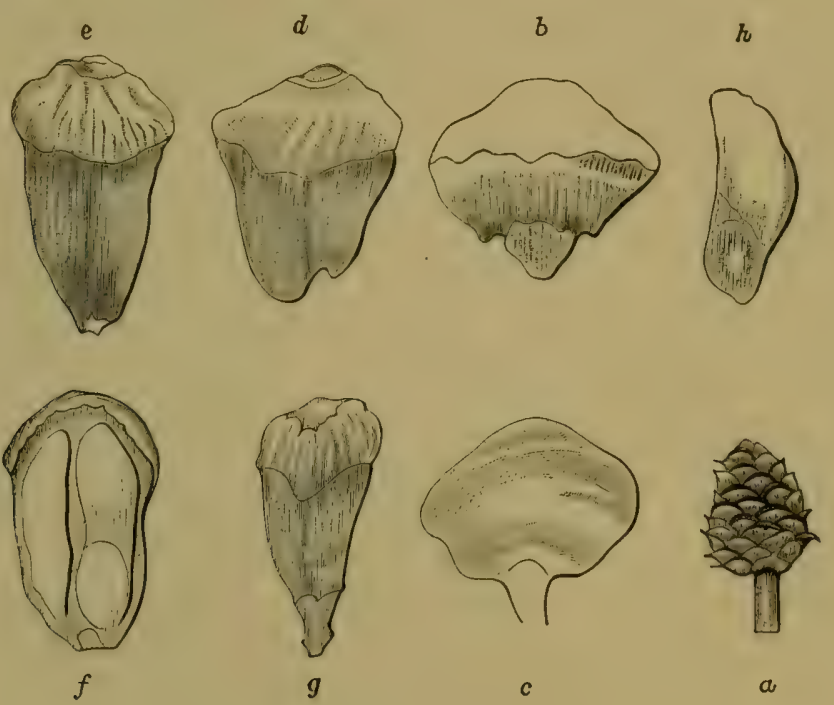

Fig. 9. Pinus fomosence IIryata.

$a-a$ very young cone; $b-n$ scale of the same cone, maguified; $c$ - the same, seen from a different side; $d-\Omega$ scale detacher from the basal portion of a mature cone; $e-a$ scale detached from the midale portion of the same cone; $f$ - the same, seen from the inner side; $g-a$ scale detachel from the apical portion of the same cone; lt- a seert.

Hab. Shōhakulin, et inter Hokkōkei et Horisha, C. Owatari, Jan. 1898 ; Taichū: Yagatayama, leg. G. Nakahara, Feb. 1907 ; ad 
summam montis Hanrizan, ad 8000 ped. alt., leg. Mrrata, Sept. 1897 ; Taitō: Bunshiseki, leg. T. Kawanıı et U. Mori, Dec. 1906 , (No. 2090).

Distrib; An allied species, P. parviflora S. et Z., occurs in Japan.

The present Pinus is very near Pinus parriflora S. et Z.; but differ's from that in the shape of the cones. The scales of the cones of this new plant are usually reflexed and especially so in the scales of the basal part. The wings of the seeds are much larger than those of $P$. parviflora $\mathrm{S}$. et $\mathrm{Z}$.

According to Mr. G. Nakahara, the plant grows in the mountainous districts of the Taichū prefecture, at an altitude of $1500 \mathrm{~m}$. Forming a forest along a valley, making lines parallel to the camphor forests, this pine gives a most remarkable feature to the vegetation of this spot. Attaining a height of about $15 \mathrm{~m}$., and a diameter of a little less than $1 \mathrm{~m}$., it describes an outline of a conical form, stretches out its branches quite loosely upwards from the middle of the trunk, and sends them down within the reach of one's arms. It grows mostly on a cliff of the clay slates, and faces itself to the valley below. The trunk presents a colour of grayish white and it is very straight like a fir, and the texture of the bark is rery similar. The distribution of the pine is rather local, the habitat being limited to the western slope of the central mountain ranges in the middle part of the island.

Pinus sp. (aff. P. Thunbergii PARL.).

HAB, in monte Morrison, leg. R. Torir, 1900.

Pinus sp, (aff. $P$. densiflora S. et $\mathrm{Z}$.)

HAB. in montibus centralibus, ad 10000 ped. alt., leg. 'T. KATAkani et U. Mont, Nor. 1906, (Nos. 2097 et 2094). 


\section{Picen Lisk.}

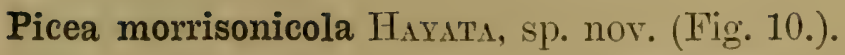

Picca Glehmi Mratsuar. in Tōkyō Dot. Mag. XV. p. 141; MLatsurr. et HAYAт, Enum. PI. Formos. p. 401, (non Fr. Schrr.).

Ramuli glabri, pulvinis oblongo-obovatis superne erectis in petiolum brevem ascendentem angustis, cicatricilus quadrangularibus. Gemmæ ovoideoconicæ, perulis scariosis ovatis obtusis. Folia linearia $6 \mathrm{~mm}-16 \mathrm{~mm}$. longa $\frac{2}{3} \mathrm{~mm}$. lata curvata quadangularia apice acuta utrinque stomatifera.

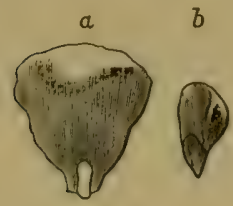

Fig. 10.

I'icee morrisonicoln MAxits "- a scale detached from the midalle portion of $\Omega$ eone; $b-$ ¿ seeci. Strobili oblongo-cylindracei $6 \mathrm{~cm}$. longi deflexi? Bracter ovato-lanceolatæ obtusæ margine fractæ $\frac{1}{+}$ squamæ fructiferæ æquantibus. Squamæ planiusculæ obovato-orbiculares basi attematr, apice truncatærotundatæ subintegræ. Semina cum alis 1 cm. longa, alis subeultriformibus obovatis semen ipsum $1 \frac{1}{2}-2$ plo superantibus.

HAB, in monte Morrison, leg. R. TonII 1900 ; Hattsukwanzan, leg. Yanishita ; in eodem monte ad 95000 ped. alt., leg. T. KawaTami et U. Mort. Nov. 1906, (No. 2108).

Strobilus (T. Kawakiur, No. 2108) $60 \mathrm{~mm}$. longus $22 \mathrm{~mm}$. latus.

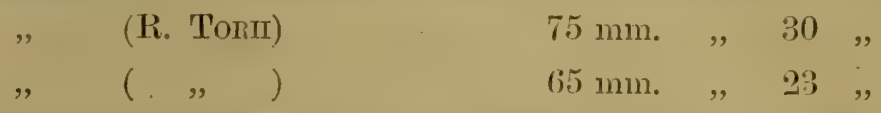

Near P. Glehnii Masters, but differs from it in having glabrous branchlets; also near $P$. Watsoniana Masters, but differ's from it in having subtruncate squamr of cones.

Distrib. An allied species, P. Glehnii Mast., oceurs in Japan and Saghalien. 


\section{Keteleevia Carr.}

Keteleeria Davidiana Brissi. val. formosana Husta, (Fig. 11.). in Gard. Chron. (1908) p. 194. Ramnli dense puberuli. Folia laxe disposita plana lineari-lanceolata 30 $11 m$. longa rel longiora $5 \mathrm{~mm}$. lata, nervis ntraque pagine prominentibus, margine parum deflexi rernicosa subtus vix pallidora apice obtusa (ramuli hornotini aristato-acuta) in pedem brevem compressum demum contortum attenuata, petiolis basi transrerse insertis. Strolili erecti cylindracei obtusi $9 \mathrm{~cm}$. longi $5 \mathrm{~cm}$. lati. Squamæ coriaceæ ovato-rotundatro r. cordatæ superne sensim attenuatre

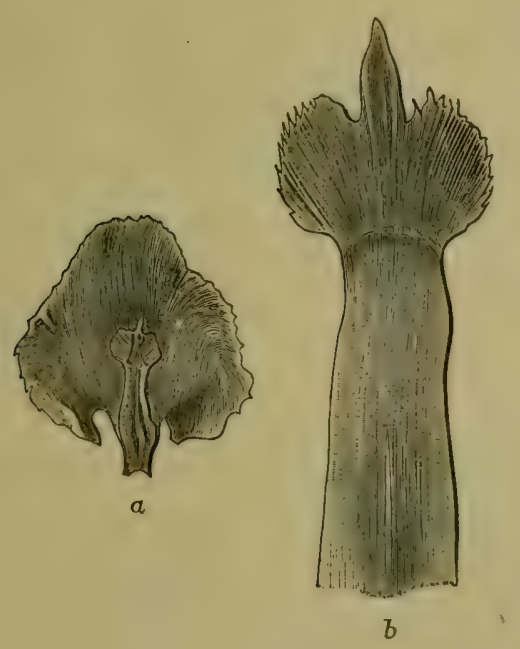

Fig. 11.

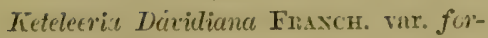
mosanc HaYATA. $\ell-\mathrm{n}$ scale of a cone; $b$ - the bract of the same seale, magnifier. apice subreflexæ basi breve unguiculatæ extus longitudinaliter striatro puberulæmargine tenues irregulariter serrulatæ. Bracter squamis duplo breviores, spathulatio membranacex dorso fusce apice cuspidatæ vel trifide irregrulariter serrulatæ subpungentes supra medium contractæ. Semina pallida fulva basi acuta, ala concolore cultriformi, squamis æquilonga, cum alis $27 \mathrm{~mm}$. in longitudine æquantia.

Haв. Shinjuki, Shinkōehō, leg. N. Konishi, Nor. 1902 ; Bunsanho, leg. T. Tashiro, Juni. 1899.

Distrib. Type: west central China.

This new variety differs from the type in haring spathulate bracts which are contracted a little ahore the middle portion. The cone is shorter and the wing of the seed is narrower. The leaf is 
acute or obtuse, but not truncate or emarginate as is the case with the type.

\section{Isuge CART.}

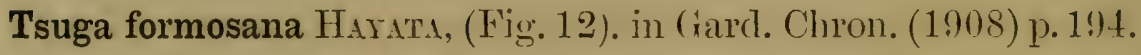
Ramuli novelli tenues glabri pallido-fuscentes. (xemme perulatx, perulis obtusis integris. Folia approximata distincte petiolata, petiolis brevibus semiteretibus parum incurvis, lincaria $8 \mathrm{~mm} .-16 \mathrm{~mm}$. longa $1 \frac{1}{2} \mathrm{~mm}$.- $2 \mathrm{~mm}$. lata apice obtusa vel emarginata integerrima

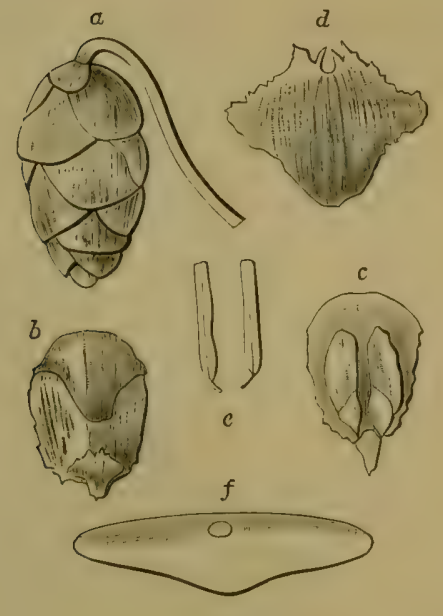

Fig. 12.

Tsugu fumosenz IFAтатA. "a cone $; b-$ a scale, seen from the outer sille; $c$ - the same, seen from the inser side; $d-$ - the bract of the same scale, magnified ; $e$ - leaves, seen from different siles; $f$ cross section of a leaf. glabra coriacea. Strobili ovoidei 2--2.3 $\mathrm{cm}$. longi $1.3 \mathrm{~cm}$. lati, squamis cire. 20 . Squamæ imbricatæ coriaceæ basi truncatæ sursum suborbiculares integræ $1 \frac{1}{2}$ $\mathrm{cm}$. longæ $1 \mathrm{~cm}$. latæ substriatæ pallidix fuscescentes. Bractex brevissimæ rhomboidex apice brevissime 2-lobatx irregulariter dentatæ. Semina parva obovata, $4 \mathrm{~mm}$. longa vel longiora, alis membranaceis tenuibus cultriformibus pallidoferrugineis $7 \mathrm{~mm}$. longis.

HAB. in monte Morrison, leg. R. TonII, 1900; Giyokusan, in montibus Morrison, ad 10634 ped. alt., leg. S. Nigasatwa, Nov. 1905, (Nos. 553 et 552); in monte Morrison, ad 8000 ped. alt., (No. 2364), et eodem monte, ad 9000 ped. alt., (No. 2110), leg. T. Kawakami et U. Mori, Oct. 1906.

Distrib. An allied species, T. diversifolia Maxni., occurs in Japan.

This T'uga very much resembles $T$. diversifolia in the shape of cones and bracts, but differs from it in the seeds having longei 
wings and in the glabrous branchlets. It also bears some resemblance to $T$. Sieboldi Carr., in the shape of the cones and secels, but is easily distinguished from the latter by the shape of the bracts, and by the shorter leaves. This new plant is, I think, just an intermediate form of $T$. Sieboldi and $T$. diversijolia.

Pseudotsuga CARr.

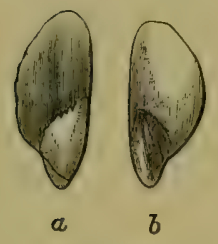

Fig. 13.

I'seutotsuga japonice Shirasatva. $a-$ a seed; $b$ - the same, seen from a different side.

Pseudotsuga japonica Shinasawa, (Fig. 13). in Tōkyō Bot. Mag. IX. p. 84; Hayata, in Tōkyō Bot. Mag. XX. p. 45 ; MAtsuxr. et Hayata, Enmm. Pl. Formos. p. 400 ; M. T. MLSTEns, in Journ. Linu. Soc. XXXVII. p. 424.

HAB. in monte Morrisón, leg. T. KawaKanI et U. Mori, Oct. 1906.

Distrib. Japan.

Abies Juss.

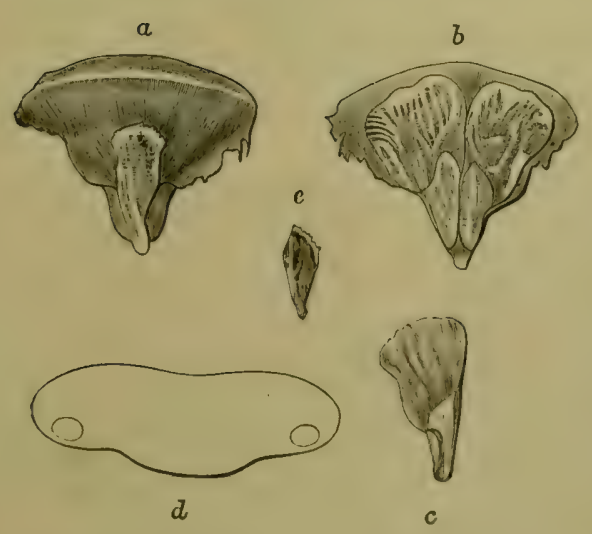

Fig. 14.

Abies Mariesii MLAst. var. Kuwolevmii MaY. TA. $a$ - a scale; $b-$ the same, seen from the inner sile ; $c-\mathbf{a}$ sead; $d$ - section of a lenf; $e-$ a seed, wing taken off.
Abies Mariesii Mast. var. Kawakamii HAYATA, n. v. (Fig. 14).

Abies Ilariesii Hayata, in MLatsum. et Hiyata, Enum. Pl. Formos. p. 400, (non MLAstens).

Ramuli ferruginei pilosissimi, pulvinis obovatis pauce prominentibus, cicatricibus depressis ovato-oblongis. Folia approximata late linearia a basi angustiora ad apicem sinsim dilata apice rotundato-obtusa et emarginata supra impressa sub- 
tus carinata inter carinam marginemque argentea stomatifera. Strobili laterali erecti orato-cylindracei apice retusi $7 \frac{1}{2} \mathrm{~cm}$. longi $4 \mathrm{~cm}$. lati. Biactere $\frac{2}{3}$ plo squamam in longitudine requantes obovatre angustie supra medium leviter constricte et transverse coloratie apice dilato-rotundatie mucronatx fracte ecostatx. Squamæ late rotundato-cuneatx $2 \mathrm{~cm}$. longæ $2 \frac{1}{2} \mathrm{~cm}$. latæ basi vix auriculatæ abrupte stipitate margine sursum integerrims deorsum fracte. Semina cum alis $18 \mathrm{~mm}$. longa, alis truncatis, seminibus alisque nigricantibus.

Hab. in monte Morrison, ad 11220 ped. alt., leg. R. Tonir, 1900 ; ibidem, leg. HoNda, 1896, (No. 98.); Seizan, in montibus Morrison, ad 11579 ped. alt., leg. S. Nagasawa, Nov. 1905, (No. 583.); in monte Morrison, ad 12000 ped. alt., leg. T. KawaKanI et U. Moni, Oct. 1906, (Nos. 2369 et 2372).

Distrib. An ally, A. Mariesii Mast., occurs in Japan.

This differs from the type in having longer cylindrical cones, black coloured wings and seeds; from A brachyphylla Maxns., this differs in having shorter cones and in the position of the resincanal lying close to the epiderm. 


\title{
Monocotyledones.
}

\author{
Orchidex.
}

The sfecies belonging to this family are about twenty in number, and must be very interesting ones. Owing to the lack of literature, I am at present obliged to put off the study of this family. The work of these orchids will be specially treated in the near future.

\section{Hæmodoraceæ.}

\section{Peliosanthes ANDR.}

Peliosanthes courtallensis W IGHT, Ic. Pl. Ind. or. t. 2051; B.trer, in Journ. Linn. Soc. XVII. p. 504; Hook. f. Fl. Brit. Ind. VI. p. 266.

Hab. Arizan, in montibus. Morrison, ad 8000 ped. alt., leg. G. Natahara, 1906 ; in monte Morrison, ad 6500 ped. alt., leg. T. Kawakami et U. Mori, Oct. 1906, (No. 2327).

Distrib. Travancore.

\section{Liliaceæ.}

\section{Smilacina DESF.}

Smilacina japonica A. Gray, Pot. Jap. p. 414; Mie. Prol. Fl. Jip. 1. 313 ; Franch. et Savat. Tnum. Pl. Jap. II. p. 53 ; M.sixns. Mrél. Biol. XI. p. 857 .

Smilacina Tirta Muxnr. Prim. FI. Amur. p. 276.

Smilacina japonice var. mandschurvice M..xñ. Mé́l. Biol. 1). XI. 1) 857. Tovaria japonica BaKen, in Journ. Linn. Soc. XIV. p. 570 ; WrighT', in Fonbes et HexsL. Ind. F1. Sin. III. p. 110.

HaB. in monte Morrison, leg. G. Namahati, Oct. 1905; in 
eodem monte, ad 13000 ped. alt., leg. T. Kawakasi et U. Monr, Oct. 1900, (No. 2384).

Dis'rrib. China and Japan.

\section{Tricyrtis WALL.}

Tricyrtis lasiocarpa MLatsex. in Tökyó Bot. Mag. XI. p. 79 ; MLıтusu. et Hiyata, Enum. Pl. Formos. p. 448.

Hab. Suizan, in montibus Morrison, ad 7703 ped. alt., (No. 730), et Ganzan, in isdem montibus, ad 9141 ped. alt., (Nos. 645 et 695), leg. S. NAg.sswa, Oct. 1905; in monte Morrison, ad 7000 ped. alt., leg. T. KAWАKaмi et U. Moni, Oct. 1906, (No. 2321).

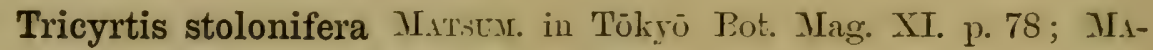
Tsux. et Hayata, Enum. Pl. Formos. p. 442.

НАв. in monte MLorrison, ad 9000 ped. alt., leg. T. KawakanI et U. Moni, Oct. 1906, (No. 2319).

\section{Metanarthecium МХХ̆.}

Metanarthecium foliatum Maxur. "Decas Pl. Nor. (1882) p. 10."

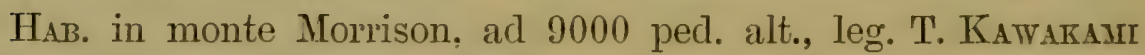
et U. Mori, Oct. 1906, (No. 2328) ; ibidem, leg. G. Naканara.

Distrib. Japan.

\section{Disporum SALISB.}

Disporum sp. nor.? Rhizoma repens crassum, caulibus simplicibus sursum foliatis. Folia alterna petiolata oblonga apice abrupte in acumen longum attenuata crassiuscula. Baccæ (ut videntur) ad axillas solitariæ longe pedunculatæ. Flores ignoti.

HAB. in monte Morrison, ad 8000 ped. alt., leg. T. Kawakain et U. Mori, Oct. 1906, (No. 2329).

Polygonatum ADAXs.

Polygonatum officinale AJ.L. var. Maximowiczii Fr.sycr. et S.tr.t. 
Maxim. in Mél. Biol. XI. p. 851 ; Patibix, Conspect. Fl. Koreæ, III. p. 10 ; Forbes et Hemst. Ind. Fl. Sin. III. p. 108 ; Matsunc. et Hayati, Enum. Pl. Formos. p. 436.

Polygonatum Maximoviczii Fr. Schxmd, Reis. Amux. p. 185, n. 449.

Polygonatum officinale ALL. $\%$ pluriflorum MiQ. Prol. Fl. Jap. p. 312.

HAB. in herbidis Taiton, ad 4000 ped. alt., leg. U. Fadrie, 1903, (No. 544).

Distrib. Type : extends from western Europe through Russia, Siberia and Mongolia to northern China and Japan. Variety: through Japan to Saghalien Manchuria and northern China.

\section{Paris Linv.}

Paris lancifolia Hıratı, (Pl. XXXIX.). in Tōkyō Bot. Mag. XX. p. 52. Rhizoma repens multinodosum. Caulis simplex circ. 20-50 $\mathrm{cm}$. longus glaberrimus. Folia ad apicem caulis 7-8 verticillata sessilia lanceolata vel lanceolato-angusta acuminata circ. $12 \mathrm{~cm}$. longa $1 \mathrm{~cm}$. lata 1-nervia, nervis superne impressis subtus prominentibus. Flores ad apicem caulis inter folia rerticillata solitarii longe pedunculati exserti, pedunculis $7 \mathrm{~cm}$. longis erectis. Segmenta perianthii distincta $\tilde{5}$-mera 2-seriata, exteriora patentissima herbacea lanceolata acuminata $5-6 \mathrm{~cm}$. longa $7-8 \mathrm{~mm}$. lata supra basin leviter contracta, interiora filiformia $2 \frac{1}{2}-3 \mathrm{~cm}$. longa. Stamina $8-10$, filamentis ovarium vix superantibus. Ovarium depresso-globosum apice truncatum concavum marginibus prominentibus ad apices carpellorum paullo cornutum, 1-loculare $\check{y}$-placentiferum, stylo כ-fido, ramis recurvis. Bacce depresso-globosa 12 mm. longæ 1.j $\mathrm{mm}$. in diametro oligospermæ.

HAB. Ganzan, in montibus Morrison, ad 9141 ped. alt., leg. S. Nigasiwa, Oct. 190);, (No.693); in monte Morrison, ad 9000 ped. alt., leg. T. Kawamam et U. Moni, Oct. 1906, (No. 1953). 


\section{Commelinaceæ.}

Aneilema R. Br.

Aneilema divergens C'trikT, in Commel. et Cyrt. Beng. t. 16, et in DC. Monogr. Phanerog. III. p. 203; Hook. f. Fl. Brit. Ind. VI. p. 376 ; Forbes et Henst. Ind. Fl. Sin. III. p. 151; Matsux. et Hayata, Enum. Pl. Formos. p. 446.

Aneilema herbaceum Kuxth, var. divergens ClariKe, in Jomn. Linn. Soc. XI. p. 448.

Hab. in monte Morrison, ad 6000 ped. alt., leg. T. KatwaKani et U. MonI, Oct. 1906, (No. 2326).

Distrib. India and South China.

\section{Cyanotis Dox.}

Cyanotis arachnoidea (TAfiKe, in DC. Monogr. Phanerog. III. p. 250 ; Ноoк. f. Fl. Brit. Ind. VI. p. 386 ; Henry, List P1. Formos. p. 99; Fonbes et Hemsi. Ind. Fl. Sin. III. p. 157; Matsums. et Hayata, Enum. Pl. Formos. p. 449.

Cyanotis pilosa Wight, Ic. Pl. Ind. or. t. 2083.

HAB. in monte Morrison, ad 3000 ped. alt., leg. T. KawaraniI et U. Monr, Oct. 1906, (No. 2325).

Distrib. India and Malay.

\section{Juncaceæ.}

\section{Lusula DC.}

Luzula effusa Buch. in ENGL. Bot. Jarhb. VI. p. 196, et XII. p. 106 ; Hoor. f. Fl. Brit. Ind. VI. p. 401 ; Drets, Fl. Centr. Chin. p. 237, et Fl. Tin ling shan, in ExGi. Bot. Jahrb. XXXIV. Beibl. p. 17; Forbes et Hexst. Ind. Fl. Sin. III. p. 161.

Hab. in monte Morrison, leg. G. Narahara, Oct. 1905 ; eodem 
monte, ad 12500 ped. alt., leg. T. KawakanI et U. Mori, Oct. 1906, (No. 2381).

Distrib. Central China and Himalaya.

Luzula spicata DC.; Hоoк. f. Fl. Brit. Ind. VI. p. 401 ; BucH. in ENGL. Bot. Jahrb. XII. p. 128 ; Sowerby, Engl. Bot. X. p. 1553.

HAB. in montibus Morrison, ad 12000 ped. alt., leg. T. Kawaramir et U. Mori, Nov. 1906, (No. 2300) ; ad summam ejusdem montis, ad 13094 ped. alt., leg. S. Nagasawa, Nov. 1905̃; (No. 599).

Distrib. Widely spread in the alpine regions of Europe, and also in Himalaya and North America; generally in the aretic Zone.

\section{June"s Lins.}

Juncus effusus Linv. Sp. Pl. ed.-2, p. 464; Кехтн, Enum. Pl. III. p. 320 ; Buch. in Evgl. Bot. Jalrrb. XII. p. 22S ; "Fravehet, Pl. David. II. p. 137 "; Ноок. f. Fl. Brit. Ind. VI. p. 392 ; Drels, Fl. Centr. Chin. p. 238; Forbes et Hexsi. Ind. FI. Sin. III. p. 163.

HAB. in montibus Morrison, ad 9000 ped. alt., leg. T. KaWAKAגI et U. Moni, Oct. 1906, (No. 1821); Ganzan, in montibus Morrison, ad 9141 ped. alt., leg. S. Nagasawa, Oct. 1906, (No. 677).

Distrib. Asia, America, Africa, Anstralia and Europe.

Juncus Maximowiczi Buch. in EXgit. Bot. Jahrb. XII. p. 394. Hab. in montibus Morrison, leg. G. Nakahara, Oct. 1905. Distrib. Japan.

\section{Aroideæ.}

Arisæma sp. (A. consanguineum Scrotr?)

HAB, in montibus Morrison, ad 7000 ped. alt., leg. 'T. KawaTiмII et U. Молі, Oet. 1906, (No. 2330). 
Alocasia (macrompliza Sснотт?)

Нав, in Kagi: Burokusha, leg. T. Kawarami et U. Moni, Oct. 1906, (No. 1752).

\section{Cyperaceæ.}

\section{Bulbostylis KUNTH.}

Bulbostylis capillaris Krxth var. trifida CLmine (Fig. 15), in Hоок. f. Fl. Brit. Ind. VI. p. 652 ; Fonbes et Hexss. Ind. Fl. Sin. III. p. 248 ; Makixo, in Tókyō Bot. Mag. IX p. 390.

Bullostylis trifida Kuxth, Enum. Pl. II. p. 213.

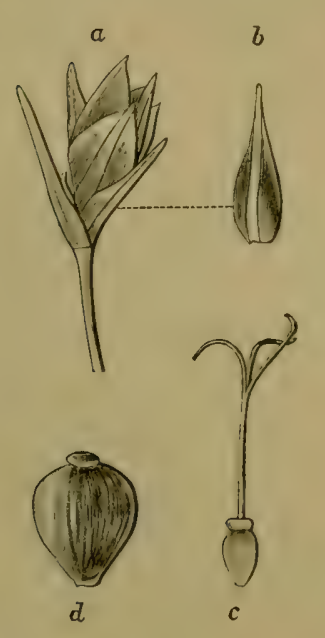

Fig. 15.

Bulbostylis capillaris КUхтн Tar. trifida Clarke. a- a spikelet ; $b-$ a glume ; $c-$ an ovary; $d-$ a seed.

Scirpus trifidus HANCE, in Journ. Bot. (1878) p. 112.

HАв. in monte Morrison, ad 8000 ped. alt., leg. T. Kawakami et U. Mori, Oct. 1906, (No. 1840).

Distrib. In tropicaI warm countries; very common in central and southern China and also in southern parts of Japan.

In the present specimens, the spikelets are very small, glumes boat-shaped, lanceolate, ovate, maculate a little above the middle portion, carinate and bearing a small bristle at the apex. Nuts truncate, oborate, longitudinally maculate. (Fig. 15.)

\section{Scirpus LixN.}

Scirpus morrisonensis Ilarata, sp. nov. (Fig. 16). Culmi plus minus fasciculati gracillimi rigiduli erecti $40-50 \mathrm{~cm}$. alti basi $1 \mathrm{~mm}$. in sectione striati teretes, raginis inferioribus scariosis oratis brevibus brumeis superioribus elongatis membranaceis transrerse 
truncatis in laminam brevem productis, laminis lanceolatis minute serrulatis. Spicula quasiterminalis oblongo-elongata leviter arcuata pallido-fusca obtusa parum compressa circ. 10-flora circ. $1 \mathrm{~cm}$. longa $0.7 \mathrm{~mm}$. lata, squamis arcte ímbricatis omnibus fertilibus mem-

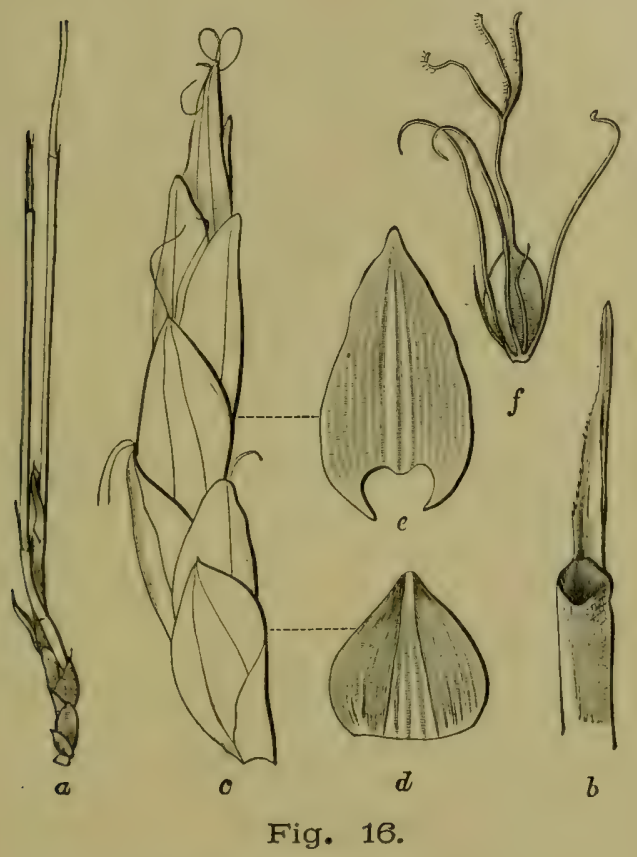

Seirpus morrisonensis Harata.

$a$ - the basal portion of caules; $b$ - a sheath; $c-a$ spikelet; $d-a$ scale detached from the basal portion of the same spikelet; $e-\Omega$ scale detached from the middle portion of the same; $f$ - a flower.

branaceis obtusis obscure carinatis superioribus ovatis basi breve decurrentibus inferioribus late ovatis basi truncatis, nervo mediano infra apicem evanido, versus apicem fusco-castaneis marginibus pallidis, binis inferioribus latioribus brevibus. Achænium obovatum v. obovato-ellipsoidale trigonum $1 \frac{1}{2} \mathrm{~mm}$. longum glabrum læve fusco-rubrum, stylo persistenti exserto apice $2-3$ fido, setis 6 capillaribus achænio brevioribus.

$\mathrm{H}_{\mathrm{AB}}$, in monte Morrison, ad 10000 ped. alt., leg. T. Kawakani et U. Mori, Oct. 1906, (No. 1843).

\section{Cavex Lins.}

The following five species were kindly examined by the Rer. Kuekenthal. Owing to the imperfectness of the specimens, as he wrote me, the identification is not easy one. Nerertheless, the rescriptions of the plants here giren may be of some interest. 
Carex sp. (aff. C. breviculmi R. BR. subsp. Royleana (NEEs) KueK.). Culmi 5-8 cm. longi firmi scabridi apice 2-3 spicas gerentes. Folia $5-7 \mathrm{~cm}$. longa $2 \mathrm{~mm}$. lata culmo breviora firma subtus carinata glaucescentia apice sæpe circinnato-curvata basi vaginata, vaginis $15 \mathrm{~mm}$. longis. Spicæ 2-3; terminalis masculina teres $7 \mathrm{~mm}$. longa $1 \mathrm{~mm}$. lata sæpe basi attenuata, squamis ovatis basi truncatis apice acutis $6 \mathrm{~mm}$. longis $2-3 \mathrm{~mm}$. latis subtrinerviis, nervis castaneis; reliquiæ fœmineæ subsessiles paucifloræ 8-9 mm. longæ, squamis late ovatis acuminatis late 1nerviis, nervo producto castaneo, partibus marginibus hyalinis. Utriculus 2-costatus ad costas minute denticulatus ovatus rostratus ore obscure bidentatus glaber. Achænium apice contractum triquetrum 3-costatum. Styli basi conico-crassati. Stigma 3-fidum.

Haв. in monte Morrison, ad 13000 ped. alt., leg. T. Kawakami et U. Mori, Oct. 1906, (No. 2383).

This Carex is very small in its habit and a floriferous culm has a very few spikes on its top. Short branches are sometimes seen at the basal portion of culms.

Carex sp. nov.?

Hab. in monte Morrison, ad 12000 ped. leg. T. Kawarami et U. Mori, Oct. 1906, (No. 2385).

The Rev. KuekenthaL informs me that this plant may be a species not yet described; but the specimen is too imperfect to draw a description of it.

Carex sp. (aff. C. Makinoensi Franch.). Rhizoma? Folia fasciculorum 20-30 cm. longa $2 \mathrm{~mm}$. lata culmo florigero paullo breviora basi vaginata, raginis $2-3 \mathrm{~cm}$. longis, oribus truncatis, supra et margine scabrida. Culmi florigeri graciles $30 \mathrm{~cm}$. longi 
3-4 foliati, foliis superioribus basi vaginatis laminis spica æquilongis. Spicæ 3-4, remote dispositæ; terminalis masculina elongata pedunculata $3 \mathrm{~cm}$. longa $2 \mathrm{~mm}$. lata, squamis obovatis vel late cuneatis marginatis, marginibus badio-fuscis; reliquia fœmineæ elongatæ, $2 \mathrm{~cm}$. longæ spica masculina latiores breve pedunculate erectre, floribus remotis, squamis ovatis marginatis acutis basi truncatis. Utriculus 2-carinatus multinervius pubescens, compressus apice rostratus distincte 2-dentatus. Stigma 3-fidum. Achænium trigonum 3-costatum glabrum apice leviter in collum brevissimum coronatum.

НАв. in monte Morrison, ad 9000 ped. alt., leg. T. KawakanI et U. Mori, Oct. 1906, (No. 2380).

I am informed by the Rev. Kuenexthat that this Carex have same resemblance to C. Makinoensis Franch.

Carex sp. (aff. C. tristachya Thunb.). Rhizoma tenue repens, culmos florigeros graciles et fasciculas steriles ad apicem rhizomatis emittens. Folia fasciculorum culmis paullo longiora $17 \mathrm{~cm}$. longa 3-4 mm. lata ntraque pagine et margine scabrida. Culmi florigeri graciles circ. $15 \mathrm{~cm}$. longi foliis brevibus instructi ; foliis superioribus longe raginatis in laminam longam vel brevem desinentibus. Spice $3-4$; terminalis masculina tenuissima breve pedunculata $15 \mathrm{~mm}$. longa $0.5 \mathrm{~mm}$. lata, squamis obovatis basi truncatis marginilus membranaceis subtrinerviis apice tenuiter ciliatis et brevissime mucronatis; reliquix fominex pedunculate lineares laxifloræ 1-2 $\mathrm{cm}$. longe, squamis pallidis late ovatis ntriculo paullo brevioribus, marginibus hyalinis subtrinerviis, nerro merlio ultra apicem in mucronem breviter producto. Utrieulus pubescens sub maturitate $3 \mathrm{~mm}$. longus tenuiter membranaceus obovatus breve stipitatus 2-costatus, multinervatus distincte rostratus, rostro bifido, ore 
scabro. Stigma trifidum. Achrnium pallidum glabrum trigonum 3-costatum stipitatum apice abrupte contractum in collum brevissimum coronatum, disco coriaceo albido styli basi conica emarcida superato.

HAB. in monte Morrison, ad 7000 ped. alt., leg. T. KawaKamI et U. Mori, Oct. 1907, (No. 1846).

This Carex somewhat resembles C. pseudo-conica Franch. et Sirat., but differs from it in the contracted apex of the achænium possessing collar-like body at the base of the style. The Rer. Kuekenthal informs me that the plant is like $C$. tristachya Thuns.

Carex sp. nov. (aff. C. japonica Thuvb.).

HaB. in monte Morrison, ad 10000 ped. alt., leg. T. KaWAKAMI et U. Mori, Nov. 1906, (No. 2998).

The Rev. Kuekenthal informs me that this Carex may be a species not yet described. It is not, however, advisable to draw a description from such an imperfect specimen.

\section{Gramineæ.}

Tsachme R. Br.

Isachne Clarkei Ноок. f. Fl. Brit Ind. VII. p. 24.

Hab. in monte Morrison, ad 8000 ped. alt., leg. 'T. Kawakanr et U. Monr, Oct. 1906, (No. 1839).

Distrib. Himalaya.

\section{Panicum Lixr.}

Panicum montanum lioxis. Fl. Ind. ed.-Carex, I. p. 313 ; Kuxth, Euum. Pl. I. p. 126 ; Bexth. Fl. Hongk. p. 412 ; Ноок. f. Fl. Brit. Ind. VII. p. 53; Fonbes et Hexsu. Ind. Fl. Sin. III. p. 331 ; MennmL, in Philipp. Journ. Scie. I. Supp. Bot. p. 27. 
Hab. in monte Morrison, ad 6500 ped. alt., leg. T. Kawakami et U. Mori, Oct. 1906, (No. 1848).

Distrib. South China, the Nalay archipelago, and from Ceylon northward to the mountains of India.

\section{Oplismenus BEAUT.}

Oplismenus undulatifolius BE.ut. var. imbecillis H.ıck.; MEnnLI, in Philipp. Journ. Scie. I. Suppl. Bot. pp. 28, et 364; HayatA, in Tōkyō Bot. Mag. XXI. p. 50.

HaB. in monte Morrison, ad 5000 ped. alt., leg. T. KawaKani et U. Mori, Oct. 1906, (No. 1845).

Distrib. Type: Japan, China and the Himalayas. Variety: the Malay archipelago.

\section{Arundinella RADDI.}

Arundinella setosa Trix.; Bexth. Fl. Hongk. p. 416 ; Hook. f. Fl. Brit. Ind. VII. p. 70 ; HACK. in Bull. Herb. Boiss. VII. (1899) p. 723 ; Fonbes et Hemsi. Ind. Fl. Sin. III. p. 342 ; Mratsur. et Hayata, Enum. Pl. Formos. p. 515.

Hab. in monte Morrison, ad 8000 ped. alt., leg. T. KawaKanII et U. Moni, Oct. 1906, (No. 184i).

Distris. South China, the Philippine islands, India and Ceylon.

\section{Miscanthus ANDERss.}

Miscanthus sinensis AxDlliss. var. formosanus ILıck. in 13ull. Herk.

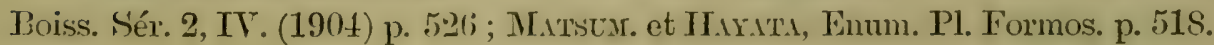

Hab. in monte Morrison, ad 8000 ped. alt., leg. T. Kawakami et U. Mori, Oct. 1906, (No. 1826) ; in montibus centralibus, leg. T. Kawatimi et U. Moni, Nov. 1906, (No. 2366).

Distrib. Type: Japan, China and the Malay archipelago. 


\section{Saccharum Lins.}

Saccharum Narenga Hıм.; НАск. Monogr. Androp. p. 119 ; Ноок. f. F1. Brit. Ind. VII. p. 120 ; Dets, Fl. Centr. Chin. p. 222 ; Fonbes et Hexss. Ind. Fl. Sin. III. p. 349 ; Matsuxr. et Hayata, Enum. PI. Formos. p. 519. Saccharum porplyyrocomum HAck. Monogi. Androp. p. 120.

Eriochrysis Narenga NeEs, ex Steud. Syn. Gram. p. 411.

Hab. Nantō: Horisha, leg. T. Kawakani et U. Mori, Nov. 1906, (No. 2379).

Distrib. India, Burma, and Sonth China.

\section{Spodiopogon TRin.}

Spodiopogon Kawakamii Hıyıtì, in Tōkyō Pot. Mag. XXI. p. 5 t. Нав. Kagi: Tappansha, leg. T. Kawakani et U. Mori; Oct. 1906, (No. 1837).

Spodiopogon tainanensis H.ı.tT, in Tökyō Lot. Mag. XXI. p. 53. Haв. Kagi : Kishirei, leg. T. KawaKani et U. Moni, Oct. 1906 , (No. 1855).

\section{Pollinire Trin.}

Pollinia ciliata Trn. ; Steud. Syn. Gram. p. 410 ; HAcr. Monogr. Autrop. p. 176, et in Bull. Herb. Roiss. VIr. (1899) p. 723 ; Hoor. f. Fl. Brit. Ind. VII. p. 116 ; Fonbes et Hearsu. Ind. Fl. Sin. III. p. 354.

var. Wallichiana HAck. Monogr. Androp. p. 177, et in Bull. Herb. Boiss. VII. (1899) p. 723 ; Matsun. et HAYATA, Enum. Pl. Formos. p. 521.

Нав. in monte Morrison, ad 6500 ped. alt., leg. T. Kawakami et U. Mori, Oct. 1906, (No. 1848).

Distrib. South China, India, and Nalay.

\section{Cymbopogon SPRENG.}

Cymbopogon Nardus liexdre subsp. marginatus var. Gœringii 
Riexdle, in Forbes et Hemsi. Ind. Fl. Sin. III. p. 376 ; Matsumr. et Hayata, Enum. Pl. Formos. p. 531.

Andropogen Nardus Lins: var. Goeringii Hick. Monogr. Androp. p. 607, et in Bull. Herb. Boiss. VII. (1899) p. 642, et Sér. 2, III. (1903) p. 501; PaLibin, Conspect. FI. Koreæ, III. p. 30.

Hab. in monte Molrison, ad 8000 ped. alt., leg. T. KawaKani et U. Mori, Oct. 1906, (No. 1841).

Distris. The Philippine islands, South China, and Japan.

\section{Agrostis LinN.}

Agrostis Clarkei Hоoк. f. Fl. Brit. Ind. VIr. p. 257 ; Haystı, in Tōkyō Bot. Mag. XXI. p. 52.

HАB. in monte Morrison, ad 12500 ped. alt., Oct. 1906, (No. $2374)$; in montibus centralibus, leg. T. Kawakami et U. Moni, Nov. 1906, (No. 2365).

Distrib. The Himalayas.

Calamagrostis ADANs.

Calamagrostis arundinacea Roтн ; HАхсе, Jouru. Bot. (1878) p. 284; HAck, in Bull. Herb. Bioss. VII. (1899) p. 652, et Sér. 2, III. (1903) p. 502. Deyeuxia sylvatica Кчмтн, Enum. Pl. I. p. 243 ; Ноок. f. Fl. Brit. Ind. VII. p. 266 ; Fonbes et Hexsi. Ind. Fl. Sin. III. p. 395.

Hab. in monte Morrison, ad 8000 ped. alt., leg. T. KawaKani et U. Mori, Oct. 1906, (Nos. 1838 et 1820).

Distrib. Japan, the Himalayas, Temperate Asia, and Europe. var. nipponica HAck. in Bull. Herb. Boiss. VII. (1899) p. 652, et Sér. 2, IV. (1904) p. 523.

Calamagrostis nipponica Franch. et Sarat. Enum. PI. Jap. II. p. 168 et 599.

Hab. ad monte Morrison, 12000 ped. alt., leg. 'I. Kawaram et U. Mori, Oct. 1906, (No. 2373).

Distris. Japan and the Philippine islands ? 


\section{Deschampsia BEAUV.}

Deschampsia cæspitosa BEaur.; Hick. in Bull. Herb. Boiss. VII. (1899) p. 703 ; MLAxur. Prim. Fl. Amur. p. 323 ; Hoor. f. Fl. Brit. Ind. VI. p. 273 ; Fonbes et Hearsu. Ind. Fl. Sin. III. p. 399.

Aira ccespitosa Linn.; Strud. Syn. Gram. p. 219.

Haв, in monte Morrison, ad 12500 ped. alt., leg. T. KawaKanI et U. Mori, Oct. 1906, (No. 2375).

Distrib. Central China, Japan, Himalaya; generally in the temperate and cold regions of the northern and southern hemispheres; and also in the alpine regions in the Tropics.

Deschampsia flexuosa Trix.; Leder. Fl. Ross. IV. p. 420 ; H.tck. in Bull. Herb. Boiss. VII. (1899) p. 702.

Airce flexuosa Lisns. var. montance Franch. et Savat. Enum. Pl. Jap. II. p. 172.

HaB. in monte Morrison, ad 13000 ped. alt., leg. T. KawakanI et U. Mori, Oct. 1906 ; in montibus centralibus, leg. T. KawaKanI et U. Mori, Nov. 1906, (No. 2364).

Distrib. Japan; the arctic regions of Asia and Eirope.

\section{Trisetum PERS.}

Trisetum subspicatum Beadv; STeud. Syn. Gram. p. 225 ; Hack. in Bull. Herb. Boiss. VII. (1899) p. 703 ; Fonbes et Henss. Ind. Fl. Sin. III. p. 400 ; Вемтн. FJ. Austral. VII. p. 588 ; MaкıNo, in Tōkyō Bot. MIag. XX. p. 44.

Avena subspicata Clannv.; Hook. f. Fl. Brit. Ind. VII p. 278; Thoné, Fl. Dent. Öst. Sch. I. p. 145.

Нав. in monte Morrison, ad 12500 ped. alt., leg. T. Kawakani et U. Mori, Oct. 1906, (No. 2378).

Distris. The Himalayas, central China and the Kurile islands ; generally in the alpine and frigid regions. 


\section{Arumdo LinN.}

Arundo formosana HAck. in Bull. Herb. Boiss. VII. p. (1899), p. 724 ; Fonbes et Hexss. Tud. Fl. Sin. III. p. 408; Matsum, et Hayata, Enum. Pl. Formos. p. 540.

HАв. Taitō: Taiwnkōsha, leg. T. Kawakaıi et U. Moni, Nov. 1906, (No. 2305).

Distrib. An endemic plant.

\section{Brachypodium BEAUV.}

Brachypodium Kawakamii HAXATג, (Pl. XL.) in T'ōkyō Bot. Mag. XXI. p. 51. Perennis ascendens subcæspitosa circ. $20 \mathrm{~cm}$. alta. Folia convoluto-teretia, laminis $5-6 \mathrm{~cm}$. longis $6-7$-nerviis, extus glaberrimis intus scabris pance hirsutis, vaginis $2 \mathrm{~cm}$. longis, ligulis latioribus brevibus leviter ciliolatis. Spiculæ pancæ sæpe ad unam terminalem reductæ longe pendunculatæ sæpe cermux, pedunculis filiformibus, 6-7-floræ compressæ $2 \mathrm{~cm}$. langæ $3 \mathrm{~mm}$. latæ, rhachillis inter flores articulatis hirsutissimis ; floribus hermaphroditis sæpe superioribus imperfectis. (Alumæ. 2 inferiores racuæ 7-nerviæ florentibus minores et breviores muticæ subglabræ; gl. [I.] 7-mm. longa ; gl. [II.] longior. (iluma florens rigidula angusta dor'so rotundata 7-9-nervia integra in aristam rectam $4 \mathrm{~mm}$. longam desinens; palea gluma vix brevior $7 \mathrm{~mm}$. longa latiuscula ㄴ-carinata, carinis ciliatis, apice truncata et emarginata. Stamina 3. Lorlicule 2 oblongæ obtusa basi oblique stipitatx margine ciliolatre, lateribus interioribus basi callosis. Ovarium obovatum apice appendicula brevi villosa coronatum. Styli longinsenli, stigmatibus laxe plumosis. Achenia angusta oblonga a dorso compressa antice late sulcata palea adherentia.

$\mathrm{H}_{\mathrm{AB}}$. in monte Morrison, ad 12500 ped. alt., leg. T. KawaKAMII et U. Мов, Oct. 1906, (No. 2377). 
As a complete description of the present species was not previously made, I hare taken this occasion to give a full account of the plant. It is perhaps the smallest species of Brachypodium, which species is very remarkable for its terete leaves and its simplest form of an inflorescence reduced into one spicule. The leaves are very slender and they measure but $1 \mathrm{~mm}$. in diameter, and $3 \mathrm{~mm}$. in circumference.

Brachypodium sylvaticum Beatr.; Mro. Prol. Fil. Jap. p. 174; Franch. et Savat. Enum. Pl. Jap. II. p. 185; Hack. in Bull. Herb. Boiss. XII. (1899), p. 714 ; Hook. f. Fl. Brit. Ind. VII. p. 363 ; Ledeb. Fl. Ross. IV. p. 346 ; Fories et HeMst. Ind. Fl. Sin. III. p. 431.

HAB. in monte Morrison, ad 12000 ped. alt., leg. T. KaWATAMI et U. Mori, Nov. 1906, (No. 2302).

Distrib. North Asia, and the mountains of India and Europe.

\section{Festuca Lins.}

Festuca ovina Lins.; MirQ. Prol. Fl. Jap. p. 170 ; Fraxch. et SAvat. Enum. Pl. Jap. II. p. 181; Thomé, Fl. Deut. Öst. Sch. I. p. 114, t. 53 ; Wagner, Deut. Fl. ed-3, p. 82 ; Forbes et Herrst. Ind. FI. Sin. III. p. 429 ; var. vulgaris Kocr ; HAck. in Bull. Herh. Boiss. VII. (1899) p. 713, et Sér. 2, III. (1903), p. 506; Hayata, in Tōkyō Bot. Mag. XXI. p. 51.

$\mathrm{H}_{\mathrm{AB}}$. in monte Morrison, ad 12500 ped. alt., leg. T. KawaKaMI et U. MonI, Oct. 1906, (No. 2376).

Distrib. North Asia; Japan and China.

\section{Amundinaria МicH.}

Arundinaria niitakayamensis HAYATı, in Tōkyō Bot. Mag. XXI p. 49. HАв. in monte Morrison, ad 9000 ped. alt., (No. 1842), et ad 8500 ped. alt., (No. 1849), leg. T. Kawakami et U. Moni, Oct. 1906. 


\title{
Cryptogamiæ.
}

\author{
Lycopodiaceæ.
}

\section{Lycopodium LrNx.}

Lycopodium clavatum Lisx. Sp. Pl. ed.-2, p. 1564; Hook. Brit. Fern. t. 49 ; Baker, Fern All. p. 26 ; Thuns. Fl. Jap. p. 341 ; Mro. Prol. Fl. Jap. p. 348 ; Maxir. in Mél. Biol. VII. p. 341 ; Franch. et Savat. Enum. Pl. Jap. II. p. 197 ; Luerss. in Engu. Bot. Jahrb. IV. p. 366 ; DieLs, Fl. Centr. Chin. p. 210 ; Ware. Mons. I. p. 97 ; Hayata, in Tōkyō Bot. Mag. XX. p. 20.

Hab. in monte Morrison, leg. S. Nagasawa, T. Kawairame et G. Nakahara, Nov. 1905.

DistrIB. West and East China and Japan; aretic and alpine zones of both hemispheres; also mountains of Tropical Asia, Africa and America.

Lycopodium complanatum Irxx. Sp. Pl. ed.-2, p. 15fit; BAKEl, Fern All. p. 28 ; DieLs, Fl. Centr. Chin. p. 210.

var. Chamæcyparissus A. Br. " in DeLL, Rhein Flora p. :36”; I3..ker, Fem All. p. 29 ; Maxnr. in Mél. Biol. VII. p. 341 ; Franch. et Sarat. Enum. P1. Jap. II. p. 198; HAYaTA, in Tōkyō Rot. Mag. XX. p. 21.

Hab. in monte Morrison, leg. S. Nagasawa, T. KawaKami et G. Nakahara, Nov. 1905.

Distrib. Japan and central and southern China ; generally in the temperate zone of both hemispheres; also in some tropical regions of Asia and America; subcosmopolitan.

Lycopodium obscurum L_Nx: Sp. P1. ed-2, p. 1566; 13.kikli, Fern 
All. p. 24 ; Hayatג, in Tōkyō Bot. Mag. XX. p. 21; Kovanov, Fl. Manshur. I. p. 159 .

Lycopodium japonicum Thunb. Fl. Jap. p. 341 ; Maxnм. in Mél. Biol. VII. p. 341 ; Franch. et Savat. Enum. Pl. Jip. II. p. 197.

Lycopodium dendroideum Мrснх. ; Мro. Prol. Fl. Jap. pp. 348 et 390 ; Hoor. Exot. Fern. t. 7.

Hab. in monte Morrison, leg. T. Kawakam, S. Nagasawa et G. Nathahara, Nov. 1905.

Drstriв. Japan, Kamtchatka, Siberia, Manchuria, and North America. Not yet known from central and southern China.

Lycopodium serratum Thuns. Fl. Jap. p. 341 ; A. Gnir, Bot. Jap. pp. 422 et 436 ; Miq. Prol. Fl. Jap. pp. 348 et 390 ; Baken, Fern All. p. 12 ; Maxmr. in Mél. Biol. VII. p. 341 ; Drels, Fl. Centr. Chin. p. 210 ; Warb. Mons. I. p. 96 ; Hayata, in Tōkyō Bot. Mag. XX. p. 20.

HaB. in monte Morrison, ad 13094 ped. alt., leg. S. NAGASAWA, (No. 735) ; ibidem, leg. T. Kawarami et G. Nakahara, Nov. 1905.

Distrib. In the tropical or subtropical regions, and in some temperate countries as Japan and China.

This Morrison plant is of a form having much broader leaves than the Japanese species.

\section{Polypodiaceæ.}

\section{Polystichum Rотн.}

Polystichum amabile Sxr. Diels, in Nat. Pfl.-fam. I-1, p. 193; Matsum. et Hayata, Enum Pl. Forms. p. 582.

Aspidium amabile Blume; Metr. in Anu. Mus. Bot. Lugd.-Bat. I. p. 227 ; MrQ. Prol. Fl. Jap. pp. 340 et 389 : Ноoк. Sp. Fil. IV. p. 25, t. 225 ; Hook et Baker, Syn. Fl. p. 254; Franch. et Savat. Enum. Pl. Jap. II. p. 232. 
HAB. Suizan, in montibus Morrison, ad 7702 ped. alt., leg. S. Nagasaiva, Oct. 1905, (No. 659).

Distrib. Japan and central and eastern China and the Malay archipelago.

Polystichum niitakayamense HAYAtı, (Pl. XLI.) in 'Tōkyō Bot. Mag. XXI. p. 14.

Stipites 10-12 cm. longi fusco-pallidi paleacei leviter canaliculati basin teretes, paleis oblongis vel linearibus. Frondes 25-30) $\mathrm{cm}$. longæe $2 \mathrm{~cm}$. latæ erectæ circumscriptione lineares pinnatæ, pinnis $7-8 \mathrm{~mm}$. longis $5 \mathrm{~mm}$. latis approximatis horizontaliter patentibus oblongis vel oblongo-quadrangularibus angulo inferiore affixis, basi superiore transverse truncatis auriculatis, basi superiore et apice aristatis, margine obscure crenulatis. Indusium 0. Sporangium fuscum longe pedicellatum. Sporæ oblongæ tuberculatæ.

HaB. Ganzan, in montibus Morrison, ad 9141 ped. alt., leg. S. Nagasatwa, 1905, (No. 698).

\section{Asplenium Lĩns.}

Asplenium laciniatum Don. "Prodr. Fl. Nep. p. 8"; Hoor. Sp. Fil. III. p. 164, t. 200, A ; Ноок. et Baker, Syn. Fil. p. 211 ; Cl.inke, Rev. Fern. North Ind. p. 481 ; Bedd. Fern. South Ind. p. 49, t. 145; Hirata, in 'Tōkyō Bot. Mag. XXI. p. 12.

HAB. Suizan, in montibus Morrison, ad 7702 ped. alt., leg. S. Nagasawa, 1905, (No. 656).

Distrib. The temperate regions of the IImalayas and Japan. Not yet found in the Philippines or China.

Asplenium Trichomanes IIINN. ; Iook. Sp. Fil. III. 1). 131; ot Brit. Fern. t. 29; МЕтт. in Anu. MIns. Bot. Lugd.-Bat. II. p. 234; Мro. Prol. Fl. Jap. p. 337 ; Jook. et Baker, Syn. Fil. p. 196 ; Christ, Farn. Erd. p. 192 ; Bedd. Fern. Sonth Ind. p. 49, t. 147; Hayatı, in Tökyō Bot. Mag. XXI. p. 14; Diels, 11. Contr. Chin. p. 198. 
Asplenium anceps Sor; Ноoк. et Grev. Ic. Fil. t. 195.

Hab, in monte Morrison, leg. G. Nakahara, 1906.

Distrib. Subcosmopolitan in the temperate and cold regions of both hemispheres.

\section{Coniogramme FÉE.}

Coniogramme fraxinea (Don.) Fís; Diels, in Nat. Pfl.-fam. I.-4. p. 262; Copeland, Folyp. Philipp. p. 66 ; Hayata, in Tōkyō Bot. Mag. XXI. p. 15.

Gymnogramme jaranica Buune, Fl. Jav. II. p. 95, t. 41 ; Hoor. et Baker, Syn. Fil. p. 381; Mte. Prol. Fl. Jap. p. 335 ; Franch. et Sarat, Euum. Pl. Jap. II. p. 248 : Henry, List Pl. Formos. p. 116.

Hab. Sanchōkei, leg. S. Nagasawa, 1905, (No. 721).

Distris. Tropics in the old world.

\section{Plagiogyria Kunzì.}

Plagiogyria glauca (Budme) MEтt. Pligiog. p. 273; Bedd. Fern. Brit. Ind. t. 90.

Lomaria glanca Blune, "Enum. Fl. Javil. Fil. p. 204"; Clarke, Rev. Ferm. North Ind. p. 472; Ноок. Sp. Fil. III. p. 22; Hooк et Baker, Syu. Fil. p. 182.

rar. philippinensis Christ, in Bull. Herb. Boiss. VI. (1898) p. 151; Coperand, Polyp. Philipp. p. 98 ; Hayata, in Tökyō Dot. Mag. XX. p. 22.

Hab. Ganzan, in montibus Morrison, ad 9141 ped. alt., leg. S. NAGASAWA, 1905 .

Distrib. The Philippine islands.

Plagiogyria Matsumureana Makino, in Tơkjō Bot. Mag. VIII.

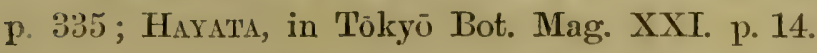

Lomaria Mlatsumureana Makino, in Tōkyō Bot. Mag. VIII. p. 90.

Hab. Rakurakusha, leg. G. Nakahara, 1905, (No. 458).

Distrib. Japan. 
Pteridium GLED.

Pteridium aquilinum Kunv. var. lanuginosum Boriy Copelind, Polyp. Philipp. p. 104; Hayata, in Tōkyō Bot. Mag. XXI. p. 12.

$\mathrm{H}_{\mathrm{AB}}$. Ganzan, in montibus Morrison, ad 9141 ped. alt., leg. S. Nagasawa, 1905, (No. 676).

Distrib. Tropies.

\section{Polypodium Linv.}

Polypodium lineare Thuxb. var. ? Hayatı, in Tōkyō Bot. Miag. XXI. p. 13.

Hab. in monte Morrison, leg. G. Nakahara, 1905.

Distrib. Type: Tropics of the old world; Japan and China. 



\section{CONTENTS.}

\section{INTRODUCTION}

1) Elements of the Flora of the Tontane $70 n$ Page.

List of the Elements of the Montane Zone.. $\quad \ldots \quad \ldots \quad \ldots \quad$.

a) Arctic Elements. . .

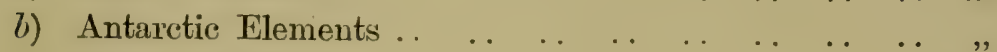

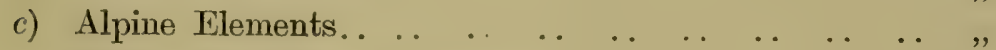

d) Tropical American Elements..

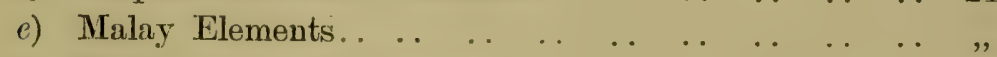

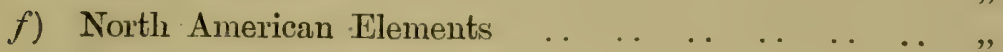

g) Himalayan Elements.. . .

h) Elements of central and southern China $\ldots$. $\quad \ldots \quad 26$

i) Japanese Elements .

j) Elements of northern China..

k) Endemic Elements .

l) General Character of the Elements . . $\quad \ldots \quad \ldots \quad \ldots 29$

2) Floristic Relationship between Formosa and other Neighbouring countries ..

3) General Aspect of the Vegetation . .

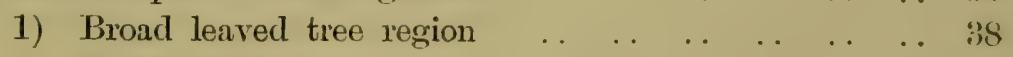

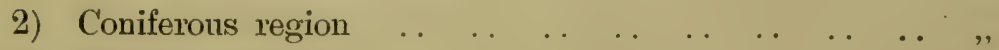

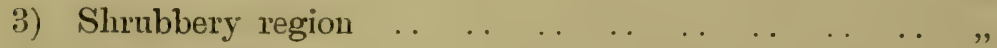

4) Grass region .

4) Enumeration of the Plants..

$\begin{array}{llllllllllll}\text { Dicotyledones } & . & \ldots & \ldots & \ldots & \ldots & \ldots & \ldots & \ldots & \ldots & \ldots & ,\end{array}$

$\begin{array}{lllllllllll}\text { Polypetalie. } & \ldots & \ldots & \ldots & \ldots & \ldots & \ldots & \ldots & \ldots & \ldots & ,\end{array}$

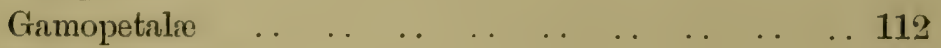

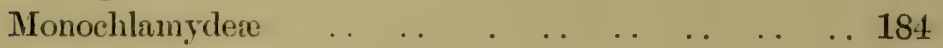

Gymmospermex.. . .

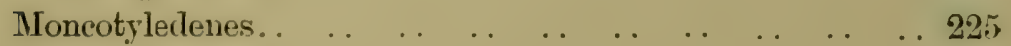

$\begin{array}{llllllllllll}\text { Cryptogamice } & \ldots & \ldots & \ldots & \ldots & \ldots & \ldots & \ldots & \ldots & \ldots & \ldots & 241\end{array}$ 



\section{IN D EX.}

Orders, tribes, sections and topics in capital type; yenera and species in romen type; synonyms and species incidentally mentioned in italic type.

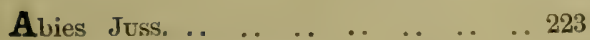

" Jrachyphylla MIAxmr. . . . .

", Mariesii Hayata.

" Mariesii MLast. ..

. Mariesii MAst. var. Kawakamii

Haysta.

ACANTHACE.E

Acanthopanax Dcne et Pu. . . . $\quad$. .

" aculeatum SEEM... . . . 104

Acer Lrvs.

$\begin{array}{llllll} & \text { cratargifolium S. et Z. } Z \text {.. } & \text {. } & \text {.. } & \text {. } & 71\end{array}$

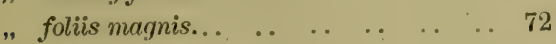

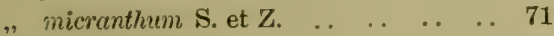

", pictum Тнunв.

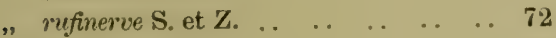

Actinidi̊ LindL. ..

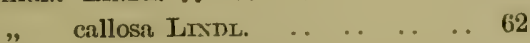

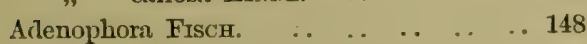
polymorpha LEDEB. var. coro.

nopifolia Trautv.

" polymorpha LEDEB. var. Lamar-

ckii Trautr. verticillata FISCH... ... . . . 148

$" \quad$ " var. linearis IIAXata.. 148

Adenostemm viscosum Forst .. $\quad . . \quad$.. 121

Agalma octophyllum SEEMr. $\ldots$. .

" racemosum SEEM.

Agerntum Lins.

" conyzoides LINN. . . . . . 121

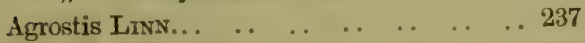

, Clarkei Ноok. f. .. . . . . . 237

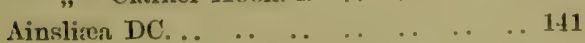

elegans HAYATA .

macroclinidioides HaYATA... 141

morrisonicola HaYATA.. . . . 142

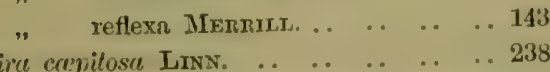

flexuose Liss. var. montame F̂raxch.

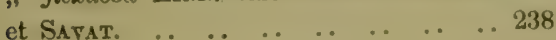

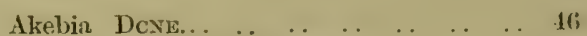

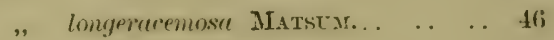

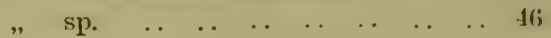

Aleurites Fonst. ..

" cordata Steud. $\quad . \quad \ldots \quad \ldots \quad \ldots 191$

Alnus GæmTN. maritima Notr. var. formosana

BuRKILI. ..

Alocasia (macror\%hiza Sснотт.

Aluptaf Elements. . .

Avacardiace. .

Anaphalis DC.

" margaritacen BeNTH, et Hoor. f. 128

. Margaritacer Bexrr. et НоoK. $f$.

var. nngustifolia (Fraxch. et SATAT.). . 128

forma morrisonicola .

Nagasawai Haxata, (Pl. XVIII.) 129

Andropogen Nardus Lnss. Far. Goeringii

$\begin{array}{llllllllll}\text { HACK. }_{\text {A }} & \ldots & \ldots & \ldots & \ldots & \ldots & \ldots & \ldots & \ldots & 2: 37\end{array}$

Aneilema R. BR. . . $\quad$.

divergens Clatike. . . . . . . 2.2S

" herbacem Кихтн. var, divergens

$\begin{array}{llllllllll}\text { Clatre } & \ldots & \ldots & \ldots & \ldots & \ldots & \ldots & \ldots & 2.24\end{array}$

Anemone Livs.

$\begin{array}{llllll}\text { " luzoniensis RoLFE } & \ldots & \ldots & \ldots & \text { :3!) }\end{array}$

, vilifolia Haм. . . . . . . . . . :3!)

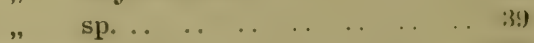

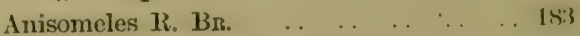

ovatr R. 13r. . . . . . . . . 18's

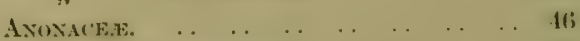

Antaibctic Lilements. . . . .

Antenurie japonica MiQ.

, maryaritacese 13. 131: .. . . . . 12k

Ambis Inisx. . .

" albill Stev.

, elpine Liss.

", Memose Scop. . .

", pterospermu LDGEW. . . . . . . 19

" turaxicifolia AxDEls. . . . . . . 4 !) 
$\begin{array}{lllllllll}\text { Arabis sp. } & \ldots & \ldots & \ldots & \ldots & \ldots & \ldots & \ldots & 50\end{array}$

AtaltaceE.

$\begin{array}{lllllll}\text { Aralia octophylla Lour. } & \text {.. } & \text {.. } & \text {.. } & \text {. } & 107\end{array}$

Aт.стіс ER.емеNтs...

$\begin{array}{llllllllll}\text { Ardisi\% } & . . & . . & . & \ldots & \ldots & \ldots & \ldots & \ldots & 157\end{array}$

Arisæma sp. (A. consunguineum Бснотт ?) 229

$\begin{array}{llllllll}\text { AtrisToLochiacki... . . } & \ldots & \ldots & \ldots & \ldots & \ldots & 187\end{array}$

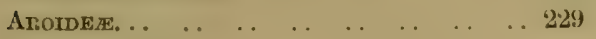

Artemisia Livs.

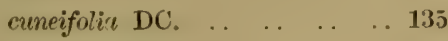

". japonica Тнunв.

" niitaknyamensis HaYata. . . 136

$\begin{array}{llllll} & \text { ". oligocarpa Hayata } & \ldots & \ldots & \ldots & 137\end{array}$

" parviflora Весн... . . . .

". salsoloides WILID. . . . . . . 187

" scoparia WaLdst. et Kíts. . . 188

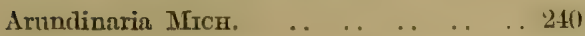
$\begin{array}{lllllll}\text { niitrkayamensis HaYata } & \ldots & 2: 10 \\ \text { Raddi. . . } & \ldots & \ldots & \ldots & \ldots & 2: 35\end{array}$

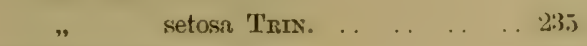

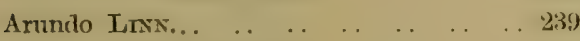

, formosinn НАСK.

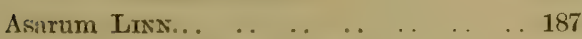

, macranthum Hook. f. . . . . . . 187

$\begin{array}{lllllllll}\text { AsCLEPLIDENE. . . } & \text {. } & \ldots & \ldots & \ldots & \ldots & \ldots & \ldots & 102\end{array}$

Aspidium amabile BLUMe. $\quad . \quad$..

Asplenium Lrsx. . .

$\begin{array}{lllllll} & \text { anceps Sor. } & \ldots & \ldots & \ldots & \ldots & 243\end{array}$

" lacinintum Dow. . . . . . . 243

$\begin{array}{lllll} & \text { " Trichomanes LNN. . . . . . } & 243\end{array}$

Aster Lixx. . .

$\begin{array}{lllllll} & \text { ageratoides Turcz. } & \ldots & \ldots & \ldots & \ldots & 125 \\ \end{array}$

$\begin{array}{llllll} & \text { " baccharoides STEETZ.. } & \ldots & \ldots & \ldots & 124\end{array}$

$\begin{array}{llllllll} & \text { " seaber Тнusв. } & . & \ldots & \ldots & \ldots & \ldots & 125\end{array}$

" trinervins RoxB. $\quad$.

Astilbe HAנr. . .

" chinensis Franch. et Savat.. . . 86

" chinensis Franch, et SAv. var.

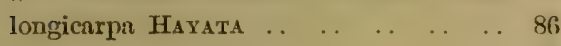

$\begin{array}{lllllll} & \text { macroflori HAYATA.. } & . & . & . & 8 & 86\end{array}$

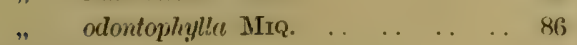

Aucuba Lins...

" chinensis BEATH. . . . . . . . . 111

$\begin{array}{llllll} & \text { " limalaicet Ноок. f. . } & \text {. . } & \text {.. } & \text {.. } & 111\end{array}$

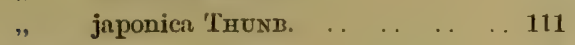

$\begin{array}{lllllll}\text { Avena subspicatu CLAIRv. } & \text {. } & \text {. } & \text {. } & \text {. } & 2: 38\end{array}$

$\begin{array}{llllllll}\text { B.LANOPHEæ. }_{\text {a }} & \ldots & \ldots & \ldots & \ldots & \ldots & \ldots & 192\end{array}$

Balanophora Fonst. $\quad$.. parvior HAYATA. $\ldots$. $\quad \ldots \quad 192$
Balanophora spicata HAтатA. .. $\quad$. $\quad$.. 192

Barthea Ноок. f. ..

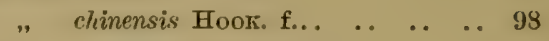

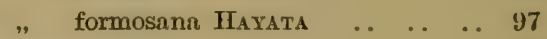

$\begin{array}{lllllllll}\text { BEGONTACEE. } & . . & . . & . . & . & \ldots & . & \ldots & 101\end{array}$

BetrerideE.E. ..

Berberis LiNx.

$\begin{array}{llllll} & \text { " barandane VIDAL. . } & \text {.. } & \ldots & \text {.. } & 48\end{array}$

" Bealei Fontune

" $\quad$ nepalensis Sprext. $\quad . \quad$.

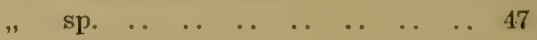

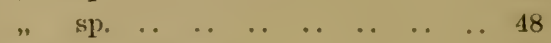

IBiotica discolm Maxıм. ..

$\begin{array}{lllllllll}\text { BRINEE. .. } & . . & . . & . . & \ldots & \ldots & \ldots & \ldots & 5 t\end{array}$

Blumen DC. ..

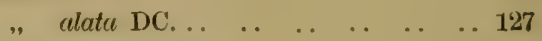

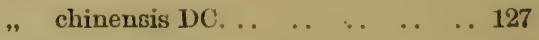

Bonnninghausenia ReтсHн. . . .

albiflora REICH13... .. 67

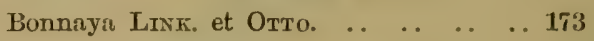

" veronicrefolia Sprexg. $\quad . \quad \ldots \quad 173$

Botagrinez.

Brachypodium BeAUr. .. $\quad$.. $\quad$.. $\quad$.. $\quad$.. 239

, Kawakamii Hayata. . . . 239

" sylvaticum BeAuv. . . . 240

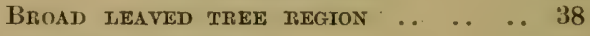

Bulbostylis KUNTH. $\quad$.

" capillaris Kuntr. var. trifida

$\begin{array}{lllllllll}\text { Charke. } & . . & . & \ldots & . & . & . . & . & 230\end{array}$

$\begin{array}{llllll} & \text { " trifule KUNTH. .. } & . & . . & . . & 230\end{array}$

Buxus Lnvs. . .

$\begin{array}{lllllll} & \text { "japonica Muell. } & \text {.. } & \text {. } & \text {. } & \text {.. } & 193\end{array}$

", sempervirens LINN. Tar. juponica

$\begin{array}{lllllllll}\text { Makino } & \ldots & \ldots & \ldots & \ldots & \ldots & \ldots & \ldots & 193\end{array}$

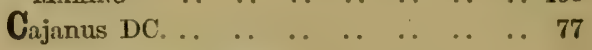

$\begin{array}{lllllll} & \text {, indicus Sprexg. } & . & \ldots & \ldots & \ldots & 77\end{array}$

Calımngrostis ADANS.

, arundinacen Rотн. . . . 237

" arrudinncen Roтн. .. . . 237

" $\quad$ " vir, nipponicr

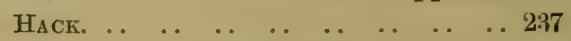

" nipponicu Franch, et SATAT. 237

Calocedrus macrolepis Kunz. .. .. .. 207

Camellia caudate WALL.

Campanulace.e

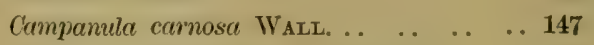

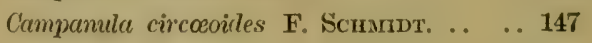

Crmprnumoer BL。 -

axillaris OLT. 
Campanumoer japonica Mraxm. . . . . . . 147 " javanica BuUsL... . . . . 117

$\begin{array}{llllllll}\text { Captifoltacez. } & \ldots & \ldots & \ldots & \ldots & \ldots & \ldots & 112\end{array}$

$\begin{array}{lllllllll}\text { C'upsicum... } & \ldots & \ldots & \ldots & \ldots & \ldots & \ldots & \ldots & 172\end{array}$

Cardamine Lrsw. . .

$\begin{array}{llllll}\text { " asarifolix LINN. } & \text {. . . } & \text {. } & 51\end{array}$

" reniformis HaYata... . . . . 50

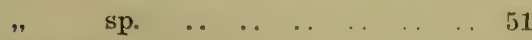

Cardinndra Sieb. et Zucc. ..

" formosance HaYATA... . . . . 92

$\begin{array}{llllll} & \text { sinenses HeMsL. } & \ldots & \ldots & \ldots & 92\end{array}$

Cardiospermum LrNs. . .

" Hilicncabum Lixx... .. 73

, microcarpum H. B. K. . . 73

Carex Lrss. . .

"breviculmis R. BR. subsp. lioyleanz.

(NeEs). . .

"tristachye Thunb.

"M Makinoensis Franch. . . . . 233

"p peudo-conica Fraxch, et Savat, . . 231

„, sp. nov.? ..

"sp. nov.? (aff. c. japonica THuNB) . 231

C'estanea indica Roxв. . . . . . . . . . 20z

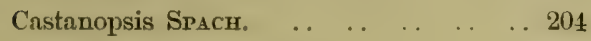

" Ifystrix A. DC. . . . . . 206

" $\quad$ indicr A. DC. . . . . . . . . 204

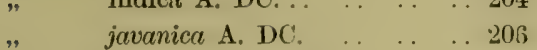

" taiwnniana HaYATA. . . . 205

" tribuloides var. formoscmu

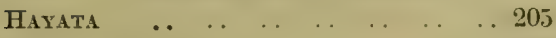

Carpesium Lrns.

acutum HAYATA. . . . . . 133

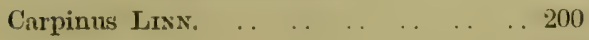

$\begin{array}{lllllllll}\text {,. } & \text { sp. . } & \ldots & \ldots & \ldots & \ldots & \ldots & \ldots & 200\end{array}$

$\begin{array}{llllllll}\text { Catyophylles: } & \ldots & \ldots & \ldots & \ldots & \ldots & \ldots & 55\end{array}$

$\begin{array}{lllllllll}\text { Celastrineaz. .. } & \ldots & \ldots & \ldots & \ldots & \ldots & \ldots & 69\end{array}$

Celastrus Lrsw.

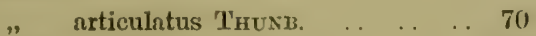

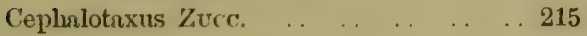

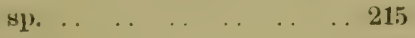

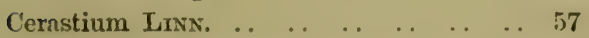

" grandiflonum WAT.D. et KrT. . 58

" morrisonense Havats. . . . 57

$\begin{array}{llllll} & \text { pilcsum LEDEB... } & \ldots & \ldots & \ldots & 58\end{array}$

Chamacyparis Spaci. .. $\quad \ldots \quad \ldots \quad \ldots \quad \ldots 208$

". formosensis Matsux. . . . 208

, obtusn Sie13, et Zucc. . . . 208

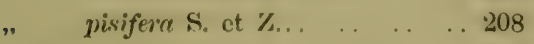

Chiritr Hàr. .
Chirita anachorata HAxcE. . . . . . .. 179

Chlotanthacel. . .

Chloranthus Sw.

serratus Roear. et SchulT. . 188

Chrysanthemum LnN. . . . $\quad . \quad \ldots 135$

" indicum Ltxx. . . . 135

Chrysosplenium Lrsw...

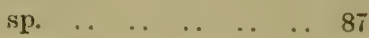

Cinnnmomum BunMax: $\ldots$.

Camphorn Nefs et EBenar. 189)

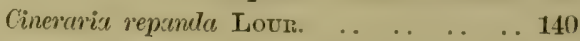

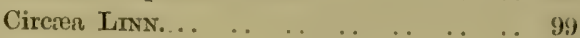

„, alpina Lins. .

, Wallichii DC. . .

Clematis LiNN.

" barbellate EDGEW... . . . . . . 42

" crassifolia BeNtн... . . . . . 44

" lasiandre Maxnr... .. .. . . 42

" lasiandra Maxint, var. Nagasawai

IIAYATA.

" longiseprla Haxata. . . . . . 41

, Morii Haуata. . . . . . . . . 42

" smilacifolia WALL. .

" tozanensis Hayata. . . . . . 42

" Wightiann WaLJ. .

$\begin{array}{llllllllll} & \text { sp. } & \ldots & \ldots & \ldots & \ldots & \ldots & \ldots & \ldots & 44\end{array}$

C'leyern llubi Chàrs. ..

$\begin{array}{llllllll}\text {, fragrans. } & . & \ldots & \ldots & \ldots & \ldots & \ldots & 60\end{array}$

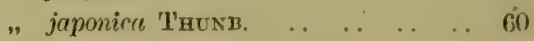

Cnicns LmN. .

, Wallichii DC. .. . . . . $\quad \ldots \quad \ldots 140$

Codonacantlus Nees. .

" pruciflorus NEES. . . . 180

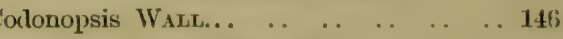

corlata Hook. f. . . . . . 147

truncate WAII... ... . . . 14ti

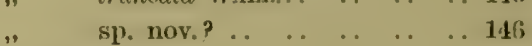

Commetisaceni.

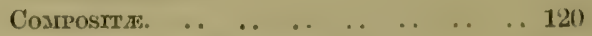

Conanilron SieB. et ZuCc. . . $\quad \ldots \quad \ldots \quad \ldots 179$ ramondioides SiF.B. et ZuCC... 179

$\begin{array}{lllllllll}\text { Conmera. } & \ldots & \ldots & \ldots & \ldots & \ldots & \ldots & \ldots & 217\end{array}$

Contremous migrox.

Coniogrnmme Fé.

" fraxiacr (Dos.) Fée .. . . 244

Comyzu chinensis Lrxx... $\quad \ldots \quad \ldots \quad \ldots \quad \ldots 127$

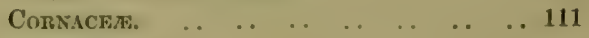

Corydrlis DC...

$\begin{array}{llllllll}\text { sp. . } & \ldots & \ldots & \ldots & \ldots & \ddots & \ldots & 49\end{array}$ 
$\begin{array}{llllllllll}\text { Crassulacem } & \ldots & \ldots & \ldots & \ldots & \ldots & \ldots & \ldots & 9\end{array}$

Crawfurdia WALL... fasciculata Waxx. . . . . 164 japonicn Sreb, et Zucc... . . 164

Crotalaria Lins.

, formosana Mlatsuarols. . . . . 74

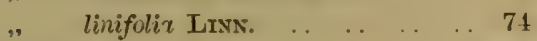

$\begin{array}{llllllllll}\text { Cructferte. } & \ldots & \ldots & \ldots & \ldots & \ldots & \ldots & \ldots & 19\end{array}$

Ciryptoganize.

Cryptomerix juprenice HaYATA. . . . . . 215

Cncubalus Livs. . .

$\begin{array}{lllllll} & \text {, baceifer Lins. } & \text {. } & \ldots & \ldots & \ldots & 57\end{array}$

$\begin{array}{lllllllll}\text { Cucurbitaces. } & \ldots & \ldots & \ldots & \ldots & \ldots & \ldots & 100\end{array}$

Cunninghamia R. Br... . . . . . . . 213

, Fonishii HardrA. . . . 213

$\begin{array}{llllllllll}\text { Cupultanes. } & \ldots & \ldots & \ldots & \ldots & \ldots & \ldots & \ldots & 199\end{array}$

$\begin{array}{llllllll}\text { Cynnotis Dox... } & \ldots & \ldots & \ldots & \ldots & \ldots & \text {. . } & 228\end{array}$

". arachnoider CíARKE. . . . . . 228

" pilosa Wight. . . . . . . . 228

Cyclocolon truncatus Hook. f. et THoss. 146

Cymbopogon Spreng. .. $\quad \ldots \quad \ldots \quad \ldots \quad . .236$

Nardus Rexduk, subsp. margi-

natus var. Goeringii Revdle: .. . . . . 236

$\begin{array}{lllllllll}\text { Crperacese. } & \ldots & \ldots & \ldots & \ldots & \ldots & \ldots & \ldots & 230\end{array}$

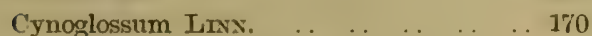

" furcatum WALL. $\quad \ldots \quad \ldots \quad \ldots 170$

" micranthum DEsF. . . . 170

". racemosum Roxi... . . . 170

Damnacanthus GærTs. $\quad \ldots \quad \ldots \quad \ldots \quad \ldots 113$

" angustifolius HaYAs. . 113

, indicus GERTN. f. . . . . 114

" macrophyllus SIEB. . . . 114

" mrijor StEB, et Zucc. . 114

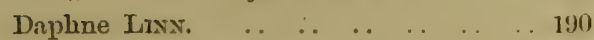

" Championi Bestн. .. . . . . $1 ! 0$

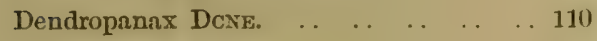

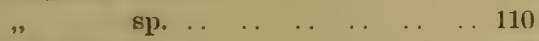

Dentzia Тнuмв.

$\begin{array}{llllll} & \text { crenat } \ell \text { SIEB. et } Z_{U C C} \ldots & \ldots & \ldots & 92\end{array}$

" erenuta $\delta$ trivomensis Maxrar. . . $\quad 92$

" senbra Тнохв.

Deschrmpsia Beauv. . .

caspitosa Beaur... . . . 2:38

flexuosa Trix. . . . . . $2: 38$

Desmodium Desv... $\ldots \begin{array}{llllll} & \ldots & \ldots & \ldots & \ldots & 7 t\end{array}$

, parvifolium DC. $\quad . \quad \ldots \quad \ldots \quad 7 t$

" polycarpum DC. .. $\quad \ldots \quad$. $\quad 75$

, pulchellum Вехтн. .. . 75

" sinuatum BLUME. .. . . . 75

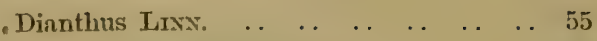

superbus Livs. . .

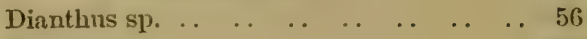

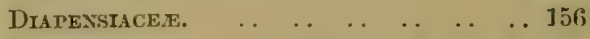

Dicotyledones. . . . . . $\quad: 39,112,181$

Diplopappus aspervimus DC. $\quad . \quad \ldots \quad$.. 125

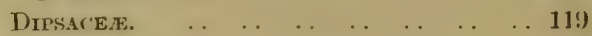

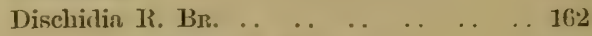

formosana Maxim. .. . . . 162

Disporum SALtSB. . .

$\begin{array}{llllllllll} & \cdots & \text { sp... } & \ldots & \ldots & \ldots & \ldots & \ldots & \ldots & 226\end{array}$

Doellingerit scalna NEEs. $\quad . \quad \ldots \quad$. .

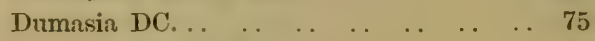

, bicolor Hayata. .. . . . . . 75

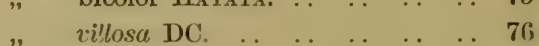

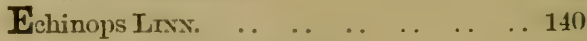

" dahuricus Frsch... . . . . . . 140

" Gmelini LEDEB. .. . . . . . . 140

, splioerocephalus MIQ... . . . 140

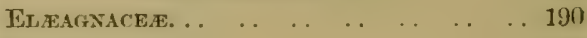

Eloongnus Lrxx. $\ldots \begin{array}{lllllll} & \ldots & \ldots & \ldots & \ldots & \ldots & 190\end{array}$

umbellata THUNB. .. . . . . 190

Elroocarpus Ltsx, . decipiens Hearst. . . . . . . 6 6it

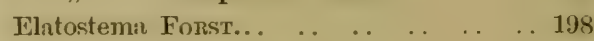
minutum Harata. . . . . . 199 ovatum. WIsHT. $\quad \ldots \quad \ldots \quad \ldots 197$ sessile Fonst. . . . . . . . 198

" $\quad$ var. cuspidatum WEDD. 198

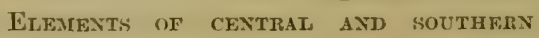

$\begin{array}{llllllllll}\text { Chrsa. .. } & \ldots & \ldots & \ldots & \ldots & \ldots & \ldots & \ldots & 26\end{array}$

Elemexts of yotthern Chisa.. . . . . 27

Elemexts of the Flora of the Montane

ZONE.

Ellisiophyllum Maxñ... $\quad \ldots \quad \ldots \quad \ldots \quad \ldots \quad$ 170)

," pimnatum Marrio. . . . 170

". reptans Maxn. . . . . .

Exdenic Eimanexts.

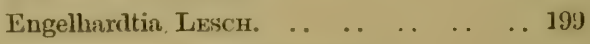

spienta BLune. .. . . . 199

" var. formosana HIIATA. 19!)

Einkylix trigyna GrTFFтt. .. . . . . . . 101

Exumeration of the Plants. . . $\quad . \quad$. . 39

Epilobium Lrsx. . . alpinum LrNs. . roseum SchrEb. $\quad \ldots \quad \ldots \quad \ldots \quad 9 J$

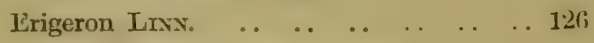
morrisonensis HaYATA. .. . 126

" Thunbergi Grax. .. . . . . . . 126 
Eriochrysis Narenga NEws. . . . . . . . . 236

$\begin{array}{llllllll}\text { Ethulia } \operatorname{Lins} . . . & \ldots & \ldots & \ldots & \ldots & \ldots & \ldots & 120\end{array}$

Ethulia angustifolis Bosms. . . . . $\quad$. . $\quad . .120$

" conyzoides LrN. . . . . . . . 120

" gracilis Delile. . .

" $\quad$ ramosa Roxв. ..

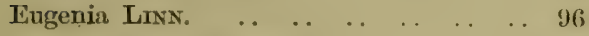

". Grijsii Haxce. . .

" pyxopleylle НАХCE... . . . . . .

" sinensis HeMrst, . . . . . . . .

Euonymus Lins. .

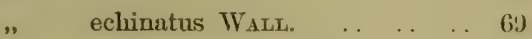

" trichocarpus Hayata. . . .

Eupatorium LINx...

, clinense LIxs... . . . . . . 12:3

., chinense var. tripartilum MIQ.. 12:3

, formosanum Hayata. . . . 122

", Lindleyanum . . . . . . . 123

, Tashiroi Harata. . . . . . 12:3

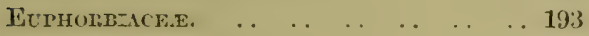

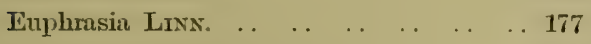

$\begin{array}{llllll} & \text { borneensis Stapi. } & \ldots & \ldots & \ldots & 177\end{array}$

$\begin{array}{llllll} & \text { petiolaris Wetsst. } & \ldots & \ldots & \ldots & 177\end{array}$

$\begin{array}{llllllll}\text { Eurya Thune, } & \ldots & \ldots & \ldots & \ldots & \ldots & \ldots & 60\end{array}$

", distichophylla Hrarsi. . . $\quad . \quad$. 61

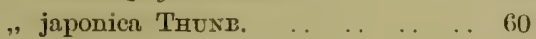

", strigillosa Harata. . . . . . . . . 61

$\begin{array}{llllllll}\text { Erodia Forst... } & \ldots & \ldots & \ldots & \ldots & \ldots & \ldots & 68\end{array}$

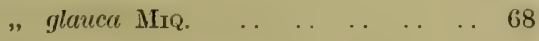

" melirfolia Bexth. . . . . . . . 68

Fagus Lins. . .

, sylvatica Lisx, var. . . . . . . . 206

Fatoun GAUD...

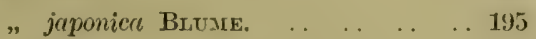

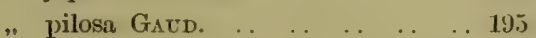

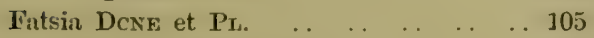

"japonice DCNe et PL. . . . . . 106

", polycarpa Hatata .. .. . . .. 105

" papyriferc Bexth. et J[ook. f. . 106

Festuca Lrnn...

" ovina var. vulgaris fiocr. .. . . 210

Ficus LiNs,

"vaccinioides Hevist. et KIre.. . . 196;

Flemingia Roxs. . . . .

, strobilifera R. 13n. .. .. . . 77

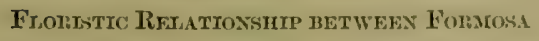

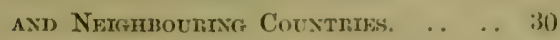

$\begin{array}{lllllllll}\text { Frngaria Lxs. } & \ldots & \ldots & \ldots & \ldots & \ldots & \ldots & 82\end{array}$

$\begin{array}{llllllllll} & \text { sp. } & \ldots & \ldots & \ldots & \ldots & \ldots & \ldots & \ldots & \text { s. }\end{array}$

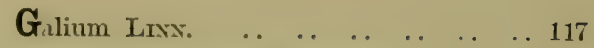

, brachyporlium Maxis. .. . . . 117

Gavotetal. . .

General Aspect of the Vegetation. .. $3: 3$

General Character. of. the Elminents. . 29

Gentianacem...

Gentiana Lisx.

" aquatica Liss. . . . . . . 16 168

", aquatica PALL. . . . . .

," cespitos mayata. . . . . 16.;

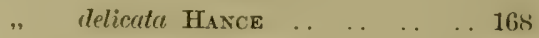

, fasciculnta Hayata . . . . . 16i)

" flavescens Harata .. . . . 166

, formosana HAYATA .. .. . . 167

" humilis Stev. . . . . . . . 167

". scabrida Hayata . . . . . . 168

" tenuissima Hayata $\ldots$. . . 167

Geinaniaces.

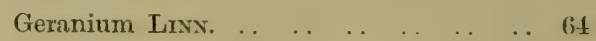

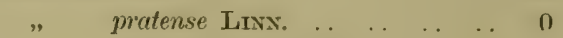

, Robertinnum Lrx. . . . . . 61

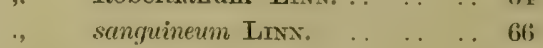

" sibiricum LrNo. . . . . . . . $666^{\circ}$

" uniflorum HaYaTa . . . .

Gerardic jeponice T'HUхв. . . . .

$\begin{array}{lllllllll}\text { Gesneraces. } & \ldots & \ldots & \ldots & \ldots & \ldots & \ldots & \ldots & 178\end{array}$

Girnrdinia GaUd. . . . . . . $\quad \ldots \quad \ldots \quad \ldots 196$

, heterophylla DcxE. .. . . . 196

" palmata GAUD. . . . . . . 197

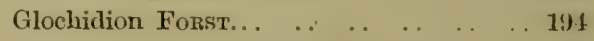

" formosnnum HaYate .. . 191

, zeylanicum A. Jusss... . . . . 194

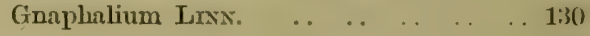

" amoyense HANCE $\quad . \quad \ldots \quad \ldots \quad 131$

, collinum LABILL. . . . . . $1: 32$

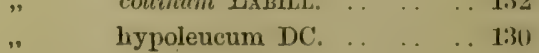

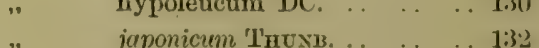

$\begin{array}{lllll}" \quad \text { linenre Hayata } & . & \ldots & \ldots & 131\end{array}$

, Iuteo-nlbum Lisx... $\quad \ldots \quad \ldots \quad 1: 32$

, margaritaceum Lisx. . . . 1:28

" margaritaceum

, var. cengustifolium (Frascru, et

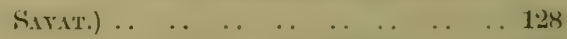

". niitaknyamense IIххатА, . . 13:2

" Thomsoni Hook. f. . . . 1:3:3

" uliginosum Lixa. . . . . . 1332

Goldfussic Cusie.NEEs... . . . . . . . . . 17!)

Golowninie joponieu Maxn. . . . . . 16if

Gonocarpus micranthus 'InUxB... . . . 95 


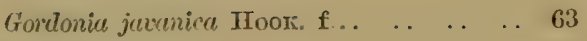

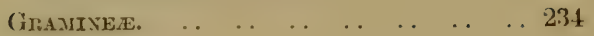

$\begin{array}{lllllllll}\text { Grass REGION... } & \ldots & \ldots & \ldots & \ldots & \ldots & \ldots & 38\end{array}$

Gymnogramme jevanica BuUxe .. .. . . 214

$\begin{array}{llllllll}\text { GMMNOSPERME } & \text {.. } & \ldots & \ldots & \ldots & \ldots & \ldots & 207\end{array}$

Gynostemma Fonst. ..

cissoides ВеNTH. et Hook. f. 101 pedatum Blume .. .. . . 100

$\begin{array}{lllllllll}\text { Gynum Cass... } & \text {. } & \text {. } & \ldots & \ldots & \ldots & \ldots & 138\end{array}$

" flava Hayata..

$\begin{array}{llllllll}\mathbf{H}_{\text {EMIODORACEX. }} & \text {. } & \ldots & \ldots & \ldots & \ldots & \ldots & 225\end{array}$

$\begin{array}{lllllllll}\text { IFALOR.AGEXE. } & \ldots & \ldots & \ldots & \ldots & \ldots & \ldots & \ldots & 95\end{array}$

$\begin{array}{llllllll}\text { Halorngis FoRST. } & . & \ldots & \ldots & \ldots & \ldots & \ldots & 95\end{array}$

$\begin{array}{lllll}\text { micrantha R. Br. } & \text {. } & . & . & 95\end{array}$

$\begin{array}{llllllll}\text { Hellera Lixx. . . . . } & \ldots & \ldots & \ldots & \ldots & \ldots & 110\end{array}$

" colchica Косн...

„ Helix Lins.

", racemosa Wıghт.

, rhombea Sies. et Zucc... . . . . 110

Helicir Lour...

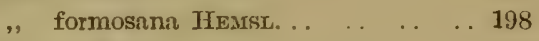

Helwingia WruLd...

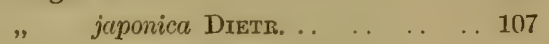

" $\quad$ rusciflora WIILD. $\quad . \quad \ldots \quad . \quad . \quad 106$

IIemiphrágma heteroplyylla WALL. . . . 164

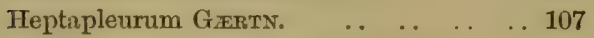

" octophyllum ВЕхтн. .. .. 107

" racemosum BEDD. . . . 107

Hrandaya Elements. .

Hockia Exgi. et Griebs. ..

"Aschersoniana ENGL, et Grasis. .. 118

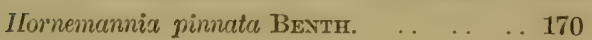

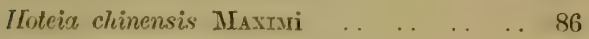

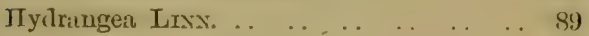

chinensis Maxm,

$\begin{array}{llllll}\text { glabm HAYATA } & . . & \ldots & . . & . & 89\end{array}$

integra Hayata. . $\quad . \quad \ldots \quad . \quad . \quad 90$

integrifolia, تАуата.. .. . . 90

involucrate Stes. .. . .

involucrata SiË.

Krawakamii FAYATA $\quad . . \quad \ldots 90$

longifolia HAXATA $\ldots$. . . . . 91

Hydrocotyle Lixx...

" hirta R. Bro, var. ceculiloba $\mathrm{F}$.

$\begin{array}{llllllllll}\text { MUELL... } & \text {. } & \text {. } & \text {.. } & \text {.. } & \text {. } & \text {.. } & \text {.. } & 102\end{array}$

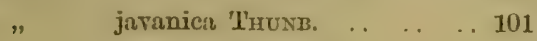

" $\quad$ nepalensis Ноок. .. .. . . 101

" . niticlula Rтсн. .. . . . . $\quad . .102$

" polycephala WigrT, et Ar... 102
Hydrocotyle puncticulalu $\mathrm{XIT}$.

rotundifolir Roxis. . . . . . 102

setulosa Hayata $\ldots$. $\quad \ldots 102$

Sïthorpioides LaM. $\quad \ldots \quad \ldots 102$

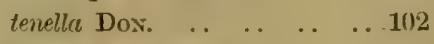

Wilfordi MLAxтs. $\quad . \quad \ldots \quad \ldots 103$

$\begin{array}{llllll}\text { zeylanice DC. . } & \text {.. } & \text {. } & \text {.. } & 102\end{array}$

Zollingeri MoLzENB. . . $\quad$. 102

$\begin{array}{llllllll}\text { HYDROPHYLLACE.Æ. . } & \ldots & \ldots & \ldots & \ldots & \ldots & 170\end{array}$

$\begin{array}{lllllllll}\text { HYPERTCINEE. . . . . } & \text {.. } & \text {. } & \ldots & \ldots & \ldots & 59\end{array}$

$\begin{array}{llllllll}\text { Hypericum Lrsx. } & \ldots & \ldots & \ldots & \ldots & . & \ldots & 59\end{array}$

attenuatum CHогsу..

Hypoestes R. BR.

T " purpurea R. BR. .. $\quad . . \quad \ldots \quad$.. 181

$\begin{array}{llllllll}\text { Ilesia Maxtur. }_{\text {M. }} & \ldots & \ldots & \ldots & \ldots & . & \ldots & 54\end{array}$

"polycarpa Мнахтм.

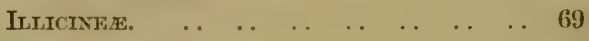

Ile:s japonica Thuхв.

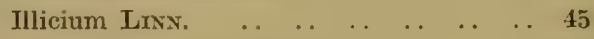

, anisatum Lrsx. $\quad . \quad$..

" Teshioi Maxmr. ..

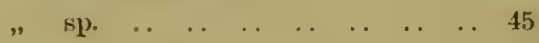

Impatiens Lrsw.

, uniflora HaYATA $\quad . \quad \ldots \quad . .66$

lsachne R. Br.

„, Clarkei Ноок. f. . .

Iaeris versicolor DC.

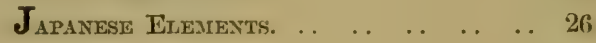

$\begin{array}{lllllllll}\text { JUGLANDACEE... } & \ldots & \ldots & \ldots & \ldots & \ldots & \ldots & 199\end{array}$

$\begin{array}{lllllllll}\text { Juglans Lrvs... } & \ldots & \ldots & \ldots & \ldots & \ldots & \ldots & 199\end{array}$

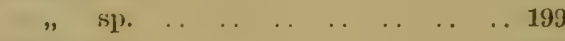

$\begin{array}{llllllllll}\text { JUNCACEN. } & \text {.. } & \ldots & \ldots & \ldots & \ldots & \ldots & . & 228\end{array}$

$\begin{array}{lllllllll}\text { Jnneus Lrvs. . . } & \ldots & \text {. } & \ldots & \ldots & \ldots & \ldots & 229\end{array}$

$\begin{array}{lllllll} & \text { " effusus Lisx. .. } & \text {.. } & \text {. } & \text {. } & \text {.. } & 229\end{array}$

, Mnximowiczi Buch. . .

Juniperus LINN.

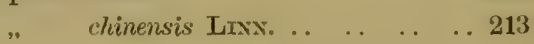

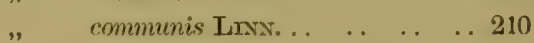

" $\quad$ formosana Hayata $\ldots$.. $\quad$.. 209

" $\quad$ morrisonicola HAYATA

" rigida Sire. et Zucc. . . $\quad$. 210

" taxifolie HaYATa $\quad . \quad$.. $\quad . .209$

" taxifolia, Hook et Ars. .. . . 210

$\begin{array}{lllllllll}\text { Justicia Lrnn... } & \ldots & \ldots & \ldots & \ldots & \ldots & \ldots & 180\end{array}$

" procumbens Lisx... . .

$\begin{array}{lllllllll}\mathbf{K}_{\text {adsura Juss. }} & \ldots & . . & \ldots & \ldots & . . & . . & 45\end{array}$

$\begin{array}{lllllll} & \text { chinensis HAXCE } & . & . & . & . & 46\end{array}$

" japonica Lisx.

Knalanchoë Adans... 


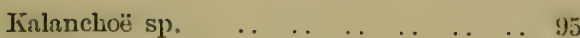

Tieteleerir CARr.

Davidiana BEISSY, var. formosana Haxata.

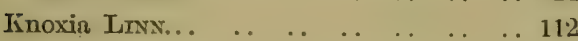

corymbosa T'HLD... 112

$\begin{array}{lllllllll}\text { Lamiate... } & \ldots & \ldots & \ldots & \ldots & \ldots & \ldots & \ldots & 181\end{array}$

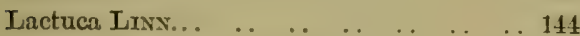

, versicolor Scr.-Brr, $\quad \ldots \quad \ldots \quad \ldots 144$

$\begin{array}{llllllll}\text { Laggern ScH.-BrP... } & \ldots & \ldots & \ldots & \ldots & \ldots & 127\end{array}$

" alata Sch.-Bip. $\quad \ldots \quad \ldots \quad \ldots \quad \ldots 127$

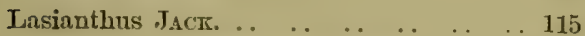

, formosensis Matsex. . . . 115

LAURINEE.

Laurus Camphora Lisw. $\quad . \quad$. . $\quad . \quad$.. 189

Lecanthus WEDD...

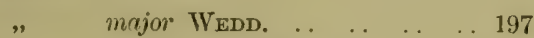

, peduncularis WEDD... . . . . 197

,. Wallichii WEDD. .. . . . . 197

$\begin{array}{llllll} & \text {, Wightii WEDD... } & \ldots & \ldots & \ldots & 197\end{array}$

LEGUMINOSE. . .

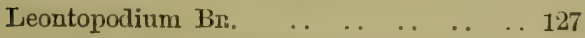

Lene? $\mathrm{R}, \mathrm{Br}$

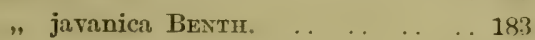

Libocedrus EXDL. .

macrolepis BENTH. . . . . . .07

LiLtacex.

Iindernix japonica Thuxв... . . . . . 173

Lobelia Lins...

", aftinis WALL. . . . . . . . .

, Davidi Fraxchet. . . . . . . . 115

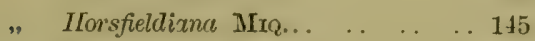

" pyramidalis WALL. .. $\quad . . \quad \ldots 145$

Lodhra spicate Mrens... . .

$\begin{array}{lllllllll}\text { LOGANLICE.J. } & \ldots & \ldots & \ldots & \ldots & \ldots & \ldots & \ldots & 162\end{array}$

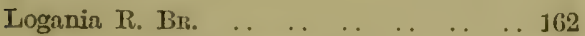

" lentatr Hayata. . . . . . . . . 1.62

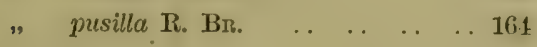

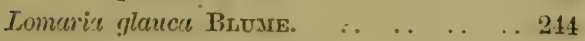

". Matsumureant М.ккхо. _. . 214

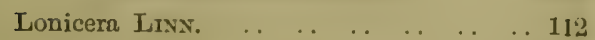

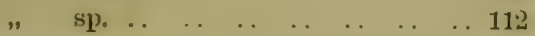

LoRaxthace:.

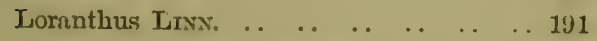

Owatarii Hayata. . . . . . 191

Yadoriki SrFm. . . . . . . . 191

Luzuli DC:

, eftusa Buch. $\ldots$.

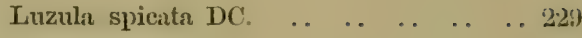

LYCOPODIACEA.

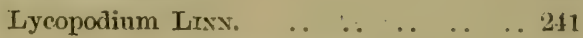

, elavatum Lrsx. . . . . 241

, complanatum Lrsv. var.

Chamxeyparissus A, Br. .. .. .. $2 \dot{41}$

" dendroideum Mтснх. .. . . 242

" juponicum THUxB... .. . . 242

" obscurum LrNx. . . . . 211

", serratum THUNB. . . . . . 242

$\begin{array}{lllllll}\text { Lysimnchin Lrxs. . . } & \ldots & \ldots & \ldots & \ldots & \ldots & 157\end{array}$

„. silkokinn $\mathrm{MIr}$... . . . . . 157

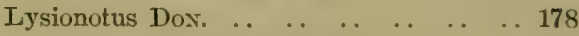

" pauciflorus MIaxis. . . . . . 178

Macroclinitium robustum Maxта. . . . 142

$\begin{array}{llllllllll}\text { Yasa. } & \ldots & \ldots & \ldots & \ldots & \ldots & \ldots & \ldots & \ldots & 157\end{array}$

MAGMOLIACEE,

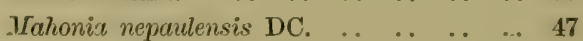

Matay Elements...

Mallotus Lovr.

cochinchinensis Lour. . . . 195

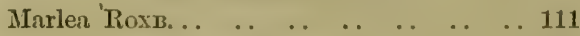

, begonirefolin Roxs... .. . . . 111

Mazus Lour. . .

" japonica O. Кíuntze. .. $\quad . . \quad \ldots 173$

" rugosus Lour... . . . . . . . . 17:3

", vandellioides HANCE. .. .. .. 173

Megubotrya meliafolic HAxce. .. . . . . 68

Metastomacez.

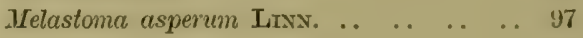

.Melodorum Dun. . .

Oldhami HFarst. $\quad \ldots \quad \ldots \quad \ldots \quad 46$

Mercurialis Lrxw. .

, leiorcarpa SIeb, et Zucc. . 194

Mesona 13Lume.

, elegans Harata. . . . . . . . . 181

" procumbens HeNsiL。 . . . . 181

Metanarthecium Maxiм.

, foliatum Mraxnr. . . . 226

Misennthus Axperss. sinensis AxDETss. var, formos-

anus HACK...

$\begin{array}{lllllllll}\text { Mitell } & \text { Lrxx... } & \ldots & \ldots & \ldots & \ldots & \ldots & \ldots & 88\end{array}$

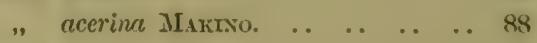

" japonica MIr...

" juponica Mro. var. integripetalu.

Minivo.

MoNocrLaMTPDFE. . . . . . . . . . . . . 181

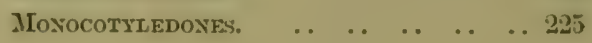


MIorns Lrvx. , alba Lisxa. . . Moseleya pinnata HEXr.is. $\begin{array}{llllllll}\text { Mrurraya Lisx. } & . . & \ldots & \ldots & \ldots & \ldots & \ldots & 68\end{array}$

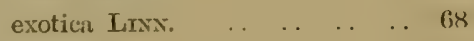

$\begin{array}{llllllll}\text { IIyriactis LeEss. } & \ldots & \ldots & \ldots & \ldots & \ldots & \ldots & 124\end{array}$

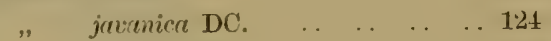

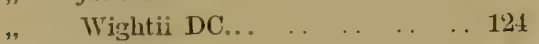

$\begin{array}{lllllll}\text { Myriophyllum Lns. } & \ldots & \ldots & \ldots & \ldots & \ldots & 95\end{array}$

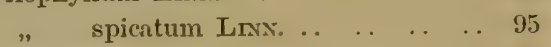

$\begin{array}{lllllllll}\text { Myrtace.e. } & \ldots & \ldots & \ldots & \ldots & \ldots & \ldots & \ldots & 96\end{array}$

$\begin{array}{lllllllll}\text { MIXSTINeE. } & \ldots & \ldots & \ldots & \ldots & \ldots & \ldots & \ldots & 157\end{array}$

$\begin{array}{lllllll} & \text { Nertera Bayss et Sou. } & \text {. } & \text {.. } & \text {. . } & \text {. } & 115\end{array}$ $\begin{array}{lllllll} & \text { " dentata Euxier. } & \text {.. } & \ldots & \ldots & \text {. } & 162\end{array}$ " depressa Baxks et Sot. . . . 116 " nigrienrpa Hayata . . . . . . 11.

North Amertcan Eledents. $\quad \ldots \quad$. .

$\begin{array}{lllllllll}\mathbf{0}_{\text {Leacfir. }} & \ldots & \ldots & \ldots & \ldots & \ldots & \ldots & \ldots & 161\end{array}$

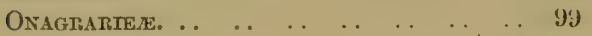

$\begin{array}{llllllll}\text { Ophiorrhiza Linx... } & \ldots & \ldots & \ldots & \ldots & \ldots & 112\end{array}$ pumila Снaмr. $\quad . \quad \ldots \quad \ldots 112$

Oplismenus BEAUN. ..

$$
\text { , undulatifolius Beavv. var. }
$$

imbecillis HACK.

OR. HIDE⿸广.

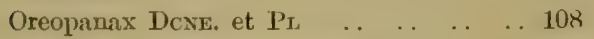

" formosana Harata. . . . . . 108

$\begin{array}{llllllll}\text { Origanum Lrsw. } & . & \ldots & \ldots & \ldots & \ldots & \ldots & 182\end{array}$

$\begin{array}{lllllll} & \text { " creticum Lour. .. } & . & \ldots & . & \text {. } & 182\end{array}$

, heracleoticum Lovi. . . . . . . 182

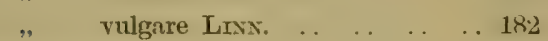

$\begin{array}{lllllllll}\text { Orobaxchacez. } & \text {. } & \ldots & \ldots & \ldots & \ldots & \ldots & 177\end{array}$

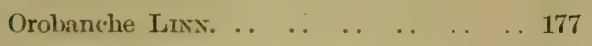

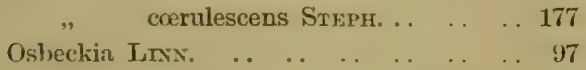

$\begin{array}{lllllll} & \text { aspern Blume. } & . & \ldots & \ldots & \ldots & \$ 7\end{array}$

Osmanthus Lovr...

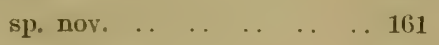

Osyris japonice Thuxb. $\quad \ldots \quad \ldots \quad \ldots \quad \ldots 107$

Oxalis Lrss.

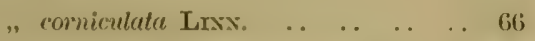

„Grifhithii EDGEw. et Iook. f. . . 66;

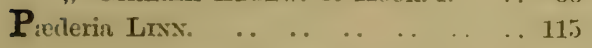

, chinensis HAxcE. . . . . . . . 115

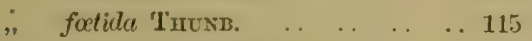

" tomentosa Butru. . . . . . 115

P'anara aculeatum ArT. ..

"Loureirirmem DC. . . . . . . . 105

Papatimaces.
Paratropice cantoniensis Hoor. et ArN. . 107

$\begin{array}{lllllllll}\text { Paris Lrsx. } & \text {. } & \text {. . } & \text {. } & \text {. } & \text {. } & \text {. . } & \text {. } & 227\end{array}$

, lancifolia Hayata.

l'arnassia Lress.

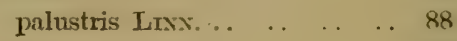

$\begin{array}{lllllllll}\text { Patrinia Juss... } & . & \ldots & . & . . & . & . & 118\end{array}$

$\begin{array}{lllllll} & \text {, ovata BUNGE. . } & . & \ldots & \ldots & \text {. . } & 118\end{array}$

" parviflora STEB. et Zucc. . . . 118

" seabiosxefolia Frsch. . . . . . . . 118

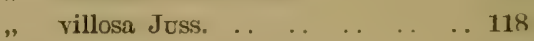

$\begin{array}{lllllll}\text { Peliosanthes Axpr. } & . . & \ldots & \ldots & . & . & 225\end{array}$

" courtallensis Wтянт. .. . . 22.5

$\begin{array}{lllllll}\text { Peperomia R. et PAT. . } & \ldots & \ldots & \ldots & \text {. } & 187\end{array}$

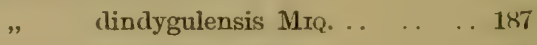

" $\quad$ Nakaharai Hayata. . . . . . 188

". reflexa A. Dietr. . . . . . . $18 x$

Peracarpa Ноок. f. et Thомs. . . . . . . 147

" carnosa Hook. f. et 'THosss. .. 147

" circcooides H. FEET. . . . . . . 147

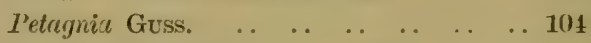

Pestalozzix pedata ZoLL. et MoI. . . . . 101

Petasites G正rta. . .

, tricholobus Fraxchet. . . . 138

Phtheirospermum Buxge. .

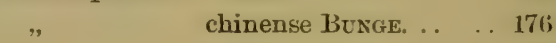

" japonicum KantTz. .. 177

$\begin{array}{lllllllll}\text { Picea Lins. } & \ldots & \ldots & . . & . & . . & . & . . & 220\end{array}$

" Glehni MAST. . .

" Glehni Mـatsumo..

" morrisonicola Havita. $\quad . \quad$. . . . 220

Picris LnN.

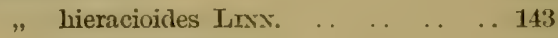

, japonica Тнимв..

liddingtonia Arummularia DC. .. . . . 145

Piler LINDL. ..

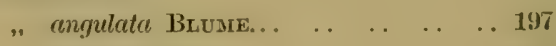

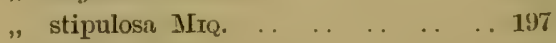

$\begin{array}{lllllll} & \text { "Wattersii Haxce. } & \text {. } & \ldots & \ldots & \ldots & 197\end{array}$

Pinus Livs.

". Armandi Fraxchet ..

" , var. Mastersiann Hayata.. 217

"densiflora $S$. et $Z$.

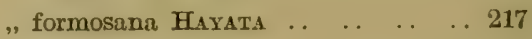

", morrisonicola HaYATA .. .. ... 217

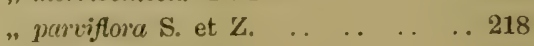

"Thunbergii Part.

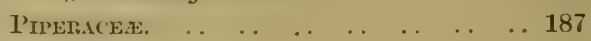

Piper LiN.

„Futokadsura Sies, et Zucc. . . . 187 
I'lectroniu clinensis Lovr. .. . . . . . 105 Pligiogyria KuNze. .. . . . . . . . . 214 glaucn (BLUМE) МЕTT. .. .. 244 " " var. philippinensis $\begin{array}{llllllllll}\text { Christ... } & \ldots & \ldots & \ldots & \ldots & \ldots & \ldots & \ldots & 214\end{array}$

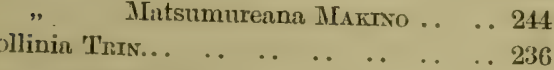
, ciliata Tris. . . " " var. Wallichiana HACK, .. 236 $\begin{array}{lllllllll}\text { Polygalee. } & \text {. } & \ldots & \ldots & \ldots & \ldots & \ldots & \ldots & 54\end{array}$

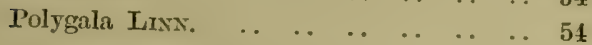

, arcuata Hayata .. .. . .

, japonica Hovtr. .. ..

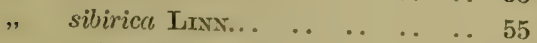

, Wattersii HANCE. .. .. . . . . 55

$\begin{array}{lllllllll}\text { Polygonaceme . . } & \ldots & \ldots & \ldots & \ldots & \ldots & \ldots & 184\end{array}$

Polygonatum ADAxs. $\ldots \begin{array}{llllll}\text {. . } & \ldots & \ldots & \ldots & 226\end{array}$

Maximowiczii Fr. Sснмтот. .. 227 officinale ALu. var. Maxim-

omiczii Franch et Savat... . . . . . 226

efficinale ALI. $\gamma$ pluriflon

$\begin{array}{llllllllll}\text { Miq. } & \ldots & \ldots & \ldots & \ldots & \ldots & \ldots & \ldots & \ldots & 227\end{array}$

Polygonum Lrxx. . . . .

clatum; ПІалг. .. ... .. .. 186

biconvexum IАуата . . . 184

chinense Lrax... . . . . . . 184

cuspidatum Sxeb, et Zucc. . 185

mionocephala Dox. .. . . . . 186

minutum HAYATA .. ... . 185

morrisonense HAYATA .. . 185

Posumbu Hax.. . . . . . . . 186

sinense Hoor, et Arix. . . . . 181

Thunbergii S. et $Z$. . . . . . 181

$\begin{array}{lllllllll}\text { Polypetal } & \text {... } & \text {. } & \text {. } & \ldots & \ldots & \ldots & \ldots & 39\end{array}$

$\begin{array}{lllllllll}\text { PULPODACE. } & \ldots & \ldots & \ldots & \ldots & \ldots & \ldots & 242\end{array}$

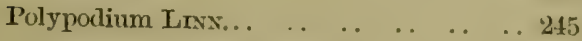

" lineare 'Тнuмs. . . . . . 245

polypremum Lins. ..

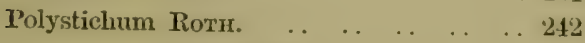

amabile Sur. $\quad . \quad \ldots \quad \ldots \quad \ldots 242$

niitakayamense IIAYATA .. 243

Potentilla LrNs.

" gelida C. A. MEx. . . . . . $\quad 83$

" grandiflora Lins. .. $\quad . \quad . \quad 83$

, leuconole Dox. . . . . . . . . 84

, leuconota Dox. var, morrisoni-

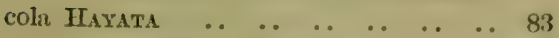

leuconota Hayata. . . . . . 83

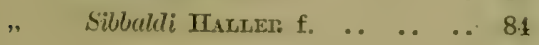

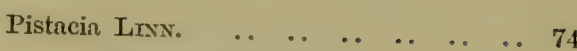

" chinensis Bunce. . .

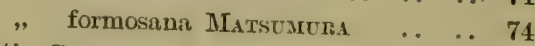

Prati̊ GAUD. ..

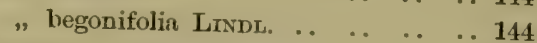

Prenanthes chinensis Trund. .. $\quad$.. $\quad$. . 144

Primulacez. ..

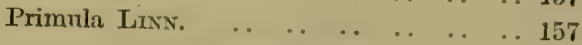

" Cochlumienu HeMsi. . . . . . 157

$\begin{array}{llllllllll} & \text { sp. } & \ldots & \ldots & \ldots & \ldots & \ldots & \ldots & \ldots & 157\end{array}$

Procris Juss. . .

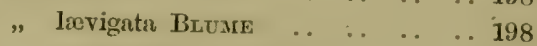

", sessilis Hook, et Arx. .. . . . . 198

$\begin{array}{lllllllll}\text { Proteaceze. } & \text {. } & \ldots & \ldots & \ldots & \ldots & \ldots & \ldots & 189\end{array}$

$\begin{array}{llllllll}\text { Prunus Lins... } & \ldots & \ldots & \ldots & \ldots & \ldots & \ldots & 77\end{array}$

" campanulata Maxis. $\quad . \quad \ldots \quad . \quad \ldots 77$

" japonica Thusm. ..

$\begin{array}{lllll}\text {, Kawakamii Hayata } & \ldots & \ldots & \ldots & 77\end{array}$

$\begin{array}{lllllll}\text { Psendotsuga CArir... } & \ldots & \ldots & \ldots & \ldots & \ldots & 223\end{array}$

japonica SHMisawa $\ldots \quad \ldots 223$

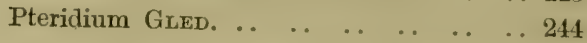

" aquilinum К

sum Borr ..

Pyrethrum indicum CAss. ..

Pyrola Lrsx. . . . . . .

" elliptice Nutr. var. morrisonensis

HaYATA . . . . . . . . . . . . . 155

" morrisonensis HAYATA .. . . . j55

, rotundifolia Lrsx. . . . . . . 156

Quercus Lns.

" amygdalifolia Skax. . . . . 200

" brevicaudata Sxax. . . . . . . 201

" Carlesii Hearst. . . . . . . . 204

, crespilata Тнunв. .. ..

" dentata Thuxв. .. . . . . . 200

, formosana Skix. . . . . . . . 200

, glauca Тншхт. . . . . . . . . . . 200

, Junghuhnii MrQ. . . . . . . . 20*3

, Kawnkamii IIxata . . . . 201

, Konishii Hayata . . . . . . 201

," obovata Bunge. . . .

, serratr Tircxi... . . . . . . . 203

, spicate Sxirrir. . . .

" Trrinbilis BLUME. . . . . . . . 204

$\begin{array}{llllllllll} & \text { " sp. } & \ldots & \ldots & \ldots & \ldots & \ldots & \ldots & . & 204\end{array}$

RANUNCULACEE。

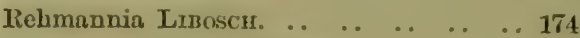

" Oldhami Hearsi. $\quad . \quad \ldots . \quad \ldots 174$

Retinispora obtusa Srew, et Zucc. . . . 208 
RHAMNEE.

Phamaus Liss. arguta MAxin, var. Niknlarai

MaYATA. . . Rhodorlenchon Lrxix. chrysanthum PALL. .. . . . 155 Nakaharai HAyata .. . . .. 153 Oldhami MAxnr.. . . . . . . 153 Oldhami Maxn. .. . . . . . . 154

" " vier. gliundulosum

HäAтA . ", jseudo-chrysanthum Híat... . . . 154 " serpyllifolium ПАХАTA .. 153

Thluus Lrxw.

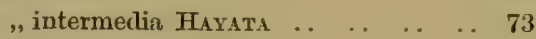

„, Toxicodendron Liss. . . . . . . . . 74

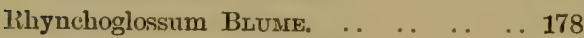

$\begin{array}{lllllll} & \text { Blumei } & \ldots & \ldots & \ldots & \ldots & 178\end{array}$

" obliquum BLUMe .. . 178

Ribes Lrwx. "formosanum H.AYATA ..... . . 93

ROSACEE.

Rosa Livn.

"Willmottia Henss.

„, xantlina Lindu. . . . .

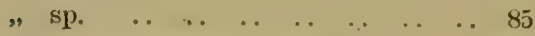

Tostellularia procumbens NEFS .. . . . 180

Rubtacez.

Rubin Irvs. . .

", cordifolir Lrnx. .. . . . . . . . 116

, lanceolata Hatata . . . . . . 117

"Schumaniana E. Prxtzel. . . . 117

Inubus Lrsx.

" corchorifolius Liss. f. var. glaber

$\begin{array}{llllllllll}\text { Matsuar. } & \ldots & \ldots & \ldots & \ldots & \ldots & \ldots & \ldots & 79\end{array}$

, elegans Hayata .. $. . \quad \ldots \quad . .79$

, fraxinifolius PoIm. .. $\quad . . \quad \ldots . \quad . .80$

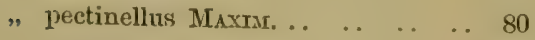

", peutalobus Hayata.. .. . . . 80

" Rolfei Vidal var. lanatus Haxata. 81

" rosafolius Sxr. var. hirsutus HAya-

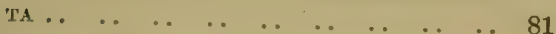

$\begin{array}{lllllllll}\text { Rutackis. } & \ldots & \ldots & \ldots & \ldots & \ldots & \ldots & \ldots & 81 \\ & & \ldots & \ldots & 67\end{array}$

$\begin{array}{llllllll}\text { Rungia NEes... } & \ldots & \ldots & \ldots & \ldots & \ldots & \ldots & 180\end{array}$

" parviflora NeEs var. pectinata

Clabrike. . .

" pectinata NeEs.. .. .. . . . . 180

" polygonoiles NEES.. .. .. . . 180

$\begin{array}{lllllll}\text { Saccharum Lrsx. .. } & \ldots & \ldots & \ldots & \ldots & \ldots & 236\end{array}$

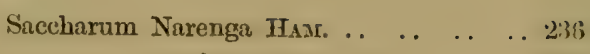

porphyrocomum НАск. .. .. 236

$\begin{array}{lllllllll}\text { S.thCaCEIE. } & \ldots & \ldots & \ldots & \ldots & \ldots & \ldots & \ldots & 206\end{array}$

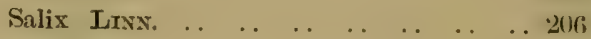

$\begin{array}{llllllllll} & \text { sp. . . } & \ldots & \ldots & \ldots & \ldots & \ldots & \ldots & \ldots & 206\end{array}$

Salvia LrNs.

", scapiformis HaNCE. . . . . . . 182

" " var. pinnata Hatata. 18:

Sanicula Lrsv. 1 .

" petagnioides Hayata .. .. .. 103

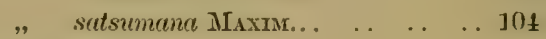

SiPINDACE.E. . .

Sarcopyramis WALL.

" lanceolata WALL. . . . . .

" nepalensis WALL. . . . . .

Sanssurea DC. $\quad \ldots$. .

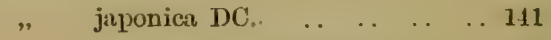

" $\quad$ var. longicephala Hayata.. 111

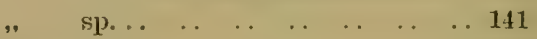

$\begin{array}{llllllll}\text { SAXmmagace. } & \ldots & \ldots & \ldots & \ldots & \ldots & \ldots & 85\end{array}$

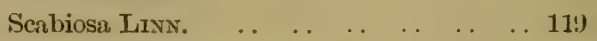

, lacerifolia ILiyata _. . . . 119

Schima Rerww.

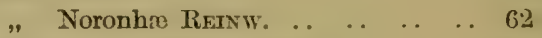

Schizocodon rotundifolius MIAxMr, . . . 156

Scirpus Lins...

" trifudus HANCE. . . . . . . . 230

" morrisonensis HaYata. . . . . 230

Scrophulartinete. ..

Scrophularia Lrss: $\quad \ldots \quad$.

" alata A. GRAY var. duplicato-ser-

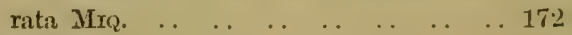

Scutellaria Lrvs. $\ldots \begin{array}{lllllll} & \ldots & \ldots & \ldots & \ldots & \ldots & 18: 3\end{array}$

luzonica RoLFe. . . . . . 18:3

Sedum Lixx. .

" morrisonense Hayata. . . . . 94

" Roborocoskii Maxiar... . . . . . . 91

$\begin{array}{llllllllll} & \text { sp. } & \ldots & \ldots & \ldots & \ldots & \ldots & \ldots & \ldots & 95\end{array}$

Senecio Lins...

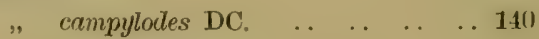

" chinensis DC. ..

" intermedius Wrart. . . . . . 140

," monnnthus DreLs... . . . . . 139

", scandens Harr.

" stipulatus WALL. .. $\quad \ldots \quad \ldots \quad \ldots 140$

" Wightianus DC. . . . . . . . 110

Shortir Torr. et Gr. . . . . $\quad$. $\quad$. . . . 156

" rotundifolir (MLamin.) MañNo. .. 156

Shrubbery recror... 
Sibballia Lrss.

" cuneata Kuxze. . . . . . .

" procumbens Lrxs. . . . . . 81

Silhthorpia pinnata Вехтн. .. .. .. $\quad .170$

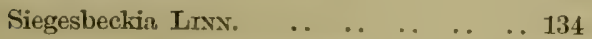

orientalis Livs. . . . . . 134

Silene Lrxx.

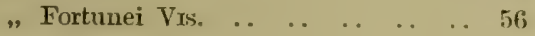

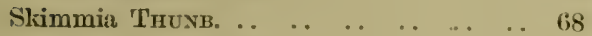

" japonica 'Thunв. .. . . . . . . 68

Smilacina DESB.

, lirta Maxme . . . . . . 225

", japonica A. GRAY. . . . . . 225

" japonica var. mandschurica

$\begin{array}{lllllllll}\text { Mахта... } & \ldots & \ldots & \ldots & \ldots & \ldots & \ldots & . & 225\end{array}$

Solayacez.

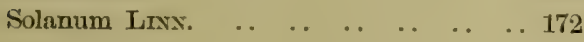

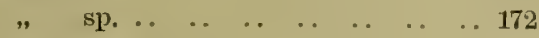

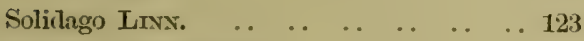

, cantoniensis. ..

" decurrens Lour. . . . . . . 123

". Virga-nurea Lrsw... . . . . . . 123

Sopubia Hax... . .

" formosana Havaia. . . . . . 175

, trifula Hax. . . ..

Spermacoce teres Rохв.

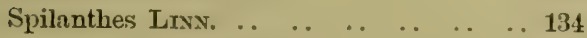

" Acmella Lixx. . . . . . . . 131

, tinctorius Lour... . . . . . 121

Spiræa Lisx. .

, prunifolia SIEB. et ZuCC. .. . . 78

$\begin{array}{llllllllll} & \text {, sp. } & \ldots & \ldots & \ldots & \ldots & \ldots & \ldots & \ldots & 78\end{array}$

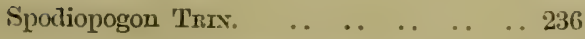

" Trawakamii Haxata. . . . 236

" trinanensis HiYata. . . . 236

Strachyurus SrEB. et Zucc. . . . . . .

" himalaicus Hоок. f. et Trons. 62

" pracox Sreb, et Zucc. . . . 62

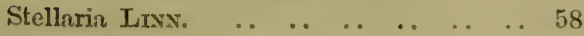

" saxatilis Buch.-HAхr. . $\quad . . \quad \ldots 59$

" stellato-pilosa Hirata. . . . 58

Steller Lrxx...

, Chamajasme Lrxx. . . . . 190

Strobilanthes Brume. . . .

" Championi T. AvDERS. .. 179

" flaccidifolins NEES. . . . 179

Stylidium chinense Loum. .. . . . . . 111

$\begin{array}{lllllllll}\text { Strmace.e. . . } & \ldots & \ldots & \ldots & \ldots & \ldots & \ldots & 15\end{array}$

Sivertin Lrsw...
Swertia alnta HAYATA... $\ldots$. . . . . 16

, tetrapterc Мाхтм. . . ..

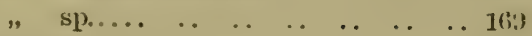

Symplocos Lrvs.

" confusa Brand...

, modesta Brand... . . . . . 15?)

". morrisonicola Hayata. . . . 160

" myrtacea HENSL. . . . . . 159

" spicata RoxB. . . . .

Syzygium buxifolium Hоок. . . . . . . . . 96

Tiviwania Hayata. .

eryptomerioides HaYAT1. . . . 215

Trancebo japonica Szysz. $\quad \ldots \quad$. .

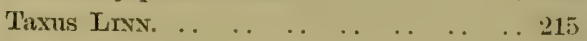

$\begin{array}{llllllllll} & \text { sp. } & \ldots & \ldots & \ldots & \ldots & \ldots & \ldots & \ldots & 215\end{array}$

$\begin{array}{llllllll}\text { Ternstremandez... } & \ldots & \ldots & \ldots & \ldots & \ldots & 60\end{array}$

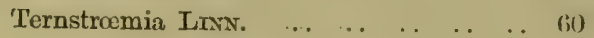

, japonica Thuxb. . . . . .

Thalictrum Lisn. . .

" $\quad$ Fauriei Haxata. .

, Lrbaini Matsumuna. .. $\quad . \quad 11$

Then Irsx.

" brevistyla Hayata. . . . . . . .

, caudata (WALL) .

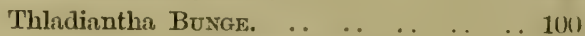

, formosana Hayata. . . . 10)

, mudiflora Hemss. . . . . . 100

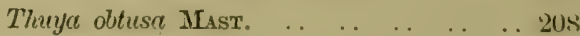

$\begin{array}{llllllll}\text { ThyMelance. } & \ldots & \ldots & \ldots & \ldots & \ldots & \ldots & 190\end{array}$

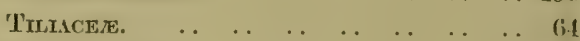

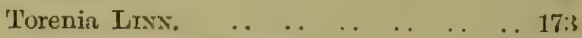

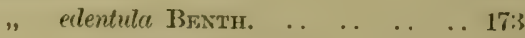

" peduncularis BeNтir. . . . . . . 173

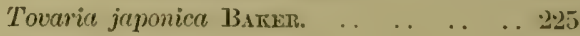

Trevesit Vrs. .

Iricyrtis WALL.

" lasiocarpa Matsum. . . . . . 226

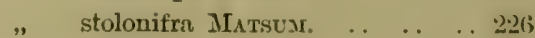

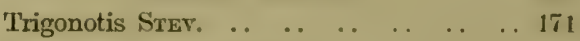

, formosnna HAYATA. . . . . 171

Trisetum Pens. subspicatum Besuv. . . . . 2:3s

Triumfettr Lrsw. .. pilos̊ Rоти. ... ... ... .. (i1

'Гrochocarses.

Trochodendron Siru, et Zucc. . . . . . 11 arrlioides Srazs, et Zucc... If

'Liropical Ameretcax Elmments... . . . . 24

'T'sugn C'n:1.. 
Tsuga formosana IIAYata. . . . . . . . 222

"diversifolia MLaxm. ..

, Sieboldi Carsi. . . . . . ..... .. 223

$\begin{array}{llllllll}\text { UnbeLLTHERג. } & \ldots & \ldots & \ldots & \ldots & \ldots & \ldots & 101\end{array}$

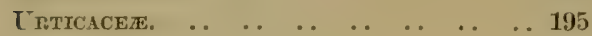

Crticr Lms. . .

„ Clioica Thuns, .. .. . . . . . . 196

, heterophylla VAHL. . . . . . . . 197

", Thunbergiana Srem... .. . . . 196

Liaria japonica InNw. . . . .

$\begin{array}{llllllll}\mathbf{V}_{\text {alemianez.... }} & \ldots & \ldots & \ldots & \ldots & \ldots & \ldots & 118\end{array}$

Tuleriana villosa Thuns. $\quad . \quad \ldots \quad \ldots \quad$.. 118

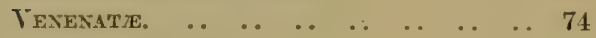

Ternonia Schreb. . . . . . .

" Andersoni Clanke. .. . . . 120

" cinerea LEss. . . . . . . . . 120

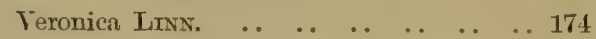

" angustifolia Fiscr. . . . . . 175

" morrisonicoln HAYATA. .. . 174

" paniculate Lins. . . . . . . 175

" serpyllifolia Lrs. . . . . . 175

, spuria LnNא... . . . . . . . . 175

$\begin{array}{lllllllll}\text { Tibumum, } & \ldots & \ldots & \ldots & \ldots & \ldots & \ldots & \ldots & 112\end{array}$

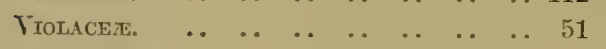

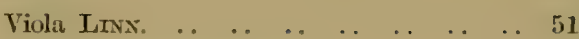

", fomosune Hayata. . . . .

" japonica LANGSD. .

" juponice var. pekinensis Maxrm. . 52

" lamtscluatica var. pelinensis REGFi. 52

, Kawakamii Hayata. . . . . . . 52

" Nagasawai Makino et Hayata. . $5: 3$

, tozanensis Hayata. . .

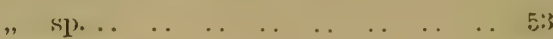

Visctum LrNN.. .

, articulatum Bris... . . . . . 191

", orientale WILLD. var. multinerve

HaYAtA. .

Wallenbergia Scmiad, $\quad \ldots \quad \ldots \quad \ldots \quad \ldots 145$

$\begin{array}{llllll}n & \text { agrestis A. DC. . . . . } & \text {. } 140\end{array}$

, gracilis A. DC. . . . . 14.

$\begin{array}{lllll} & \text { deliscens A. DC... . . } & \text {. } 1116\end{array}$

" quatrifida A. DC. . . . 11t;

", lavandulafolia A. DC. . . 14;

, Sieberi A. DC, .. . . . 111;

Youngix clinensis DC... . . $\quad \ldots \quad$.. $\quad \ldots 111$

Zanonic cissoides WALL. $\ldots$..

" pedata Miq. .. . . . . . . . . 101

Zanthoxylum trifoliatum Lrs. $\quad . . \quad \ldots \quad$. 10 , 
B. MAYATA.

FLORA MONTANA FORMOSE.

PLATE I. 


\section{PLATE I.}

\section{Polygala arcuata Hayata.}

Fig. 1. The plant.

2. A flower.

3. The same, sepals taken off, showing patals.

t. A pistil.

5. The superior sepal of the exterior series.

6 and 7. The inferior sepals of the exterior saries.

8. A sepal of the interior series.

9. Petals and stamens seen from above, patals a little expanded.

10. Stamens, seen from various sides.

11. A capsule:

12. A seed, seen from the dorsal side.

13. The same, seen from the lateral side.

14. The same, seen from the ventral side.

15. An albumen.

16. An embryo. 


\section{PLATE II.}

Stellaria stellato-pilosa HAYATA.

Fig. 1. A branch.

2. A leaf detached from an upper portion of a stem.

3. Transrerse section of a leaf, showing stellate hairs on both sides of the leaf.

4. A stellate hair on the upper surface of a leaf.

5. A superposed stellate hair on the under surface of a leaf.

(i. A flower.

7. A pistil.

8. A eapsule with persistent sepals and petals.

9. A capsule after dehiscence.

10. A placenta in a capsule

11. A seed.

12. An embryo. 
B. HAY ATA.

FLORA MONTANA FORMOSE.

PLATE III. 


\section{PLATE III.}

Thea trevistyla HaYATA.

Fig. 1. A branch.

2. A flower.

3. Vertical section of a flower, upper parts of the petals taken off.

4. Stamens, one seen from the ventral side, and the other, from the dorsal side.

5. Vertical section of an orary.

6. Cross section of the same orary. 


\section{PLATE IV.}

\section{Rubus elegans Hayata.}

Fig. 1. The plant.

2. A leaf, detacher from the basal portion of a stem.

3. A flower seen from the under side.

4. A petal.

5. Fruits.

6. Vertical section of the same.

7. A stamen, seen from the inner side.

8. The same, səen from the outer side.

9. A fruit.

10. Another one, highly magnified. 
13. HА Y А А.

FLORA MONTANA FORMOSE.

\section{PLATE V.}




\section{PLATE V.}

Potentille Tenconote Dor. var. morrisonicole HaYaTA.

Fig. 1. The plant.

2. A rạdical leaf.

3. A flower, seen from the upper side.

4. Another one, seen from the under side.

5. Stamens, one seen from the ventral side, and the other, from the dorsal side.

6. A pistil.

7. Fruits on a receptrcle.

8. A fruit.

9. The same, in vertical section.

10. An embryo. 
B. HAYATA.

FLORA MONTANA FORMOSE.

PLATE VI. 


\section{PLATE VI.}

Hycliangea glabra Hayata.

Fig. 1. The plant.

‥ A fruit.

:. The same, in cross section.

4. A seed.

5. An embryo. 


\section{PLATE VII.}

\section{Hydrangece integra HaYaTA.}

Fig. 1. The plant.

2. A fruit.

3. The same, in vertical section.

4. A seed.

5. An embryo. 
B. HAYATA.

FLORA MONTANA FORMOSE.

\section{PLATE VIII.}




\section{PLATE VIII.}

Hydrangea Kavakamii HAYATA.

Fig. 1. The plant.

2. A fruit.

3. The sime, in cross section.

4. A seer.

5. An embryo. 
I3. HAY A' A.

FLORA MONTANA FORMOSE.

\section{PLATE IX.}




\section{PLATE IX.}

Ribes formosanum HaYATA.

Fig. 1. A branch.

2. Calyx and petals, laid opæn.

:i. Styles.

t. A fruit.

5. Seeds.

6. A ssed (highly magnified)

7. An embryo. 
B. HAYATA.

FLORA MONTANA FORMOSE.

\section{PLATE X.}




\section{PLATE $\mathrm{X}$.}

\section{Barthea formosana HAYATA.}

Fig. 1. A branch.

2. A flower-but.

3 A flower.

4. A petal.

5. A portion of the upper margin of a petal

6. A longer stamen, (dorsal riew.)

7. The same, (lateral view.)

'8. A shorter stamen, (lateral view.)

9. An ovary, in vertical section.

10. The same, in cross section, stamens are seen in the holes of the wall of the calyx tube.

11. A capsule, a part of the calyx taken off.

12. Seeds, seen from different sides.

13. An embryo. 
B. HAYATA.

FLORA MONTANA FORMOSE.

PLATE XI. 


\section{PLATE XI.}

\section{Thladianthe formosane HAYATA.}

Fig. 1. A branch.

2. A fiower, seen from abore.

:3. The same, seen from below.

4. A flower-bud.

5. Stamens and glands at the base of filaments. 
B. HAY ATA.

FLORA MONTANA FORMOSE.

PLATE XII. 


\section{PLATE XII.}

Sanicula petaynioides Hayata.

Fig. 1. The plant.

2. An numbel.

3. A male flower, seen from side.

4. The same, seen from below.

5. The same, seen from above.

(i. A stamen, seen from within.

7. The same, seen from without.

8. A perfect flower.

9. A fruit.

10. The same, in cross section. 
B. HAYA'A.

FLORA MONTANA FORMOSE.

PLATE XIII. 


\section{PLATE XIII.}

Fatsia polycarpa HaYata.

Fig. 1. The plant.

2. An umbel, not yet unfolded.

3. The same, seen from a different side.

4. An outer larger bract.

5. 'Two inner smaller bract.

6. A flower-bud.

7. The same, in vertical section.

8. An ovary, in cross section.

9. Stamens, seen from different sides.

10. A flower.

11. The apэx of a style.

12. A petal. 
B. HAXATA.
FLORA MONTANA FORMOS.
PIA TE XIV.

B. HAXATA.
FLORA MONTANA FORMOS.
PIA TE XIV.

B. maYATA.
FLORA MONTANA Formose. 


\section{PLATE XIV.}

\section{Oreopanax formosana HiуатA.}

Fig. 1. A branch.

2. A head.

3. The same, in vertical section.

4. A flower, in vertical section.

5. An ovary, in cross section.

6. An ovary, in a more advanced stage.

7. Stamens, seen from different sides.

8. A head of fruits.

9. A ruminate albumen.

10. The same, in vertical section, the embryo is seen.

11. The same embryo, more enlarged.

12. Section of a fruit; a seed is ripa, while the other is abortive.

13. A seed (lateral view).

14. The same (dorsal view).

15. An inferior bract.

16. A lateral bract.

17. Hairs covering all over the plant.

18. A stellats hair dotting the surface of a leaf and inflorescence. 
13. HAYATA.

FLORA MONTANA FORMOSE.

\section{PLATE XV.}




\section{PLATE XV.}

Demnacanthus angustifotius Hayıtx.

Irig. 1. A brimch.

2. A flower.

3. Corolla and stamens, laid opəu.

4. A flower showing the ovary, corolla and stamens taken off.

5. An ovary, in vertical section.

6. The same, in cross section.

7. An ovule.

s. A stamen (đorsal view).

9. The same (lateral view).

10. A fruit.

11. 'The same, in cross section.

12. A seed (ventral view).

1:3. Tho same (dorsal view).

14. An albumen, in section, ths embryo seen.

15 and 16. An embryo, seen from different sides. 
B. HAYATA.

FLORA MONTANA FORMOSE.

PLATE XVI. 


\section{PLATE XVI.}

Scabiosa Tacerifolia Hayata.

Fig. 1. The plant.

2. A marginal flower.

3. A disc-flower.

4. Cross section of an involucel.

5. A bract of a flower.

6. Corolla and stamens, laid open.

7. Stamens, seen from different sides.

8. An inferior ovary.

9. A fruit in the involucel.

10. The same, the inrolucel taken off.

11. Cross section of the same.

12. An allbumen.

13. An embryo. 
13. HAYATA.

FLORA MONTANA FORMOSEE.

PLATE XVII. 


\section{PLATE XVII.}

Leontopodium microphyllum HAYATA.

Fig. 1. The plant.

2. A cyme of lieads.

3. A head.

4. A bract of an involncre.

5. A female and fertile flower.

6. A perfect and sterile flower.

7. A female fertile flower, pappus taken off, corolla seen.

S. Stamans of a perfect and sterile flower.

9. A style of the same flower.

10. A stamen, more magnified.

11. An apical portion of a style.

12. Setre of a pappus. 
13. HAYATA.

FLORA MONTANA FORMOSE.

\section{PLATE XVIII.}




\section{PLATE XVIII.}

Anaplatis Nagasazai Hayata.

Fig. 1. The plant.

2. An inner bract.

3. An onter-most bract.

4. An inner-most bract.

5. A perfect and sterile flower.

6. A female and fertile flower.

7. The apical portion of the style of a perfect flower.

8. A stamen of a perfect flower.

9. The apical portion of the style of a female flower.

10. The apical portion of the corolla of a femal flower.

11. An achene.

12. A bristle of the pappus of a perfect flower.

13. A bristle of the pappus of a female flower. 
B3. HAYATA.

FLORA MONTANA FORMOS正.

\section{PLATE XIX.}




\section{PLATE XIX.}

Gnapliatium lineare HaYats.

Fig. 1. The plant.

2. A head.

3. A female flower.

4. A perfect flower.

5. A stamen of the same flower:

6. The apical portion of the style.

-7. The apical portion of the corolla of $a$ female - flower.

8. An achene.

9. A bristle of a pappus.

10. An outer-most bract.

11. An inner-most bract. 
B. HAY ATA.

FLORA MONTANA FORMOSE.

\section{PLATE XX.}




\section{PLATE XX.}

Artemisia niitalayamensis HaYATA.

Fig. 1. The plant.

2. A head.

3. The same, seen from a little below.

4. An outer-most bract.

5. An inner bract.

6. An inner-most bract.

7. A perfect flower.

8. A stamen of the sime flower.

9. The apical portion of the style.

10. A female flower.

11. The apical portion of the style of the same flower.

12. An achene.

13. The same, in cross section.

14. Another achene.

15. The same, in cross section.

16. A seed.

17. An embryo 
B. HAYATA.

FLORA MONTANA FORMOSE.

PLATE XXI. 


\section{PLATE XXI.}

Artemisia oligocarpa HaYaTA.

Fig. 1. The plant.

2. A head.

3. A female flower.

4. A perfect and sterile flower.

5. The stamens of the same flower.

6. The upper portion of the style of the same flower.

7. The same, more magnified.

8. A stamen of the same flower.

9. The upper portion of the corolla.

10. An Achene.

11. An embryo. 
B. HAYATA.

FLORA MONTANA FORMOSE.

PLATE XXII. 


\section{PLATE XXII.}

Ains?ice macroclinidioides HАYаTА.

Fig. 1. The plant.

2. Roots.

3. A part of an inflorescence.

4. A cleistogamous flower.

5. The corolla of the same flower.

6. The strmens of the same flower.

7. The style of the same flower.

8. The upper portion of the same style.

9. The basal portion of the same style.

10. A bristle of a pappus.

11. A stamen, much more maguified.

12. Cioss section of an achene.

13. An embryo. 
B. HAYATA.

FLORA MONTANA FORMOSA.

\section{PLATE XXIII.}




\section{PLATE XXIII.}

Ains?ioen movisonicole HAYATs.

Fig. 1. The plant.

2. A cleistogamous flower.

$\therefore$ A radical leaf.

4. Corolla.

5. Stamens.

(i. A stamen, much more maguified.

7. A style.

S. A bristle of a pappus.

9. Cross section of an achene.

11. A seed.

11. An embryo. 
B. HAYATA.

FLORA MONTANA FORMOSE.

PLATE XXIV. 


\section{PLATE XXIV.}

\section{Vaccinium Merrillianm HaYaT.}

Fig. 1. A branch.

2. Seeds (natural size)

3. A seed, highly maguified.

4. An albumen.

5. An embryo. 
B. HAYA'A.

FLORA MONTANA FORMOSF.

PLATE XXV. 


\section{PLATE XXV.}

\section{Geuttierice Itoance HAYATA.}

Fig. 1. The plant.

2. A flower-bud.

3. A flower.

4. A stamen (ventral view).

5. The same (dorsal view).

6. The same (lateral view).

7. An ovary.

8. The same, in cross section.

9. A fruit.

10. The same, in vertical section.

11. Seeds.

12 and 13. The same, seen from different sides, highly magnified.

14. An albumen.

15. An embryo, in the same proportion.

16. The same, much more magnified. 
13. HAYATA.

FLORA MONTANA FORMOSE.

PLATE XXVI. 


\section{PLATE XXVI.}

Rhododendron pseudo-chrysanthum НАхата.

Fig. 1. A branch.

2. An inflorescence.

3. An inner-most bract.

$4,5,6,7,8,9$ and 10. Eracts of different series.

11. An outer-most bract.

12. A corolla, laid cpen.

13. Stamens.

14 and 15. The same, seen from different sides.

16. An ovary.

17. The same, much more magnified.

18. The upper portion of a style.

19. Cross section of an ovary.

20. A capsule, in vertical section.

21. Cross section of a capsule.

22. Seeds (natural size).

23. A seed highly magnified.

24. An embryo. 
I3. HAYATA.

FLORA MONTANA FORMOSE.

PLATE XXVII. 


\section{PLATE XXVII.}

Symplocos monvisonicole HaYATA.

Fig. 1. A branch.

2. A flower-bud.

3. A flower.

4. Corolla and stamens laid open.

5. A stamen, seen from withont.

6. The same, seen from within.

7. A calyx.

8. The same, in vertical section.

9. Cross section of an ovary.

10. An ovile. 
13. HAYATA.

FLORA MONTANA FORMOSE.

\section{PLATE XXVIII.}




\section{PLATE XXVIII.}

Lagania dentata (ELMER) HAYATA.

Fig. 1. The plant.

2. A deformed flower.

3. A flower.

4. The same, a part of the calyx taken off, the corolla seen.

5. The same corolla, laid open.

fi. A stamen, seen from without.

7. An ovary.

8. The same, in vertical section,much more magnified.

9. The same, in cross section.

10. A capsule.

11. The same, after opening.

12. A seed.

1:). An embryo. 
13. HAYATA.

FLORA MONTANA FORMOSE.

PLATE XXIX. 


\section{PLATE XXIX.}

Veronica morrisonicola HAYATA.

Fig. 1. The plant.

2. A flower, seen from side.

3. The same, seen from above.

4. A corolla, laid open.

5. An ovary.

6. A branch bearing fruits.

7. A fruit.

8. The same, in rertical section.

9. The seeds (natural size).

10. A seed, magnified.

11. An embryo. 
B. HAY ATA.

FLORA MONTANA FORMOSE.

PLATE XXX. 


\section{PLATE XXX.}

\section{Polygonem minutum HaYATA.}

Fig. 1. The plant.

$\because$ A sheath.

:3. A flower on a brinchlet.

4. A flower.

5. A periantl, laid opэn.

(i. An ovary.

7. Stamens, seen from different sides.

8. An ovitry.

9. A fruit.

10. The same, in vertical section, showing the embryo in it.

11. The embryo. 
13. HAYATA.

FLORA MONTANA FORMOSE.

PLATE XXXI. 


\section{PLATE XXXI.}

\section{Polygonum mon'risonense HaYATA.}

Fig. 1. The plant.

2. A branch, showing sheaths.

$\therefore$ A hear.

t. Bracts, seon from different sides.

5. A flower.

(i. A perianth, laid open.

7. An ovary.

s. A stamen with a gland at the bass of the filament.

9. A fruit.

10. A seed.

11. An embryo. 
13. HAXATA.

FLORA MONTANA FORMOS年。

PLATE XXXII. 


\section{PLATE XXXII.}

\section{Peperomia Naticharai Hayata.}

Fig. 1. The plant.

2. A spike.

3. A female flower, seen from the dorsal side.

4. The same, seen from literal side.

5. The same, in a more advanced stage.

6. A fruit. 
B. HAYATA.

FLORA MONTANA FORMOSE.

PLATE XXXIII. 


\section{PLATE XXXIII.}

Balanophos'ce spicata HaYaTa.

Fig. 1. A male plant.

2. A female plant.

3. A male flower.

4. A stipe with female flowers.

5. A female flower. 
13. HAYATA.

FLORA MONTANA FORMOS庄.

\section{PLATE XXXIV.}




\section{PLATE XXXIV.}

Balanophora parvior HaYaTA.

Fig. 1. A male plant.

2. A female plant.

3. A male flower.

4. A stipe with female flowers.

5. A female flower. 
13. HAYATA.

FLORA MONTANA FORMOSE.

PLATE XXXV. 


\section{PLATE XXXV.}

Ficus vaccimioides Hewst. et KING.

Fig. 1. The plant.

2. A dwarf.

3. A leaf with stipules.

4. An axil, showing stipules.

5. A leaf.

6. A receptacle.

7. The same, seen from below.

8. Another form of a receptacle.

9. A receptacle, in vertical section.

10. Bracts at the month of the same receptacle.

11. A male flower.

12. A stamen.

13, 14, 15 and 16. Various forms of female flowers and gall flowers.

17 and 18. Oraries, seen from different sides. 
13. HAYA'TA.

FLORA MONTANA FORMOSE.

PLATE XXXVI. 


\section{PLATE XXXVI.}

Elatostoma minutum HaYATA.

Fig. 1. The plant.

2. A receptacle in an axil.

3. The same, detached from the asil.

4. A fruit. 


\author{
B. HAYATA.
}

FLORA MONTANA FORMOSE.

\title{
PLATE XXXVII.
}




\section{PLATE XXXVII.}

Quercus (Pasania) Konishii Hayata.

Fig. 1. A branch.

2. A male flower.

3. Stamens, seen from different sides.

4. Female flower.

5. A bract at the base of a flower.

6. A rudimental stamen in a scale of a flower.

7. Rudimental stamens, seen from different sides, more magnified.

8. A female flower, in vertical section.

9. A fruit, seen from a little above.

10. The same, seen from a little below.

11. The same, cup taken off, seen from below.

12. The same, showing the under side of the glans. 
B. HAYATA.

FLORA MONTANA FORMOSE.

\section{PLATE XXXVIII.}




\section{PLATE XXXVIII.}

Juniperus formosane HaYATA.

Fig. 1. A branch.

2. A leaf, seen from the inner side.

3. The same, seen from the outer sicle.

4. A fragment of a branch.

5. A fruit.

6. Bracts at the base of a fruit, magnified.

7. A seed (lateral view).

8. The same (dorsal view).

9. The same, in cross section.

10. A fruit showing vestiges upon it.

11. An albumen.

12. An embryo. 
13. HAY ATA.

FLORA MONTANA FORMOSÆ.

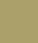

\section{PLATE XXXIX.}




\section{PLATE XXXIX.}

\section{Paris Tancifolic Hayata.}

Fïg. 1. A full grown plant.

2. A small form.

:3. A flower, a little magnified.

4. An ovary.

5. Cross section of an ovary.

6. An embryo (magnified).

7. An albumen (natural size).

8. The same albumen, in the same proportion as the embryo shown in Fig. 6. 
B. HAYATA.

FLORA MONTANA FORMOSE.

PLATE XL. 


\section{PLATE XL.}

\section{Brachypodium Kanvakamii HaYaTA.}

Fig. 1. The plant.

2. A flowering glume.

3. The same, the palea and rhachilla are taken off.

4. A palea, its section is figured near it.

5. An ovary with its lodicules.

6. The same ovary, more magnified.

7. The same, seen from a different side.

8. Two lodicules belonging to an ovary.

9. A stamen.

10. An achene, seen from different sides.

11. Cross section of the same.

12. The basal portion of the same achene.

13. The basal portion of a culm.

14. A part of a leaf, showing its ligule. 


\section{PLATE XLI.}

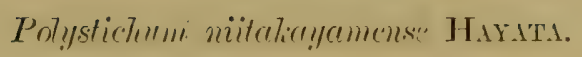

Fig. 1. Fronds of the plant.

2. A chaff, detached from the basal portion of a frond.

3. Another one, detached from the middle portion of a frond.

4. A pinna.

5. Section of a sorns.

6. Sporangia.

7. Section of the basal portion of a frond.

8. Section of the middle portion of a frond. 
Jour. Sci. Coll., Vol. XXV., Art. XIX., PI.I.

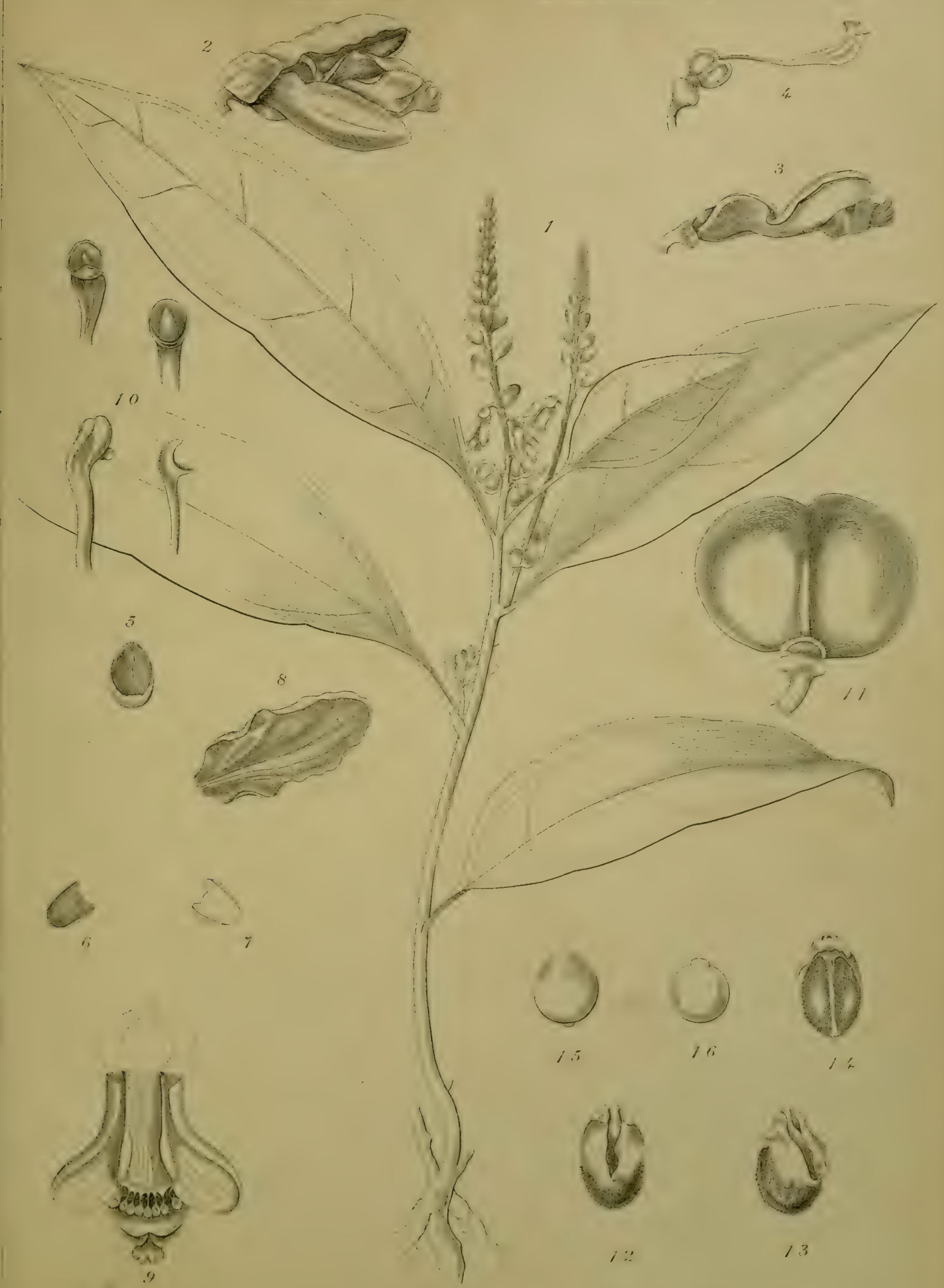

13. Hivalat del. 


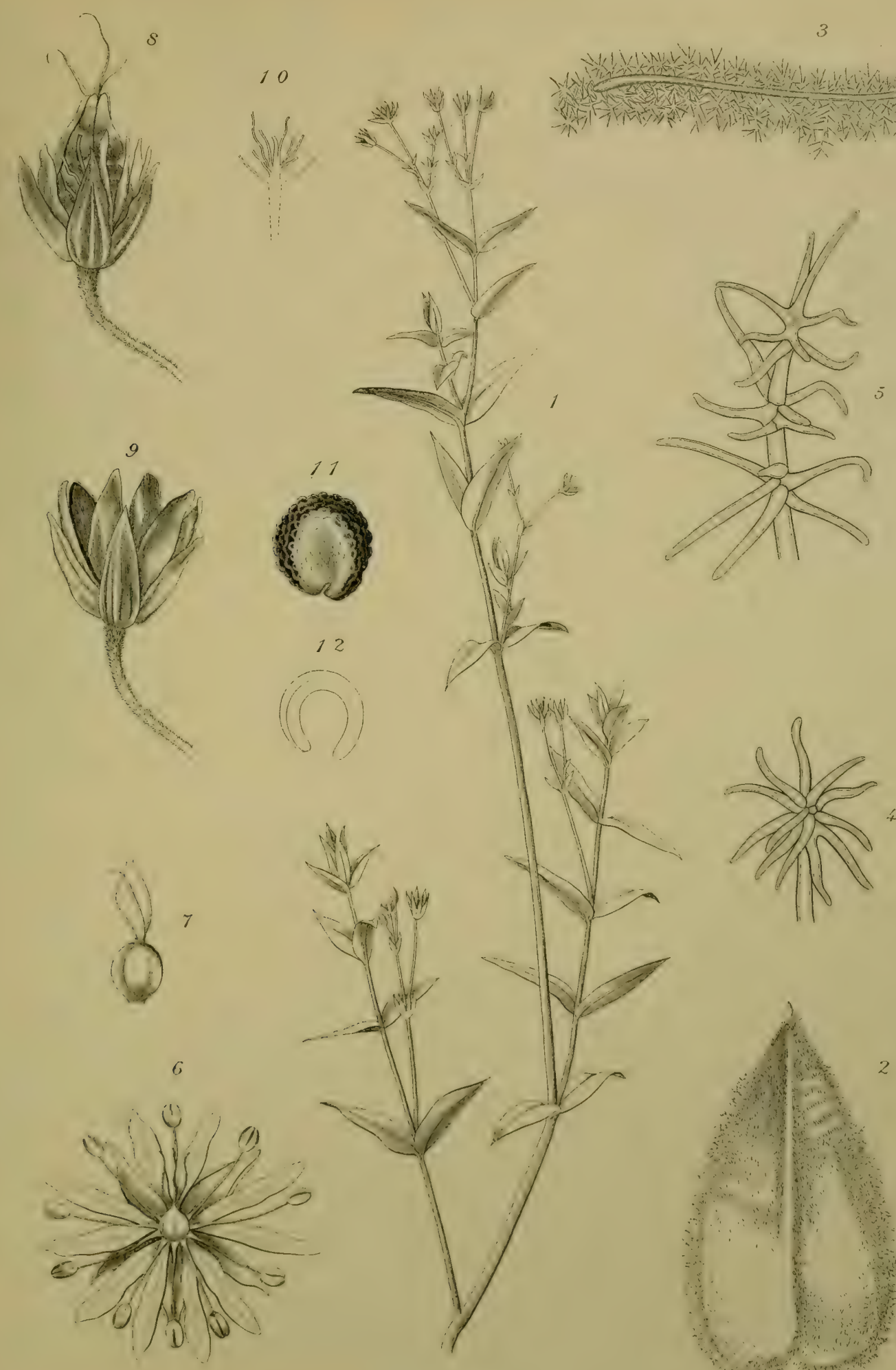

3
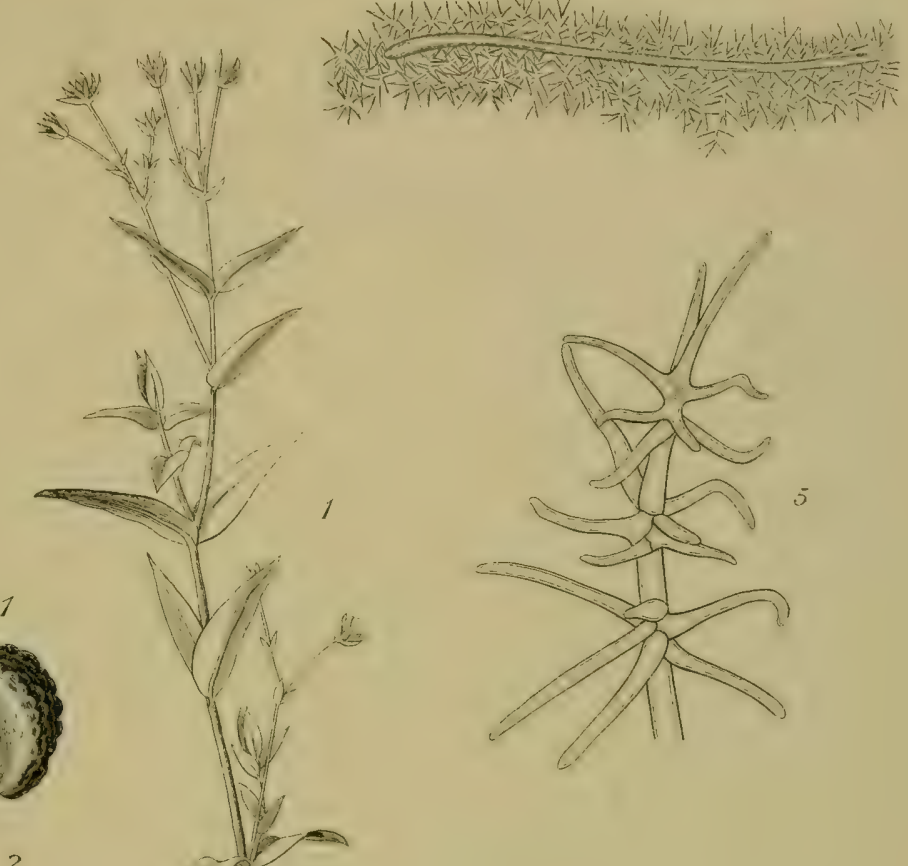



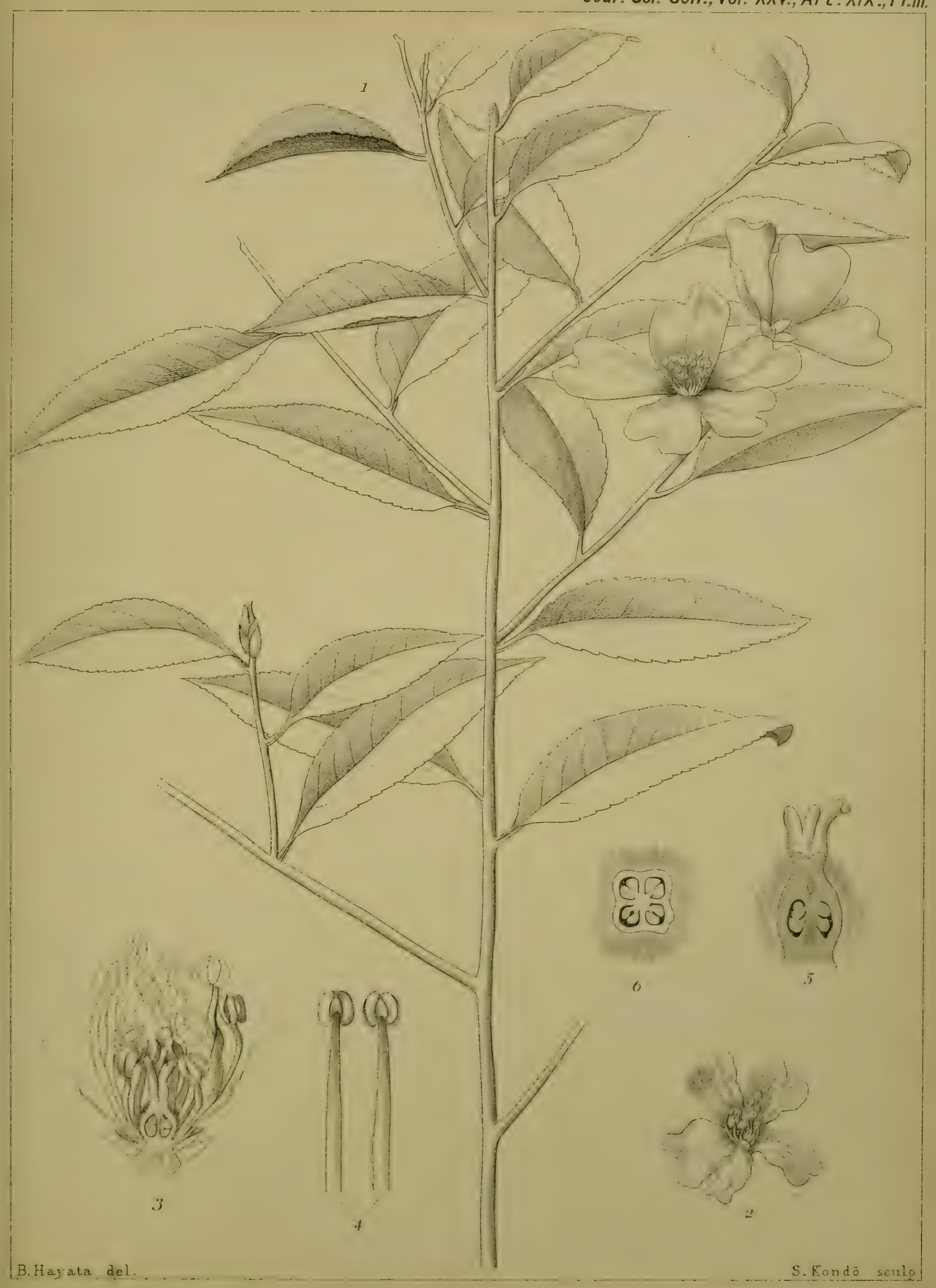



Jour. Sci. Coll., Vol. XXV., Art. XIX.,PI.IV.
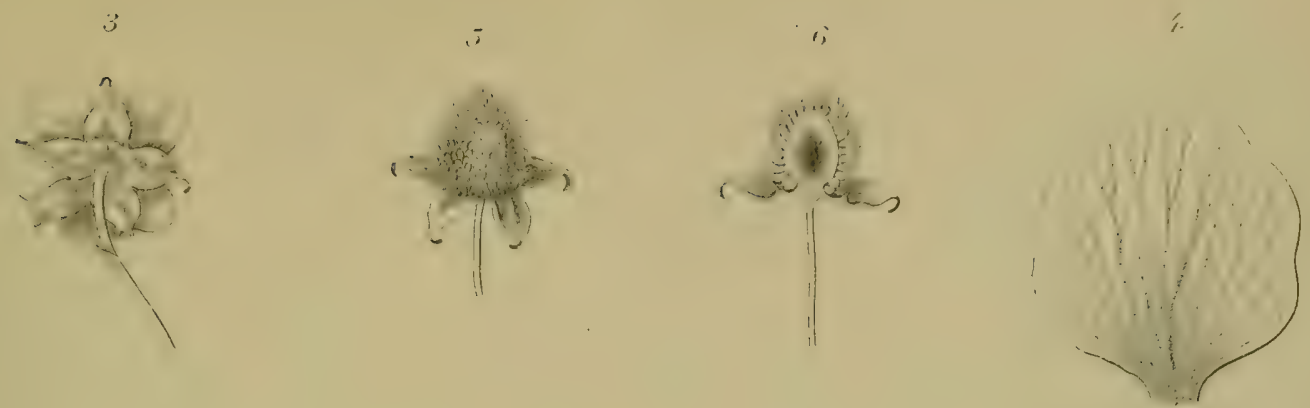

is
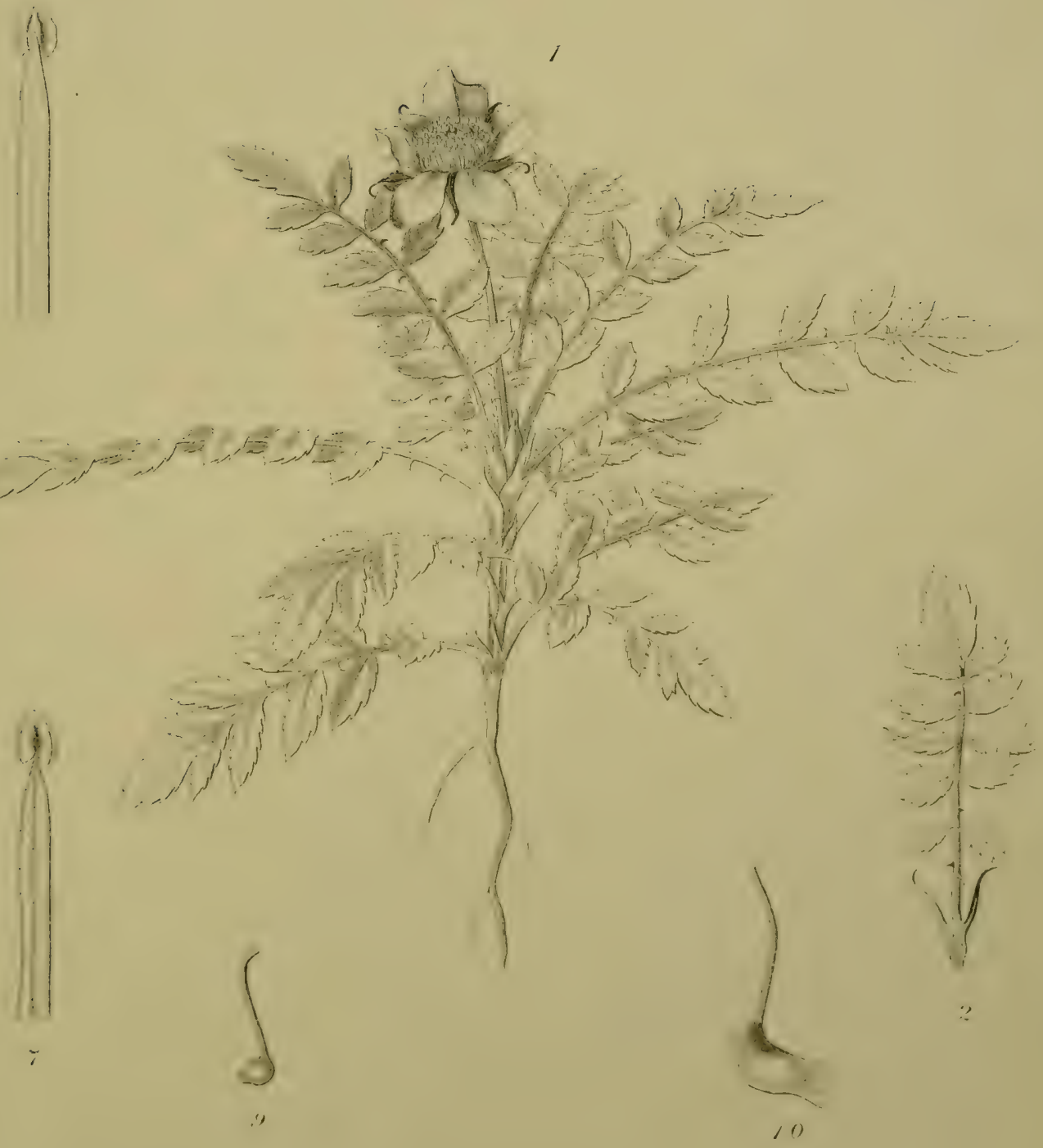

Jour. Sci, Coll., Vol. XXV., Art. XIX., PI.V.

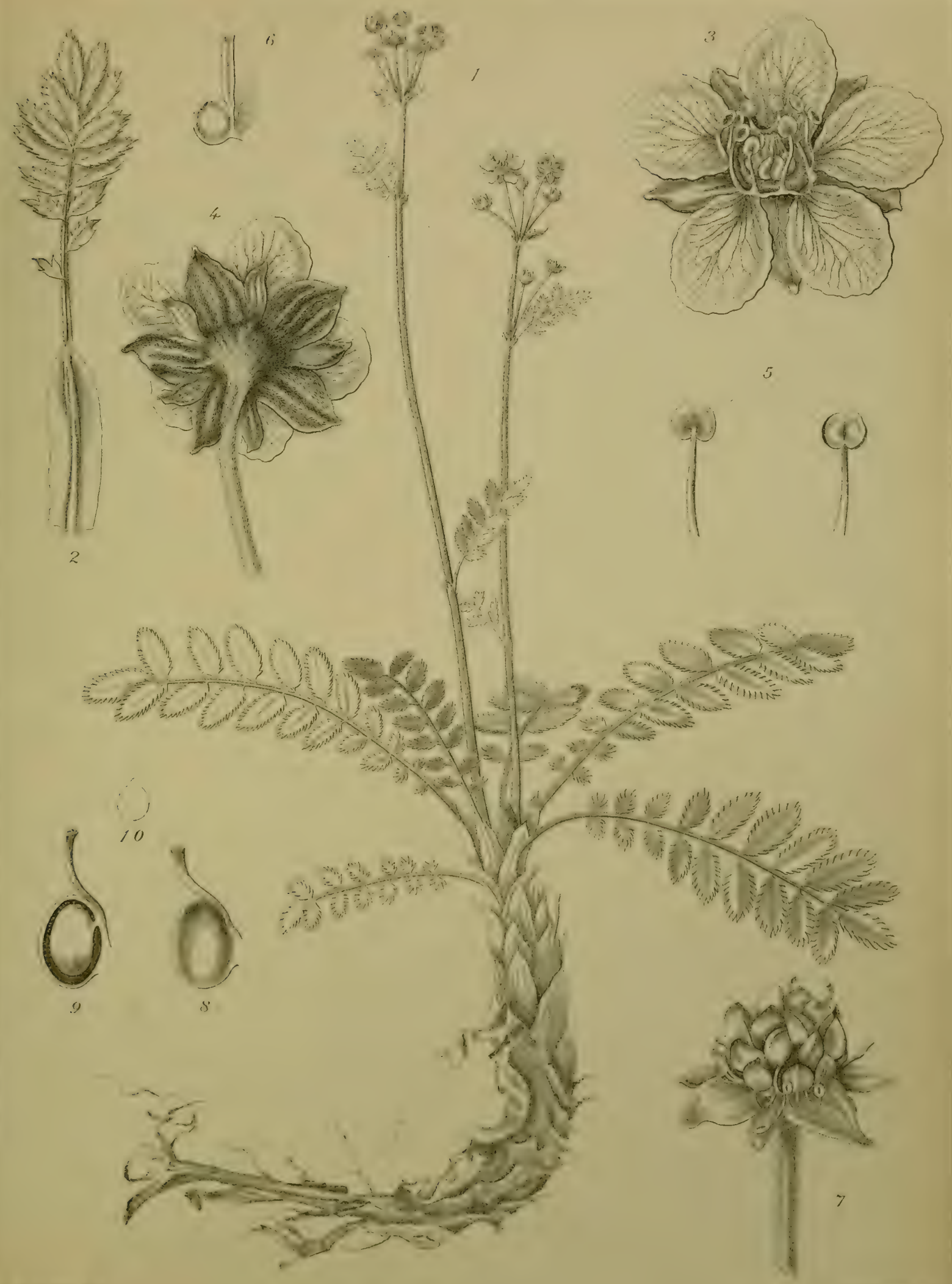

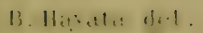



Jour. Sci. Coll., Vol. XXV., Art. XIX., PI.VI.

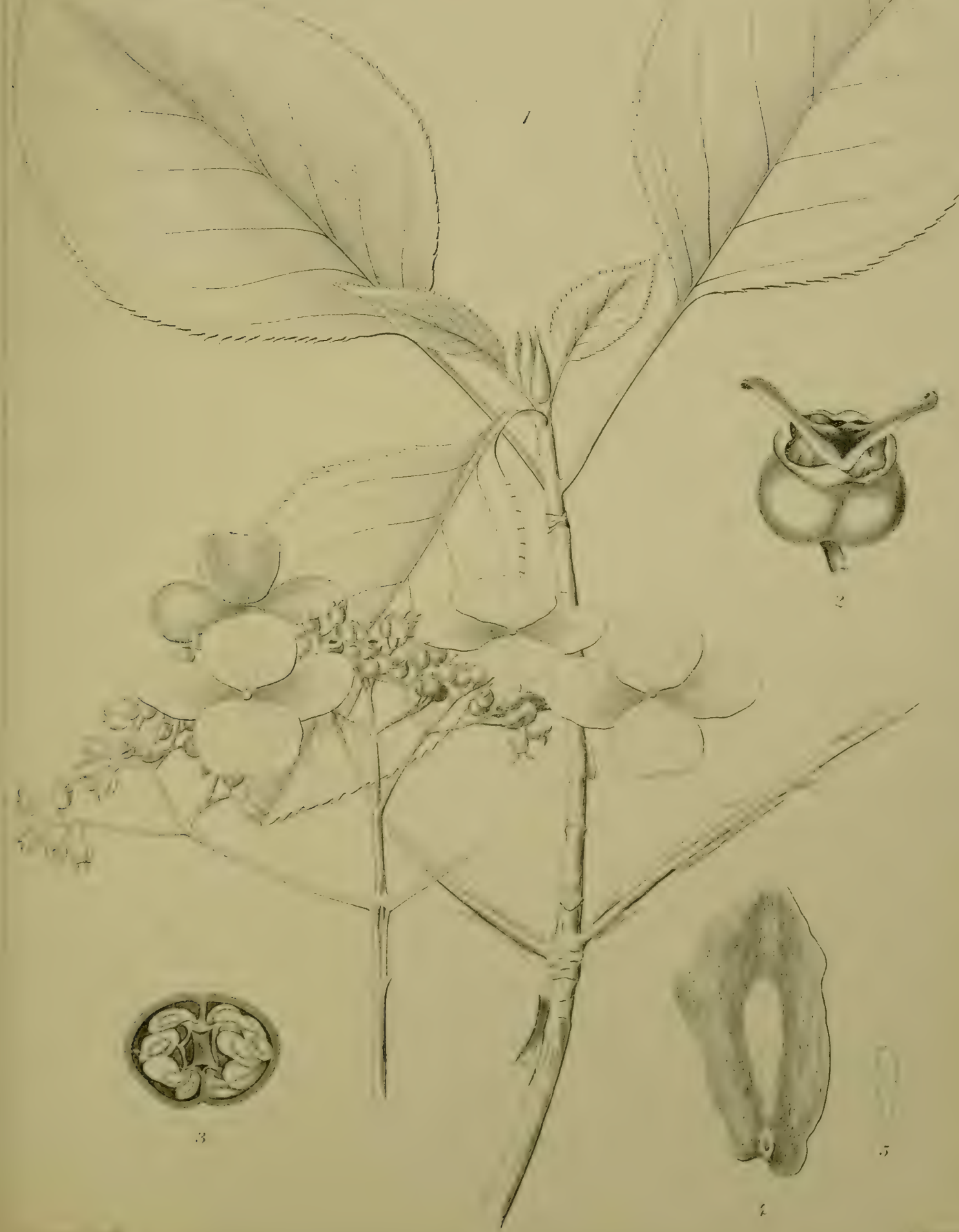



Jour. Sci. Coll., Vol. XXV., Art. XIX., PI.VII.

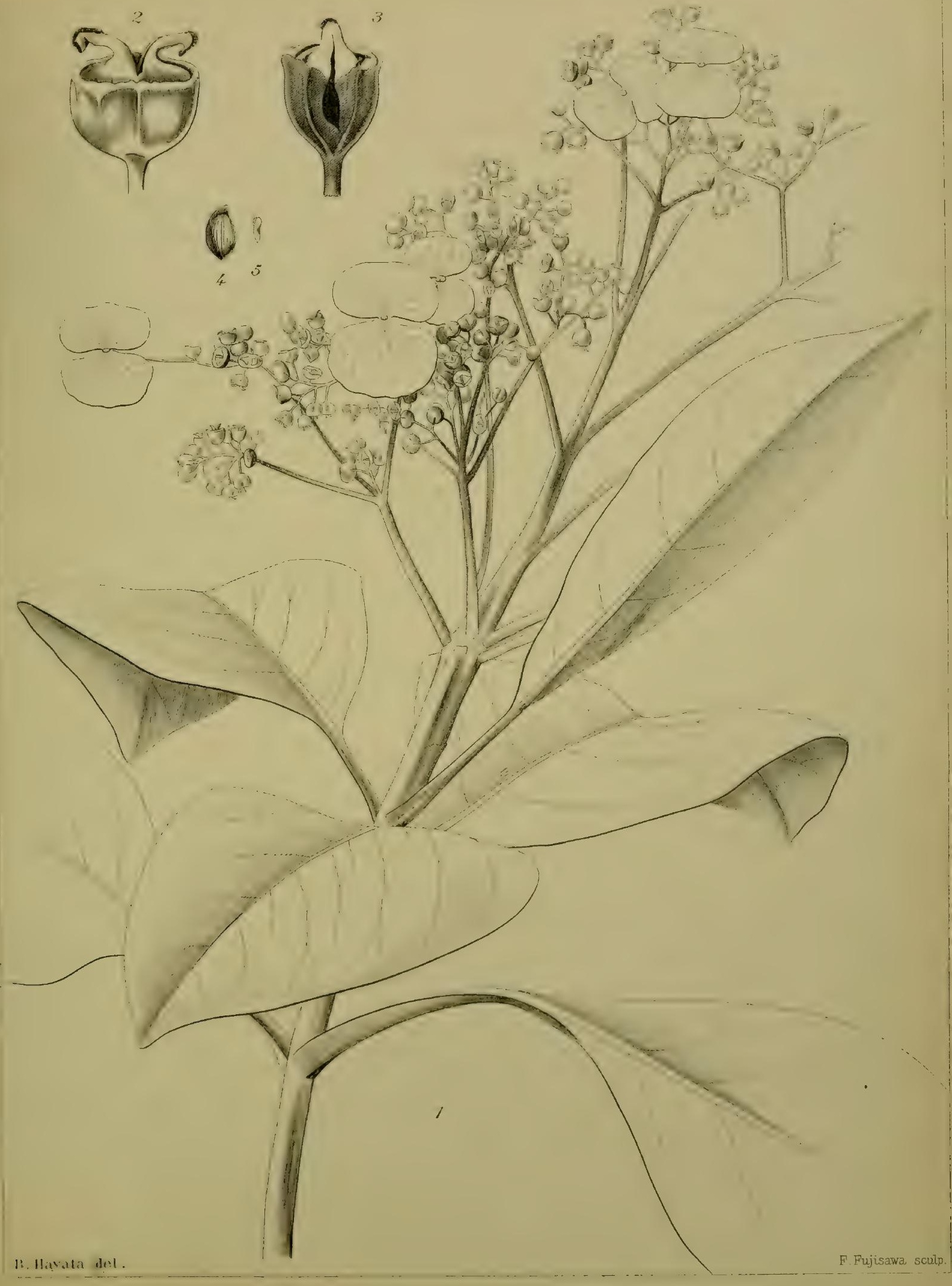



Jour. Sci. Coll., Vol. XXV., Art. XIX., PI.VIII.

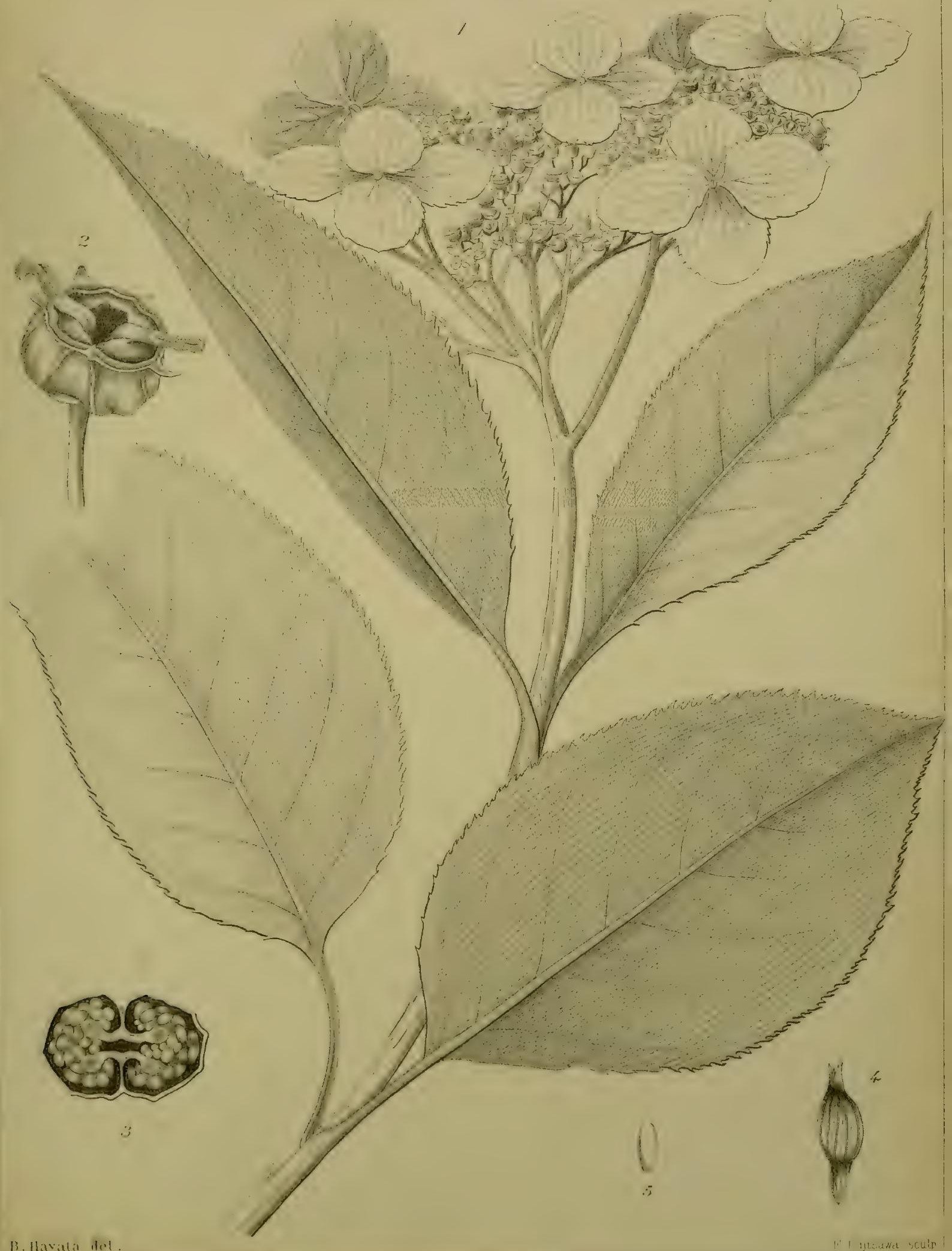

B. Havila Mel 

Jour. Sci. Coll., Vol. XXV., Art. XIX., PI.IX.
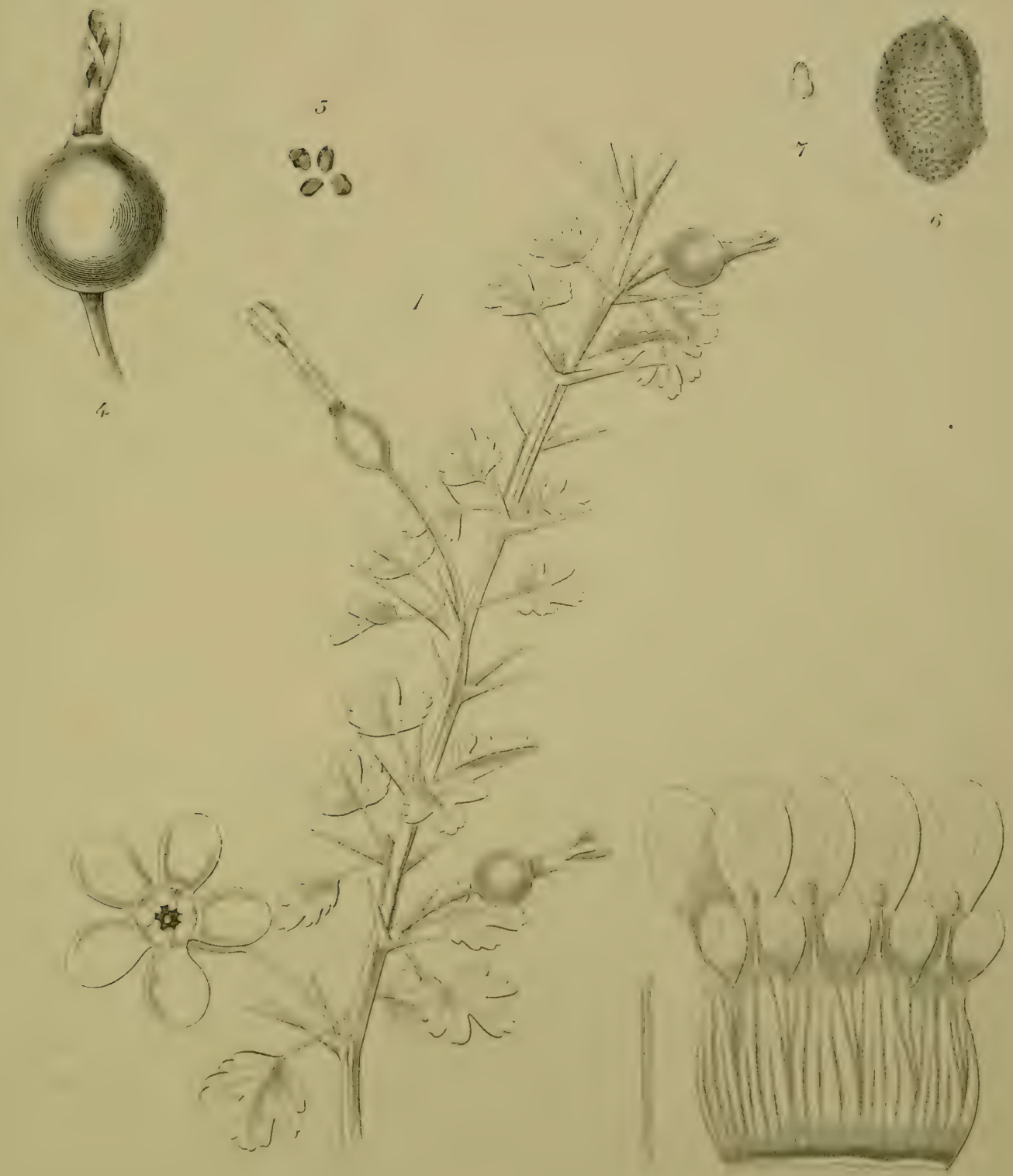

Jour. Sci. Coll., Vol. XXV., Art. XIX.,PI. X.

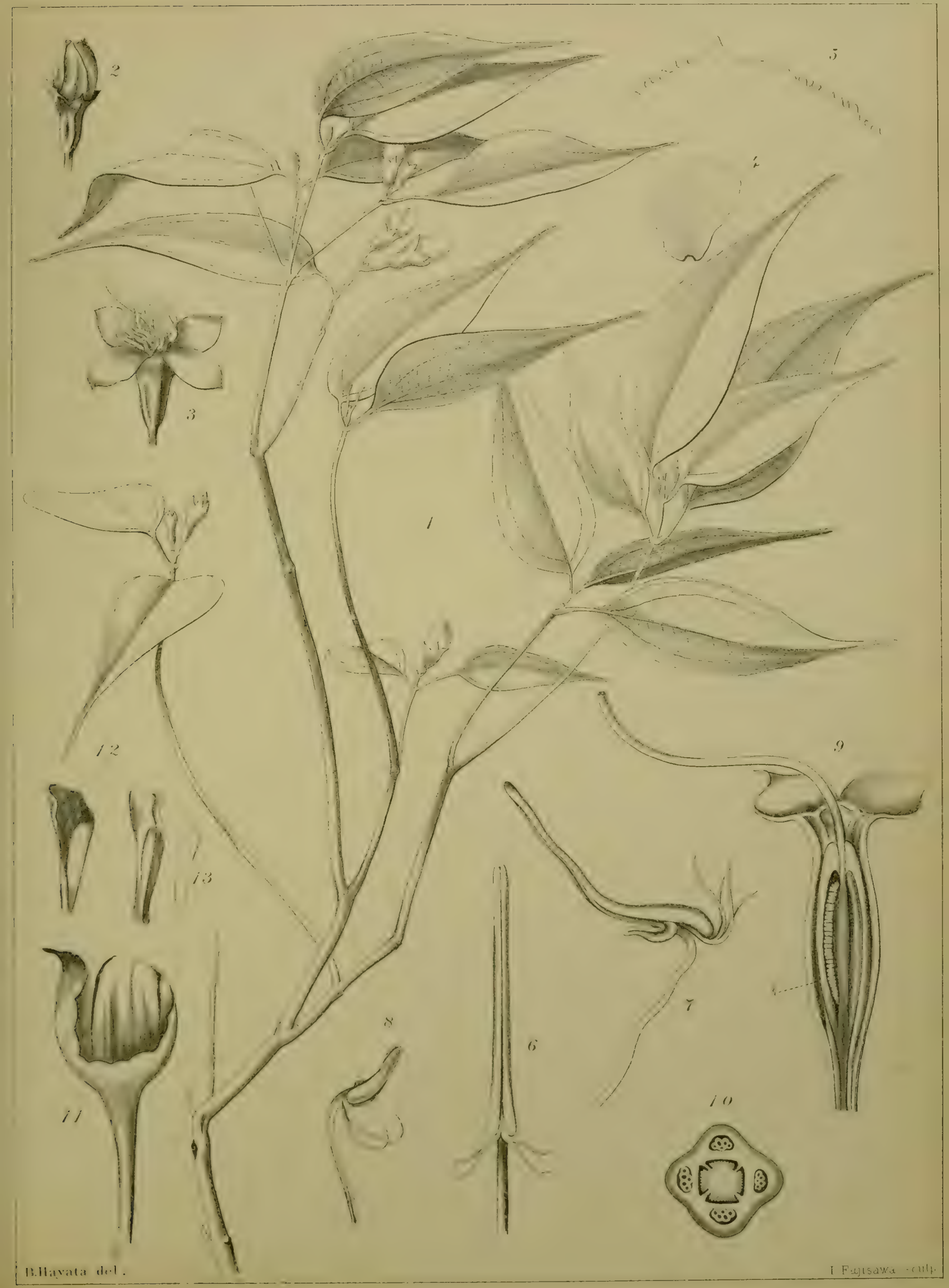





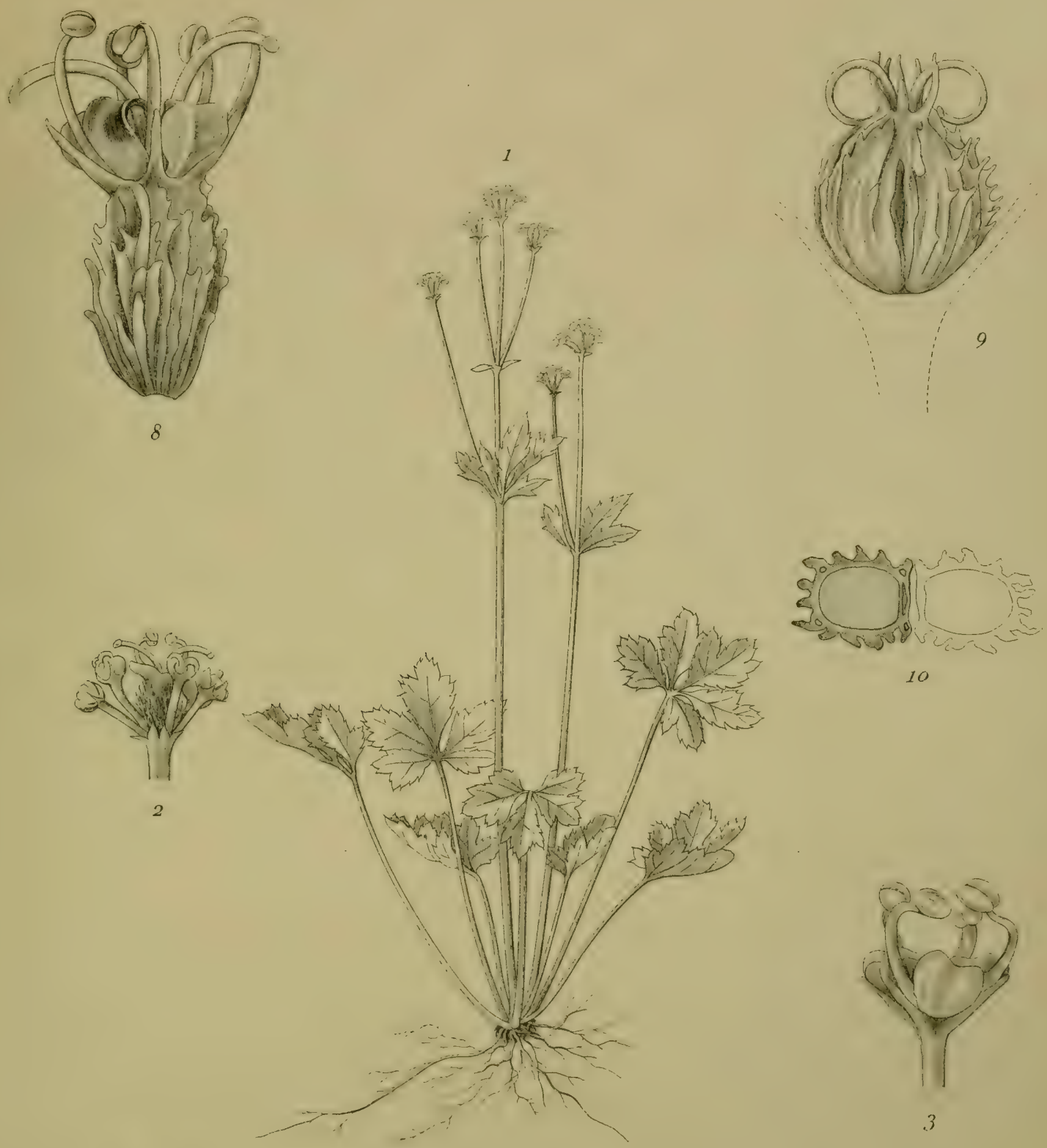

2
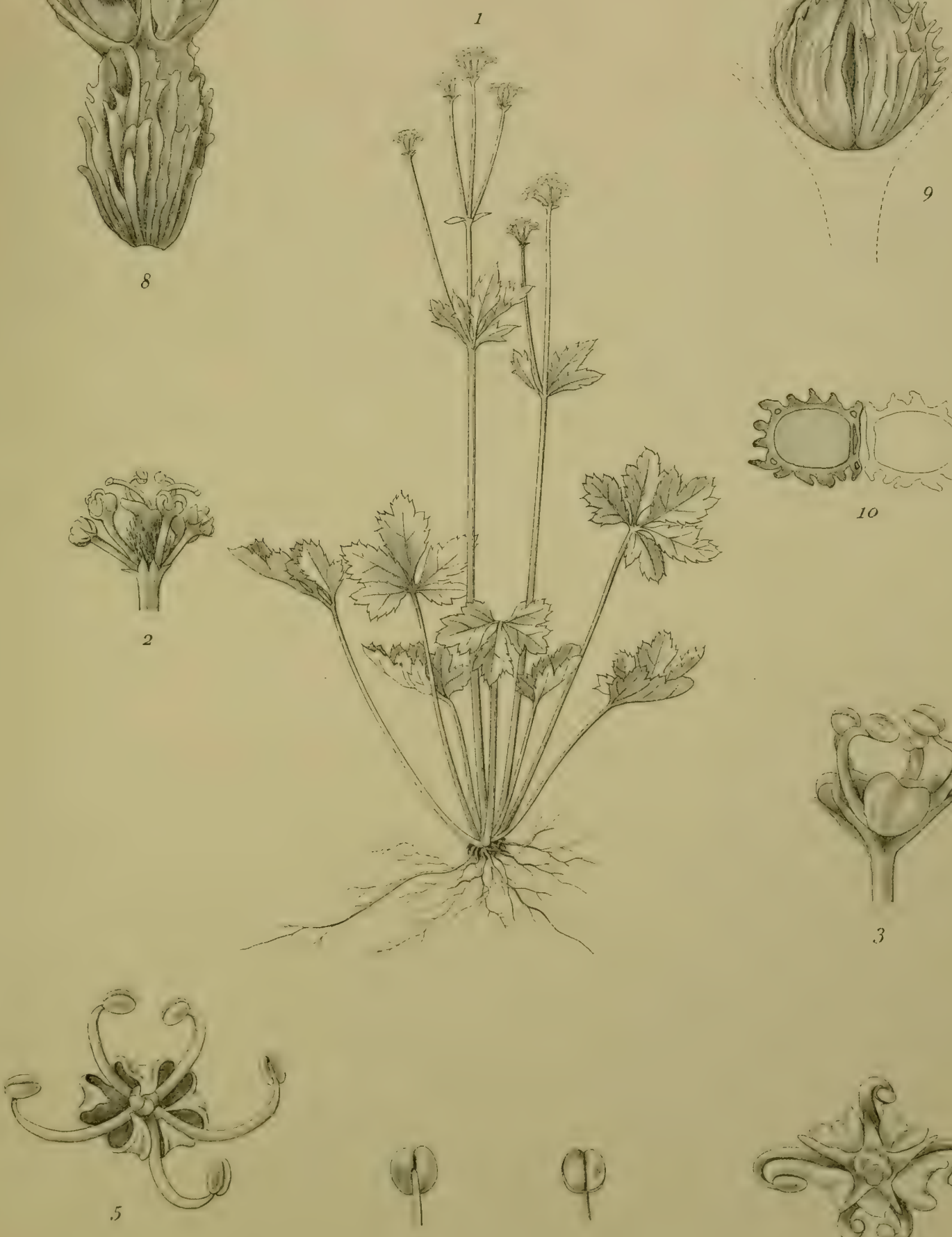

7
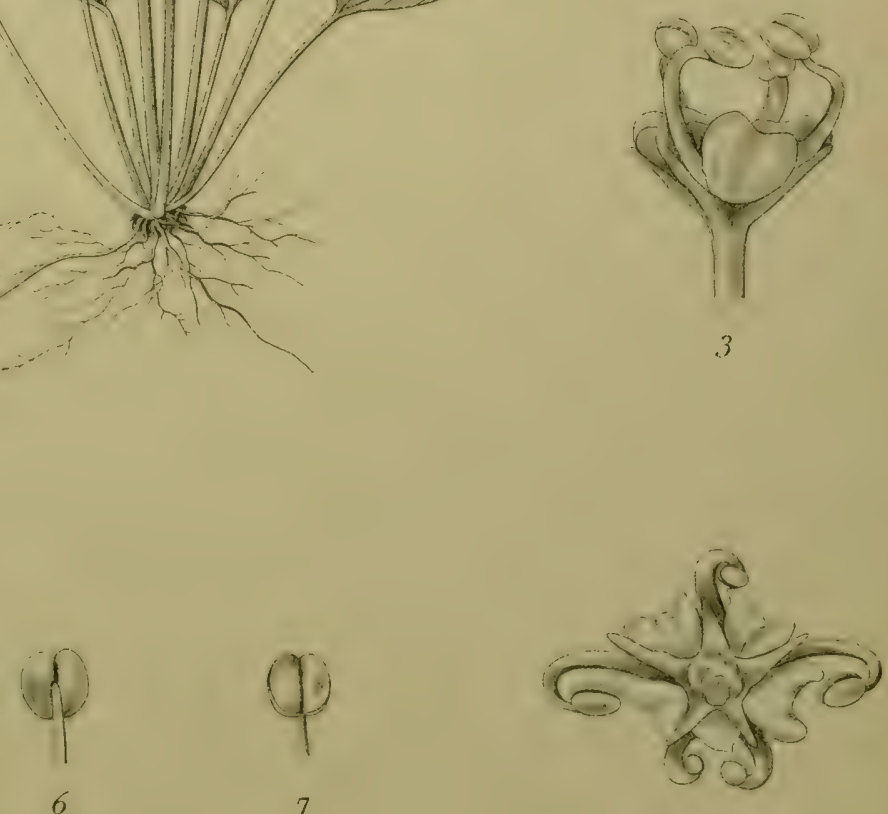

4 

Jour. Sci. Coll., Vol. XXV., Art. XIX.,PI.XIII.

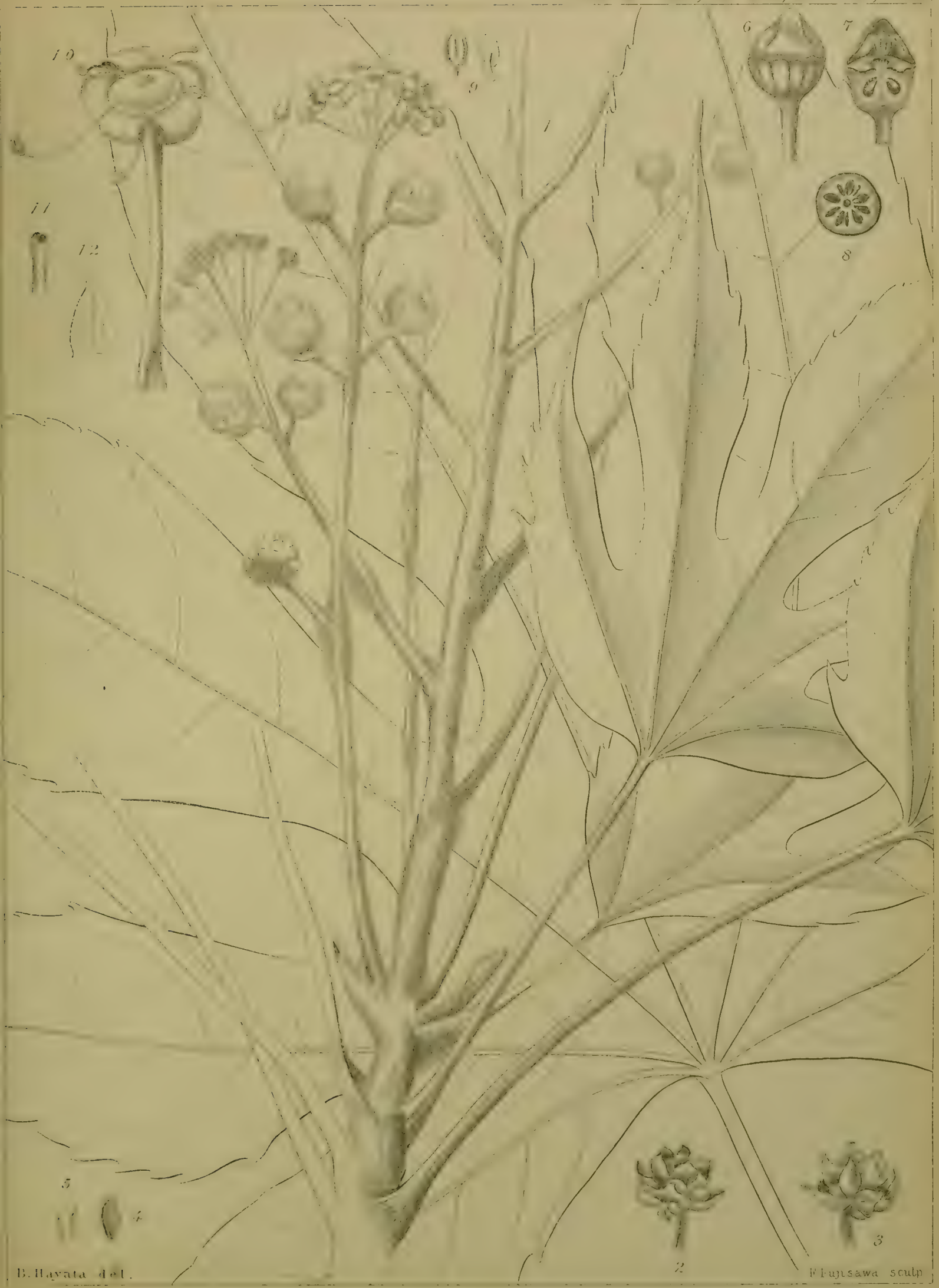





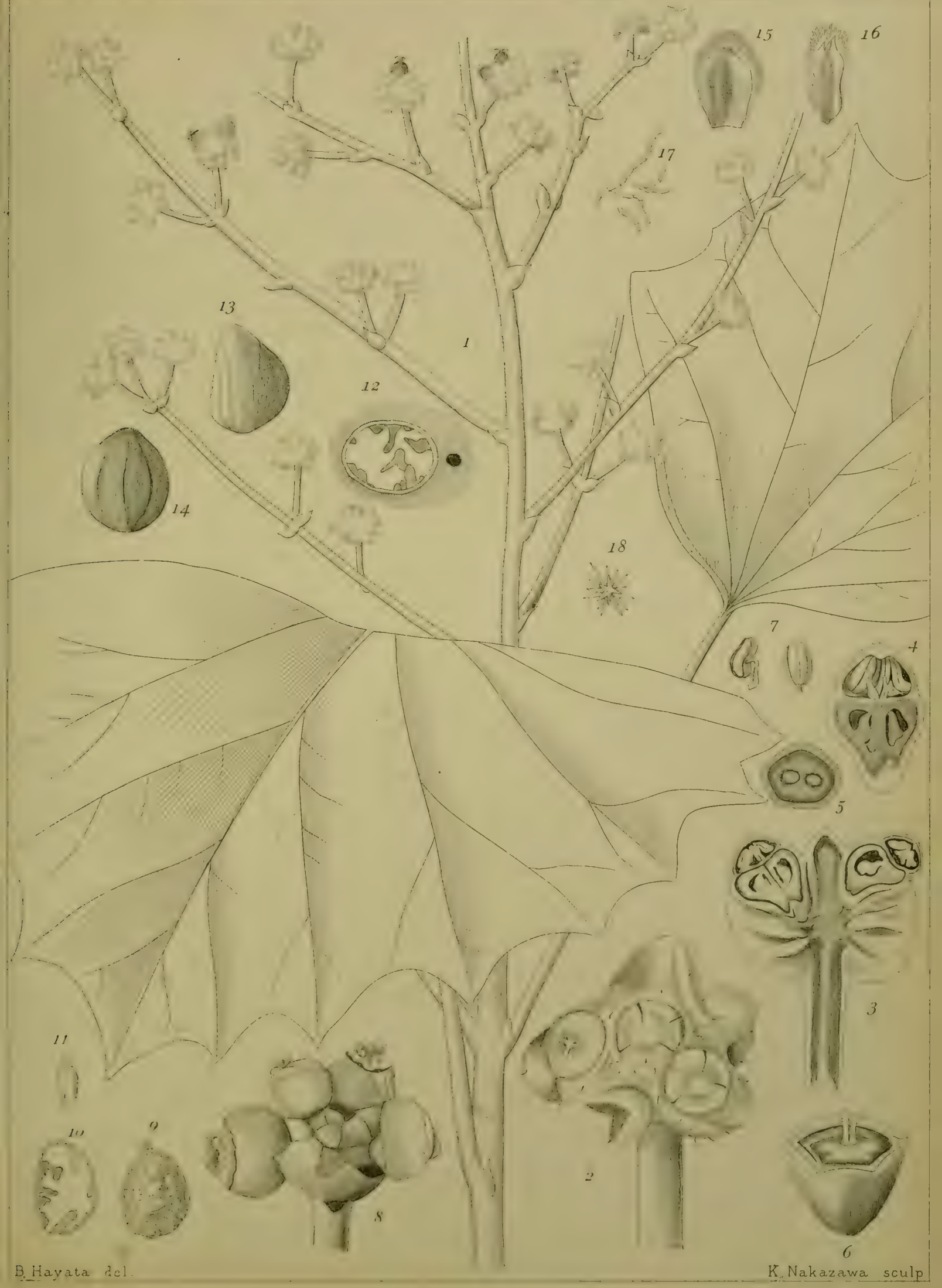



Jour. Sci. Coll., Vol. XXV., Art. YIX..PI.XV.

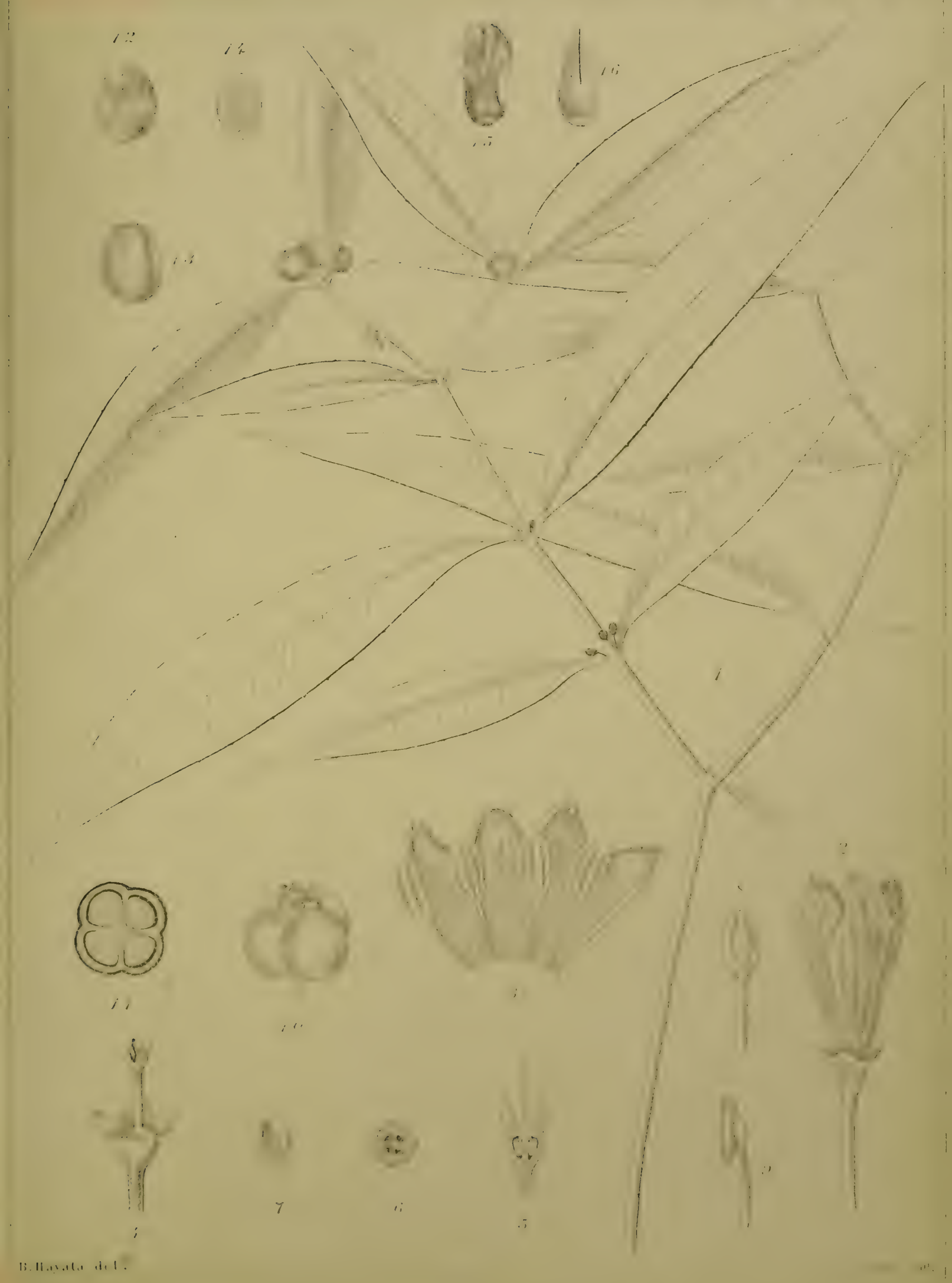





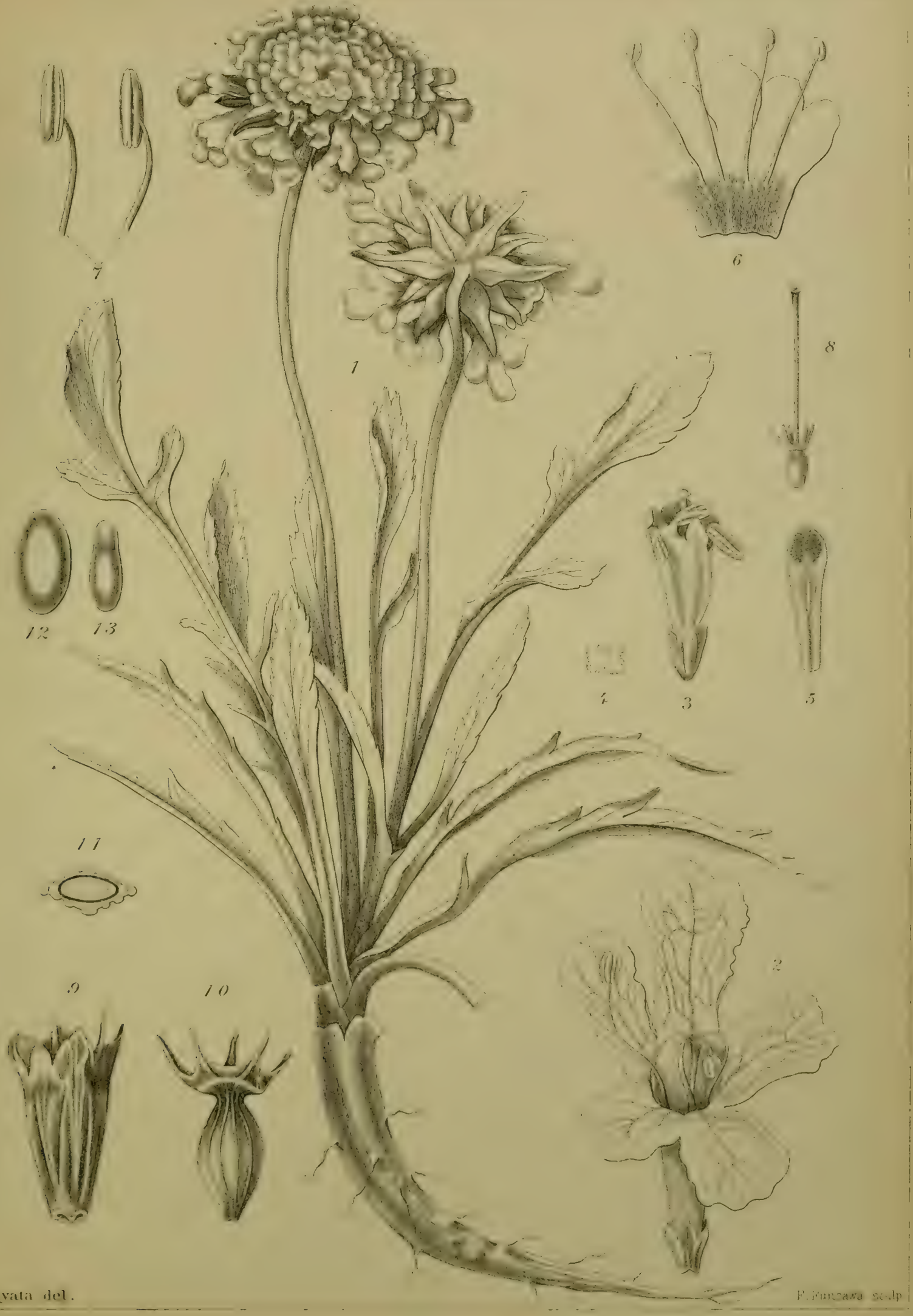





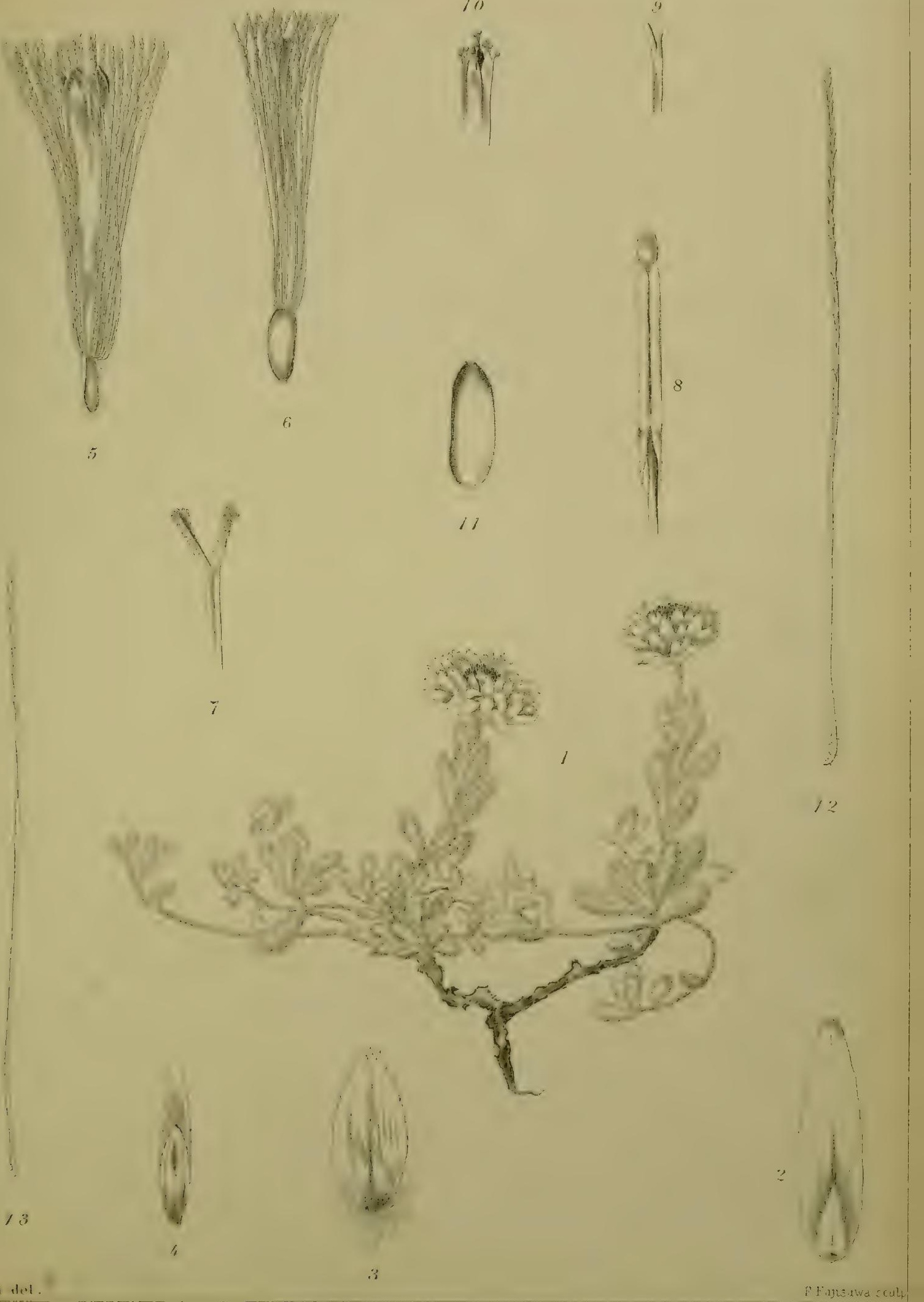




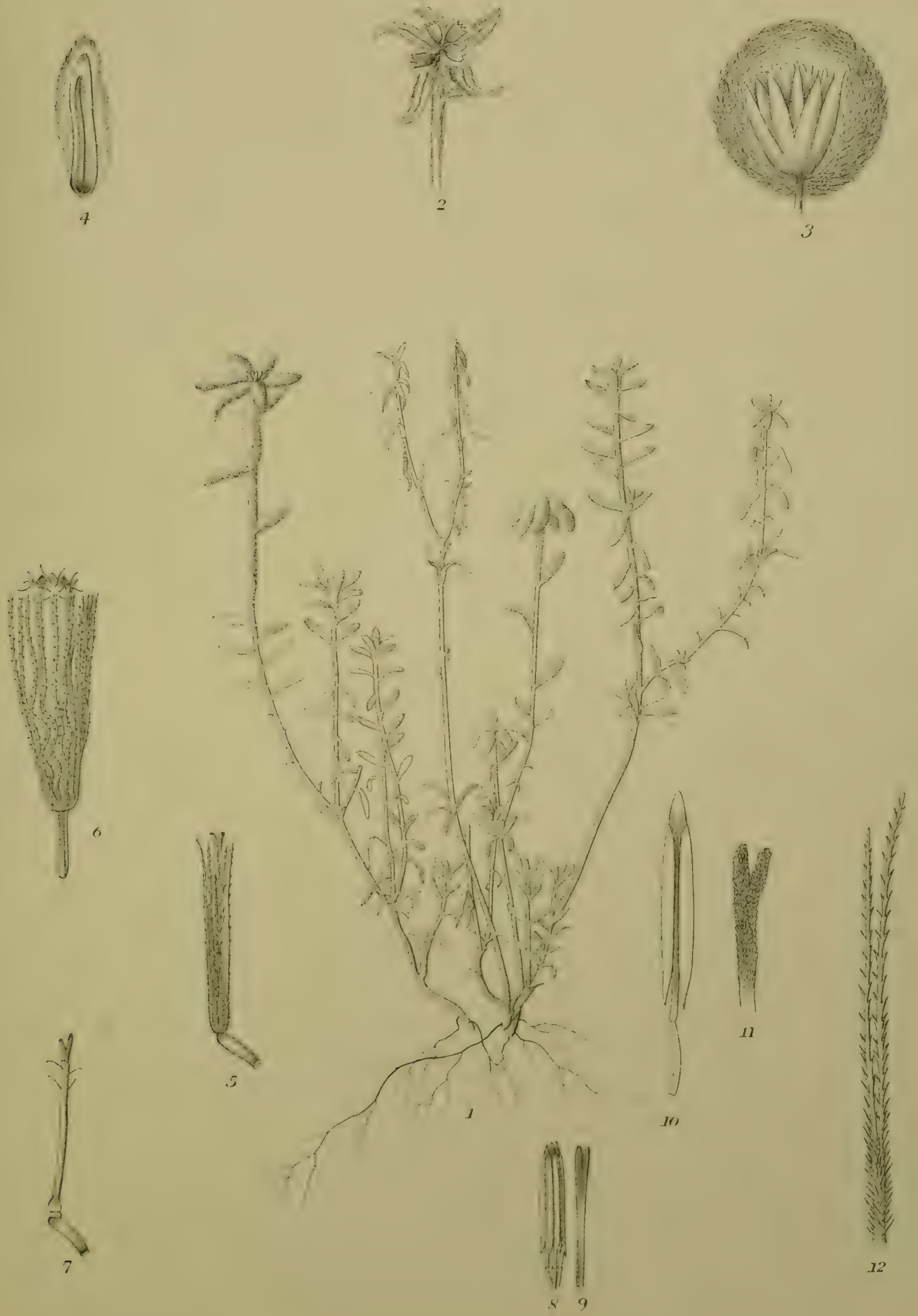



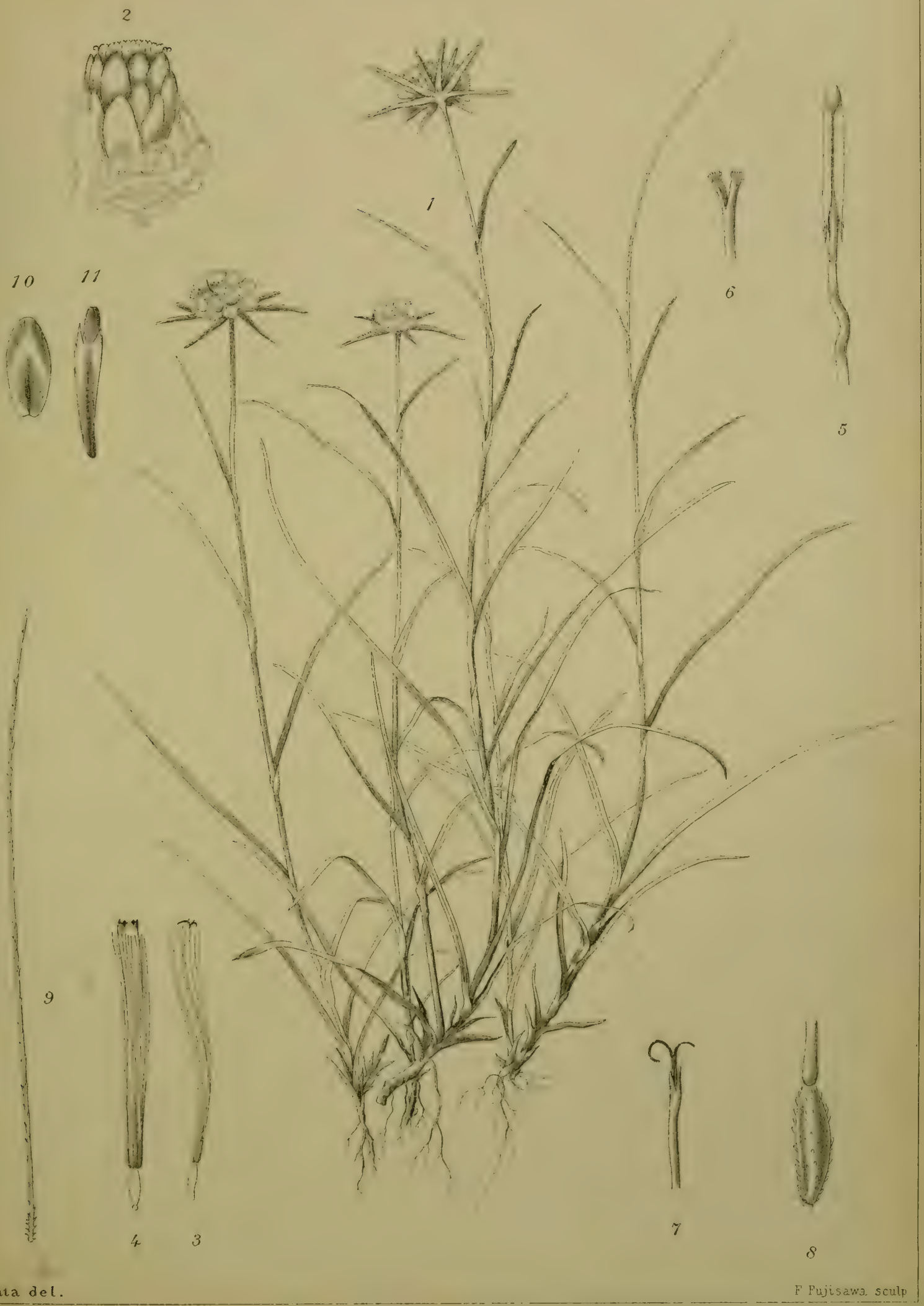



Jour. Sci. Coll., Vol. XXV., Art. XIX., PI.XX.

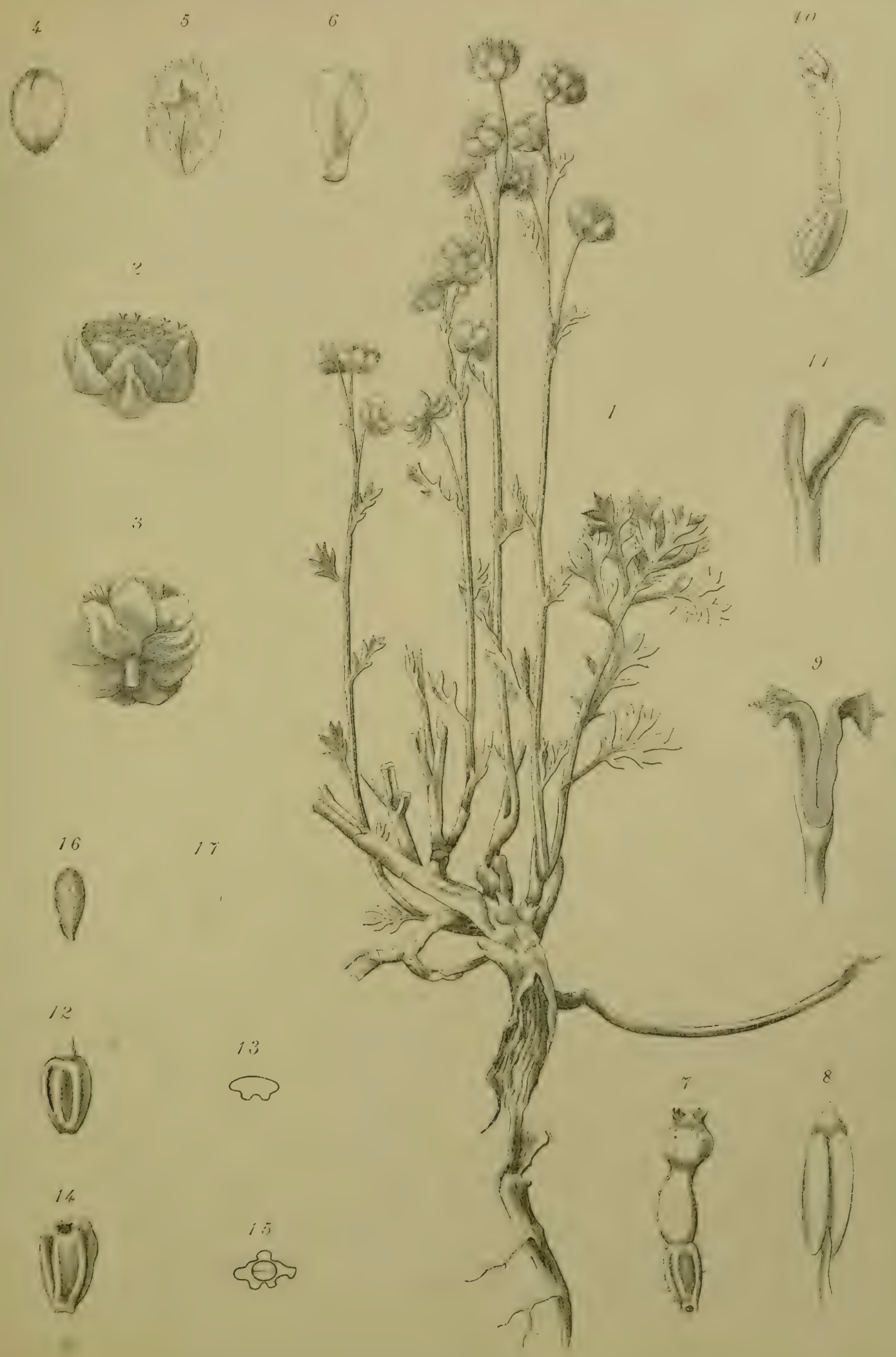



Jour. Sci. Coll., Vol. XXV., Art. XIX.,PI.XXI.

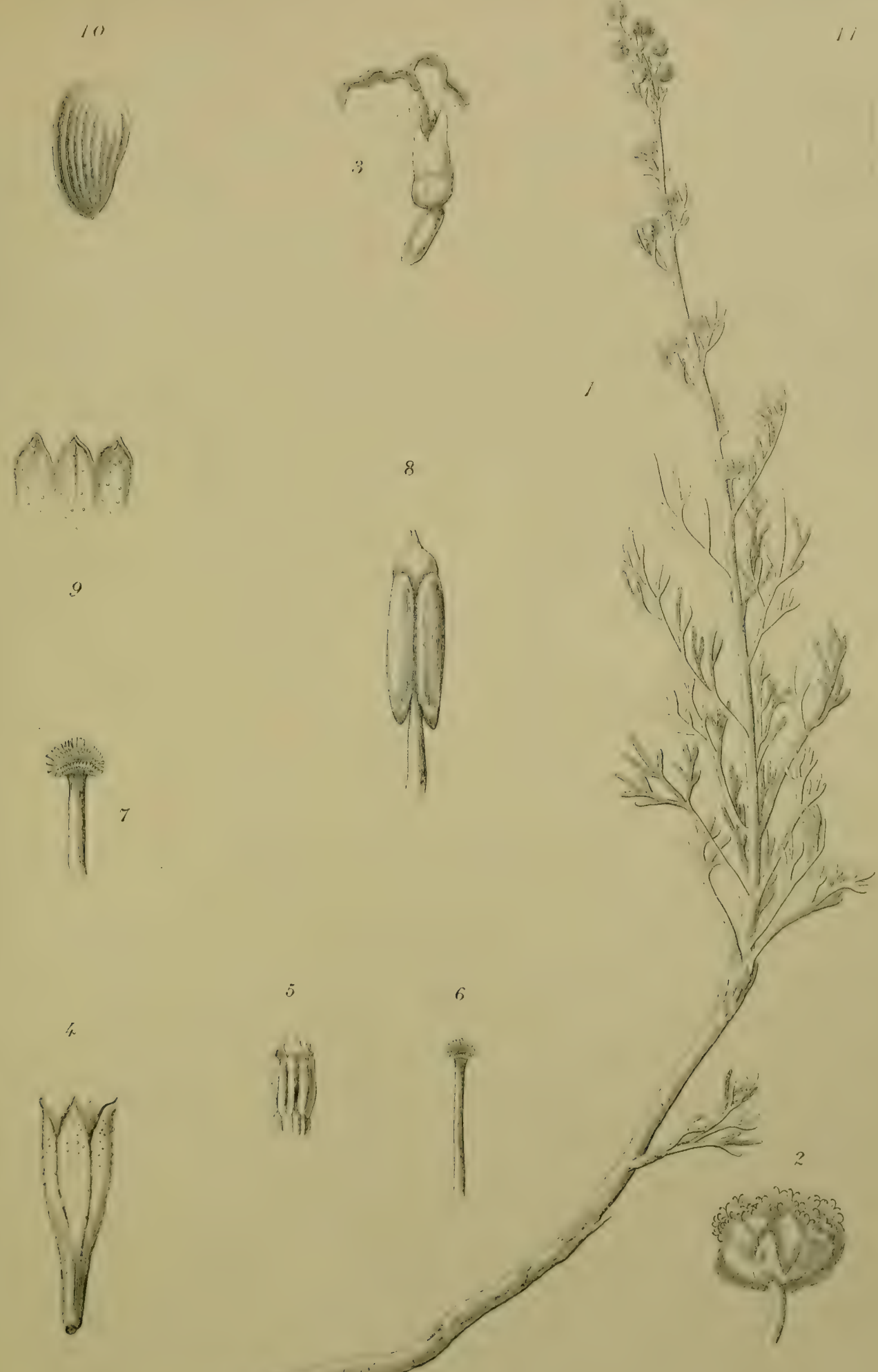





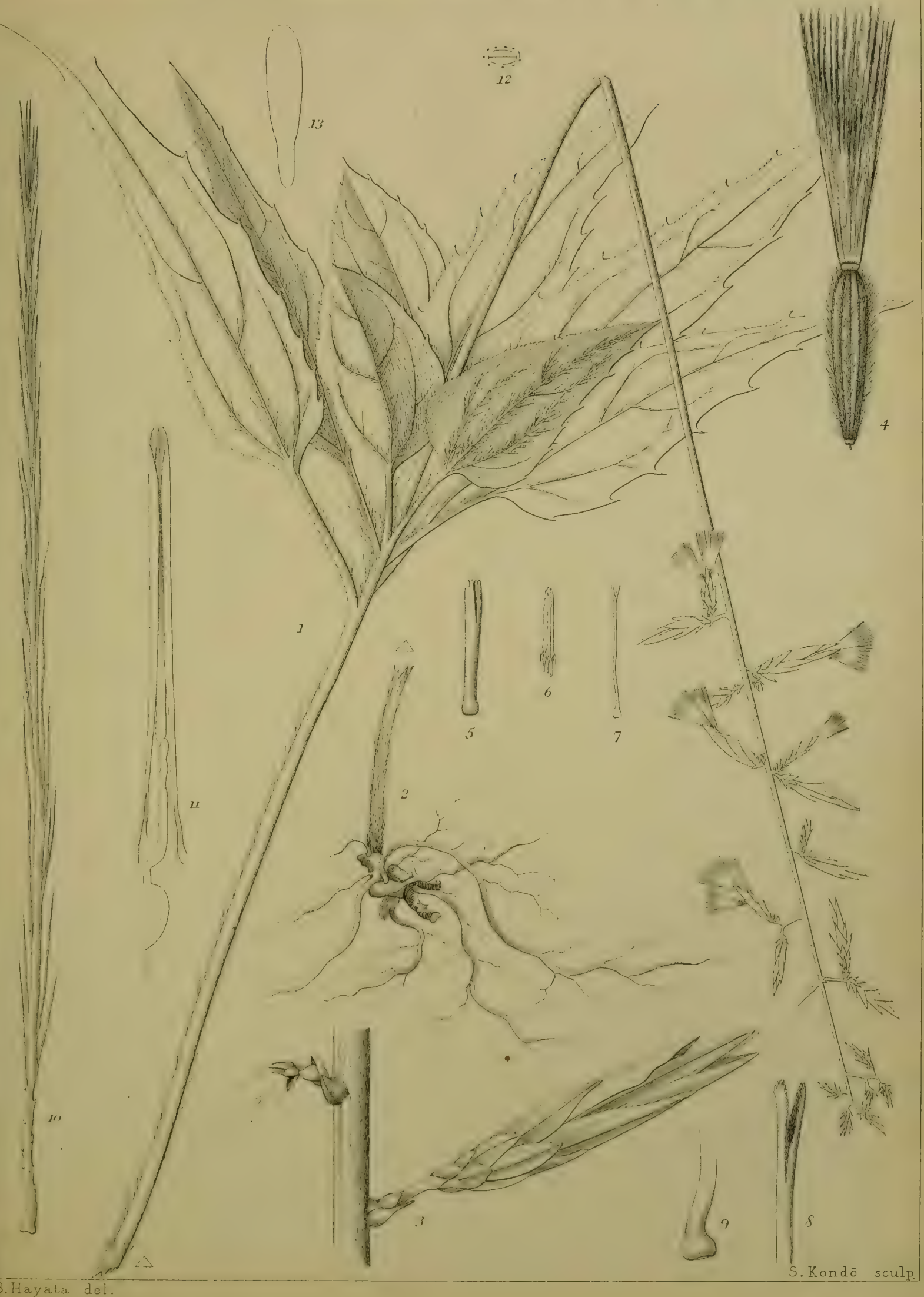



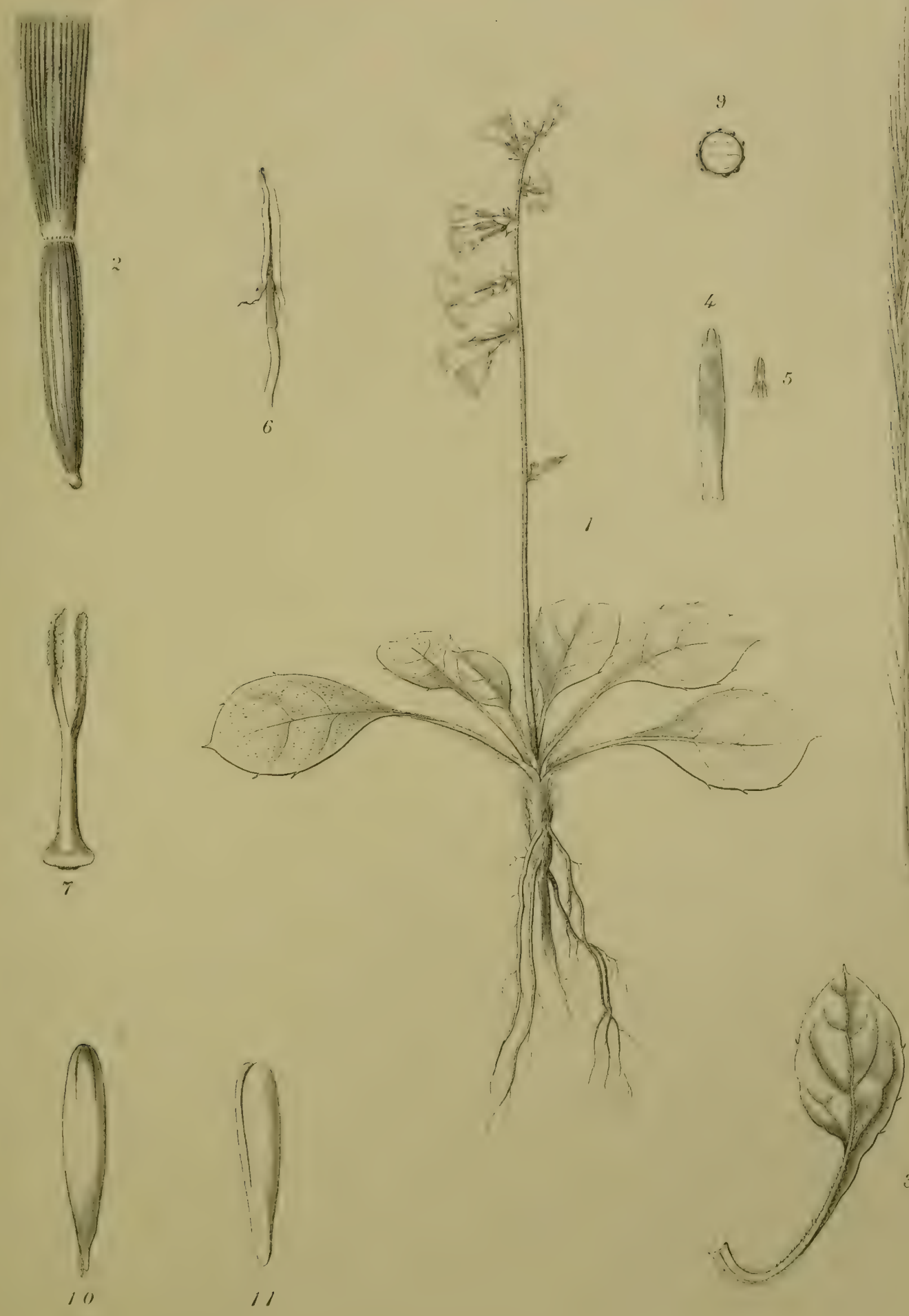



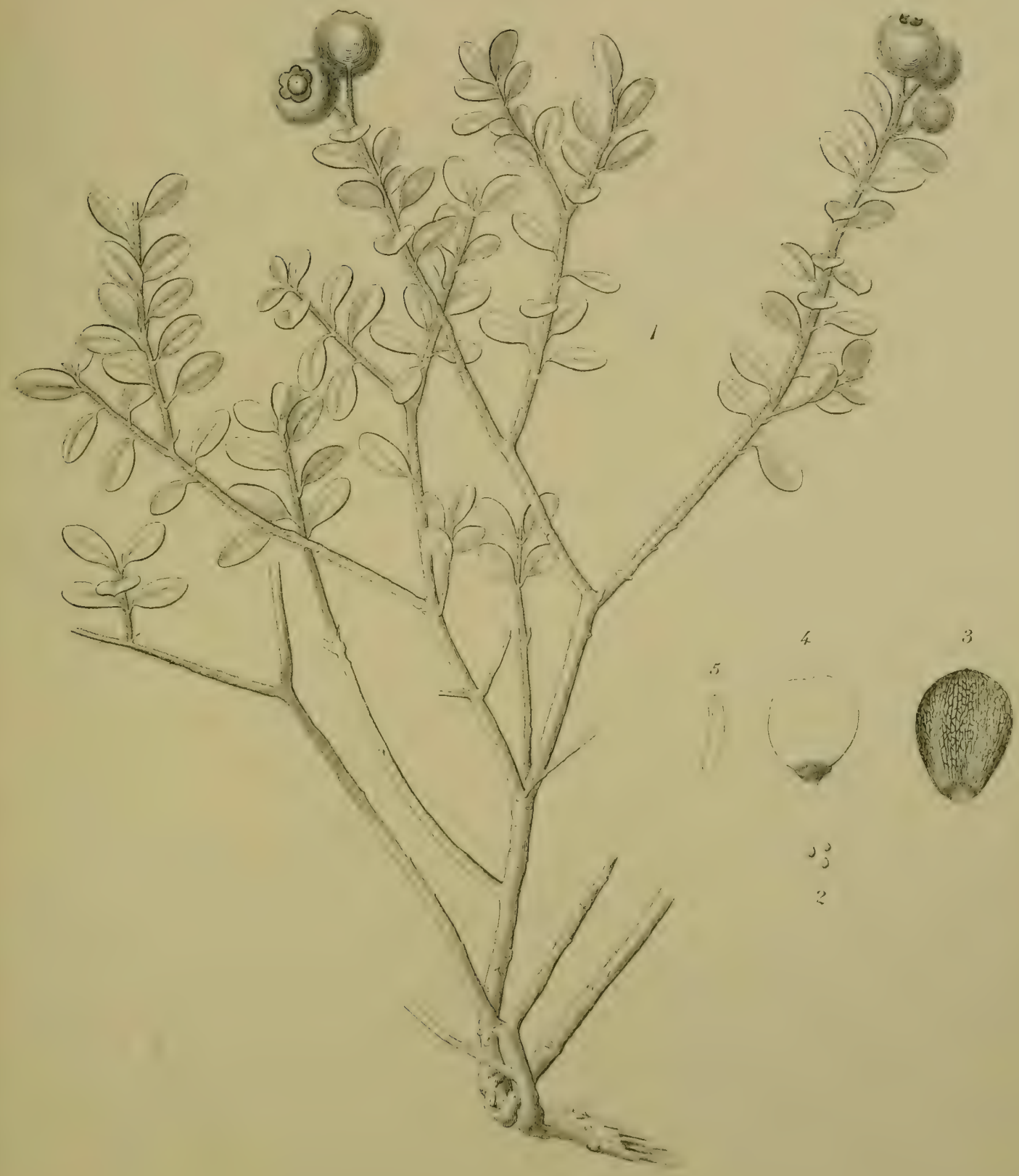





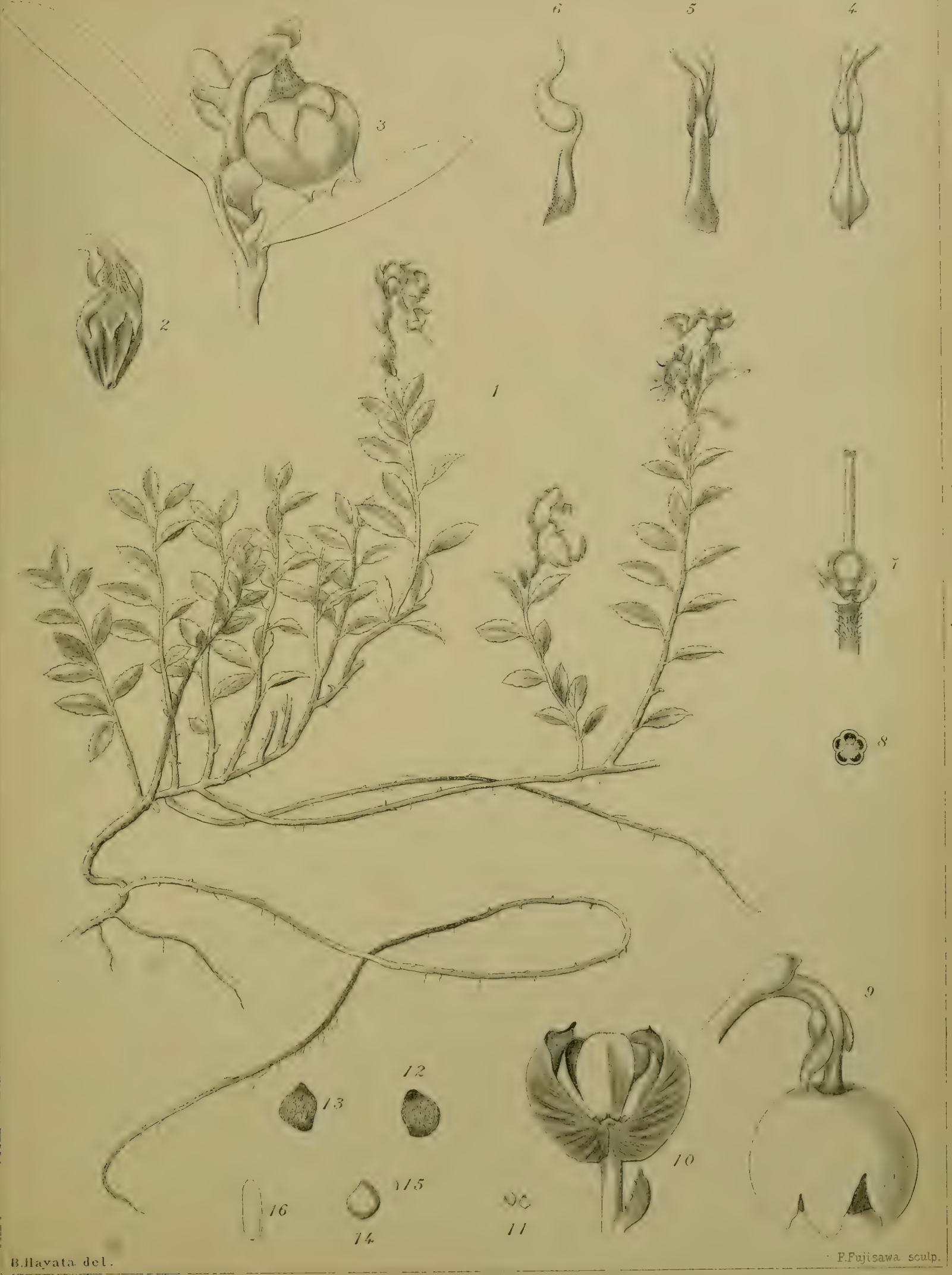



Jour. Sci. Coll., Vol. XXV., Art. XIX., PI.XXVI.

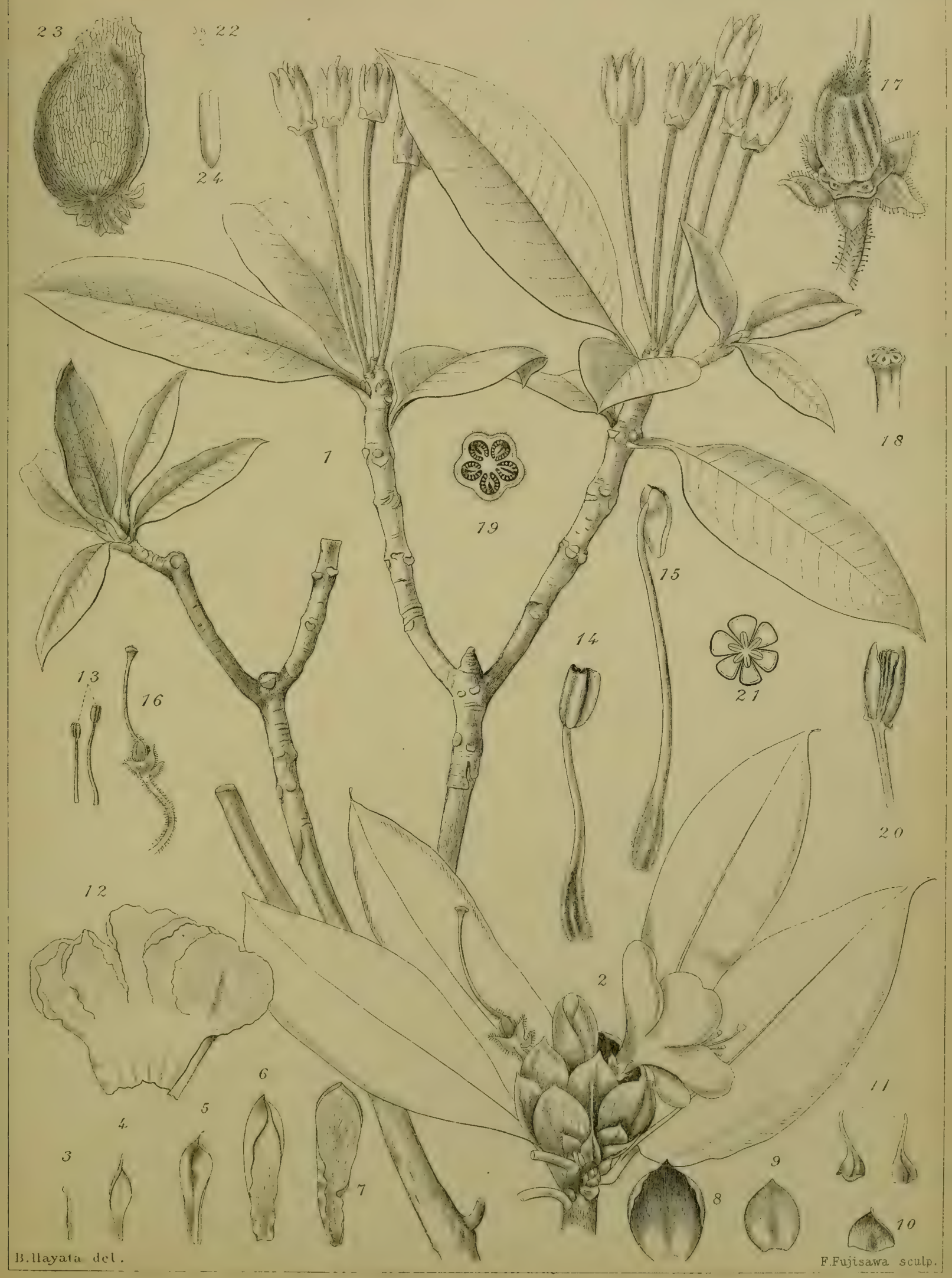



Jour. Sci. Coll., Vol. XXV., Art. XIX., PI.XXVII.

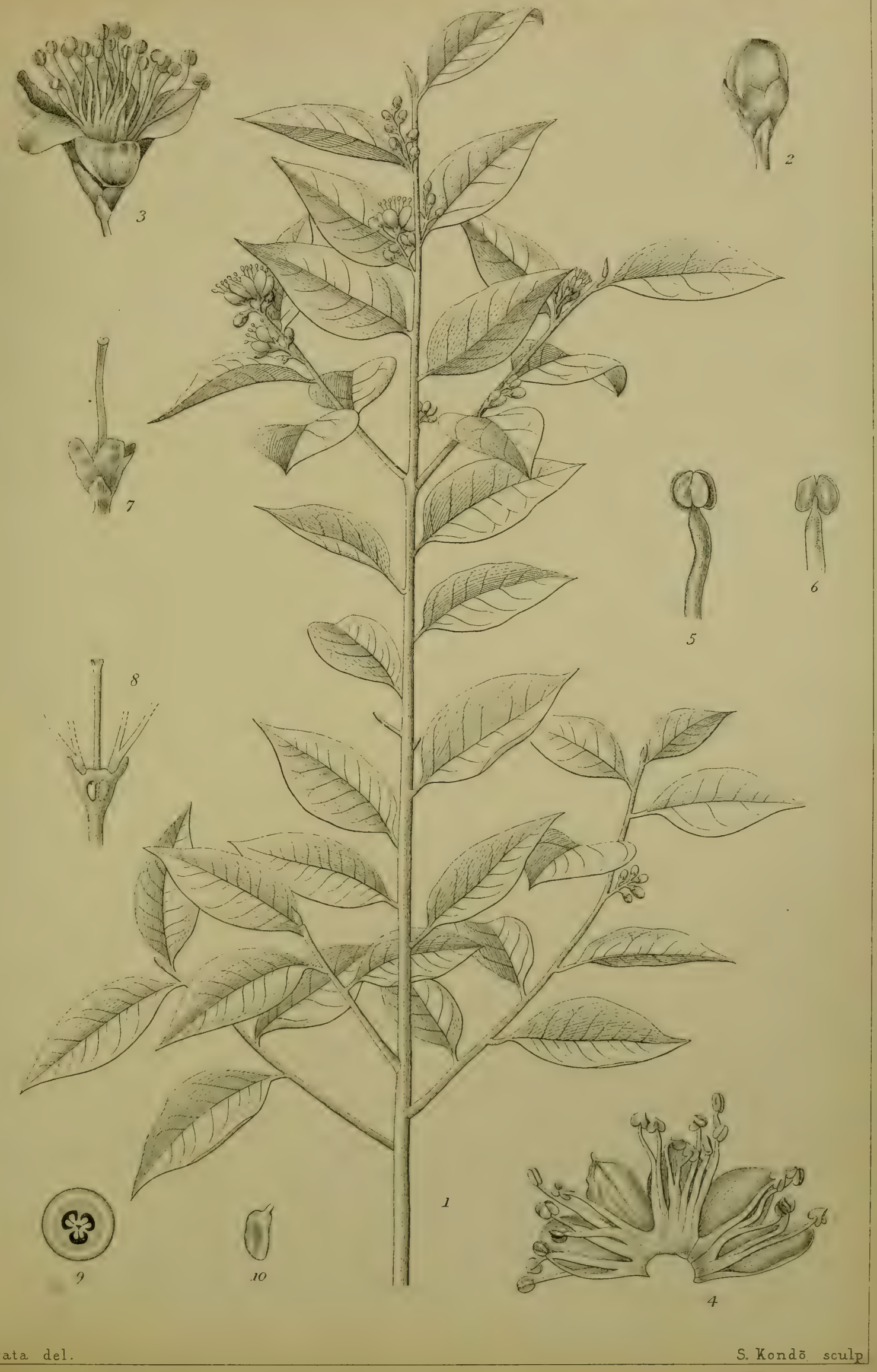




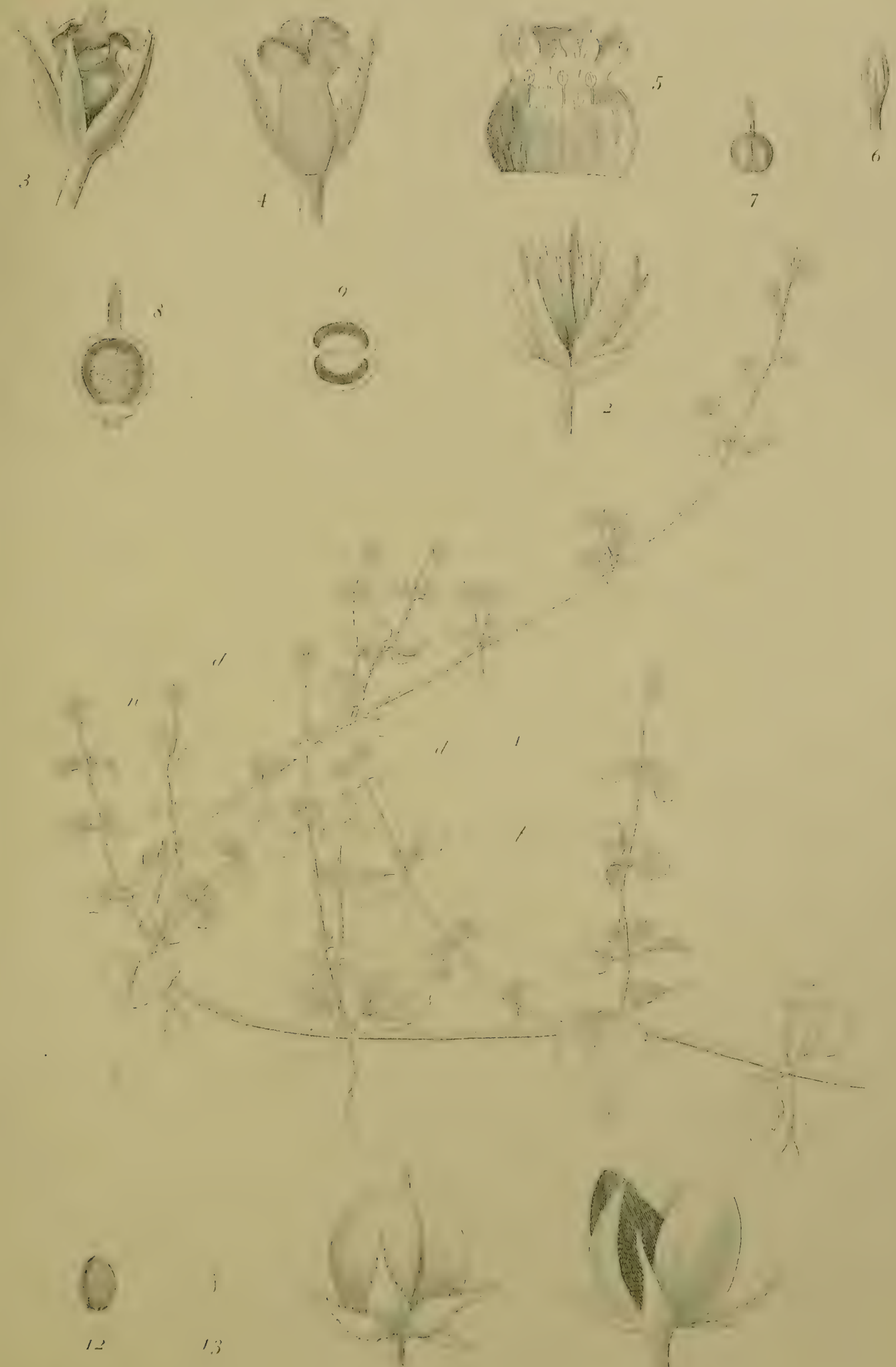

B. Hayata del.
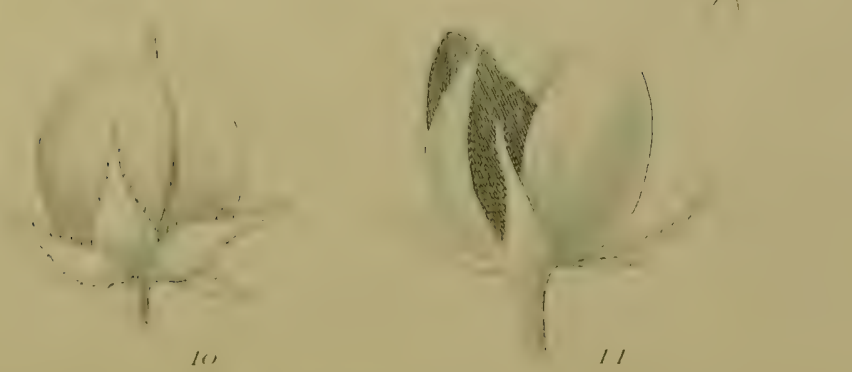

K. Nakazawa scuip : 


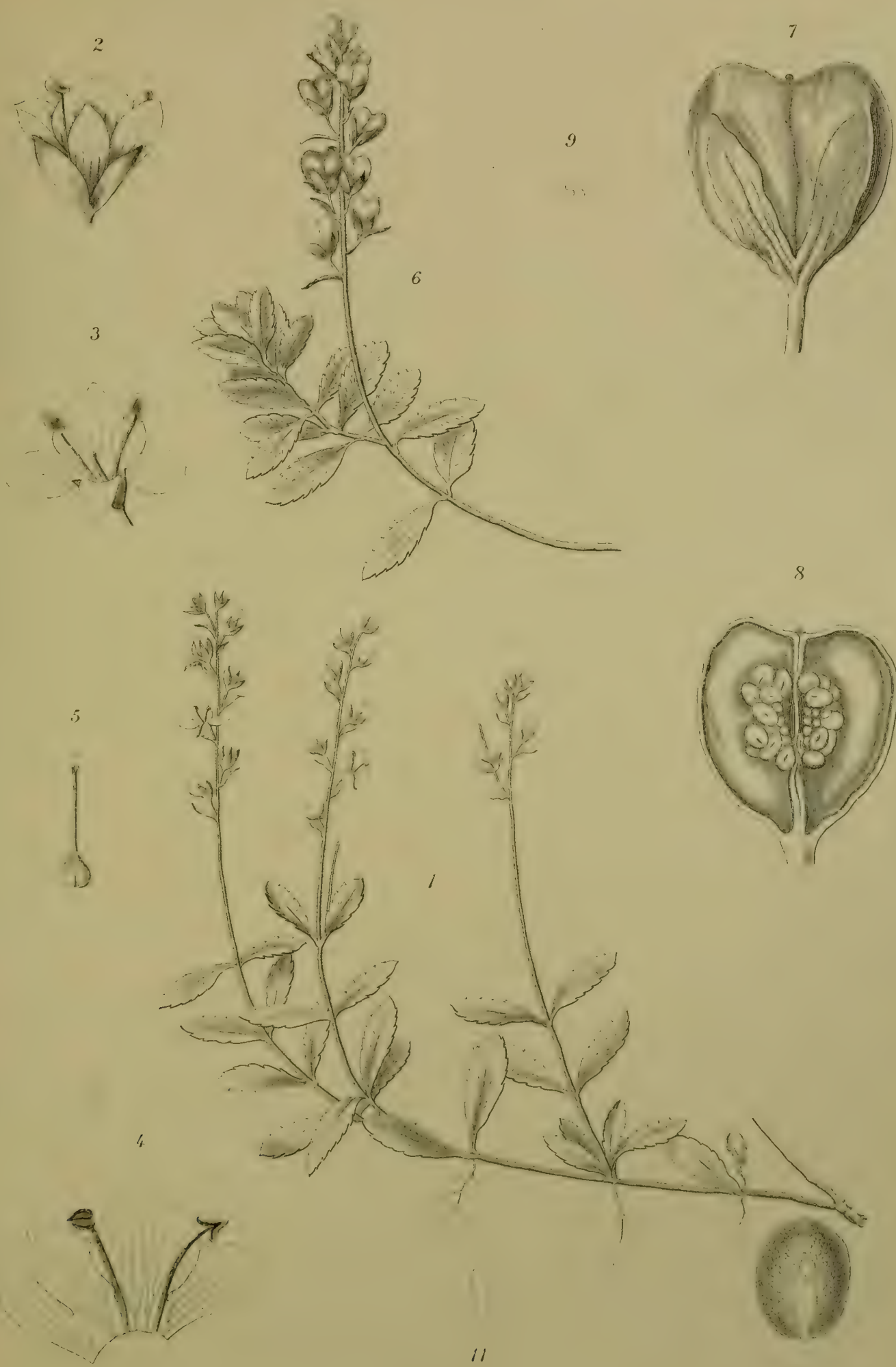


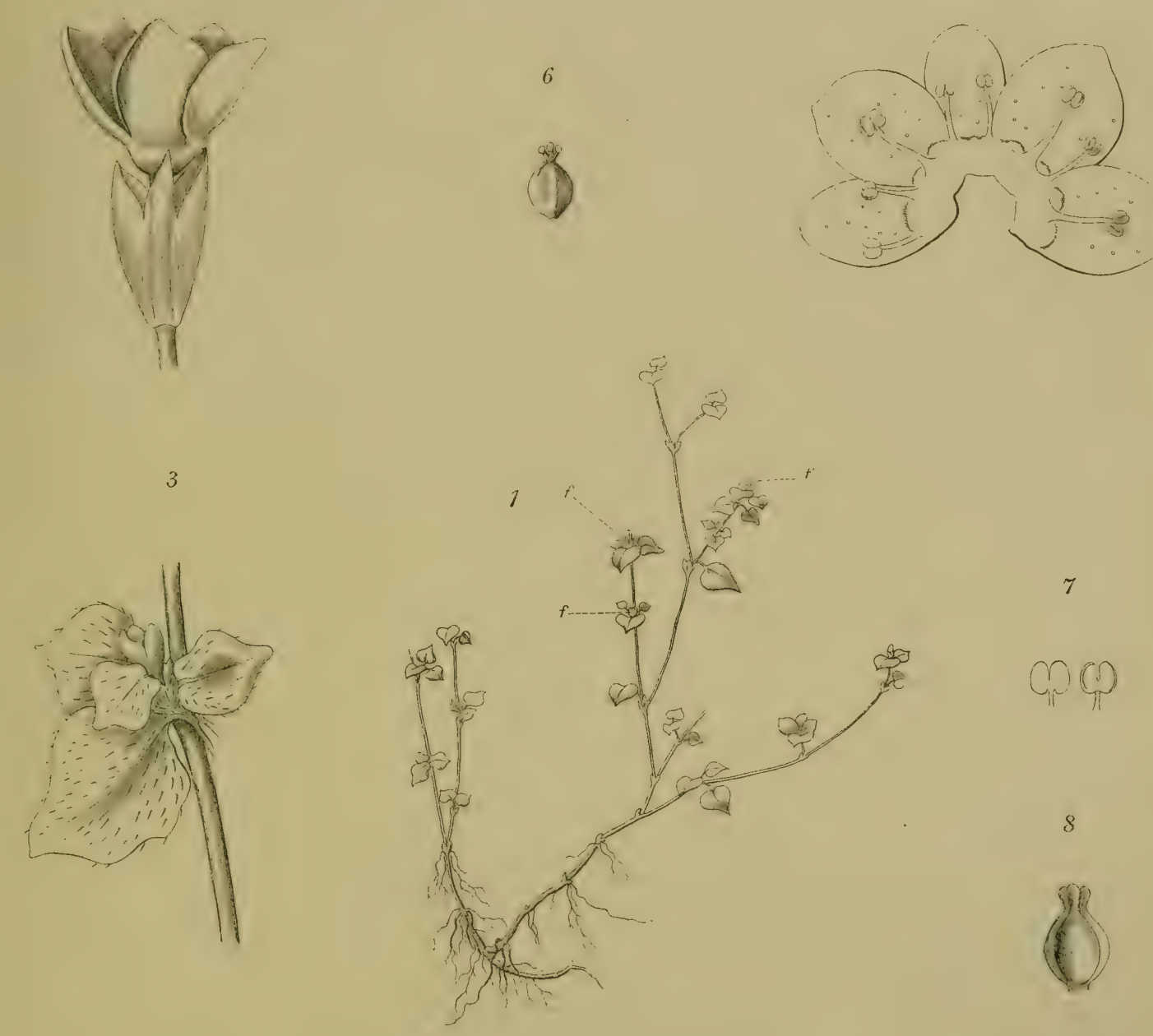

7

$\mathrm{C}\left(\bigcap_{\pi}\right.$

8

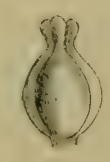

2
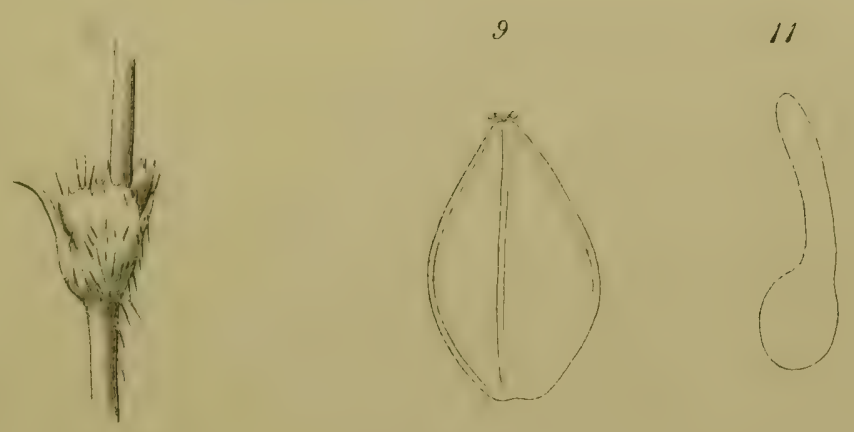

10 



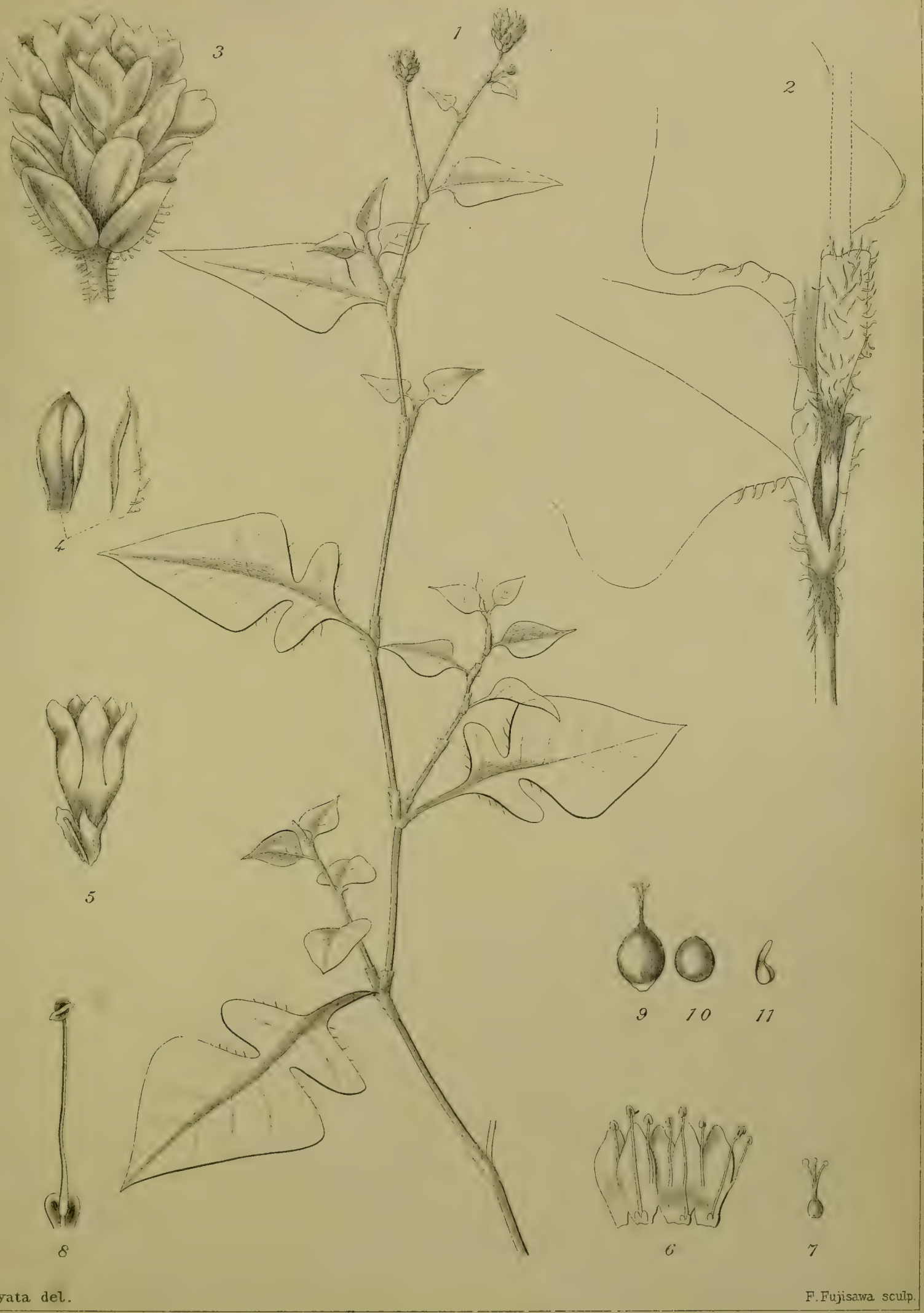





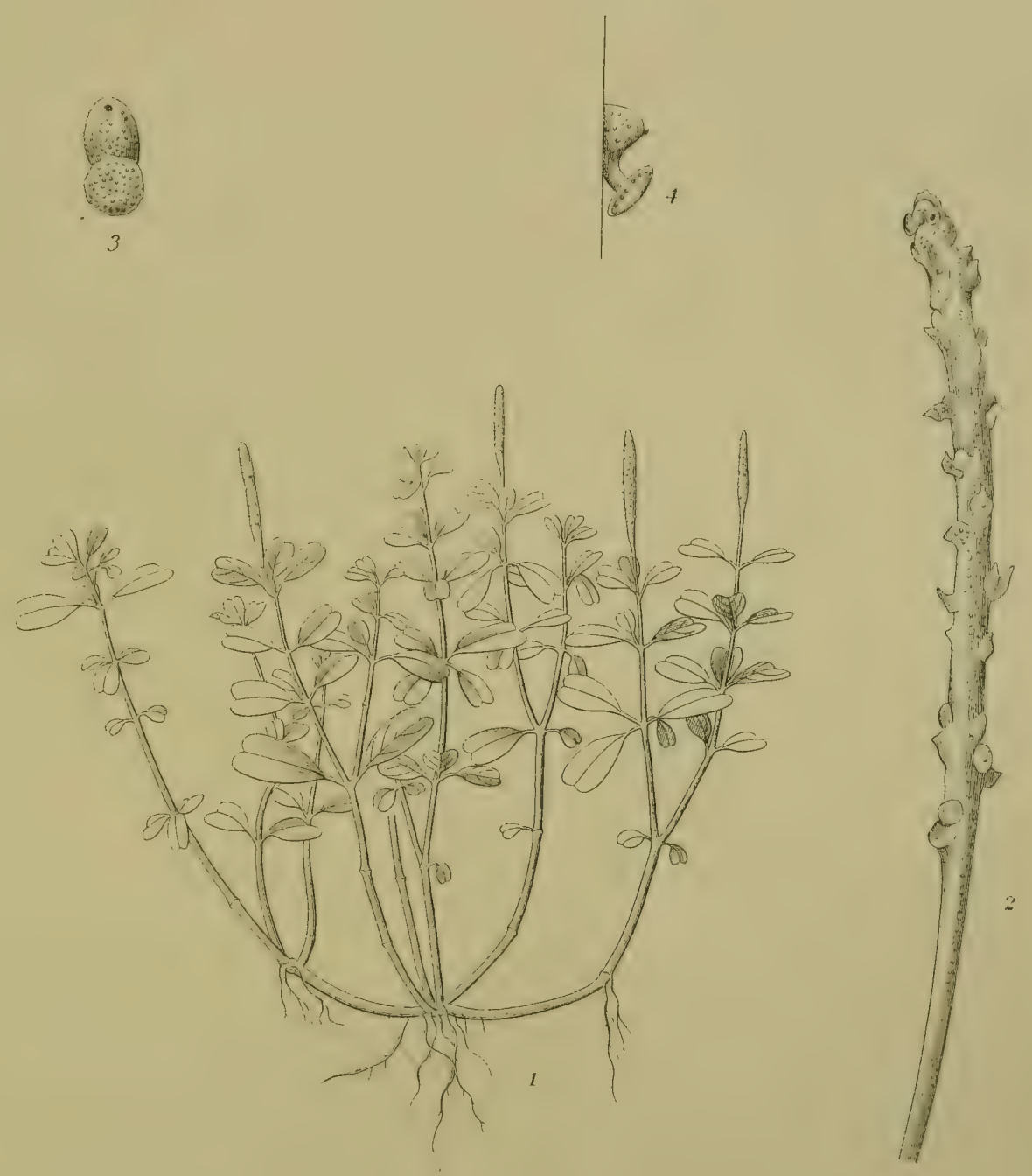





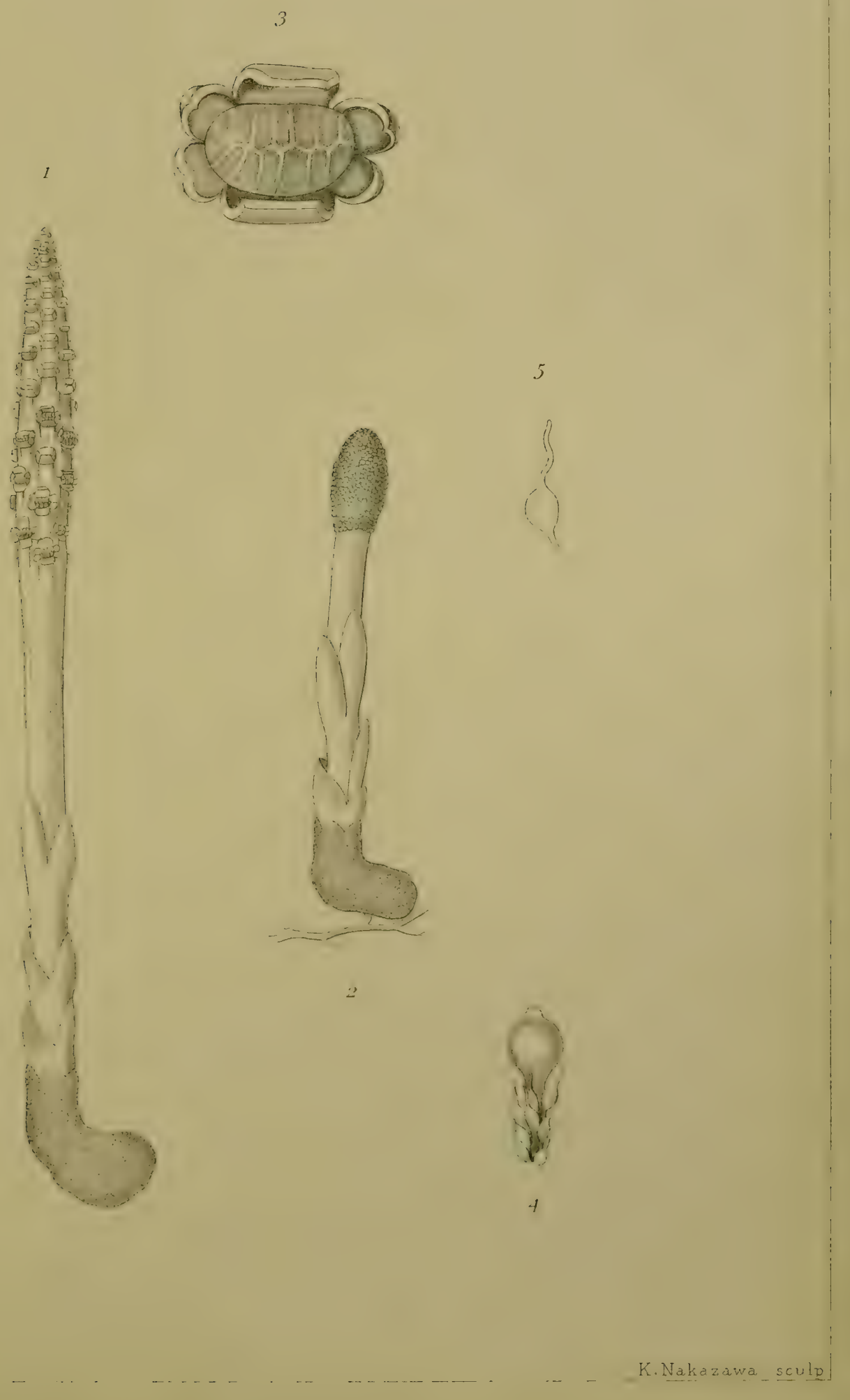





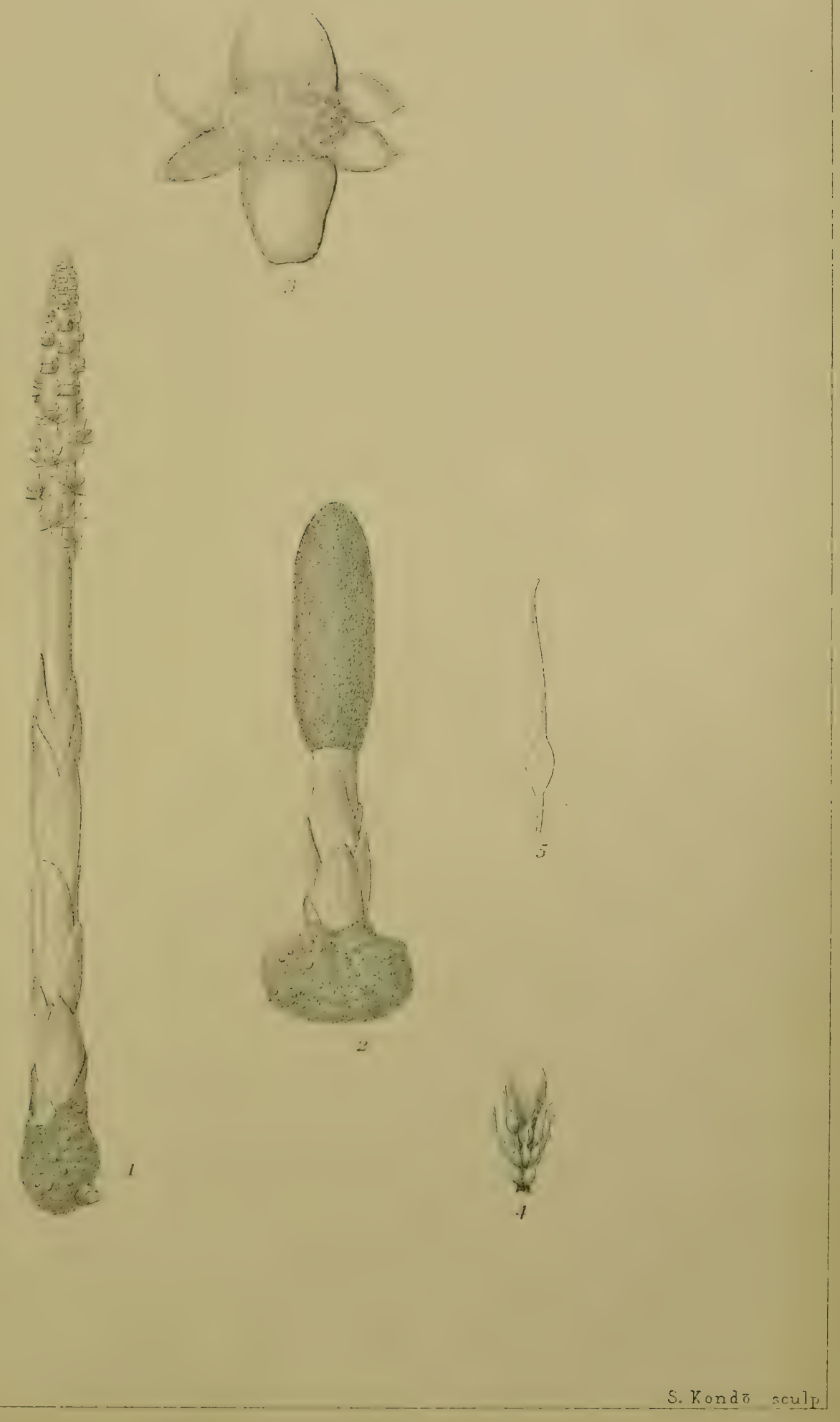



Jour. Sci. Coll., Vol. XXV., Art. XIX.,PI.XXXV.

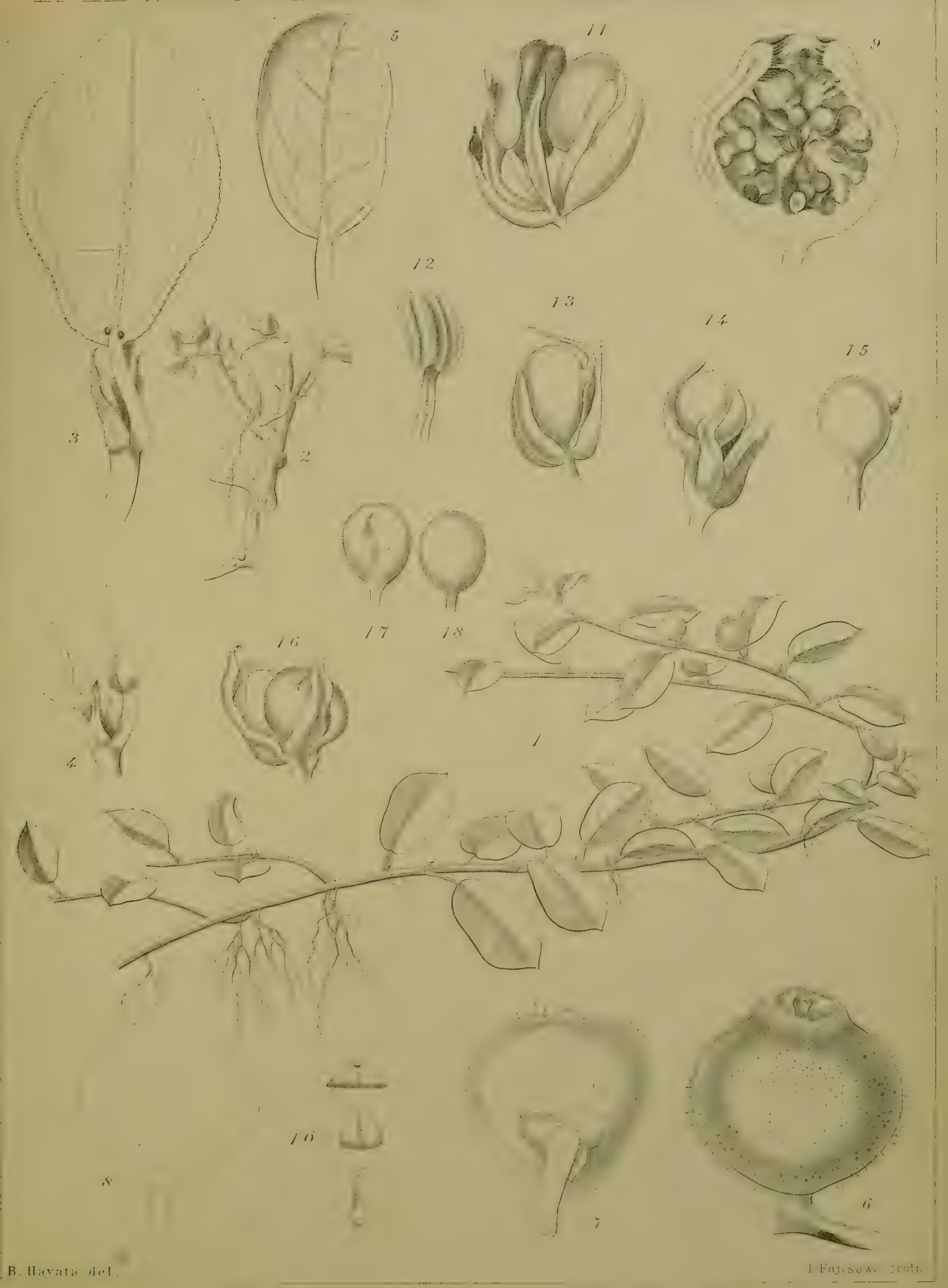



Jour. Sci. Coll., Vol. XXY., Art. XIX., PIXXXVI.
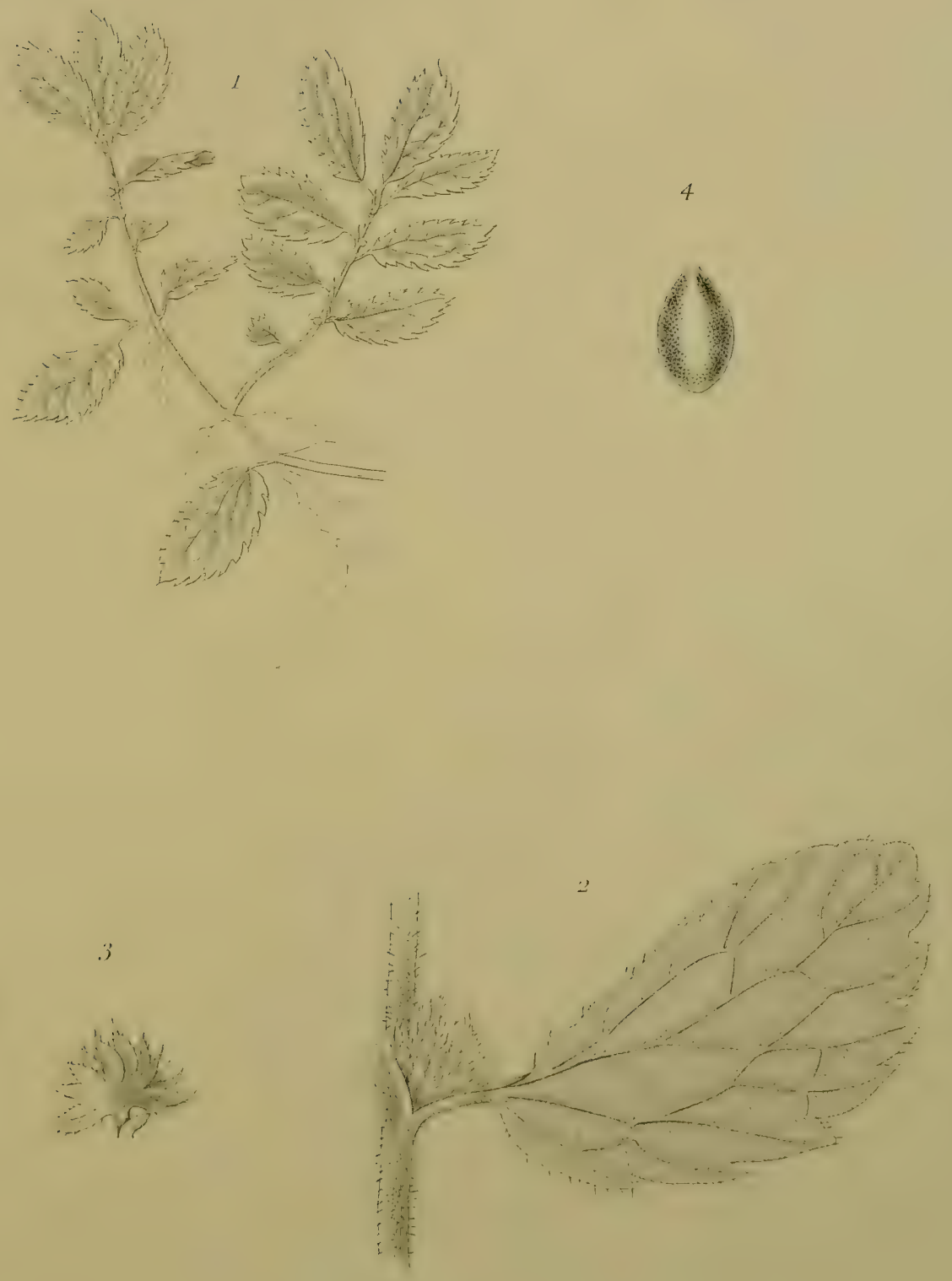




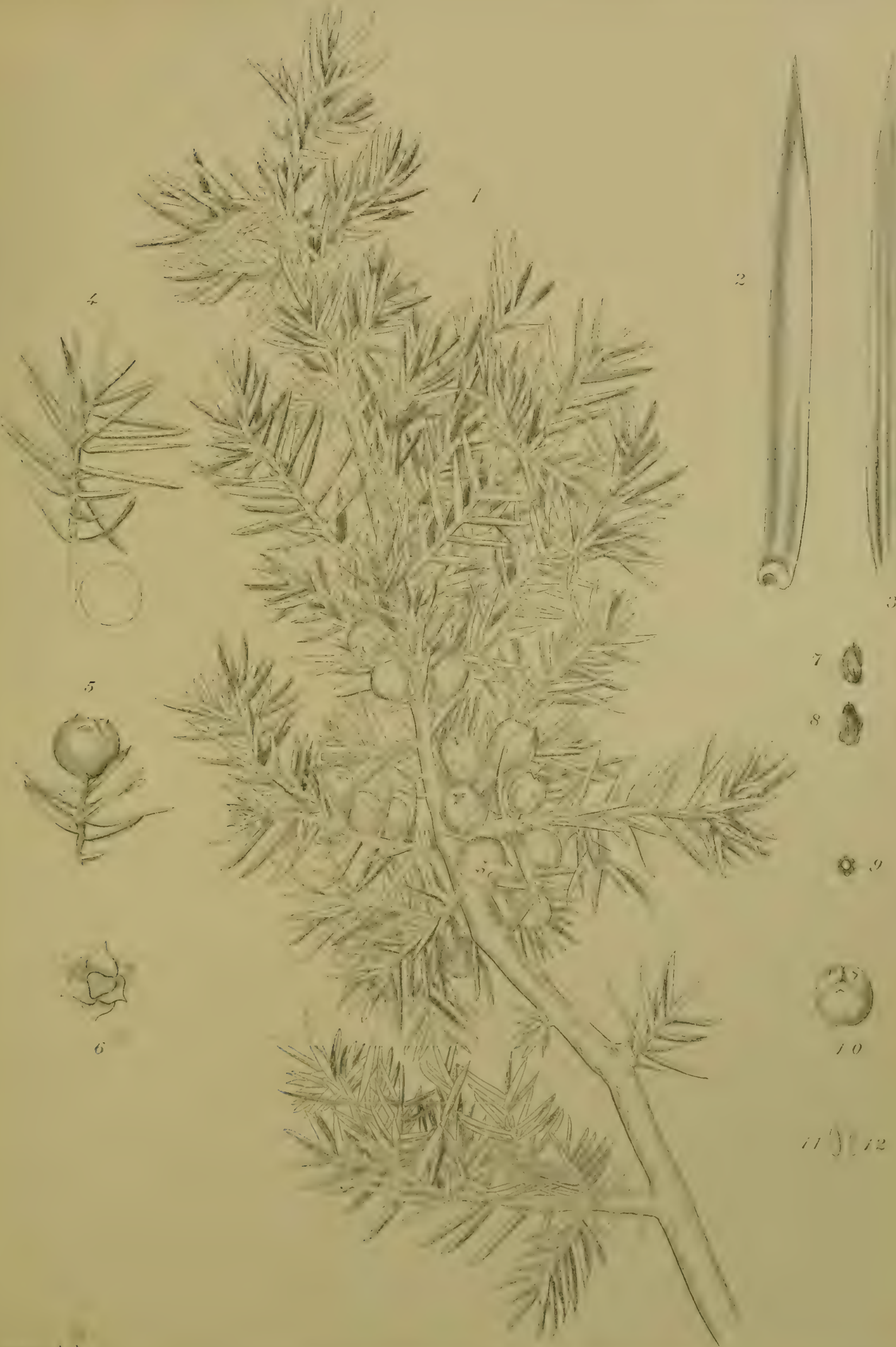



Jour. Sci. Coll., Vol. XXV., Art. XIX.,PI.XXXIX.

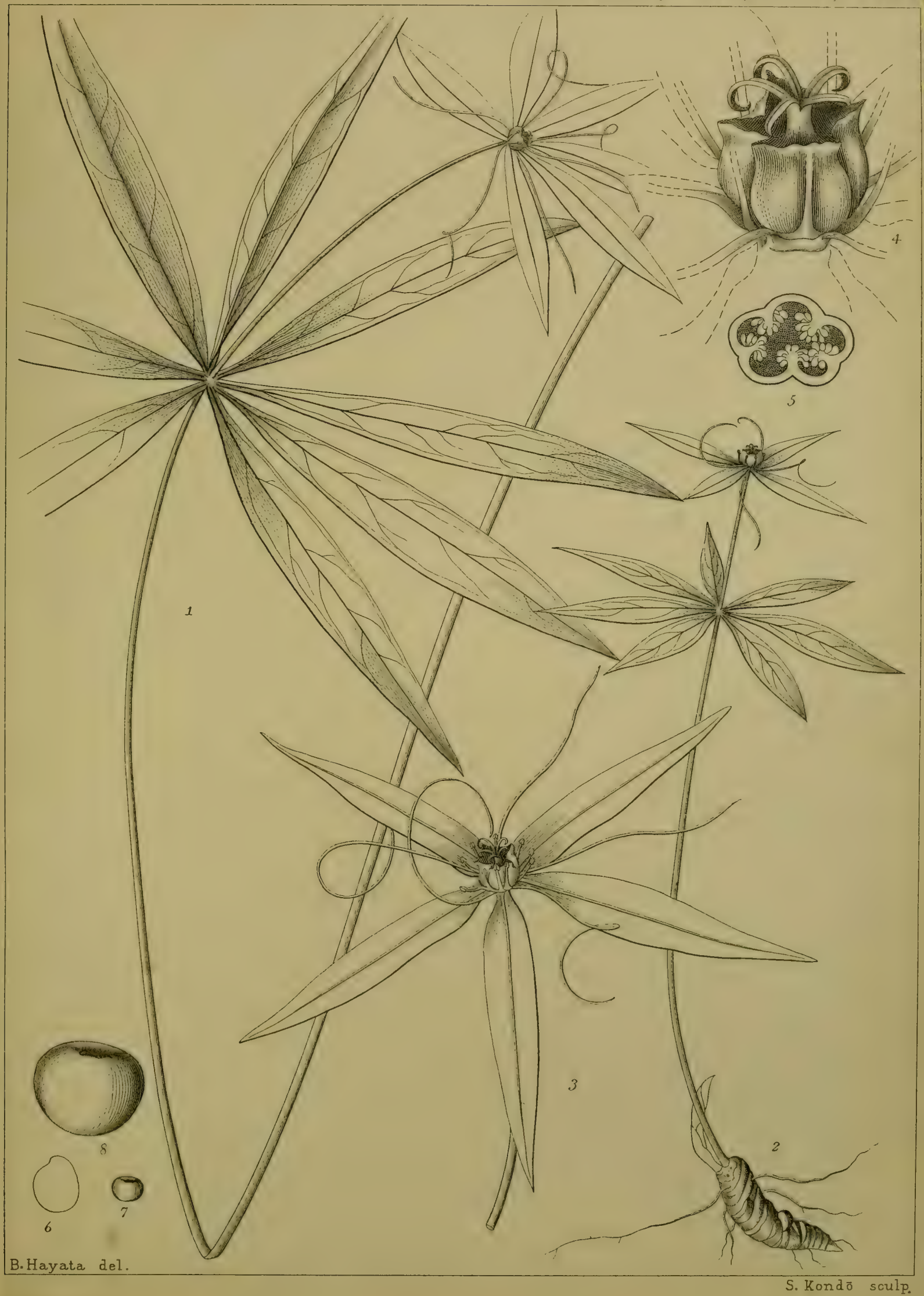



Jour. Sci. Coll., Vol. XXV., Art. XIX., PI:XL.

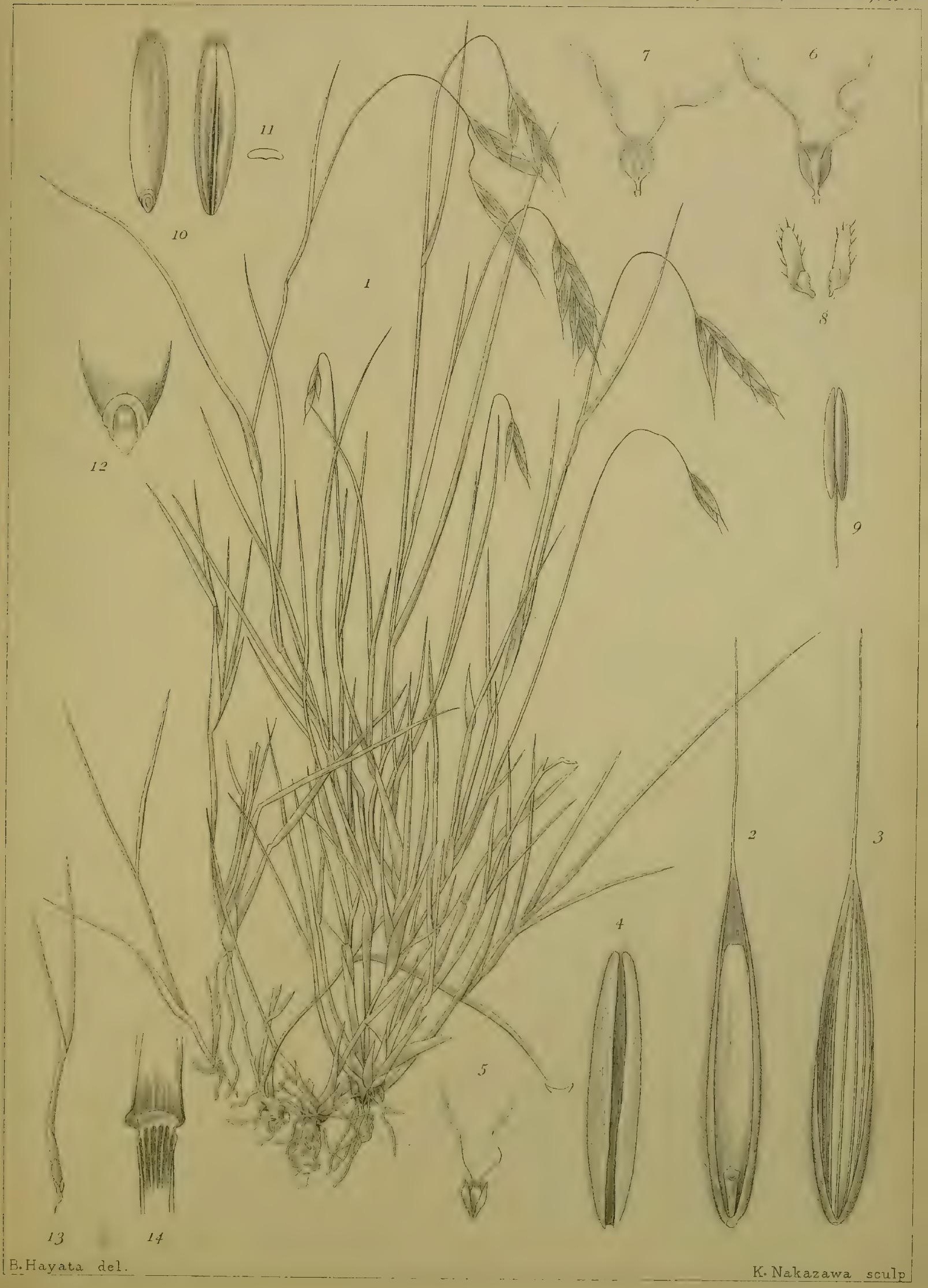





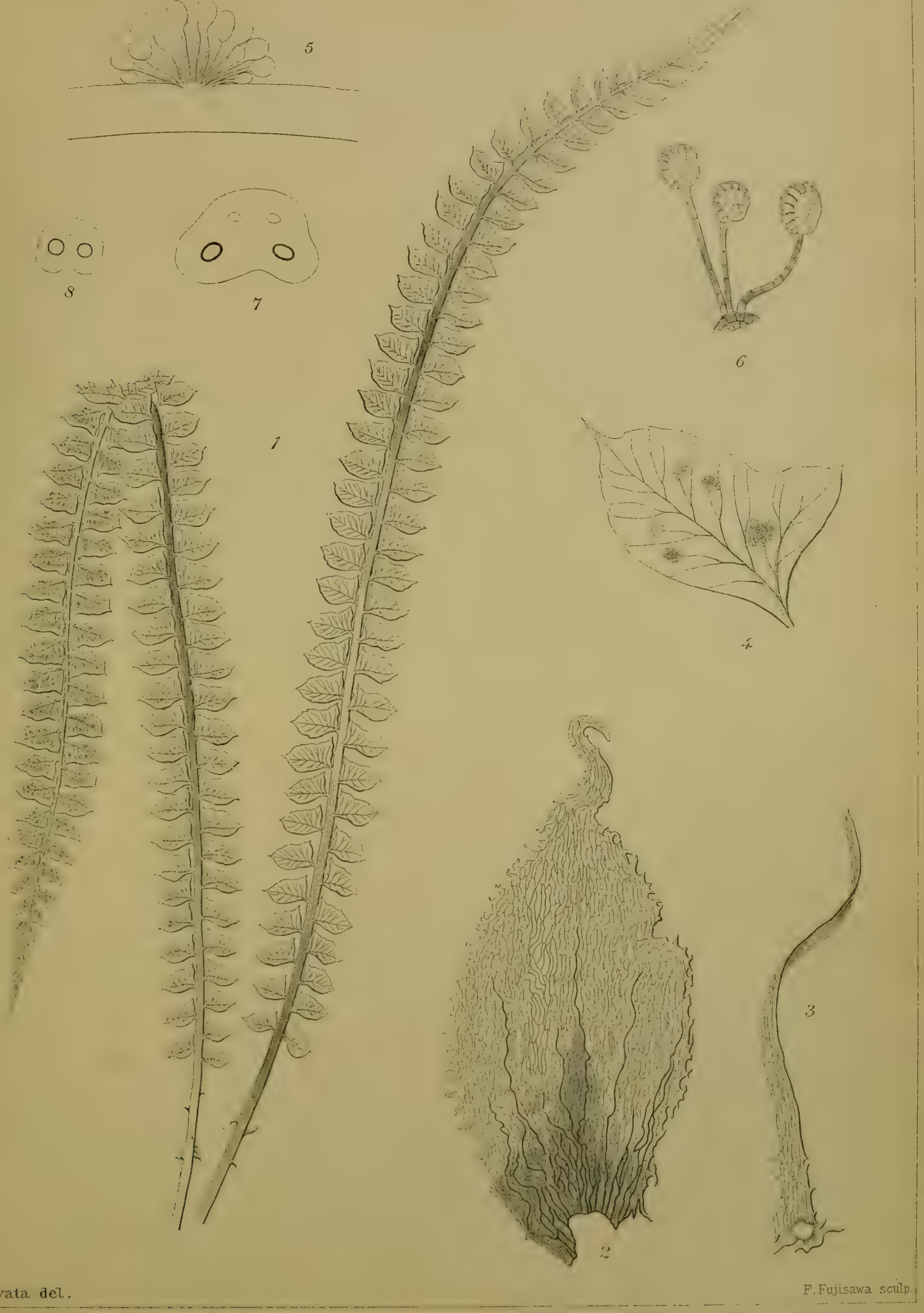



.

175 


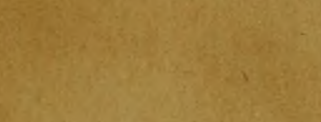


(3)

-

4.

13

(n)

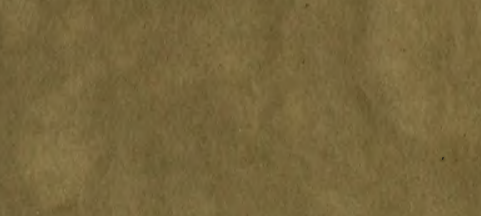

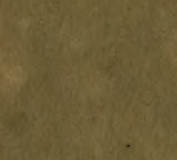

(x)

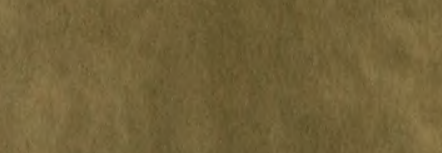

4.

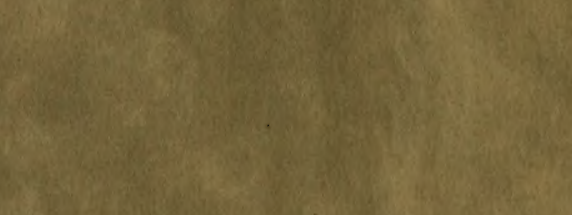

4. 7

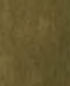

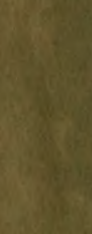

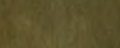

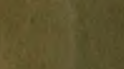

- +2 (2.

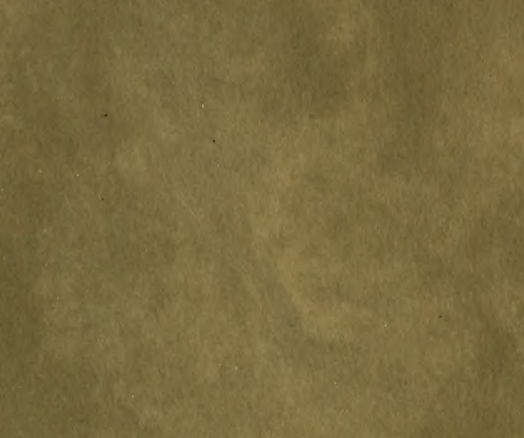

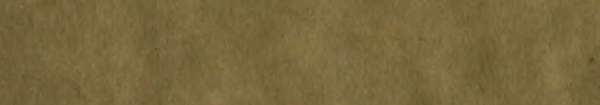
4

6858

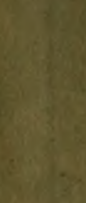

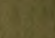

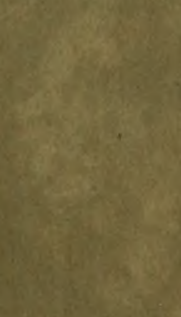

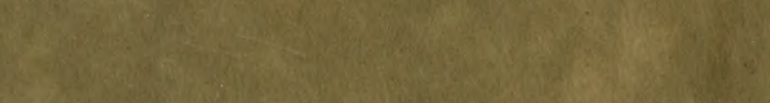
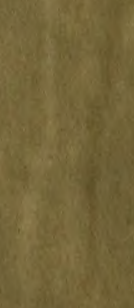

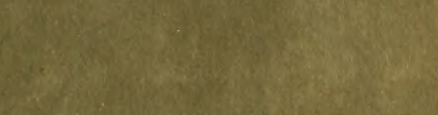




\section{OK 367.9. H295
Hayata, Bunzo/Flor}

ayata, Bunzo/Flora montana formosae; an

||||||||||||||||||||||||||||||||||||||||||||||||||||||||||||

5185000604072 
